\title{
Results from the TARC experiment: spallation neutron phenomenology in lead and neutron-driven nuclear transmutation by adiabatic resonance crossing
}

\author{
The TARC Collaboration
}

\begin{abstract}
We summarize here the results of the TARC experiment whose main purpose is to demonstrate the possibility of using Adiabatic Resonance Crossing (ARC) to destroy efficiently Long-Lived Fission Fragments (LLFFs) in accelerator-driven systems and to validate a new simulation developed in the framework of the Energy Amplifier programme. An experimental set-up was installed in a CERN PS proton beam line to study how neutrons produced by spallation at relatively high energy $\left(E_{\mathrm{n}} \geq 1 \mathrm{MeV}\right)$ slow down quasi adiabatically with almost flat isolethargic energy distribution and reach the capture resonance energy of an element to be transmuted where they will have a high probability of being captured. Precision measurements of energy and space distributions of spallation neutrons (using $2.5 \mathrm{GeV} / c$ and $3.5 \mathrm{GeV} / c$ protons) slowing down in a $3.3 \mathrm{~m} \times 3.3 \mathrm{~m} \times 3 \mathrm{~m}$ lead volume and of neutron capture rates on LLFFs ${ }^{99} \mathrm{Tc},{ }^{129} \mathrm{I}$, and several other elements were performed. An appropriate formalism and appropriate computational tools necessary for the analysis and understanding of the data were developed and validated in detail. Our direct experimental observation of ARC demonstrates the possibility to destroy, in a parasitic mode, outside the Energy Amplifier core, large amounts of ${ }^{99} \mathrm{Tc}$ or ${ }^{129} \mathrm{I}$ at a rate exceeding the production rate, thereby making it practical to reduce correspondingly the existing stockpile of LLFFs. In addition, TARC opens up new possibilities for radioactive isotope production as an alternative to nuclear reactors, in particular for medical applications, as well as new possibilities for neutron research and industrial applications.
\end{abstract}




\section{The TARC Collaboration}

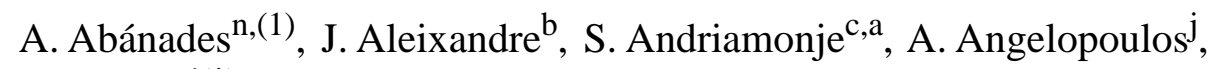
A. Apostolakis ${ }^{j(\ddagger)}$, H. Arnould ${ }^{\mathrm{a}}$, E. Belle ${ }^{\mathrm{g}}$, C.A. Bompas ${ }^{\mathrm{a}}$, D. Brozzi ${ }^{\mathrm{c}}, \mathrm{J}$. Bueno ${ }^{\mathrm{b}}$, S. Buono ${ }^{\text {c,h,(2) }}$, F. Carminati ${ }^{\text {c }}$, F. Casagrande ${ }^{\text {ce,e,(3) }}$, P. Cenninic , J.I. Collar $^{\text {c,(4), }}$, E. Cerro ${ }^{b}$, R. Del Moral ${ }^{\mathrm{a}}, \mathrm{S} . \mathrm{Díez}^{\mathrm{m}},(1)$, L. Dumps $^{\mathrm{c}}$, C. Eleftheriadis ${ }^{1}$, M. Embid ${ }^{\mathrm{i}, \mathrm{d}}$, R. Fernández ${ }^{\text {c,d }}$, J. Gálvezi , J. García ${ }^{\text {n,d }}$, C. Gelès ${ }^{\text {c }}$, A. Giorni ${ }^{\mathrm{g}}$, E. González $^{\mathrm{d}}$,

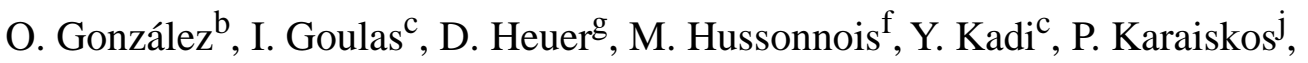
G. Kitis ${ }^{1}$, R. Klapisch ${ }^{\text {c,(5) }}$, P. Kokkas ${ }^{\mathrm{k}}$, V. Lacoste ${ }^{\mathrm{a}}$, C. Le Naour ${ }^{\mathrm{f}}$, C. López ${ }^{\mathrm{i}}$, J.M. Loiseaux ${ }^{\mathrm{g}}$, J.M. Martínez-Val ${ }^{\mathrm{n}}$, O. Méplan ${ }^{\mathrm{g}}$, H. Nifenecker $^{\mathrm{g}}$, J. Oropesa ${ }^{\mathrm{c}}$, I. Papadopoulos ${ }^{1}$, P. Pavlopoulos ${ }^{\mathrm{k}}$, E. Pérez-Enciso ${ }^{\mathrm{i}}$, A. Pérez-Navarro ${ }^{\mathrm{m},(1)}$,

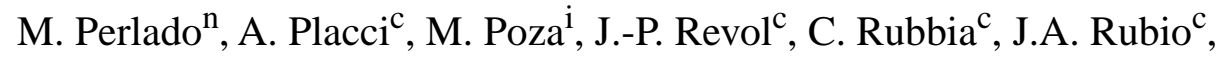
L. Sakelliou ${ }^{j}$, F. Saldaña ${ }^{c}$, E. Savvidis ${ }^{1}$, F. Schussler ${ }^{\mathrm{g}}$, C. Sirvent ${ }^{\mathrm{i}}$, J. Tamarit ${ }^{\mathrm{b}}$,

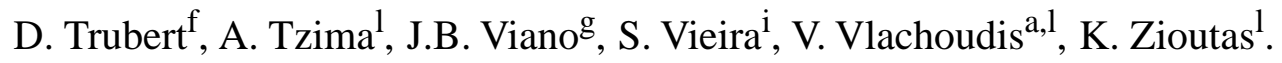

(a) CEN, Bordeaux-Gradignan, France; (b) CEDEX, Madrid, Spain; (c) CERN, Geneva, Switzerland; (d) CIEMAT, Madrid, Spain; (e) INFN, Laboratori Nazionali di Frascati, Italy; $(f)$ IPN, Orsay, France; (g) ISN, Grenoble, France; (h) Sincrotrone Trieste, Trieste, Italy; (i) Universidad Autónoma de Madrid, Madrid, Spain; (j) University of Athens, Athens, Greece; $(k)$ University of Basel, Basel Switzerland; (l) University of Thessaloniki, Thessaloniki, Greece; (m) Universidad Alfonso X el Sabio, Madrid, Spain; (n) Universidad Politécnica de Madrid, Madrid, Spain.

\footnotetext{
(1) Present address LAESA, Zaragoza, Spain

(2) Present address CRS4, Cagliari, Italy

(3) Present address MIT, Cambridge, USA

(4) Present address Université Paris VI, Paris, France

(5) Also at CSNSM, IN2P3, Orsay, France

(†) Deceased
} 


\section{Contents}

1 Introduction 1

2 Experimental Set-up 3

$\begin{array}{ll}\text { 2.1 Lead assembly } & 3\end{array}$

2.1.1 Mechanics 3

2.1.2 Lead purity 4

$\begin{array}{ll}\text { 2.2 Beam line } & 7\end{array}$

2.2.1 Experimental area $\quad 7$

2.2.2 Fast extraction beam 8

2.2.3 Slow extraction beam 11

2.3 Data AcQuisition (DAQ) 12

2.3.1 Hardware scheme of the DAQ 13

3 Development of Simulation Tools for TARC 14

$\begin{array}{ll}3.1 \text { Introduction } & 14\end{array}$

$\begin{array}{ll}\text { 3.2 Physics modelling } & 14\end{array}$

3.3 Neutron cross-sections $\quad 15$

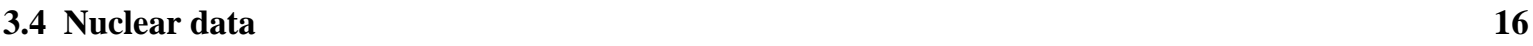

$\begin{array}{ll}\text { 3.5 TARC general Monte Carlo code } & 17\end{array}$

3.5.1 Systematic errors and technical checks on the Monte Carlo flux calculation 18

3.5.2 TARC geometry description 23

4 Study of the Energy-Time Correlation with $\mathrm{CeF}_{3}$ Counters 24

4.1 Introduction $\quad 24$

4.2 Analysis of the simulated correlation function 25

4.3 Experimental parameters of the correlation function $\quad 28$

$\begin{array}{lll}4.4 & \text { Conclusion } & 31\end{array}$

5 Neutron Fluence Measurements $\quad 31$

5.1 Neutron fluence and neutron flux $\quad 31$

5.1.1 Definition of neutron fluence 31

5.1.2 Properties of lead 32

5.2 Low-energy neutrons $\left(E_{\mathbf{n}} \leq 50 \mathrm{keV}\right)$ using energy-time correlation 33

5.2.1 ${ }^{6} \mathrm{Li}^{233} \mathrm{U}$ target silicon detectors 33

5.2.2 ${ }^{3}$ He scintillation counter $\quad 42$

5.2.3 Triple-foil activation 51

5.2.4 Thermoluminescence detectors 51 
5.3 High-energy neutrons ( $10 \mathrm{keV}$ to $2 \mathrm{MeV})$ with ${ }^{3} \mathrm{He}$ ionization counters 52

5.3.1 Introduction 52

5.3.2 Description of the counter 53

5.3.3 The signals from the counter 55

5.3.4 Calibration of the counter with monochromatic neutrons and simulation of the detector response to neutrons $\quad 57$

5.3.5 Detector resolution 58

5.3.6 TARC experimental data set 58

5.3.7 Monte Carlo simulation of the experiment 58

5.3.8 Fluence determination 58

5.3.9 Conclusion 62

$\begin{array}{lr}5.4 \text { High-energy neutrons with activation methods } & 63\end{array}$

5.4.1 Fissions in ${ }^{232} \mathrm{Th}$, and ${ }^{237} \mathrm{~Np}$

5.5 General conclusion on neutron fluence measurements $\quad 65$

6 Neutron Capture Cross-Section Measurements as a Function of Energy 68

$\begin{array}{ll}\text { 6.1 Detecting neutron capture with } \mathrm{AeF}_{3} \text { detector } & 68\end{array}$

$\begin{array}{lr}6.2 & \text { Transmutation rate } \\ & 69\end{array}$

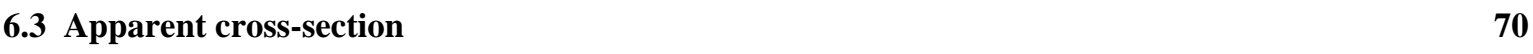

6.3.1 Absolute values 71

6.3.2 Error analysis 71

6.4 Results $\quad \mathbf{7 2}$

6.5 Evaluation of the ${ }^{99} \mathrm{Tc}$ cross-section $\quad 74$

6.6 Results for other targets $\quad 75$

$\begin{array}{ll}\text { 6.7 Conclusion } & 75\end{array}$

7 Test of Transmutation of Long-Lived Fission Fragments 75

$7.1{ }^{99}$ Tc rabbit integral transmutation rate measurements $\quad 75$

7.1.1 Motivation 75

$\begin{array}{ll}\text { 7.1.2 Experimental set-up } & 76\end{array}$

7.1.3 Germanium detectors 80

7.1.4 HPGe detector spectra analysis 81

7.1.5 Energy calibration and efficiency of the HPGe detectors 82

7.1.6 Analysis of experimental data 82

7.1.7 Monte Carlo simulation and comparison with the data 93

7.1.8 Systematic studies and other special measurements 94

$7.2{ }^{129}$ I and ${ }^{127}$ I integral transmutation rate measurements $\quad 97$

7.2.1 Iodine capture and decay schemes 98

7.2.2 Data analysis 99

7.2.3 Experimental results 106 
7.2.4 Comparison with the Monte Carlo simulation 107

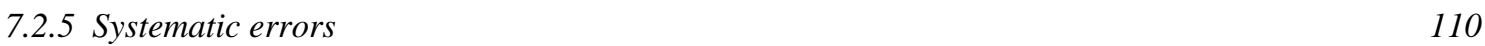

$\begin{array}{ll}\text { 7.2.6 Conclusion } & 111\end{array}$

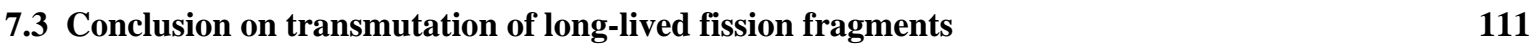

8 Other Integral Measurements $\quad 113$

8.1 Production rates of ${ }^{233} \mathrm{U}$ and ${ }^{239} \mathrm{Pu} \quad 113$

8.1.1 Production rate of ${ }^{233} U \quad 113$

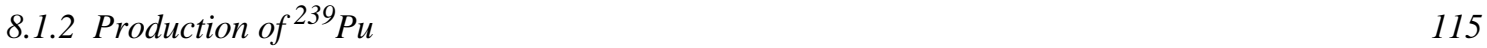

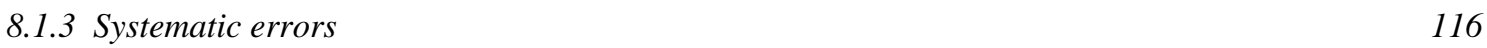

8.1.4 Protactinium $\left({ }^{233} \mathrm{~Pa}\right)$ half-life 116

8.2 The ${ }^{232}$ Th $(n, 2 n)^{231}$ Th reaction rate 117

$\begin{array}{ll}\text { 8.2.1 Motivation } & 117\end{array}$

$\begin{array}{ll}\text { 8.2.2 Photon spectra } & 118\end{array}$

9 Medical Applications of ARC $\quad 120$

$\begin{array}{lr}9.1 \text { General strategy for medical applications of ARC } & 120\end{array}$

\begin{tabular}{ll}
9.1 .1 & Introduction \\
\hline
\end{tabular}

9.1.2 Selected examples of procedures for an activator $\quad 121$

9.1.3 Characterization of the spallation neutron source 124

$\begin{array}{ll}\text { 9.1.4 Performance of a typical activator } & 124\end{array}$

9.2 Measurement of ${ }^{99}{ }^{9}$ Tc production rate from natural molybdenum 125

9.2.1 Sample configuration and preparation $\quad 125$

9.2.2 Molybdenum capture and decay schemes 126

9.2.3 General summary of the irradiation data 130

9.2.4 Data analysis 130

9.2.5 Monte Carlo simulation 134

9.2.6 Various checks on the data 137

$\begin{array}{ll}\text { 9.2.7 Conclusion } & 137\end{array}$

10 Energy Deposition in Lead by Neutrons 138

$\begin{array}{lr}10.1 \text { Introduction } & 138\end{array}$

$\begin{array}{lr}10.2 & 139\end{array}$

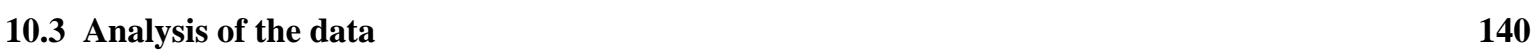

10.3.1 Analysis of the data in quasi-adiabatic conditions 140

10.3.2 Analysis of the data in non-adiabatic conditions 142

10.4 Experimental results $\quad 142$

10.4.1 Adiabatic measurements 143

10.4.2 Contact or non-adiabatic measurements 145 
10.5 Monte Carlo simulations

10.6 Conclusion

11 Practical scheme for an Incineration Device Based on ARC

12 General conclusion of the TARC study

Acknowledgements .

References 153 


\section{Introduction}

Transmutation by Adiabatic Resonance Crossing (TARC) is part of a broader experimental programme designed to test directly some of the basic physics concepts applicable to the field of radioactive waste elimination. A first experiment (FEAT) [1] was performed to test the concept of energy gain in the Energy Amplifier (EA) system proposed by C. Rubbia [2], a system designed to destroy all actinide elements by fission. As a consequence, the EA is also producing energy while it is destroying the TRansUranium (TRU) content of nuclear reactor waste. The EA is a fast-neutron subcritical system driven by a proton accelerator, using natural thorium as fuel and lead as a neutron spallation target, a neutron moderator, a heat extraction agent and a neutron confinement medium. In its present version, the EA is optimized for the destruction of TRU [3].

In a system such as the EA, where TRUs are destroyed by fission, long-term ( $\geq 500$ years $\left.{ }^{1}\right)$ radiotoxicity of the waste is dominated by long-lived fission fragments (LLFFs) which can, in practice, only be destroyed by nuclear decay following neutron capture.

The main goal of TARC is to test a new idea put forward by one of us [4] and relying on the properties of spallation neutrons diffusing in lead, the use of Adiabatic Resonance Crossing for transmutation, to destroy efficiently LLFFs. More generally, TARC is a systematic study of the phenomenology of spallation neutrons in pure lead.

Furthermore, in a system which transforms LLFFs into short-lived or stable elements, it is also possible to transform stable elements into radioactive elements. Therefore, TARC has important applications to several other fields.

For instance, in medicine, radioactive elements are increasingly used for diagnostics, therapy, and pain relief. These elements can be produced through neutron capture on stable elements in an accelerator-driven activator as an alternative to nuclear reactor production [4], using the 'inverse' process invoked for the destruction of LLFFs.

In all cases, it is very important to optimize the efficiency of the neutron capture process. The specific neutron capture rate, $R_{\text {capt }}$, can be enhanced by maximizing each of the relevant factors in $R_{\text {capt }} \propto \int \phi(E) \sigma(E) \mathrm{d} E(E$ is the neutron energy), i.e.

(a) the neutron flux $[\phi(E)]$ (Fig. 1) by the choice of a dense neutron 'storage' medium (lead) with high atomic mass, high neutron elastic cross-section (mean free path: $\lambda_{\mathrm{el}} \sim 3 \mathrm{~cm}$ ) and high neutron transparency (the doubly magic nature of the dominant isotope ${ }^{2}{ }^{208} \mathrm{~Pb}$ nucleus, makes natural lead one of the most transparent elements below $1 \mathrm{keV})$;

(b) the capture cross-section $[\sigma(E)]$ (Fig. 1) of the element to be transmuted by an efficient use of resonances made possible by the very small lethargic steps of neutrons slowing down in lead. Indeed, neutrons have an interesting behaviour in lead:

(1) a small average lethargy $\xi$ due to the high atomic mass of lead:

$$
\xi \equiv 1+\frac{\alpha}{1-\alpha} \ln (\alpha) \approx 9.6 \times 10^{-3} \quad \text { where } \alpha \equiv \frac{\left(m_{\mathrm{Pb}}-m_{\mathrm{n}}\right)^{2}}{\left(m_{\mathrm{Pb}}+m_{\mathrm{n}}\right)^{2}} \approx 0.98 ;
$$

\footnotetext{
1. It takes 500 years for the radiotoxicity of the waste to reach the level of the radiotoxicity of coal ashes corresponding to the production of the same amount of energy.

2. Natural lead contains $1.4 \%{ }^{204} \mathrm{~Pb}, 24.1 \%{ }^{206} \mathrm{~Pb}, 22.1 \%{ }^{207} \mathrm{~Pb}$, and $52.4 \%{ }^{208} \mathrm{~Pb}$.
} 
(2) a high and nearly energy-independent elastic scattering cross-section;

(3) a long 'storage' time because, below the capture resonances $\left(E_{\mathrm{n}} \leq 1 \mathrm{keV}\right)$ and down to epithermal energies, the elastic scattering process is nearly isotropic and the transparency to neutrons is very high (it takes about $3 \mathrm{~ms}, 1800$ scatterings and a path in lead of $60 \mathrm{~m}$ to thermalize a $1 \mathrm{MeV}$ neutron).

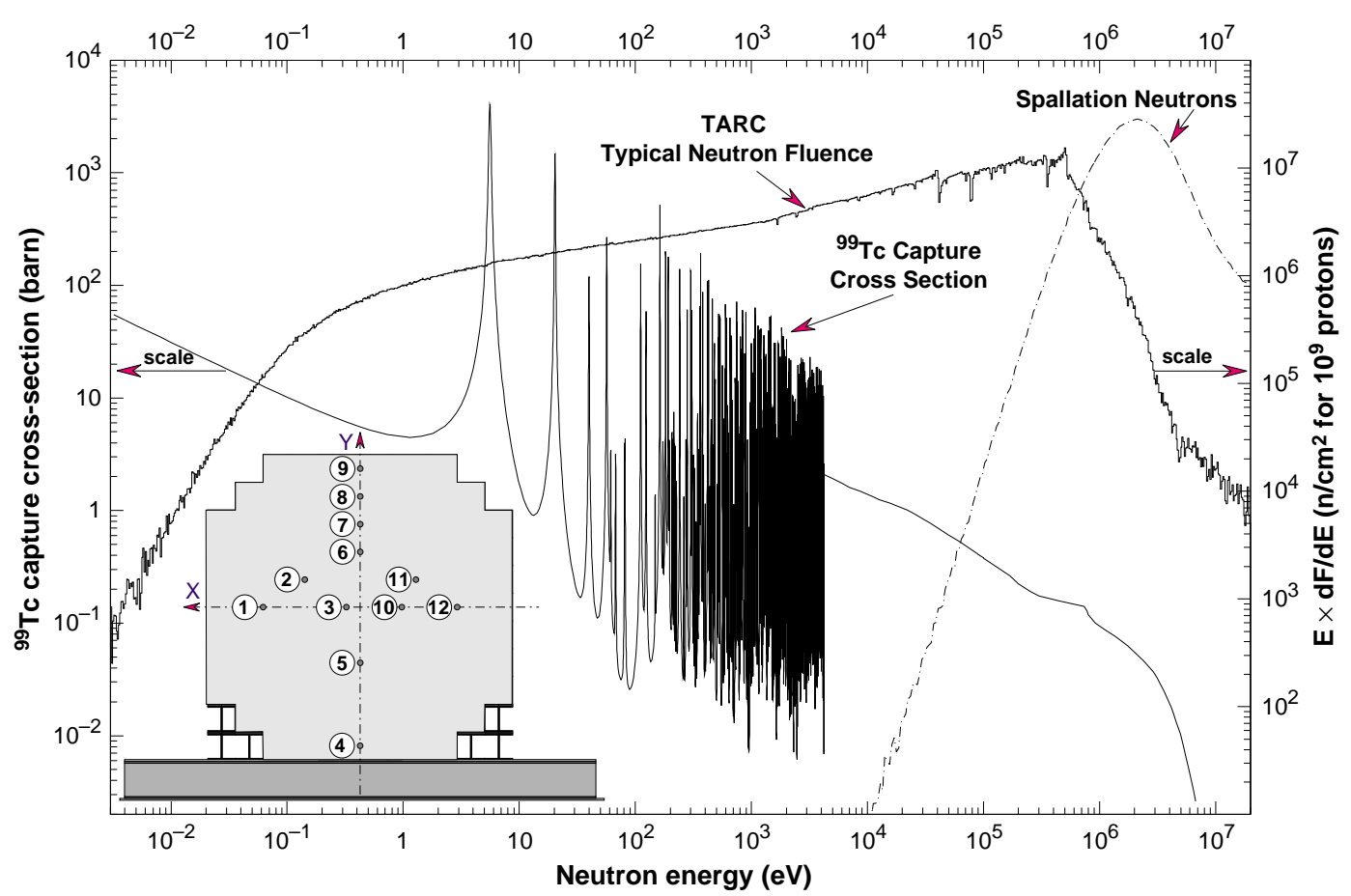

Fig. 1: ${ }^{99}$ Tc neutron capture cross-section (JENDL-3.2 database [5]), as a function of neutron energy (left-hand scale); typical neutron fluence energy distribution in TARC (hole $10, z=+7.5 \mathrm{~cm}$ ), as a function of neutron energy in isolethargic bins, for $3.5 \mathrm{GeV} / c$ protons (right-hand scale). Energy distribution of neutrons from the spallation process shown in arbitrary units.

As a result, neutrons produced by spallation at relatively high energy $\left(E_{\mathrm{n}} \approx\right.$ few $\left.\mathrm{MeV}\right)$, after having been quickly moderated by $(n, x n),\left(n, n^{\prime}\right)$ reactions down to energies of a few hundred $\mathrm{keV}$, will slow down quasiadiabatically with small isolethargic steps and reach the capture resonance energy of an element to be transmuted where it will have a high probability of being captured. The resonance width is usually larger than the average lethargic step. This is the case of ${ }^{99} \mathrm{Tc}$, which has a strong neutron capture resonance at $5.6 \mathrm{eV}$ (4000 b) (Fig. 1), covering four average lethargy steps. The ${ }^{99} \mathrm{Tc}$ resonance integral is $310 \mathrm{~b}$ while the cross-section at thermal/epithermal neutron energies $\left(E_{\mathrm{n}} \leq 1 \mathrm{eV}\right)$ is only of the order of $20 \mathrm{~b}$. Neutron capture on ${ }^{99} \mathrm{Tc}\left(t_{1 / 2}=2.11 \times 10^{5} \mathrm{yr}\right)$ produces ${ }^{100} \mathrm{Tc}\left(t_{1 / 2}=15.8 \mathrm{~s}\right)$ which then decays to ${ }^{100} \mathrm{Ru}$, a stable element. Thus, the radiotoxicity can be eliminated in a single neutron capture and, since ${ }^{100} \mathrm{Ru}$ has a small neutron capture cross-section and both ${ }^{101} \mathrm{Ru}$ and ${ }^{102} \mathrm{Ru}$ are stable, essentially no new radioactive elements are produced. ARC should be most efficient for elements with strong capture resonances, such as ${ }^{99} \mathrm{Tc}$ and ${ }^{129} \mathrm{I}$ (which together represent $95 \%$ of the total LLFF radiotoxicity inventory).

The above general considerations resulted in the new approach put forward in Ref. [4] consisting in making use of the ARC concept. TARC was specifically proposed as an experimental study of the corresponding phenomenology of spallation neutrons in a large lead volume. Experiment PS211 [6], known as TARC, was set up at the CERN PS. Even though the 
main goal of TARC was to demonstrate the efficiency of ARC in the transmutation of LLFFs, the experiment was designed in such a way that several basic processes involved in Accelerator-Driven Systems (ADS) could be studied in detail:

(a) neutron production by $\mathrm{GeV}$ protons hitting a large lead volume;

(b) neutron transport properties, on the distance scale relevant to industrial applications (reactor size);

(c) efficiency of transmutation of LLFFs in the neutron flux produced by spallation neutrons.

All of this programme was achieved by performing mainly two general types of measurements:

(a) neutron fluence measurements with several complementary techniques, providing redundancy, using detectors built by the TARC Collaboration and operated over a broad neutron energy range, from thermal up to a few $\mathrm{MeV}$;

(b) neutron capture rate measurements on ${ }^{99} \mathrm{Tc}$ (both differential and integral measurements) and on ${ }^{129} \mathrm{I}$ and ${ }^{127} \mathrm{I}$ (integral measurements). For ${ }^{99} \mathrm{Tc}$, in addition, a high statistics measurement of the ${ }^{99} \mathrm{Tc}$ apparent neutron capture cross-section was obtained, up to $\sim 1 \mathrm{keV}$, an energy below which $85 \%$ of all captures occur in a typical TARC neutron spectrum. Many other capture or fission measurements relevant to the design parameters of the EA or to various other applications were also performed.

Every effort was made to check carefully the systematics of the various measurements to provide the best possible experimental precision. This implied great care in the construction of the lead assembly, the development of detectors using state-of-the-art techniques, and, throughout the duration of the experiment, a systematic use of redundant techniques to allow internal cross-checks of the results.

Some of the first results of TARC were published in Ref. [7]. An extensive description of the experiment can be found in Ref. [8].

\section{Experimental Set-up}

\subsection{Lead assembly}

\subsubsection{Mechanics}

The 334 ton lead assembly, with approximate cylindrical symmetry about the beam axis, was the result of an optimization between sufficient neutron containment, acceptable background conditions, and affordable cost. A good compromise was found with an approximately cylindrical volume of diameter $3.3 \mathrm{~m}$ and length $3 \mathrm{~m}$ (Fig. 2), the axis being aligned with the beam line. With such a large lead volume $\left(29.3 \mathrm{~m}^{3}\right)$, about $70 \%$ of neutrons produced by spallation from proton interactions near the centre are contained within the volume, and there is a large central region, with a radius $\sim 1 \mathrm{~m}$, where the neutronics is little affected by background neutrons produced by reflection from the environment (background $\leq 2 \%$ ). In order to minimize neutron reflection from the environment, we 'pushed' the concrete shielding of the experimental area as far away as possible. For the floor, we chose to support the lead assembly with a steel I-beam platform (Fig. 3) $44 \mathrm{~cm}$ high, on top of which we introduced a $3 \mathrm{~cm}$ thick layer of special cement (Masterflow) containing $20 \%$ of $\mathrm{B}_{4} \mathrm{C}$. Because of the presence of ${ }^{10} \mathrm{~B}, \mathrm{~B}_{4} \mathrm{C}$ tends to protect the lead assembly from neutrons which have 
thermalized in the floor concrete, while it is relatively transparent to the outgoing flux of higher energy neutrons escaping the lead assembly [9]. The size of the platform was conditioned by the stability of the floor (maximum authorized load, $20 \mathrm{t} / \mathrm{m}^{2}$ ). The lead assembly was completed by mid-April 1996 and dismounted in August 1997. The lead assembly was constructed as a modular structure to allow handling and also to provide, if necessary, flexibility in the choice of configuration. There are three types of parallelepipedal blocks [type $A$ and $B$ with dimensions of $30 \times 30 \times 60 \mathrm{~cm}^{3}$ and type $C$ with dimensions $15 \times 30 \times 60 \mathrm{~cm}^{3}$ ]. Each block (weighing $613 \mathrm{~kg}$ for type A) could be positioned with high precision by using a suction device $^{3}$. In this way, handling could be done without deforming the lead blocks which are made of relatively soft lead (no hardening element), and also without introducing any extra structure or materials which could destroy the homogeneity of the lead assembly. To avoid costly machining, the holes necessary for the introduction of activation samples $\left({ }^{99} \mathrm{Tc},{ }^{129} \mathrm{I}\right.$, etc.) and for the introduction of detectors were obtained directly from the moulding process, by introducing a half-cylindrical volume at the bottom of the mould [10]. In practice, the construction and dismounting of the 580 block lead assembly could be done quickly (two weeks for dismounting) and we were able to obtain excellent alignment of the instrumental and beam holes. The beam is introduced through a $77.2 \mathrm{~mm}$ diameter blind hole, $1.2 \mathrm{~m}$ long, in such a way that the neutron shower is approximately centred in the middle of the $3 \mathrm{~m}$ long lead volume.

\subsubsection{Lead purity}

Pure lead (99.99\%) [11] was chosen to ensure that impurities have a negligible effect on the neutron flux. In practice, the experiment calls for high-purity lead devoid of such known impurities as silver, antimony, and cadmium. The $4 \mathrm{~N}$ quality lead offered by Britannia Refined Metals (UK) [12] was subjected to thorough chemical analyses as samples were sent to several independent laboratories world-wide. All the measurements were combined to obtain the best estimate of the impurity concentrations in our lead.

We obtained information on the concentration levels for 60 elements [11]: (1) measured values for 11 elements ( $\mathrm{Na}, \mathrm{Mg}, \mathrm{Al}, \mathrm{Cu}, \mathrm{Ag}, \mathrm{Cd}, \mathrm{Sb}, \mathrm{Te}, \mathrm{Tl}, \mathrm{Au}$ and $\mathrm{Bi}$ ) (Table 1) and (2) upper limits for 49 other elements (Fig. 4). The lead ingots had to be transformed into blocks in order to construct the assembly with the required geometry. A manufacturing process was devised with the goal of avoiding the introduction of impurities but also keeping the price acceptable. This was based on moulding followed by a quick machining pass to take away the surface crust and was done by Calder Industrial Materials (UK) [10] with help from the CERN workshop. We have checked the chemical composition of the blocks as compared to the initial chemical composition of the ingots and conclude that the manufacturing process did not significantly alter the lead purity. If impurities were introduced during moulding they presumably were near the surface of the blocks. The machining to bring the blocks to acceptable dimensions must have removed whatever was present.

3. The dimensions of the lead blocks were chosen by optimization between surface, volume, and availability of suction devices on the market. 


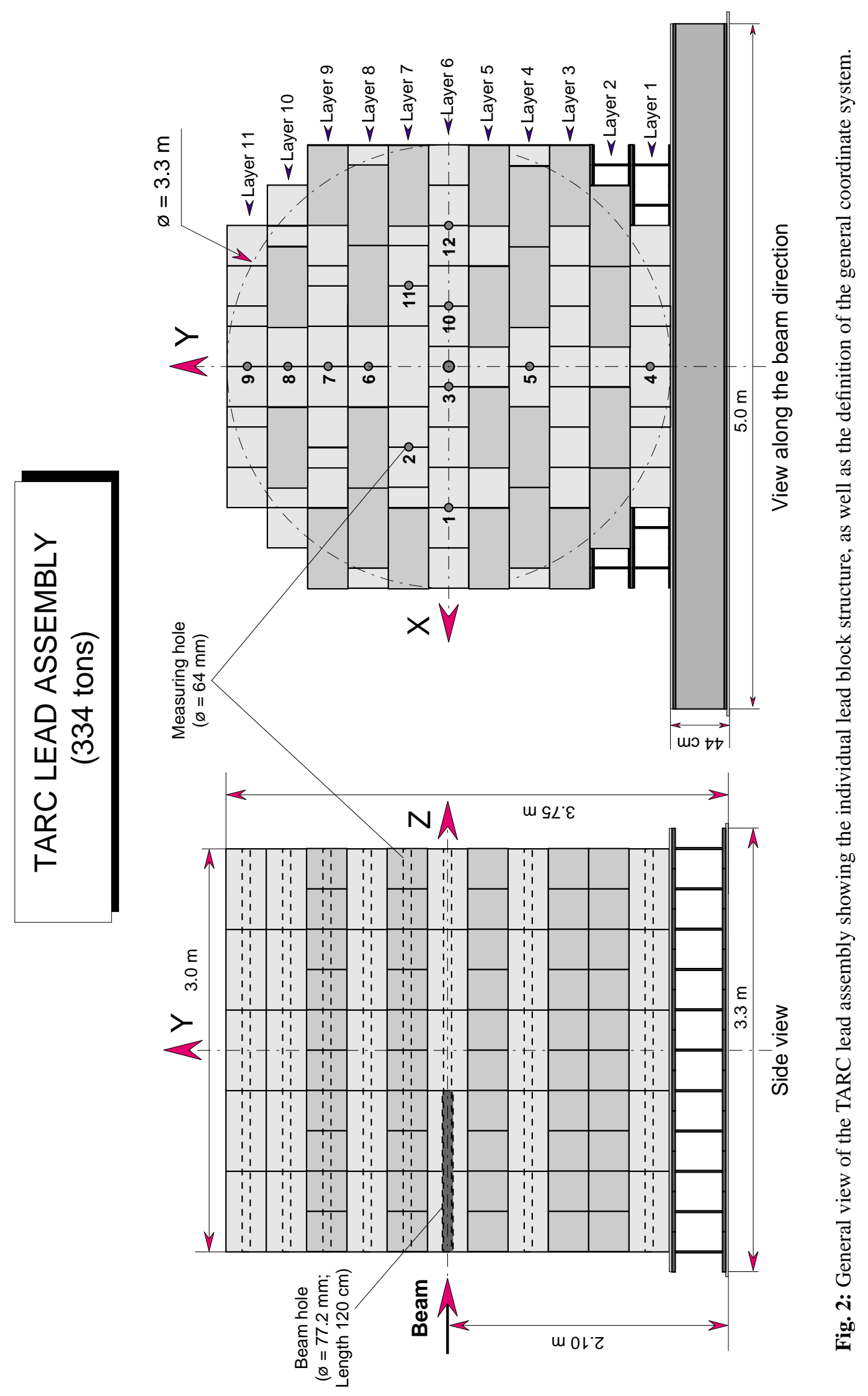




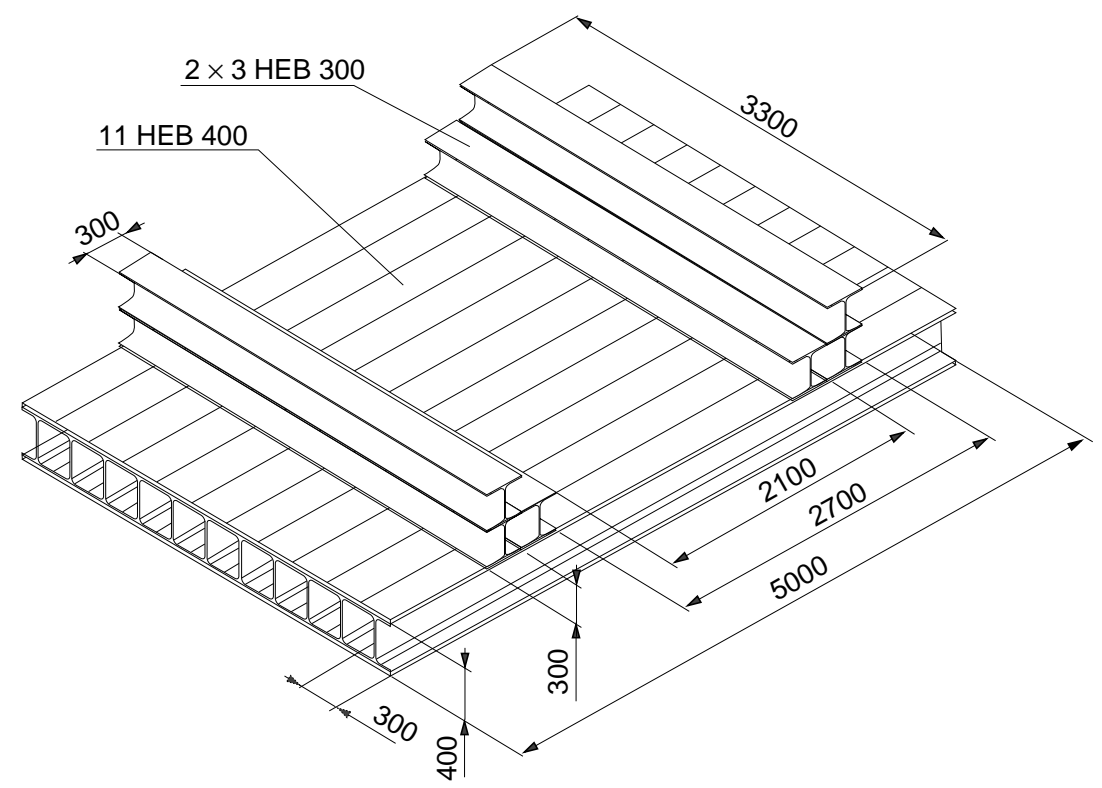

Fig. 3: Structure of the metallic platform supporting the TARC lead assembly (dimensions in mm). The various I-beams were welded together before a Masterflow layer was deposited.

Table 1: Summary of impurity concentrations for manufactured lead blocks. The systematic error is the error quoted by the laboratory which performed the measurement, the third column shows the spread between all measurements of a given sample. The total error is the quadratic sum of the two contributions.

\begin{tabular}{c|c|c|c|c}
\hline Element & $\begin{array}{c}\text { Concentration } \\
\text { (ppmw) }\end{array}$ & $\begin{array}{c}\text { Error spread } \\
\text { (ppmw) }\end{array}$ & $\begin{array}{c}\text { Syst. error } \\
\text { (ppmw) }\end{array}$ & $\begin{array}{c}\text { Total error } \\
\text { (ppmw) }\end{array}$ \\
\hline $\mathrm{Na}^{*}$ & 0.006 & 0.007 & 0.0001 & 0.0075 \\
$\mathrm{Mg}^{*}$ & 0.003 & 0.005 & 0.0006 & 0.005 \\
$\mathrm{Al}^{*}$ & 0.02 & 0.01 & 0.004 & 0.01 \\
$\mathrm{Cu}$ & 0.09 & 0.2 & 0.01 & 0.2 \\
$\mathrm{Ag}$ & 3.78 & 0.6 & 0.07 & 0.6 \\
$\mathrm{Cd}$ & 0.09 & 0.04 & 0.006 & 0.045 \\
$\mathrm{Te}$ & 0.215 & 0.09 & 0.025 & 0.09 \\
$\mathrm{Sb}$ & 0.14 & 0.14 & 0.006 & 0.14 \\
$\mathrm{Tl}$ & 4.6 & 1.3 & 0.3 & 1.4 \\
$\mathrm{Bi}$ & 19.0 & 0.8 & 0.3 & 2.8 \\
$\mathrm{Au}$ & 0.0008 & 0.002 & 0.0035 & 0.0004 \\
\hline
\end{tabular}

* Some upper limits were included in the determination. A simple average was used.

Among the main elements which could affect neutronics silver is a priori the most relevant in the framework of Adiabatic Resonance Crossing because of the presence of a strong resonance in the neutron capture cross-section at $5.2 \mathrm{eV}\left(\sigma=10^{4}\right.$ barn $)$. However, this capture resonance is $0.4 \mathrm{eV}$ below that of ${ }^{99} \mathrm{Tc}$. Therefore, we can afford, in principle, a moderate silver concentration. Nevertheless, because of the significant overlap between ${ }^{99} \mathrm{Tc}$ and silver resonances, an effort was made to have a low silver content: $3.8 \pm 0.6 \mathrm{ppm}$ (even so $1.4 \%$ of all captures are expected on silver). The largest contaminant present in our lead is bismuth $(19 \mathrm{ppm})$, which is obviously of no consequence, as its properties are very similar to those of lead. Thus we have demonstrated that $4 \mathrm{~N}$ quality commercial lead is adequate for ARC use and have identified a process whereby the required purity level can survive manufacturing of blocks. 


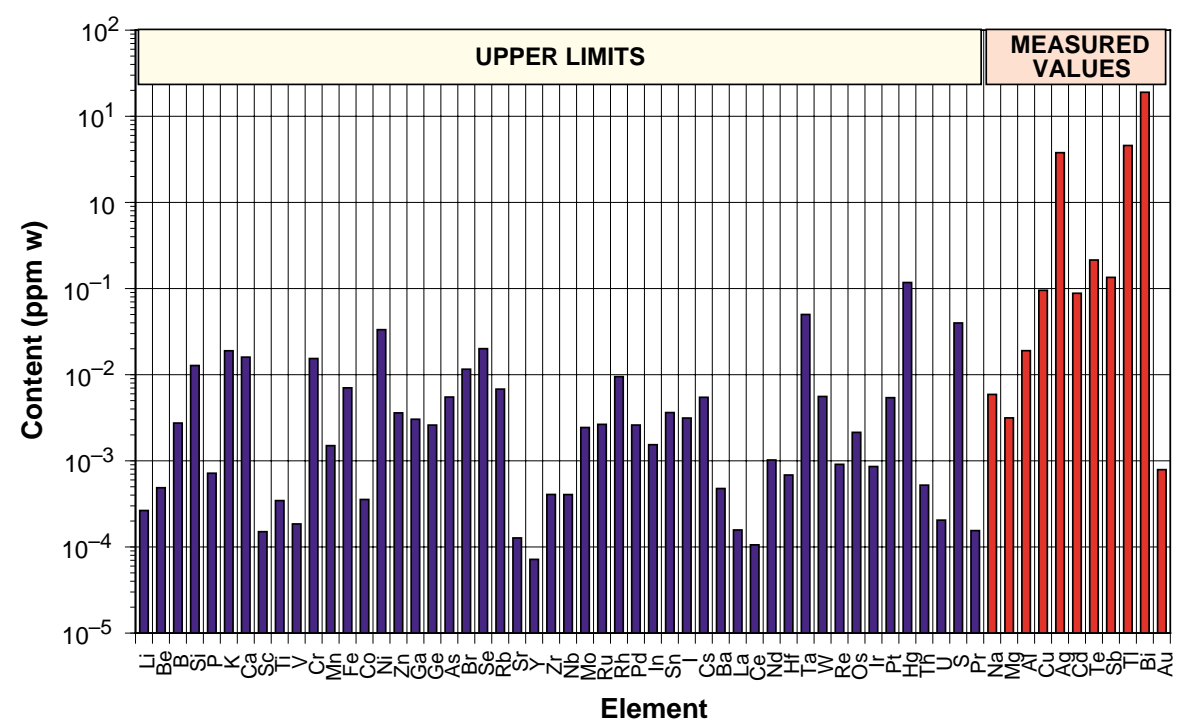

Fig. 4: Impurity content (ppm by weight) of the TARC lead blocks: (a) measured values for the 11 elements on the right-hand side and (b) upper limits for the others.

\subsection{Beam line}

\subsubsection{Experimental area}

The TARC experiment was installed in the T7 beam line at the CERN Proton Synchrotron (PS) East Area. The first run started in April 1996, and most of the data taking was completed by November 1996. A small additional run took place in May 1997. The beam was provided in two different modes: (a) the fast extraction mode used for activation experiments (high intensities, up to $10^{10}$ protons per PS shot) and for the measurements of neutron fluxes relying on the energy-time relation; (b) the slow extraction mode which was used for the operation of the ${ }^{3} \mathrm{He}$ ionization chambers, for which a very low beam intensity was needed (1000 protons per PS extraction). Hence the range of proton beam intensities in TARC covered seven orders of magnitude.

Most of the fast extraction data have been collected with a proton momentum of $3.5 \mathrm{GeV} / c$. The FEAT experiment [1] has shown that an optimum use of the proton kinetic energy in a EA is obtained for kinetic energies above about $900 \mathrm{MeV}$. There was no strong physics reason to choose a particular beam energy for TARC; $3.5 \mathrm{GeV} / c$ was selected mainly because it is a standard PS extracted beam momentum for which we could expect an excellent PS duty factor, therefore maximizing the volume of data we could collect. We did run the fast extraction system at a lower proton momentum $(2.5 \mathrm{GeV} / c)$ in May 1997 to provide additional checks of our data calibration (for proton energies larger than $1 \mathrm{GeV}$ the neutron yield is proportional to the proton kinetic energy), but also to allow lower neutron flux intensities, to explore earlier neutron times (hence higher neutron energies) for some of the neutron detectors $\left({ }^{6} \mathrm{Li} /{ }^{23} \mathrm{U}\right.$ and ${ }^{3} \mathrm{He}$ scintillation). The spallation neutron energy spectrum, dominated by the evaporation of the target nuclei, is essentially the same for proton energies in the range considered here. In the slow extraction mode, most of the data were taken at $2.5 \mathrm{GeV} / c$.

A main consideration for the design of the experimental area was the need to minimize neutron reflections from the surrounding concrete walls, ceiling, and floor. As a result, the roof was raised to about $1.9 \mathrm{~m}$ above the lead volume, the side walls were $1.1 \mathrm{~m}$ away from the vertical sides of the lead assembly (limited by the availability of lateral space in T7) (Fig. 5), 
back and front walls were several metres away from the lead assembly (Figs. 5 and 6) but the floor was only $43 \mathrm{~cm}$ away, set by the size of the steel I-beams available for the supporting platform, requiring a $\mathrm{B}_{4} \mathrm{C}$ shield as already discussed in Section 2.1.1.

The counting room, where the rabbit hyperpure germanium measuring station was also situated (see Section 7.1 for details of the rabbit set-up), was chosen to be far enough away from the beam area and with adequate shielding in order to minimize neutron background in the germanium counters.

A general orthonormal co-ordinate system was defined in the following way. Origin at the centre of the lead volume; $z$-axis along the beam direction; the $y$-axis upwards in the vertical direction; and the $x$-axis is such a way that the system is orthonormal.

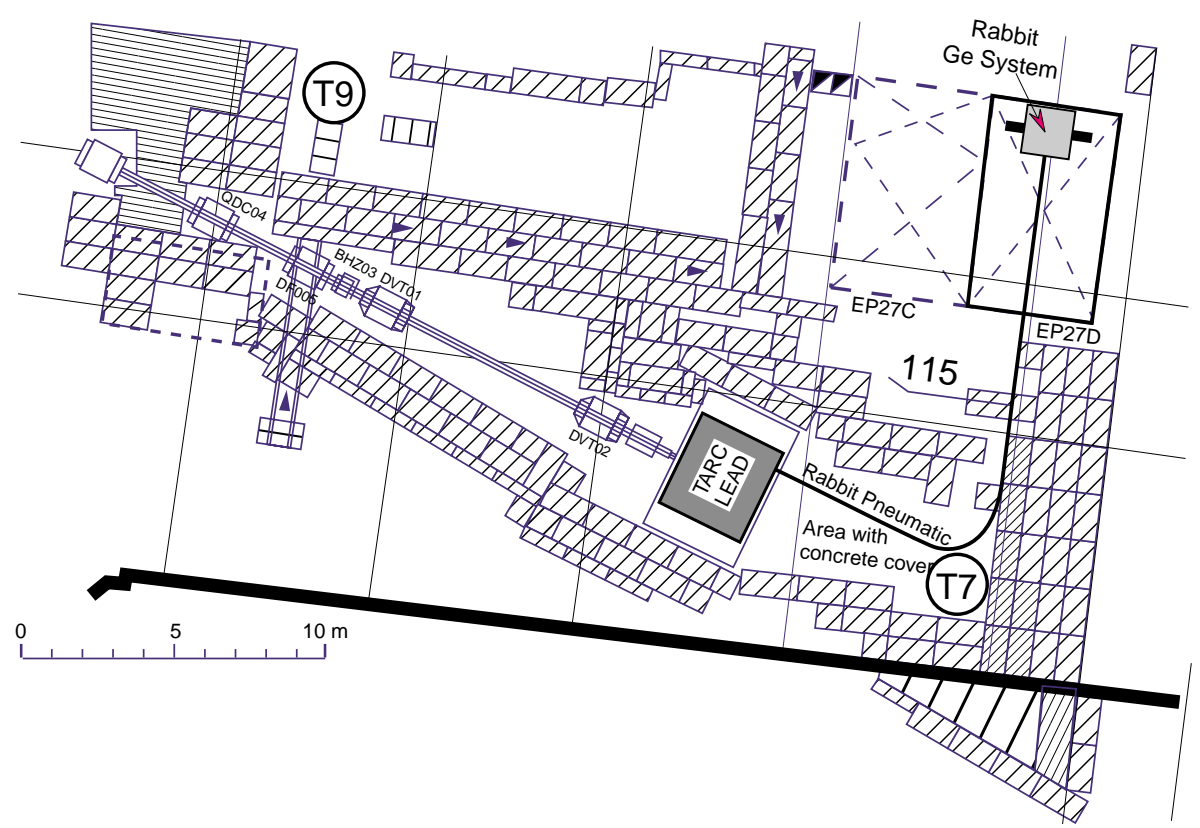

Fig. 5: General layout of the CERN T7 experimental area: view from the top showing the beam line, the lead assembly, the concrete shielding, and the rabbit system of which the germanium counters are located inside the counting room (labelled EP27D).

\subsubsection{Fast extraction beam}

The beam is extracted all at once by the fast rise of a magnetic kicker. In this mode, the structure of the beam in the PS machine is preserved, namely bunches 20-30 ns wide recurring normally every 14.4 seconds. For part of the time we could benefit from additional fast extraction within the usual $14.4 \mathrm{~s}$ PS supercycle. We were able to obtain, at the end of the T7 beam line, intensities ranging from $3 \times 10^{7}$ to $2 \times 10^{10}$ protons per shot. Of special concern is the accurate measurement of the intensity of the beam. Two beam transformers were used to measure the number of protons from the signal induced by the beam charge in a coil mounted around a vacuum pipe. One beam transformer, situated $13.4 \mathrm{~m}$ from the lead assembly, is the same as the one used in the FEAT experiment. The other one, situated immediately in front of the lead assembly, is an improved beam transformer developed for our purpose by industry [13]. A new design of the induction loop through which calibrated charges simulating the beam are injected allows a more linear behaviour of the calibration system [14]. The beam transformers measure the beam intensity of each PS shot (Fig. 7) providing the detailed history of the experimental runs. 


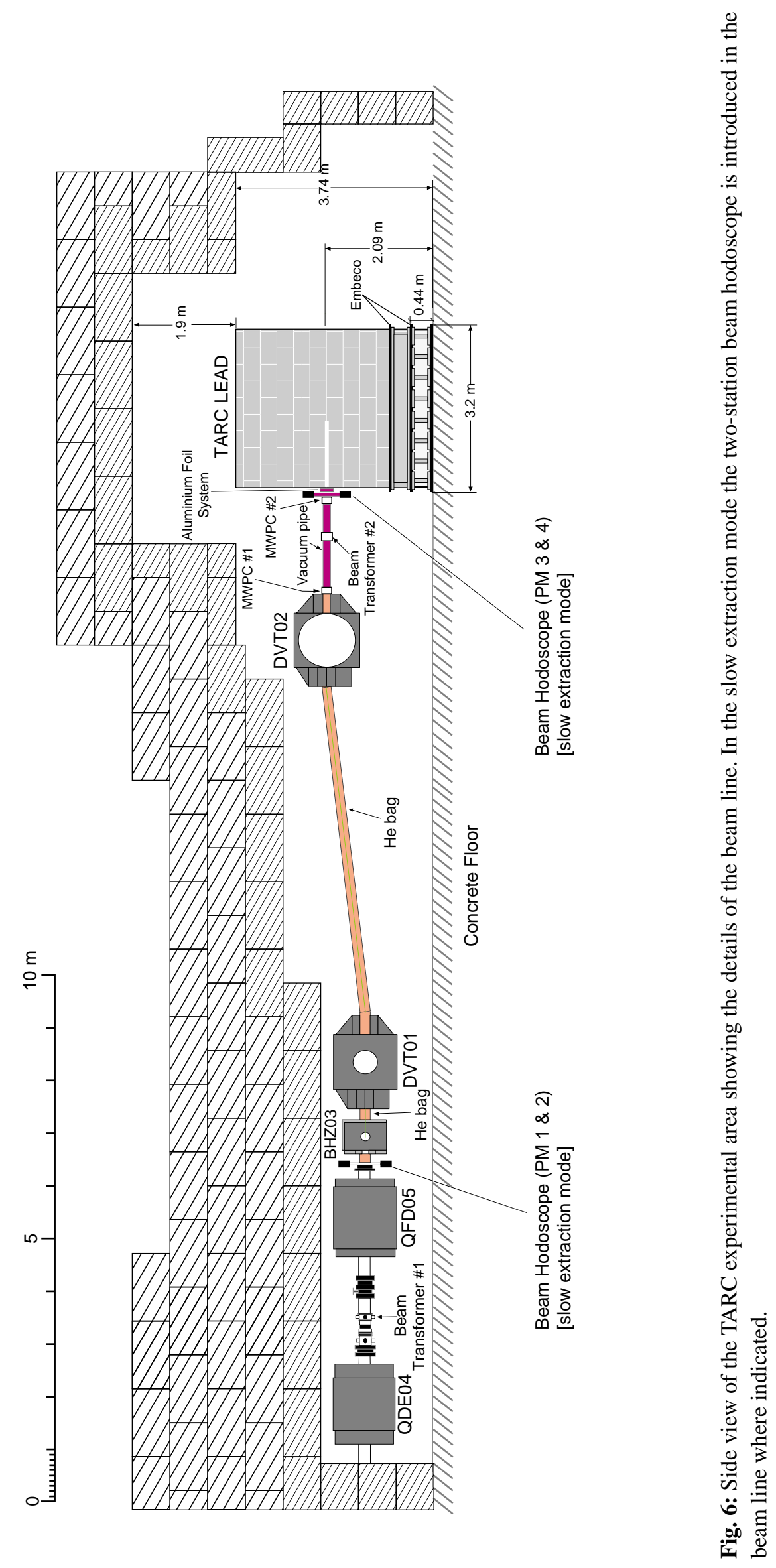




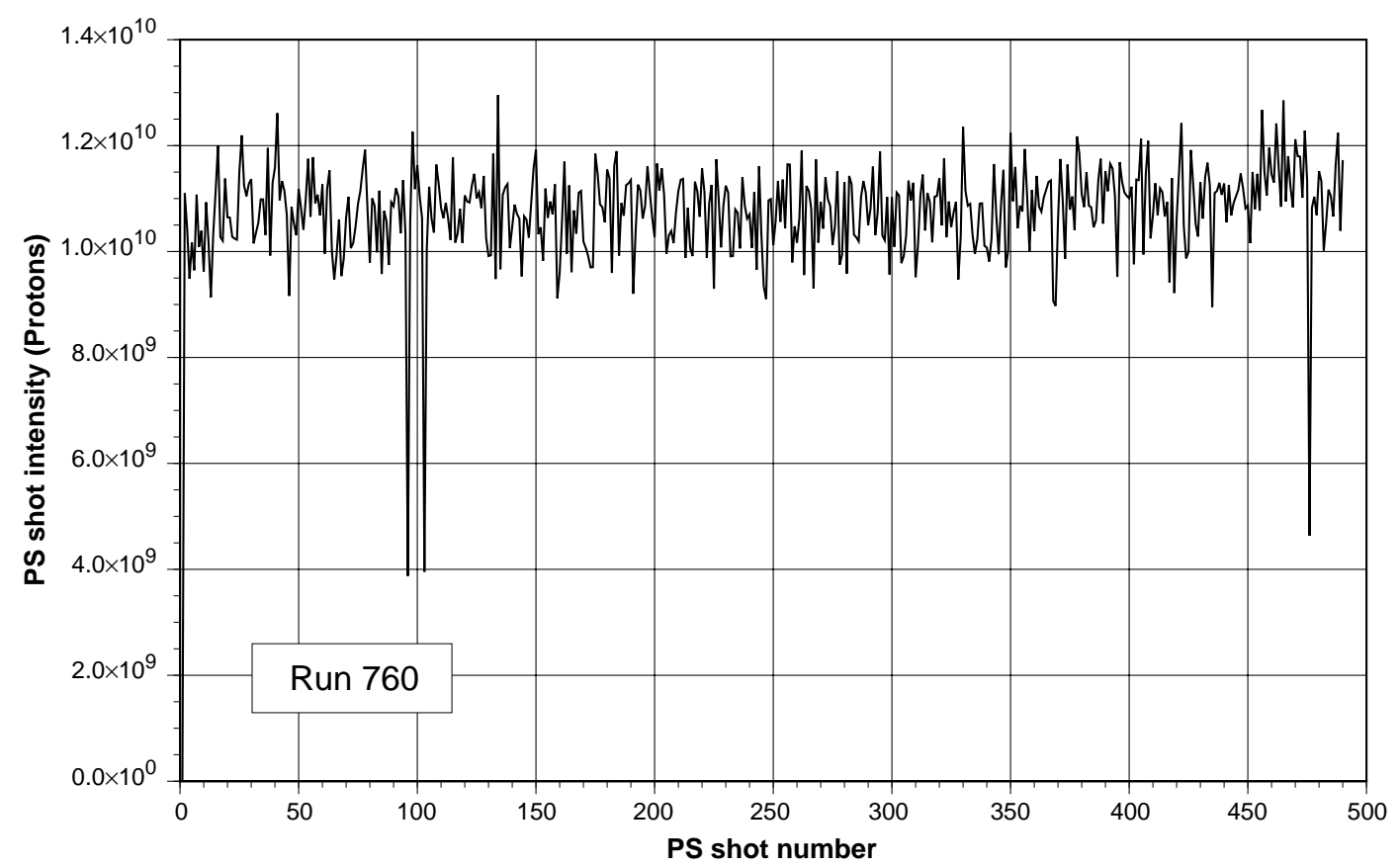

Fig. 7: Example of measurement of the beam intensity (run 760) showing the number of protons for each PS shot.

An important issue was to check the absolute calibration of the beam transformers. This was done by bombarding aluminium foils with the beam, and then comparing the number of protons obtained by counting the number of ${ }^{24} \mathrm{Na}$ produced with the number of protons predicted by the beam transformers. The irradiation of aluminium foils is a classic technique, involving the formation of ${ }^{24} \mathrm{Na}$ by the reaction ${ }^{27} \mathrm{Al}(\mathrm{p}, 3 \mathrm{pn}){ }^{24} \mathrm{Na},{ }^{24} \mathrm{Na}$ being detected via its $1368.45 \mathrm{keV}$ gamma ray emission. There were two types of analysis [15]: the 'global' mode compared the integrated number of protons during a run with the ${ }^{24} \mathrm{Na}$ produced, implicitly assuming that the beam intensity is constant pulse to pulse. The 'shot' mode took into account the variation of the proton intensity shot per shot. The results are shown in Fig. 8. We note that the method is limited by a systematic error of $6.9 \%$ of which the largest components are the uncertainty on the ${ }^{24} \mathrm{Na}$ production cross-section of the order of $4 \%$ and the absolute calibration of the ${ }^{125} \mathrm{Eu}$ source used to calibrate the Ge detector (5\%). We conclude from the studies made of the two types of calibrations performed and from the systematic comparisons between the results of the two beam transformers that the overall uncertainty on the beam intensity is better than $5 \%$.

Finally, the control of the beam implies also the control of its position and direction. This was done using two Multi Wire Proportional Chambers (MWPC), $1.6 \mathrm{~m}$ apart, mounted at the two ends of a vacuum pipe situated right in front of the beam hole in the lead assembly [16] (Fig. 6). The position of the two chambers was precisely measured (0.2-0.3 $\mathrm{mm}$ precision), and they provided a measurement of the position of the beam impact at the end of the beam hole, $1.2 \mathrm{~m}$ from the front face of the lead assembly. Generally, during the experiment, the beam was centred with a precision of $2-4 \mathrm{~mm}$. Moreover, in the case of the electronic detector measurements, the MWPC information was used to reject occasional bad shots of the PS, in order to ensure a good quality of the beam information. The fraction of bad shots was generally extremely small $(\leq 1 \%)$. 


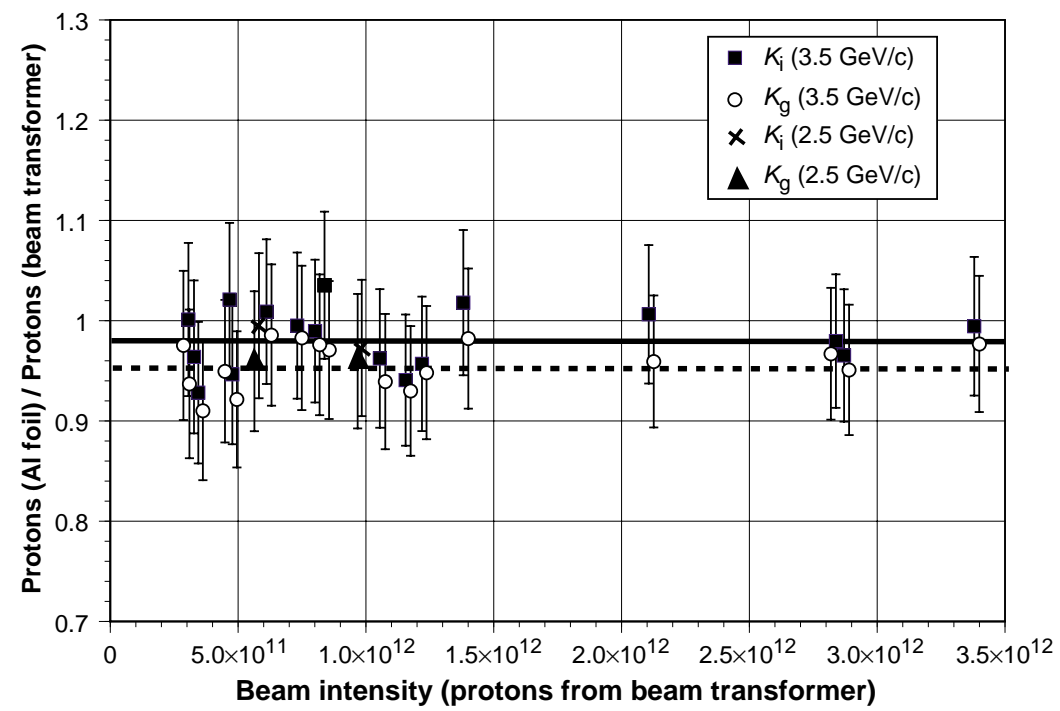

Fig. 8: Ratio $(K)$ of aluminium foil to beam transformer measurements using global $\left(K_{\mathrm{g}}\right)$ and individual $\left(K_{\mathrm{i}}\right)$ methods as explained in the text [15]. Both $3.5 \mathrm{GeV} / c$ and $2.5 \mathrm{GeV} / c$ data are included. The lines are fit to the data (full line: individual shot method, dotted line: global method).

\subsubsection{Slow extraction beam}

The ${ }^{3} \mathrm{He}$ ionization chambers required a very low beam intensity of $\sim 10^{3}$ protons $\left(\sim 5 \times 10^{3}\right.$ particles $)$ per PS ejection which could not be obtained with a fast extraction because of the impossibility of controlling and monitoring such a low-intensity beam in the PS accelerator system. Therefore, a low-intensity beam was prepared by collecting secondary particles produced by $24 \mathrm{GeV} / c$ primary protons hitting a target (usually made of two parts, one in aluminium the other in tungsten), allowing beam intensities down to about $5 \times 10^{3}$ particles per pulse. Primary protons were extracted progressively, by resonant extraction, over a $350 \mathrm{~ms}$ period, every $14.4 \mathrm{~s}$. However, the secondary beam contains a mixture of pions (60\%), protons (20\%), electrons and muons (20\%), etc. (of the same momentum) which need to be distinguished. A time-of-flight hodoscope was built. Protons and heavier particles could be separated from pions, muons, etc. from the difference in time of flight over a distance of $13.4 \mathrm{~m}$. The time resolution was about $0.5 \mathrm{~ns}$, and this forced us to select a low enough beam momentum of $2.5 \mathrm{GeV} / c$, to have $\sim 5$ standard deviations separation between proton and pion time distributions [Figs. 9(a) and 9(b)]. At such a momentum, the beam contains about $20 \%$ of protons, and the time difference between protons and pions is 3 ns. Pions could not be separated from lighter particles. The scintillator hodoscope (Fig. 6) was made of two counter stations: one situated just in front of the BHZ03 magnet [one single scintillator with horizontal and vertical dimensions $80 \mathrm{~mm}$ and $60 \mathrm{~mm}$, respectively, read out by two photomultipliers (PM 1 and 2)], the other one situated just in front of the beam hole in the lead assembly made of two overlapping scintillators (horizontal and vertical dimensions $60 \mathrm{~mm}$ and $45 \mathrm{~mm}$, respectively), each read out by one photomultiplier (PM 3 and 4).

Four time coincidences were built out of the two sets of signals (PM 1, 2) and (PM 3, 4). The redundancy of information was used to (a) monitor the beam condition; (b) reject accidental hits in the counters; and (c) measure the hodoscope efficiency. With this hodoscope, proton fathers of neutrons recorded in the ${ }^{3} \mathrm{He}$ chambers could be identified. At $10 \mathrm{keV}$ (the lower end of the neutron energy spectrum covered by that detector), the time between the beam shot and the interaction of the neutron is about $3.75 \mu \mathrm{s}$. 
The details of the operation and performance of the slow extraction beam hodoscope are described in Ref. [17]. Additional information can be found in Ref. [18].

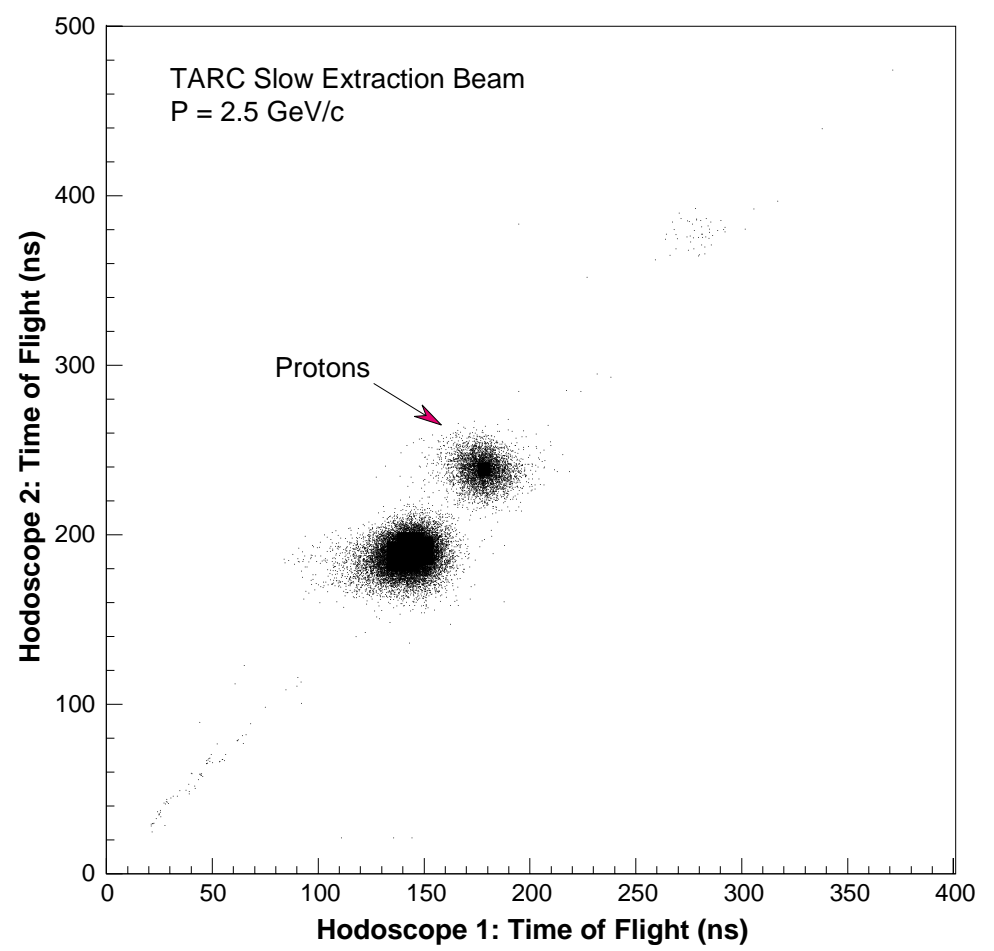

Fig. 9(a): Time differences measured by hodoscope 1 (PM 1 \& 3) versus hodoscope 2 (PM 2 \& 4), showing the $5 \sigma$ separation between protons and lighter particles.

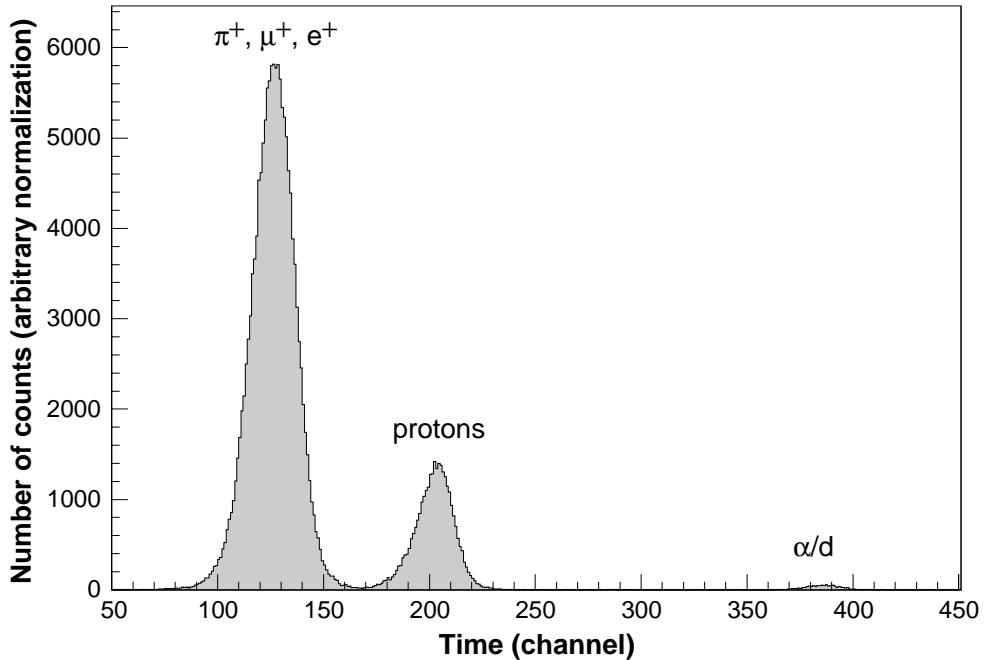

Fig. 9(b): Separation of protons from other particles, by time of flight, using PM 1 \& 4, at a beam momentum of $1.866 \mathrm{GeV} / c$.

\subsection{Data AcQuisition (DAQ)}

In TARC, neutron fluence measurements, covering a range from thermal energies to a few $\mathrm{MeV}$, together with transmutation measurements involve the use of many different types of detectors. These detectors have been developed in a number of collaborating institutes and had to be integrated in the DAQ system at the time they became available. Furthermore, the experiment had to operate in several distinct modes: synchronous to the beam short pulses, sparse data collection during spills, calibrations with radioactive sources, etc. An adaptable and 
evolvable DAQ system was designed to cover all the different detector combinations, allowing them to run both separately or jointly during the full lifetime of the experiment at CERN. In the following paragraphs only the general aspects of the DAQ will be described. Details specific to the different detectors can be found in the sections where those detectors are described.

\subsubsection{Hardware scheme of the DAQ}

The TARC DAQ system was designed as a network of Versa Module Europe (VME) crates controlled by a UNIX workstation (Fig. 10). Each VME crate includes a Motorola 68040 CPU @ $25 \mathrm{MHz}$ in a FIC8234 module running under the OS9 operating system. The DAQ software for TARC was developed using the CASCADE package provided by CERN [19]. In the CASCADE model the DAQ is a distributed system with a unified central RUN CONTROL. The software is divided into pieces, called stages, some of them running on the VME CPUs and others running in the RUN CONTROL and MONITORING UNIX workstations.

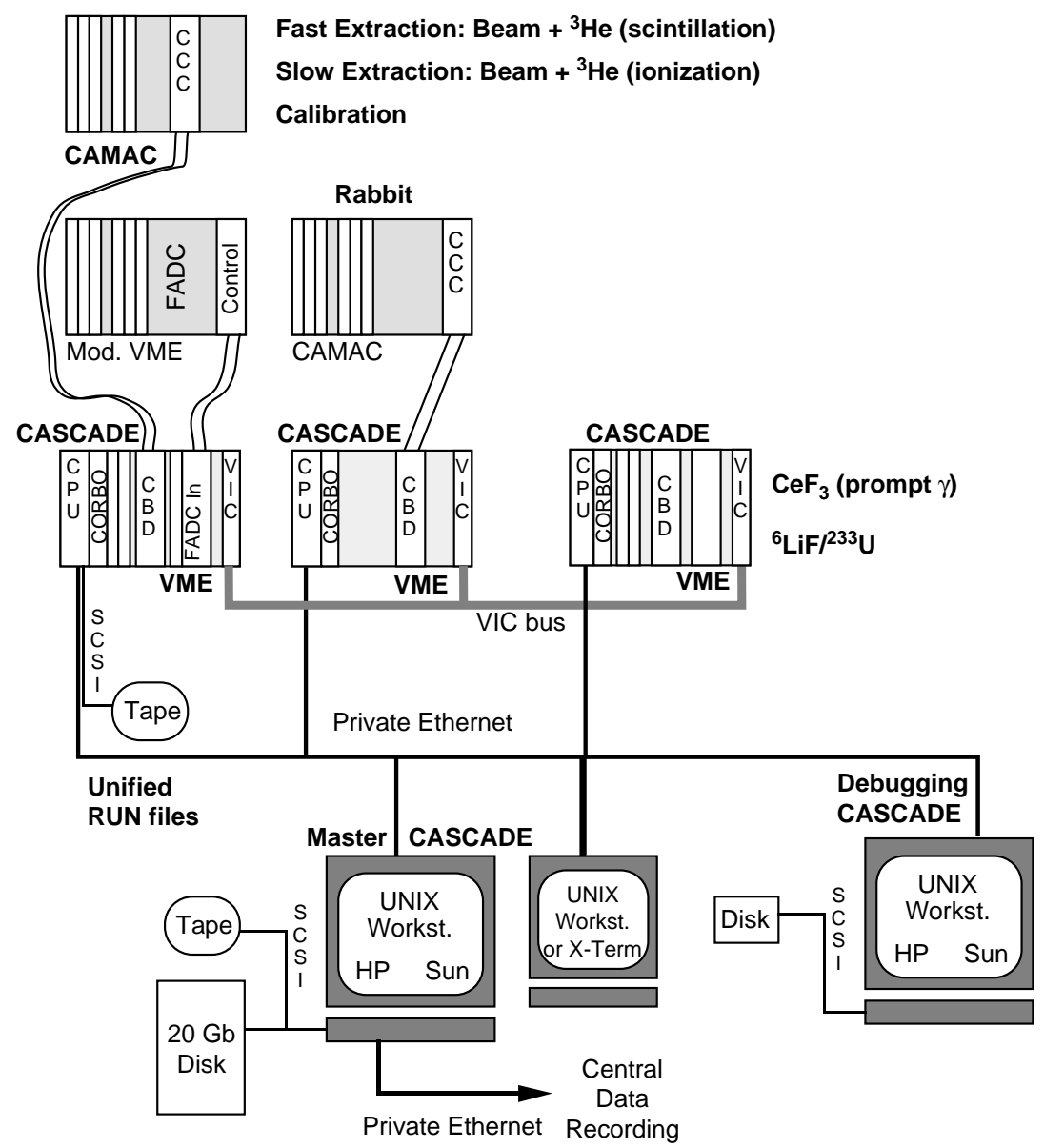

Fig. 10: Scheme of the TARC data acquisition system architecture.

All the data from TARC were collected in VME crates, by means of ADC modules and other I/O devices, either installed directly in the VME bus or using CAMAC, modified-VME buses, and RS232 interfaces. The front-end hardware readout and the manager of the event buffer were the main, but not the only, stages running on the VME modules. Several trigger signals are used by the DAQ system and in some subdetectors, such as the rabbit and the ${ }^{3} \mathrm{He}$ ionization chambers, organized in two levels of triggers. These trigger signals were collected in a CORBO unit at each VME crate and handled by the specific drivers included in the CASCADE system. The VME crates are linked together by a VIC bus and at the same time use 
a TCP/IP network, in a private Ethernet segment, to communicate with the UNIX workstations. In TARC, even when it was possible to use standalone VME crates with disk and other mass storage units, the VME crates were diskless. Hence, these crates had to use the BOOTP protocol to load the operating system from the UNIX workstation disks. Once booted, they used NFS to access the remote workstation file system as their main file system, however, a faster specific client-server protocol has been used to send the data collected, from the VME crates to the workstation disks.

More technical details about the DAQ can be found in Ref. [8].

\section{Development of Simulation Tools for TARC}

\subsection{Introduction}

The sequence of phenomena ranging from a high-energy proton-induced cascade in lead, to the production of neutrons that subsequently interact until they are finally absorbed or escape the system is rather complex. For a correct simulation of the Energy Amplifier (EA) it is essential to understand the fine details of the physics related to all these phenomena. This is one of the main purposes of the TARC experiment, which is aimed at the study of the spallation neutron phenomenology in a large lead volume.

A complete understanding of this complex phenomenology can only be claimed if it is possible to predict correctly the observed behaviour of the entire system. In order to do so, an innovative simulation was developed at CERN for the EA by C. Rubbia [2], [8], using Monte Carlo techniques. This code allows the description of complicated geometries and material compositions. Special attention was devoted to the development of techniques (for kinematic calculation, cross-section evaluation, etc.) to minimise the computer time in order to provide sufficient statistics. In the case of the TARC simulation, a computing time of $20 \mu \mathrm{s} /$ neutron/ process per single processor was achieved on our Convex SPP1200 with eight parallel processors.

Since this simulation represents a new approach and is highly complex it needs to be validated. In this respect, TARC is an ideal benchmark not only for our new simulation but for any ADS simulation, since it provides many different measurements generally more precise than previously available simulation predictions.

The Monte Carlo code used to simulate the TARC experiment is a version of the EA Monte Carlo where the time evolution has been switched off [32].

\subsection{Physics modelling}

The spallation neutron source distribution is generated by FLUKA-96 [20], [21]. Neutrons are transported by FLUKA down to an energy of $19.6 \mathrm{MeV}$ and then are written into a history file. The energy of $19.6 \mathrm{MeV}$ is selected for neutrons as being just below the upper limit $(20 \mathrm{MeV})$ of neutron cross-section data sets, thereby easing the transition to fast neutron transport codes.

The transport of neutrons below $19.6 \mathrm{MeV}$ is performed with the EA Monte Carlo code (Fig. 11).

Since the precision of a Monte Carlo simulation depends strongly on the three-dimensional geometrical description of the system simulated, a large effort has been devoted to the development of computable geometrical models (Fig. 12) [32]. 


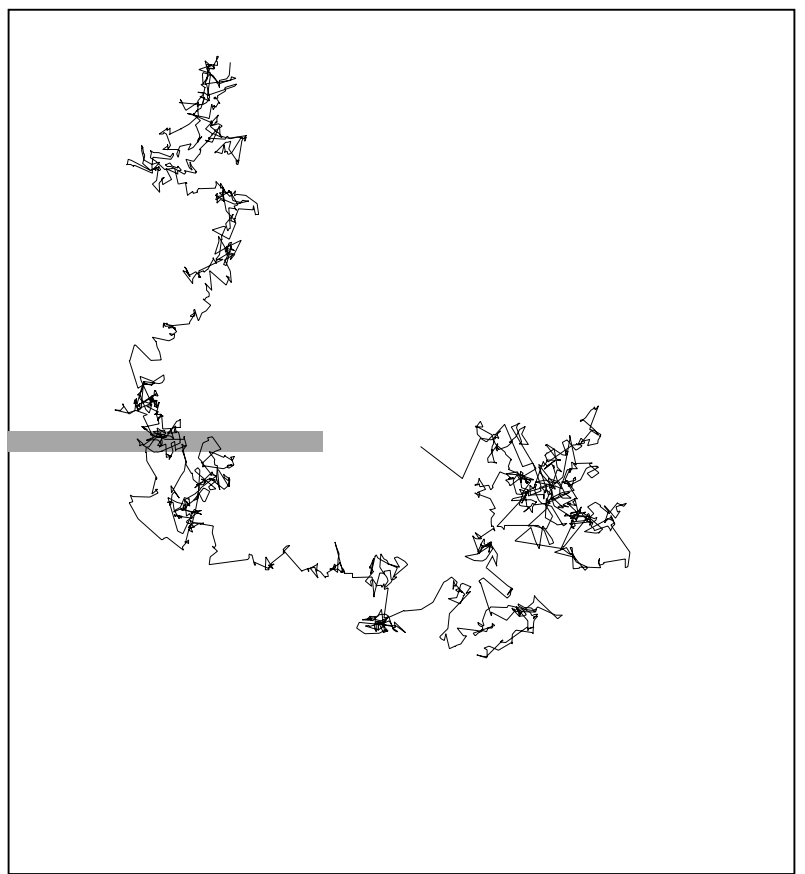

Fig. 11: Complete simulation of the interactions within the TARC lead volume of a single evaporation neutron produced by a $3.5 \mathrm{GeV} / c$ primary proton and transported down to thermal energies.

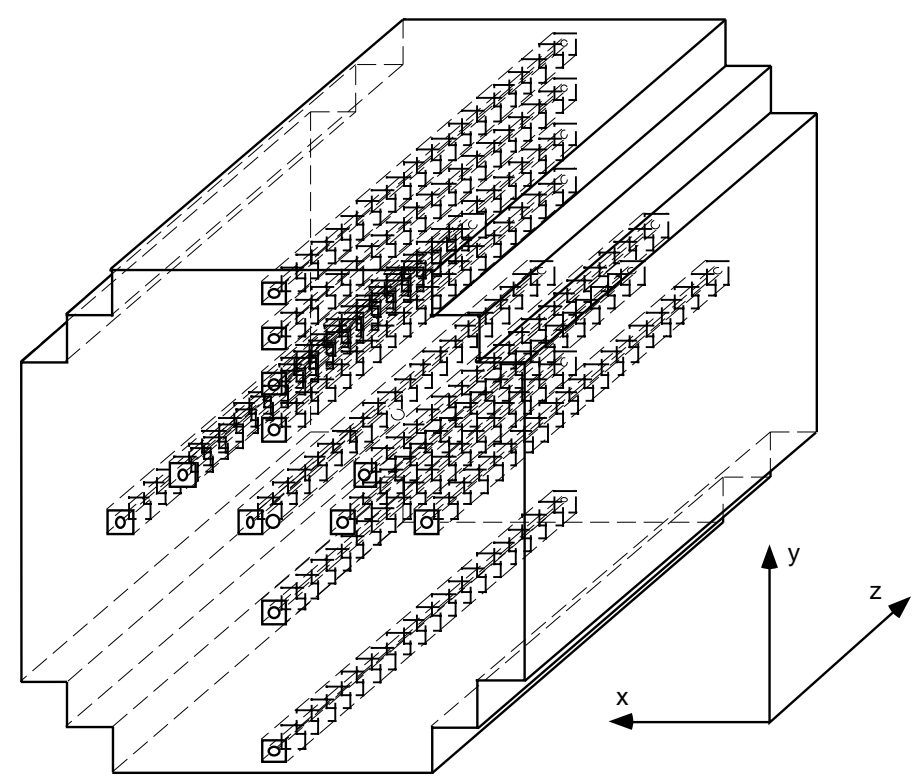

Fig. 12: Picture of the simulated TARC geometry, showing the segmentation into local blocks, $15 \times 15 \times 15 \mathrm{~cm}^{3}$, used in a second stage as local neutron generators for the various detector simulations (see Section 3.5).

\subsection{Neutron cross-sections}

Since a simulation is never better than the quality of the input data which are used, a special effort was made to provide the best possible neutron cross-section data. Our neutron cross-section data selection is taken from the latest compilations available [22]: ENDF/B-VI 4 (USA), JENDL-3.2 (Japan), JEF-2.2 (Europe), EAF-4.2 (Europe), CENDL-2.1 (China), EFF-2.4 (Europe) and BROND-2 (Russia). 
For each nuclide we have selected one evaluation out of those available on the basis of a systematic comparison [23]. In practice, the selection was done isotope by isotope according to the evaluation of the resonances and the number of reaction cross-sections, as shown in Fig. 13 for ${ }^{137} \mathrm{Cs}$ for instance. When both the resonance region and the number of cross-sections evaluated are similar, then the most recently evaluated cross-section was selected. This resulted in a database of 800 nuclides with reaction cross-sections out of which 400 also have the elastic cross-section available. For all nuclides the corresponding information on isomeric states exists, and isomer-dependent reactions are treated correctly whenever they are available.

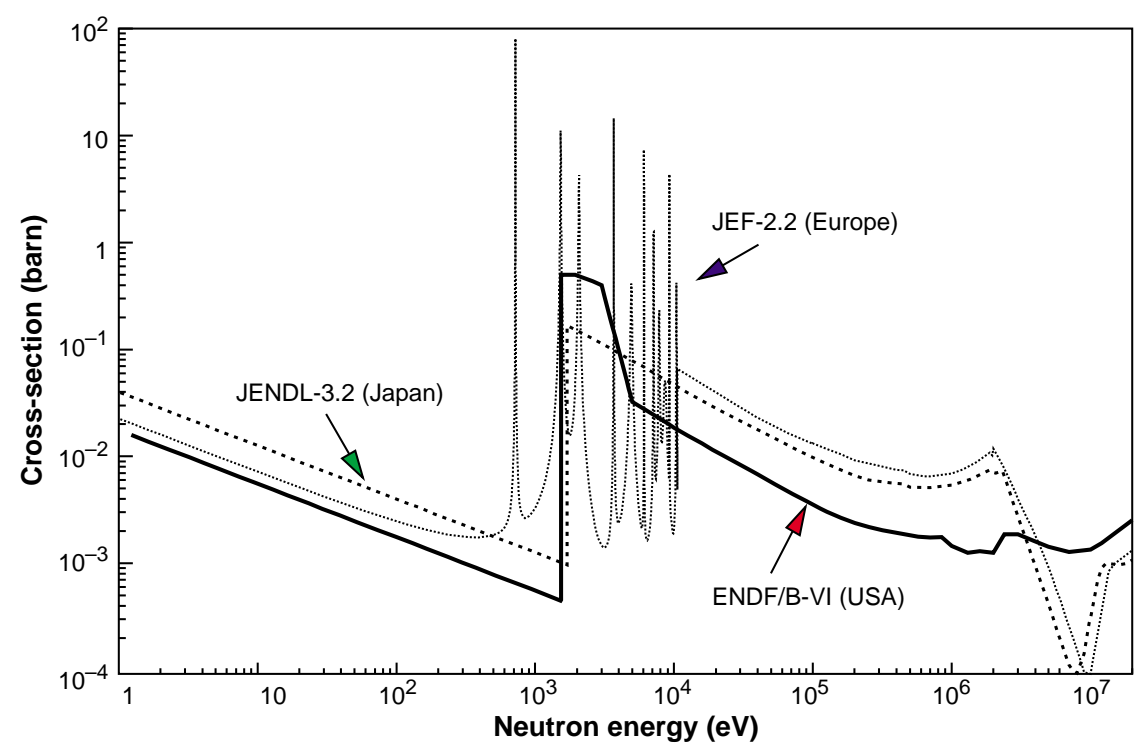

Fig. 13: Comparison of the ${ }^{137} \mathrm{Cs}$ capture cross-section in the different databases available. The one chosen in this case for the EA Monte Carlo corresponds to the European Database (JEF-2.2).

All cross-section files have been processed and checked for inconsistencies with the standard PREPRO-96 [24] code suite (LINEAR, RECENT, SIGMA1 and FIXUP) including EAF-4.2.

All cross-section files produced by PREPRO-96 have been subsequently processed with a specially written code package PROCESS [25] to create a direct-access library containing neutron cross-sections, cumulative secondary neutron energy distributions, and cumulative neutron angular distributions (optionally).

\subsection{Nuclear data}

For nuclear transmutation studies, accurate mass and decay tables are needed. Here again we have decided to go to the source of the data and we have created our own nuclear database from the most up-to-date compilations available. The EET Nuclear Database selection [26] has been assembled via a careful comparison of several sources. In particular, we have used the Brookhaven nuclear database [27], NUBASE [28], the National Radiological Protection Board (NRPB)database [29], the ICRP database [30] and nuclear data information from ENDF files.

For each isomer we store the atomic number, the chemical symbol, the mass number, the isomeric level, the isospin and parity, the mass excess, the half-life, the decay modes, branching ratios and decay-values where applicable, the natural isotopic abundance, and the inhalation and ingestion radiotoxicities [31]. 
This database represents a major improvement over all existing ones for use in a Monte Carlo simulation. All available information about isomeric state decay and production has been included. Nuclear data have been extensively checked for inconsistencies. In particular, all decay paths are closed (i.e. terminate in a nuclide present in the database) and all branching ratios are consistent (i.e. sum $=100 \%$ ).

The effort to have consistent databases was partly driven by practical considerations which come naturally if those databases are to be used in a simulation code. As a result, the fact of having such an innovative code and such a reliable nuclear database makes our simulation program unique.

\subsection{TARC general Monte Carlo code}

In order to reduce the computing time needed to simulate the TARC experiment, it has been decided to factorize the problem into a number of transport steps. In the first step the neutrons generated by the high-energy proton beam described by the FLUKA program are followed in the lead block without detectors ('Cube' program) until they escape the block or are captured (Fig. 14).

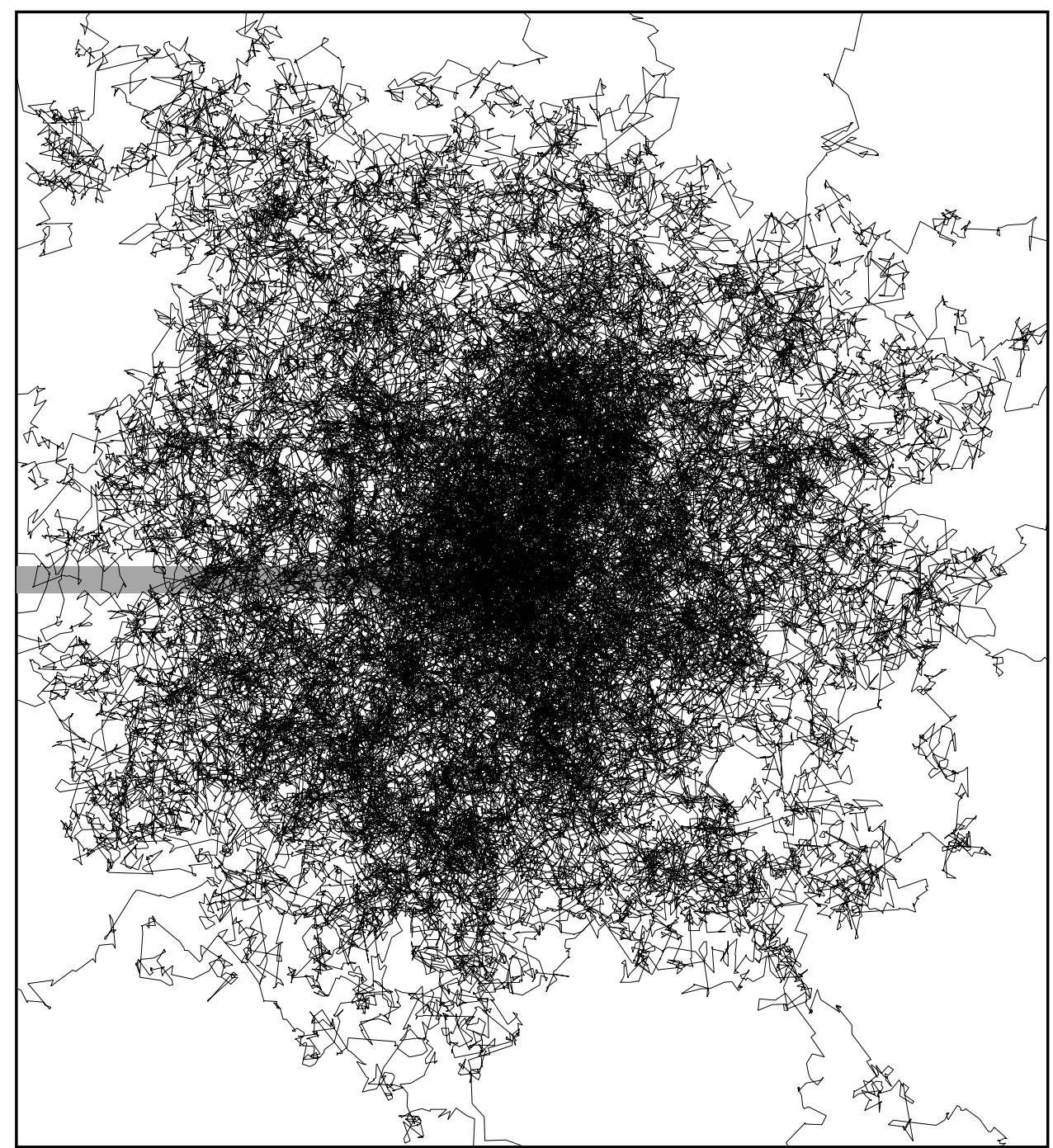

Fig. 14: Complete simulation of the secondary neutron shower produced by a single $3.5 \mathrm{GeV} / \mathrm{c}$ primary proton and transported in the entire TARC lead assembly until all the neutrons are captured or escape from the volume. 
During this transport, a Data Summary Tape (DST) is produced. This is a condensed description of the neutrons escaping from the system or crossing internal boundaries 4 providing source terms for further calculations. Creating such a tape has the advantage of allowing repeated and various analyses with the same neutron source.

All crossings of the same neutron in and out of a block are recorded. In the successive transport steps ('Detector' program), the effect of the surrounding lead can thus be neglected and all incoming neutrons can be used as independent source neutrons and neutrons exiting the block must be discarded. This simplifies the problem of the simulation of specific detectors. This approximation is correct only if the perturbation introduced by the detector is negligible. The magnitude of this perturbation can be evaluated by comparing the unperturbed and the perturbed exiting neutron spectrum which was done for each detector used in this study (see Section 5).

While simulating a detector in a single instrumented block, the same neutron sample is run more than once. This introduces some correlation in the events, but if the neutron sample is large enough to be representative of the true neutron population, the error should be negligible as the neutron histories will be different because of different random numbers.

The size of $15 \mathrm{~cm}$ around the instrumentation hole has been chosen to be large enough to allow the randomization of the neutron flux due to scattering in lead and to minimize the perturbations due to the presence of detectors.

All the neutrons escaping the lead assembly are recorded. These neutrons are used as independent source neutrons in subsequent Monte Carlo calculations ('CAVE' program) where they are propagated into the area surrounding the lead assembly (hereafter called cave). Those scattered back onto the lead assembly are recorded on a special history tape and used to recompute the detector response and evaluate the effect of background coming from neutron reflections on the concrete walls surrounding the experimental hall.

\subsubsection{Systematic errors and technical checks on the Monte Carlo flux calculation}

The TARC flux measurements have to be compared to the prediction of the TARC Monte Carlo chain: FLUKA for the spallation followed by Cube for neutron transport below 19.6 MeV and finally by Detector, a specific code simulating the detector response. This detailed comparison is an important prerequisite step for the understanding of Adiabatic Resonance Crossing. It is therefore crucial to assess precisely the agreement between the data and the prediction. This requires not only technical checks of the Monte Carlo calculation, ensuring that the whole chain, from FLUKA to the detector simulation is handled in a consistent way, but also an evaluation of the systematic errors.

\subsubsection{Systematics errors on the Monte Carlo simulation}

The main systematic error contributions to the calculation of the neutron flux come from:

(1) The simulation of the spallation process in FLUKA and the neutron transport down to 19.6 $\mathrm{MeV}$ for which the major sources of uncertainties can be summarized as follows:

- Cross-sections: (mostly reaction cross-sections) for protons and neutrons in the energy range of interest $(20 \mathrm{MeV}$ to $3 \mathrm{GeV})$ : The r.m.s. deviation between the

4. Each time the surface of one of the $24015 \times 15 \times 15 \mathrm{~cm}^{3}$ boxes around the instrumented holes is crossed by a neutron, an entry is logged into the binary DST file. 
cross-section adopted in FLUKA and available experimental data for lead is below $10 \%$. The error introduced in the calculations is significantly smaller in the bulk of the cascade since at the energies of TARC almost all protons interact inelastically before being ranged out by ionization losses. Larger errors could arise at a few interaction lengths from the build-up region where, however, TARC results are mostly dominated by the diffusion of low-energy neutrons, rather than by local production due to the tails of the high-energy cascade.

- Neutron production model: The model used in FLUKA for the description of nuclear interactions has been extensively benchmarked against several sets of experimental data. Double differential data about neutron production in lead are available for energies from $20 \mathrm{MeV}$ to $3 \mathrm{GeV}$. The predicted double differential spectra agree with the experimental data within a factor two and often much better over the full range of energies and angles. The resulting agreement on angle integrated spectra which are expected to be most relevant for TARC is typically within 10-20\% over most energy spectra. The total neutron multiplicity can be predicted with errors not exceeding $10 \%$. These errors are significantly reduced in a thick target cascade owing to a significant averaging among different energies and to overall constraints (like energy conservation).

We can take $15-20 \%$ as an estimate of the systematic error coming from the simulation of the spallation process.

(2) The simulation of neutron physics in lead below $19.6 \mathrm{MeV}$ (mainly uncertainties in neutron cross-sections for lead). This contribution is estimated to be of the order of $10 \%$.

(3) The knowledge of the chemical composition of the lead (the effect of the various impurities).

In this case, the TARC simulation was used to study the effect, on the neutron flux, of the various impurities contained in the lead. Four different lead qualities were considered: special TARC lead of purity $99.99 \%$, standard $99.99 \%$ lead purity, and purities of $99.985 \%$ and 99.97\%. Table 2 gives the detailed concentration actually used [33]. For the TARC lead, we used the measured concentrations, for the other lead qualities we changed the concentration for all the elements for which the vendor gives the concentration and for the others which are not known we kept the same concentrations as for TARC.

We find that within a distance of $1.5 \mathrm{~m}$ from the centre of the lead assembly, the average change in fluence over the entire neutron energy range is smaller than $10 \%$ for qualities 99.99 and 99.985. For quality 99.97 the decrease in flux below $5 \mathrm{eV}$ reaches $30 \%$ (Fig. 15).

In order to assess the systematic error contribution from the uncertainty in the impurity content of the TARC lead, we have run the simulation with concentrations modified in the following way: (1) all concentrations increased by two standard deviations $\left(\mathrm{C}=\mathrm{C}+2 \sigma_{\mathrm{c}}\right)$; (2) all concentrations decreased by two standard deviations $\left(\mathrm{C}=\mathrm{C}-2 \sigma_{\mathrm{c}}\right)$. Then we computed the fluence ratio between the two cases (Fig. 16). The change of fluence is generally small, negligible above $1 \mathrm{eV}$, and it reaches $6 \%$ at thermal neutron energy. This implies that the effect on the TARC neutron fluence measurement of the uncertainty in the impurity concentration in the lead is negligible, over the energy range of interest. 
Table 2: Details of the impurity contents for the various qualities of lead used in this study

\begin{tabular}{|c|c|c|c|c|}
\hline Element & TARC content (appm) & $99.99 \%$ & 99.985\% & $99.97 \%$ \\
\hline $\mathrm{Ag}$ & 3.78 & 10 & 15 & 50 \\
\hline${ }^{27} \mathrm{Al}$ & $1.9 \mathrm{E}-2$ & 0 & 5 & 5 \\
\hline${ }^{75} \mathrm{As}$ & $5.5 \mathrm{E}-3$ & 1 & 5 & 10 \\
\hline${ }^{197} \mathrm{Au}$ & 7.9E-4 & 7.9E-4 & 7.9E-4 & 7.9E-4 \\
\hline${ }^{11} \mathrm{~B}$ & $2.7 \mathrm{E}-3$ & $2.7 \mathrm{E}-3$ & $2.7 \mathrm{E}-3$ & $2.7 \mathrm{E}-3$ \\
\hline${ }^{138} \mathrm{Ba}$ & $4.8 \mathrm{E}-4$ & $4.8 \mathrm{E}-4$ & $4.8 \mathrm{E}-4$ & $4.8 \mathrm{E}-4$ \\
\hline${ }^{9} \mathrm{Be}$ & $4.9 \mathrm{E}-4$ & $4.9 \mathrm{E}-4$ & $4.9 \mathrm{E}-4$ & $4.9 \mathrm{E}-4$ \\
\hline${ }^{209} \mathrm{Bi}$ & 19 & 50 & 110 & 250 \\
\hline${ }^{79} \mathrm{Br}$ & $1.2 \mathrm{E}-2$ & $1.2 \mathrm{E}-2$ & $1.2 \mathrm{E}-2$ & $1.2 \mathrm{E}-2$ \\
\hline C & 1.2 & 1.2 & 1.2 & 1.2 \\
\hline $\mathrm{Ca}$ & $1.6 \mathrm{E}-2$ & 0 & 10 & 10 \\
\hline $\mathrm{Cd}$ & $8.8 \mathrm{E}-2$ & $8.8 \mathrm{E}-2$ & $8.8 \mathrm{E}-2$ & $8.8 \mathrm{E}-2$ \\
\hline${ }^{140} \mathrm{Ce}$ & $1.1 \mathrm{E}-4$ & $1.1 \mathrm{E}-4$ & $1.1 \mathrm{E}-4$ & $1.1 \mathrm{E}-4$ \\
\hline${ }^{35} \mathrm{Cl}$ & $1.5 \mathrm{E}-2$ & $1.5 \mathrm{E}-2$ & $1.5 \mathrm{E}-2$ & $1.5 \mathrm{E}-2$ \\
\hline${ }^{59} \mathrm{Co}$ & $3.6 \mathrm{E}-4$ & 0 & 5 & 5 \\
\hline $\mathrm{Cr}$ & $1.5 \mathrm{E}-2$ & 0 & 5 & 5 \\
\hline${ }^{133} \mathrm{Cs}$ & $5.5 \mathrm{E}-3$ & $5.5 \mathrm{E}-3$ & $5.5 \mathrm{E}-3$ & $5.5 \mathrm{E}-3$ \\
\hline $\mathrm{Cu}$ & $9.5 \mathrm{E}-2$ & 10 & 10 & 10 \\
\hline${ }^{19} \mathrm{~F}$ & $2.3 \mathrm{E}-3$ & $2.3 \mathrm{E}-3$ & $2.3 \mathrm{E}-3$ & $2.3 \mathrm{E}-3$ \\
\hline $\mathrm{Fe}$ & $7.0 \mathrm{E}-3$ & 1 & 15 & 15 \\
\hline${ }^{69} \mathrm{Ga}$ & $3.0 \mathrm{E}-3$ & $3.0 \mathrm{E}-3$ & $3.0 \mathrm{E}-3$ & $3.0 \mathrm{E}-3$ \\
\hline${ }^{74} \mathrm{Ge}$ & $2.6 \mathrm{E}-3$ & $2.6 \mathrm{E}-3$ & $2.6 \mathrm{E}-3$ & $2.6 \mathrm{E}-3$ \\
\hline $\mathrm{Hf}$ & $6.8 \mathrm{E}-4$ & $6.8 \mathrm{E}-4$ & $6.8 \mathrm{E}-4$ & $6.8 \mathrm{E}-4$ \\
\hline${ }^{202} \mathrm{Hg}$ & $1.2 \mathrm{E}-1$ & $1.2 \mathrm{E}-1$ & $1.2 \mathrm{E}-1$ & $1.2 \mathrm{E}-1$ \\
\hline${ }^{127} \mathrm{I}$ & $3.1 \mathrm{E}-3$ & $3.1 \mathrm{E}-3$ & $3.1 \mathrm{E}-3$ & $3.1 \mathrm{E}-3$ \\
\hline In & $1.5 \mathrm{E}-3$ & $1.5 \mathrm{E}-3$ & $1.5 \mathrm{E}-3$ & $1.5 \mathrm{E}-3$ \\
\hline $\mathrm{Ir}$ & $8.6 \mathrm{E}-4$ & $8.6 \mathrm{E}-4$ & $8.6 \mathrm{E}-4$ & $8.6 \mathrm{E}-4$ \\
\hline K & $1.9 \mathrm{E}-2$ & $1.9 \mathrm{E}-2$ & $1.9 \mathrm{E}-2$ & $1.9 \mathrm{E}-2$ \\
\hline${ }^{139} \mathrm{La}$ & $1.6 \mathrm{E}-4$ & $1.6 \mathrm{E}-4$ & $1.6 \mathrm{E}-4$ & $1.6 \mathrm{E}-4$ \\
\hline${ }^{7} \mathrm{Li}$ & $2.6 \mathrm{E}-4$ & $2.6 \mathrm{E}-4$ & $2.6 \mathrm{E}-4$ & $2.6 \mathrm{E}-4$ \\
\hline $\mathrm{Mg}$ & $3.2 \mathrm{E}-3$ & $3.2 \mathrm{E}-3$ & 5 & 5 \\
\hline${ }^{55} \mathrm{Mn}$ & $1.5 \mathrm{E}-3$ & $1.5 \mathrm{E}-3$ & 5 & 5 \\
\hline Mo & $2.4 \mathrm{E}-3$ & $2.4 \mathrm{E}-3$ & $2.4 \mathrm{E}-3$ & $2.4 \mathrm{E}-3$ \\
\hline${ }^{14} \mathrm{~N}$ & 0.99 & 0.99 & 0.99 & 0.99 \\
\hline${ }^{23} \mathrm{Na}$ & $5.9 \mathrm{E}-3$ & $5.9 \mathrm{E}-3$ & $5.9 \mathrm{E}-3$ & $5.9 \mathrm{E}-3$ \\
\hline${ }^{93} \mathrm{Nb}$ & $4.1 \mathrm{E}-4$ & 4.1E-4 & $4.1 \mathrm{E}-4$ & $4.1 \mathrm{E}-4$ \\
\hline${ }^{142} \mathrm{Nd}$ & $1.0 \mathrm{E}-3$ & $1.0 \mathrm{E}-3$ & $1.0 \mathrm{E}-3$ & $1.0 \mathrm{E}-3$ \\
\hline $\mathrm{Ni}$ & $3.3 \mathrm{E}-2$ & 1 & 5 & 10 \\
\hline${ }^{16} \mathrm{O}$ & 3.97 & 3.97 & 3.97 & 3.97 \\
\hline Os & $2.1 \mathrm{E}-3$ & $2.1 \mathrm{E}-3$ & $2.1 \mathrm{E}-3$ & $2.1 \mathrm{E}-3$ \\
\hline${ }^{31} \mathrm{P}$ & $7.2 \mathrm{E}-4$ & $7.2 \mathrm{E}-4$ & 7.2E-4 & 7.2E-4 \\
\hline${ }^{106} \mathrm{Pd}$ & $2.6 \mathrm{E}-3$ & $2.6 \mathrm{E}-3$ & $2.6 \mathrm{E}-3$ & $2.6 \mathrm{E}-3$ \\
\hline${ }^{141} \mathrm{Pr}$ & $1.5 \mathrm{E}-4$ & $1.5 \mathrm{E}-4$ & $1.5 \mathrm{E}-4$ & $1.5 \mathrm{E}-4$ \\
\hline${ }^{194} \mathrm{Pt}$ & $5.4 \mathrm{E}-3$ & $5.4 \mathrm{E}-3$ & $5.4 \mathrm{E}-3$ & $5.4 \mathrm{E}-3$ \\
\hline${ }^{85} \mathrm{Rb}$ & $6.8 \mathrm{E}-3$ & $6.8 \mathrm{E}-3$ & $6.8 \mathrm{E}-3$ & $6.8 \mathrm{E}-3$ \\
\hline $\mathrm{Re}$ & $9.1 \mathrm{E}-4$ & $9.1 \mathrm{E}-4$ & $9.1 \mathrm{E}-4$ & $9.1 \mathrm{E}-4$ \\
\hline
\end{tabular}


Table 2: Details of the impurity contents for the various qualities of lead used in this study (Continuation)

\begin{tabular}{c|c|c|c|c}
\hline Element & TARC content (appm) & $\mathbf{9 9 . 9 9 \%}$ & $\mathbf{9 9 . 9 8 5 \%}$ & $\mathbf{9 9 . 9 7 \%}$ \\
\hline${ }^{103} \mathrm{Rh}$ & $9.5 \mathrm{E}-3$ & $9.5 \mathrm{E}-3$ & $9.5 \mathrm{E}-3$ & $9.5 \mathrm{E}-3$ \\
${ }^{102} \mathrm{Ru}$ & $2.7 \mathrm{E}-3$ & $2.7 \mathrm{E}-3$ & $2.7 \mathrm{E}-3$ & $2.7 \mathrm{E}-3$ \\
${ }^{32} \mathrm{~S}$ & $4.0 \mathrm{E}-2$ & 1 & 15 & 5 \\
$\mathrm{Sb}$ & $1.4 \mathrm{E}-1$ & 1 & 15 & 5 \\
${ }^{45} \mathrm{Sc}$ & $1.5 \mathrm{E}-4$ & $1.5 \mathrm{E}-4$ & $1.5 \mathrm{E}-4$ & $1.5 \mathrm{E}-4$ \\
${ }^{80} \mathrm{Se}$ & $2.0 \mathrm{E}-2$ & $1.0 \mathrm{E}-2$ & 10 & 10 \\
$\mathrm{Si}$ & $1.3 \mathrm{E}-2$ & 1 & $1.3 \mathrm{E}-2$ & $1.3 \mathrm{E}-2$ \\
$\mathrm{Sn}$ & $3.6 \mathrm{E}-3$ & $1.3 \mathrm{E}-4$ & 5 & 10 \\
${ }^{88} \mathrm{Sr}$ & $1.3 \mathrm{E}-4$ & $5.0 \mathrm{E}-2$ & $5.0 \mathrm{E}-2$ & $1.3 \mathrm{E}-4$ \\
${ }^{181} \mathrm{Ta}$ & $5.0 \mathrm{E}-2$ & 5 & 5 & $5.0 \mathrm{E}-2$ \\
${ }^{128} \mathrm{Te}$ & $2.2 \mathrm{E}-1$ & $5.2 \mathrm{E}-4$ & $5.2 \mathrm{E}-4$ & 5 \\
${ }^{232} \mathrm{Th}$ & $5.2 \mathrm{E}-4$ & 1 & 10 & $5.2 \mathrm{E}-4$ \\
$\mathrm{Ti}$ & $3.5 \mathrm{E}-4$ & 4.6 & 10 & 10 \\
${ }^{205} \mathrm{Tl}$ & 4.6 & $2.0 \mathrm{E}-4$ & $2.0 \mathrm{E}-4$ & 10 \\
${ }^{238} \mathrm{U}$ & $2.0 \mathrm{E}-4$ & $1.8 \mathrm{E}-4$ & $1.8 \mathrm{E}-4$ & $1.0 \mathrm{E}-4$ \\
$\mathrm{~V}$ & $1.8 \mathrm{E}-4$ & $5.6 \mathrm{E}-3$ & $5.6 \mathrm{E}-3$ & $5.6 \mathrm{E}-3$ \\
$\mathrm{~W}$ & $5.6 \mathrm{E}-3$ & $7.2 \mathrm{E}-5$ & $7.2 \mathrm{E}-5$ & $7.2 \mathrm{E}-5$ \\
${ }^{89} \mathrm{Y}$ & $7.2 \mathrm{E}-5$ & 1 & 2 & 10 \\
$\mathrm{Zn}$ & $3.6 \mathrm{E}-3$ & $4.1 \mathrm{E}-4$ & $4.1 \mathrm{E}-4$ & $4.1 \mathrm{E}-4$ \\
$\mathrm{Zr}$ & $4.1 \mathrm{E}-4$ & & \\
\hline
\end{tabular}

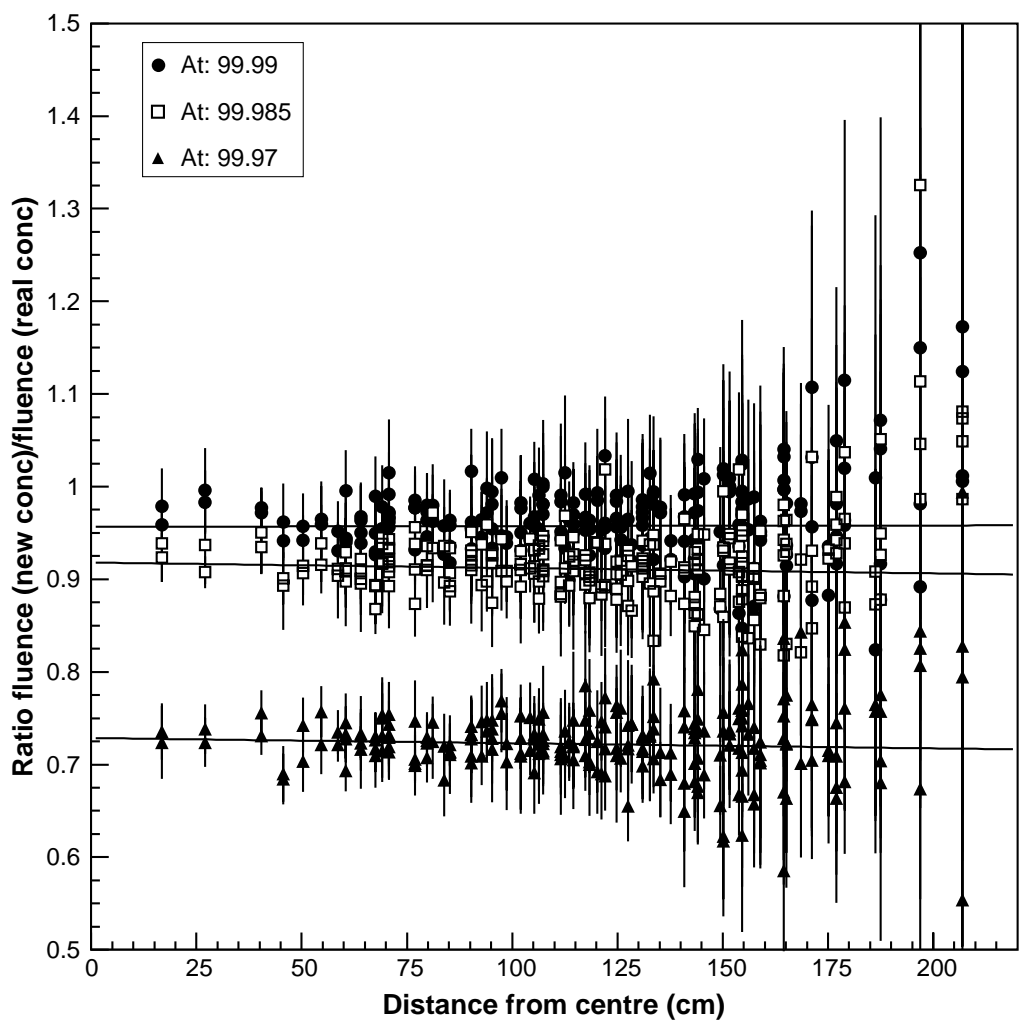

Fig. 15: Variation of the ratio of neutron fluence in TARC lead to neutron fluence in lead with different impurity contents as a function of distance to the centre of the lead assembly. The neutron fluence is considered in the energy range from $1.0 \mathrm{eV}$ to $5.0 \mathrm{eV}$. 


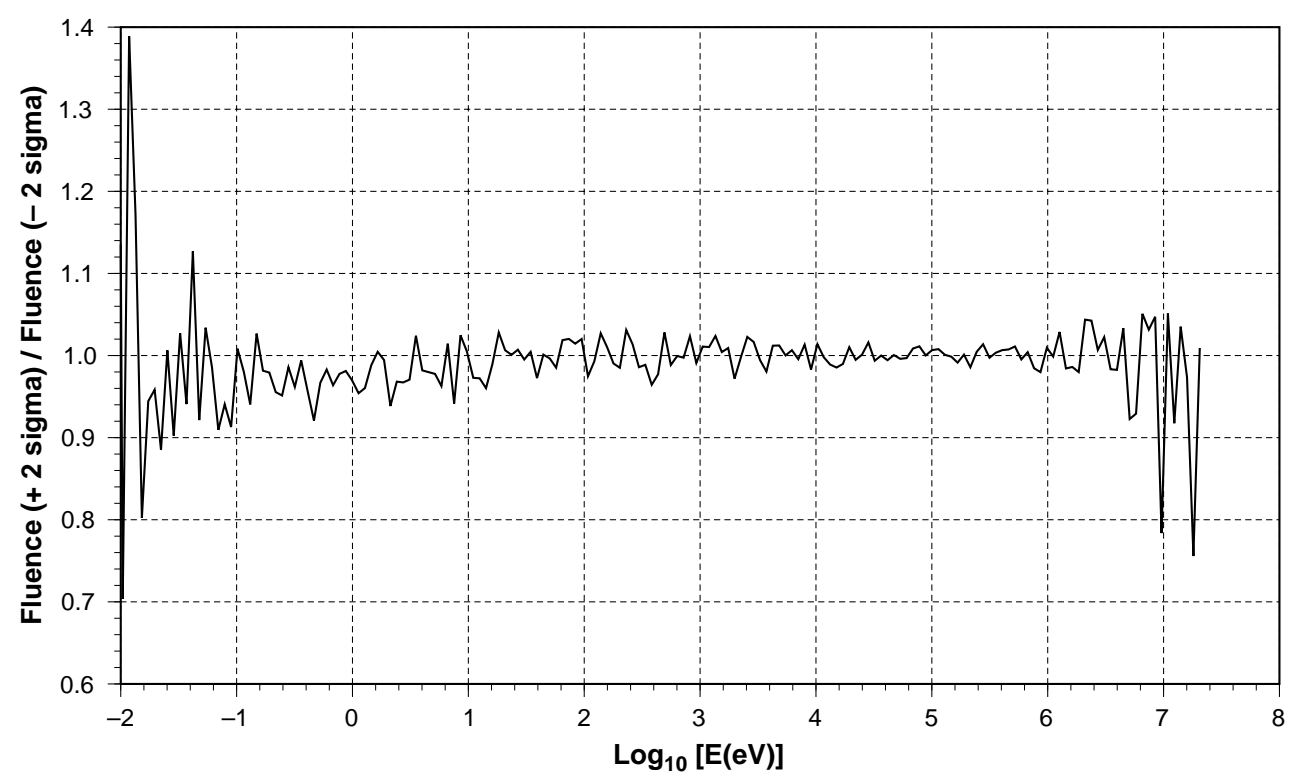

Fig. 16: Ratio of neutron fluences obtained with modified concentrations by \pm 2 standard deviations with respect to TARC nominal concentrations.

In order to study more specifically the effect of silver, we have run the simulation for a silver concentration increased by a factor $10[\mathrm{C}(\mathrm{Ag})=37.8 \mathrm{ppm}]$. The ratio of fluences with the increased silver concentration to the normal TARC concentration shows no effects down to neutron energies of $50 \mathrm{eV}$. A spectacular effect is found, as expected, below the main silver resonance of $5.2 \mathrm{eV}$, where the fluence drops by $20 \%$ (Fig. 17). This allows the effect of silver to be quantified. A variation of the silver concentration by 2 standard deviations, implies a maximum flux change by $0.7 \%$, well below the other main sources of systematic errors.

We conclude that the error coming form the uncertainty in impurity concentrations in the TARC lead is safely negligible.

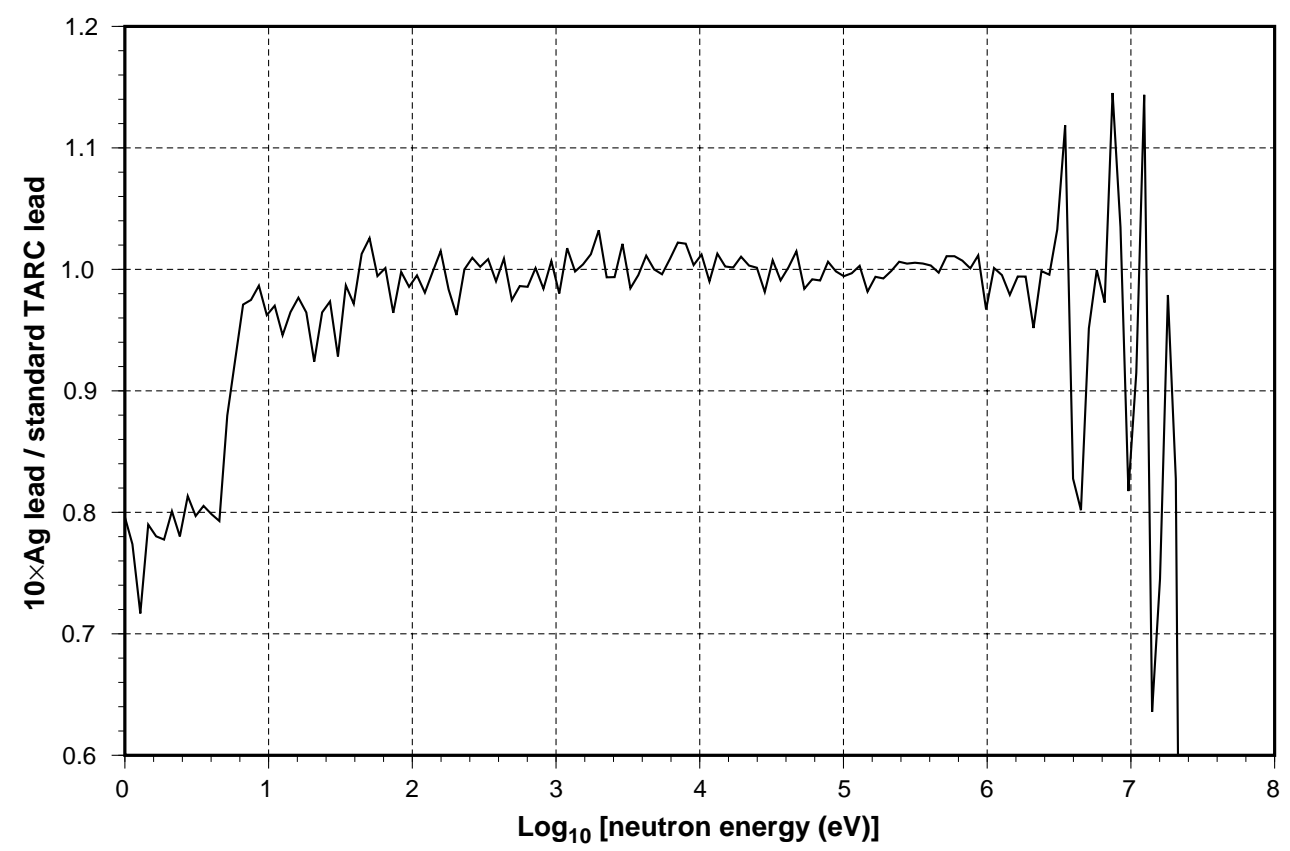

Fig. 17: Ratio of neutron fluences obtained with a modified silver concentration by a factor 10 with respect to the TARC nominal value. 
The total systematic uncertainty in the flux calculation, over the neutron energy range covered by TARC, from thermal neutrons to a few $\mathrm{MeV}$, even though hard to pin down precisely, is estimated to be not larger than $25 \%$.

\subsubsection{Comparison between fluences from $2.5 \mathrm{GeV} / \mathrm{c}$ and $3.5 \mathrm{GeV} / \mathrm{c}$ protons as function of} the neutron energy

It has always been assumed that the ratio of neutron fluences produced by protons of different energies is independent from neutron energy. We have verified, using our simulation, that this is indeed the case, at least in the neutron energy range between thermal and a few $\mathrm{MeV}$ (Fig. 18).

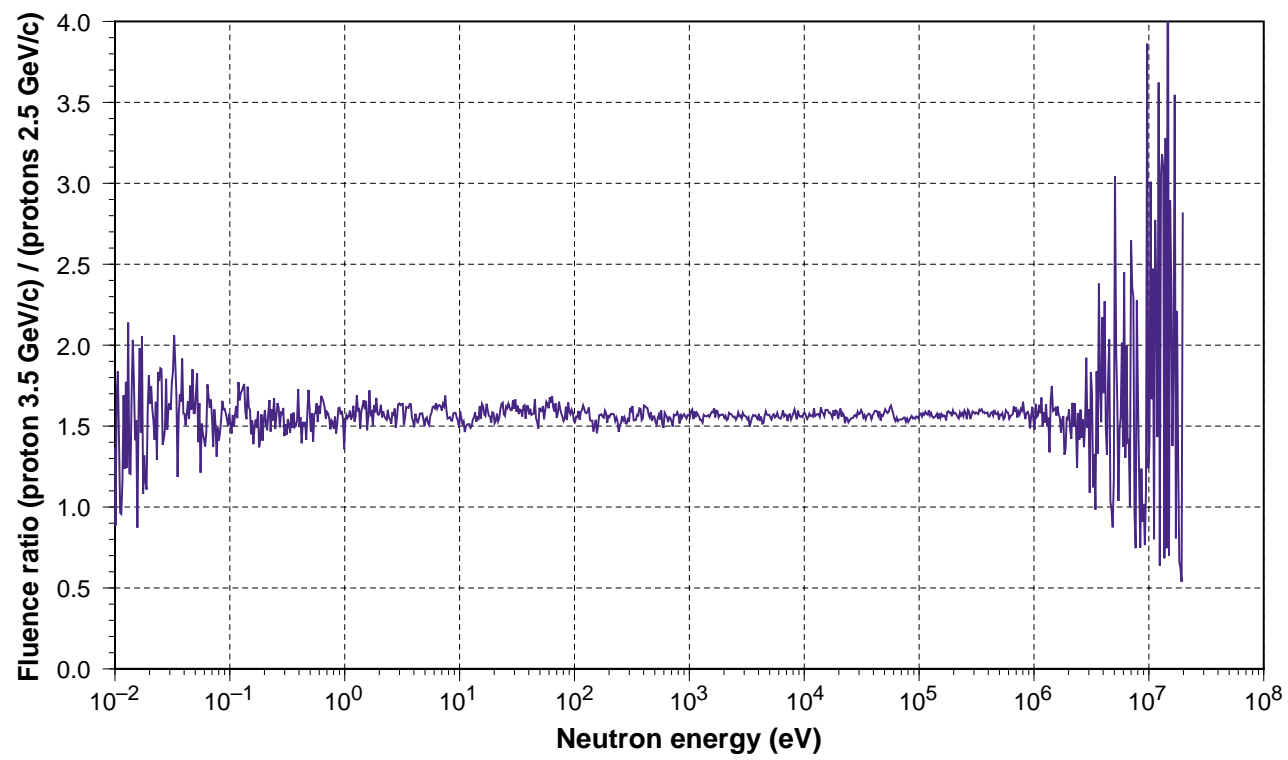

Fig. 18: Ratio of fluences produced by $3.5 \mathrm{GeV} / c$ and $2.5 \mathrm{GeV} / c$ protons in TARC, as a function of neutron energy (hole $10, z=+7.5 \mathrm{~cm}$ ).

These systematic checks, together with many more trivial checks, allowed us to develop confidence in the results obtained, and gave us a good understanding of the detailed neutron behaviour in lead, which is one of the main objectives of the TARC experiment.

\subsubsection{TARC geometry description}

The centre of the TARC reference system is at the geometrical centre of the lead volume and the beam is along the positive $z$ axis. The $y$ axis is along the vertical direction. The general layout of the assembly is shown in Fig. 19.

The 12 instrumentation holes and the beam hole have been included in the simulation. A virtual box of $15 \times 15 \mathrm{~cm}^{2}$ in cross-section and $300 \mathrm{~cm}$ in length is placed around each instrumented hole (diameter $64 \mathrm{~mm}$ ). This box is further subdivided into 20 slices along $z$, each one $15 \mathrm{~cm}$ long. The coordinates of the centres of the blocks in the TARC Monte Carlo reference system are listed in Table 3.

Concerning the computer simulation of the back-scattered neutrons from the surrounding case, a detailed description of the area surrounding the lead assembly is used [34] (see also Section 2).

A more complete description of the Monte Carlo technique developed both for the Energy Amplifier and for the TARC experiment can be found in Ref. [8]. 


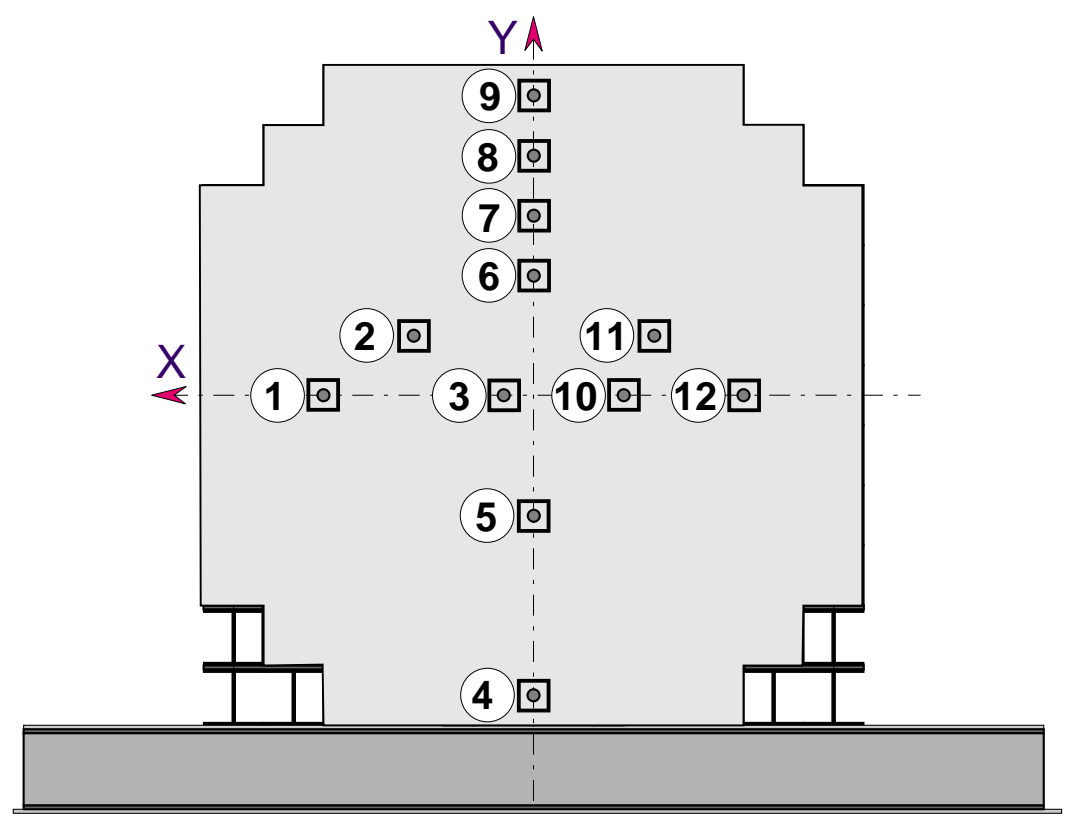

Fig. 19: Schematic view of the simulated lead assembly. The $z$-axis is along the beam direction (into the page).

Table 3: Summary of the coordinates of the centres of the 12 instrumented holes in the 334 ton lead assembly volume. The beam is introduced through a $77.2 \mathrm{~mm}$ diameter and $1.2 \mathrm{~m}$ long blind hole.

\begin{tabular}{c|c|c|c|c|c}
\hline Hole no. & $\boldsymbol{x}(\mathbf{m})$ & $\boldsymbol{y}(\mathbf{m})$ & Hole no. & $\boldsymbol{x}(\mathbf{m})$ & $\boldsymbol{y}(\mathbf{m})$ \\
\hline 1 & 1.05 & 0.00 & 2 & 0.60 & 0.30 \\
3 & 0.15 & 0.00 & 4 & 0.00 & -1.50 \\
5 & 0.00 & -0.60 & 6 & 0.00 & 0.60 \\
7 & 0.00 & 0.90 & 8 & 0.00 & 1.20 \\
9 & 0.00 & 1.50 & 10 & -0.45 & 0.00 \\
11 & -0.60 & 0.30 & 12 & -1.05 & 0.00 \\
\hline
\end{tabular}

\section{Study of the Energy-Time Correlation with $\mathrm{CeF}_{3}$ Counters}

\subsection{Introduction}

In the TARC slowing-down lead spectrometer, a correlation develops between the time at which a neutron is observed and its velocity, hence its kinetic energy [35]-[38]:

$$
t=\frac{2 \lambda_{\mathrm{s}}}{\xi}\left(\frac{1}{v}-\frac{1}{v_{0}}\right)
$$

where $\xi$, the average lethargy change, is given by:

$$
\xi \equiv 1+\frac{\alpha}{1-\alpha} \ln (\alpha) \approx 9.6 \times 10^{-3} \quad \text { with } \quad \alpha \equiv \frac{\left(m_{\mathrm{Pb}}-m_{\mathrm{n}}\right)^{2}}{\left(m_{\mathrm{Pb}}+m_{\mathrm{n}}\right)^{2}} .
$$

The diffusion mean free path $\lambda_{\mathrm{s}}$ of a neutron in the lead medium is practically constant over the energy range between $0.1 \mathrm{eV}$ and a few $\mathrm{keV} ; v$ is the velocity of the observed neutron at time $t$, and $v_{0}$ is the initial velocity corresponding to the energy $E_{0}$ at which the neutron was created at time $t=0 ; m_{\mathrm{n}}$ and $m_{\mathrm{Pb}}$ are respectively the neutron and lead nucleus masses. This 
relation can be rewritten in a slightly different form, relating the time of observation to the mean energy of the neutron:

$$
\bar{E}(t)=\frac{K}{\left(t+t_{0}\right)^{2}} \quad \text { where } \quad K=\frac{2 m_{\mathrm{n}} \lambda_{\mathrm{s}}^{2}}{\xi^{2}} \quad \text { and } \quad t_{0}=\frac{\lambda_{\mathrm{s}}}{\xi} \sqrt{\frac{2 m}{E_{0}}} .
$$

It is relation (4) which is used by slowing-down spectrometers, in particular for several detectors in TARC $\left(\mathrm{CeF}_{3}\right.$ counter, ${ }^{3} \mathrm{He}$ scintillation counter and ${ }^{6} \mathrm{Li} /{ }^{233} \mathrm{U}$ counter), to obtain the energy of neutrons from the measurement of the time between the arrival of the beam pulse, and the time of the neutron interaction in the detector.

The actual value of the $K$ parameter has been experimentally determined (see Section 4.3). The quantity $t_{0}$ can be considered as a time correction owing to the fact that the initial neutron is not created at infinite energy but at energy $E_{0}$ and at velocity $v_{0}$. In practice, all spallation neutrons are not created at the same energy, nor at the same place, nor at exactly the same time and, in addition, the first part of the slowing down process is dominated by inelastic scattering. Therefore, $t_{0}$ is a phenomenological constant which has to be estimated experimentally and, in our case, was checked using our simulation code and verified experimentally.

\subsection{Analysis of the simulated correlation function}

In practice, the energy-time relation is not a one-to-one relation but the mean value of a correlation function $C(E, t)$. The energy-time correlation function obtained by Monte Carlo simulation is presented in Fig. 20(a) and 20(b). These Monte Carlo data are displayed in a different way in Fig. 21, where one can observe that the quantity $\left(t+t_{0}\right) \sqrt{\bar{E}}=\sqrt{K}$ is almost constant over the energy range $0.1 \mathrm{eV}$ to $10 \mathrm{keV}$, with $K=173.3 \mathrm{keV} \times \mu \mathrm{s}^{2}$ and $t_{0}=0.37 \mu \mathrm{s}$. It is instructive to study more specifically the dispersion of $K$ by showing several slices at fixed energies of the distribution obtained (Fig. 22).

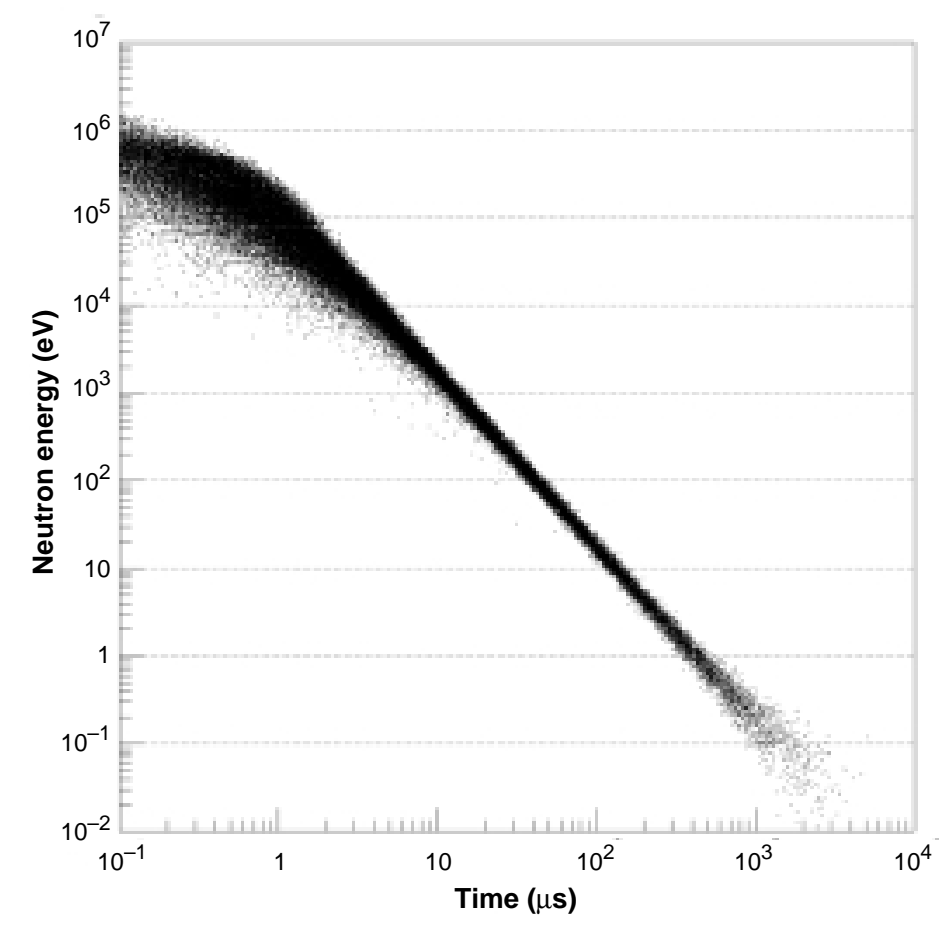

Fig. 20(a): Distribution of neutron energies and times from the Monte Carlo simulation. 


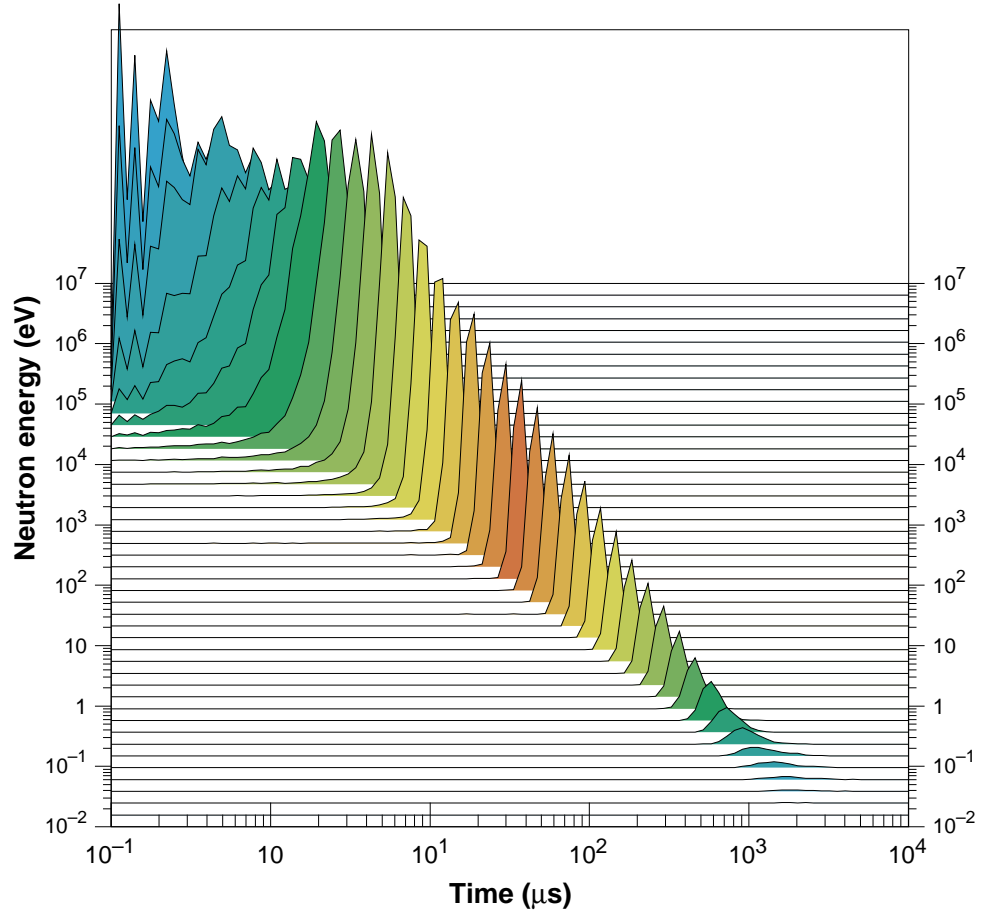

Fig. 20(b): Evolution of the width of the correlation function shown in part (a), as a function of time (time distribution for various neutron energy slices).

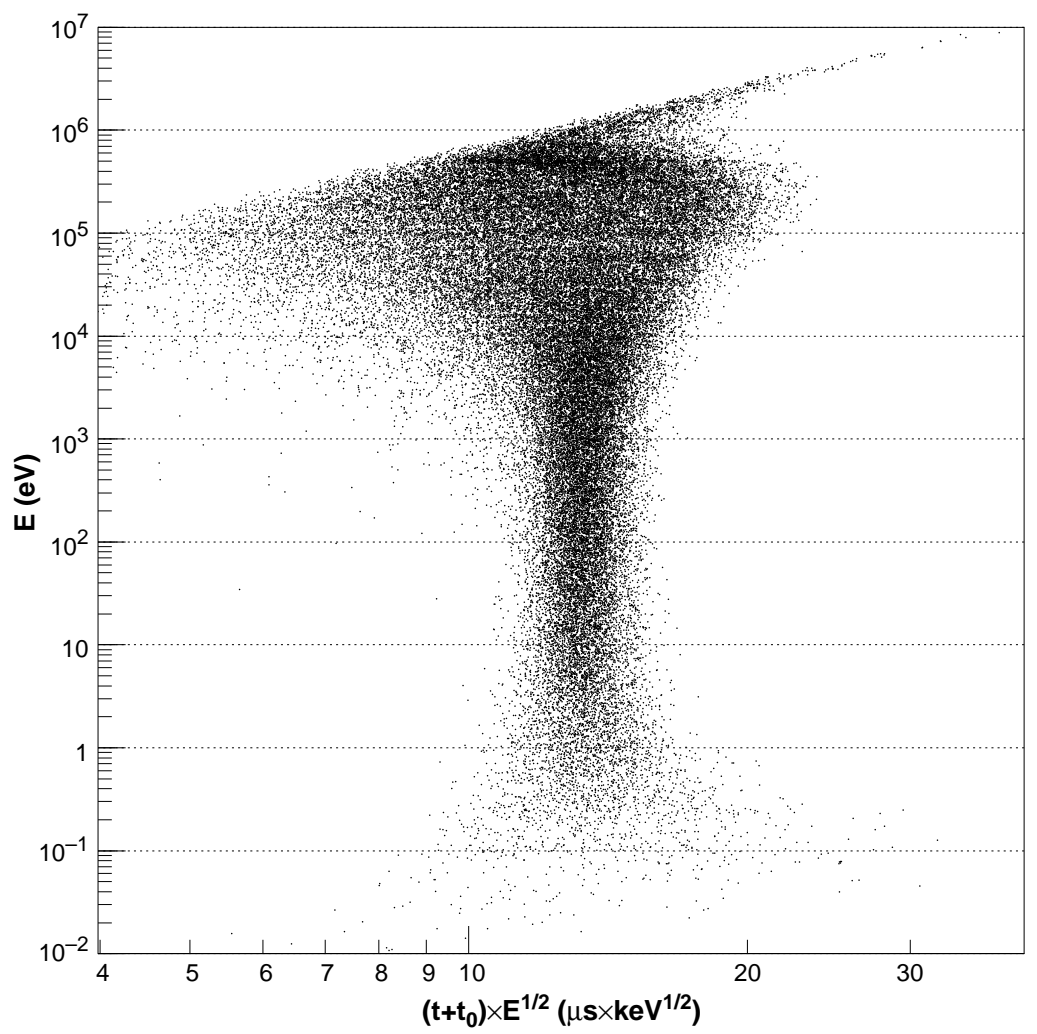

Fig. 21: Correlation function between energy $E$ and the quantity $\left(t+t_{0}\right) \sqrt{E}$ as obtained from our Monte Carlo simulation. 


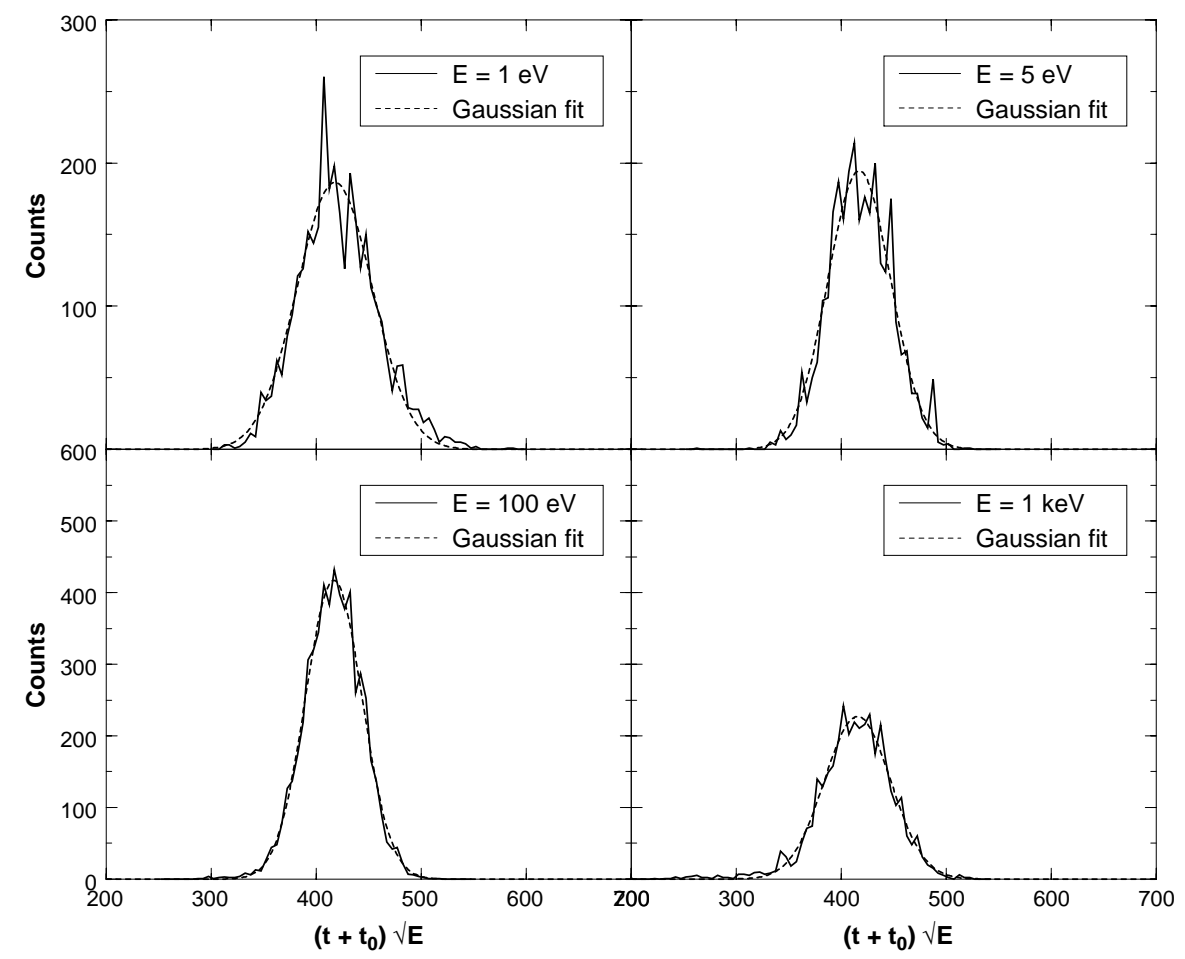

Fig. 22: Energy slices of the correlation function displayed in Fig. 21, as a function of $\left(t+t_{0}\right) \sqrt{E}=\sqrt{K}$ illustrating that $K$ is indeed constant and showing the dispersion of the energy-time correlation. Gaussian fits are superimposed.

A cut at fixed $t$ in the $C(E, t)$ correlation function gives a quasi-Gaussian energy curve with a typical spread $\sigma_{\mathrm{E}} / E=0.13$ between $3 \mathrm{eV}$ and $1 \mathrm{keV}$. A cut at fixed energy $E$ also gives a quasi-Gaussian time distribution with a typical spread $\sigma_{\mathrm{t}}(E)$. Assuming that the shape at fixed energy is given by:

$$
C(\bar{E}, t) \propto e^{-\frac{(t-\bar{t})^{2}}{2 \sigma_{\mathrm{t}}^{2}}} \quad \text { where } \quad \bar{t} \equiv \sqrt{\frac{K}{\bar{E}}}-t_{0}
$$

the variation of $\sigma_{\mathrm{t}}(E)$ with $E$ can be parametrized in the following way:

$$
\sigma_{\mathrm{t}}=\frac{25.23}{\sqrt{E}} \sqrt{1+\frac{1.7}{E}} \text { for } E<4 \mathrm{eV}
$$

and

$$
\sigma_{\mathrm{t}}=\frac{30.54}{\sqrt{E}}\left[1+0.306 \times \ln \left(\frac{E}{896}\right)\right] \text { for } 4 \mathrm{eV}<E<1600 \mathrm{eV}
$$

where $E$ is in $\mathrm{eV}$ and $\sigma_{\mathrm{t}}$ in $\mu \mathrm{s}$.

From the energy-time relation, in the low-energy region $E<4 \mathrm{eV}$, we can derive:

$$
\frac{\sigma_{\mathrm{E}}}{E}=2 \frac{\sigma_{\mathrm{t}}}{t}=0.121 \sqrt{1+\frac{1.7}{E(\mathrm{eV})}}
$$

which is consistent with the theoretical calculation derived by Bergmann [37] which gives:

$$
\frac{\sigma_{\mathrm{E}}}{E}=0.116 \sqrt{1+\frac{1.89}{E(\mathrm{eV})}} .
$$




\subsection{Experimental parameters of the correlation function}

When measuring a neutron interaction between times $t$ and $t+\Delta t$ its corresponding energy is located at $\bar{E}(t)$ within a quasi-Gaussian distribution. Using these properties we can characterize experimentally the energy-time correlation by measuring $K$ and the time dispersion $\sigma_{t}$ for well-defined neutron energies. Hence, for well-isolated strong and narrow known neutron capture resonances, $K$ and $\sigma_{\mathrm{t}}$ can be determined by detecting the time distribution of neutron captures at these resonances.

For instance, a resonance at $5 \mathrm{eV}$ with a width of $0.1 \mathrm{eV}$ is located at $t \approx 200 \mu$ s with a time-width of $16 \mu \mathrm{s}$. The $\mathrm{CeF}_{3}$ detector described below (see Section 7) detects the prompt $\gamma \mathrm{s}$ following the neutron captures with a time resolution of typically $0.2 \mu \mathrm{s}$. An example of a recorded time spectrum is given in Fig. 23 for a tantalum target.

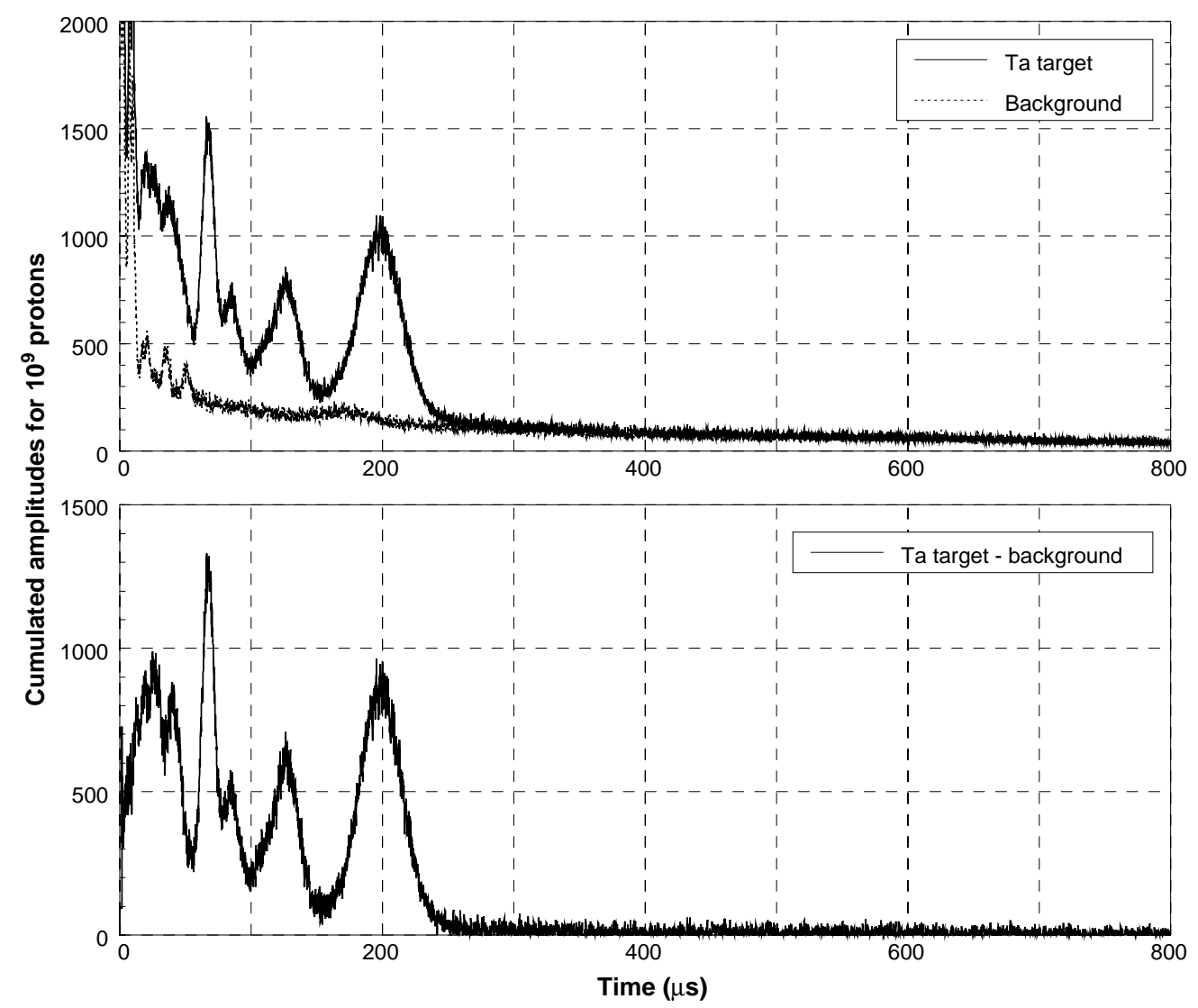

Fig. 23: Time spectra of neutron captures recorded with the $\mathrm{CeF}_{3}$ detector with and without a tantalum target. The lower spectrum corresponds to the captures in tantalum obtained after background subtraction.

In the case of tantalum, for the determination of $K$ and $\sigma_{t}$ we selected only the first well-isolated resonance at $4.28 \mathrm{eV}(t \approx 200 \mu \mathrm{s})$. After background subtraction, the selected peak is fitted with a Gaussian distribution superimposed onto a local exponential level:

$$
F(t)=\alpha e^{-\frac{t}{\tau}}+\beta e^{-\frac{\left(t-t_{\mathrm{i}}\right)^{2}}{2 \sigma_{\mathrm{t}}^{2}\left(t_{\mathrm{i}}\right)}} .
$$

This type of fit gives, for the resonance studied, at energy $E_{\mathrm{i}}$, both the associated time $t_{\mathrm{i}}$ and the dispersion $\sigma_{t}\left(t_{\mathrm{i}}\right)$. 
The following targets ${ }^{\text {nat }} \mathrm{Ta},{ }^{\text {nat }} \mathrm{Au},{ }^{\text {nat }} \mathrm{Ag},{ }^{\text {nat }} \mathrm{In},{ }^{\text {nat }} \mathrm{Mn}$ and ${ }^{99} \mathrm{Tc}$ have been used. For these targets the selected resonances are given in Table 4 with the positions in the lead volume where the measurements were made.

Table 4: Experimental parameters for the determination of the energy-time correlation function, including the position at which the measurement was performed

\begin{tabular}{l|c|c|c|c}
\hline $\begin{array}{c}\text { Target } \\
(\text { resonance energy) }\end{array}$ & $\begin{array}{c}\text { Time recorded } \\
(\mu \mathbf{s})\end{array}$ & $\begin{array}{c}\text { Width } \\
(\mu \mathbf{s})\end{array}$ & $\begin{array}{c}\text { Position } \\
{[\text { Hole, } \boldsymbol{z}(\mathbf{c m})]}\end{array}$ & $\begin{array}{c}\boldsymbol{K} \text { value } \\
\left(\mathbf{k e V} \times \mu \mathbf{s}^{\mathbf{2}}\right)\end{array}$ \\
\hline${ }^{181} \mathrm{Ta}(4.28 \mathrm{eV})$ & $199.5 \pm 1$ & $16 \pm 2.7$ & $10,7.5$ & $171 \pm 1.8$ \\
${ }^{197} \mathrm{Au}(4.906 \mathrm{eV})$ & $187.1 \pm 1$ & $17.5 \pm 2.8$ & $5,82.5$ & $172.4 \pm 1.8$ \\
${ }^{109} \mathrm{Ag} \quad(5.19 \mathrm{eV})$ & $180.7 \pm 1$ & $13.1 \pm 2.1$ & $12,7.5$ & $170.2 \pm 1.8$ \\
${ }^{99} \mathrm{Tc} \quad(5.584 \mathrm{eV})$ & $175.2 \pm 0.9$ & $14.7 \pm 2.8$ & $10,7.5$ & $172.2 \pm 1.8$ \\
${ }^{115} \mathrm{In} \quad(9.07 \mathrm{eV})$ & $136.9 \pm 0.7$ & $8.84 \pm 1.1$ & $5,82.5$ & $170.9 \pm 1.9$ \\
${ }^{107} \mathrm{Ag} \quad(16.30 \mathrm{eV})$ & $102.3 \pm 0.6$ & $6.0 \pm 0.7$ & $12,7.5$ & $171.9 \pm 2$ \\
${ }^{55} \mathrm{Mn}(337 \mathrm{eV})$ & $22.4 \pm 0.3$ & $1.9 \pm 0.4$ & $5,82.5$ & $174.4 \pm 5$ \\
\hline
\end{tabular}

The extracted energy-time correlation is shown in Fig. 24. Using relation (4) the $K$ coefficient can be determined for each individual resonance. Figure 25 shows the variation of the extracted values obtained in different positions in the lead block as a function of the resonance energy. Throughout this study we fixed the $t_{0}$ parameter at $0.37 \mu \mathrm{s}$, the value given by a full Monte Carlo simulation. Note that for all resonances except that at $337 \mathrm{eV}$ of ${ }^{55} \mathrm{Mn}$ the $K$ determination is almost insensitive to $t_{0}$, as expected since their $t_{\mathrm{i}}$ values are large compared to $t_{0}$ (larger that $90 \mu \mathrm{s}$ ).

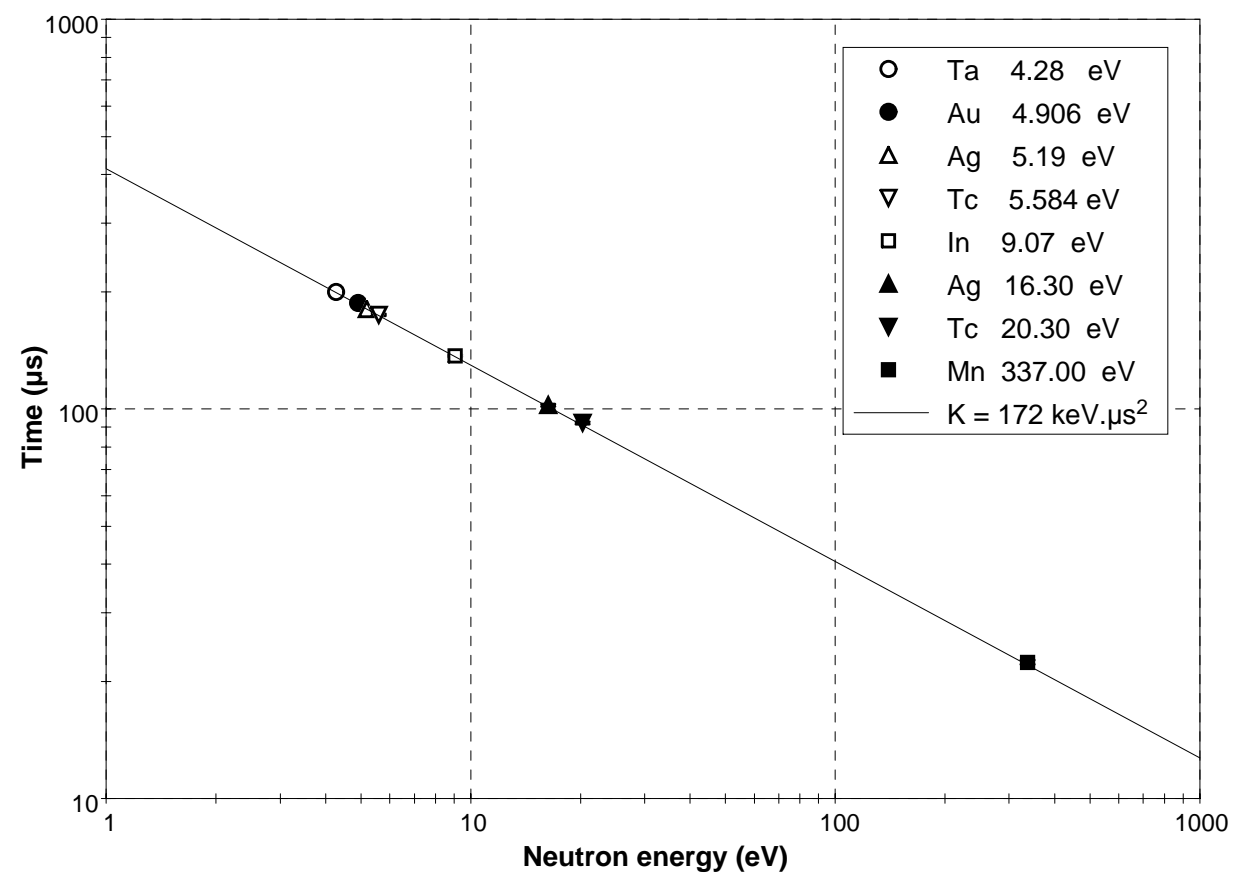

Fig. 24: Time vs energy for the selected resonances. Note that the error bars are smaller than the symbols. The straight line is the best $\log -\log$ fit. 


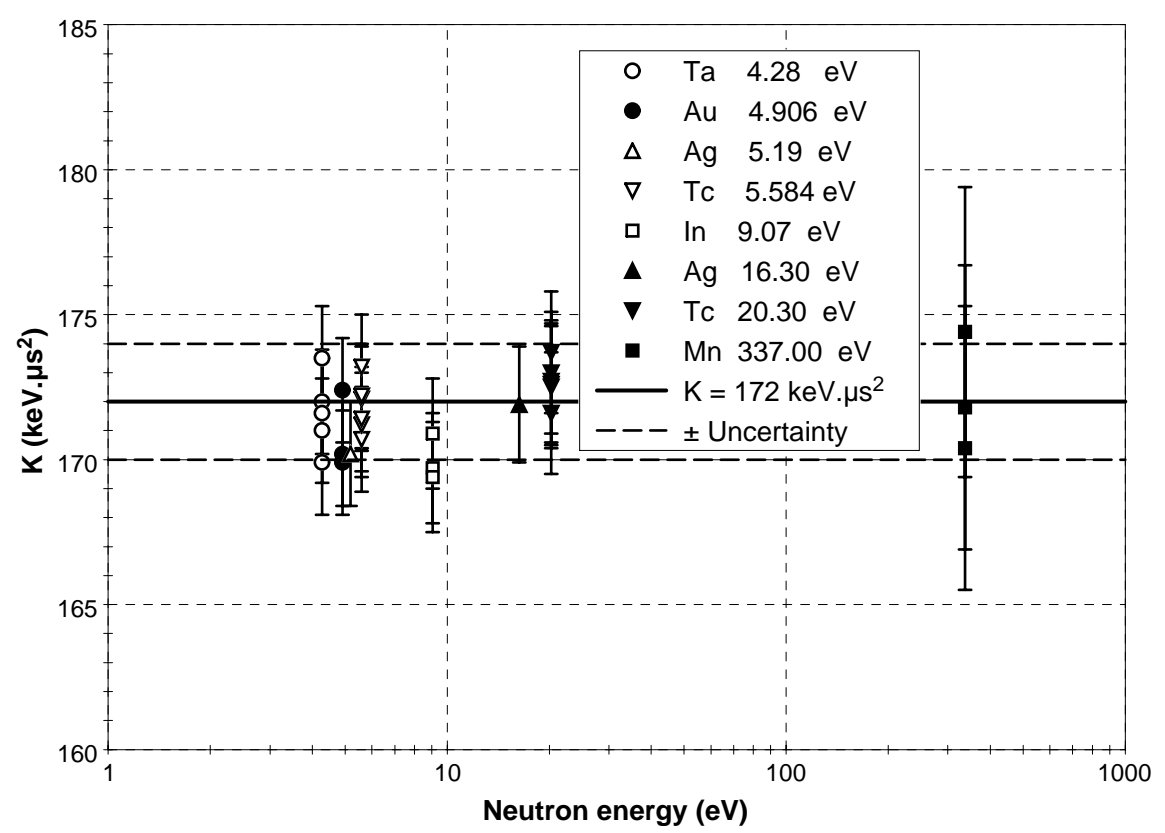

Fig. 25: Extracted experimental $K$-values for different energies at different positions in the lead volume.

From all $K$ determinations, using the analysis of the various resonances mentioned above, the final experimental value of $K$ is:

$$
K_{\text {exp }}=172 \pm 2 \mathrm{keV} \times \mu \mathrm{s}^{2}
$$

in agreement with the value extracted from fitting the corresponding simulation data:

$$
K_{\text {simulation }}=173 \pm 3 \mathrm{keV} \times \mu \mathrm{s}^{2} .
$$

Note that this experimental value of $K$ is somewhat different from the estimation of $K=165 \mathrm{keV} \times \mu \mathrm{s}^{2}$ used by Chou and Werle [39] and we can only speculate that the difference is due to their much smaller lead volume since they do not describe their energy-time calibration nor the time distribution of their neutron source and the exact chemical purity of their lead.

We could not measure $t_{0}$ with high precision, even with the $337 \mathrm{eV}$ resonance of ${ }^{55} \mathrm{Mn}$. The corresponding $t+t_{0}$ value is $22.6 \mu \mathrm{s}$ and the time accuracy of the $t_{\mathrm{i}}$ determination is estimated to $0.2 \mu \mathrm{s}$. We checked that the value $t_{0}=0.37 \mu$ s corresponds to the central value of ${ }^{55} \mathrm{Mn}$ measurements. The error on $t_{0}$ is then set at $\pm 0.2 \mu$ s and is included in the time measurement error.

Using the same well-separated neutron capture resonances in eight different targets it has been possible to measure the $\sigma_{\mathrm{t}}$ dispersion at eight different neutron energies. After conversion of the time dispersion $\sigma_{\mathrm{t}}$ to energy dispersion $\sigma_{\mathrm{E}}$, the relative energy dispersion $\sigma_{\mathrm{E}} / E$ was plotted versus the neutron energy (Fig. 26). The comparison with the simulation results shows a good overall agreement, although experimental values of $\sigma_{\mathrm{E}}$ seem to be $15 \%$ higher than the simulation data. This small discrepancy may be associated with flux perturbations due to the presence of the detector material and target and is of negligible consequence for the use of the energy-time relation for TARC. 


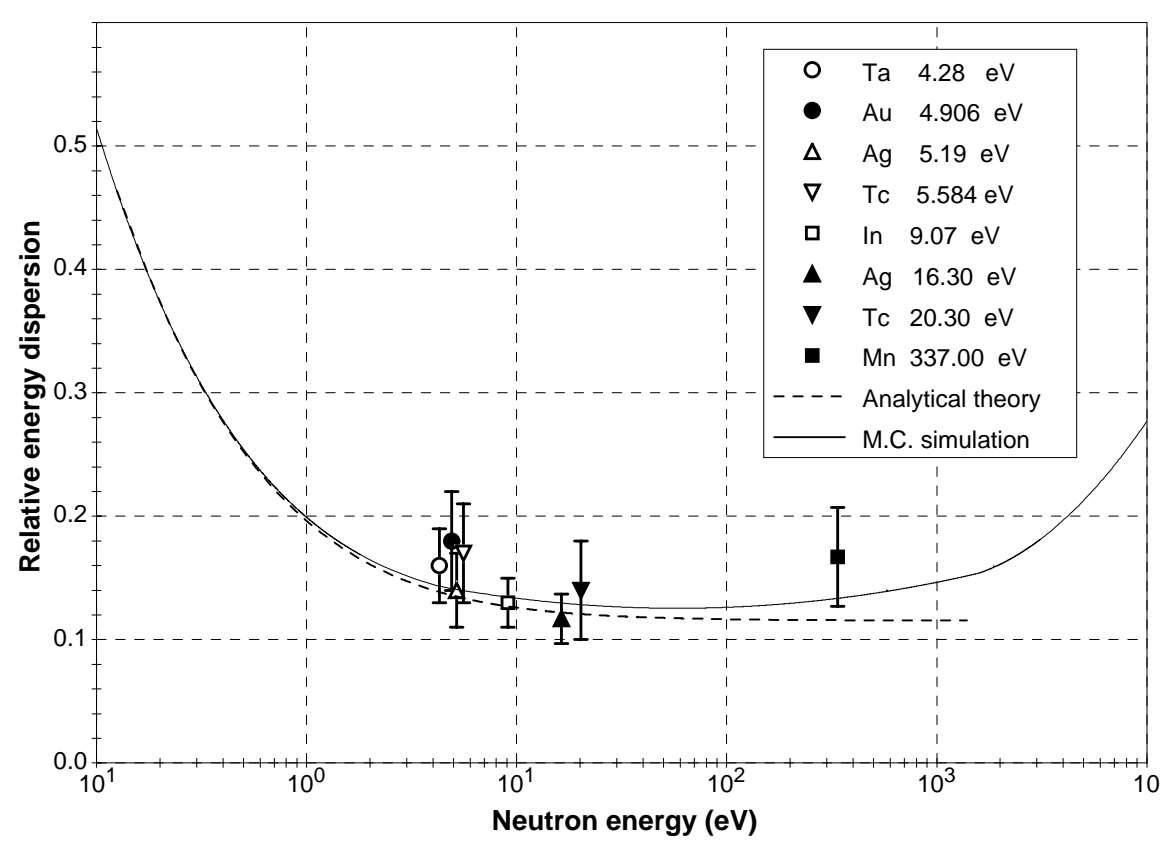

Fig. 26: Extracted values of the correlation function dispersion $\sigma(E) / E$ for different resonance energies, compared to the simulation and the analytical calculation from Ref. [37].

\subsection{Conclusion}

The energy-time correlation function has been experimentally determined for a number of neutron energies and positions in the lead volume. Experimental results are in very good agreement with complete simulation calculations. Recalling that the time width of the neutron source is very small (20 ns) this systematic study shows that the actual TARC lead volume is a very efficient neutron spectrometer with a precisely determined energy-time correlation function, well reproduced by Monte Carlo simulation. The energy-time calibration obtained here is then used by the other detectors measuring time to obtain neutron energy $\left({ }^{6} \mathrm{Li} /{ }^{233} \mathrm{U}\right.$, ${ }^{3} \mathrm{He}$ scintillation mode).

\section{Neutron Fluence Measurements}

\subsection{Neutron fluence and neutron flux}

\subsubsection{Definition of neutron fluence}

For a number of people not expert in neutron physics, it is easy to get confused by the definition of neutron fluence and neutron flux. Therefore, in an attempt to save time and effort for the reader, we propose a few practical remarks on the notion of neutron fluence and neutron flux, some of them inspired from Ref. [40].

First, we must apologize for still using sometimes the word 'flux' which the International Commission for Radiological Units (ICRU) has banned and replaced by 'fluence rate', but which we still find quite convenient.

Let us recall here that for monoenergetic neutrons of velocity $V(\mathrm{~m} / \mathrm{s})$, and of density $n$ (neutrons $/ \mathrm{m}^{3}$ ), the neutron flux is defined as $\phi=V \times n$ and is a quantity such that, upon multiplying by the macroscopic cross-section $\Sigma$ for the process considered ( $\Sigma \equiv N \times \sigma$, where $N$ is the number of scattering centres per unit volume, and $\sigma$ the cross-section for the process), one obtains the neutron reaction rate per unit volume. 
This flux quantity must not be confused with the rate of particles crossing a surface element, which is a 'current' and which depends on the orientation of the surface with respect to the direction of the particles.

We call 'fluence' the integral of the flux over a certain period of time, which is what we measure in TARC. We are interested in the neutron fluence produced by a proton of a given energy. In practice, we measure the 'fluence' per number of incident protons on the lead assembly. Most of our results are given as a fluence per $10^{9}$ protons, in practice, integrating the flux over time from zero to infinity.

In TARC we used several different practical ways to compute the neutron fluence, in some cases as a cross-check that the procedures were correct:

(1) $\mathrm{d} N / \mathrm{d} S_{\text {perp }}$ is calculated as the number of neutrons crossing a small surface element $\mathrm{d} S$, with $\mathrm{d} S_{\text {perp }}=\mathrm{d} S \times \cos \theta$, where $\theta$ is the angle between the neutron direction and the normal to the surface element. One can note that in this process, considering all neutron directions, if the small surface is a disk, then it will describe a spherical surface in space of area $4 \times \mathrm{d} S$. The neutron flux $\phi$ will be the number of neutrons penetrating this spherical surface per unit time from all sides (in an isotropic flux, one has $\phi / 2$ neutrons penetrating a $1 \mathrm{~cm}^{2}$ disk surface per second).

(2) It is more practical in Monte Carlo calculations to compute the average fluence in a volume element $\mathrm{d} V$ as $\mathrm{d} \ell / \mathrm{d} V$, where $\mathrm{d} \ell$ is the total track length of neutrons in the volume element $\mathrm{d} V$. If the space distribution of neutrons does not change too abruptly, this allows one, by selecting appropriate volume elements, to obtain better statistics. It is clear that the larger the region, the larger the systematic uncertainty on the flux. In TARC, we have tested several volumes (sphere with diameter equal to the diameter of one hole, cylinder of length $15 \mathrm{~cm}$ with the same diameter as a hole, etc.) and found that the difference in flux obtained was negligible compared to usual statistical errors.

(3) In the data we usually record the number of interactions in a detector, therefore, we compute the neutron fluence as the quantity $(1 / \Sigma) \times \mathrm{d} N / \mathrm{d} V$, where $\mathrm{d} N$ is the number of interactions in volume element $\mathrm{d} V$.

Of course, the three methods are equivalent. In this TARC report, we usually quote the quantity $F$ for fluence and $\phi$ for flux. Please note also that the isolethargic distribution $\mathrm{d} F / \mathrm{d} \ln (E)$ is equal to $E \times \mathrm{d} F / \mathrm{d} E$ which in practice means that one is taking bins which have a constant width in the natural logarithm of energy.

\subsubsection{Properties of lead}

Lead plays the major role in ARC, by providing neutrons by spallation, by having the appropriate properties already discussed in Section 1, in particular, its transparency to neutrons and its very uniform and high elastic scattering cross-section shown in Fig. 27, and its heavy atomic mass. It is these properties which determine the characteristics of the neutron fluence in TARC. 


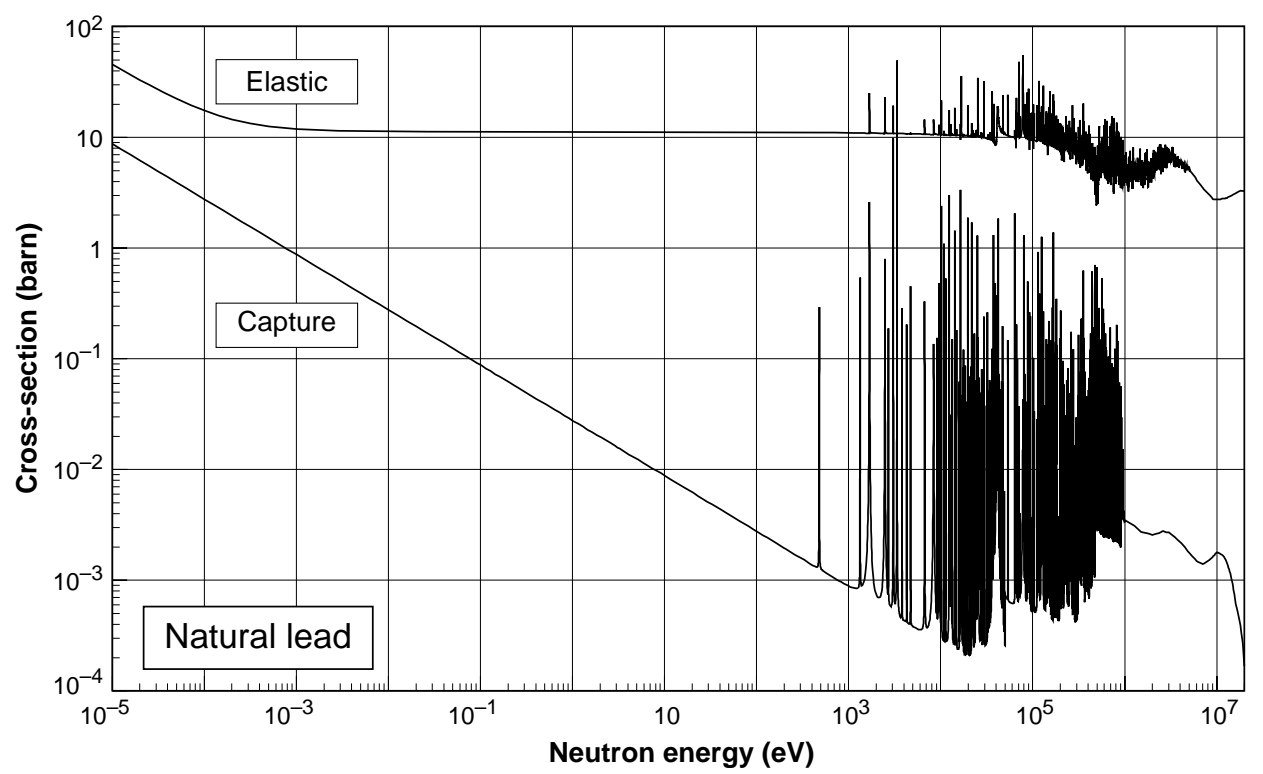

Fig. 27: Neutron capture and neutron elastic scattering cross-sections in natural lead (from the JENDL-3.2 database).

\subsection{Low-energy neutrons $\left(E_{\mathbf{n}} \leq 50 \mathrm{keV}\right)$ using energy-time correlation}

\subsection{1 ${ }^{6} \mathrm{Li} /{ }^{233} \mathrm{U}$ target silicon detectors}

The spallation neutrons, generated at time zero by $\mathrm{GeV}$ protons hitting the lead volume, give at position $\vec{r}$ a neutron flux $\phi(E, t, \vec{r})$ which is measured with a detection system using the exothermic reactions ${ }^{6} \mathrm{Li}(\mathrm{n}, \mathrm{t}) \alpha$ or ${ }^{233} \mathrm{U}$ (n,fission). The reaction rate versus time is proportional to the quantity $\phi(E, t, \vec{r}) \sigma(E)$. Provided the cross-section $\sigma(E)$ is known, the measurement of the reaction rate gives an experimental access to the quantity $\phi(E, t, \vec{r})$ as explained in detail below.

\subsubsection{The silicon detector and data recording}

The charged particles produced in the reactions ${ }^{6} \mathrm{Li}(\mathrm{n}, \mathrm{t}) \alpha(Q=4780 \mathrm{keV})$ or ${ }^{233} \mathrm{U}(\mathrm{n}$,fission) $(Q=180 \mathrm{MeV})$ are recorded by two silicon detectors (Fig. 28) (with surface of $10 \times 10 \mathrm{~mm}^{2}$ and thickness $300 \mu \mathrm{m}$ ). Two thin-foil targets of $1 \times 1 \mathrm{~cm}^{2}$ separated by a $0.1 \mathrm{~mm}$ foil of lead, are inserted between two silicon detectors. The ${ }^{6} \mathrm{Li}$ target is made of a lithium $\left(95 \%\right.$ of $\left.{ }^{6} \mathrm{Li}\right)$ fluoride (LiF) layer of either $50 \mu \mathrm{g} / \mathrm{cm}^{2}$ or $100 \mu \mathrm{g} / \mathrm{cm}^{2}$ deposited on a $50 \mu \mathrm{g} / \mathrm{cm}^{2}$ polyethylene backing. The ${ }^{233} \mathrm{U}$ target of $200 \mu \mathrm{g} / \mathrm{cm}^{2}$ is pure ${ }^{233} \mathrm{U}$ electro-deposited on a $200 \mu \mathrm{m}$ thick aluminium foil.

The output signal from the charge-preamplifier is encoded, after amplification, by an 8-bit flash-ADC (8192 channels, $0.1 \mu \mathrm{s} / \mathrm{channel}$ ). Examples of flash records are shown on Fig. 29 where alpha particles, tritons or fission fragments can be clearly identified. The beam intensity in this experiment ranges typically from $3 \times 10^{7}$ to a few $10^{8}$ protons/shot.

A threshold on the amplitude spectra (Fig. 30) selects without ambiguity the charged particle signals: $\alpha$ 's, tritons or fission products. After processing of the data, time spectra of detected reactions by unit time $n_{\text {det }}(t)$, normalized to $10^{9}$ protons are obtained (Fig. 31 ). 


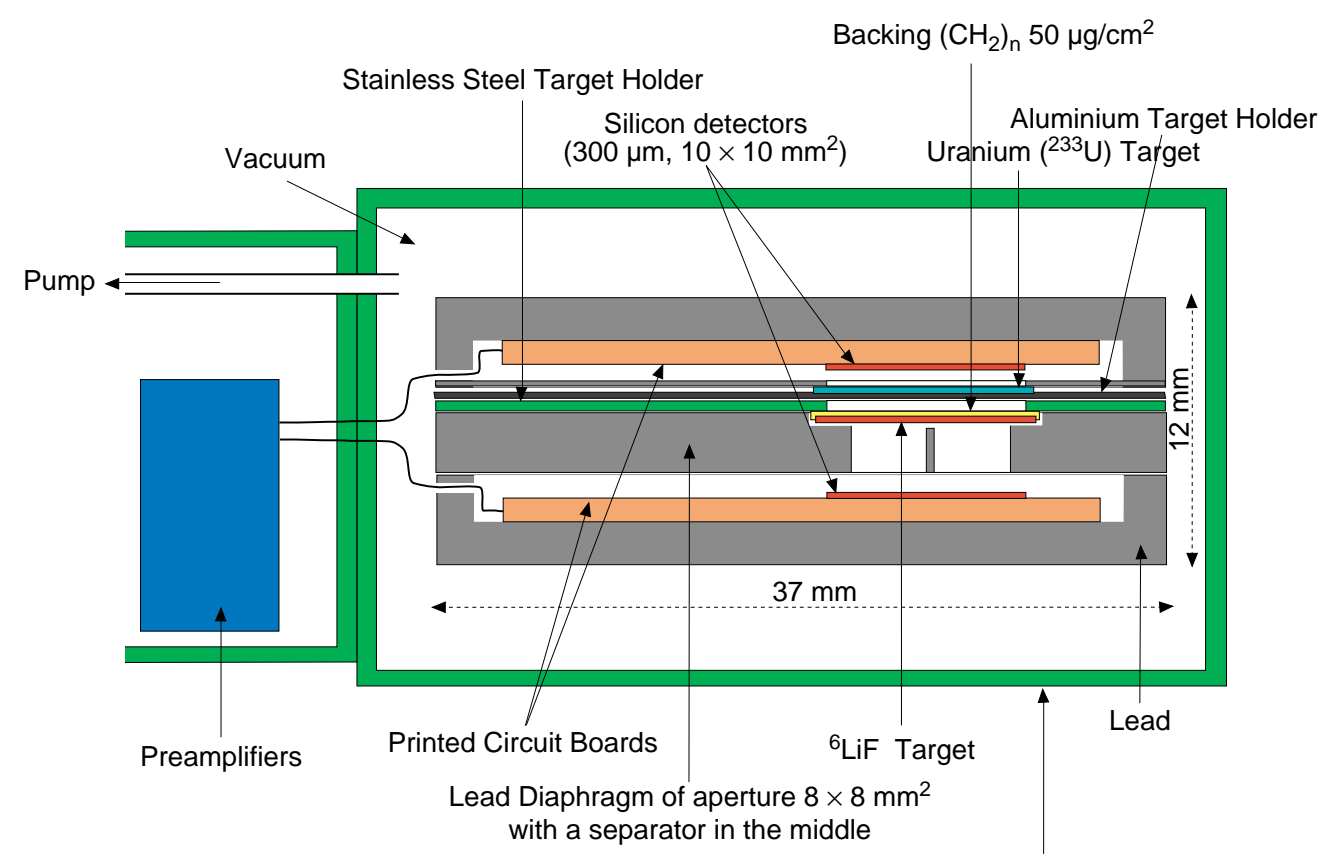

Stainless Steel Cylinder of $\varnothing 25 \mathrm{~mm}$

Fig. 28: Schematics of the silicon-detector ensemble showing both the ${ }^{233} \mathrm{U}$ and ${ }^{6} \mathrm{LiF}$ targets.

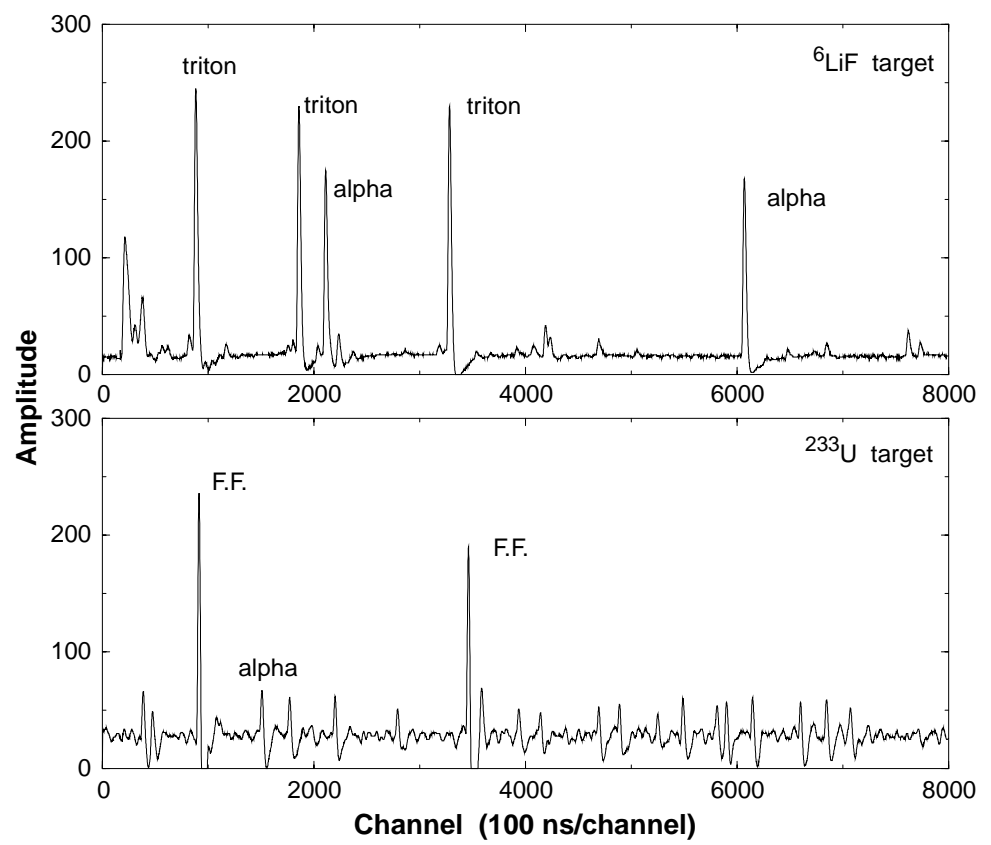

Fig. 29: Examples of flash-ADC recordings for ${ }^{6} \mathrm{Li}$ and ${ }^{233} \mathrm{U}$ targets. In the ${ }^{233} \mathrm{U}$ target flash, small amplitude pulses correspond to $\alpha$-particles emitted by ${ }^{233} \mathrm{U}$. The electronic gain used with the ${ }^{233} \mathrm{U}$ target is lower than for the ${ }^{6} \mathrm{LiF}$ target. The counting of $\alpha$ 's from ${ }^{233} \mathrm{U}$ was made with special runs with a higher gain to separate better the $\alpha$ 's from the noise. 


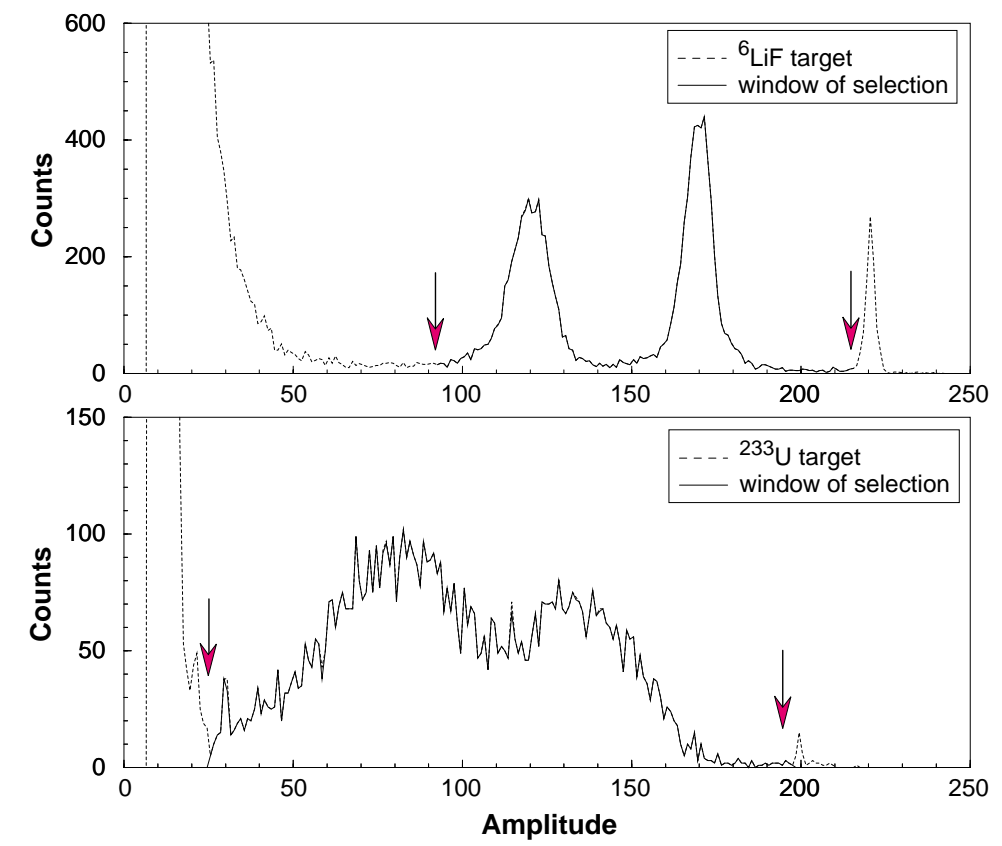

Fig. 30: Amplitude spectra for ${ }^{6} \mathrm{Li}$ (upper histogram) and ${ }^{233} \mathrm{U}$ (lower histogram) targets, showing the selection windows. The higher energy peak in the upper spectrum is produced by a pulser.

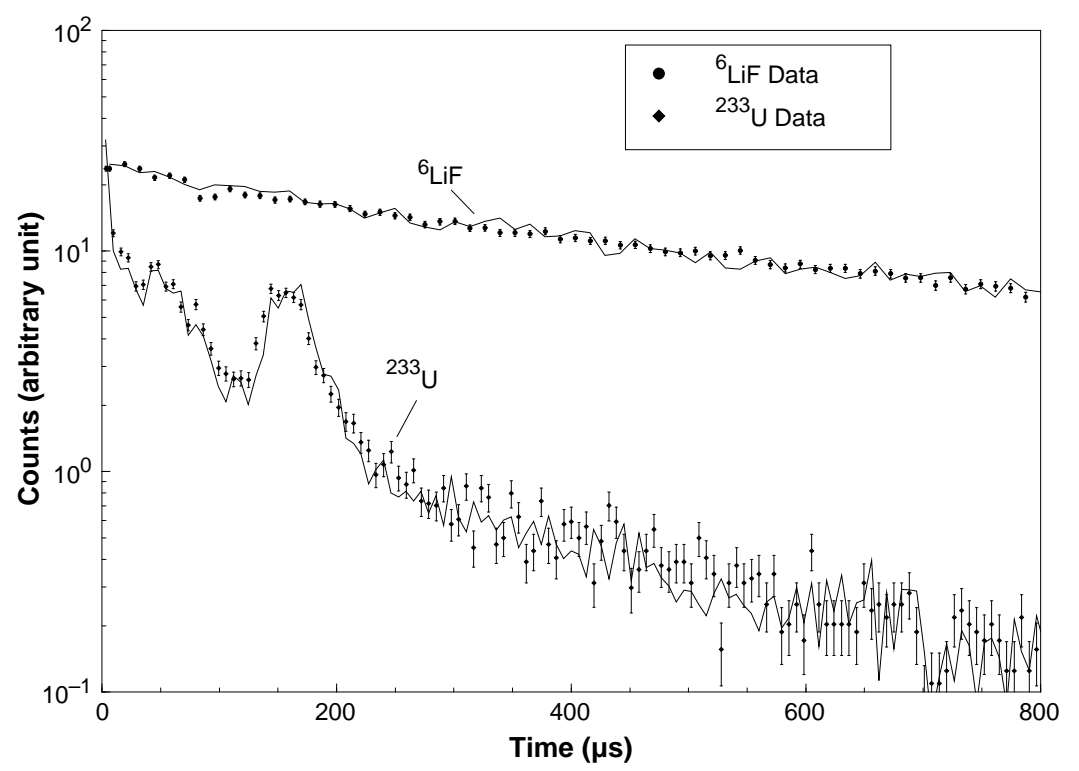

Fig. 31: Typical ${ }^{6} \operatorname{Li}(\mathrm{n}, \mathrm{t})$ and ${ }^{233} \mathrm{U}(\mathrm{n}, \mathrm{f})$ reactions time spectra recorded with the silicon detector and comparison with the simulation (lines).

\subsubsection{Extraction of neutron fluences from experimental measurements}

The number of detected reactions $\Delta n_{\text {det }}=n_{\operatorname{det}}(t, \vec{r}) \Delta t$ detected in the time interval $(t, t+\Delta t)$, for a given number of incident protons $\left(10^{9}\right)$, is given by:

$$
\Delta n_{\text {det }}(t, \vec{r})=\varepsilon N_{\text {target }} \Delta t \int_{0}^{\infty} \phi(E, t, \vec{r}) \sigma(E) \mathrm{d} E,
$$

where $N_{\text {target }}$ is the number of target atoms, $\phi(E, t, \vec{r})$ is the mean neutron flux in the target volume at position $\vec{r}, \sigma(E)$ is the ${ }^{6} \mathrm{Li}(\mathrm{n}, \mathrm{t})$ or ${ }^{233} \mathrm{U}(\mathrm{n}, \mathrm{f})$ reaction cross-section. Because of the target's small thickness and the relatively small amount of detector material, with small 
neutron cross-sections, the local flux is not significantly perturbed by the target itself. This has been checked explicitly by Monte Carlo simulation.

The expression (11) shows that for a time interval $\Delta t$ around $t$, the number of detected reactions depend on the integral of $\phi(E, t, \vec{r}) \sigma(E)$ over all neutron energies present at $t$ in the interval $\Delta t$. We define, for a given $t$ value, the flux $\phi(E, t, \vec{r})$ as:

$$
\phi(E, t, \vec{r}) \Delta t=\Delta F(t, \vec{r}) M(E, t),
$$

where $M(E, t)$ is a normalized probability distribution with $\int_{0}^{\infty} M(E, t) \mathrm{d} E=1$ which describes the energy distribution of neutrons detected in the interval $(t, t+\Delta t)$. From relation (12) one deduces:

$$
\Delta F(t, \vec{r})=\Delta t \frac{\int_{0}^{\infty} \phi(E, t, \vec{r}) \sigma(E) \mathrm{d} E}{\int_{0}^{\infty} M(E, t) \sigma(E) \mathrm{d} E},
$$

$\Delta F(t, \vec{r})$ appears to be the differential of the neutron fluence in the $\Delta t$ interval (integrated on $E)$. The relation (11) can now be written as:

$$
\Delta n_{\text {det }}(t, \vec{r})=\varepsilon N_{\text {target }} \Delta F(t, \vec{r}) \int_{0}^{\infty} M(E, t) \sigma(E) \mathrm{d} E .
$$

We then define a mean cross-section $\bar{\sigma}$ :

$$
\bar{\sigma}=\int_{0}^{\infty} M(E, t) \sigma(E) \mathrm{d} E .
$$

This mean cross-section at time $t$ is assumed to be the mean cross-section at energy $\bar{E}(t)$. This convolution is computed using the analytical parametrization of $M(E, t)$ (see Section 4) and the JENDL database cross-section for $(\mathrm{n}, \mathrm{t})$ or $(\mathrm{n}, \mathrm{f})$ reactions. The fluence $\Delta F(t, \vec{r})$ can be converted into $\Delta F(\bar{E}, \vec{r})$ using the $\bar{E}(t)$ relation [Eq. (4), Section 4]. It corresponds to the fluence measured in the interval $(t, t+\Delta t)$. The differential neutron fluence $f(\bar{E}, \vec{r})$ at energy $\bar{E}(t)$ is then given by:

$$
f(\bar{E}, \vec{r})=\frac{\Delta F(t, \vec{r})}{\Delta \bar{E}}=\frac{1}{\varepsilon N_{\text {target }}} \frac{n_{\mathrm{det}}(t, \vec{r})}{\bar{\sigma}}\left|\frac{\Delta t}{\Delta \bar{E}}\right| .
$$

In order to determine absolute values of fluences, we still have to determine the quantity $\varepsilon N_{\text {target }}$.

5.2.1.2.1 Experimental determination of $\varepsilon N_{\text {target }}$

This determination is made by using the ${ }^{233} \mathrm{U}$ target-Si detector ensemble, in which the $\mathrm{Si}$ junction is detecting both $\alpha$ 's coming from natural decay of ${ }^{233} \mathrm{U}$ and fission fragment (ff) with respective efficiency $\varepsilon_{\alpha}$ and $\varepsilon_{\mathrm{ff}}$. The number of $\alpha$ 's detected per second is given by:

$$
n_{\alpha}=\frac{\Delta N_{\alpha}}{\Delta t}=\lambda_{\mathrm{U} 3} \varepsilon_{\alpha} N_{\mathrm{U} 3}
$$

where $\lambda_{\mathrm{U} 3}$ is the radioactive ${ }^{233} \mathrm{U}$ decay constant and $N_{\mathrm{U} 3}$ the number of ${ }^{233} \mathrm{U}$ atoms. The number $n_{\alpha}$ can be measured precisely, $\lambda_{\mathrm{U} 3}$ is well known.

The number of fission fragments detected per unit of time (shown in Fig. 31) is given by:

$$
n_{\mathrm{det}}(t, \vec{r}) \Delta t=\varepsilon_{\mathrm{ff}} N_{\mathrm{U} 3} \bar{\sigma}(\bar{E}) f(\bar{E}, \vec{r}) \Delta \bar{E} .
$$

The efficiencies $\varepsilon_{\mathrm{ff}}$ and $\varepsilon_{\alpha}$ can be taken as equal to a common value $\varepsilon$ because the ${ }^{233} \mathrm{U}$ target is very thin $\left(200 \mu \mathrm{g} / \mathrm{cm}^{2}\right)$ and because collimators in front of the Si detector stop the $\alpha$ 
and fission fragments with large incident angles. The quantity $\varepsilon \times N_{\mathrm{U} 3}$ is extracted from relation (17). Using relation (18), $f(\bar{E}, \vec{r})$ is given by:

$$
f(\bar{E}, \vec{r})=\frac{\lambda_{\mathrm{U} 3}}{n_{\alpha}} \frac{n_{\mathrm{det}}(t, \vec{r})}{\bar{\sigma}}\left|\frac{\Delta t}{\Delta \bar{E}}\right| .
$$

The detection efficiency of the ${ }^{6} \mathrm{Li}$-target-Si junction ensemble was determined assuming that the same neutron flux is measured both by ${ }^{233} \mathrm{U}$ and ${ }^{6} \mathrm{Li}$ detectors located in the same position. This normalization procedure introduces a systematic error estimated at $6 \%$.

\subsection{Errors on fluence determination with ${ }^{6} \mathrm{Li} /{ }^{233} \mathrm{U}$ silicon detector}

The differential fluence $f(\bar{E}, \vec{r})$ is determined by time measurement using relation (19). This formula can be expressed as a function of time using the energy-time relation determined with the $\mathrm{CeF}_{3}$ measurements (Section 4). Assuming that for the main part of the measurements, the cross-section $\bar{\sigma}$ (both for ${ }^{6} \mathrm{Li}$ or ${ }^{233} \mathrm{U}$ ) is given by

$$
\bar{\sigma} \approx \frac{A}{\sqrt{E}},
$$

where $A$ is a constant, we get

$$
\bar{\sigma} \approx \frac{A t}{\sqrt{K}}
$$

neglecting $t_{0}$. The term

$$
\left|\frac{\Delta t}{\Delta \bar{E}}\right| \text { is } \frac{t^{3}}{2 K}
$$

and then $f(\bar{E})$ becomes:

$$
f(\bar{E}) \approx \frac{\lambda_{\mathrm{U} 3} n_{\mathrm{det}}(t)}{2 n_{\alpha}} \quad \frac{t^{2}}{A \sqrt{K}} .
$$

In this relation $n_{\text {det }}(t)$ represents the normalized number of captures per $10^{9}$ protons. Then $n_{\text {det }}(t)=10^{9} n_{\text {measured }}(t) / I$ where $I$ is the total number of protons in the run. Errors on $t, K$, and $A$ are independent, then the uncertainty on $f$ writes as:

$$
\left[\frac{\delta f}{f}\right]^{2}=\left[\frac{\delta n_{\text {measured }}}{n_{\text {measured }}}\right]^{2}+\left[\frac{\delta I}{I}\right]^{2}+\left[\frac{\delta n_{\alpha}}{n_{\alpha}}\right]^{2}+\left[\frac{\delta A}{A}\right]^{2}+\left[2 \frac{\delta t}{t}\right]^{2}+\left[\frac{\delta K}{2 K}\right]^{2} .
$$

In this expression the first term $\delta n_{\text {measured }} / n_{\text {measured }}$ represents the relative statistical error and the others describe the relative systematic errors:

- the relative error on $n_{\alpha}$ is estimated to be $6 \%$ mainly due to the normalization procedure used to calibrate the ${ }^{6} \mathrm{Li}$ target measurement;

- the relative error on the beam intensity $\delta I / I$ is $5 \%$;

- the relative error on the coefficient $A, \delta A / A$, is estimated to be $10 \%$;

- the error on time determination is $\delta t=0.5 \mu \mathrm{s}$ (including the error on $t_{0}$ ). Then $\delta t / t$ ranges from $13 \%$ to $0.1 \%$ between $10 \mathrm{keV}$ and $1 \mathrm{eV}$;

- The relative error $\delta K / K$ has been estimated to be $1.2 \%$.

Consequently, the experimental results for fluence determinations from thermal energies up to $10 \mathrm{keV}$ have a relative systematic uncertainty of 14 to $28 \%$. 


\subsubsection{Fluence energy and spatial dependence}

A mapping was made in 72 positions scanning distances to the centre of the lead volume from $30 \mathrm{~cm}$ to $190 \mathrm{~cm}$.

Typical fluence energy distributions from $3.5 \mathrm{GeV} / c$ protons collected at several positions in the lead volume are presented in Fig. 32. Figure 33 shows the spatial distribution of all flux measurements for some selected neutron energy intervals $(0.1 \mathrm{eV}$ to $1 \mathrm{keV})$ as function of the detector radial distance to the centre of the lead volume (negative values of $r$ correspond to $z<0)$. These distributions are well fitted by Gaussian shaped curves.

The fluence shape is in agreement with the theoretical fluence for mono-energetic point-like neutron source $f_{0}(E, \vec{r})$ given by the Fermi age theory modified by an energy-dependent absorption term:

$$
f_{0}(E, \vec{r})=\frac{a}{E u^{3 / 2}} \exp \left(-\frac{r^{2}}{b u}\right) \exp \left(-\frac{c}{\sqrt{E}}\right) ; \quad u=\ln \frac{E_{0}}{E} .
$$

Indicative values of the parameters, for $3.5 \mathrm{GeV} / c$ protons, are:

$$
\begin{array}{cc}
E_{0} \approx 1.5 \mathrm{MeV} & a \approx(8 \pm 1) 10^{7} \text { neutrons } / \mathrm{cm}^{2} \text { per } 10^{9} \text { protons } \\
b \approx 10^{3} \mathrm{~cm}^{2} & c \approx 0.5 \mathrm{eV}^{1 / 2}
\end{array}
$$

In fact the $b$ parameter has to be adjusted to fit the data at different energies. Its variation has been parametrized by a function $b_{\text {exp }}$ changing slowly with the neutron energy $E$. The theoretical value $\left(b_{\mathrm{th}}\right)$ is given as a function of the lethargy and of the neutron mean free path $\lambda_{\mathrm{s}}$ by:

$$
b_{\text {th }}=\frac{4 \lambda_{\mathrm{s}}^{2}}{3 \xi} \quad \text { with } \quad \lambda_{\mathrm{s}} \approx 2.76 \mathrm{~cm} .
$$

The experimental value $\left(b_{\exp }\right)$ has been found slowly energy-dependent with the parametrization:

$$
\sqrt{\frac{b_{\exp }}{b_{\text {th }}}}=(0.922+0.0181 \ln E) .
$$

A comparison of this model with experimental results is shown in Fig. 34.

Note that this representation provides a simplified and compact way to describe all the experimental data assuming that fluences have a quasispherical symmetry, although this is not strictly exact for short radial distances near the spallation source centre or large radial distances near the edge of the lead volume. In principle the parameter $a$ can lead to the number of neutrons created per proton by the spallation process. But the $a$ value is extracted by fitting the flux for neutron energies below $1 \mathrm{keV}$ and at this stage there has been sizeable absorption, leakage out of the lead assembly, and (n,xn) production.

While most of the measurements have been made using a proton beam of $3.5 \mathrm{GeV} / c$, a few measurements have also been made at $2.5 \mathrm{GeV} / c$. The associated fluence energy spectra for both beam energies are compared. As expected these fluences are proportional to each other, in the measured energy range. This is illustrated in Fig. 35 and leads to the measured ratio:

$$
R=\left\langle\frac{\Phi_{3.5 \mathrm{GeV} / c}(E)}{\Phi_{2.5 \mathrm{GeV} / c}(E)}\right\rangle=1.44 \pm 0.05 \text { (stat.) . }
$$




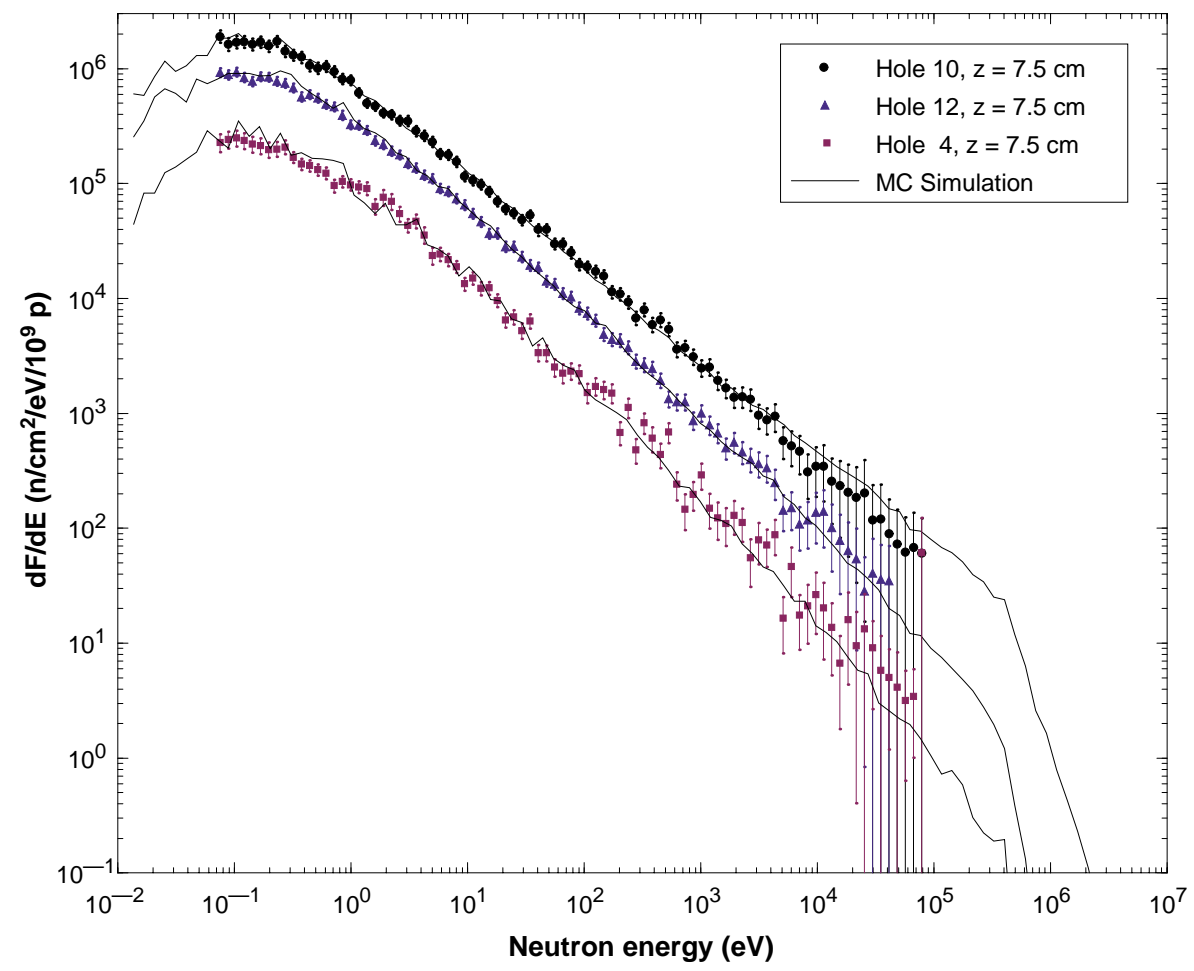

Fig. 32: Neutron fluence measured at different positions with silicon detectors for $10^{9}$ incoming protons at 3.5 GeV/c compared to the simulation.

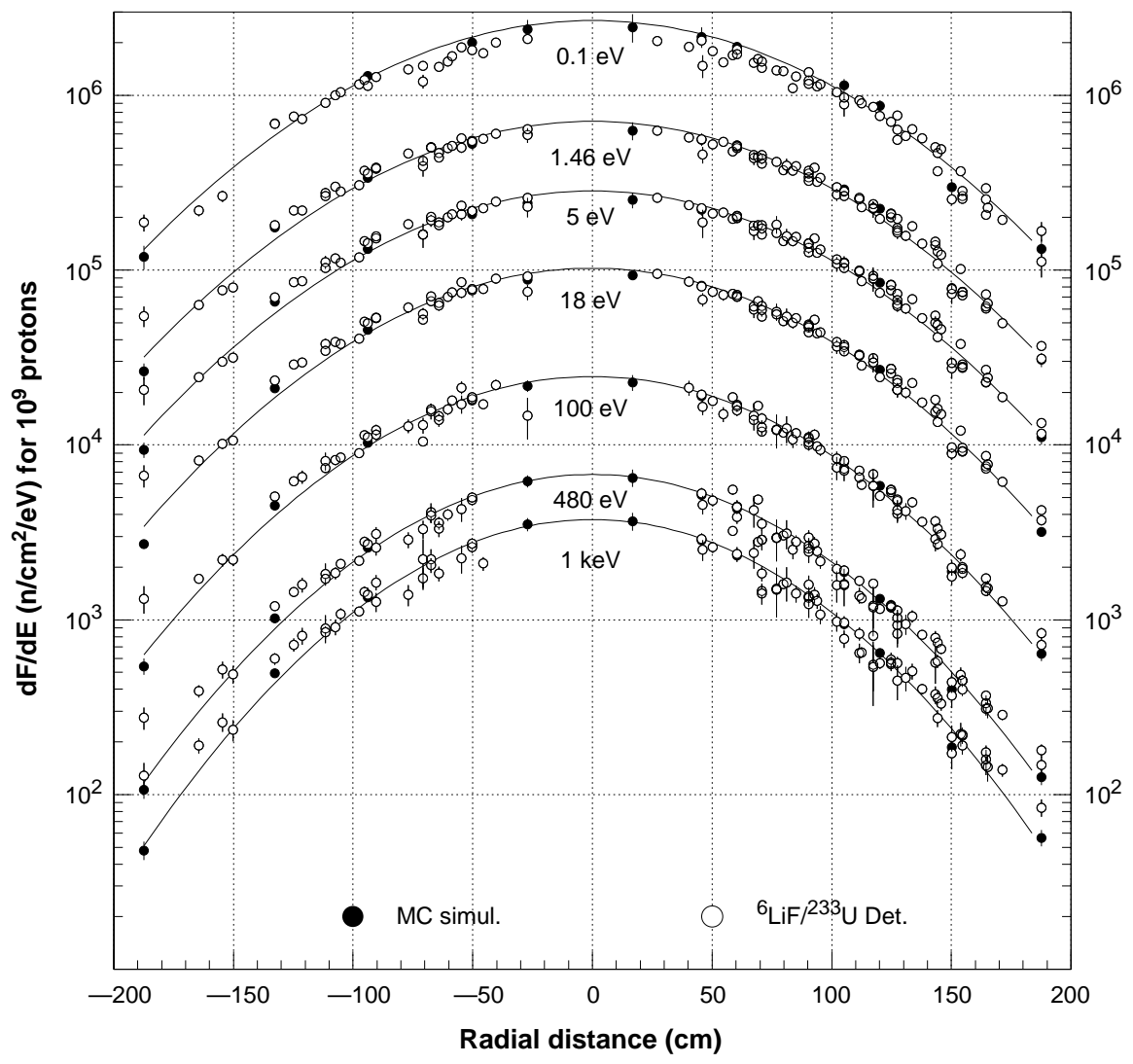

Fig. 33: ${ }^{6} \mathrm{LiF}$ and ${ }^{233} \mathrm{U}$ experimental and simulated neutron fluences for some fixed energies as a function of the radial distance for $10^{9}$ incoming protons at $3.5 \mathrm{GeV} / c$. The solid lines correspond to fits with the Fermi age theory function using the parametrization from formula (24). 


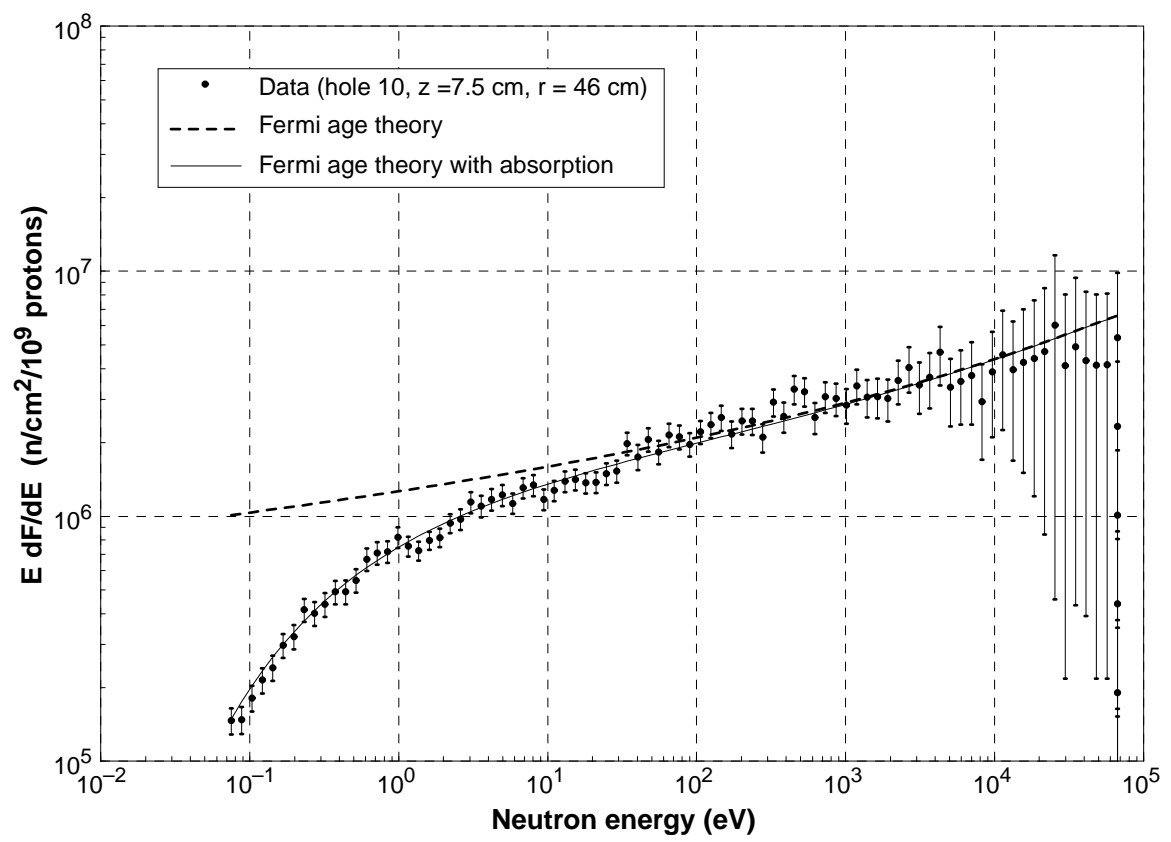

Fig. 34: Isolethargic fluence experimental spectrum $E \mathrm{~d} F / \mathrm{d} E$ compared with the Fermi age theory, with and without the absorption term.

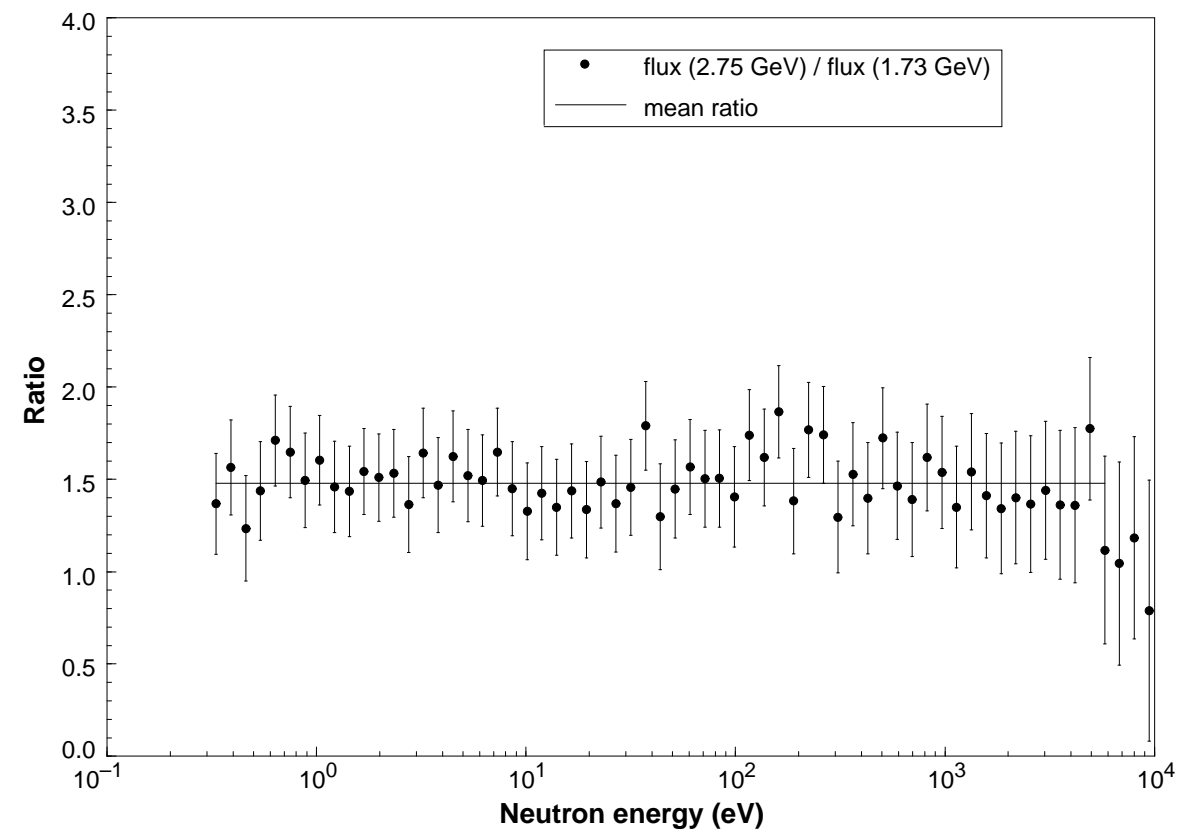

Fig. 35: Example of ratio of differential fluences obtained with the two beam momenta $(3.5 \mathrm{GeV} / c$ and $2.5 \mathrm{GeV} / c$ ) both measured at the same position (hole $10, z=+7.5 \mathrm{~cm}$ ) inside the lead volume.

This result is not too far off the expected value coming from the ratio of beam kinetic energies $R_{\text {kinetic }}=1.59^{5}$, if the number of primary spallation neutrons is proportional to the proton kinetic energy.

5. We have data for proton momenta of $3.568 \mathrm{GeV} / c$ and $2.499 \mathrm{GeV} / c$. The kinetic energy is then $T=2.75 \mathrm{GeV}$ and $T=1.73 \mathrm{GeV}$, respectively. 


\subsection{Comparison with our simulation}

The measured fluence at various positions was compared to Monte Carlo simulations (Fig. 36). In order to show a global comparison of simulation and data, we calculated the ratio $r=\langle$ data/MC $\rangle$. The bracket means that the averaged value is made over the energy range $1 \mathrm{eV}$ to $1 \mathrm{keV}$. The distribution of the residuals, $1-r$, obtained for all runs (Fig. 37), shows excellent average agreement with the simulation with a narrow dispersion of the $r$ values $(8 \%$ consistent with the run-to-run fluctuations).

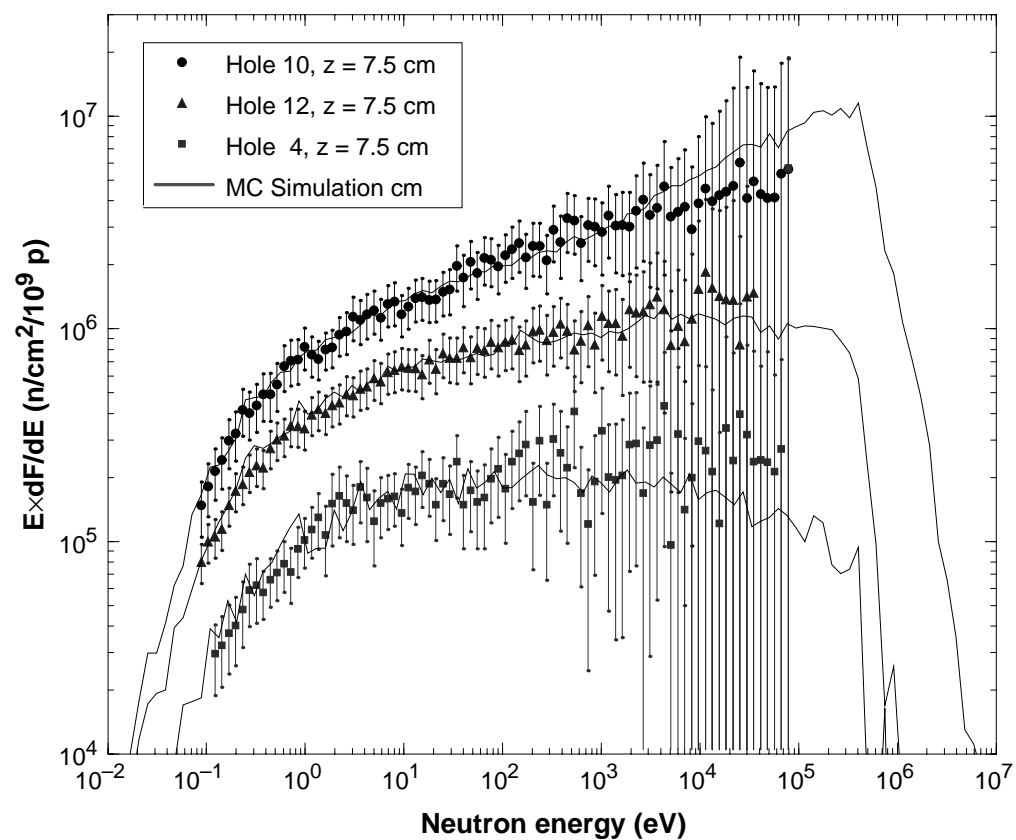

Fig. 36: Isolethargic fluence $E \times \mathrm{d} F / \mathrm{d} E$ at $3.5 \mathrm{GeV} / c$ at different positions compared with Monte Carlo simulation.

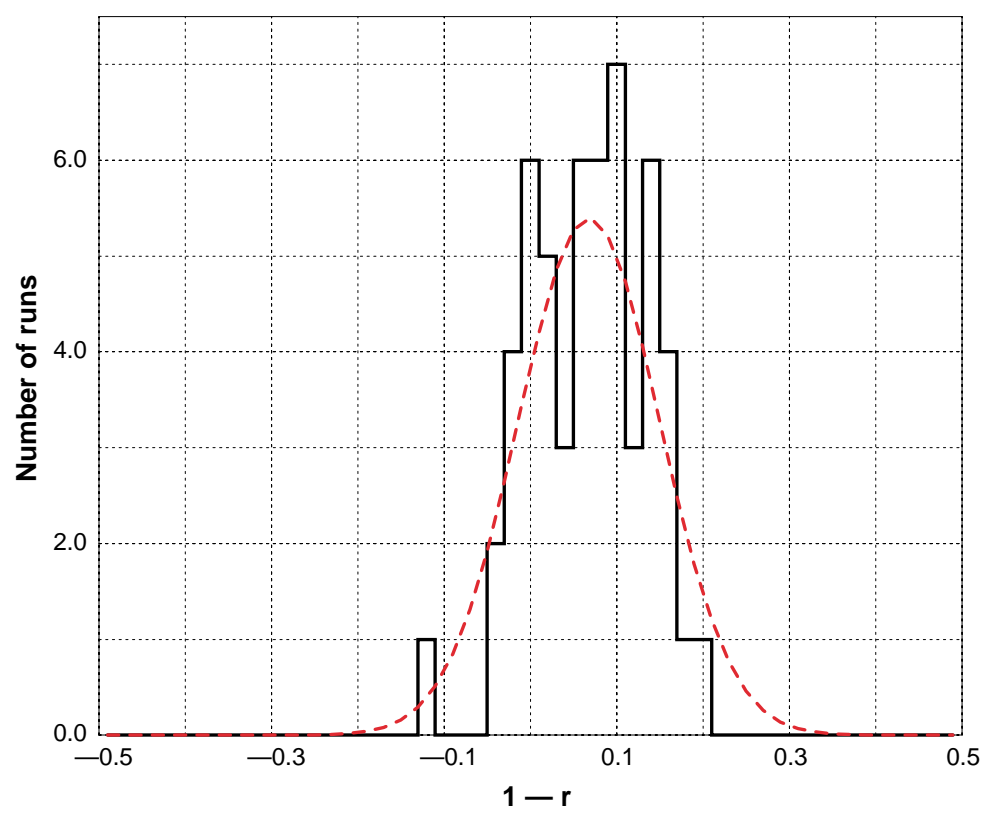

Fig. 37: Number of runs versus $1-r$ (see text). $1-\langle r\rangle=0.05 \pm 0.09$. A Gaussian fit is also shown. The mean value of the distribution is found to be $0.05 \pm 0.09$. 


\subsection{2 ${ }^{3}$ He scintillation counter}

\subsubsection{Description of the counter}

The ${ }^{3} \mathrm{He}$ scintillation detector uses the ${ }^{3} \mathrm{He}(\mathrm{n}, \mathrm{p})^{3} \mathrm{H}$ reaction which has a positive $Q$ value of $0.764 \mathrm{MeV}$ [41].

The energy lost by the charged products of the reaction slowing down in the gas mixture leads to the emission of light with an intensity proportional to the energy deposited in the gas. The photons emitted in the atomic de-excitation and recombination processes, following the electromagnetic interactions with the electrons of the gas atoms through which the reaction products lose their kinetic energy, have an energy of about $20 \mathrm{eV}(62 \mathrm{~nm})$ [42]. In order to increase the scintillation light output, instead of using pure ${ }^{3} \mathrm{He}$ we used a mixture of ${ }^{3} \mathrm{He}$ with $\mathrm{Xe}$ since the number of scintillation photons per $\mathrm{MeV}$ for $\mathrm{Xe}$ is three times more than for $\mathrm{He}$ and since a mixture of at least $10 \% \mathrm{Xe}$ (the rest is $\mathrm{He}$ ) gives as much light output as pure $\mathrm{Xe}$ [42]. Xenon produces light at $172 \mathrm{~nm}$ and is also used as a wavelength shifter that shifts part of the ${ }^{3} \mathrm{He}$ scintillation photons from $62 \mathrm{~nm}$ to $172 \mathrm{~nm}$ [42].

In order to increase further the light output and match it to the maximum response of the photomultiplier photocathode used to observe the gas scintillation (above $300 \mathrm{~nm}$ ), a layer of p-terphenyl [41] was vacuum-deposited on a highly reflective coating of $\mathrm{MgO}$ [43] on the inner walls and on the base of the chamber. P-terphenyl is a wavelength shifter that absorbs the ultraviolet emissions at $172 \mathrm{~nm}$ and part of the $20 \mathrm{eV}$ photons, re-emitting at $340 \mathrm{~nm}$ [44]. P-terphenyl is also evaporated on the internal surface of the quartz window of the chamber (cut-off at $160 \mathrm{~nm} \mathrm{[45])} \mathrm{to} \mathrm{wavelength-shift} \mathrm{the} \mathrm{direct} \mathrm{photons.}$

The ${ }^{3} \mathrm{He}$ scintillation chamber (Fig. 38) is cylindrical with internal diameter $40 \mathrm{~mm}$ and length $59 \mathrm{~mm}$ and is made of stainless steel. For the optical connection to the photomultiplier we used a quartz window $4 \mathrm{~mm}$ thick, sitting on a copper ring with $27 \mathrm{~mm}$ internal diameter and $4 \mathrm{~mm}$ width.

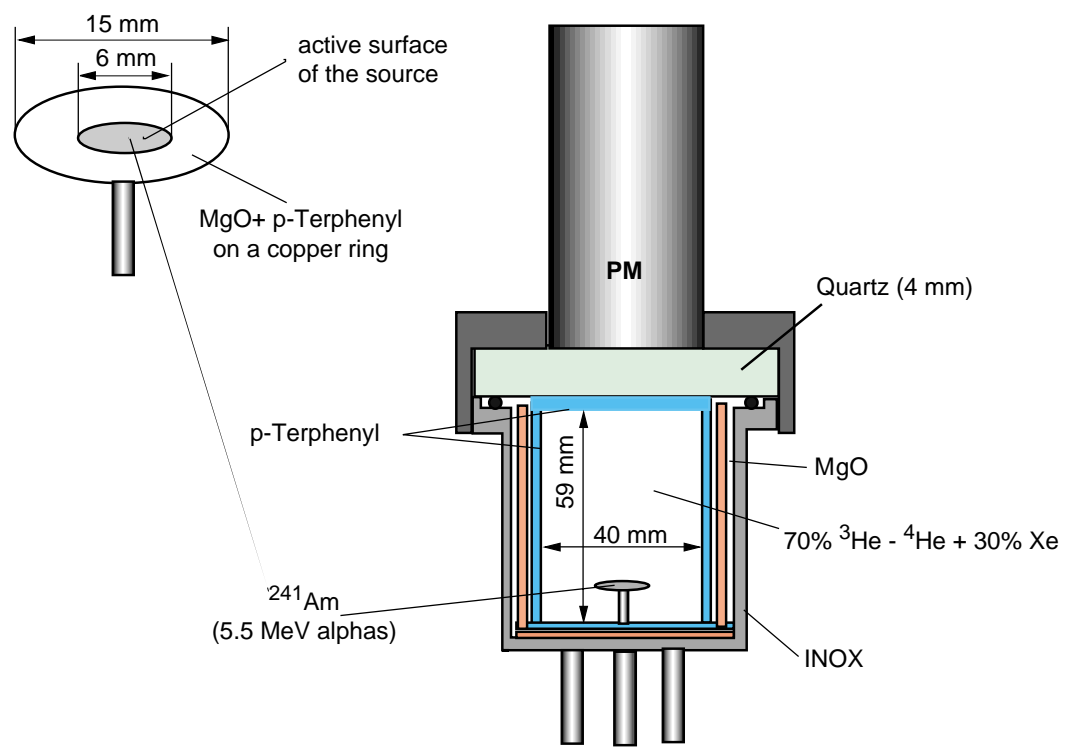

Fig. 38: Schematics of the ${ }^{3} \mathrm{He}$ scintillation chamber.

We note that p-terphenyl evaporates at room temperature and becomes an impurity in the gas, thus quenching the photon emission [42]. In order to calibrate continuously the variation of the efficiency of the gas mixture with time, we installed a $5.5 \mathrm{MeV}^{241} \mathrm{Am}$ alpha source at 
the bottom of the chamber (in the centre). The gas deterioration, due to the evaporation of the p-terphenyl, is observed as the displacement of the alpha photopeak with time (Fig. 39). The diameter of the active surface of the source is $6 \mathrm{~mm}$ while the diameter of the whole surface is $15 \mathrm{~mm}$. In order to minimize shadowing effects from the source we installed around the active surface of the source a copper ring on which $\mathrm{MgO}$ and p-terphenyl layers were deposited.

The range of $1.5 \mathrm{~cm}$ for $5.5 \mathrm{MeV} \alpha$ particles [46] and the corresponding mixture were chosen because both the total pressure $\left(3.93\right.$ bar) of the mixture and the consumption of ${ }^{3} \mathrm{He}$ are low, while the light output is satisfactory, according to the results of the measurements shown in Fig. 40.

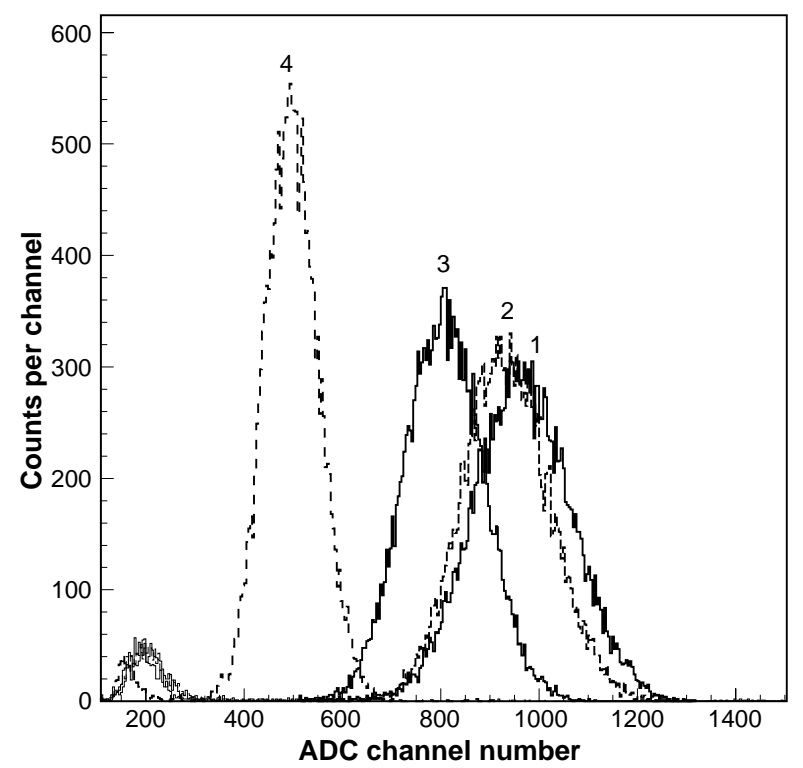

Fig. 39: Monitoring the ${ }^{3} \mathrm{He}$ counter efficiency with the ${ }^{241} \mathrm{Am} 5.5 \mathrm{MeV}$ alpha photopeak for the same filling of the chamber. Curves 1, 2, 3 and 4 correspond to times $t=0,1 \mathrm{~h}, 2 \mathrm{~h}$ and $4 \mathrm{~h}$ respectively.

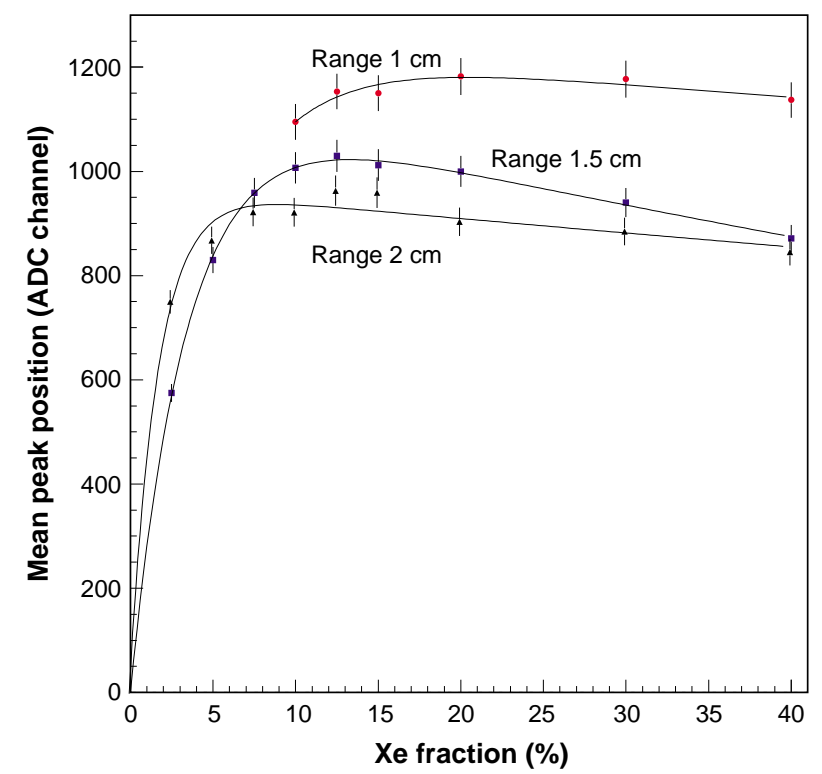

Fig. 40: Mean position of the $5.5 \mathrm{MeV}$ alpha photopeak as a function of Xe percentage for a ${ }^{4} \mathrm{He}-\mathrm{Xe}$ mixture. The lines are guides for the eye (made with a polyonymial fit), while the error bars represent the sigmas of the distribution of every photopeak (as calculated with Gaussian fits). 


\subsubsection{Measurements and data analysis}

There are two sets of measurements: data taken in October 1996 at the proton momentum of $3.5 \mathrm{GeV} / c$ and in May 1997 at the proton momentum of $2.5 \mathrm{GeV} / c$. Table 5 shows all the positions studied during the two sets of measurements.

Table 5: Summary of positions (hole number and z-coordinate) at which measurements were made in TARC with the ${ }^{3} \mathrm{He}$ scintillation counter. For the definition of holes and coordinates see Section 2 . The unit is centimetres.

\begin{tabular}{c|c|c|c|c|c|c}
\hline $3.5 \mathrm{GeV} / c$ & $3.5 \mathrm{GeV} / c$ & $3.5 \mathrm{GeV} / c$ & $2.5 \mathrm{GeV} / c$ & $2.5 \mathrm{GeV} / \boldsymbol{c}$ & $2.5 \mathrm{GeV} / \boldsymbol{c}$ & $2.5 \mathrm{GeV} / \boldsymbol{c}$ \\
\hline Hole 3 & Hole 10 & Hole 1 & Hole 3 & Hole 10 & Hole 1 & Hole 8 \\
\hline $\begin{array}{c}+7.5 \\
+37.5\end{array}$ & +7.5 & +7.5 & +7.5 & +7.5 & +7.5 & +7.5 \\
+67.5 & +67.5 & +67.5 & +67.5 & +67.5 & +67.5 & \\
+97.5 & & +112.5 & +112.5 & & +112.5 & \\
+112.5 & & & & & & \\
\hline
\end{tabular}

To obtain the time spectrum for the above positions, the signal of the photomultiplier (PM) is directed to a $200 \mathrm{MHz}$ channel PM amplifier and then to an 8-bit ICARUS FADC with a sampling rate of $100 \mathrm{~ns}$.

For each of the above positions we also took measurements using the mixture (1.18 bar Xe, 2.75 bar ${ }^{4} \mathrm{He}$ ) for background subtraction. These background measurements have been normalized to the same proton beam intensity and subsequently subtracted from the spectra.

In order to evaluate the deterioration of the gas mixture with time (due to evaporation of p-terphenyl), we made at the beginning of every run one calibration measurement using the internal $\alpha$ source in the chamber. Then by fitting the $\alpha$ photo-peak to a Gaussian we extracted the number of photoelectrons (the number of photoelectrons is equal to the square of the ratio mean over sigma of the Gaussian), corresponding to the $\alpha$ photopeak for every calibration measurement. In this way, for each run we could obtain the gas calibration factor.

For each run of the first set of measurements in $1996(3.5 \mathrm{GeV} / c)$ we accepted the events with a PS shot intensity between $3 \times 10^{7}$ and $9 \times 10^{7}$ protons while for the second set of measurements in $1997(2.5 \mathrm{GeV} / c)$ we accepted the events with shot intensity between $10^{7}$ and $5 \times 10^{7}$ protons. We note here that for the second set of measurements $(2.5 \mathrm{GeV} / c)$ the beam intensity was a factor of two smaller. We normalized every shot to a beam average of $6 \times 10^{7}$ protons per shot (for both sets) and we also corrected for the gas deterioration using the calibration measurements. For every run we added all the pulses bin by bin, divided by the number of shots and thus calculated the mean pulse (Fig. 41). The same procedure was applied in order to obtain the mean pulse for every background run (Fig. 41). The background subtraction was made using the calculation of the mean pulses of the signal $\left({ }^{3} \mathrm{He}\right)$ and of the background $\left({ }^{4} \mathrm{He}\right)$ runs for all corresponding runs (Fig. 42).

The ratios of data taken with $3.5 \mathrm{GeV} / c$ protons over data taken with $2.5 \mathrm{GeV} / c$ protons presented in Table 6 are expected to follow the ratio of the kinetic energies of the beams which is 1.59. The fit of the ratio of data for hole 3 , at $z=+67.5 \mathrm{~cm}$ for the two different proton momenta (Fig. 43) shows that indeed this ratio is constant over the neutron energy range considered and consistent with the expected value. 


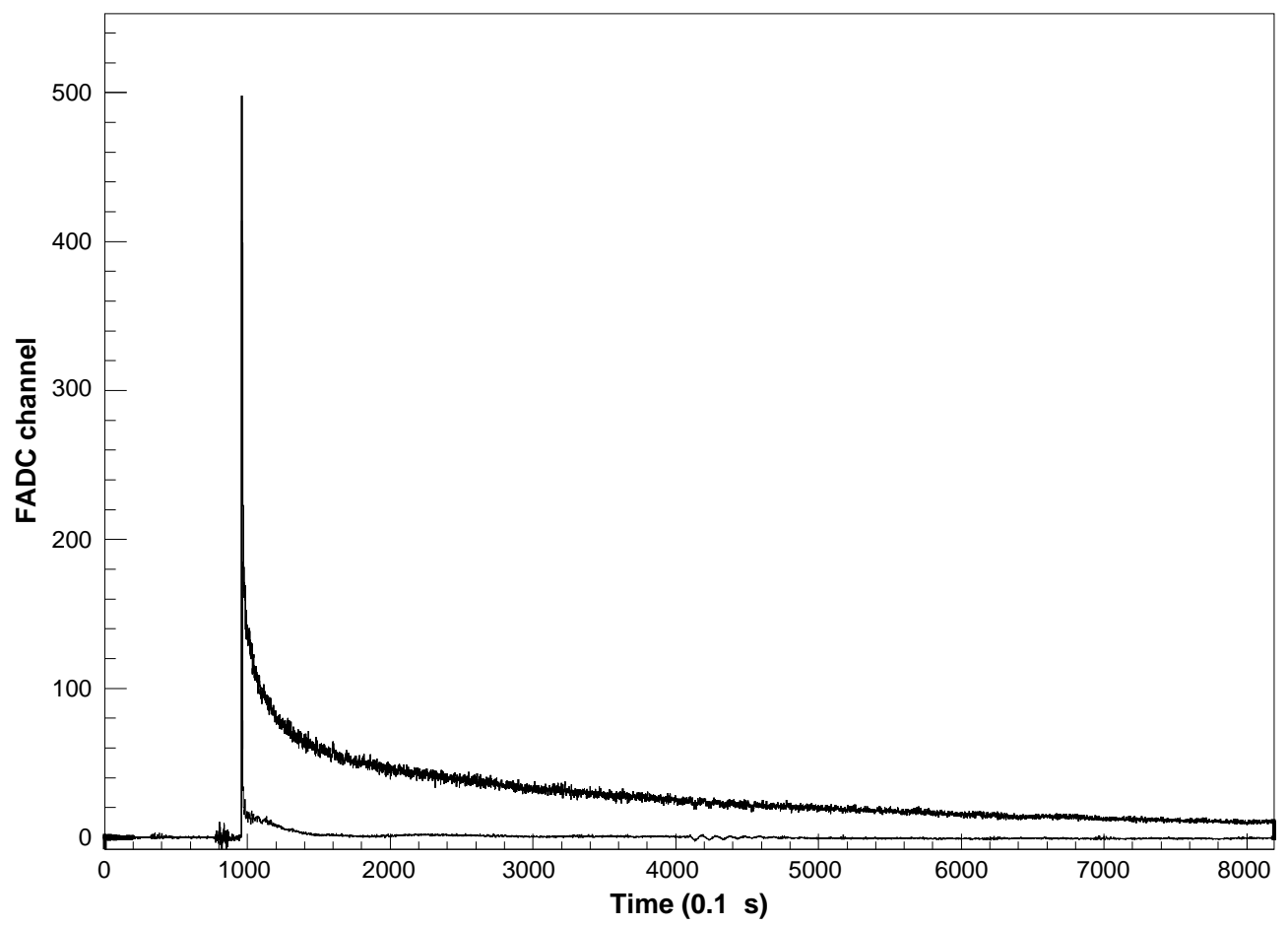

Fig. 41: Mean signal i.e. integrated charge (FADC channel) $\left({ }^{3} \mathrm{He}\right)$ [upper curve] and mean background $\left({ }^{4} \mathrm{He}\right)$ [lower curve] pulses with $100 \mathrm{~ns}$ binning at hole $10, z=+7.5 \mathrm{~cm}, 500 \mathrm{~V}(2.5 \mathrm{GeV} / c$ proton run).

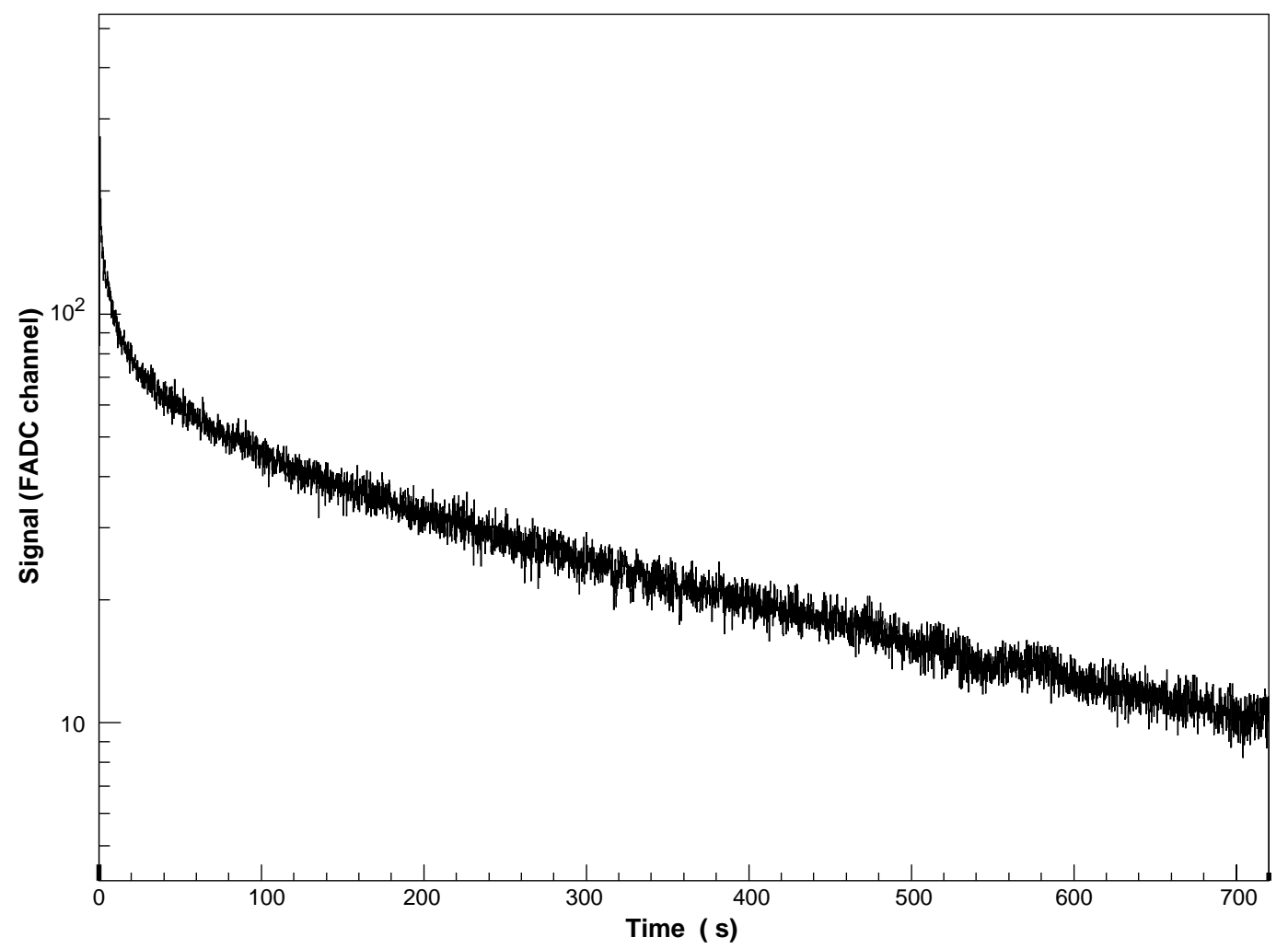

Fig. 42: ${ }^{3} \mathrm{He}$ signal i.e. integrated charge (FADC channel) minus background $\left({ }^{4} \mathrm{He}\right)$ with $100 \mathrm{~ns}$ binning at hole $10, z=+7.5 \mathrm{~cm}, 500 \mathrm{~V}(2.5 \mathrm{GeV} / c$ proton run $)$. 


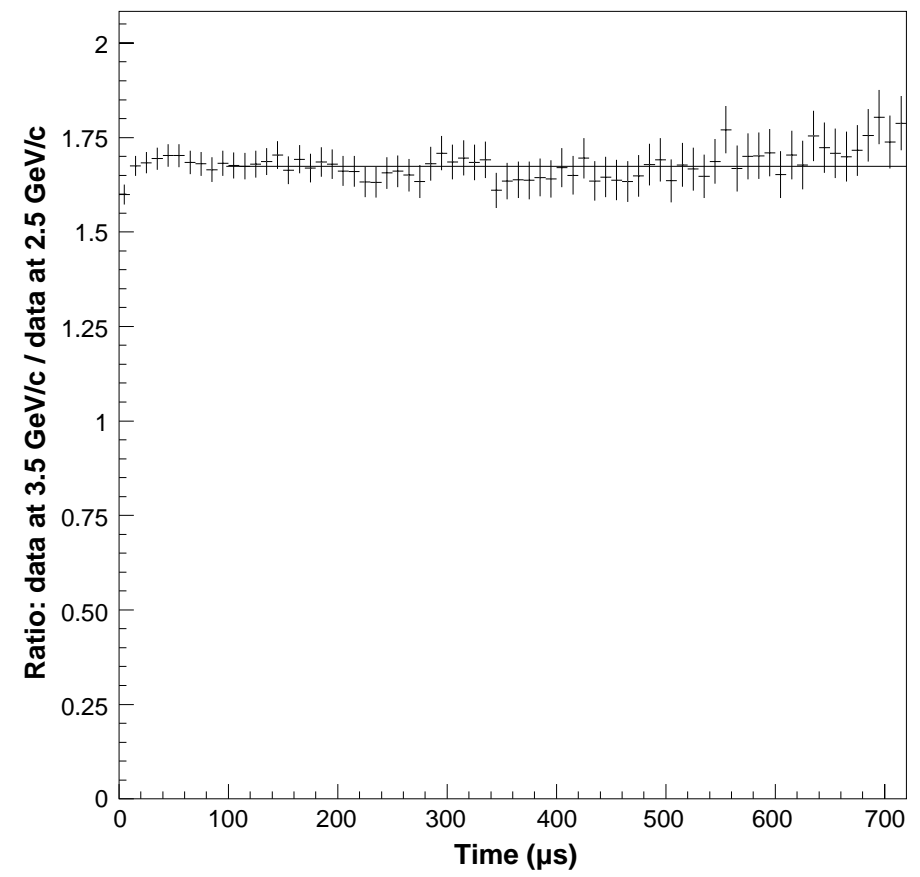

Fig. 43: Example of ratio of fluence with $3.5 \mathrm{GeV} / c$ protons over fluence with $2.5 \mathrm{GeV} / c$ protons for hole $10, z=+7.5 \mathrm{~cm}$. The fitted ratio 1.67 is consistent with the value 1.59 ratio of the kinetic energies of the proton beam.

Table 6: Ratio of ${ }^{3} \mathrm{He}$ signal i.e. integrated charge (FADC channel) minus background $\left({ }^{4} \mathrm{He}\right)$ for $3.5 \mathrm{GeV} / \mathrm{c}$ proton runs over the signal for $2.5 \mathrm{GeV} / c$ proton runs as calculated by fitting the ratios of the corresponding data for various holes and positions. (The errors shown are statistical while the systematic error is $6 \%$.)

\begin{tabular}{c|c|c}
\hline Hole & $\boldsymbol{z}$ position $(\mathbf{c m})$ & Ratio \\
\hline 3 & +7.5 & $1.422 \pm 0.005$ \\
3 & +37.5 & $1.482 \pm 0.005$ \\
3 & +67.5 & $1.618 \pm 0.004$ \\
3 & +112.5 & $1.528 \pm 0.006$ \\
10 & +7.5 & $1.676 \pm 0.005$ \\
10 & +67.5 & $1.719 \pm 0.005$ \\
1 & +7.5 & $1.636 \pm 0.006$ \\
1 & +67.5 & $1.456 \pm 0.006$ \\
\hline
\end{tabular}

\subsubsection{Comparison of data with Monte Carlo simulation}

We used the TARC Monte Carlo simulation code developed by the CERN EET group [32] in order to make a comparison with the measurements. The complete geometry of the ${ }^{3} \mathrm{He}$ scintillation chamber has been described in the simulation. The device was placed in the middle of a DST volume $\left(15 \times 15 \times 15 \mathrm{~cm}^{3}\right.$ lead box with a hollow cylinder of radius $3.2 \mathrm{~cm}$ and length $11 \mathrm{~cm}$ that contains air).

Average ratios from ${ }^{3} \mathrm{He}$ measured signals i.e. integrated charge (FADC channel) corrected for background $\left({ }^{4} \mathrm{He}\right)$ to Monte Carlo simulation for the different positions and holes and for the three different sets of measurements corresponding to PM voltages of 450, 500 and $550 \mathrm{~V}$ (Table 7) show that the data are consistent with the simulation of the entire set of operating parameters. An example of ratio distribution is shown in Fig. 44. 
Table 7: Ratios of ${ }^{3} \mathrm{He}$ signal i.e. integrated charge (FADC channel) background $\left({ }^{4} \mathrm{He}\right)$ subtracted to Monte Carlo for various positions and PM voltages for $3.5 \mathrm{GeV} / c$ protons

\begin{tabular}{c|c|c|c|c}
\hline Hole & $\boldsymbol{z}$ position $(\mathbf{c m})$ & $\mathbf{4 5 0} \mathbf{V}$ & $\mathbf{5 0 0} \mathbf{~}$ & $\mathbf{5 5 0} \mathbf{~}$ \\
\hline 3 & +7.5 & $1.013 \pm 0.022$ & $0.990 \pm 0.021$ & $0.977 \pm 0.024$ \\
3 & +37.5 & $1.017 \pm 0.017$ & $0.981 \pm 0.016$ & $1.026 \pm 0.020$ \\
3 & +67.5 & $0.875 \pm 0.012$ & $0.928 \pm 0.013$ & $0.940 \pm 0.013$ \\
3 & +112.5 & $0.967 \pm 0.012$ & $0.951 \pm 0.011$ & $0.958 \pm 0.010$ \\
10 & +7.5 & $1.077 \pm 0.016$ & $1.112 \pm 0.016$ & $1.098 \pm 0.019$ \\
10 & +67.5 & $1.079 \pm 0.014$ & $1.006 \pm 0.012$ & $0.974 \pm 0.012$ \\
1 & +7.5 & $0.964 \pm 0.011$ & $1.012 \pm 0.010$ & $1.000 \pm 0.095$ \\
1 & +67.5 & $0.964 \pm 0.010$ & $0.981 \pm 0.009$ & $0.953 \pm 0.085$ \\
\hline
\end{tabular}
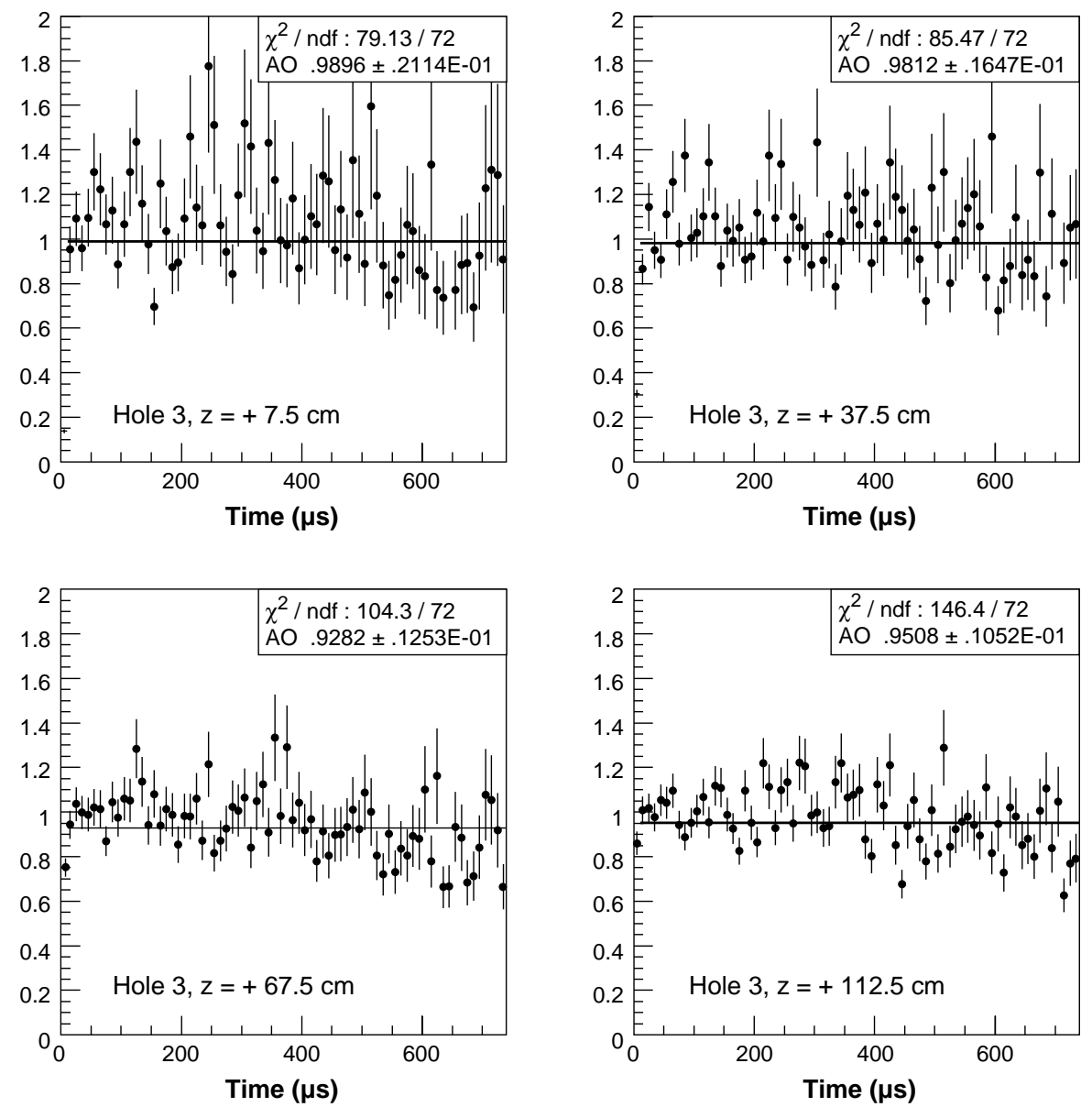

Fig. 44: Fits of the ratios of ${ }^{3} \mathrm{He}$ signal i.e. integrated charge (FADC channel) background $\left({ }^{4} \mathrm{He}\right)$ subtracted to Monte Carlo for various positions in hole 3, a PM voltage of $500 \mathrm{~V}$ and for $3.5 \mathrm{GeV} / c$ protons.

We also made tests of the ${ }^{3} \mathrm{He}$ scintillation chamber using neutrons produced by the $t(p, n)^{3} \mathrm{He}$ reaction at the Bordeaux van de Graaff accelerator. The aim of these measurements (Fig. 45) was to determine the ratio of the light emission from the ${ }^{3} \mathrm{He}(\mathrm{n}, \mathrm{p})^{3} \mathrm{H}$ reaction with thermal neutrons to the light emission of the $5.5 \mathrm{MeV}^{241} \mathrm{Am}$ alpha source.

The ratio of the thermal neutron peak over the alpha peak was found from these measurements to be $0.137 \pm 0.002$, which is compatible with the expected value of the ratio $0.764 \mathrm{MeV} / 5.5 \mathrm{MeV}=0.139$. 

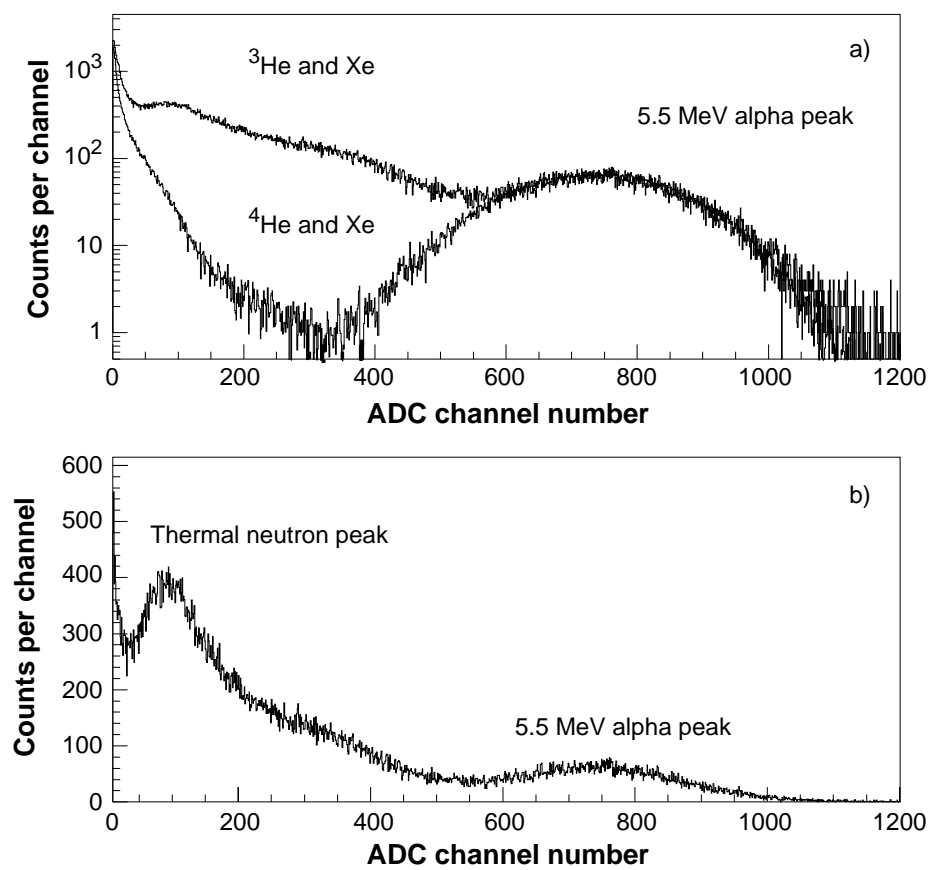

Fig. 45: (a) Signal and background measurements in logarithmic scale for thermal neutrons; (b) Signal after background subtraction in linear scale (without subtracting the alpha peak).

\subsubsection{Neutron fluence calculation}

The neutron fluence was calculated using the relation:

$$
N_{\text {events }}(E)=\sigma(E) \times F(E) \times N_{\text {atoms }}
$$

where $N_{\text {events }}(E)$ is the number of events as a function of energy in the counter volume, $\sigma(E)$ the cross-section for the ${ }^{3} \mathrm{He}(\mathrm{n}, \mathrm{p})^{3} \mathrm{H}$ reaction, $F(E)$ the neutron fluence, and $N_{\text {atoms }}$ the number of ${ }^{3} \mathrm{He}$ atoms in the counter volume equal to $11.017 \times 10^{20}$ atoms for the gas mixture we used. The number of events as a function of time is obtained by converting the data spectrum via the calibration factor of the FADC for a given photomultiplier voltage. The data spectrum is the result of the subtraction of the mean background spectrum [the one obtained using only ${ }^{4} \mathrm{He}$ i.e. integrated charge (FADC channel) as a function of time] from the mean signal spectrum (the one obtained using the mixture of ${ }^{3} \mathrm{He}$ and ${ }^{4} \mathrm{He}$ ).

In order to obtain the number of events as a function of energy we use the mean energy-time relation:

$$
E[\mathrm{eV}]=\frac{172000}{(t[\mu \mathrm{s}]+0.37)^{2}} .
$$

For a given binning in energy (100 equal lethargy bins from $10^{-2} \mathrm{eV}$ up to $10^{5} \mathrm{eV}$ ), for each energy bin the corresponding time window is calculated via the mean energy-time relation. The contents of the time bins within this time window are taken from the data spectrum (100 ns binning) and are subsequently converted, via the FADC calibration factor for a given photomultiplier voltage, to number of events.

Selected from all the positions measured in the lead assembly (Table 5), Fig. 46 presents typical fluence measurements as a function of neutron energy for hole 3 , as obtained from the data for a photomultiplier voltage of $450 \mathrm{~V}$ with a binning of $100 \mathrm{~ns}$ at a proton momentum of $3.5 \mathrm{GeV} / c$. 


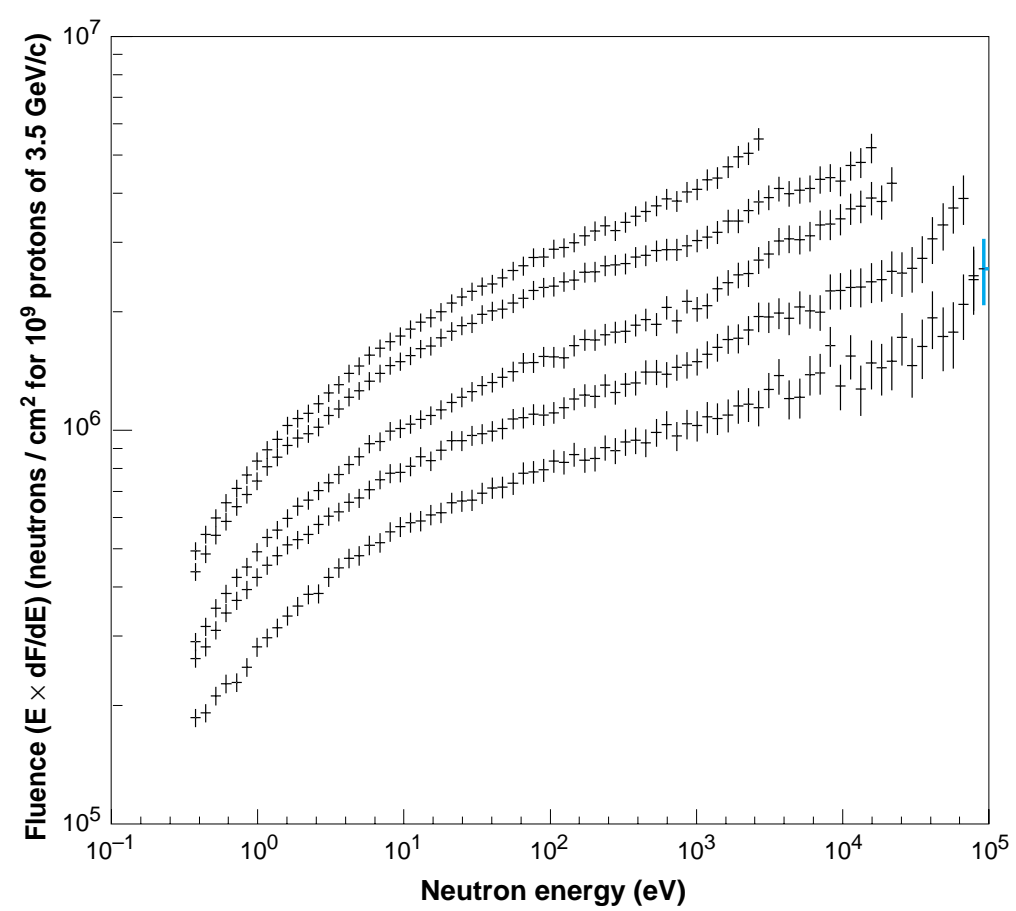

Fig. 46: Neutron fluences $(E \times \mathrm{d} F / \mathrm{d} E)$ versus neutron energy, for $10^{9}$ protons of $3.5 \mathrm{GeV} / c$ in hole 3 . The curves correspond, from top to bottom, to the positions: $z=+7.5 \mathrm{~cm},+37.5 \mathrm{~cm},+67.5 \mathrm{~cm},+97.5 \mathrm{~cm}$, and $+112.5 \mathrm{~cm}$.

\subsubsection{Systematic errors}

We estimate that the systematic errors are:

- $5 \%$ in the proton intensity, mainly related to the $\mathrm{Al}(\mathrm{p}, 3 \mathrm{pn})^{24} \mathrm{Na}$ cross-section uncertainty, used for the beam transformer calibration.

- 3\% due to the uncertainty in the absolute gas pressure. The precision of the absolute pressure meter is 0.001 bar. However, the uncertainty of $10^{\circ}$ due to the cooling trap induces an error of approximately $3 \%$.

- $0.6 \%$ due to error on the estimation of $K$ in the energy-time relation from $\Delta k / k=1.2 \%$.

- $12 \%$ due to the conversion of the FADC channels to number of events. The factors contributing to this uncertainty are $2.5 \%$ from the range of the FADC ( 256 channels correspond to $3.00 \pm 0.075 \mathrm{mV}$ ); $7 \%$ from the amplification precision of Lecroy $612 \mathrm{~A}$ with an amplification factor $10 \pm 0.07 ; 5 \%$ from the precision of the 11-bit Lecroy ADC [47], $512 \pm 25.6 \mathrm{pC}$, and $12 \%$ in the gain due to the error in the value of the coefficient $\alpha$ set by the dynode material and to the geometry of the photomultiplier. (The error in the gain depends on the voltage and is $12 \%$ for $450 \mathrm{~V}, 10 \%$ for $500 \mathrm{~V}, 8.4 \%$ for $550 \mathrm{~V}$ and $6.7 \%$ for $600 \mathrm{~V}$.

- Error on the value of $t$ and $t_{0}$, dominant at high energy region (8\% at $1 \mathrm{keV}, 54 \%$ at $50 \mathrm{keV})$.

From the above contributions the error in the proton intensity is common for all detectors in the TARC experiment, while the errors on the energy-time relation and on the value of $t$ and $t_{0}$ are common for the ${ }^{3} \mathrm{He}$ scintillation detector and the ${ }^{6} \mathrm{LiF}$ detectors. In summary the systematic error ranges from $13.5 \%$ to $55 \%$ for neutron energy from $0.1 \mathrm{eV}$ to $50 \mathrm{keV}$. 


\subsubsection{Consistency with other flux measurements in the TARC experiment}

In this section we present a comparison with the data taken with the lithium fluoride $\left({ }^{6} \mathrm{LiF}\right)$ detectors (Figs. 47 and 48), (see Section 5.2.1). These detectors have a neutron energy range similar to that of the ${ }^{3} \mathrm{He}$ scintillation detector.

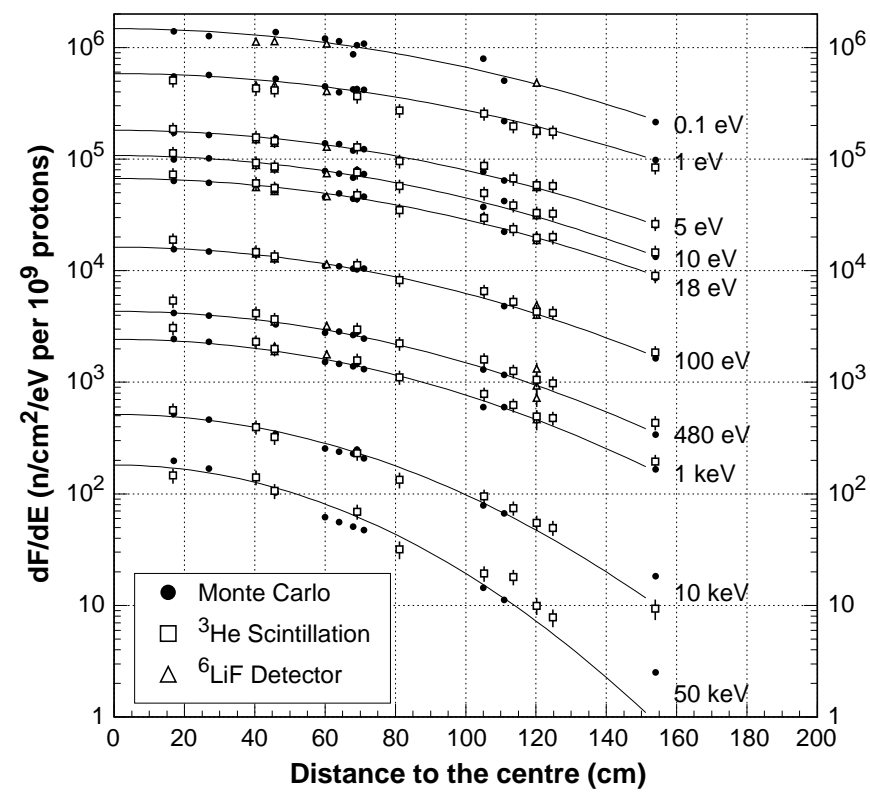

Fig. 47: Neutron fluence per electronvolt for $10^{9}$ protons at $2.5 \mathrm{GeV} / \mathrm{c}$ as a function of distance from the centre of the lead volume at energies $0.1 \mathrm{eV}, 1 \mathrm{eV}, 5 \mathrm{eV}, 10 \mathrm{eV}, 18 \mathrm{eV}, 100 \mathrm{eV}, 480 \mathrm{eV}, 1 \mathrm{keV}, 10 \mathrm{keV}$ and $50 \mathrm{keV}$ from the ${ }^{3} \mathrm{He}$ scintillation detector, ${ }^{6} \mathrm{LiF}$ silicon detectors and Monte Carlo. Lines represent Gaussian fits to the Monte Carlo points.

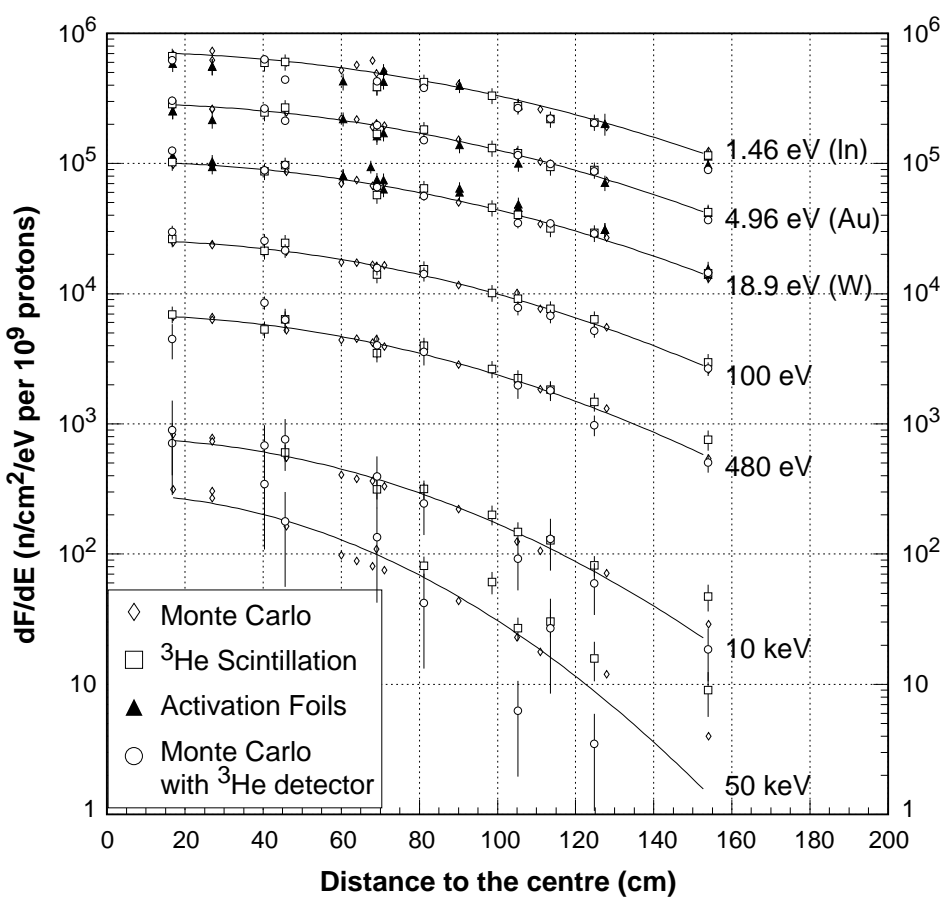

Fig. 48: Neutron fluence per electronvolt for $10^{9}$ protons at $3.5 \mathrm{GeV} / \mathrm{c}$ as a function of radial distance from the centre of the lead volume at energies $1.46 \mathrm{eV}, 4.96 \mathrm{eV}, 18.9 \mathrm{eV}, 100 \mathrm{eV}, 480 \mathrm{eV}, 10 \mathrm{keV}$ and $50 \mathrm{keV}$ from activation foils, the ${ }^{3} \mathrm{He}$ scintillation detector and Monte Carlo for two cases: with and without the presence of the ${ }^{3} \mathrm{He}$ detector. Lines represent Gaussian fits to the Monte Carlo points (diamond shape) without detector. 
The energies $1.46 \mathrm{eV}, 4.96 \mathrm{eV}$, and $18.9 \mathrm{eV}$ have been chosen because at these energies the gold, indium, and tungsten used in activation foils exhibit strong resonances in their neutron absorption cross-sections (see Section 5.2.3). The energy of $480 \mathrm{eV}$ is of particular interest, since it is the energy of the ${ }^{98}$ Mo capture resonance.

As seen from all the above plots, the data from different detectors are consistent between themselves and are also consistent with the Monte Carlo predictions.

\subsubsection{Triple-foil activation}

Some additional checks were made of the neutron fluence at low energies, using the activation foil technique [48]. The method consists of activating in the TARC lead volume triple foils of a material such as typically gold $\left({ }^{197} \mathrm{Au}\right)$, with a very high resonance in its neutron capture cross-section (27 405 barns at $4.9 \mathrm{eV}$ in the case of gold). The foil thickness is chosen so that the outer foils are essentially opaque to neutrons at the resonance energy. The difference between the average activity of the outer foils and the activity of the central foil, provides a measurement of the neutron fluence at the resonance energy. As an example, in the case of gold, we find that both the average activity of the outer foils and the difference between outer and central foils are well reproduced by our Monte Carlo simulation (Fig. 49). This implies that the neutron fluence at $4.9 \mathrm{eV}$ is correct. Similarly the neutron fluence at $1.46 \mathrm{eV}$ and $18.9 \mathrm{eV}$ was checked with ${ }^{115} \mathrm{In}$ and ${ }^{188} \mathrm{~W}$ foils respectively.

A summary of neutron fluence measurements from all the low energy detectors $\left({ }^{6} \mathrm{Li} /{ }^{233} \mathrm{U}\right.$, ${ }^{3} \mathrm{He}$ scintillation mode and activation foils) (Fig. 48) shows that all the measurements are consistent with one another and with the Monte Carlo prediction. We estimate that in the case of activation foils the systematic error on the fluence measurement is about $10 \%$.

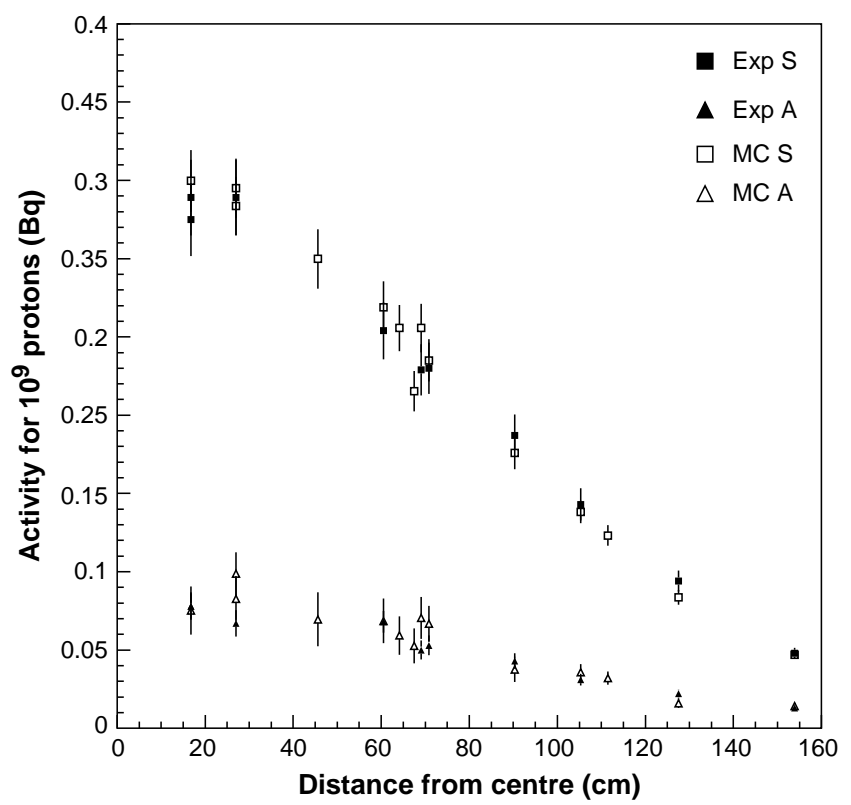

Fig. 49: Experimental and Monte Carlo mean activity $S$ and resonance activity $A$ for gold foils.

\subsubsection{Thermoluminescence detectors}

A further test was made using the thermoluminescence technique to probe the neutron fluence below $0.5 \mathrm{eV}$ [49]. The technique is well known for measuring radiation doses. In our case we irradiated pairs of ${ }^{6} \mathrm{LiF}-{ }^{7} \mathrm{LiF}$ sandwiches, either covered with cadmium to shield from the neutrons below $0.5 \mathrm{eV}$ or uncovered. From the measurements of the neutron dose (from the 
difference between ${ }^{6} \mathrm{Li}$ and ${ }^{7} \mathrm{Li}$ ), and taking advantage of the fact that the $\mathrm{LiF}$ material was thick enough to totally absorb neutrons below $0.5 \mathrm{eV}$, the integral neutron fluence through the detector could be evaluated (Fig. 50), and was found consistent with the simulation. This completes our low energy checks of the neutron fluence in TARC.

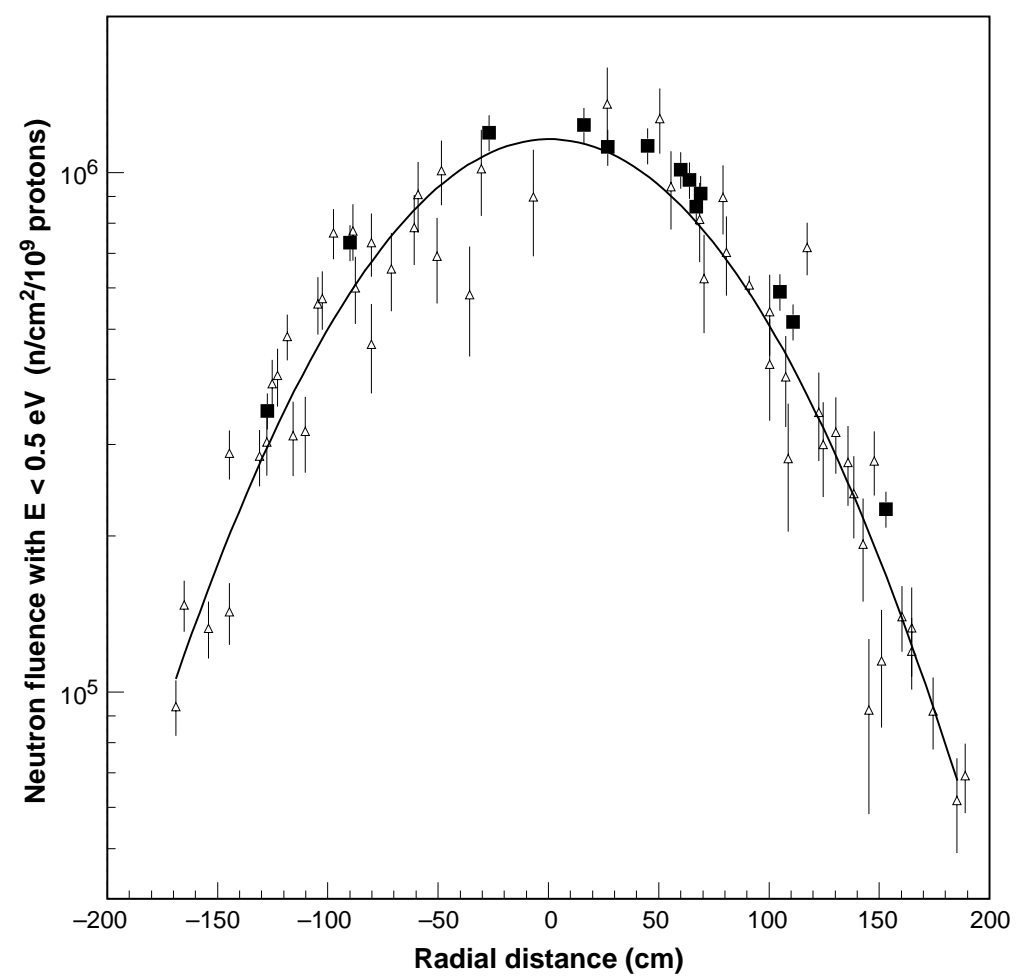

Fig. 50: Thermal neutron fluence $(E<0.5 \mathrm{eV})$ as a function of the radial distance in the lead block. The line is an eye guide connecting the experimental points (triangles). The black squares are Monte Carlo simulation.

\subsection{High-energy neutrons ( $10 \mathrm{keV}$ to $2 \mathrm{MeV}$ ) with ${ }^{3} \mathrm{He}$ ionization counters}

\subsubsection{Introduction}

As already explained in previous sections, it is neutrons in the low-energy range that are most important for long-lived fission fragment transmutation. Neutron energies up to $10 \mathrm{keV}$ are covered by several detectors, mainly the ${ }^{3} \mathrm{He}$ scintillation counter and the $\left({ }^{6} \mathrm{LiF},{ }^{233} \mathrm{U}\right) \mathrm{Si}$ detectors. However, the birth of neutrons takes place in the high-energy range, where the competition between capture resonances, elastic and inelastic collisions, and multiplicative $(\mathrm{n}, \mathrm{xn})$ reactions will determine the available neutron flux at lower energies. In addition, the knowledge of the neutron flux in the region of the spallation cascade, dominated by the high-energy neutrons, is very important for the design of the spallation target, one major item of any ADS design, in particular for the Energy Amplifier. The ${ }^{3} \mathrm{He}$ ionization chamber is the main TARC detector covering the neutron spallation energy region.

Ionization chambers filled with ${ }^{3} \mathrm{He}$ are standard detectors for fast neutron spectroscopy i.e. in the energy range from tens of $\mathrm{keV}$ to a few $\mathrm{MeV}$ [50]. Neutrons entering such detectors interact with the ${ }^{3} \mathrm{He}$ gas through the exothermic reaction:

$$
\mathrm{n}+{ }^{3} \mathrm{He} \rightarrow \mathrm{p}+{ }^{3} \mathrm{H}+Q \quad \text { where } \quad Q=764 \mathrm{keV} .
$$

The simplicity of the well-known cross-section together with the noble gas properties and the high $Q$-value make ${ }^{3} \mathrm{He}$ detectors well-suited for spectrometry both in the proportional or 
ionization modes. In the latter case, in most of the applications, a Frisch grid (Figs. 51 and 52) has been added in order to maximize the energy resolution. Resolution is the most crucial parameter when one is trying to have the lower end of the detectable neutron energy spectrum separated from the overwhelming thermal peak background given by the $(n, p)$ reaction. The most important factors that affect the energy resolution are general parameters such as amplifier noise, pulse shaping, mechanical vibrations, homogeneity of the electric field, and peculiar ones linked to neutron detection such as wall effects and recoil effects at high energies.

\subsubsection{Description of the counter}

Since the best results have been obtained with gridded ionization chambers of the Cutler-Shalev type [51], our ${ }^{3} \mathrm{He}$ spectrometer is based on the same principle with, however, a major additional implementation: good signals can be selected to correspond only to tracks fully contained in a well-defined fiducial gas volume, determined by a system of guard electrodes and proportional counters which allow the suppression of unwanted events by anticoincidence (veto) (Fig. 51).

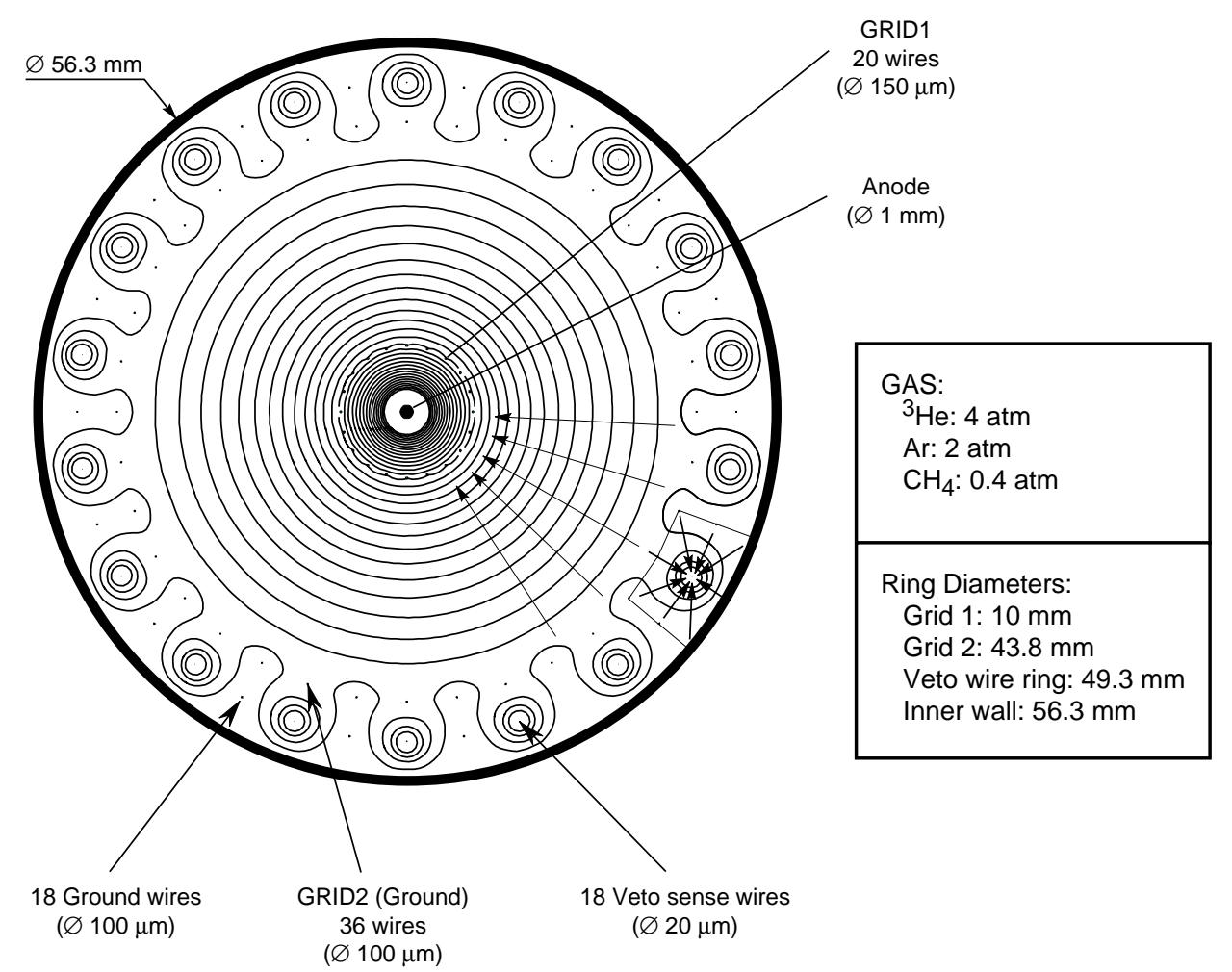

Fig. 51: Transverse view of the ${ }^{3} \mathrm{He}$ ionization detector, with indication of the equipotential lines.

Since ${ }^{3} \mathrm{He}$ has a very large neutron capture cross-section for low and intermediate energies the counter length of $140 \mathrm{~mm}$, defining a total internal volume of $\sim 330 \mathrm{~cm}^{3}$, was chosen to have a sensitive volume giving a counting rate not exceeding a few kilohertz, and to minimize perturbation of the gradual slowing down of neutrons in lead.

A detailed description of the counter geometry can be found in Ref. [8].

The counter mechanics (Fig. 52) basically consists of two flanges spaced and kept together by two semi-cylindrical shells $0.3 \mathrm{~mm}$ thick (also made of stainless steel 304L), having a diameter of $54.8 \mathrm{~mm}$ and a length of $88 \mathrm{~mm}$. Each flange, holding the counter's wires, is made of two parts: 
(a) an external aluminium ring, $8 \mathrm{~mm}$ thick, with an outer diameter of $55.4 \mathrm{~mm}$ and an internal diameter of $25 \mathrm{~mm}$;

(b) an internal insulating (Stesalite) disk, $5 \mathrm{~mm}$ thick, inserted in the aluminium ring and supporting the central detecting anode and the cylindrical Frisch grid.

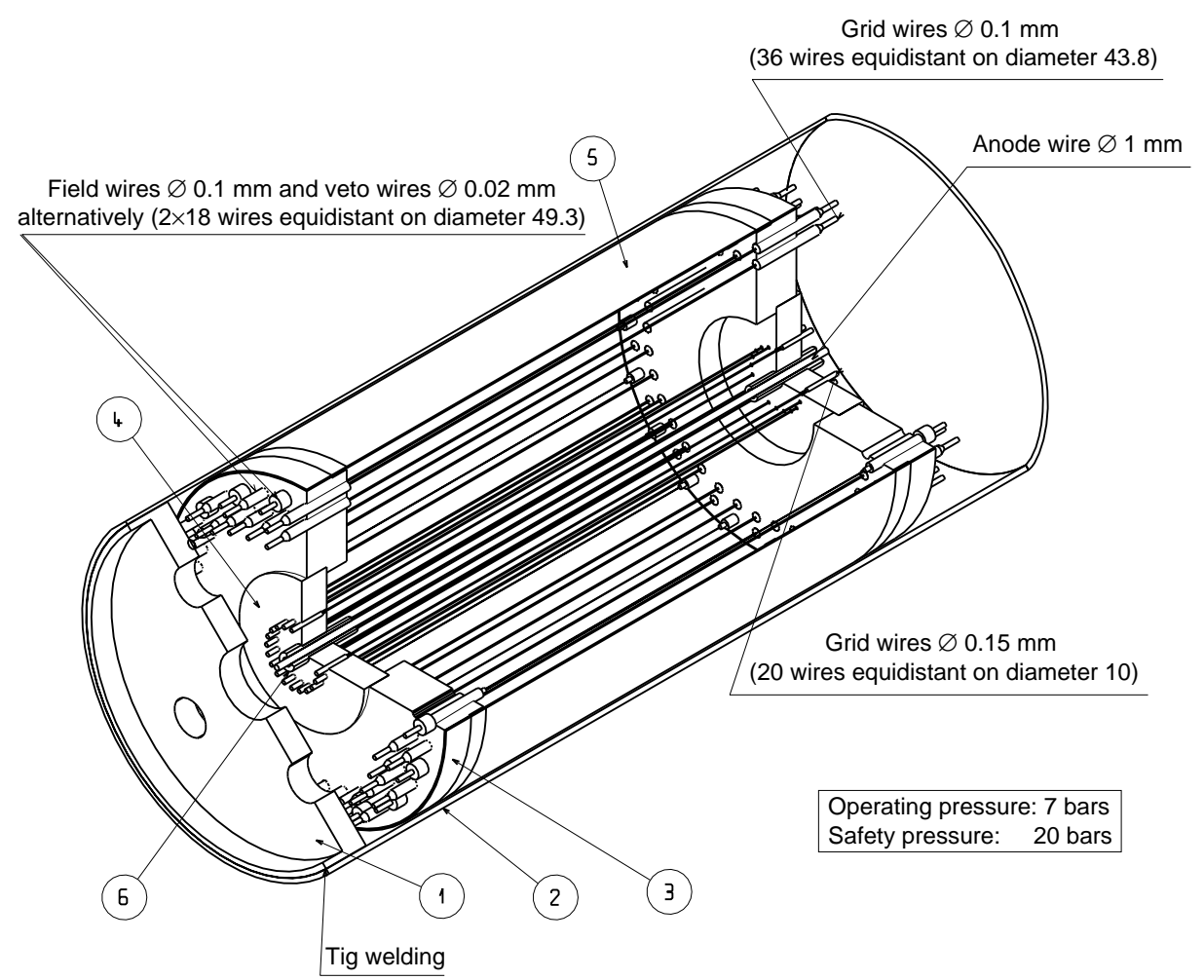

Fig. 52: 3D sketch of the ${ }^{3} \mathrm{He}$ ionization detector. 1) Stainless steel flange (welded); 2) stainless steel container; 3) Al bouchon ring; 4) Stesalite insulator; 5) insulating layer; 6) bouchon veto.

A first innovation with respect to most ${ }^{3} \mathrm{He}$ neutron counters is represented by veto-guard end tubes surrounding the anode edges having a diameter of $3 \mathrm{~mm}$ and a length in the active area of $8 \mathrm{~mm}$. These veto tubes allowed the detection of pulses induced by neutron tracks near to or in the end flanges.

The anode and guard tubes are surrounded by the Frisch circular grid (10 mm diameter) made of a ring of $20 \mathrm{Cu}$-Be wires, each with a diameter of $150 \mu \mathrm{m}$. The choice of the above geometrical parameters results in an electrical shielding inefficiency of $\sim 7 \%$ and imposes a ratio $V_{\text {anode }} / V_{\text {grid }}>3$ to have $\sim 100 \%$ transparency for electrons [52].

The anticoincidence, with respect to the external cylindrical surface, is provided by a system of proportional wires stretched on the external aluminium ring. It consists of two rings of wires:

(a) an internal ring (Grid 2) having a diameter of $43.8 \mathrm{~mm}$ made of $36 \mathrm{Cu}-\mathrm{Be} 100 \mu \mathrm{m}$ wires all grounded to the aluminium plates, which defines the outer diameter of the effective detecting volume;

(b) an external ring with a diameter of $49.3 \mathrm{~mm}$, made of 36 wires, alternatively one $20 \mu \mathrm{m}$ gold-plated tungsten anode and one grounded $\mathrm{Cu}-\mathrm{Be} 100 \mu \mathrm{m}$ wire.

Assuming a full efficiency for the anticoincidence counters and including the effect of the lateral guard tubes, the total effective sensitive volume, to which the neutron spectra can be normalized, is $\sim 118 \mathrm{~cm}^{3}$, with a geometrical ratio $\operatorname{Vol}_{\text {grid }} / \operatorname{Vol}_{\text {total }} \sim 5 \%$. 
The ${ }^{3} \mathrm{He}$ ionization counter was filled with a gas mixture of 4 atm of ${ }^{3} \mathrm{He}, 2$ atm of Ar, and 0.4 atm of $\mathrm{CH}_{4}$.

The counter works in induction mode and, therefore, the input signal to the preamplifier is the direct charge produced by ionization by the products of the neutron interaction in the gas mixture. Since the electron yield is one electron per $30 \mathrm{eV}$, the ionization for a thermal neutron delivering $764 \mathrm{keV}$ corresponds typically to $\sim 25500$ electrons or $4 \mathrm{fC}$. It clearly appears that the electronic thermal noise introduced by the first stage becomes the most important parameter concerning the energy resolution. A low noise preamplifier from the ICARUS experiment [53] was used.

\subsubsection{The signals from the counter}

\subsubsection{Signals recorded}

The signals from the detector are recorded through four FADC channels, corresponding to the anode, grid, and the radial and longitudinal (guard end tube) vetoes.

The anode amplitude is proportional to the energy released in the fiducial volume, in the form of gas ionization, by the charged particles resulting from the neutron reactions with the gas nuclei. The neutron energy is computed from this signal. Figure 53 shows the shape of a typical recorded anode signal.

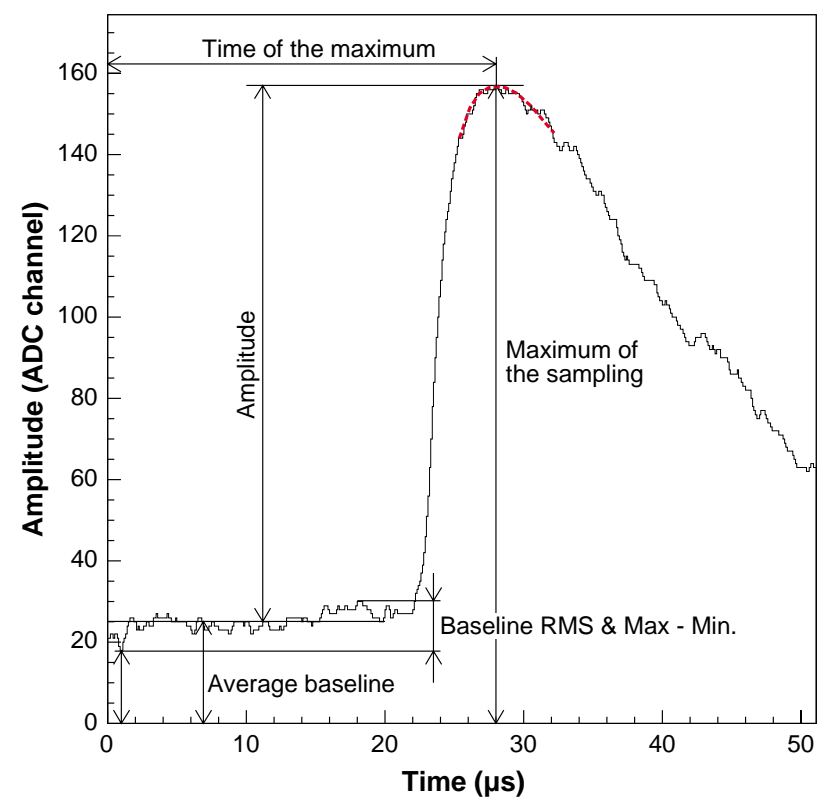

Fig. 53: Typical anode signal (signal actually recorded at TARC).

The FADC memory is locked when the anode signal becomes larger than a predefined trigger level (typically $20 \%$ of the signal size in the thermal peak). The time with respect to the arrival at the TARC lead volume of the closest preceding proton (or pion) candidate as father of the neutron which generated the signal is computed combining the anode signal information with the beam hodoscope 4-fold coincidences signals (see the beam instrumentation, Section 2.2.3) recorded by FADC modules synchronized to the ${ }^{3} \mathrm{He}$ counter. This time difference is used to select events produced by high-energy neutrons. Events are selected to have a maximum delay from the father proton candidate of $5 \mu \mathrm{s}$. In practice, a hardware selection at $10 \mu \mathrm{s}$ is applied during data-taking and the final $5 \mu$ s cut is applied by the offline event selection. Figure 54 shows the effects of this selection on the reduction of the epithermal peak. 


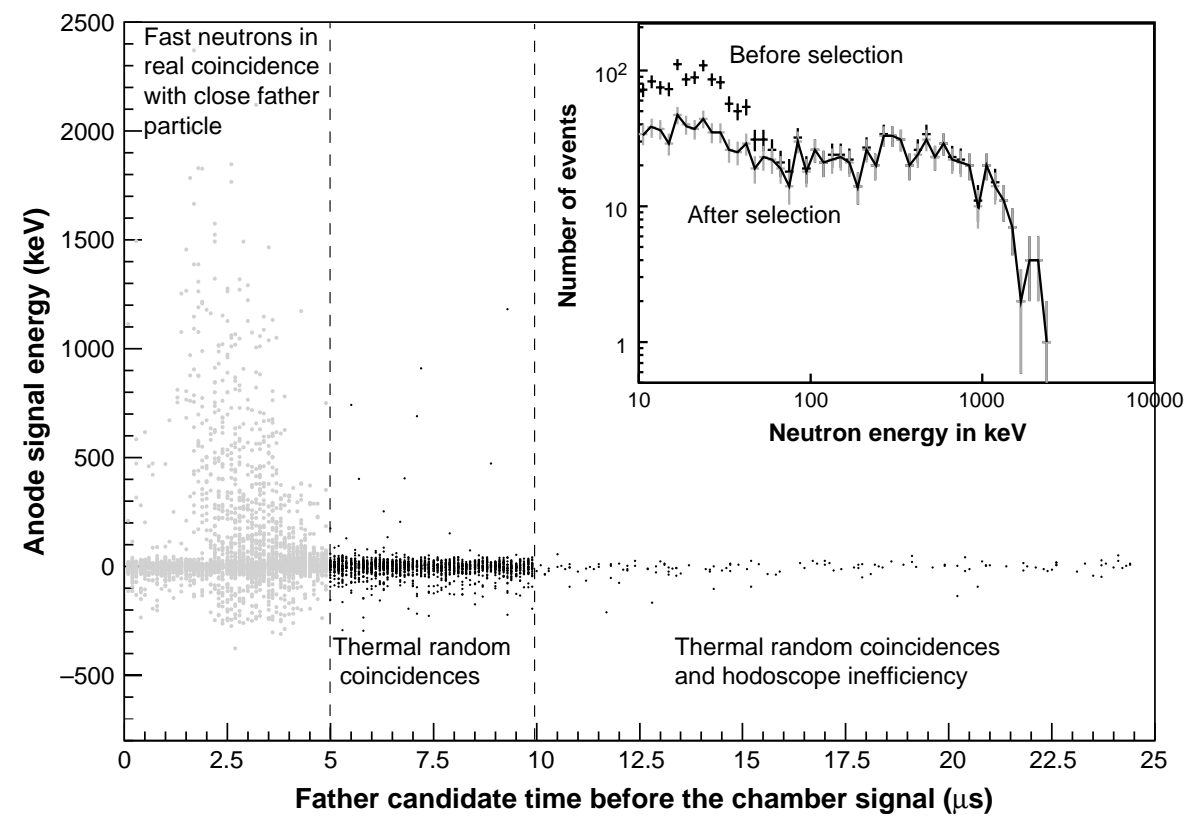

Fig. 54: Selection of high-energy neutrons from the difference between the time of arrival of the father candidate and time of detection of the anode signal (run 1622). The inset shows the projection on the neutron energy axis using a logarithmic scale for the number of events.

For a general event with the particle tracks randomly oriented but well within the fiducial volume, the grid signal is expected first to rise significantly and then to fall down, at least down to the baseline. Following these principles, a complex analysis has been applied to the grid signal (Fig. 55) to select only events whose tracks are well inside the fiducial volume (excluding the grid) and with no significant loss of energy.

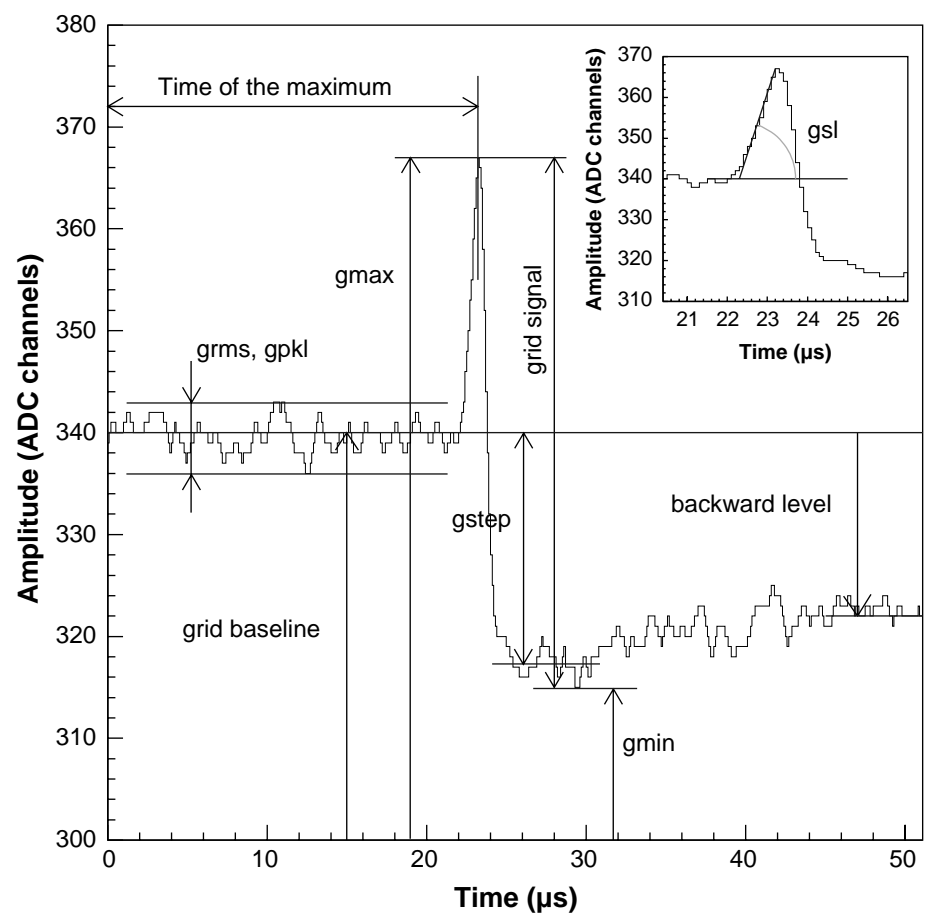

Fig. 55: Typical grid signal and the various parameters used in the event analysis and selection. The inset is a blown-up region between 20.5 and $26.5 \mu$ s showing how gsl is defined. 
The two vetoes collect signals only if a fraction of the ionization is produced outside the fiducial volume. The presence of any significant signal in these channels indicates the possibility of incomplete signal collection on the anode channel. A more detailed description of the event selection criteria can be found in Ref. [54].

\subsubsection{Calibration of the counter with monochromatic neutrons and simulation of the detector response to neutrons}

The ${ }^{3} \mathrm{He}$ counter response to monoenergetic neutrons was measured in the calibration experiments performed in the CEN-Bordeaux-Gradignan Van de Graaff accelerator. In these experiments a monochromatic proton beam was sent on thin targets of tritium and ${ }^{7} \mathrm{Li}$. In the targets the $(\mathrm{p}, \mathrm{n})$ reaction produces a monochromatic neutron beam (for a fixed angle with respect to the proton beam). The counter was illuminated with these neutron beams at zero degree and the response function was directly measured (Fig. 56).

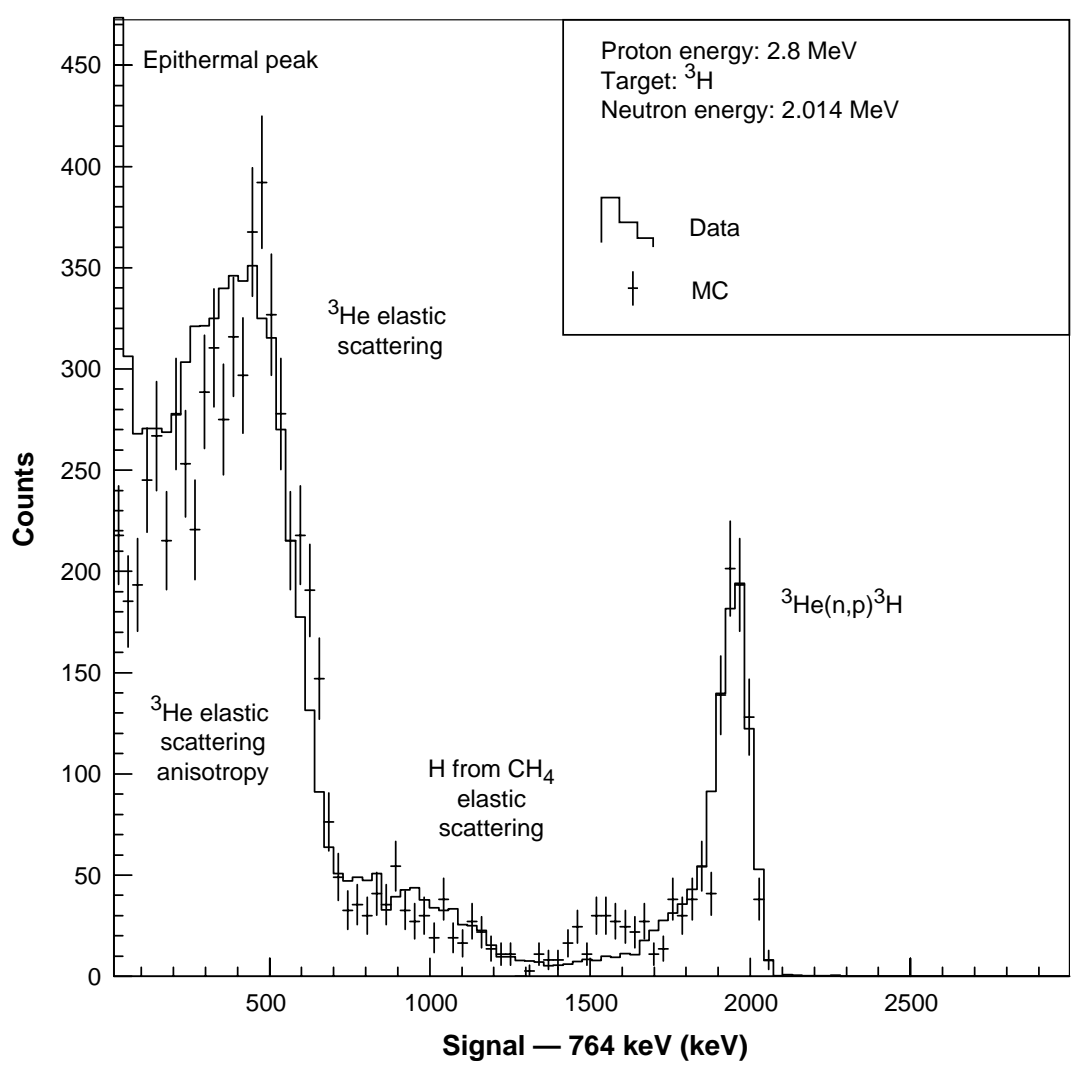

Fig. 56: Detector response to a monochromatic neutron beam of $2014 \mathrm{keV}$ at the CEN-BordeauxGradignan van de Graaff accelerator.

It was also possible to compute, from the Bordeaux calibration, the efficiency of the detector in the $(\mathrm{n}, \mathrm{p})$ peak as the number of signals for a given neutron fluence and to compare with the quasitransparent approximation for the detector response (Fig. 57). In this quasitransparent approximation, the detector efficiency is obtained as the product of the ${ }^{3} \mathrm{He}(\mathrm{n}, \mathrm{p})$ reaction cross-section, times the total number of ${ }^{3} \mathrm{He}$ nuclei in the fiducial volume, times the probability for the event to be accepted by the selection cuts (mainly the geometrical efficiency), times the probability of collection of the complete ionization charge released in the chamber.

The error bars in Fig. 57 correspond only to the statistical fluctuations. In addition, the uncertainties on the proton intensity $(5 \%)$, the exposure time $(2 \%)$, the production reaction 
cross-section (2.5\%), the thickness of the $\left({ }^{3} \mathrm{H}\right.$ and $\left.{ }^{7} \mathrm{Li}\right)$ targets $(2 \%)$, the effect of the position of the ${ }^{3} \mathrm{He}$ counters with respect to the targets $(8 \%)$, and the possible dead time (2\%) produce a total systematic uncertainty on the detector efficiency calibration of $10 \%$. The combination of the statistical and systematic errors add from 10 to $15 \%$ to peak efficiency uncertainties. Similar considerations apply to the uncertainties on the convolution matrix.

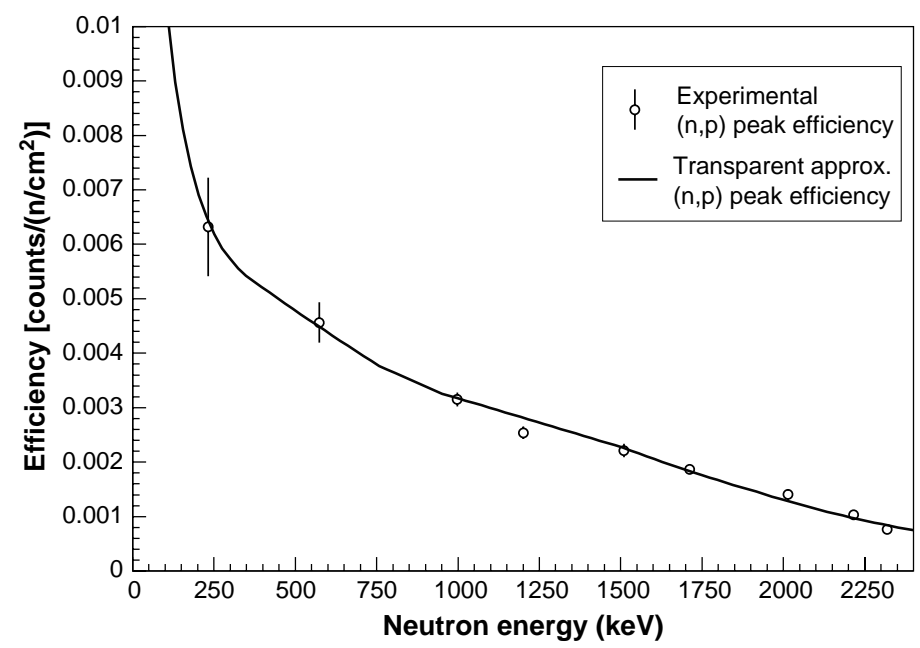

Fig. 57: (n,p) peak efficiency of the ${ }^{3} \mathrm{He}$ counter versus the neutron energy.

\subsubsection{Detector resolution}

As already mentioned when describing the electronic chain, the thermal noise in the preamplifiers is the largest component in chamber resolution. Using electronic pulses corresponding to 25500 electrons (equivalent to $764 \mathrm{keV}$ ) injected into the preamplifier input, the resolution is measured to be $4.9 \%$ (FWHM).

The actual resolution of the chamber obtained with the help of an ${ }^{241} \mathrm{Am}-\mathrm{Be}$ (alpha,n) neutron source, after thermalizing the neutrons by surrounding the counter with $10 \mathrm{~cm}$ of paraffin and screening against background neutrons with a thick layer of $\mathrm{B}_{4} \mathrm{C}(\sim 5 \mathrm{~cm})$ is $5.1 \%$ (FWHM), confirming that the resolution is dominated by the electronics.

During the actual data taking at the TARC experiment the resolution obtained by fitting the thermal peak with a Gaussian function is typically 5.9\% (FWHM).

\subsubsection{TARC experimental data set}

A secondary PS beam [17] collected behind a target hit by the $26 \mathrm{GeV}$ protons from the PS provided typical pulses of 5000 to 10000 particles $(2.5 \mathrm{GeV} / \mathrm{c})$, during a $400 \mathrm{~ms}$ spill, every $14.4 \mathrm{~s}$. A time-of-flight system was able to identify protons with $5 \sigma$ separation but it was not possible to identify pions from other light particles.

\subsubsection{Monte Carlo simulation of the experiment}

The full flux measurement experiment with the ${ }^{3} \mathrm{He}$ ionization counter has been simulated combining the standard TARC simulation with the detailed detector simulation.

\subsubsection{Fluence determination}

For each position the collected signal spectra have been computed and normalized to the beam intensity. Because of the complex response function of the ${ }^{3} \mathrm{He}$ ionization detector to monoenergetic neutrons, the fluence energy spectra cannot in general be obtained with high precision, by simple division of the observed signal spectra by the counter efficiency. A 
deconvolution process is required. However, it should be noted that given the very rapidly decreasing flux with increasing energy in the high-energy range in TARC, the error on the direct flux calculation method can be acceptable for some positions and energy ranges.

Both methods have been used for the analysis of the TARC data as both, after small corrections, provide measurements with reduced and different systematic uncertainties, resulting in complementary information.

The simplest method to extract an estimate of the fluence from the signal, that we shall call the peak efficiency method, consists of dividing, bin by bin, the histogram of signal amplitudes by the detector efficiency in the (n,p) peak (Fig. 57), after applying a small correction dependent on the detector position. In the direct peak efficiency, for each energy bin we ignore the contributions from elastic collisions of neutrons at higher energies resulting in an energy deposited corresponding to the bin under study. At TARC, the fluence decreases very steeply at high energies and, as a consequence, the fraction of signals in a given energy bin coming from neutrons with energies inside the same energy bin, through direct $(n, p)$ reaction, is very high for energies sufficiently low (typically below about $2 \mathrm{MeV}$ ) (Figs. 58 and 59). The fraction of (n,p) signals is, within errors, larger than $80 \%$ for all positions. An average energy-independent correction factor has been computed to take into account this difference, for each individual measurement position. The values for the corrections are in all cases smaller than $10 \%$ and the maximum systematic error left from this analysis method is also $10 \%$ (much smaller at far positions).

The second method used to extract the fluence is what we call the deconvolution method. It is described in detail in Ref. [54]. It is an attempt to take into account additional (n,p) contributions coming from recoiling nuclei produced by neutron elastic scattering mainly on hydrogen (contained in methane) and on ${ }^{3} \mathrm{He}$.

Figures 58 and 59 show the ratios, computed by Monte Carlo simulation, between signals produced in a specific energy bin by the neutrons with less than $2.4 \mathrm{MeV}$ with respect to the complete neutron spectra. The ratio is very close to 1 for low neutron energies but can be as low as $80 \%$ for neutron energies close to $2 \mathrm{MeV}$. Similarly to what was done in the first analysis method, an energy-independent correction factor has been computed for each individual position. This correction is again always smaller than $10 \%$ and the residual systematic uncertainty is also smaller than $10 \%$.

One additional consequence of both analysis methods is that the fluence can only be computed with small uncertainties up to a maximum energy varying for each measuring position. In any case, the statistics available at higher energies is very small and the statistical errors prevent any evaluation of the neutron fluence, above $\sim 2 \mathrm{MeV}$. The systematic errors on the fluence measurements are estimated to be 16 to $18 \%$.

The validity of these methods of fluence extraction from the signal histograms has been checked with the full TARC Monte Carlo simulation.

Figure 60 shows the measured neutron fluence energy spectra at different positions extracted from the signal histogram associated to proton-generated neutrons, using the two analysis methods and compared with the direct Monte Carlo predictions for those positions. The error bars include both the statistical and the systematic error. A generally good agreement is found at low energies, that extends up to $1.5 \mathrm{MeV}$ for positions not too close to the spallation cascade, whereas for closer positions the measurements indicate larger neutron fluences at high energies than what was predicted by the Monte Carlo simulation. 

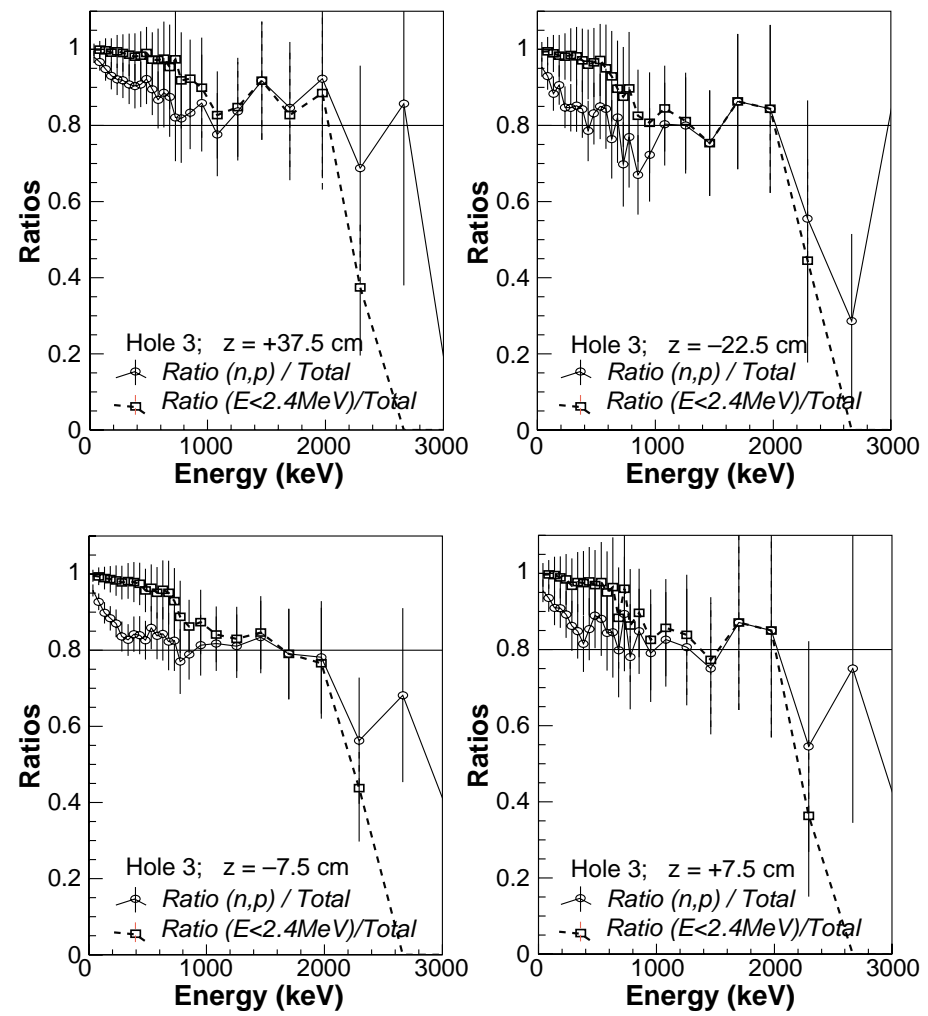

Fig. 58: Fraction of $(n, p)$ signals in all events and fraction of signals (all $n, p$ included) from neutrons of energy lower than $2.4 \mathrm{MeV}$ for several positions in instrumentation hole 3 obtained from our Monte Carlo simulation (close to the centre of the lead volume where the neutron energy spectrum is harder).
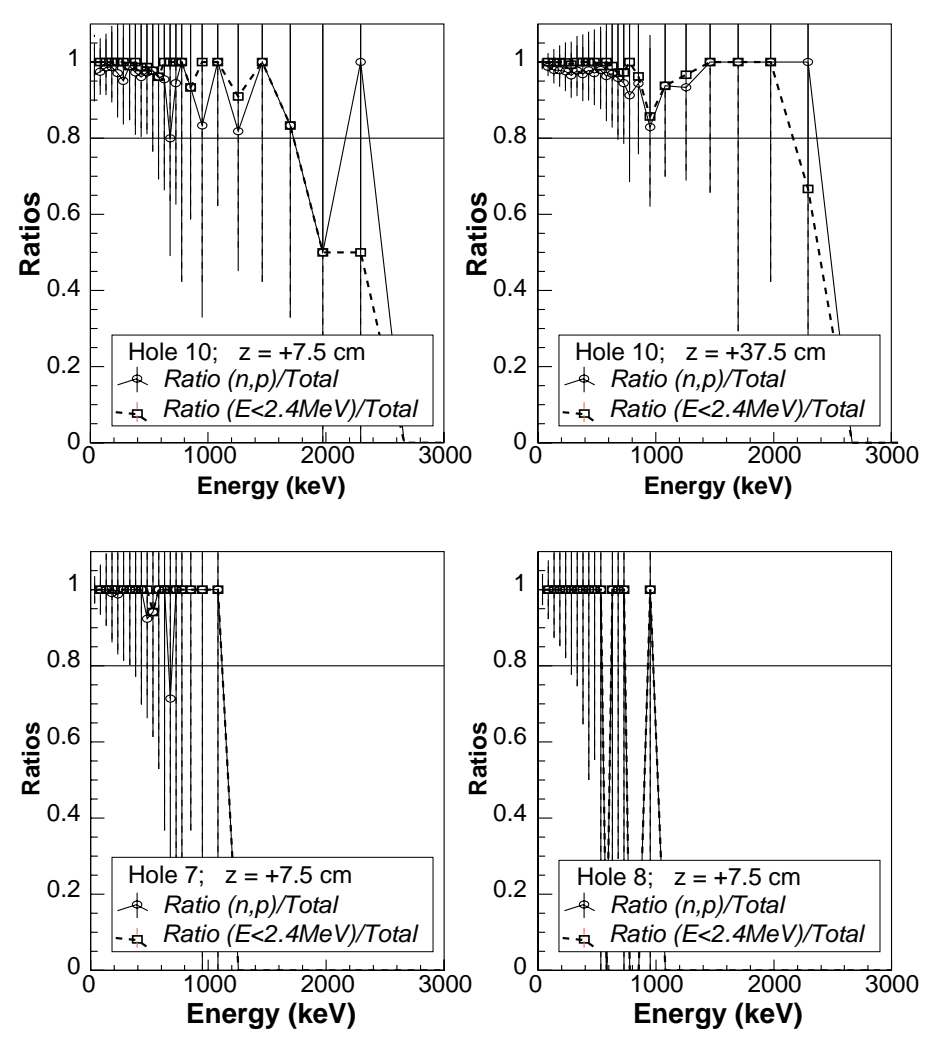

Fig. 59: Fraction of (n,p) signals in all events and fraction of signals (all $n, p$ included) from neutrons of energy lower than $2.4 \mathrm{MeV}$ for several positions in the instrumentation holes 10, 7, and 8 obtained from our Monte Carlo simulation (close to the centre of the lead volume where the neutron energy spectrum is harder). 

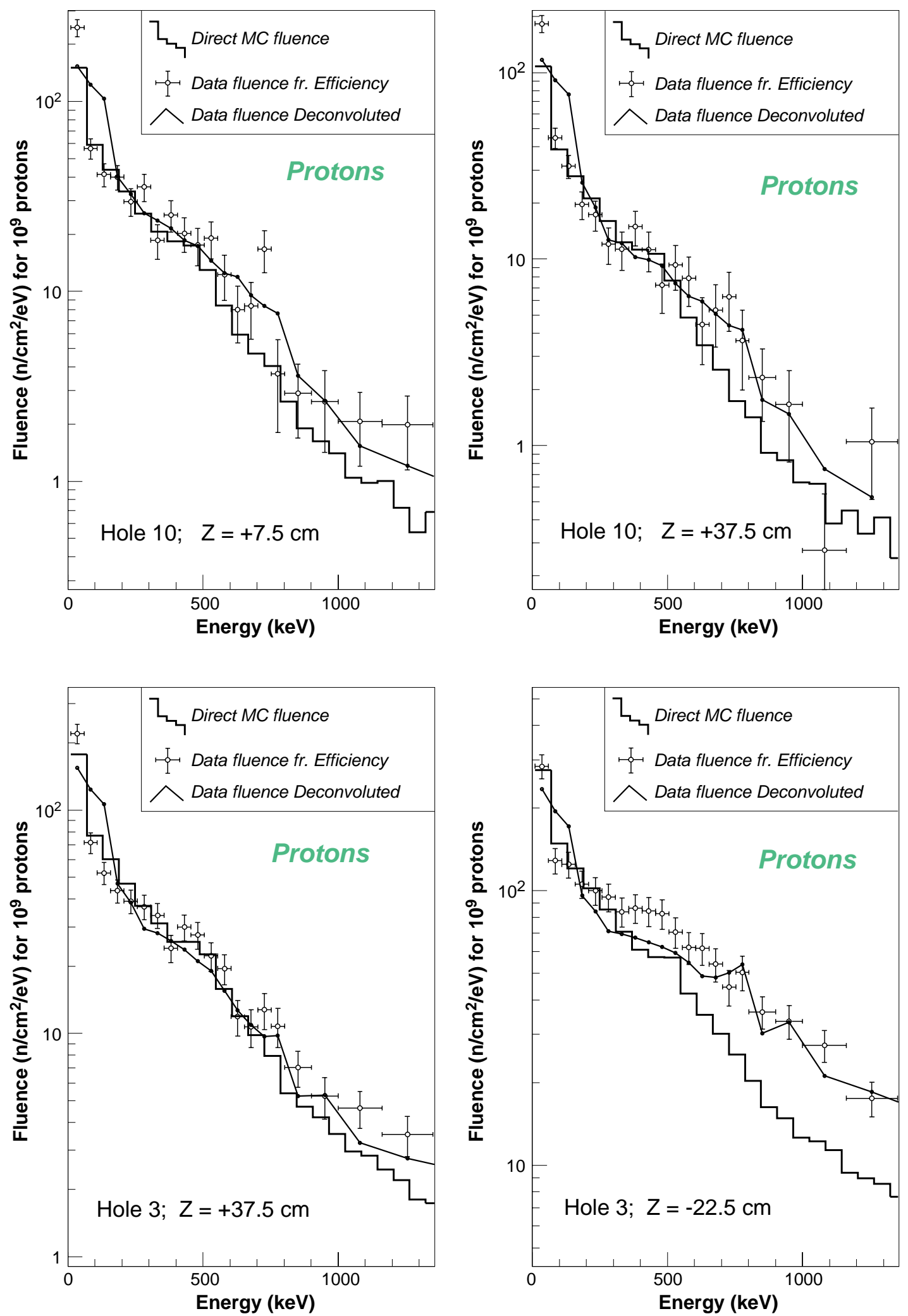

Fig. 60: Measured neutron fluence spectra produced by $2.5 \mathrm{GeV} / c$ protons, at different positions in the TARC lead assembly.

For positions sufficiently far from the spallation cascade, $40 \mathrm{~cm}$ or more, there is good agreement between data and Monte Carlo (Fig. 61). On the other hand, for close positions the agreement is good up to $200 \mathrm{keV}$, getting worse for higher energies (Fig. 61). In this last case 
the experimental values for the fluence are always larger than the neutron fluence from the Monte Carlo. One possible explanation for part of this difference is the fact that the positions where there are discrepancies are deeply inside the spallation cascade and this has two consequences. First, some very high energy neutrons $(E>20 \mathrm{MeV})$ are present but not included in the Monte Carlo estimation of the signals histogram, and consequently in the required corrections for the analysis methods. This is because neutrons above $20 \mathrm{MeV}$ are treated in the FLUKA step, and their contribution to the fluence is not retained. Second, at these positions, in addition to neutrons, there is also a substantial fluence of high-energy photons and charged particles. The detector is designed to have a high rejection factor for signals coming from these particles, but residuals will contribute to the observed differences.

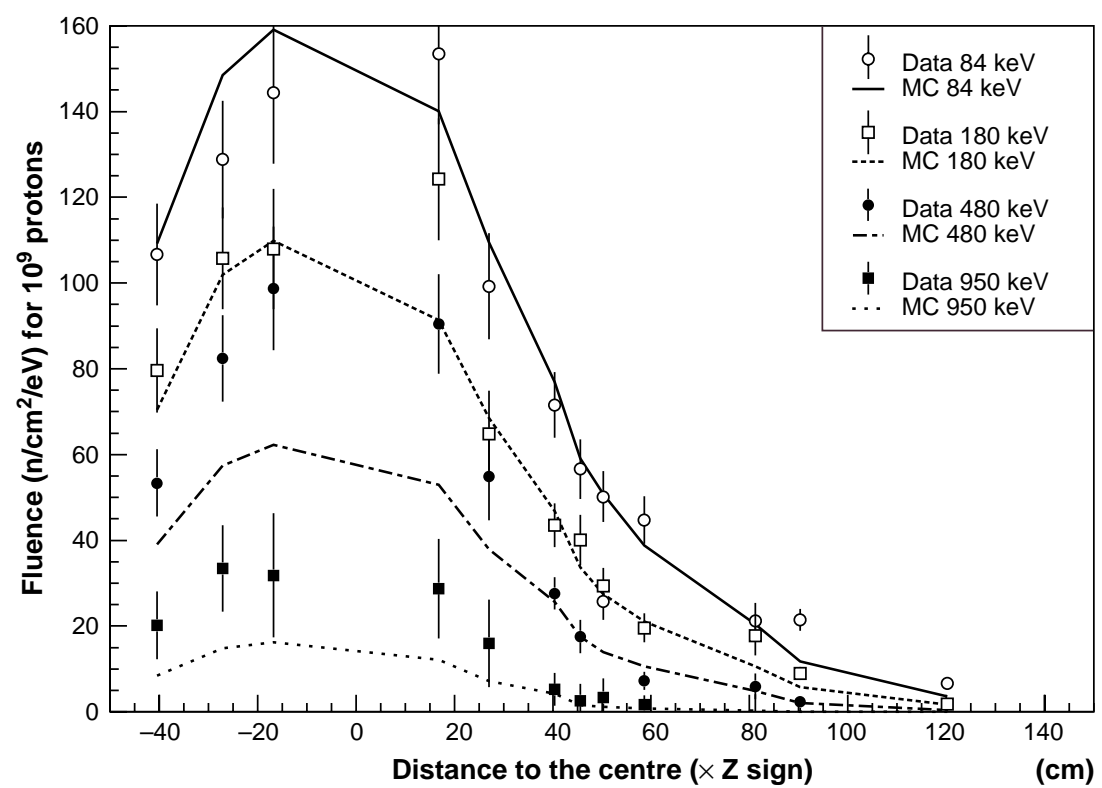

Fig. 61: Distribution of the neutron fluence as a function of distance with respect to the center of the TARC lead volume, at different energies measured by the ${ }^{3} \mathrm{He}$ ionization counter. The minus signs indicate positions at $\mathrm{z}<0$.

\subsubsection{Conclusion}

An innovative system of signal collection has allowed us to use the full anode and grid signal shapes for the data analysis, achieving enhanced rejection of unwanted events such as pile-ups, events produced outside the fiducial volume, and events with incomplete energy collection.

The energy spectra of the neutron fluence were measured in the range from a few tens of $\mathrm{keV}$ up to about $1.5 \mathrm{MeV}$. The precise range of reliable measurements depends on the hardness of the neutron fluence spectra and consequently is a function of the distance from the measurement position and the spallation target.

As the statistics is generally limited we tried to combine the signals coming from protons with those coming from pions [54]. This is possible because of the very similar shape of the neutron fluence energy spectra produced by the two types of particles with the same nominal momentum at TARC $(2.5 \mathrm{GeV} / c)$. This is confirmed both by our experimental data and by the Monte Carlo simulation. However, the very large uncertainty on the pion beam intensity prevents this merging of data from being quantitatively significant. 
In spite of the many difficulties in performing such measurements, a reasonable agreement is found with our Monte Carlo predictions both for the neutron flux energy and space distributions, especially good for positions outside the spallation cascade core extending over $40-50 \mathrm{~cm}$ from the centre of the cascade.

The ${ }^{3} \mathrm{He}$ ionization counter measurements are unique because they cover the energy region where the shape of the fluence energy distribution changes very rapidly and is very sensitive to the properties of the spallation process (Fig. 62). This gives us at the same time a direct test of the neutron transport in the energy region dominated by the lead capture resonances.

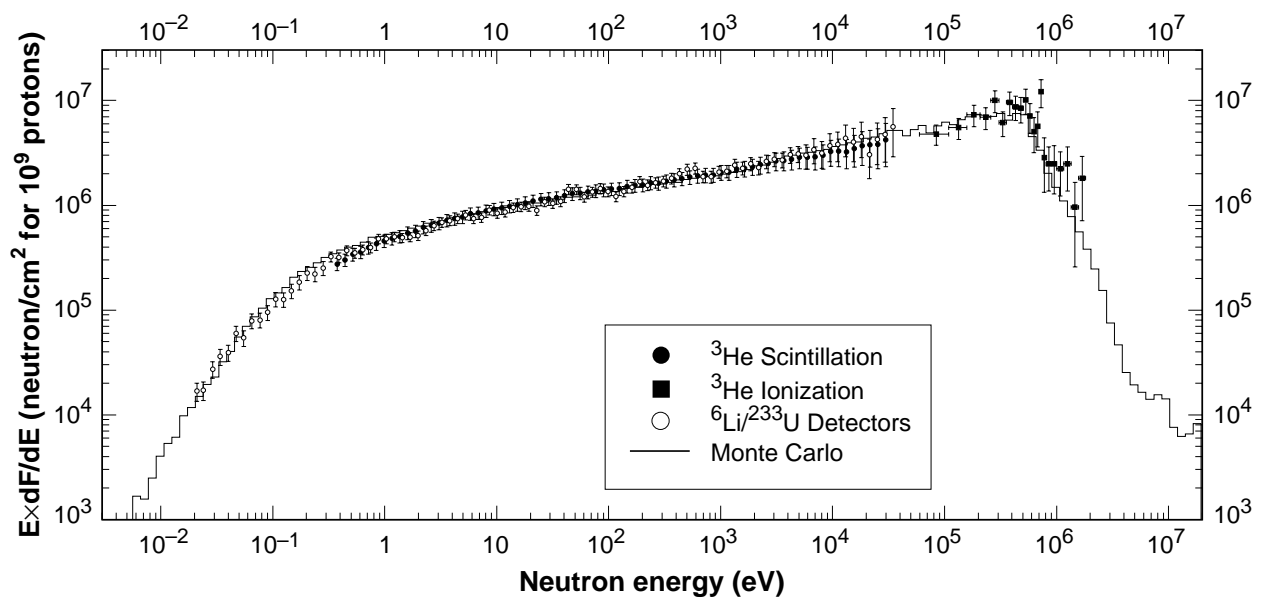

Fig. 62: Distribution of the neutron fluence versus its energy measured by the ${ }^{3} \mathrm{He}$ ionization detector in the TARC lead volume (hole $10, z=+7.5 \mathrm{~cm}$ ). The other data come from ${ }^{3} \mathrm{He}$ scintillation and ${ }^{6} \mathrm{Li} /{ }^{23} \mathrm{U}$ detectors. The histogram is the Monte Carlo simulation. All data were taken with protons of $2.5 \mathrm{GeV} / \mathrm{c}$ momentum.

\subsection{High-energy neutrons with activation methods}

\subsubsection{Fissions in ${ }^{232} \mathrm{Th}$, and ${ }^{237} \mathrm{~Np}$}

The distribution of fission rates in the TARC lead volume was measured using fissionable materials $\left({ }^{232} \mathrm{Th}, \mathrm{U}_{\text {natural }},{ }^{235} \mathrm{U},{ }^{239} \mathrm{Pu}\right.$ and $\left.{ }^{237} \mathrm{~Np}\right)$ in close contact with Lexan track-etch detectors, which can detect the produced fission fragments. Integral measurements of the high-energy neutron fluence $\left(E_{\mathrm{n}}>1.4 \mathrm{MeV}\right)$ obtained from fission on ${ }^{232} \mathrm{Th}$, provide the most significant additional check of the neutron fluence at high energy.

Only ${ }^{232} \mathrm{Th}$ and ${ }^{237} \mathrm{~Np}$ measurements are reported here. The details of the technique used as well as the other measurements can be found in Ref. [55].

The fission cross-section of ${ }^{232} \mathrm{Th}$ is negligible for neutrons with energies $E_{\mathrm{n}}<1.4 \mathrm{MeV}$ but becomes significant for neutrons with higher energies. This makes the ${ }^{232} \mathrm{Th}$ a good tool to study the integral neutron fluence for energies above $1.4 \mathrm{MeV}$. Our data are in excellent agreement with the Monte Carlo prediction (Fig. 63).

For ${ }^{237} \mathrm{~Np}$ this is not the case (Fig. 64) pointing out a potential problem with the ${ }^{237} \mathrm{~Np}$ fission cross-section database.

The error on the simulation was estimated to be $10 \%$ coming mainly from the Monte Carlo simulation statistical error and from the uncertainty on cross-section data from different databases. The second stage of TARC Monte Carlo simulation processes only neutrons with energy $E<19.6 \mathrm{MeV}$. However, there is a small contribution of neutrons with higher energies $(E>19.6 \mathrm{MeV})$ in the case of ${ }^{232} \mathrm{Th}$ and ${ }^{237} \mathrm{~Np}$ which was calculated in the FLUKA stage and 
found to represent typically $12 \%$ and $10 \%$ of the neutrons with energy $1.4 \mathrm{MeV}<E_{\mathrm{n}}<19.6 \mathrm{MeV}$, at $z=7.5 \mathrm{~cm}$ in holes 3 and 10 , respectively. For ${ }^{232} \mathrm{Th}$ the average fission cross-section of $0.27 \mathrm{~b}$ for neutrons with energy $E_{\mathrm{n}}>1.4 \mathrm{MeV}$ (used as a reference for the fluence measurements of neutrons with $E_{\mathrm{n}}>1.4 \mathrm{MeV}$ ) and the cross-section from the ENDF/B-VI database were compared (Fig. 63). Both gave similar results within the above errors although the Monte Carlo results using the ENDF/B-VI database are slightly underestimated, since they do not include neutrons above $19.6 \mathrm{MeV}$.

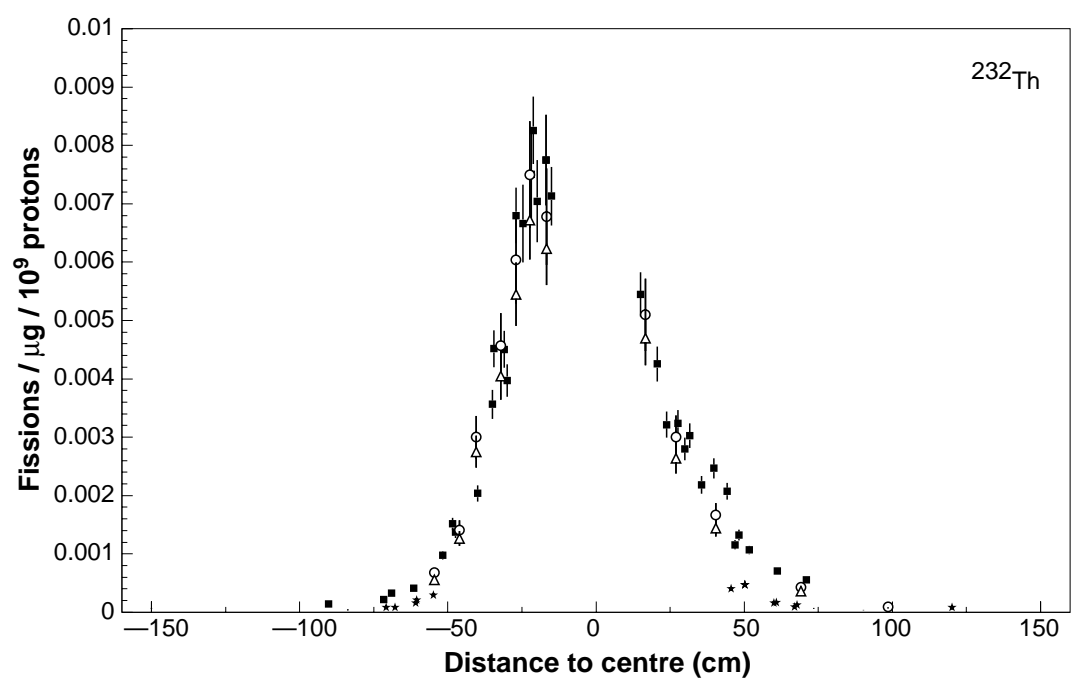

Fig. 63: Measurement of fission rates in ${ }^{232} \mathrm{Th}$ as a function of distance from the centre of the lead volume, in hole 3 (black squares), in other holes (stars) and comparison with the TARC Monte Carlo simulation using ENDF/B-VI (open circles) and using a step function cross-section ( $0.27 \mathrm{~b}$ ) (open triangles).

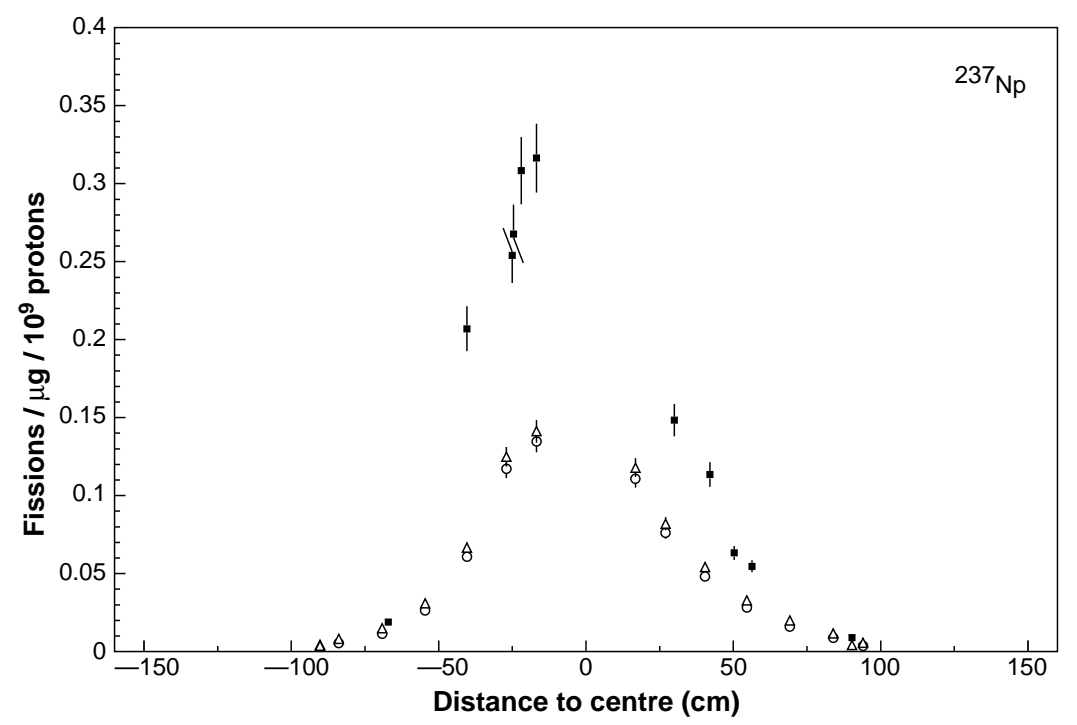

Fig. 64: Measurement of fission rates in ${ }^{237} \mathrm{~Np}$ as a function of distance from the centre of the lead volume, in hole 3 (black squares) and comparison with the Monte Carlo simulations using ENDF/B-VI (open circles), JENDL-3.2 (open triangles).

In the case of ${ }^{237} \mathrm{~Np}$ the fission rates predicted by ENDF/B-VI and JENDL-3.2 show differences ranging from $11 \%$ near the centre of the lead volume to a factor 3 larger for JENDL-3.2 at a distance $1.5 \mathrm{~m}$ from the centre. This indicates large inaccuracies in the ${ }^{237} \mathrm{~Np}$ fission cross-section (Fig. 65). However, this does not explain the discrepancy with our data which are a factor 2 higher than the prediction (Fig. 64). 


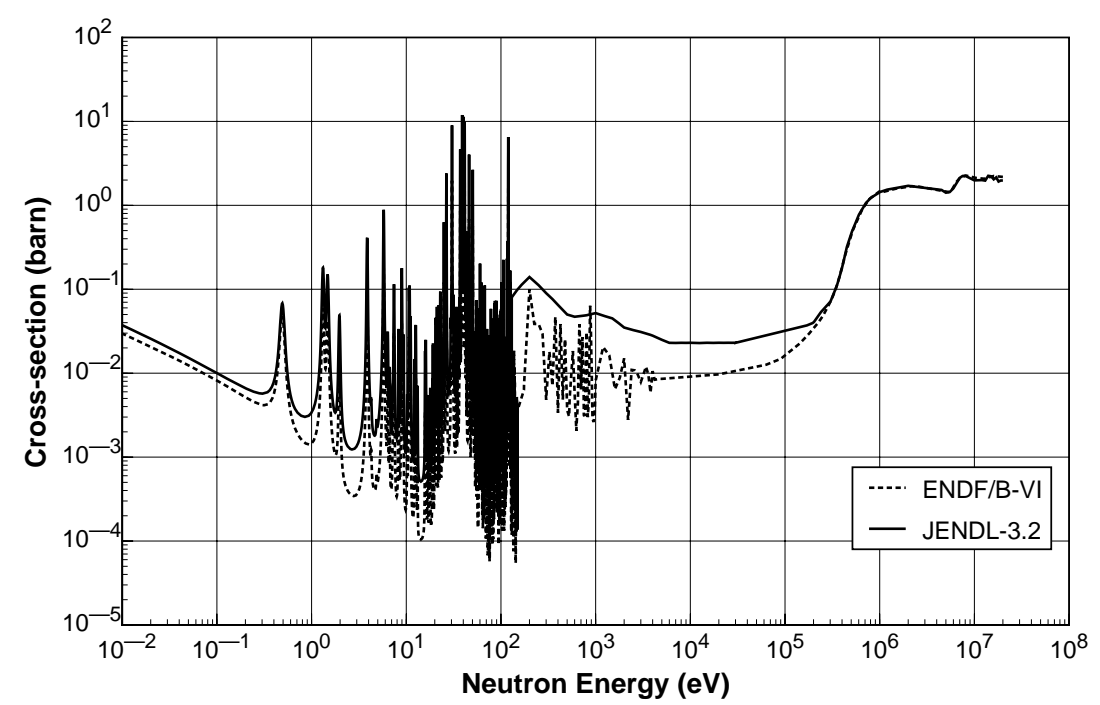

Fig. 65: The fission cross-section of ${ }^{237} \mathrm{~Np}$ from the JENDL-3.2 (full line) and ENDF/B-VI (dotted line) data libraries as a function of neutron energy.

In conclusion, Lexan is an integral fission fragment detector. It measures fission fragments produced in the converter by the whole energy spectrum of incident neutrons. However, owing to the properties of the fission cross-section of the ${ }^{232} \mathrm{Th}$ converter and its neutron energy threshold of about $1.4 \mathrm{MeV}$ for fission, fluences of fast neutrons with energy greater than 1.4 MeV can be measured using the fission measurements with ${ }^{232} \mathrm{Th}$ (see Ref. [55]). These fast neutron fluences measurements are in agreement with the corresponding Monte Carlo calculations, providing an important check of the high energy component of the neutron fluence, which in addition has important implications in the design parameters of the Energy Amplifier (fast neutron damage to structural material).

Further studies of the neutron fluence at high energies were performed with ${ }^{12} \mathrm{C}(\mathrm{n}, 2 \mathrm{n}){ }^{11} \mathrm{C}$ and ${ }^{12} \mathrm{C}(\mathrm{n}, 3 \mathrm{n}){ }^{10} \mathrm{C}$ reactions. This was done using the carbon shuttle in the rabbit measurement (Section 7.1). The reader is referred to Ref. [8] for a complete description of these measurements.

\subsection{General conclusion on neutron fluence measurements}

We have used several techniques (Fig. 66) in TARC to measure neutron fluences over the desired energy range from thermal to $\mathrm{MeV}$ neutrons.

The ${ }^{6} \mathrm{Li} /{ }^{233} \mathrm{U}$ detectors and the ${ }^{3} \mathrm{He}$ detector in the scintillation mode cover the neutron energy range from thermal up to about $100 \mathrm{keV}$ and the ${ }^{3} \mathrm{He}$ ionization detector covers the higher energy range from $10 \mathrm{keV}$ to $2 \mathrm{MeV}$. These differential measurements were complemented by measurements at specific neutron energies with triple-foil activation methods. Several additional cross-checks of the neutron fluence were performed, outside the energy range covered by the electronic detectors both at low energies $(<0.5 \mathrm{eV})$ using thermoluminescence techniques and at high energies $(>1.4 \mathrm{MeV})$ using fission measurement in ${ }^{232} \mathrm{Th}$. At higher energies threshold reactions $\left[{ }^{12} \mathrm{C}(\mathrm{n}, 2 \mathrm{n}){ }^{11} \mathrm{C}\left(E_{\mathrm{n}}>22 \mathrm{MeV}\right)\right.$ and $\left.{ }^{12} \mathrm{C}(\mathrm{n}, 3 \mathrm{n}){ }^{10} \mathrm{C}\left(E_{\mathrm{n}}>34 \mathrm{MeV}\right)\right]$ have also been studied.

In all cases, our measurements are found to be in very good agreement with our simulation (Fig. 67). We find also, in particular with the fission measurements in ${ }^{232} \mathrm{Th}$, that the spatial distribution of the high-energy component of the neutron fluence shows a significant 
forward-backward asymmetry and dies away quickly (within $50 \mathrm{~cm}$ from the centre of the cascade generated by the beam protons) which is a very useful practical consideration for the design of the EA where fast-neutron damage in structural materials must be minimized.

The data corresponding to Fig. 67 are given in the Appendix, so that they can be useful for benchmaking of future simulation codes.

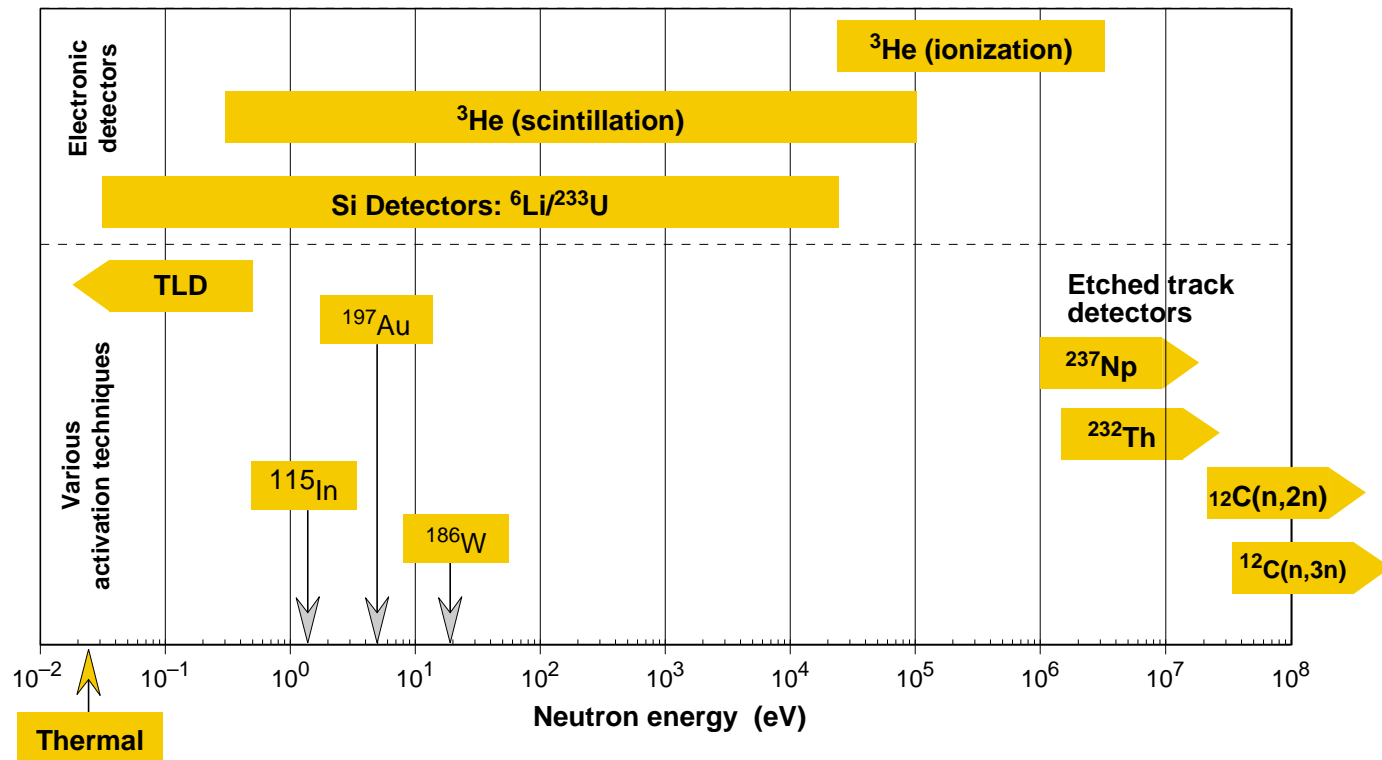

Fig. 66: Illustration of the energy ranges covered by the different detector techniques used in TARC to measure neutron fluences.

We can use the simulation to investigate further the behaviour of neutrons in lead. We can, for instance, switch off the neutron capture process in the simulation and observe the modification of the neutron fluence (Fig. 68). We find that indeed, in our lead, the effect of captures is relatively small, down to neutron energies of $1 \mathrm{eV}$. For instance, the fluence $(\mathrm{d} F / \mathrm{d}[\ln (E)]$ decreases only by a factor 10 from $1 \mathrm{MeV}$ to $1 \mathrm{eV}$, while if we switch off lead captures, this factor is reduced to 5. This remaining decrease in fluence is due in part to the fact that the lead volume is not infinite and also to a diffusion effect well accounted for in the Fermi age theory.

We have obtained a large coherent set of neutron fluence measurements, with redundant methods especially in the energy range below $\sim 10 \mathrm{keV}$ where we want to use the ARC method. This is the first important element in our demonstration of the efficiency of the ARC effect confirming the high transparency of lead over the whole neutron energy range. The fact that the simulation of the neutron fluence at any point within the TARC lead volume agrees with the data, confirms that both the spallation process (FLUKA) and the neutron diffusion and transport (cross-sections) are well described, and constitutes a precise validation of the overall simulation chain developed by our group (see Section 3).

Another interesting aspect of these neutron fluence measurements is that they provide a check of the complete chain of processes from neutron production by spallation to neutron transport until capture or escape from the lead assembly. If we assume that neutron transport is known with good accuracy ( $10 \%$, see Section 3$)$, then our measurements provide a check of the spallation process. We can note that the number of neutrons produced and transported down to $19.6 \mathrm{MeV}$ is found to be 98 for a proton momentum of $3.5 \mathrm{GeV} / c$. 

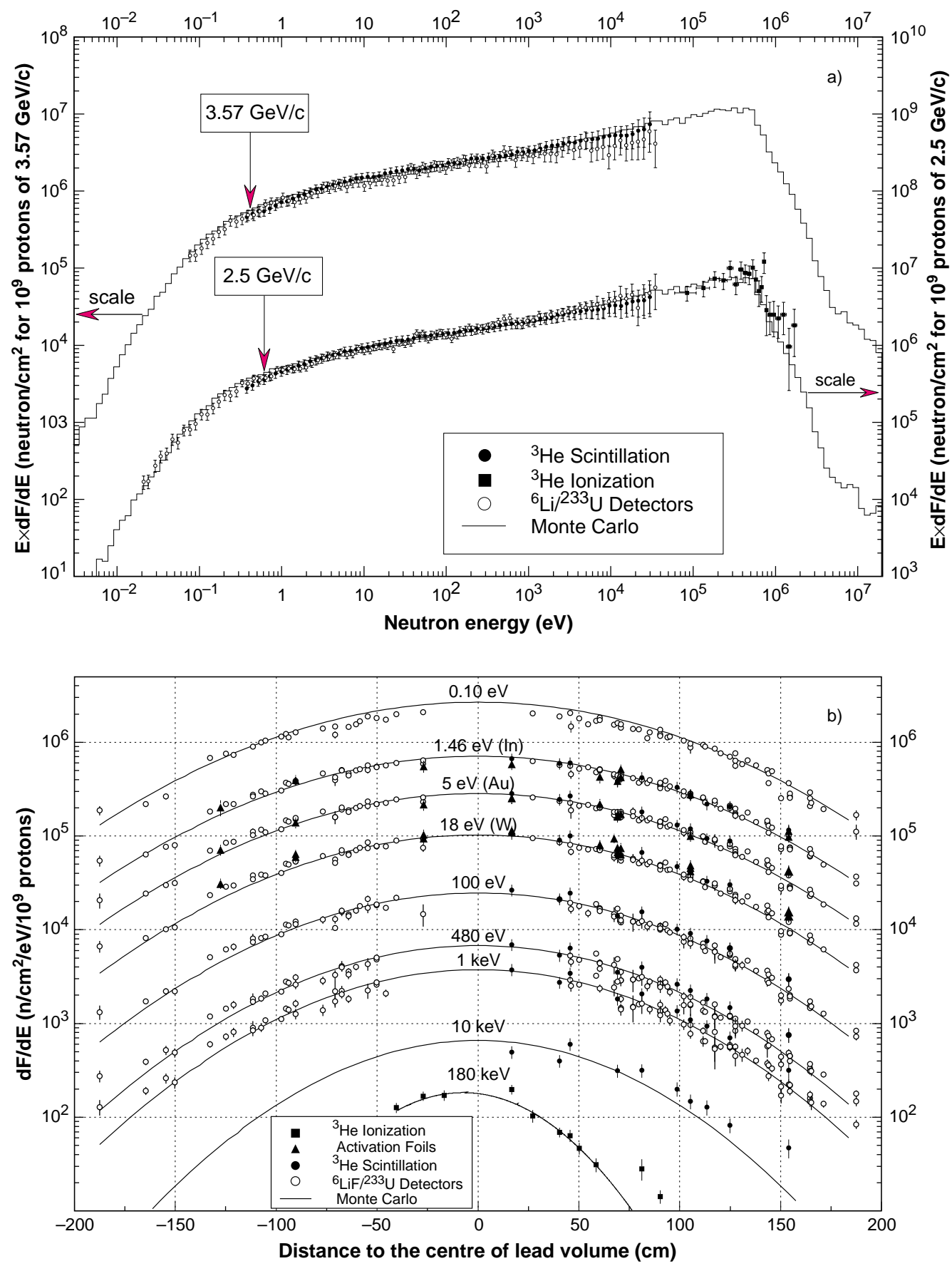

Fig. 67: a) Example of TARC measurements of the neutron fluence. $E \times \mathrm{d} F / \mathrm{d} E$ is shown as a function of neutron energy, from 2.5 and $3.57 \mathrm{GeV} / \mathrm{c}$ protons in hole number $10(z=7.5 \mathrm{~cm}$, at a distance of $45.6 \mathrm{~cm}$ from the centre of the lead volume). The Monte Carlo predictions are shown as histograms. The data are from ${ }^{6} \mathrm{Li} /{ }^{233} \mathrm{U}$ detectors (open circles), ${ }^{3} \mathrm{He}$ in the scintillation mode (full circles) and ${ }^{3} \mathrm{He}$ in the ionization mode (full squares). The error bars include both statistical and systematic errors added in quadrature;

b) Variation of the neutron fluence as a function of distance from the centre of the lead volume (the minus sign indicates negative $z$ positions). $\mathrm{d} F / \mathrm{d} E$ is shown for a few selected energy bins. The Monte Carlo predictions are shown as lines obtained from a Gaussian fit to Monte Carlo points. The data are shown with the same convention as in part (a) with, in addition, data from triple-foil activations shown as full triangles. 


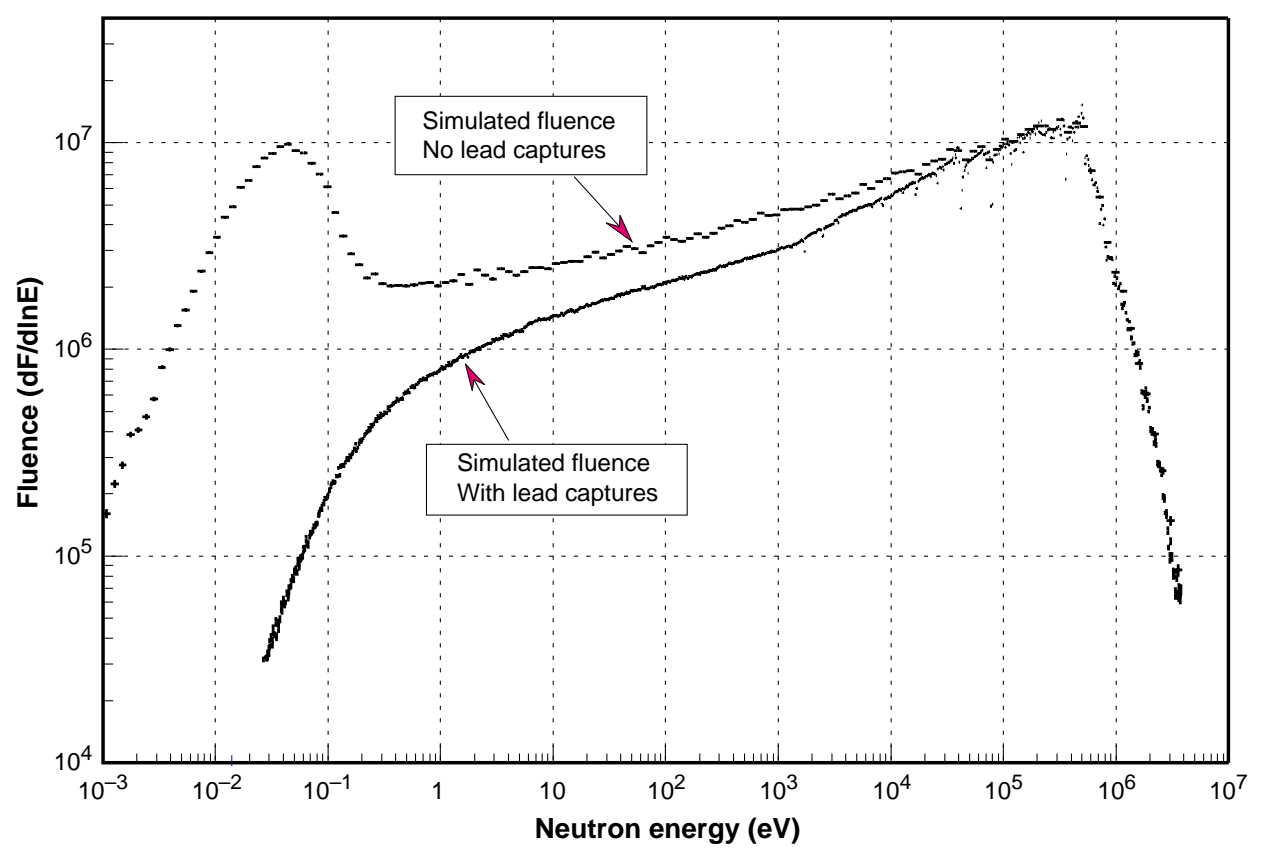

Fig. 68: Comparison of the neutron fluence in hole number $10, z=+7.5 \mathrm{~cm}$, with and without neutron captures in lead.

\section{Neutron Capture Cross-Section Measurements as a Function of Energy}

\subsection{Detecting neutron capture with a $\mathrm{CeF}_{3}$ detector}

The prompt $\gamma$-rays of the cascade following a neutron capture are detected using a $\mathrm{CeF}_{3}$ scintillator coupled to a quartz window photomultiplier (Philips XP-2020Q). The $\mathrm{CeF}_{3}$ has been chosen because the neutron capture cross-section in this material is very small and its scintillation light has fast decay-time constants (5 and $30 \mathrm{~ns}$ ). In practice, two detectors were assembled with scintillators cross-sections of $2 \times 2 \mathrm{~cm}^{2}$ and thicknesses of $2 \mathrm{~mm}$ and $4 \mathrm{~mm}$ respectively. The scintillator (Fig. 69) is coupled to the photocathode by a film of optical grease, and a thin $5 \mu \mathrm{m}$ aluminium foil is used as light reflector on the opposite face. The light tightness was achieved with black adhesive tape. The target, usually a metallic foil, is maintained on the scintillator with two very small pieces of tape and is covered by an aluminium foil. The output signal from the photomultiplier is amplified by a fast amplifier (integration time of $0.5 \mu \mathrm{s}$ ), whose output is encoded by an 8-bit flash-ADC (8192 channels, $0.1 \mu \mathrm{s} /$ channel). An example of a flash-ADC record for a tantalum target is given in Fig. 70 .

Immediately after the arrival of the proton beam burst, a high reaction rate is produced in the detector which saturates the photomultiplier for a few microseconds. Therefore, the counting rate for times $t<5 \mu \mathrm{s}$ is not reliably measured. This effect induces a limitation in our measurements. Reliable data have been obtained only for neutron energies smaller than $\approx 6 \mathrm{keV}$. As can be seen from Fig. 70, the counting rate was rather high and precise measurement of the single-pulse time distribution was not possible. We analysed the data by adding together all flash records, which turns out to be equivalent to a current integration method.

The data processing is described in detail in Ref. [8]. A typical accumulated time spectrum obtained with our ${ }^{99}$ Tc sample is shown in Fig. 71. 


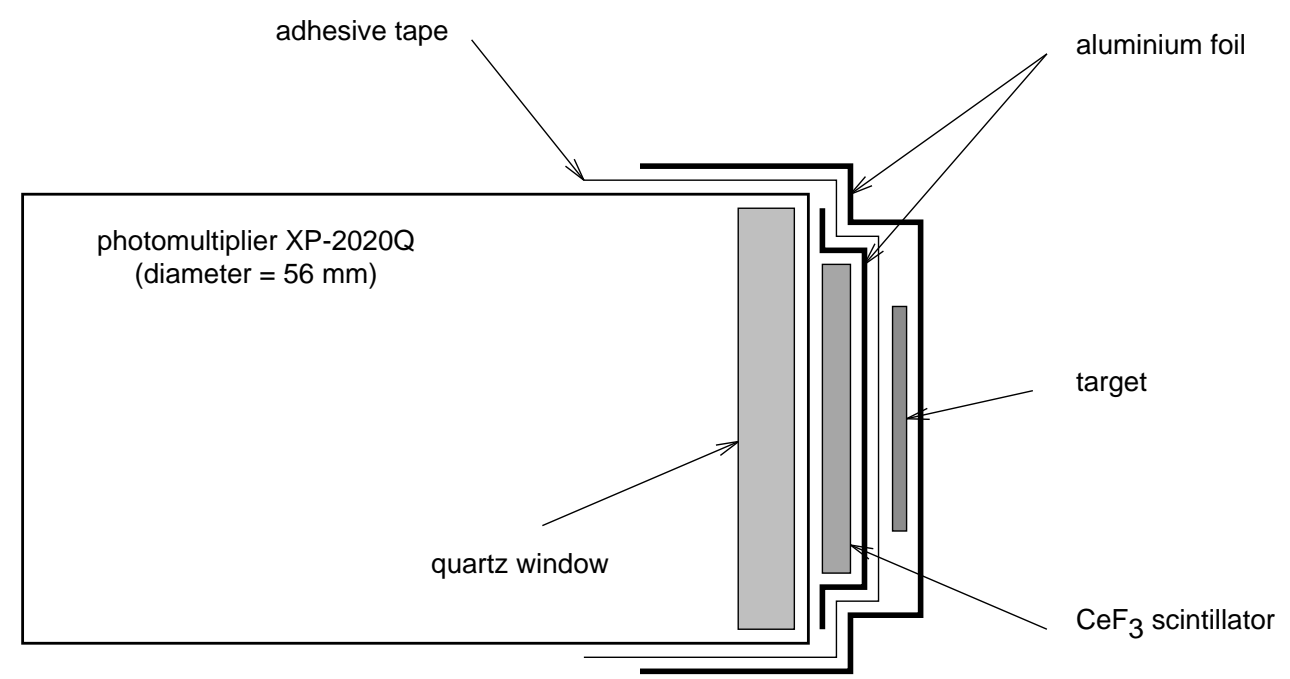

Fig. 69: Schematic view of the $\mathrm{CeF}_{3}$ detector. The thicknesses of the black adhesive tape and of the aluminium foil are $0.2 \mathrm{~mm}$ and $5 \mu \mathrm{m}$ respectively.

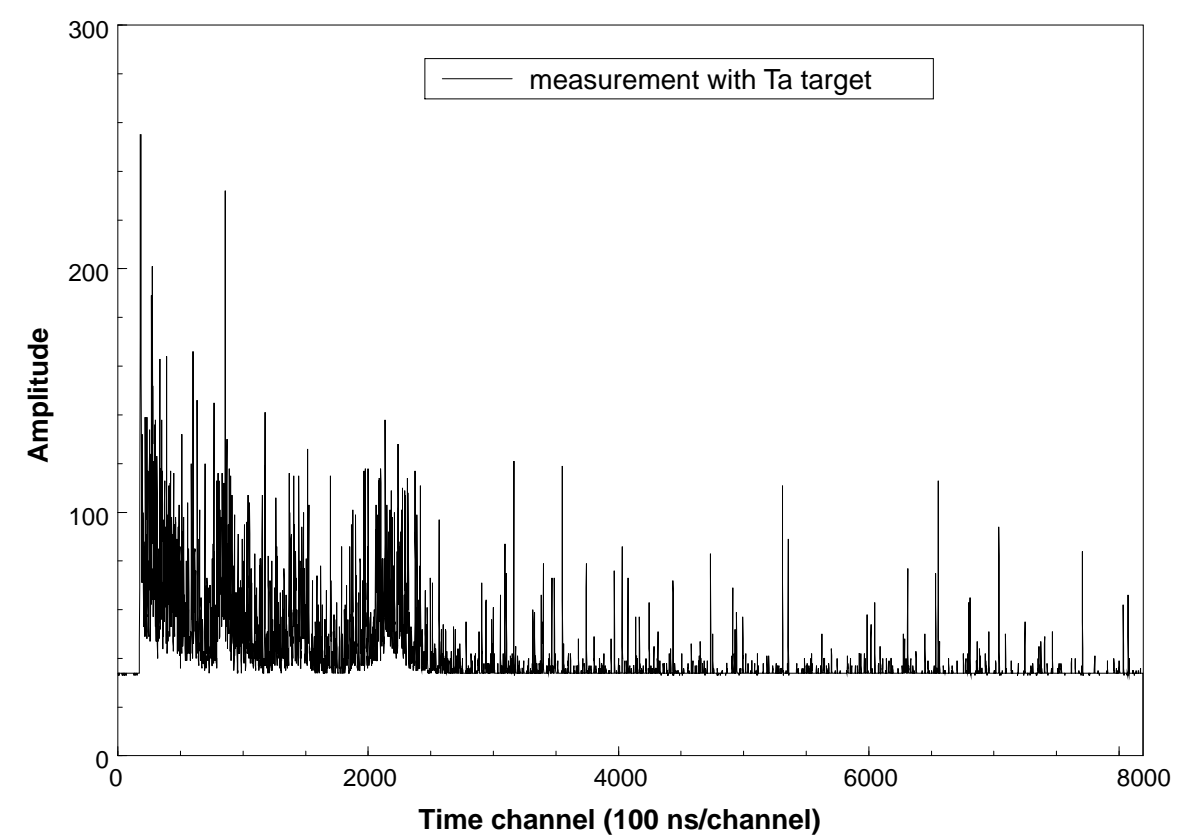

Fig. 70: Example of a flash-ADC record for a tantalum target. This is the data corresponding to one single proton pulse of intensity of $\approx 5 \times 10^{7}$ protons.

\subsection{Transmutation rate}

The transmutation rate is given by the time distribution of the number of captures per incident proton. The measurement gives the time differential transmutation rate $n(t, \vec{r})$ through the relation $n(t, \vec{r})=a(t, \vec{r}) / \alpha$. This spectrum is related to the energy differential transmutation rate $\operatorname{tr}(\bar{E})$ per energy unit:

$$
\operatorname{tr}(\bar{E})=n(t) \times\left|\frac{\Delta t}{\Delta \bar{E}}\right|
$$

For some targets $\left({ }^{99} \mathrm{Tc}, \mathrm{Ag}, \mathrm{Au}\right)$ the scaling coefficient $\alpha$ is found by comparison of the cumulated transmutation rate to the total transmutation rate $t r_{\text {tot }}$ determined by the integral measurement made by activation methods (see Section 5.2.1 and Section 7.1 for details). 


$$
t r_{\mathrm{tot}}=\int n(t) \mathrm{d} t=\frac{1}{\alpha} \int a(t) \mathrm{d} t
$$

if $t r_{\text {tot }}$ is determined from activation measurements, then $\alpha$ can be obtained.

For the other targets, the scaling coefficient $\alpha$ 's were found by adjustment of the experimental results on the Monte Carlo calculation using the JENDL database and the full experimental set-up description.

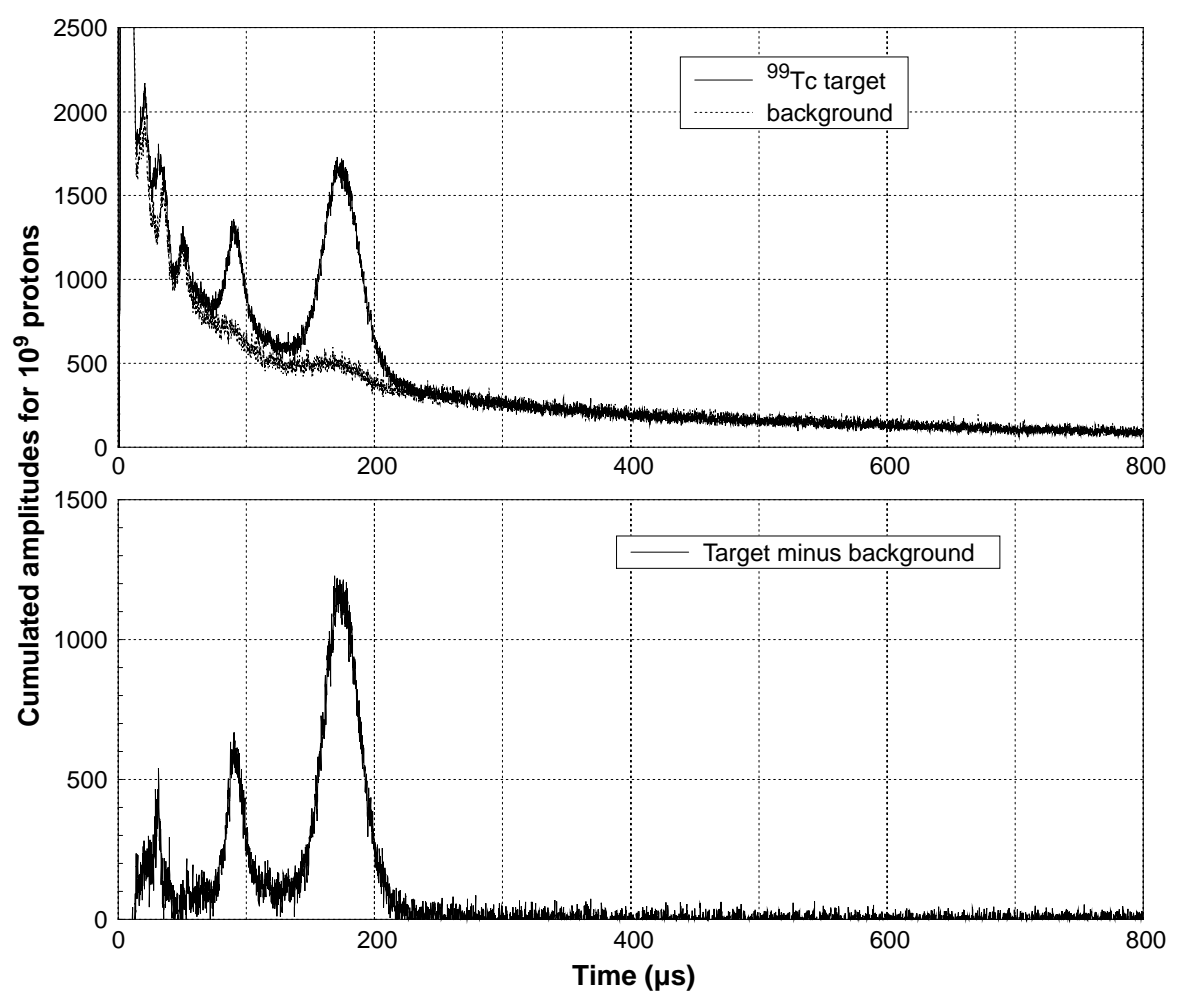

Fig. 71: Experimental time spectra obtained for technetium. The upper figure shows spectra taken with and without the target sample. The lower figure shows the signal spectrum obtained by subtraction of the two upper spectra.

\subsection{Apparent cross-section}

It has been shown in Section 5.2.1.2 that the reaction rate can be written as:

$$
\Delta n(t, \vec{r})=n(t, \vec{r}) \Delta t=\frac{N_{\text {target }}}{V} \int_{\text {target volume }} \mathrm{d} \vec{x} \Delta F(t, \vec{x}) \int M(E, t) \sigma(E) \mathrm{d} E
$$

where the neutron flux at a point $\vec{x}$ in the target volume $V$, located around the position $\vec{r}$ in the lead is defined as:

$$
\phi(E, t, \vec{x}) \Delta t=\Delta F(t, \vec{x}) M(E, t) .
$$

In this equation the correlation function $M(E, t)$ is a normalized probability

$$
\int_{0}^{\infty} M(E, t) \mathrm{d} E=1
$$

Using the energy-time $\bar{E}(t)=K /\left(t+t_{0}\right)^{2}$ relation, Eq. (31) can be written in an equivalent manner: 


$$
\phi(E, t, \vec{x}) \Delta t=\Delta F(\bar{E}(t), \vec{x}) M(E, \bar{E}(t))
$$

With these definitions, Eq. (30) reads:

$$
\Delta n(t, \vec{r})=\frac{N_{\text {target }}}{V} \int_{\text {target volume }} \mathrm{d} \vec{x} \Delta F(\bar{E}(t), \vec{x}) \bar{\sigma}(\bar{E})
$$

or, since the fluence can be considered as constant over the volume of the target

$$
\Delta n(t, \vec{r})=\frac{N_{\text {target }}}{V} V \Delta F(\bar{E}(t), \vec{r}) \bar{\sigma}(\bar{E}) .
$$

Because of self-screening effects, this complete formulation cannot be avoided. The actual mean fluence $\Delta F(\bar{E}, \vec{x})=f(\bar{E}, \vec{x}) \Delta \bar{E}$ can be significantly affected by the presence of the target itself. Then we define an apparent cross-section by the following equation:

$$
\Delta n(t, \vec{r})=N_{\text {target }} \Delta F_{0}(\bar{E}, \vec{r}) \sigma_{\text {app }}(\bar{E})
$$

where $\Delta F_{0}(\bar{E}, \vec{r})=f_{0}(\bar{E}, \vec{r}) \mathrm{d} \bar{E}$ is the unperturbed fluence measured at position $\vec{r}$. This apparent cross-section would be the quantity defined in Section $5,\langle\sigma\rangle=\int M(E, t) \sigma(E) \mathrm{d} E$ in the case of a very thin target without any self-screening effect. In this apparent cross-section, both self-screening effects and averaging by the $M(E, t)$ function, are included. Using this formulation the unperturbed fluence measured by ${ }^{6} \mathrm{Li} /{ }^{233} \mathrm{U}$ or ${ }^{3} \mathrm{He}$ counters $f_{0}(E, \vec{r})$ is used to extract $\sigma_{\text {app }}(\bar{E})$. The apparent cross-section can then be written as:

$$
\sigma_{\text {app }}(\bar{E})=\frac{\Delta n(t, \vec{r})}{N_{\text {target }} \Delta F_{0}(\bar{E}, \vec{r})}=\frac{a(t, \vec{r})}{\alpha N_{\text {target }} f_{0}(\bar{E}, \vec{r})}\left|\frac{\Delta t}{\Delta \bar{E}}\right|
$$

\subsubsection{Absolute values}

To obtain the apparent cross-sections given by Eq. (36) one assumes explicitly that the efficiency, which enters the $\alpha$ coefficient, is constant over the observed neutron energy range for a given target. This hypothesis which implies that the $\gamma$ cascade is fairly independent of the neutron energy seems rather reasonable, since between few $\mathrm{keV}$ and $0.1 \mathrm{eV}$ the neutron angular momentum with respect to the target nucleus remains mostly $L=0$, the same excited states of the compound nucleus are formed, and therefore the $\gamma$ cascade can be supposed to be independent of neutron energy. Typical energies of the cascade gammas are of the order of 1 to $2 \mathrm{MeV}$; therefore, our small scintillator $\left(2 \times 2 \times 0.2 \mathrm{~cm}^{3}\right)$ has a low intrinsic efficiency for detecting these gamma rays, but it is more efficient for the low-gamma energies ending the cascade. Hence we expect the efficiency to be independent of the neutron energy $(0.1 \mathrm{eV}$ to few $\mathrm{keV}$ ) but dependent on the type of target element. As a consequence neither the efficiency nor the $\alpha$ coefficient can be known simply in an absolute manner.

So the $\mathrm{CeF}_{3}$ measurements alone will determine only the energy profiles of transmutation rates, or apparent cross-sections which are sample-dependent. For the absolute values of ${ }^{99} \mathrm{Tc}$ transmutation rates or $\sigma_{\text {app }}(E)$ we will normalize the measured relative values using the activation measurements made with the rabbit system.

\subsubsection{Error analysis}

In Eq. (36), $|\Delta t / \Delta \bar{E}|$ can be replaced by $\left(t+t_{0}\right)^{3} /(2 K)$. Then the errors on $\sigma_{\text {app }}$ can be calculated as follows: 


$$
\left[\frac{\Delta \sigma}{\sigma}\right]^{2}=\left[\frac{\delta a(t)}{a(t)}\right]^{2}+\left[\frac{\delta f_{0}}{f_{0}}\right]^{2}+\left[\frac{\delta I}{I}\right]^{2}+\left[\frac{\delta\left(\alpha N_{\text {target }}\right)}{\alpha N_{\text {target }}}\right]^{2}+\left[\frac{\delta K}{K}\right]^{2}+\left[\frac{3 \delta t}{t}\right]^{2}
$$

in which $(\delta a / a)$ is the statistical error on the measurement and the other terms are systematic errors.

From TARC fluence measurements we have $\left(\delta f_{0} / f_{0}\right)=15 \%$, the beam intensity precisions $(\delta I / I)=5 \%$ and $(\delta K / K)=1.2 \%$. The error $\delta\left(\alpha N_{\text {target }}\right) /\left(\alpha N_{\text {target }}\right)$ on the normalization procedure is estimated to $15 \%$.

The error on the energy coming from the uncertainty on the time measurement (taken here as $\delta t=0.5 \mu$ s because these measurements are involved: with target, without target and flux measurement), ranges from $0.2 \%$ to $12 \%$ in the energy interval of interest $(0.1 \mathrm{eV}$ to $1 \mathrm{keV})$.

The total systematic error ranges from $24 \%$ at low neutron energy up to $26 \%$ around $1 \mathrm{keV}$.

\subsection{Results}

The samples used are mixtures of aluminium and technetium: $0.085 \mathrm{~g}$ of ${ }^{99} \mathrm{Tc}$ in $1.7 \mathrm{~g}$ of Al. They have square shape of $2 \mathrm{~cm} \times 2 \mathrm{~cm}$ with a thickness of $1.5 \mathrm{~mm}$. The background is measured using a pure aluminium sample of same dimensions. Measurements with the same sample have been made at different positions in the lead volume (holes 3,5,10 at $z=7.5 \mathrm{~cm}$, and hole 12 at $z=7.5 \mathrm{~cm}$ and $97.5 \mathrm{~cm}$ ). The total transmutation rate:

$$
t r_{\mathrm{tot}}=\frac{1}{\alpha} \int a(t) \mathrm{d} t
$$

compared with the integral measurement by activation, in hole 10 at $z=7.5 \mathrm{~cm}$ gives for the normalization constant the value $\alpha=0.45 \pm 0.01$. Figure 72 shows the differential transmutation rate $t r_{\text {exp }}(E)$; in fact $E \times t r_{\text {exp }}(E)$ is compared with the Monte Carlo simulated $E \times t r_{\text {sim }}(E)$ using the JENDL database cross-section. Note that $t r_{\text {sim }}(E)$ is obtained in the same way as $\operatorname{tr}_{\exp }(E)$ from the $n_{\text {sim }}(t)$ spectrum.

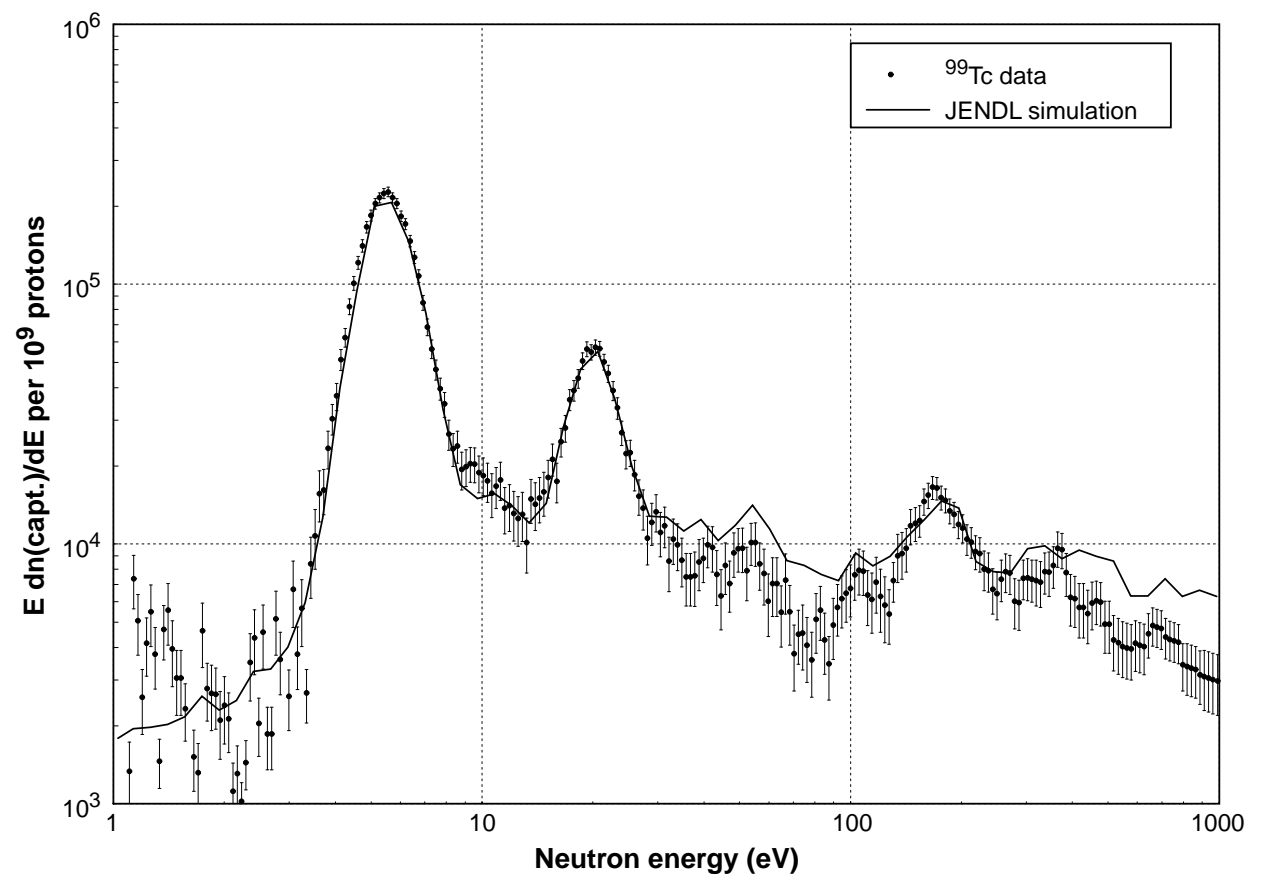

Fig. 72: Transmutation rate for technetium $\left({ }^{99} \mathrm{Tc}\right)$ compared with the simulation using the JENDL-3.2 database. The sample is $0.085 \mathrm{~g}$ of ${ }^{99} \mathrm{Tc}$ diluted in a $2 \mathrm{~cm} \times 2 \mathrm{~cm} \times 1.5 \mathrm{~mm}$ Al parallelepiped. 
The cross-section data (Fig. 73) are not extrapolated to zero thickness. An estimation of this correction would give a value 50\% higher for the larger resonance at $5.58 \mathrm{eV}, 18 \%$ for the resonance at $22.3 \mathrm{eV}$ and no significant correction for other energies. We checked that the cross-section data are, as expected, independent of position. The relative systematic error is, on average, in the order of $15-20 \%$.

Note that the general shape of the experimental apparent cross-section is in good agreement with the full Monte Carlo simulation using the JENDL cross-section (Fig. 73). Our results are compared with the previous measurement (which is extrapolated to zero thickness) of J.C. Chou and H. Werle [39] in Fig. 74.

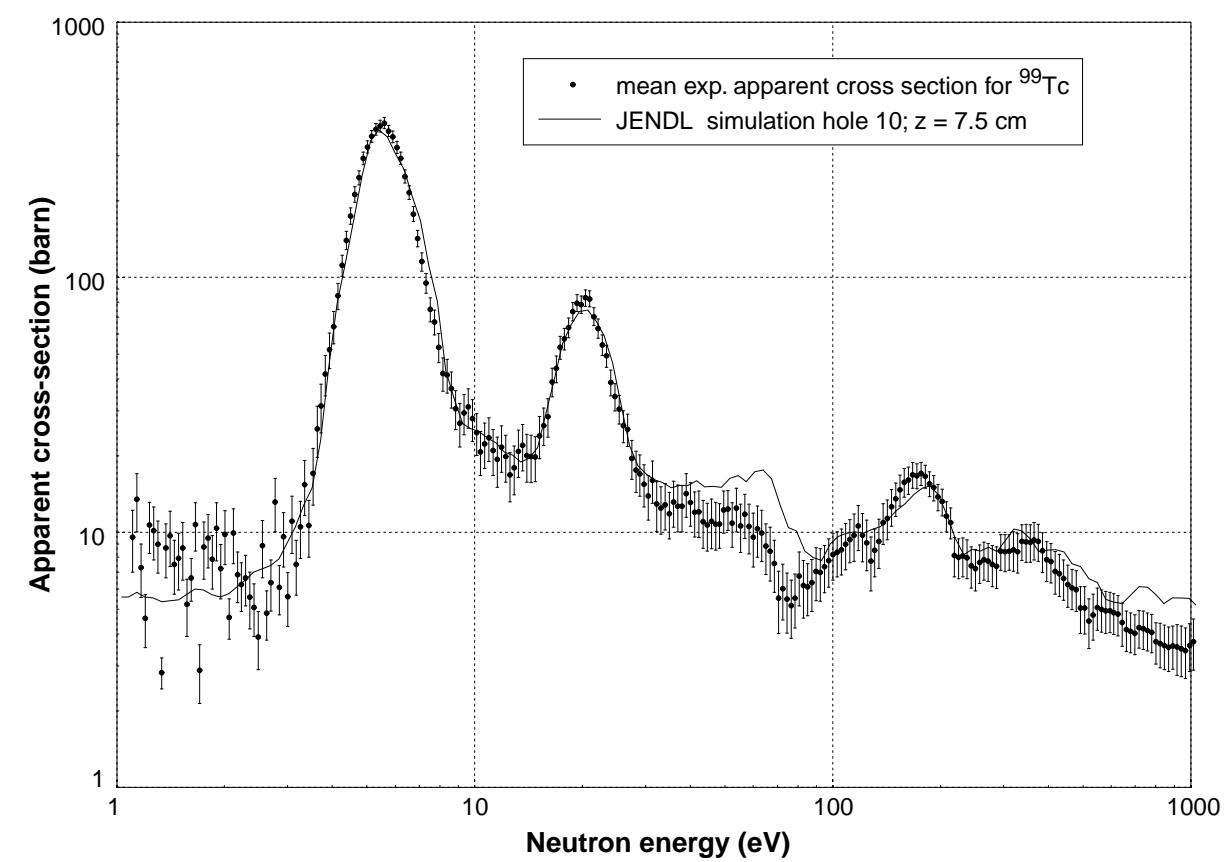

Fig. 73: Averaged TARC $(\mathrm{n}, \gamma)$ apparent cross-section for ${ }^{99} \mathrm{Tc}$ compared with the simulation using the JENDL-3.2 database.

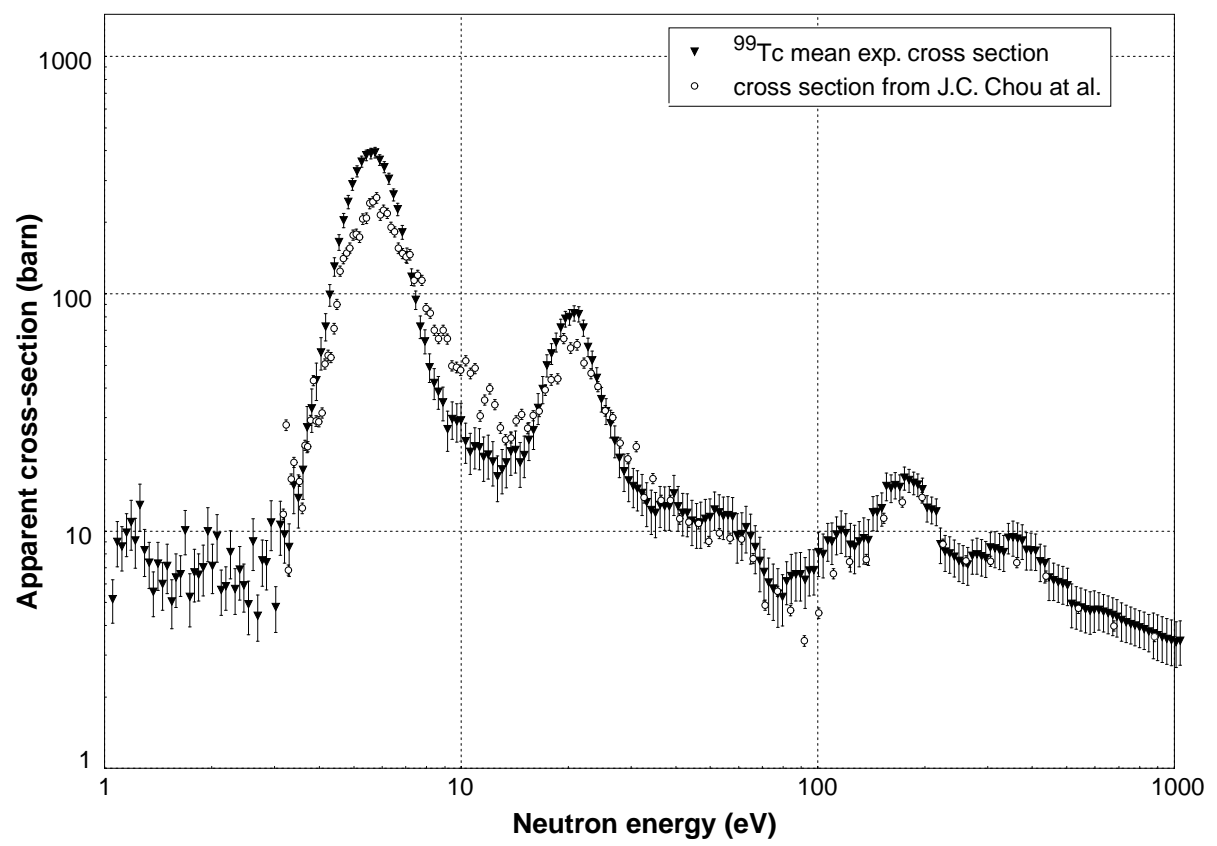

Fig. 74: Averaged TARC $(\mathrm{n}, \gamma)$ apparent cross-section for ${ }^{99} \mathrm{Tc}$ compared with Chou and Werle's data [39]. 
These experimental results on the energy dependence of transmutation rates obtained (with only $85 \mathrm{mg}$ of ${ }^{99} \mathrm{Tc}$ ), normalized by the activation measurement (rabbit), give a quite complete experimental check of the validity of the JENDL database neutron capture cross-section for ${ }^{99}$ Tc. It implies also that the resulting simulation is validated in detail and gives strong confidence in the simulation of other transmutation applications.

\subsection{Evaluation of the ${ }^{99} \mathrm{Tc}$ cross-section}

We have evaluated the Geel data [56] using the SAMMY code [57] and found that integrating the neutron capture rate over the neutron energy range covered by the evaluation $(0.7$ to $617 \mathrm{eV}$ ) leads to a $+3 \%$ increase in the predicted number of captures when compared with JENDL-3.2, which is a small difference compared with the size of the errors quoted for instance in the rabbit analysis. Therefore, we conclude that the new Geel data do not make any significant change in the various predictions of the number of captures on ${ }^{99} \mathrm{Tc}$ in TARC (most of the captures in TARC occur on the two main resonances of ${ }^{99} \mathrm{Tc}$ ).

We have corrected the apparent TARC cross-section for both the effect of the hydrogen contained in the plastic tape surrounding the photomultiplier tube and the effect of the ${ }^{99} \mathrm{Tc}$ sample thickness $\left(21.2 \mathrm{mg} / \mathrm{cm}^{2}\right)$, using the SAMMY code. The sample thickness effect is most significant at the two main capture resonances, at $5.6 \mathrm{eV}$ and $22.3 \mathrm{eV}$ where its magnitude is $50 \%$ and $18 \%$, respectively.

In both cases (JENDL-3.2 and GEEL) there is a good agreement with the cross-sections obtained by TARC (Fig. 75), within the estimated overall systematic error of about $25 \%$. Even though it was not the purpose of TARC to measure cross-sections, the fact that good quality cross-section data could be obtained from our studies of ${ }^{99} \mathrm{Tc}$ transmutation is a check of the general high quality of TARC data.

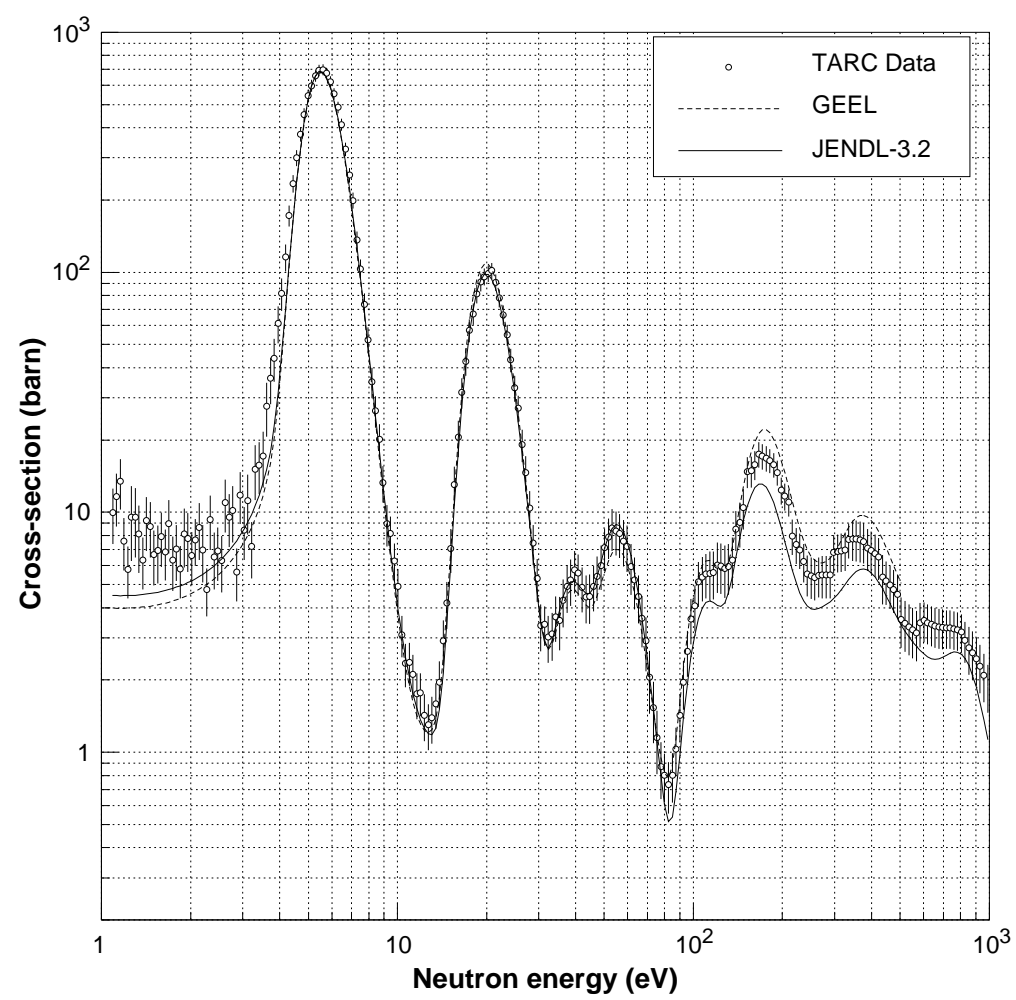

Fig. 75: Comparison of the TARC ${ }^{99} \mathrm{Tc}(\mathrm{n}, \gamma)$ cross-section data with an evaluation based on GEEL data and an evaluation based on the JENDL-3.2 data library. In both cases the lines correspond to the cross sections at $293 \mathrm{~K}$ and have been broadened for the slowing-down resolution $(\Delta \mathrm{E} / \mathrm{E} \sim 12 \%)$. 


\subsection{Results for other targets}

Apparent $(\mathrm{n}, \gamma)$ cross-sections for ${ }^{181} \mathrm{Ta},{ }^{197} \mathrm{Au},{ }^{\text {nat }} \mathrm{Ag}$, and ${ }^{\text {nat }}$ In targets have also been measured and are reported in Ref. [8].

\subsection{Conclusion}

TARC has shown that the energy variation of the ${ }^{99} \mathrm{Tc}(\mathrm{n}, \gamma)$ transmutation cross-section can be validated quite accurately with short measurement times and small quantities of material. The concept of transmutation by adiabatic resonance crossing is fully understood in a low lethargy medium (lead). The self-shielding effect, which modifies the local flux inside the sample volume, and the flux perturbation due to the detector itself are well taken into account by the Monte Carlo simulation.

The transmutation rate (tr) as a function of neutron energy was measured with a good precision using small samples of material. Reliable energy profiles have been obtained between $1 \mathrm{eV}$ up to $1 \mathrm{keV}$ which represents more than $80 \%$ of the total $t r$. These data combined with activation measurements provide absolute apparent cross-section measurement for given samples. From a more general point of view, this method can be considered as very well adapted for checking the validity (or non-validity) of present database cross-sections for transmutation calculation. Finally, it can be stressed that in this experiment neutron fluences and transmutation rates have been measured independently and both agree well with our Monte Carlo simulations.

\section{Test of Transmutation of Long-Lived Fission Fragments}

\section{$7.1{ }^{99}$ Tc rabbit integral transmutation rate measurements}

One of the main goals of the TARC experiment is the study of transmutation of Long-Lived Fission Fragments (LLFFs) using Adiabatic Resonance Crossing (ARC). After having demonstrated that the neutron flux in the lead volume has the expected properties, we have measured directly the transmutation rate for the most important LLFFs in order to complete the demonstration that ARC works effectively, choosing two LLFFs of major relevance: ${ }^{99} \mathrm{Tc}$ and ${ }^{129}$ I (which represent about $95 \%$ of the LLFF radiotoxicity). In a system such as the Energy Amplifier (EA) [2], where the actinides from PWR spent fuel are incinerated, LLFFs such as ${ }^{99} \mathrm{Tc}$ and ${ }^{129} \mathrm{I}$ generate the main contribution to the long-term radiotoxicity (> 500 years). A spallation neutron, surviving the lead capture resonances, cannot miss a given resonance energy such as $5.6 \mathrm{eV}$ in ${ }^{99} \mathrm{Tc}$. In its 'adiabatic' slowing down process, a neutron will sweep all energies with very small steps of constant $\Delta E / E$, until it is captured with high probability on an 'impurity' with a high resonance in its capture cross-section, as is the case for ${ }^{99} \mathrm{Tc}$ (Fig. 76). As already explained in Section 1 the TARC effect maximizes the efficiency of the process.

In the case of ${ }^{99} \mathrm{Tc}$ the two main resonances at $5.6 \mathrm{eV}$ and $22.3 \mathrm{eV}$ correspond, because of the energy-time correlation (Section 4), to neutron captures occurring respectively 175 and $87 \mu$ s (Fig. 76) after the arrival of the proton beam.

\subsubsection{Motivation}

By neutron capture the ${ }^{99} \mathrm{Tc}\left(2.1 \times 10^{5}\right.$ years half-life $)$ becomes ${ }^{100} \mathrm{Tc}(15.8 \mathrm{~s}$ half-life $)$ which in turn decays into the excited states of ${ }^{100} \mathrm{Ru}$, finally stable. The 'rabbit system' method consists in evaluating the number of ${ }^{99} \mathrm{Tc}$ nuclei transmuted by counting of the ${ }^{100} \mathrm{Tc}$ de-excitation photons. Since the ${ }^{99} \mathrm{Tc}$ itself is radioactive, and given the complex structure of the photon energy spectra produced by the irradiated ${ }^{99} \mathrm{Tc}$ sample, we use a pair of 
high-efficiency, High-Purity germanium (HPGe) detectors. They provide sufficient photon energy resolution to identify and count the number of photons in each individual characteristic line. The two germanium counters provide redundancy of information allowing detailed systematic studies. To reduce the radiation background and avoid damage to the HPGe by the fast neutrons present in the irradiation area, the sample is transferred to a remote counting station by a pneumatic system (described in Section 7.1.2).

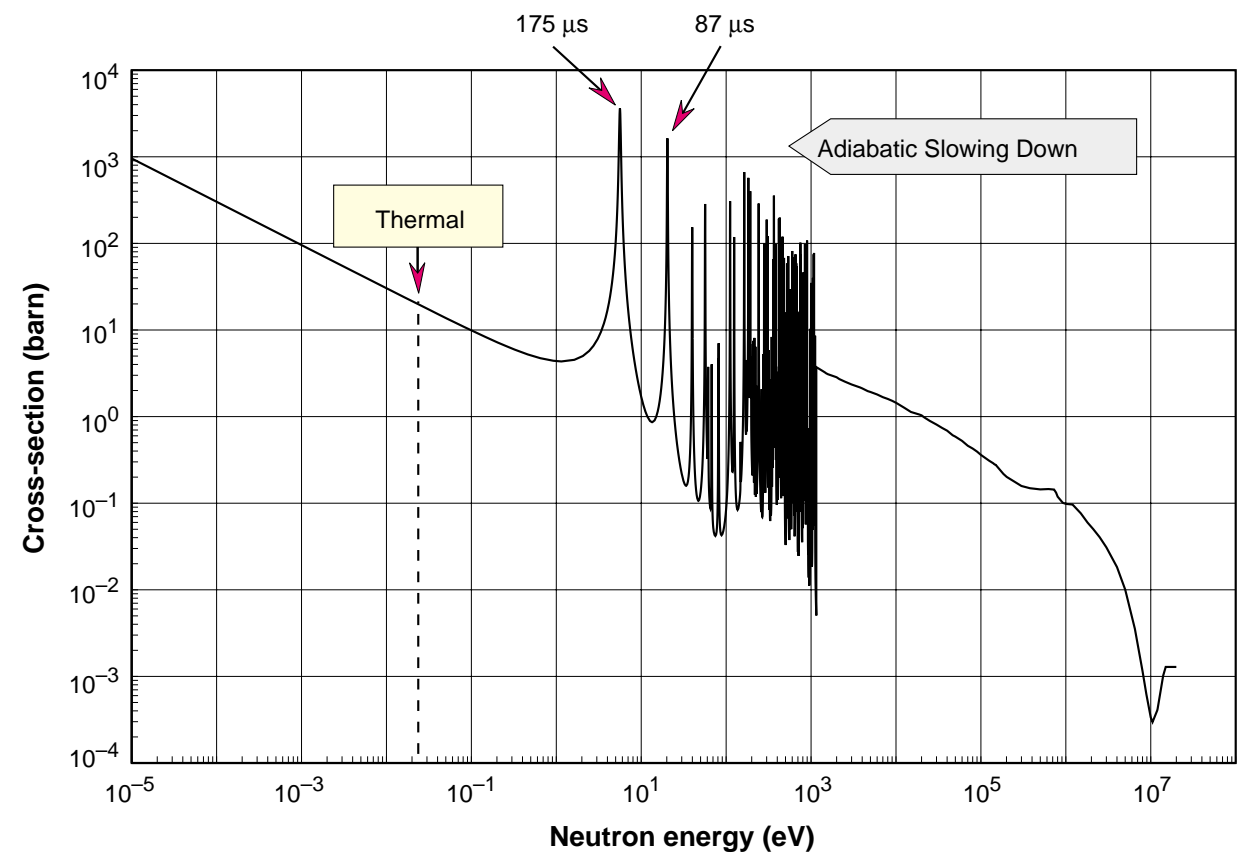

Fig. 76: ${ }^{99} \mathrm{Tc}$ neutron capture cross-section as a function of neutron kinetic energy (from the JENDL-3.2 database). The times from the arrival of the proton pulse corresponding to the two main capture resonances are indicated.

\subsubsection{Experimental set-up}

A primary fast extracted proton beam with $2.75 \mathrm{GeV}$ of kinetic energy $(3.5 \mathrm{GeV} / \mathrm{c}$ momentum) and an intensity of a few times $10^{9}$ protons per pulse was used, with a repetition period of $14.4 \mathrm{~s}$. A proton pulse, incident on a large volume of lead, produces by spallation a neutron cascade. These spallation neutrons diffuse and thermalize inside the lead volume. A ${ }^{99} \mathrm{Tc}$ sample, contained in a transport shuttle (rabbit), is placed at different positions in the lead assembly, before and at least until $0.5 \mathrm{~s}$ after the beam arrives (long enough to make sure that all spallation neutrons have been produced and captured or escaped from the lead). Some ${ }^{99} \mathrm{Tc}$ nuclei will capture a neutron and become ${ }^{100} \mathrm{Tc}$, with a $15.8 \mathrm{~s}$ half-life. The shuttle is then transferred by a pneumatic system to a measurement station (Fig. 77). This station consists of two large HPGe detectors inside a massive shielding system. The photons produced by the transition from the ${ }^{100} \mathrm{Ru}$ excited states (produced in ${ }^{100} \mathrm{Tc}$ decay) to the ground state are collected during most of the time interval between beam pulses ( $9 \mathrm{~s}$ out of $14.4 \mathrm{~s}$ ). Each single photon or photon coincidence between the two germanium counters is registered in amplitude and time by an appropriate DAQ system, which also registers the beam intensity for each accelerator beam shot and monitors the proper operation of the pneumatic system and the correct positioning inside the measurement station of the shuttle containing the sample. After the measurement, the sample is sent back to the irradiation position, a fraction of a second before the next beam shot arrives. This process is repeated until enough statistics are collected (typically $10^{4}$ counts in the major photon peaks). 


\subsubsection{The pneumatic system}

The pneumatic system consists of two ports, the irradiation $(R)$ and the measurement $(M)$ ports, connected by a $24 \mathrm{~m}$ long plastic tube (Fig. 77). Each port contains a set of three electromechanical valves, a couple of pressurized air reservoirs, specific pieces to define the position of the shuttle containing the samples, and one optical sensor mounted on the tube close to the port.

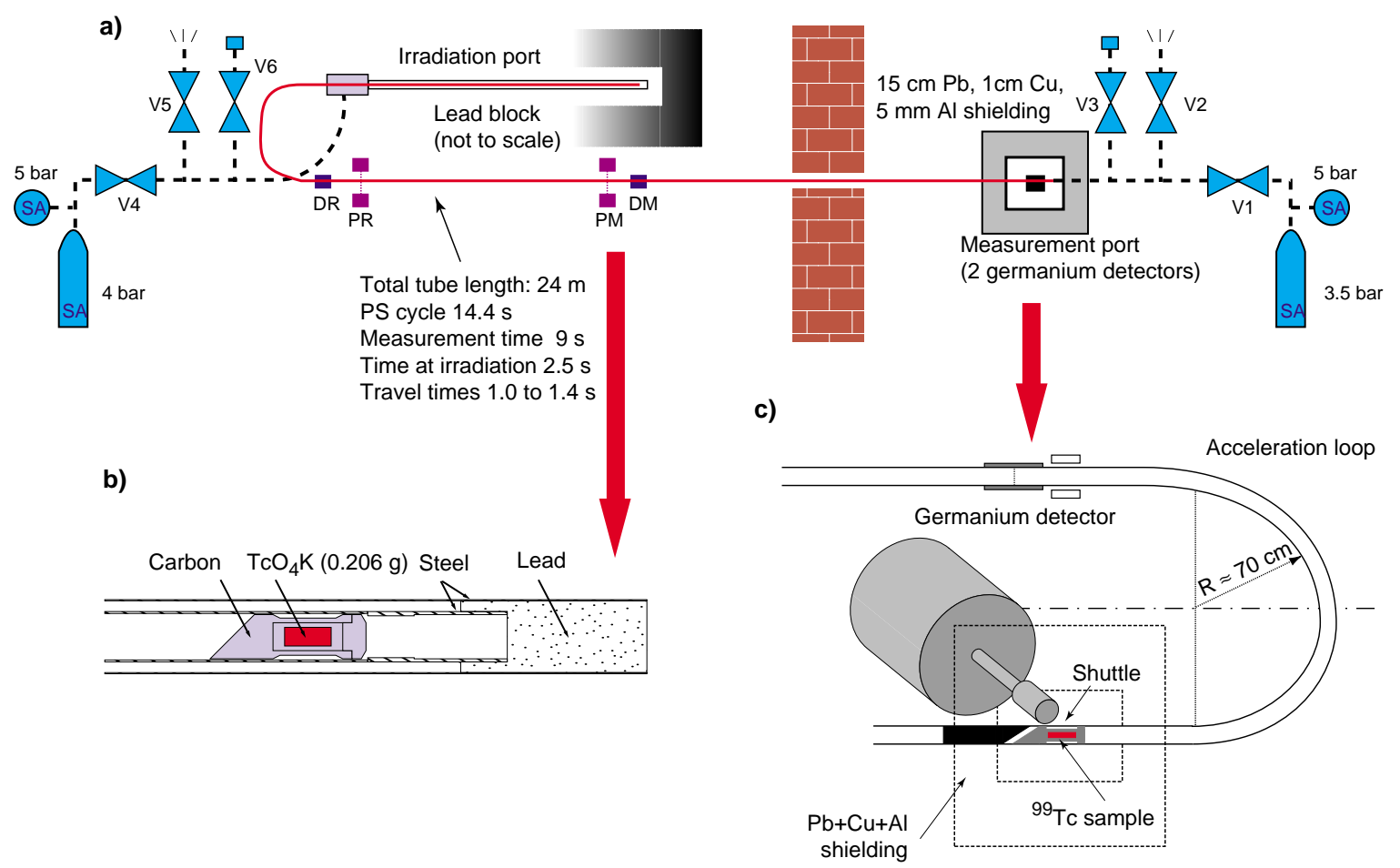

Fig. 77: a) Schematic layout of the 'rabbit' system; b) View of the carbon fibre shuttle containing the ${ }^{99} \mathrm{Tc}$ sample in the irradiation port; c) View of the gamma-ray detection station.

A specially designed electronic control box allows the state of each electrovalve to be set according to the signal received from the optical sensors, the pressure in the tube, or through external commands. These commands can be provided either by a manual switch box or by the input signal of the automatic controls.

The tube connecting the two ports is made of Polyamid $11^{6}$ and has internal and external diameters of $10 \mathrm{~mm}$ and $12 \mathrm{~mm}$, respectively. A couple of pressure sensors have also been installed in the tube. The first one is an analogic pressure transducer, for calibration and diagnostic purposes, and the second a digital sensor that produces a signal whenever the pressure in the tube crosses some predefined thresholds. The signals are used to control the pneumatic cycle and the values for these thresholds have been calibrated for each individual transport shuttle. Typical values were of the order of 1.9 and 2.75 bar.

\subsubsection{The sample irradiation shuttle}

As already mentioned, the samples are transported by the rabbit between the irradiation and the measuring ports inside a special shuttle. The function of this shuttle is to hold the sample during the transport, to position it at the two ports, and to provide the required safety in case there is an unexpectedly strong collision of the shuttle with the stoppers at any of the two ports.

6. Rilsan, Polyamid 11, Hoses for fluids and gases - Stores catalogue CERN 38.40.30.612.4. 
The length of the shuttle is $33.5 \mathrm{~mm}$ and the external diameter is $9.5 \mathrm{~mm}$. The internal cylinder length, where the sample is normally placed, is $15 \mathrm{~mm}$ with a diameter of $6 \mathrm{~mm}$ [Figs. 77(b) and 78]. The front-end shape, cut at $45^{\circ}$, combined with the stopper on the measuring port, allows the position and orientation of the sample to be defined with respect to the HPGe detectors [Fig. 77(c) and Fig. 80], determining in a precise way their geometrical efficiency.

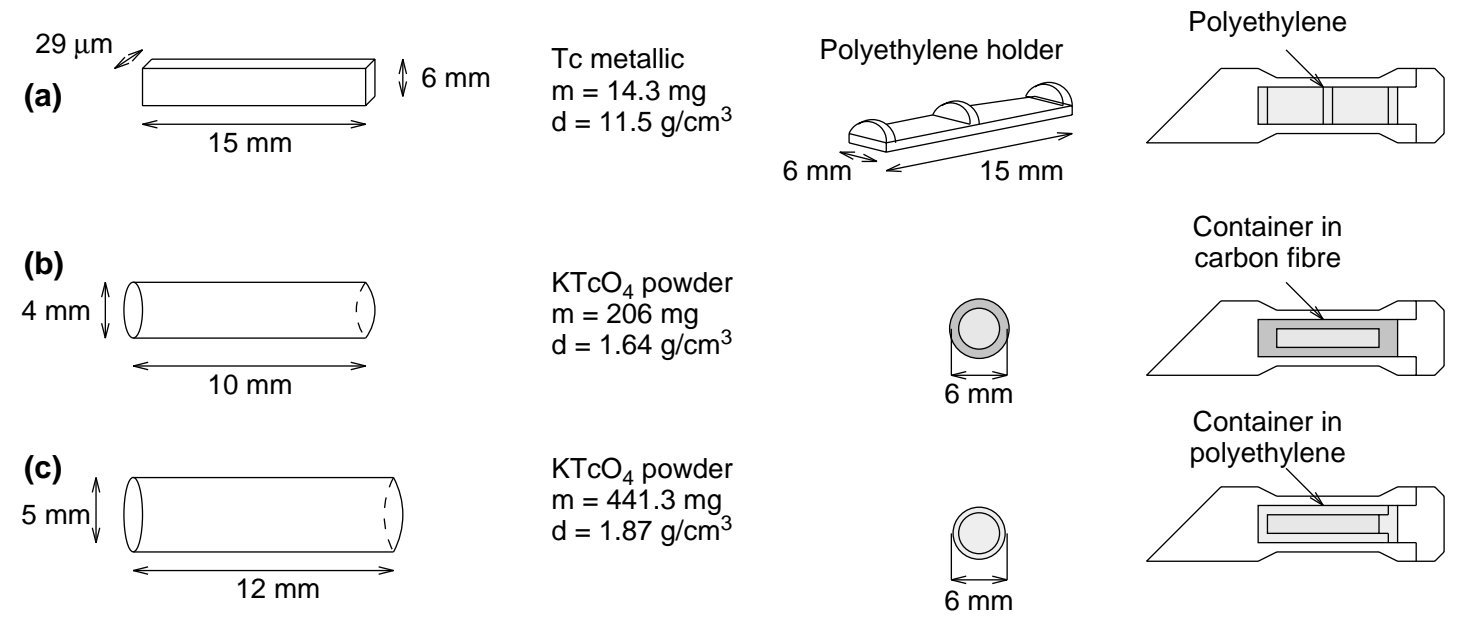

Fig. 78: (a) Schematics of the metallic ${ }^{99} \mathrm{Tc}$ arrangement inside the shuttle and the polyethylene holding pieces; (b) the carbon-fibre cylinder holding the $206 \mathrm{mg}$ of ${ }^{99} \mathrm{TcO}_{4} \mathrm{~K}$ sample; (c) the polyethylene cylinder holding the $441.3 \mathrm{mg}$ of ${ }^{99} \mathrm{TcO}_{4} \mathrm{~K}$ sample.

\subsection{Choice of the shuttle material}

The shuttle material was chosen according to the following requirements:

(a) Minimum perturbation of the neutron flux around the ${ }^{99} \mathrm{Tc}$ sample to be studied. This means no big resonances in the neutron cross-sections and low hydrogen content.

(b) Minimum absorption of the gamma ray of ${ }^{100} \mathrm{Tc}$, to be observed in the HPGe counters.

(c) Small gamma-ray emission rate during the measurement.

(d) Substantial mechanical strength, to be able to handle the repeated trips along the rabbit tube at a speed greater than $100 \mathrm{~km} / \mathrm{h}$, as well as the impacts at the arrival at each of the end ports.

The final choice of the material for the shuttle was carbon fibre. Carbon fibre is manufactured from textile pre-cursor yarns made of polyacrylonitrile that results in a predominantly graphite structure highly oriented in the direction of the fibre axis. This high orientation provides them with their remarkable properties which includes a small negative coefficient of thermal expansion along the axial direction as well as a high specific strength and stiffness.

The carbon fibre used in the present experiment was composed of four main elements: $\mathrm{C}(71 \%), \mathrm{H}(6 \%), \mathrm{O}(16 \%), \mathrm{N}(7 \%)$ (special material made by the Stesalit AG Company). None of these elements presents a large total cross-section for neutrons in the energy range of interest (thermal to few MeV). Furthermore, the only significant activation is coming from the $(\mathrm{n}, 2 \mathrm{n})$ and $(\mathrm{n}, 3 \mathrm{n})$ reactions with carbon at very high neutron energies. The level of activation was not a problem for the ${ }^{99} \mathrm{Tc}$ data analysis, as it produces lines of moderate intensity and clearly separated from the ${ }^{100} \mathrm{Tc}$ decay lines. In fact these lines were useful to study the amount of very fast neutrons irradiating the sample (see Sections 5.4, 5.5 and Ref. [8]). 


\subsubsection{Irradiated samples}

\subsection{The metallic ${ }^{99} \mathrm{Tc}$ sample $(14.3 \mathrm{mg})$}

A polyethylene support assures the precise positioning of the ${ }^{99} \mathrm{Tc}$ sample inside the shuttle [Fig. 78(a)]. The weight of the sample has been evaluated using a precision scale: $14.3 \pm 0.1 \mathrm{mg}$ and its thickness is about $29 \mu \mathrm{m}$. The total amount of technetium was verified with gamma ray spectroscopy. The ${ }^{99} \mathrm{Tc}$ is $100 \%$ beta-emitter. The major fraction $(99.9984 \%)$ de-excites directly to the fundamental state $\left(5 / 2^{+}\right)$of ${ }^{99} \mathrm{Ru}$. A very small fraction $(0.0016 \%)$ de-excites to the $3 / 2^{+}$excited state of ${ }^{99} \mathrm{Ru}$ [58]. The de-excitation from the $3 / 2^{+}$to $5 / 2^{+}$of ${ }^{99} \mathrm{Ru}$ is a $100 \%$ gamma decay with an energy of $89.68 \mathrm{keV}$ (see Fig. 85 extracted from reference [59]). Using a high efficiency and a high resolution HPGe this characteristic gamma ray of ${ }^{99} \mathrm{Ru}^{*}$ was measured.

A Gaussian plus an appropriate polynomial function was used to fit the shape of the bremsstrahlung spectrum in the ${ }^{99} \mathrm{Tc}$ beta emission (Fig. 79) (maximum energy equal to $293.5 \mathrm{keV}$ ). The quantity of ${ }^{99} \mathrm{Tc}$ was computed from the intensity of the $89.68 \mathrm{keV}$ using the branching ratio given in Ref. [58]. The agreement with the direct weight measurement is better than $10 \%$. A strong discrepancy (factor 100) is found when using the branching ratio value given in Ref. [59].

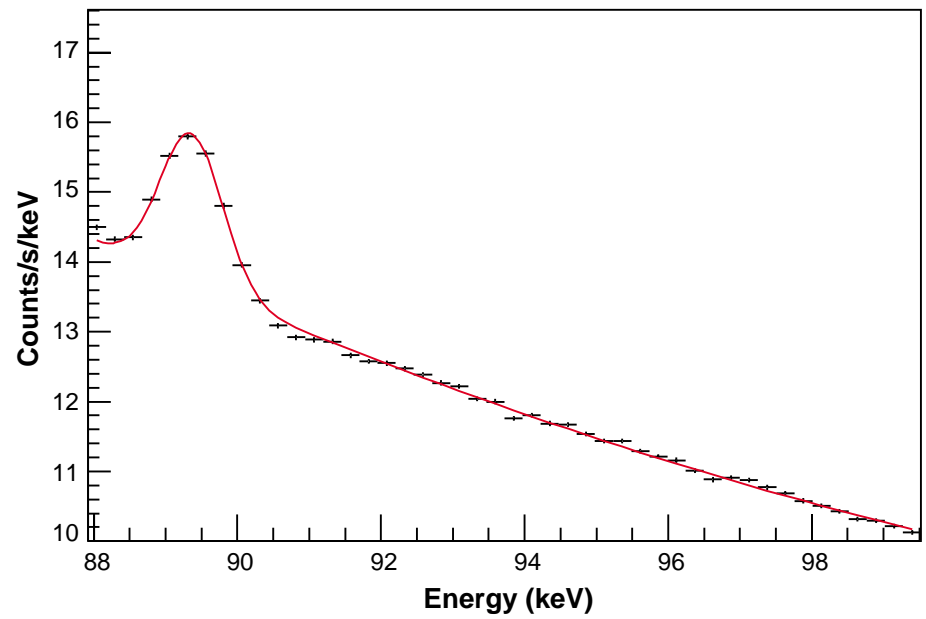

Fig. 79: Part of the gamma-ray spectrum of ${ }^{99} \mathrm{Tc}$ around $89.68 \mathrm{keV}$. The line is the result of a Gaussian plus polynomial fit used for the determination of the $89.68 \mathrm{keV}$ line intensity to verify the total ${ }^{99} \mathrm{Tc}$ mass of the metallic ${ }^{99} \mathrm{Tc}$ sample.

\subsubsection{2 $\mathrm{The}^{99} \mathrm{TcO}_{4} \mathrm{~K}$ powder samples}

Even though it is preferable to use pure ${ }^{99} \mathrm{Tc}$ samples, the only large samples available to us were in the form of ${ }^{99} \mathrm{TcO}_{4} \mathrm{~K}$ powder, from which we made two samples.

First, the $206.0 \pm 0.1 \mathrm{mg}$ of ${ }^{99} \mathrm{TcO}_{4} \mathrm{~K}$ (sample no. 1 with $100.88 \mathrm{mg}$ of ${ }^{99} \mathrm{Tc}$ ) were placed inside one carbon-fibre cylinder [Fig. 78(b)]. Second the $441.3 \pm 0.1 \mathrm{mg}$ of ${ }^{99} \mathrm{TcO}_{4} \mathrm{~K}$ (sample no. 2 with $216.11 \mathrm{mg}$ of ${ }^{99} \mathrm{Tc}$ ) were placed inside one polyethylene cylinder [Fig. 78(c)]. The carbon-fibre cylinder or the polyethylene cylinder is placed inside the shuttle. These powder samples contain two additional elements, oxygen and potassium. Their contribution to the gamma spectrum has been evaluated.

We checked that oxygen and potassium impurities will produce negligible effects on the gamma-ray spectra of interest. Their effect on the neutron flux is acceptable and taken into account in the Monte Carlo simulation. 


\subsubsection{Germanium detectors}

\subsubsection{Detector configuration and specification}

Two high-efficiency HPGe detectors were used in the experiment. The main characteristics of these detectors are given in Table 8. Each detector is equipped with a Transistor Reset Preamplifier (TRP) allowing counting rates up to $10^{6}$ counts/s. The TRP has three outputs: an analog signal, a time signal, and an inhibit signal.

(a) The analog signal from the TRP was sent to a fast and high-performance spectroscopic amplifier (Canberra model 2020) and then to a NIM-ADC (Canberra $100 \mathrm{MHz}$ model 8077). A specific CAMAC interface was built to control and read out this NIM-ADC.

(b) The time signal is sent to a shaping amplifier and a discriminator unit to provide the trigger for the photon detection events. The integration of this signal in the DAQ is explained in the following paragraph.

Table 8: Main characteristics of the germanium detectors

\begin{tabular}{l|c|c|c|c|c|c}
\hline Detector & $\begin{array}{c}\text { Relative } \\
\text { efficiency } \\
(\%)\end{array}$ & \multicolumn{2}{|c|}{$\begin{array}{c}\text { Resolution (keV) } \\
\text { (FWHM) at }\end{array}$} & $\begin{array}{c}\text { Peak/ } \\
\text { Compton }\end{array}$ & $\begin{array}{c}\text { Al window } \\
\text { thickness } \\
(\mathbf{m m})\end{array}$ & $\begin{array}{c}\text { Useful } \\
\text { crystal } \\
\text { volume }\end{array}$ \\
\hline \multicolumn{2}{l|}{} & $\mathbf{1 2 2} \mathbf{~ k e V}$ & $\mathbf{1 3 3 2} \mathbf{~ k e V}$ & & & \\
\hline HPGe1 & 73.5 & 0.97 & 2.01 & 66.1 & 1.0 & $285 \mathrm{~cm}^{3}$ \\
HPGe2 & 73.0 & 1.02 & 2.10 & 60.0 & 0.5 & $293 \mathrm{~cm}^{3}$ \\
\hline
\end{tabular}

This TRP makes periodic resets of its base line and during each of these resets there is a small dead time. The accumulated dead time from this source typically ranges between $2 \%$ and $10 \%$ depending on the HPGe noise and details of the electronic chain.

(c) The inhibit signal. The two detectors used in the experiment have been selected to have a small reset frequency of the order of $20 \mathrm{~Hz}$ for the room background. To each reset corresponds a pulse which is used to inhibit the ADC that normally receives the amplifier signal during the reset time. In this way, the dead time is minimized. Nevertheless, the dead time from this or any other source is measured run by run and can therefore be taken into account.

The environmental background was reduced to the minimum possible level, to allow the proper operation of the HPGe detectors, by:

(a) locating the HPGe detectors as far as possible from the lead assembly in order to reduce damaging neutron background ( $\sim 20 \mathrm{~m}$ away in a different room behind a concrete shielding);

(b) installing a $10 \mathrm{~cm}$ lead layer on the floor of the counting room because of radioactivity residuals from an old beam line;

(c) building a very hermetic lead-copper-aluminium shielding box (Fig. 80) around the two counters. One external ingoing photon would encounter first $15 \mathrm{~cm}$ of lead, then $1 \mathrm{~cm}$ of copper, and finally $5 \mathrm{~mm}$ of Al. With this arrangement, the signal's counting rate of one counter was reduced from about $500 \mathrm{~Hz}$ to only $2 \mathrm{~Hz}$. 


\subsubsection{Bremsstrahlung absorbers}

The electrons from the $\beta$-decays of ${ }^{99} \mathrm{Tc}$ and ${ }^{100} \mathrm{Tc}$ (after irradiation) interact with matter (the sample itself, the container, etc.) and produce a strong bremsstrahlung spectrum which constitutes a background to the detection of the $\gamma$ lines of interest. This bremsstrahlung radiation induces a high counting rate, pile-up, and increases the dead time. The main components are photons below $200 \mathrm{keV}$. To reduce them, one solution is to introduce an absorber material between the sample and the detector. Two filters were used, one made out of lead $(0.5 \mathrm{~mm})$ to cut down the spectrum below $100 \mathrm{keV}$, and the other one made out of copper $(0.5 \mathrm{~mm})$, to absorb the X-rays produced by scintillation in the lead filter. The X-rays from the copper foil are in turn absorbed by the HPGe detector aluminium window (1 or $0.5 \mathrm{~mm}$ ).

The thickness of the filters was optimized with the help of the MCNP simulation program in order to minimize the absorption of the $539 \mathrm{keV}$ and $590 \mathrm{keV}$ lines while at the same time having an acceptable counting rate. The lead and copper filters also stop efficiently the $\beta$ electrons $\left(Q_{\max }=3.2 \mathrm{MeV}\right)$ from the ${ }^{100} \mathrm{Tc}$ decays.

The HPGe crystal position was chosen to be as close as possible to the shuttle (Fig. 80), in order to cover the maximum solid angle but with the limitations of introducing only a small Compton cross-talk and avoiding that the dependency of the geometrical acceptance on the shuttle position becomes too large.

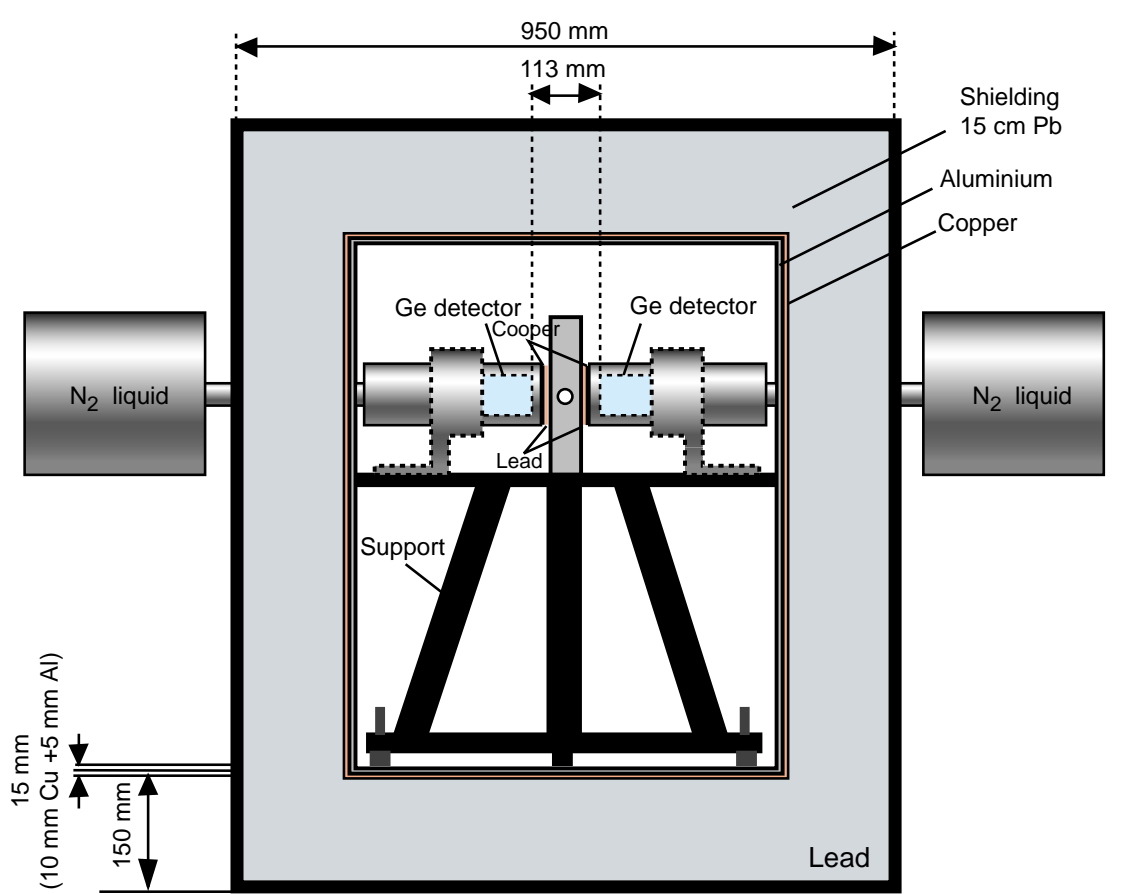

Fig. 80: View of the measurement port with the lead shielding and the two gamma-ray (HPGe) detectors.

\subsubsection{HPGe detector spectra analysis}

The analysis of the HPGe energy spectra consists of the identification of the different observed lines with the known peaks of the spectrum and the evaluation of the number of signal events in each peak after background subtraction. In the present analysis, three methods have been applied for each individual spectrum.

The first method is the simplest but is not the most precise one. It consists of a visual identification of the peaks with an evaluation of the contents in each peak by summing the 
number of entries on a range of channels around the peak, and the evaluation of the value of the continuum background by summing the entries on two ranges of channels on each side of the peak, assuming a linear evolution of the continuum.

The second method also uses visual identification of the peaks, but the content is obtained from a non-linear fit to the peak and neighbouring region by the sum of a Gaussian and a polynomial, and is described in detail in Ref. [60].

The third method, based on the algorithm developed by Mariscotti [61], allows the automatic search of the peaks, including the identification of a single line or a doublet of lines inside the peak, and a constrained fit of one or two Gaussians plus one polynomial to the peak and the surrounding channels, providing the number of photons on each line inside the peak.

This last method has been used as reference in the present analysis. However, for every measurement all the $\gamma$ lines have been analysed with the three methods and whenever different results were found, extra re-analysis was made to solve the discrepancy.

\subsubsection{Energy calibration and efficiency of the HPGe detectors}

The two HPGe detectors used in the TARC experiment were calibrated, both in energy and efficiency, with a set of four radioactive sources: ${ }^{152} \mathrm{Eu},{ }^{134} \mathrm{Cs},{ }^{88} \mathrm{Y}$ and ${ }^{85} \mathrm{Sr}$. These sources were prepared by the Radiation Protection service of the Technical Inspection and Safety Commission (TIS) at CERN and calibrated using their own HPGe spectrometer. The intensities of all the $\gamma$-lines used have been extracted from Refs. [62] and [63]. The efficiency curves obtained with standard methods are shown in Fig. 81.

The systematic error in the fit was taken as $2.5 \%$ (the biggest deviation of the original values from those given by the fit). Moreover, another 5\% coming from the systematic error in the activity of the calibration sources, was also taken into account, giving a final systematic error of $6 \%$.
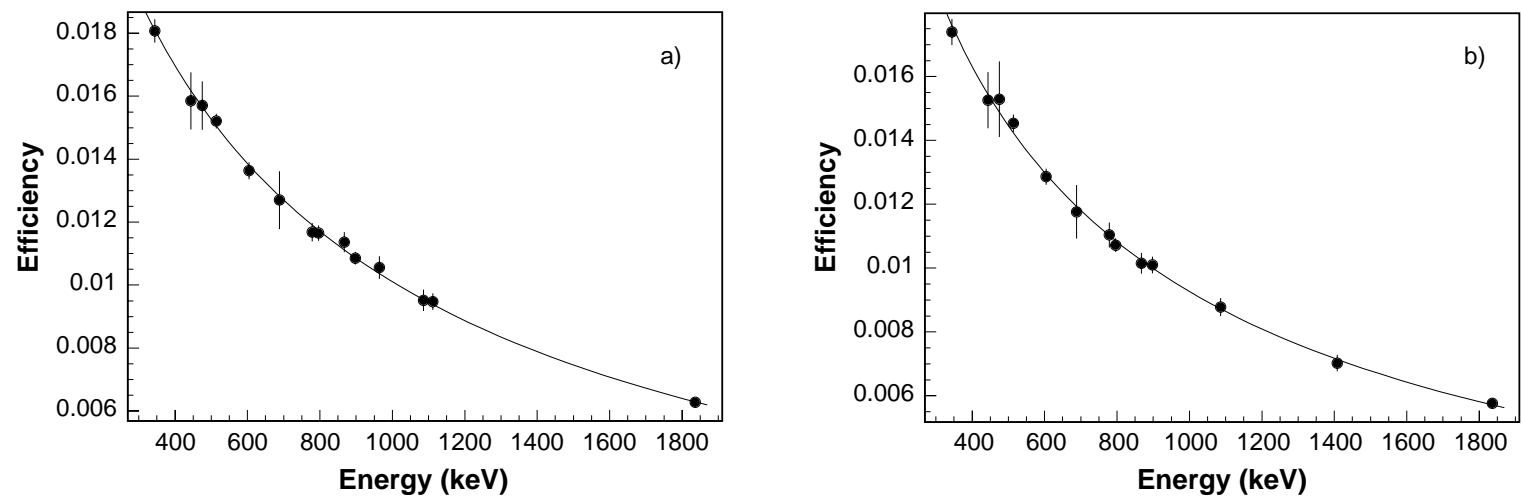

Fig. 81: Efficiency curves for the two germanium detectors (a) for GeHP1 and (b) for GeHP2. In each case, the line is the result of the fit with an exponential of a polynomial in the logarithm of energy.

\subsubsection{Analysis of experimental data}

Two groups of measurements were performed with the rabbit system: a ${ }^{99} \mathrm{Tc}$ scan of the lead volume and a set of runs devoted to the evaluation of possible systematic biases. The ${ }^{99} \mathrm{Tc}$ scan is a set of measurements in which the ${ }^{99} \mathrm{TcO}_{4} \mathrm{~K}$ sample of $441.3 \mathrm{mg}$ was irradiated at different positions inside the lead volume. The positions covered most of the volume of the lead and included longitudinal and transversal scans, as well as several pairs of symmetrical positions. The beam energy and shape were kept constant during the whole scan. Different 
positions correspond to different neutron fluxes both in intensity and in energy distribution. For each position the incineration rate was obtained. The comparison with the Monte Carlo simulation allows one to verify if the nuclear databases, the descriptions of the cross-sections and the Monte Carlo program used are adequate to describe ${ }^{99} \mathrm{Tc}$ transmutation. The large number of points helps in understanding possible differences between data and Monte Carlo simulation. Finally, combining the measurements corresponding to different spectra with the actual measurement of the neutron flux can allow the evaluation of the contribution of different regions of the neutron energy spectra, in particular, the contribution of the ${ }^{99} \mathrm{Tc}$ capture resonances to the transmutation process.

The transmutation rate of ${ }^{99} \mathrm{Tc}$ is obtained from the study of the gamma spectra of the $\mathrm{TcO}_{4} \mathrm{~K}$ sample after irradiation. There are three main components contributing to these spectra: the environmental background, the bremsstrahlung from the ${ }^{99} \mathrm{Tc} \beta$-decay, and the actual gamma spectrum both from the ${ }^{100} \mathrm{Tc} \beta$-decay and from de-excitation lines of the produced ${ }^{100} \mathrm{Ru}^{*}$.

\subsubsection{Background spectra}

Figure 82 shows the gamma spectra collected with the HPGe detectors without radioactive source, before and after shielding. The higher curve, corresponding to no shielding, shows a high activity giving 500 counts/s/detector in our T7 counting room. The spectra include the typical lines from the radon chain, the $1461 \mathrm{keV}$ line from the ${ }^{40} \mathrm{~K}$ (contained in the concrete of the walls) and a relevant $511 \mathrm{keV}$ line from the $\beta^{+}$annihilation.

The massive lead $(15 \mathrm{~cm})$, copper $(1 \mathrm{~cm})$ and aluminium $(0.5 \mathrm{~cm})$ shielding was very effective in reducing the integral background counting rate to 2 counts/s/detector, distributed according to the lower curve of Fig. 82.

This very small background rate was totally negligible in the ${ }^{99} \mathrm{Tc}$ incineration measurements.

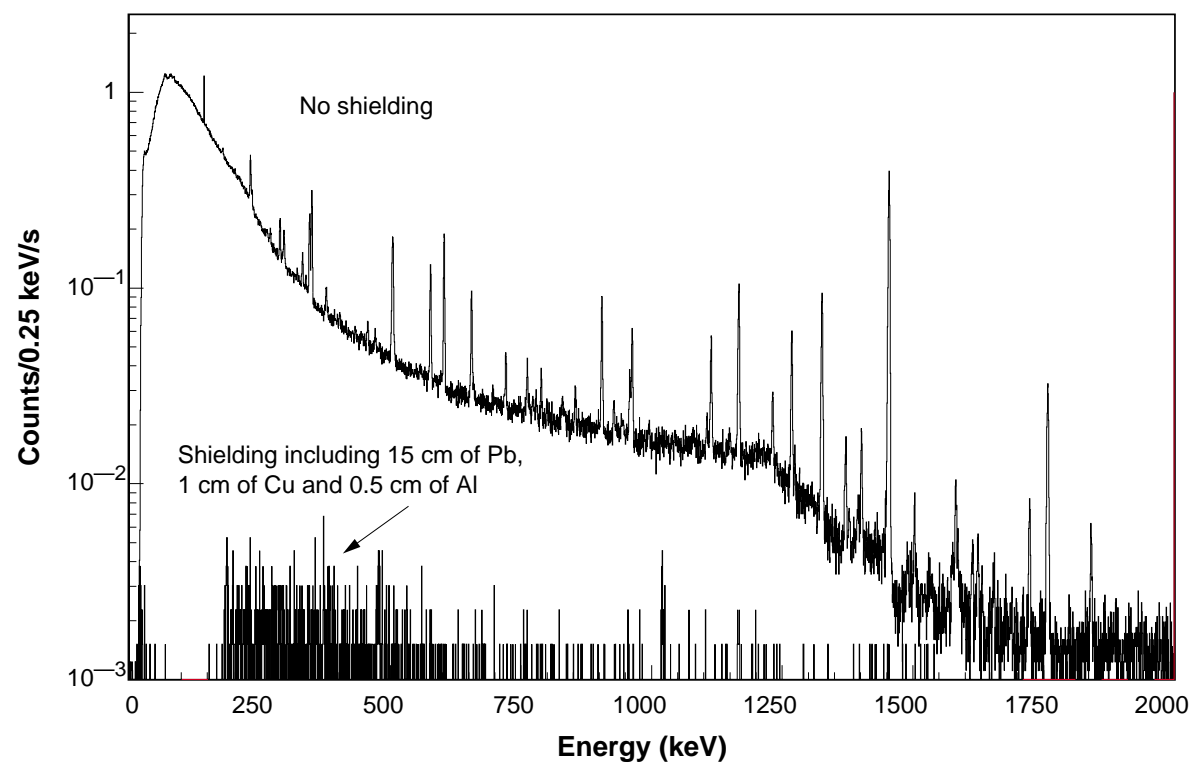

Fig. 82: Gamma-ray background before and after shielding of the HPGe counters, in the TARC counting room. 


\subsubsection{2 ${ }^{99}$ Tc spectra}

Figure 83 shows a spectrum recorded from the ${ }^{99} \mathrm{Tc}$ sample before irradiation. It includes three prominent elements.

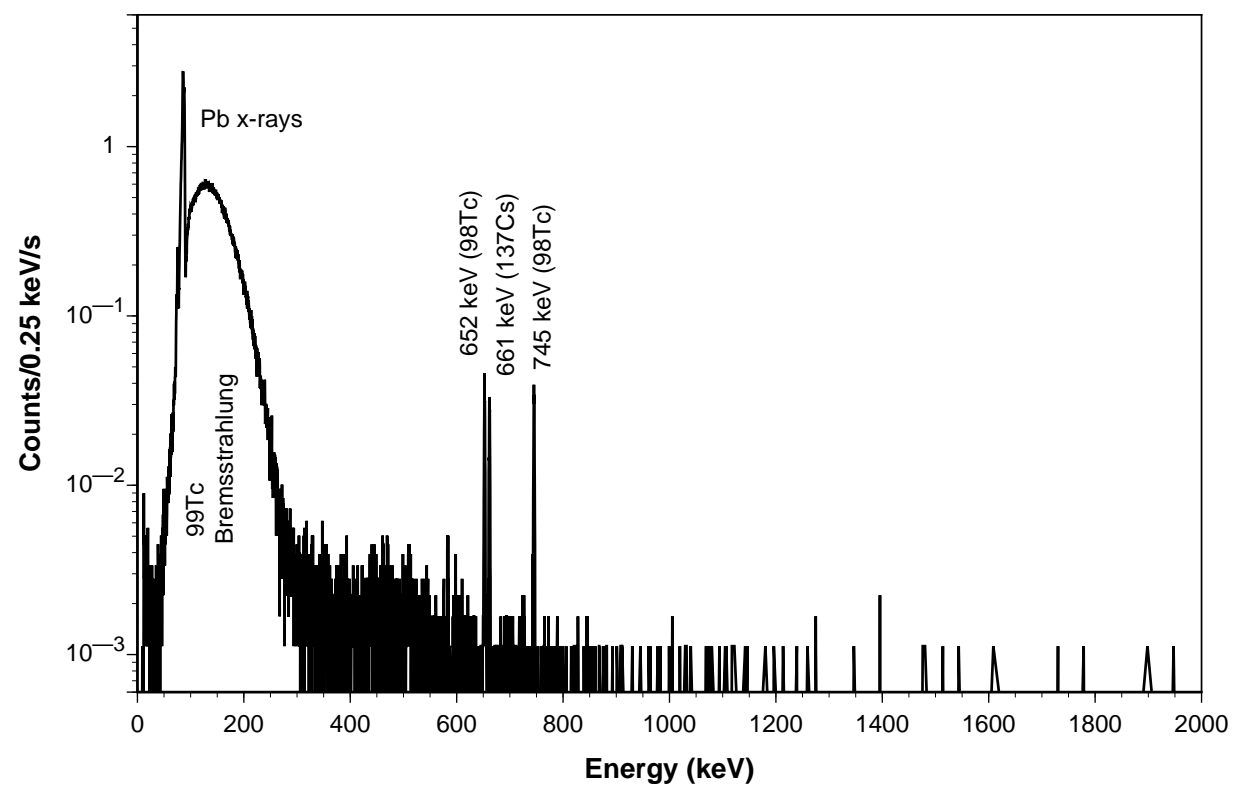

Fig. 83: Gamma-ray spectrum produced by the ${ }^{99} \mathrm{Tc}$ sample before irradiation.

First the presence of a very large activity at photon energies below $292 \mathrm{keV}$. This activity corresponds to the bremsstrahlung produced by the stopping of the large amount of $\beta$ radiation from the decay of the ${ }^{99} \mathrm{Tc}$. Most of the electrons are stopped in the sample itself and the few that escape are stopped in the carbon shuttle. The large activity is a problem since it introduces a large dead time and pile-up effects. A big part of the dead-time effect has been reduced by raising the discrimination threshold of the electronic chain during the actual transmutation experiments to $250 \mathrm{keV}$. The filters described in Section 7.1.3.2 reduce approximately by $10 \%$ the counting rate for the interesting ${ }^{100} \mathrm{Tc}$ lines. Their effect is of course naturally included in the efficiency calibration of the counters.

The second element is a peak due to $\mathrm{Pb} \mathrm{X}$-rays, mainly produced in the filters and in a ring of lead in contact with the germanium crystal used to hold this crystal in position.

The third element concerns three discrete lines: the lines at $652 \mathrm{keV}$ and $745 \mathrm{keV}$ coming from a small contamination of ${ }^{98} \mathrm{Tc}$ contained in the sample. Even though the mass fraction is very small $(\sim 8 \mathrm{ppm})$ and the half-life large $\left(4.2 \times 10^{5} \mathrm{y}\right)$ the much larger branching ratios make its contribution to the spectra visible. The intensity of these lines has been useful in order to cross-check the relative ${ }^{99} \mathrm{Tc}$ mass contained in the different samples as well as the relative normalization of spectra taken in different conditions. Finally we noted a $661 \mathrm{keV}$ line coming from the presence of a very small contamination of ${ }^{137} \mathrm{Cs}$.

\subsubsection{3 ${ }^{100}$ Tc spectra}

Figure 84 shows a typical spectrum recorded from the $\mathrm{TcO}_{4} \mathrm{~K}$ sample after irradiation (in the instrumentation hole 6 at $z=+112.5 \mathrm{~cm}$ ). The figure shows first the effect of the very high discrimination threshold, and second, nine clear new lines from the decay of ${ }^{100} \mathrm{Tc}$ in addition to the three already present in the non-irradiated ${ }^{99} \mathrm{Tc}$ spectrum, over a modest background (note the log scale). Out of these nine lines, two, the $539 \mathrm{keV}$ and the $590 \mathrm{keV}$, are used to compute the transmutation rate. 


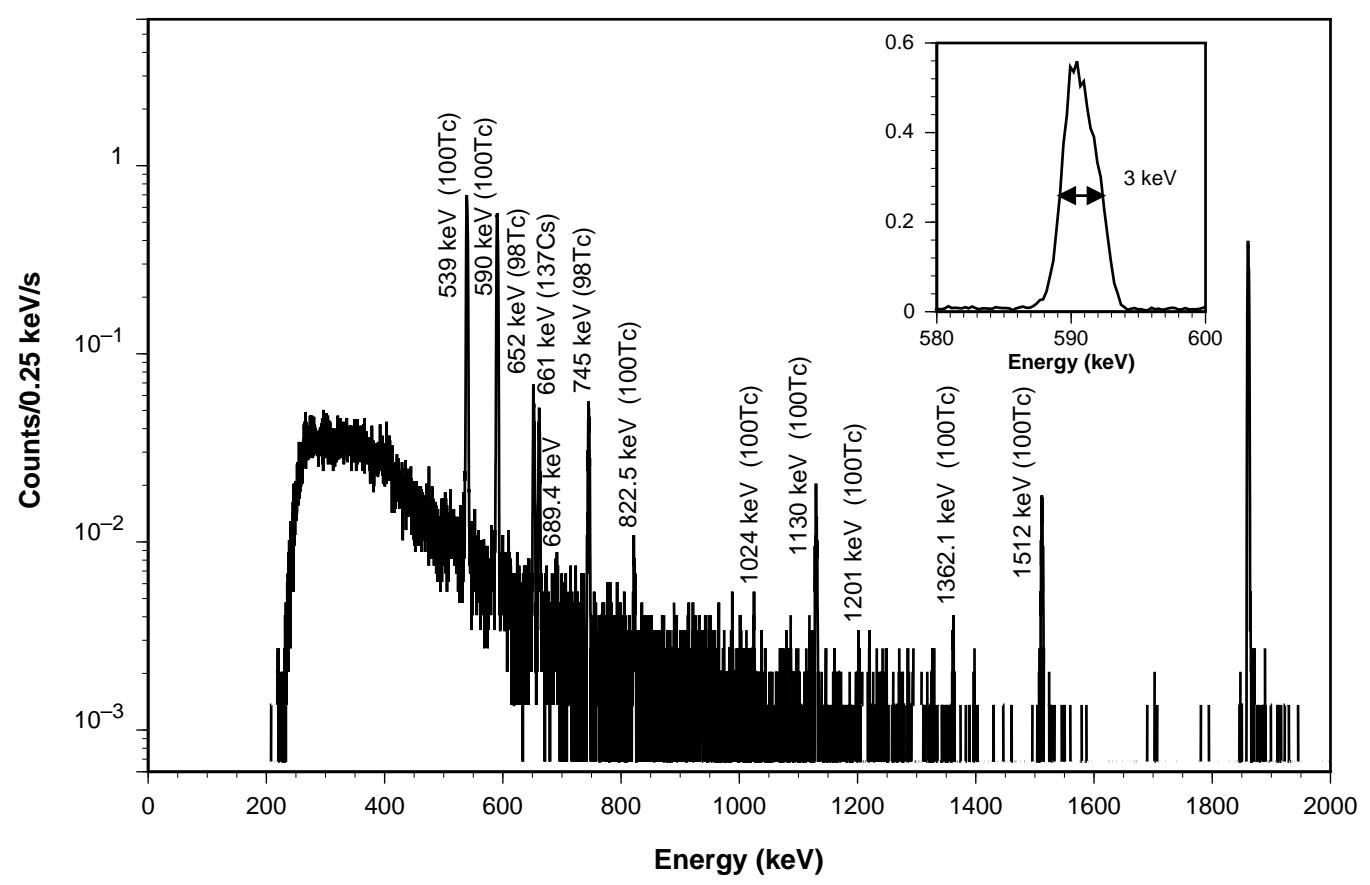

Fig. 84: Gamma-ray spectra produced by the $\mathrm{TcO}_{4} \mathrm{~K}$ sample after irradiation in the instrumentation hole 6 at $z=+112.5 \mathrm{~cm}$ (from HPGe detector 1). Note that in this case an energy threshold of about $200 \mathrm{keV}$ was applied - the $1840 \mathrm{keV}$ peak is a pulser signal.

Figure 85 which summarizes the level structure, the decay chain, and the population of each level of the ${ }^{100} \mathrm{Ru}^{*}$ from the decay of ${ }^{100} \mathrm{Tc}$ shows that there is a $590 \mathrm{keV}$ and a $539 \mathrm{keV}$ line present in $5.7 \%$ and $7 \%$ of the decays, respectively.

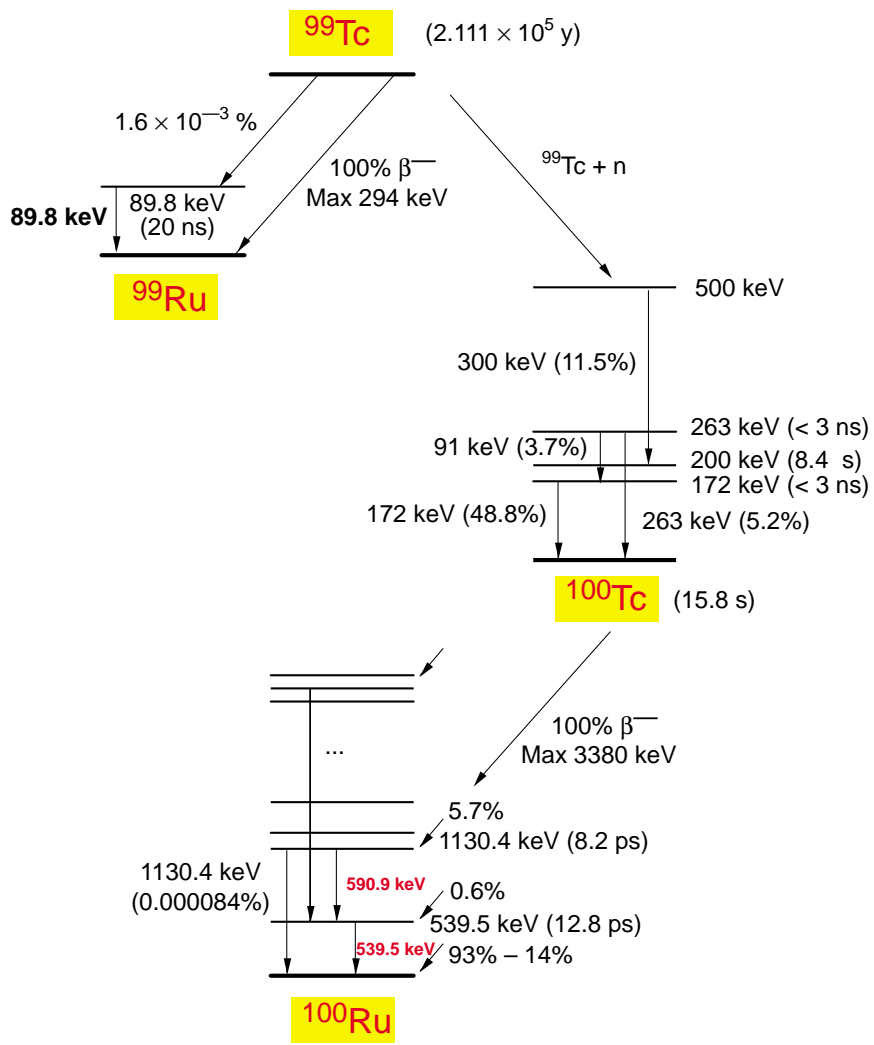

Fig. 85: Level scheme in the decay of ${ }^{99} \mathrm{Tc}$ to ${ }^{99} \mathrm{Ru}$ and partial level scheme in the decay of the ${ }^{100} \mathrm{Tc}$ formed by neutron capture on ${ }^{99} \mathrm{Tc}$ and leading to ${ }^{100} \mathrm{Ru}$. 
It must be pointed out that there is a very large (14\%) uncertainty in the absolute $\gamma / \beta$ fraction. This uncertainty will limit the precision of our transmutation rate measurement. In spite of this, a big effort was made to keep the rest of the uncertainties to a minimum, because the relative comparison of different configurations is independent of this factor and provides useful information on possible systematic deviations between the data and the Monte Carlo predictions, and also because the $\gamma / \beta$ fraction may be measured with better precision in the future.

\subsubsection{Angular correlation between the 539 and $590 \mathrm{keV}$ lines}

Figure 85 also shows that, for each $590 \mathrm{keV}$ photon (except for $0.000084 \%$ of cases), a $539 \mathrm{keV}$ photon is in coincidence. The angular correlation between these two photons must be taken into account (pile-up effect) when computing the actual number of decays observed.

References [64] and [65] report measurements of this angular correlation and show that they are in agreement with the theoretical expectation for a double-decay cascade E2-E2 between $0^{+}-2^{+}-0^{+}$levels. We have used the value published in Ref. [65] for the correlation function parameters. Finally, we should note that the internal conversion fraction is very small and has been neglected in the analysis.

Using the measured correlation between the 539 and $590 \mathrm{keV}$ gammas, the detailed geometry of the experimental set-up, a Monte Carlo simulation has been developed to estimate the correction required for this effect. In this simulation, the peak efficiency is taken from the calibration described in Section 7.1.5. The total efficiency is computed by a Monte Carlo simulation of the HPGe crystal response using MCNP [66]. The results of this simulation have been cross-checked with the recorded spectra of the ${ }^{85} \mathrm{Sr}$ and ${ }^{88} \mathrm{Y}$. From these comparisons a $10 \%$ uncertainty on the ratio between the computed total and the observed peak efficiencies has been estimated. The result obtained is that $(6.0 \pm 1.0) \%$ of the $539 \mathrm{keV}$ photons and $(6.2 \pm 1.0) \%$ of the $590 \mathrm{keV}$ photons are lost from the peaks.

\subsubsection{Photon absorption in the source}

Table 9: Photon self-absorption corrections for the ${ }^{100} \mathrm{Tc},{ }^{108,110} \mathrm{Ag}$ and ${ }^{28} \mathrm{Al}$ gamma-ray lines, relative to the absorption in the calibration shuttle.

\begin{tabular}{l|c|c}
\hline Sample or isotope & $\begin{array}{c}\text { Photon energy } \\
(\mathbf{k e V})\end{array}$ & $\begin{array}{c}\text { Self-absorption correction } \\
(\boldsymbol{\%})\end{array}$ \\
\hline $441 \mathrm{mg}{ }^{99} \mathrm{TcO}_{4} \mathrm{~K}$ & 539 & $(0.03 \pm 0.23)$ \\
& 590 & $(0.02 \pm 0.23)$ \\
\hline $206 \mathrm{mg}{ }^{99} \mathrm{TcO}_{4} \mathrm{~K}$ & 539 & $(4.47 \pm 0.30)$ \\
\hline $14.3 \mathrm{mg}$ metallic Tc & 590 & $(1.12 \pm 0.34)$ \\
\hline $25.9 \mathrm{mg}{ }^{107} \mathrm{Ag}$ & 539 & $(1.06 \pm 0.40)$ \\
\hline $24 \mathrm{mg}{ }^{109} \mathrm{Ag}$ & 590 & $(1.50 \pm 0.50)$ \\
\hline $880 \mathrm{mg}{ }^{27} \mathrm{Al}$ & 633 & $(1.53 \pm 0.50)$ \\
\hline
\end{tabular}


An additional very small correction, to be applied to the number of photons detected, is the difference in absorption in the calibration shuttle and in the actual ${ }^{99} \mathrm{TcO}_{4} \mathrm{~K}$ shuttle. In the calibration experiment a point source was held by a shuttle filled with carbon fibre and in the actual transmutation experiments the source is distributed over the ${ }^{99} \mathrm{TcO}_{4} \mathrm{~K}$ powder and the photons have to cross different materials. Using MCNP [66], a Monte Carlo simulation of the photon transport up to the germanium crystal was performed for the two photon energies (539 and $590 \mathrm{keV}$ ) and for the two shuttle configurations. The correction for the sample used in the scan $\left(441 \mathrm{mg}{ }^{99} \mathrm{TcO}_{4} \mathrm{~K}\right.$ ) (Table 9) is compatible with zero within error and has been neglected. However, this will not be the case for the measurements presented in Section 7.1.7 where different sample/shuttle configurations are used and where the corrections become relevant.

\subsubsection{Protons equivalent and capture per proton evaluation}

To compute the number of ${ }^{99} \mathrm{Tc}$ transmutations per proton we have to apply one correction which takes into account that only a fraction of all the ${ }^{100} \mathrm{Tc}$ nuclei produced by a given shot will decay inside the finite and non-continuous counting gate. This correction can be applied to the counts under the peaks or to the number of protons sent by the accelerator. Although both methods are equivalent, we prefer the second one because it allows checks on possible systematic errors. To apply the correction a quantity named 'protons equivalent' is defined for each beam shot as the sum of all the protons that can contribute to this shot (the protons from the present shot and all the preceding shots) weighted by the probability that one ${ }^{100} \mathrm{Tc}$ nucleus produced by that proton will decay within the collection gate associated to this shot.

This probability is a function of the number of protons contained in each shot, $N_{k}$, the ${ }^{100} \mathrm{Tc}$ half-life, $t_{1 / 2}=15.8 \pm 0.1 \mathrm{~s}$, normally used as $\lambda=\ln (2) / t_{1 / 2}$, the delay between the time of arrival of one beam shot and the beginning of the counting gate of the shot, $t_{\text {delay }}$, the duration

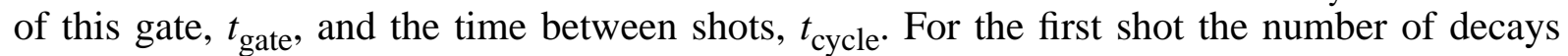
during the counting gate is proportional to $p_{1}^{\mathrm{eq}}$ :

$$
p_{1}^{\text {eq }}=\beta_{\text {gate }} N_{1} \quad \text { with } \quad \beta_{\text {gate }}=e^{-\lambda t_{\text {delay }}}-e^{-\lambda\left(t_{\text {delay }}+t_{\text {gate }}\right)}
$$

For the counting gate of the second shot, there are contributions from the protons of the first and second shots:

$$
p_{2}^{\mathrm{eq}}=\beta_{\text {gate }} \times\left(N_{2}+N_{1} e^{-\lambda t_{\text {cycle }}}\right)
$$

In general the protons equivalent of beam shot $k$ is:

$$
p_{k}^{\mathrm{eq}}=\beta_{\text {gate }} \sum_{j=0}^{k-1} N_{k-j} e^{-j \lambda t_{\text {cycle }}}
$$

From this definition, the number of counts detected on one $\gamma$-line, $\alpha$, in the window following shot $k$, can be expressed as:

$$
C_{k}^{\alpha}=p_{k}^{\mathrm{eq}} R T_{\mathrm{eff}}^{\alpha}
$$

where $R$ is the number of transmutations or captures produced per proton and

$$
T_{\mathrm{eff}}^{\alpha}=\varepsilon_{\alpha} B R_{\alpha}\left(1-A C_{\alpha}\right) \times(1-D T)
$$


with $\varepsilon_{\alpha}$ the efficiency for detecting line $\alpha, B R_{\alpha}$ the branching ratio of line $\alpha, D T$ the global dead time of the run, and $A C_{\alpha}$ the angular correlation correction for line $\alpha$. For a run with $m$ shots, the total number of events collected in line $\alpha$, is:

$$
C_{\alpha}=\sum_{k=1}^{m} C_{k}^{\alpha}=p^{\mathrm{eq}} R T_{\mathrm{eff}}^{\alpha} \quad \text { with } \quad p^{\mathrm{eq}}=\sum_{k=1}^{m} p_{k}^{\mathrm{eq}}
$$

where $p^{\mathrm{eq}}$ is the total protons equivalent of the run. From these expressions, $R$ can be computed from each line as:

$$
R=\frac{C_{\alpha}}{p^{\mathrm{eq}} \varepsilon_{\alpha} B R_{\alpha}\left(1-A C_{\alpha}\right) \times(1-D T)}
$$

All the time parameters involved in the beam and DAQ cycle are known (and constant for the selected events) with a precision better than $0.1 \%$, and have no significant contribution to the uncertainty on the total protons equivalent of the run. The half-life of ${ }^{100} \mathrm{Tc}$ is known to better than $1 \%$ and has negligible contribution to the error. The main uncertainty is coming from the beam intensity measurement of each shot. As explained in Section 2.2, the statistical uncertainties in the beam intensity measurement are small at the intensities used in the rabbit experiment, and, when added (weighted sum) to compute the total protons equivalent of the run, the statistical uncertainty is very small. However, the systematic uncertainty from the beam intensity will not average out and will dominate the final uncertainty on the total protons equivalent of the run. This uncertainty is estimated as $5 \%$ for all the rabbit runs.

\subsubsection{Data quality tests}

Before using data for the evaluation of the ${ }^{99} \mathrm{Tc}$ transmutation rate, every run and every event had to pass a set of data quality and systematic effects tests (described in detail in Ref. [8]).

These data quality tests include the ratio of the number of ${ }^{100} \mathrm{Tc}$ decays observed by the two HPGe detectors on a given gamma line, tested for both 539 and $590 \mathrm{keV}$ lines [Fig. 86(a)], the ratio of the number of ${ }^{100} \mathrm{Tc}$ decays observed on the two lines 539 and $590 \mathrm{keV}$ by the same HPGe detector [Fig. 86(b)], and the distribution of the time difference between the beam shot and the detection time for the photons in one of the two main $\gamma$-lines, collected in the following DAQ gate, accumulated for all the shots of one run (Fig. 87).

These data quality tests gave us high confidence in the reliability of the data we used and allowed us to remove obvious technical problems.
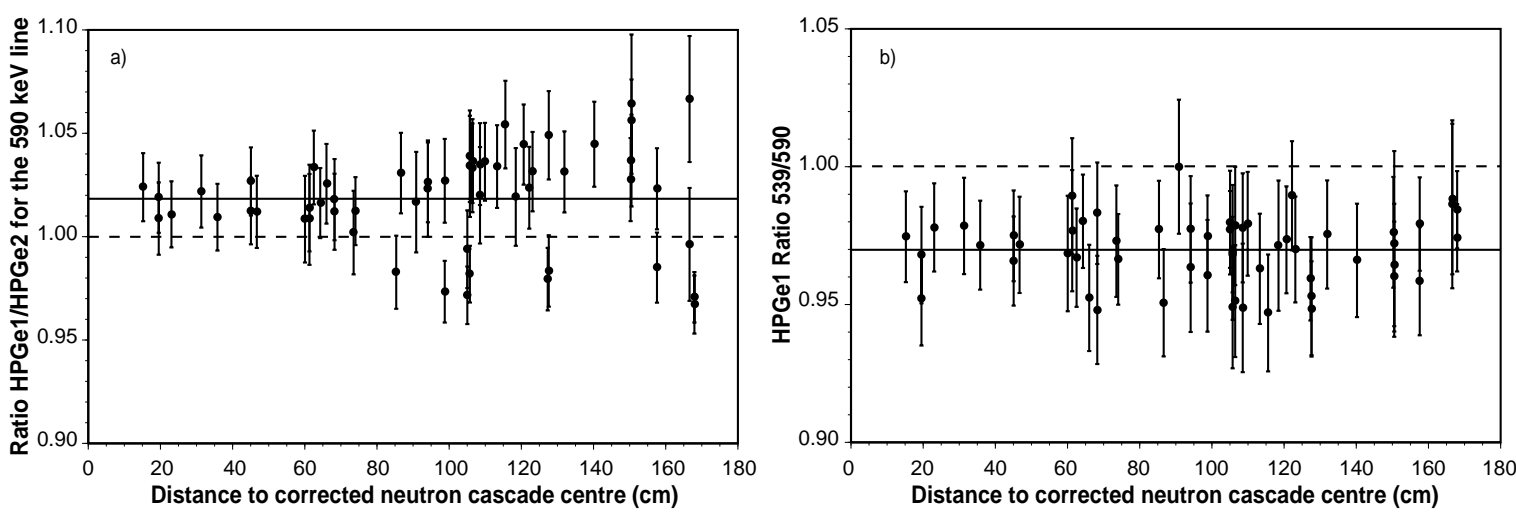

Fig. 86: (a) Ratio of the ${ }^{99} \mathrm{Tc}$ capture rates measured by the two HPGe counters on the $590 \mathrm{keV}$ line (all runs); (b) Ratio of the number of ${ }^{99} \mathrm{Tc}$ captures per proton computed from the 539 and $590 \mathrm{keV}$ lines with HPGe1 (all runs). 


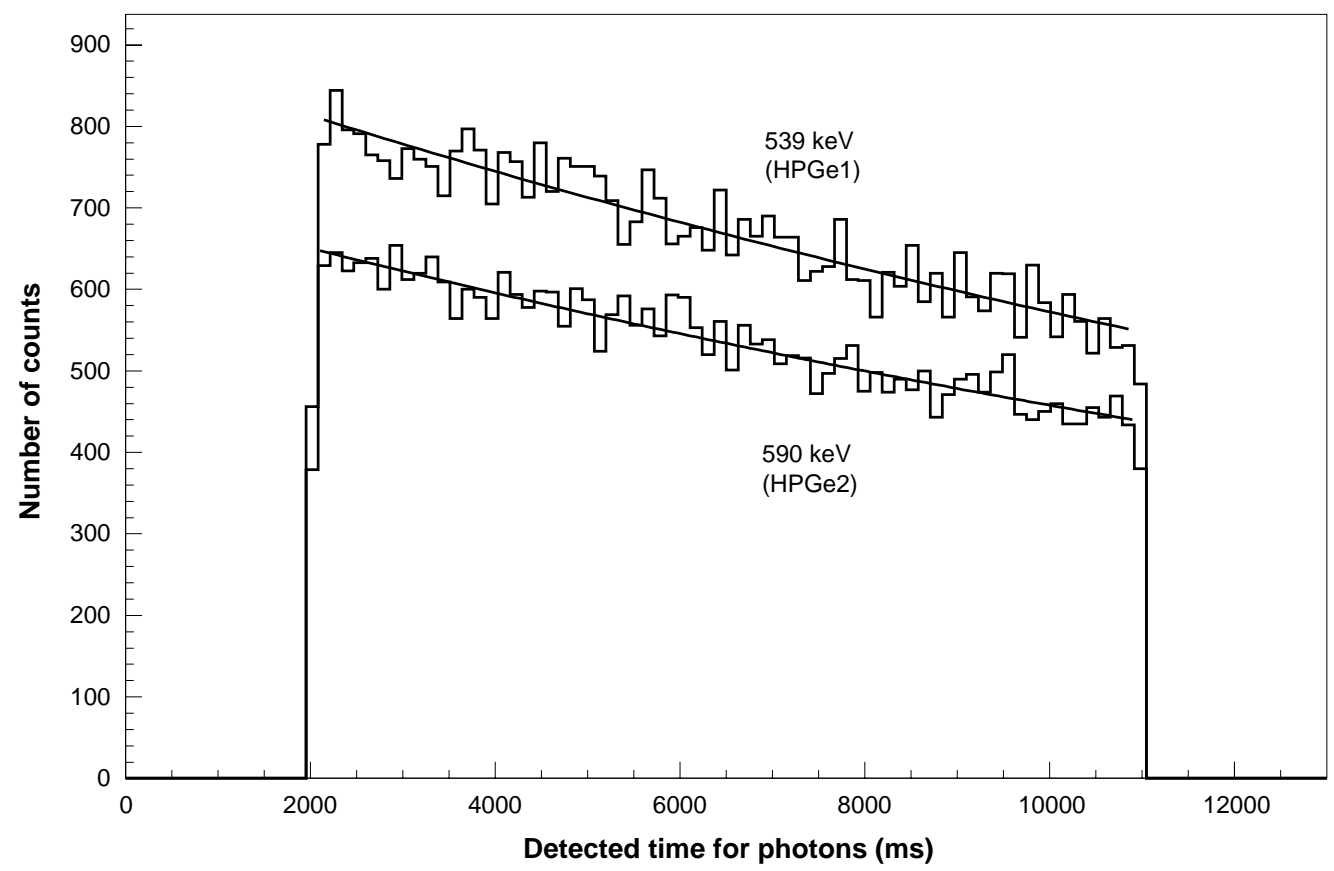

Fig. 87: Distribution of delays between beam shot and time of detection of the subevents of the corresponding shot. The continuous lines represent exponential fits with a half-life of $15.8 \mathrm{~s}$.

\subsubsection{Measurement of the half-life of ${ }^{100} \mathrm{Tc}$}

To further verify that the 539 and $590 \mathrm{keV}$ peaks were $100 \%$ produced by ${ }^{100} \mathrm{Tc}$, a set of special runs were performed to evaluate the half-life of the isotope producing these two $\gamma$-lines. During these special runs, the PS accelerator was prepared to send to the TARC lead volume only one shot every three PS cycles. In this way, the cycle time was $43.2 \mathrm{~s}$ instead of the $14.4 \mathrm{~s}$ used for most of the other runs.

Figure 88 shows the perfect description of the data by a simple exponantial fit. The half-life obtained, $15.9 \pm 0.4 \mathrm{~s}$, allows one to discard the possibility of a significant component from a different (short half-life) isotope.

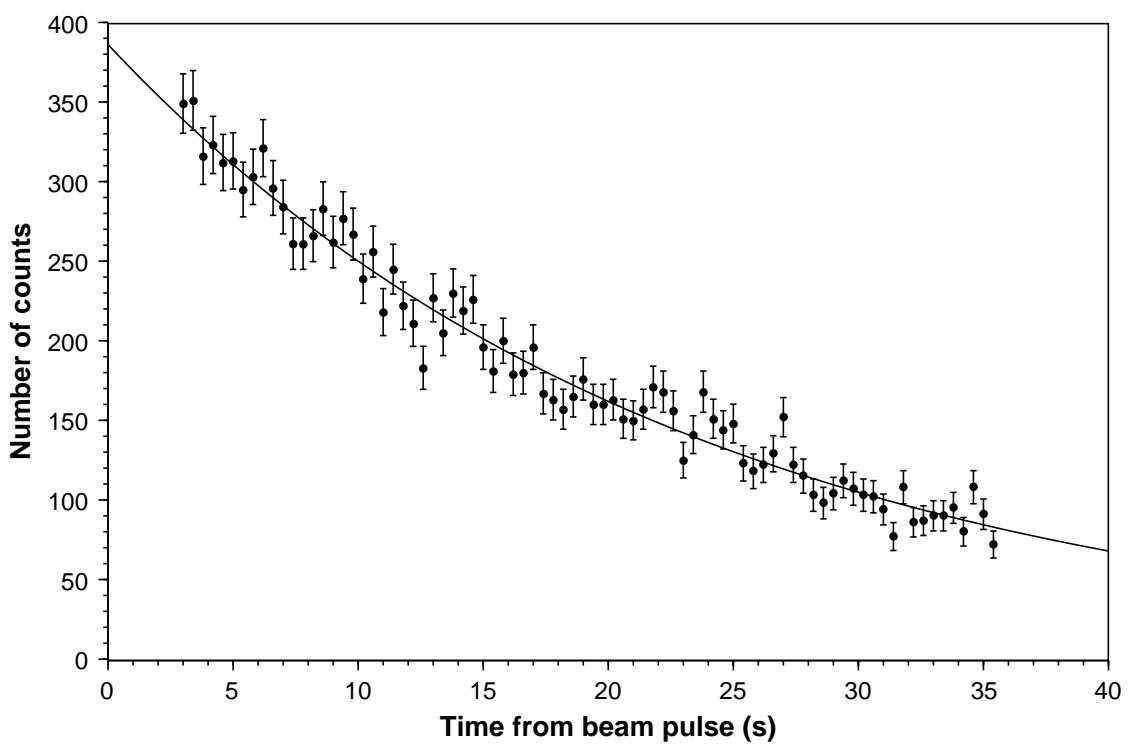

Fig. 88: Measurement of ${ }^{100} \mathrm{Tc}$ half-life with TARC. The fit gives $t_{1 / 2}=15.9 \pm 0.4 \mathrm{~s}$. 


\subsubsection{Measured number of captures per proton}

An extensive positions scan was performed with the $441.3 \mathrm{mg}{ }^{99} \mathrm{TcO}_{4} \mathrm{~K}$ sample. For every run, the number of counts collected after each selection, on each of the two HPGe counters on each of the two lines (539 and $590 \mathrm{keV}$ ) were evaluated using the weighted average from the three different methods described in Section 7.1.4.

Table 10: Measured number of captures per proton for different position measurements in the lead volume. The Error 1 column displays the uncertainties on the average number of captures per proton including all statistical and systematic errors except the $14 \%$ uncertainty on the $\gamma / \beta{ }^{100} \mathrm{Tc}$ branching ratio. The Error 2 column displays the total error on the average. The unit is $10^{-4}$ captures per proton and $\mathrm{cm}$ for $x, y, z$.

\begin{tabular}{|c|c|c|c|}
\hline$z(\mathbf{c m})$ & Average $\left(10^{-4}\right)$ & Error $1\left(10^{-4}\right)$ & Error $2\left(10^{-4}\right)$ \\
\hline $\begin{array}{c}\text { Hole } 1 ; \boldsymbol{x}=\mathbf{1 0 5} ; \boldsymbol{y}=\mathbf{0} \\
-7.5 \\
7.5 \\
67.5 \\
112.5\end{array}$ & $\begin{array}{l}1.25 \\
1.24 \\
0.84 \\
0.39\end{array}$ & $\begin{array}{l}0.10 \\
0.10 \\
0.07 \\
0.03\end{array}$ & $\begin{array}{l}0.21 \\
0.21 \\
0.14 \\
0.07\end{array}$ \\
\hline $\begin{array}{c}\text { Hole 2; } x=\mathbf{6 0} ; y=\mathbf{3 0} \\
67.5\end{array}$ & 1.46 & 0.12 & 0.25 \\
\hline $\begin{array}{c}\text { Hole } 3 ; \boldsymbol{x}=\mathbf{1 5} ; \boldsymbol{y}=\mathbf{0} \\
-112.5 \\
-67.5 \\
-37.5 \\
-22.5 \\
-7.5 \\
7.5 \\
7.5 \\
22.5 \\
37.5 \\
37.5 \\
67.5 \\
112.5\end{array}$ & $\begin{array}{l}1.04 \\
2.26 \\
3.05 \\
3.30 \\
3.43 \\
3.38 \\
3.52 \\
3.10 \\
2.83 \\
2.77 \\
2.04 \\
0.91\end{array}$ & $\begin{array}{l}0.08 \\
0.18 \\
0.24 \\
0.26 \\
0.27 \\
0.27 \\
0.28 \\
0.25 \\
0.23 \\
0.22 \\
0.16 \\
0.07\end{array}$ & $\begin{array}{l}0.18 \\
0.38 \\
0.52 \\
0.56 \\
0.58 \\
0.57 \\
0.60 \\
0.53 \\
0.48 \\
0.47 \\
0.35 \\
0.15\end{array}$ \\
\hline $\begin{array}{c}\text { Hole } \mathbf{4} ; \boldsymbol{x}=\mathbf{0} ; \boldsymbol{y}=\mathbf{- 1 5 0} \\
7.5 \\
7.5 \\
67.5\end{array}$ & $\begin{array}{l}0.29 \\
0.29 \\
0.20\end{array}$ & $\begin{array}{l}0.02 \\
0.02 \\
0.02 \\
\end{array}$ & $\begin{array}{l}0.05 \\
0.05 \\
0.03\end{array}$ \\
\hline $\begin{array}{c}\text { Hole } 5 ; \boldsymbol{x}=\mathbf{0} ; \boldsymbol{y}=\mathbf{- 6 0} \\
7.5 \\
67.5\end{array}$ & $\begin{array}{l}2.27 \\
1.48\end{array}$ & $\begin{array}{l}0.18 \\
0.12\end{array}$ & $\begin{array}{l}0.39 \\
0.25\end{array}$ \\
\hline $\begin{array}{c}\text { Hole } \mathbf{6} ; \boldsymbol{x}=\mathbf{0} ; \boldsymbol{y}=\mathbf{6 0} \\
-112.5 \\
-67.5 \\
-37.5 \\
-22.5 \\
-7.5 \\
7.5 \\
22.5 \\
37.5 \\
67.5 \\
112.5\end{array}$ & $\begin{array}{l}0.80 \\
1.70 \\
2.20 \\
2.27 \\
2.37 \\
2.31 \\
2.18 \\
2.05 \\
1.53 \\
0.68\end{array}$ & $\begin{array}{l}0.06 \\
0.14 \\
0.18 \\
0.18 \\
0.19 \\
0.18 \\
0.17 \\
0.17 \\
0.12 \\
0.05\end{array}$ & $\begin{array}{l}0.14 \\
0.29 \\
0.37 \\
0.39 \\
0.40 \\
0.39 \\
0.37 \\
0.35 \\
0.26 \\
0.12\end{array}$ \\
\hline
\end{tabular}




\begin{tabular}{|c|c|c|c|}
\hline$z(\mathbf{c m})$ & Average $\left(10^{-4}\right)$ & Error $1\left(10^{-4}\right)$ & Error $2\left(10^{-4}\right)$ \\
\hline $\begin{array}{c}\text { Hole } 7 ; \boldsymbol{x}=\mathbf{0} ; \boldsymbol{y}=\mathbf{9 0} \\
7.5 \\
67.5\end{array}$ & $\begin{array}{l}1.55 \\
1.05\end{array}$ & $\begin{array}{l}0.13 \\
0.08 \\
\end{array}$ & $\begin{array}{l}0.26 \\
0.18\end{array}$ \\
\hline $\begin{array}{c}\text { Hole 8; } \boldsymbol{x}=\mathbf{0} ; \boldsymbol{y}=\mathbf{1 2 0} \\
7.5 \\
37.5 \\
67.5 \\
112.5 \\
112.5\end{array}$ & $\begin{array}{l}0.81 \\
0.77 \\
0.60 \\
0.28 \\
0.28\end{array}$ & $\begin{array}{l}0.07 \\
0.06 \\
0.05 \\
0.02 \\
0.02\end{array}$ & $\begin{array}{l}0.14 \\
0.13 \\
0.10 \\
0.05 \\
0.05\end{array}$ \\
\hline $\begin{array}{c}\text { Hole } 9 ; \boldsymbol{x}=\mathbf{0} ; \boldsymbol{y}=\mathbf{1 5 0} \\
7.5 \\
67.5\end{array}$ & $\begin{array}{l}0.32 \\
0.23\end{array}$ & $\begin{array}{l}0.03 \\
0.02\end{array}$ & $\begin{array}{l}0.05 \\
0.04\end{array}$ \\
\hline $\begin{array}{c}\text { Hole 10; } x=-\mathbf{4 5} ; y=\mathbf{0} \\
7.5 \\
67.5\end{array}$ & $\begin{array}{l}2.67 \\
1.73\end{array}$ & $\begin{array}{l}0.21 \\
0.14\end{array}$ & $\begin{array}{l}0.45 \\
0.29\end{array}$ \\
\hline $\begin{array}{c}\text { Hole 11; } \boldsymbol{x}=\mathbf{- 6 5} ; y=\mathbf{3 0} \\
7.5 \\
67.5\end{array}$ & $\begin{array}{l}2.10 \\
1.41\end{array}$ & $\begin{array}{l}0.17 \\
0.11\end{array}$ & $\begin{array}{l}0.36 \\
0.24\end{array}$ \\
\hline $\begin{array}{c}\text { Hole 12; } \boldsymbol{x}=\mathbf{- 1 0 5} ; \boldsymbol{y}=\mathbf{0} \\
-112.5 \\
-67.5 \\
-37.5 \\
-22.5 \\
-22.5 \\
-7.5 \\
7.5 \\
7.5 \\
22.5 \\
37.5 \\
67.5 \\
112.5\end{array}$ & $\begin{array}{l}0.42 \\
0.85 \\
1.08 \\
1.13 \\
1.15 \\
1.17 \\
1.16 \\
1.15 \\
1.10 \\
1.02 \\
0.78 \\
0.37\end{array}$ & $\begin{array}{l}0.03 \\
0.07 \\
0.09 \\
0.09 \\
0.09 \\
0.09 \\
0.09 \\
0.09 \\
0.09 \\
0.08 \\
0.06 \\
0.03\end{array}$ & $\begin{array}{l}0.07 \\
0.14 \\
0.18 \\
0.19 \\
0.19 \\
0.20 \\
0.20 \\
0.20 \\
0.19 \\
0.17 \\
0.13 \\
0.06\end{array}$ \\
\hline
\end{tabular}

The uncertainty was estimated as the sum, in quadrature, of the average uncertainty of the three evaluations plus the quadratic mean of the deviations to their mean value. From these numbers of detected counts, four estimates of the total number of captures are computed per run (two lines times two detectors), applying the described corrections. The weighted average of these four estimates is used to compute the number of ${ }^{99} \mathrm{Tc}$ transmutations or captures per incident proton (Table 10).

Figure 89 displays the transmutation rate for holes 3, 6, and 12, respectively, as a function of the $z$ coordinate. Figure 90 shows the transmutation rate for all the runs in the scan as a function of the distance to a corrected centre located at $x=0, y=0, z=-5 \mathrm{~cm}$. The Gaussian fit demonstrates the high level of spherical symmetry of the distribution. This reflects the similar property of the neutron fluence at low energy.

Error 1 in Table 10 includes the statistical error plus the systematic error associated to the different evaluations of the number of counts in the peaks, typically smaller than $2 \%$; the uncertainty on the dead-time correction contributing less than $1 \%$ of the total error; the uncertainty on the detector efficiencies, 6\%, mainly systematic; the uncertainty on the correction for the angular correlation contributing, $1 \%$; and the systematic error on the beam 
intensity, $5 \%$. All these components added quadratically result in a value very close to $8 \%$ for all the runs in the scan.

Error 2 is the addition in quadrature of error 1 with the $14 \%$ common uncertainty in the absolute branching ratio of ${ }^{99} \mathrm{Tc} \gamma$-lines. In Fig. 90 only error 1 is shown.

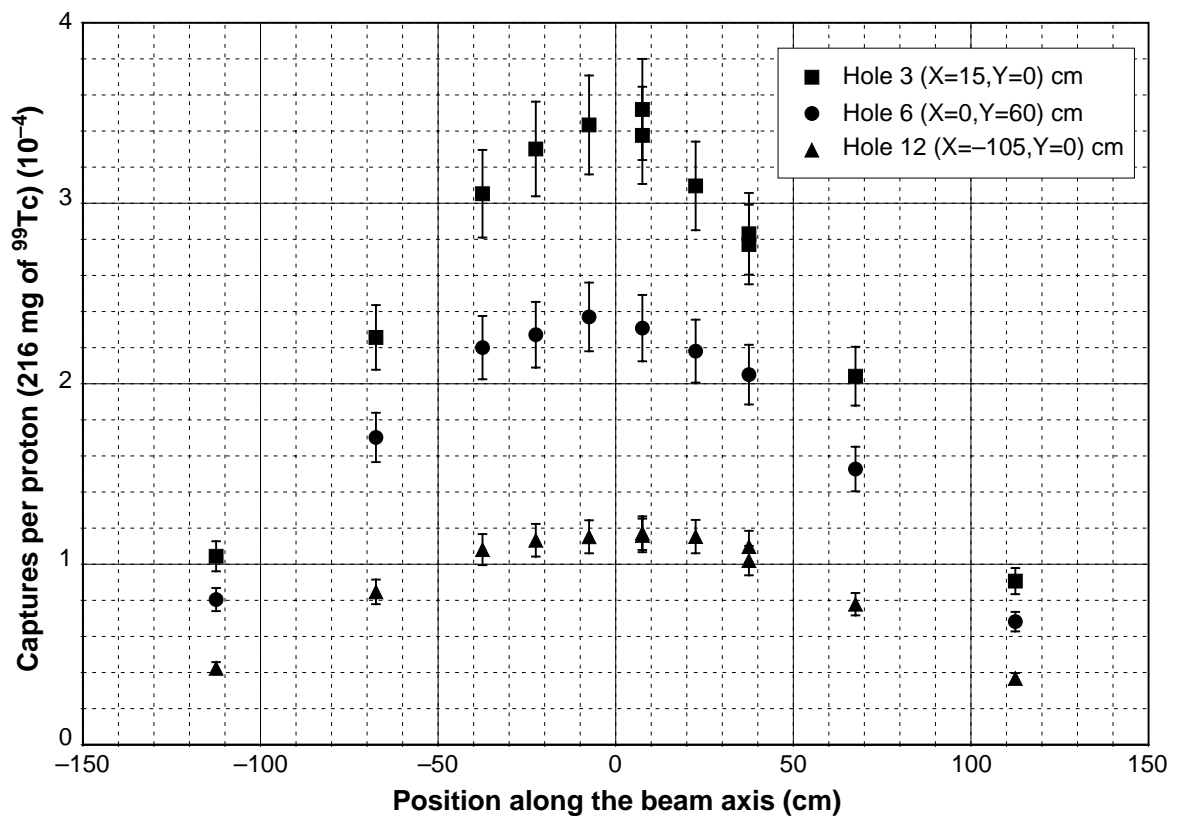

Fig. 89: Measured number of captures per incident proton in $0.216 \mathrm{~g}$ of ${ }^{99} \mathrm{Tc}$, for three different measuring holes (parallel to the beam line), and for various positions along the holes. Error bars include uncertainties from statistics, beam intensity $(\sim 5 \%)$, and dead time $(\sim 0.7 \%)$ corresponding to error 1 in Table 10.

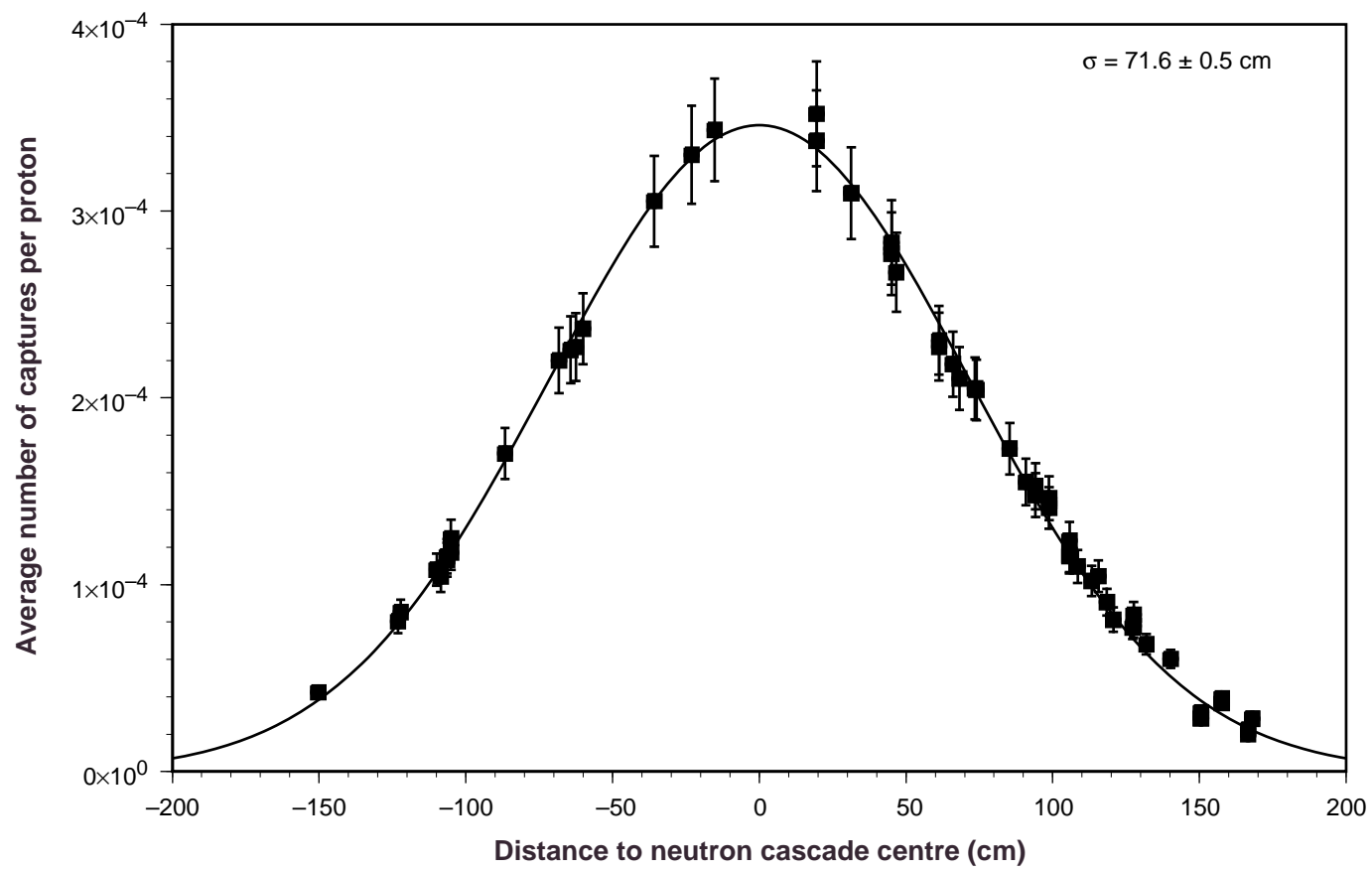

Fig. 90: Transmutation rate versus the distance to a corrected centre $(x=0, y=0, z=-5 \mathrm{~cm})$. The error bars correspond to error 1 in Table 10. The line is a Gaussian fit. 


\subsubsection{Monte Carlo simulation and comparison with the data}

The EA Monte Carlo [2] adapted for TARC is used in this study [32].

\subsubsection{Geometry of the irradiation port for the Monte Carlo simulation}

Figure 77(b) shows the geometrical description of the rabbit irradiation port used in our Monte Carlo simulation for the runs with the $441 \mathrm{mg}{ }^{99} \mathrm{TcO}_{4} \mathrm{~K}$ sample. It includes the precise geometry and material composition of the irradiation port (steel tubes and lead plugs), the shuttle made of carbon fibre, the polyethylene capsule, the ${ }^{99} \mathrm{TcO}_{4} \mathrm{~K}$ sample, and obviously the surrounding lead and hole. Similarly, the different arrangements and material in the carbon-fibre shuttles, have been simulated for the metallic ${ }^{99} \mathrm{Tc}$ [Fig. 78(a)], the $206 \mathrm{mg}$ ${ }^{99} \mathrm{TcO}_{4} \mathrm{~K}$ [Fig. 78(b)], the aluminium and the silver samples (Fig. 94).

\subsubsection{Choice of the ${ }^{99}$ Tc neutron cross-section database}

Different databases contain different descriptions of the ${ }^{99} \mathrm{Tc}(\mathrm{n}, \gamma)$ cross-section. The JENDL nuclear database was used (Fig. 76). However, it must be pointed out that this cross-section has been evaluated from the analysis of the same experimental data [39] used in most of the other cross-section databases. A component of the cross-section systematic error can be estimated from the spread of the capture rates obtained from the various cross-section evaluations all based on the same experimental data (Table 11). The differences reach $10 \%$.

Table 11: The number of captures per proton estimated by Monte Carlo using the different databases for the ${ }^{99} \mathrm{Tc}$ cross-sections at three positions in the lead volume $\left(441.3 \mathrm{mg}{ }^{99} \mathrm{TcO}_{4} \mathrm{~K}\right)$

\begin{tabular}{l|c|c|c}
\hline Database & Hole 3, $z=+\mathbf{7 . 5}$ & Hole 6, $z=+\mathbf{7 . 5}$ & Hole 12, $z=+\mathbf{+ 7 . 5}$ \\
\hline JENDL-3.2 & $0.401 \times 10^{-3}$ & $0.241 \times 10^{-3}$ & $0.123 \times 10^{-3}$ \\
\hline ENDF/B-VI & $0.372 \times 10^{-3}$ & $0.243 \times 10^{-3}$ & $0.120 \times 10^{-3}$ \\
\hline JEF-2.2 & $0.379 \times 10^{-3}$ & $0.254 \times 10^{-3}$ & $0.129 \times 10^{-3}$ \\
\hline BROND & $0.413 \times 10^{-3}$ & $0.261 \times 10^{-3}$ & $0.130 \times 10^{-3}$ \\
\hline
\end{tabular}

The second component of the cross-section systematic error is the uncertainty in the experimental data used in the evaluation. In the original paper describing those data [39] a systematic error 'below $10 \%$ between a few $\mathrm{keV}$ and $10 \mathrm{eV}$ and somewhat above $10 \%$ outside this energy region' is quoted.

\subsubsection{Quality checks of the Monte Carlo simulation}

A check is made to determine if the 'detector' modifies substantially the neutron flux in its neighbourhood. The fluxes of neutrons escaping the parallelepipedal surface around the detector computed with and without the detector are compared. In the case of the rabbit simulation the difference is found to be of the order of $2 \%$ validating the two-step procedure for the Monte Carlo simulation.

Figure 91 displays the spectra of energies for the neutrons captured by the ${ }^{99} \mathrm{Tc}$, showing the fraction of resonant and continuous captures at different positions. As expected, most of the captures occur at the two main resonances $(5.6 \mathrm{eV}$ and $22.3 \mathrm{eV})$. For the far position [Fig. 91(b)], the neutron flux is more thermalized leading to a higher fraction of captures at the two main resonances. 


\subsubsection{Comparison of data and Monte Carlo simulation}

The agreement between data and simulation for the full scan of positions is excellent (Fig. 92). The error evaluation includes the experimental data error (Table 10 - 'Error 1') and the Monte Carlo simulation statistical errors. The band around 1 describes the global systematic uncertainty in the absolute gamma branching ratio of the ${ }^{100} \mathrm{Tc}$ lines $(14 \%)$ and $10 \%$ from the systematic uncertainty of the Monte Carlo prediction.

It must be pointed out that the set of positions (from $15 \mathrm{~cm}$ to $170 \mathrm{~cm}$ ) corresponds to a broadly varying neutron flux, both in magnitude and in energy spectrum. It follows that, among other features, the Monte Carlo simulation is able to reproduce the varying contributions of the different energy regions of the neutron flux to the ${ }^{99} \mathrm{Tc}$ transmutation, for the different positions explored in the lead volume.
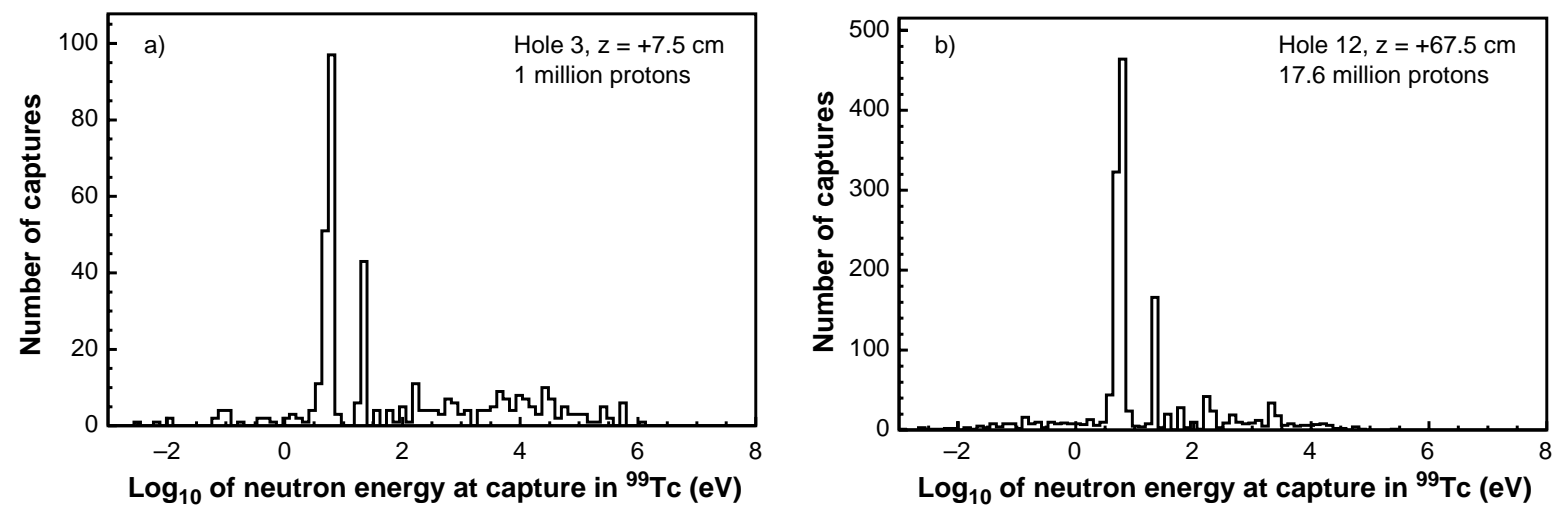

Fig. 91: Monte Carlo energy spectra of neutron captures on ${ }^{99} \mathrm{Tc}$ : (a) hole $3, z=+7.5 \mathrm{~cm}$ (close to centre); (b) hole $12, z=+67.5 \mathrm{~cm}$ (far from centre).

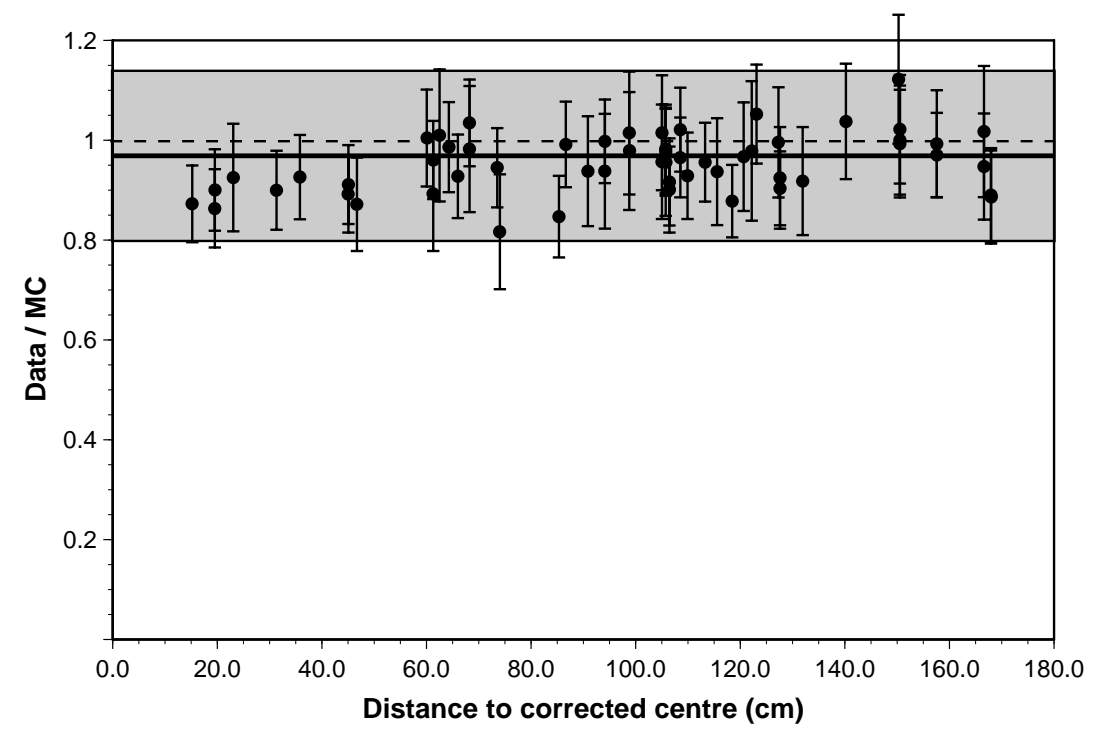

Fig. 92: Comparison of measured and predicted incineration rates in TARC for ${ }^{99} \mathrm{Tc}$. The grey band represents the overall systematic uncertainty as described in the text.

\subsubsection{Systematic studies and other special measurements}

In addition to the general scan described in Section 7.1.6, a set of special measurements with sources different from the $441 \mathrm{mg}{ }^{99} \mathrm{TcO}_{4} \mathrm{~K}$, was made to study the possible systematic effects affecting the results described in Section 7.1.6. The instrumentation hole number 10 at 
$z=+7.5 \mathrm{~cm}$ was used for these measurements. This position has been selected to have a typical spallation neutron spectrum, that means not too close to and not too far from the spallation neutron source. The different samples listed below were used: metallic ${ }^{99} \mathrm{Tc}(14 \mathrm{mg}),{ }^{99} \mathrm{TcO}_{4} \mathrm{~K}$ powder $(0.206$ and $0.441 \mathrm{~g})$, natural aluminium $(0.883 \mathrm{~g})$, and natural silver $(51.839 \%$ of ${ }^{107} \mathrm{Ag}$ and $48.161 \%$ of ${ }^{109} \mathrm{Ag}$ ).

The use of different mass, geometry, and chemical form for ${ }^{99} \mathrm{Tc}$ allows one to verify that the self-shielding and the self-absorption effects are properly simulated. The capture cross-section of aluminium is relatively well known and the experiment performed allows one to verify the full procedure used with the rabbit method. For the same reason, natural silver is also used. Of particular interest is the ${ }^{109} \mathrm{Ag}$ isotope, whose $5.2 \mathrm{eV}$ capture resonance is very close in energy to the main resonance, at $5.6 \mathrm{eV}$, of ${ }^{99} \mathrm{Tc}$. The study of the incineration rate is similar to that of ${ }^{99} \mathrm{Tc}$ with the advantages of having no initial radioactivity in the sample and a known neutron capture cross-section over a large domain of neutron energies and a known branching ratio for gamma emission.

\subsubsection{Self-screening and self-absorption tests}

Two extra samples of ${ }^{99} \mathrm{Tc}$ with different masses, chemical compositions, and shapes, already described in Section 7.1.2, were used to compare the rates of transmutation with the results obtained with the standard sample. These measurements allow one to verify if the self-shielding of the different ${ }^{99} \mathrm{Tc}$ samples is correctly predicted by the Monte Carlo simulation. The different efficiency corrections for self-absorption of photons with respect to the calibration sources have been taken into account (Table 9).

Figure 93 shows the ratio between the captures per proton per gram for the different samples of ${ }^{99} \mathrm{Tc}$ and the value for the metallic sample $\left(14.3 \mathrm{mg}{ }^{99} \mathrm{Tc}\right)$ as a function of the sample mass. The Monte Carlo prediction confirms that the self-shielding effect is large and shows good agreement with the data. The errors do not include the common $14 \%$ from the $\gamma / \beta$ ${ }^{100}$ Tc branching ratio.

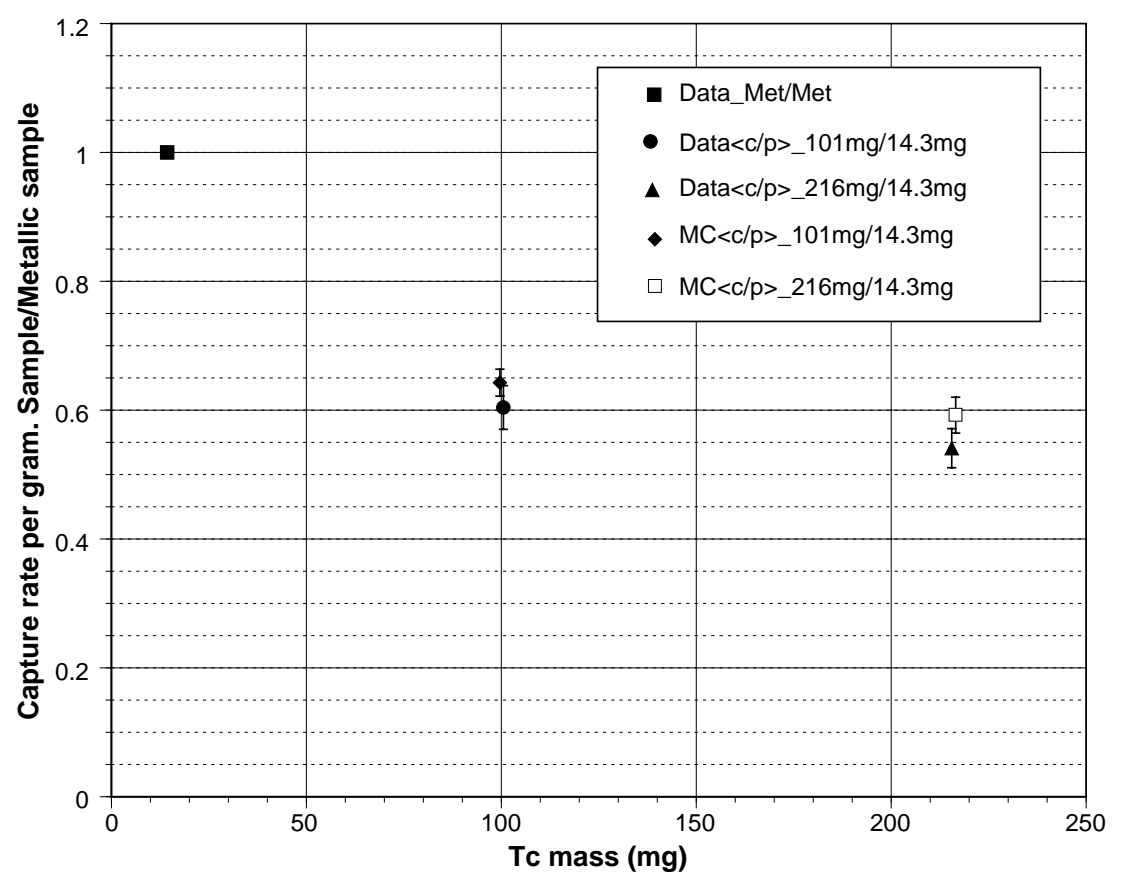

Fig. 93: Ratio of capture rates per gram of different ${ }^{99} \mathrm{Tc}$ samples to the metallic one. ${ }^{99} \mathrm{Tc}$ masses are indicated in the legend. Both data and Monte Carlo simulation are shown. 


\subsubsection{Cross-check of the rabbit method with silver and aluminium samples}

We used a silver sample, whose neutron capture properties are well known and present some similarities to those of technetium, to verify the overall procedure for the rabbit system. The silver nucleus has a resonance energy $(5.2 \mathrm{eV})$ close to that of ${ }^{99} \mathrm{Tc}(5.6 \mathrm{eV})$. The sample is a metallic foil of $50 \pm 0.1 \mathrm{mg}$ of pure $(99.99 \%)$ natural silver with the dimensions shown in Fig. 94(a). The sample is held inside the same support used for the metallic ${ }^{99} \mathrm{Tc}$.

A $880 \pm 1 \mathrm{mg}$ pure $(99.999 \%)$ natural aluminium $\left({ }^{27} \mathrm{Al}\right)$ sample has also been irradiated. Its geometry and the polyethylene support used to ensure its precise position inside the shuttle are shown in Fig. 94(b).

a)

$50 \mu \mathrm{m}$

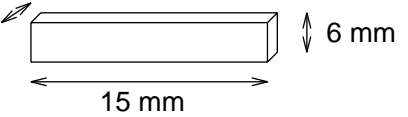

b)

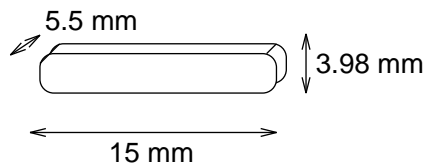

Polyethylene
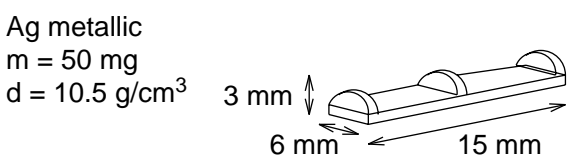

Al metallic
$\mathrm{m}=0.88 \mathrm{gr}$
$\mathrm{d}=2.69 \mathrm{~g} / \mathrm{cm}^{3}$

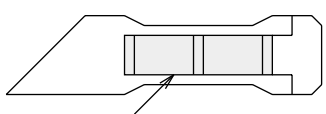

Polyethylene

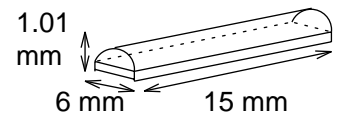

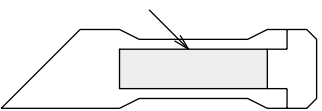

Fig. 94: Schematics of (a) the Ag sample and (b) the $\mathrm{Al}$ sample arrangements inside the shuttle and the polyethylene holding pieces.

Six gamma peaks characteristic of the de-excitation of ${ }^{108} \mathrm{Ag}$ and ${ }^{110} \mathrm{Ag}(443,614,633$, 657, 815 and $1125 \mathrm{keV}$ ) [67] are observed in the energy domain between $200 \mathrm{keV}$ and $2 \mathrm{MeV}$.

The analysis method is similar to that used for ${ }^{99} \mathrm{Tc}$ and is described in detail in Ref. [8]. The transmutation rates measured in the instrumentation hole 10 at $z=+7.5 \mathrm{~cm}$, after all corrections have been applied, are summarized in Table 12.

Table 12: Measured and simulated number of captures per proton in hole 10 at $z=+7.5 \mathrm{~cm}$ for the three configurations of the ${ }^{99} \mathrm{Tc}$ sample, the Ag and $\mathrm{Al}$ samples

\begin{tabular}{l|c|c}
\hline Sample or isotope & Measured captures per proton & MC captures per proton \\
\hline $441.3 \mathrm{mg}^{99} \mathrm{TcO}_{4} \mathrm{~K}$ & $(2.67 \pm 0.21) \times 10^{-4}$ & $(3.05 \pm 0.11) \times 10^{-4}$ \\
$206 \mathrm{mg}{ }^{99} \mathrm{TcO}_{4} \mathrm{~K}$ & $(1.38 \pm 0.11) \times 10^{-4}$ & $(1.53 \pm 0.02) \times 10^{-4}$ \\
$14.3 \mathrm{mg} \mathrm{metallic} \mathrm{Tc}$ & $(3.27 \pm 0.25) \times 10^{-5}$ & $(3.41 \pm 0.10) \times 10^{-5}$ \\
$25.9 \mathrm{mg}{ }^{107} \mathrm{Ag}$ & $(2.43 \pm 0.16) \times 10^{-5}$ & $(2.68 \pm 0.09) \times 10^{-5}$ \\
$24 \mathrm{mg}{ }^{109} \mathrm{Ag}$ & $(1.21 \pm 0.11) \times 10^{-5}$ & $(1.18 \pm 0.02) \times 10^{-5}$ \\
$880 \mathrm{mg}{ }^{27} \mathrm{Al}$ & $(5.96 \pm 0.35) \times 10^{-6}$ & $(6.28 \pm 0.42) \times 10^{-6}$ \\
\hline
\end{tabular}

In total, five samples, three of ${ }^{99} \mathrm{Tc}$, one of $\mathrm{Ag}$ and one of $\mathrm{Al}$ were used in the rabbit for transmutation measurements. Figure 95 shows the transmutation rate ratios, data over Monte Carlo simulation, for the different samples. Within their systematic error of $10 \%$ the Monte Carlo simulation results are consistent with the data. 


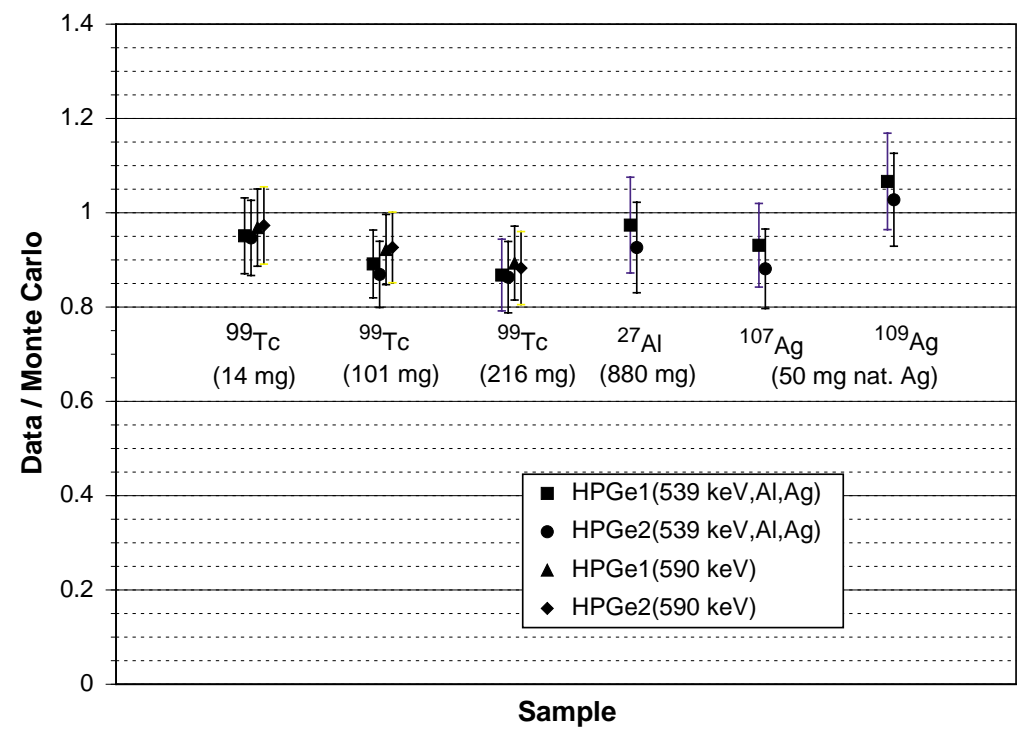

Fig. 95: Ratio of the measured number of captures per proton over the Monte Carlo predictions (the database used is JENDL-3.2 except for ${ }^{27} \mathrm{Al}$ for which we used JEF-2.2) in the instrumentation hole 10 at $z=+7.5 \mathrm{~cm}$ for the various samples used. Data from the two germanium detectors and from the two gamma lines, in the case of ${ }^{99} \mathrm{Tc}$, are shown separately.

\subsubsection{Conclusion of rabbit measurements}

With the rabbit system, a subset of the TARC experiment, the transmutation of ${ }^{99} \mathrm{Tc}$ based on the Adiabatic Resonance Crossing method, was studied using the flux of spallation neutrons created and subsequently diffusing in a large lead volume.

Transmutation of ${ }^{99} \mathrm{Tc}$ has been clearly observed from the photon spectra of the de-excitation of ${ }^{100} \mathrm{Ru}^{*}$ produced by ${ }^{100} \mathrm{Tc}$ decay. These spectra include nine $\gamma$-lines identifying uniquely this isotope. Furthermore, the half-life of the two main lines used to compute transmutation rates $(539 \mathrm{keV}$ and $590 \mathrm{keV})$ were measured providing additional confirmation of the negligible level of background to ${ }^{100} \mathrm{Tc}$ decay. A detailed and extensive study of the possible systematic uncertainties contributing to the observed transmutation rates has also been performed, and the corresponding corrections have been applied.

The ${ }^{99} \mathrm{Tc}$ transmutation rate has been measured in a large number (58) of widely distributed positions inside the lead volume. Over which the neutron flux varies both in intensity and in energy. Data coherency is further assured because at each position we made four different measurements (two HPGe detectors and two main $\gamma$-lines). This set of data constitutes in addition an excellent benchmark for any simulation program needed to study transmutation systems. In particular, it constitutes a precise validation of the simulation developed for the Energy Amplifier by the CERN EET Group with which our data are very well reproduced.

\section{2 ${ }^{129} \mathrm{I}$ and ${ }^{127}$ I integral transmutation rate measurements}

The incineration rate of the second most offending LLFF, ${ }^{129} \mathrm{I}$, has also been measured as part of our study of the Adiabatic Resonance Crossing method. In this section, we summarize the procedure used for collecting and analysing the data to measure the neutron capture rate on a sample of iodine containing an isotopic mixture of $86.1 \%$ of ${ }^{129} \mathrm{I}$ and $13.9 \%$ of ${ }^{127} \mathrm{I}$. The sample was obtained in a solid phase as $286.85 \pm 1.4 \mathrm{mg}$ of $\mathrm{PbI}_{2}$ (Fig. 96). A more detailed description of the sample can be found in Refs. [8] and [68]. 


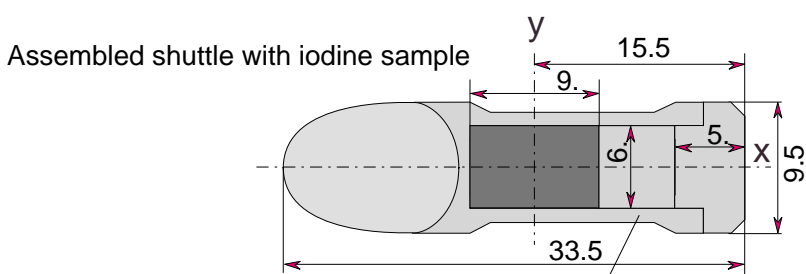

Details of sample configuration Carbon Fibre

a)

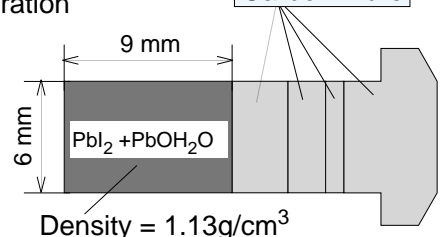

SAMPLE INFORMATION

\begin{tabular}{|c|c|}
\hline Element & $\begin{array}{c}\text { Mass and error } \\
\text { (mg) }\end{array}$ \\
\hline $129 \mathrm{I}$ & $64.70(1.00)$ \\
\hline $127 \mathrm{I}$ & $10.44(0.20)$ \\
\hline $\mathrm{Pb}$ & $190.39(2.10)$ \\
\hline $\mathrm{H}_{2}$ & $1.26(0.02)$ \\
\hline $\mathrm{O}_{2}$ & $20.06(0.30)$ \\
\hline Total & $286.85(1.40)$ \\
\hline Density $\left(\mathrm{g} / \mathrm{cm}^{3}\right)$ & $1.13(0.01)$ \\
\hline
\end{tabular}

Fig. 96: (a) Configuration of the iodine sample. The material around the iodine sample is a special carbon fibre described in Section 7.1.2.2. (b) Properties of the iodine sample.

\subsubsection{Iodine capture and decay schemes}

\subsubsection{Iodine incineration scheme}

Through neutron capture, ${ }^{129}$ I which has a half-life of $1.57 \times 10^{7} \mathrm{y}$ is transmuted into two possible states of ${ }^{130} \mathrm{I}$ with relatively short half-lives $(9 \mathrm{~min}$ for the isomer and $12.36 \mathrm{~h}$ for the ground state). Therefore, the corresponding radioactivity disappears rapidly as they both eventually lead to ${ }^{130} \mathrm{Xe}$ which is stable.

The $13.9 \%$ of stable ${ }^{127} \mathrm{I}$, contained in the sample can capture neutrons and produce ${ }^{128} \mathrm{I}$. We have used the detection of ${ }^{128} \mathrm{I}$ as a technical check of our various procedures (chemistry to prepare the sample and activation procedure).

A simplified decay scheme for ${ }^{130 m_{I}}$ and ${ }^{130 g} \mathrm{I}$ is shown in Fig. 97(a) and for ${ }^{128} \mathrm{I}$ in Fig. 97(b), where only the levels of interest to this analysis have been kept.

With high-energy neutrons, another possible channel is the $(n, 2 n)$ reaction on ${ }^{129} \mathrm{I}$ producing ${ }^{128} \mathrm{I}$ in principle in competition with $(\mathrm{n}, \gamma)$ reactions on ${ }^{127} \mathrm{I}$. The threshold is $8.8 \mathrm{MeV}$ and our Monte Carlo simulation indicates a totally negligible rate (about $0.04 \%$ of the neutron capture rate on ${ }^{129} \mathrm{I}$ at the closest position to the centre of the lead assembly). This produces additional ${ }^{128}$ I atoms to those produced in the ${ }^{127}$ I neutron capture, but the addition is at most $0.5 \%$.

The other possible $(\mathrm{n}, 2 \mathrm{n})$ reaction is on ${ }^{127} \mathrm{I}$ with a threshold at $9.1 \mathrm{MeV}$ producing ${ }^{126} \mathrm{I}$ $\left(t_{1 / 2} \sim 13.11 \mathrm{~d}\right)$. The rate corresponds to $0.05 \%$ of ${ }^{127} \mathrm{I}$ captures and can be neglected. As a check, we verified that there is no sign of $388.6 \mathrm{keV}(\mathrm{Br}=32.2 \%)$ and $666.3 \mathrm{keV}(\mathrm{Br}=31.3 \%)$ $\gamma_{\text {s f from possible }}{ }^{126}$ I decays in the high statistics Ge counting of activation in hole 3 , at $z=+7.5 \mathrm{~cm}$.

\subsubsection{Gamma branching ratios in iodine isotopes}

Table 13 gives the values of the branching ratios we used, for the various gamma lines produced in the decays of the isotopes of interest. In addition, the probability for the ${ }^{130 \mathrm{~m}}$ I state to decay to the ground state is taken as $P=84.2 \%$ [63] [Fig. 97(a)]. The decay schemes from Ref. [63] have been chosen, since it is the latest compilation available, but also because, as we shall see, it gives a good consistency between the activities obtained from each individual gamma line. 

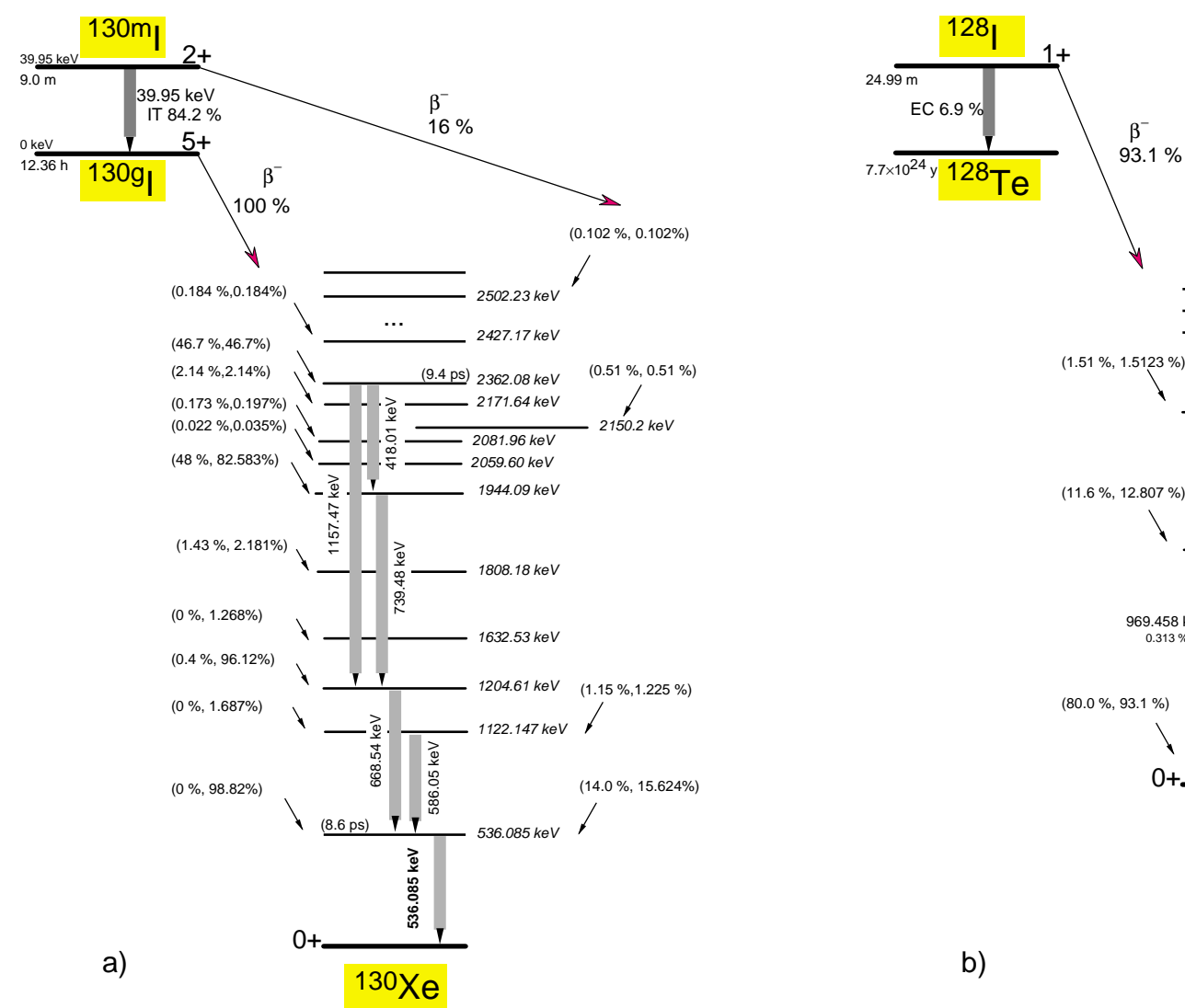

Fig. 97: Simplified decay scheme, derived from Ref. [63], for a) ${ }^{130 m_{I}}$ and ${ }^{130 g} \mathrm{I}$ and b) ${ }^{128}$ I. Inside parentheses, we indicate both the probability to reach the level directly through $\beta$-decay and the total probability to reach the level.

Table 13: Gamma branching ratios (taken from Ref. [63]) in the various decays of interest

\begin{tabular}{|c|c|c|c|c|c|c|c|c|}
\hline a) ${ }^{130 g_{I}}$ & ecay & & b) $130 \mathrm{~m}$ & lecay & & c) ${ }^{128} \mathrm{I}$ & cay & \\
\hline $\begin{array}{c}{ }^{\operatorname{130g}_{\mathbf{I}}} \\
\text { lines } \\
(\mathrm{keV})\end{array}$ & $\begin{array}{l}\text { Branching } \\
\text { ratio }(\%)\end{array}$ & $\begin{array}{c}\text { Error } \\
(\%)\end{array}$ & $\begin{array}{c}{ }^{130} \mathrm{mI} \\
\text { lines } \\
(\mathrm{keV})\end{array}$ & $\begin{array}{c}\text { Branching } \\
\text { ratio }(\%)\end{array}$ & $\begin{array}{c}\text { Error } \\
(\%)\end{array}$ & $\begin{array}{c}{ }^{128} \mathrm{I} \\
\text { lines } \\
(\mathrm{keV})\end{array}$ & $\begin{array}{c}\text { Branching } \\
\text { ratio }(\%)\end{array}$ & $\begin{array}{c}\text { Error } \\
(\%)\end{array}$ \\
\hline 418.0 & 34.57 & 0.1 & 536.1 & $15.64(*)$ & 0.1 & 442.9 & $12.81(*)$ & 0.1 \\
\hline 536.1 & 98.82 & 0.1 & 586.1 & $1.06(* *)$ & 0.01 & 526.6 & $1.20(* *)$ & 0.01 \\
\hline 586.1 & 1.46 & 0.01 & \multirow{4}{*}{\multicolumn{3}{|c|}{$\begin{array}{l}\text { (*) } 16.7 \% \text { from Ref. [69] } \\
(* *) 1.14 \% \text { from Ref. [69] }\end{array}$}} & \multirow{4}{*}{\multicolumn{3}{|c|}{$\begin{array}{l}\text { (*) } 16.0 \% \text { from Ref. [69] } \\
(* *) 1.50 \% \text { from Ref. [69] }\end{array}$}} \\
\hline 668.6 & 96.2 & 0.1 & & & & & & \\
\hline 739.5 & 82.58 & 0.1 & & & & & & \\
\hline 1157.5 & 11.41 & 0.1 & & & & & & \\
\hline
\end{tabular}

\subsubsection{Data analysis}

\subsubsection{Irradiation procedure}

We used the PS proton beam, with a momentum of $3.5 \mathrm{GeV} / c$, extracted directly from the PS in the fast extraction mode (bunch length $\sim 20 \mathrm{~ns}$ ). The number of protons for each individual PS shot is measured with beam transformers as already described in Section 1 and in Ref. [14]. The activation data are summarized in Table 14. 
Table 14: Summary of activation run information

\begin{tabular}{c|c|c|c|c|c|c}
\hline Activation & Hole & $\begin{array}{c}\boldsymbol{z} \text { pos. } \\
(\mathbf{c m})\end{array}$ & $\begin{array}{c}\text { Distance to } \\
\text { centre }(\mathbf{c m})\end{array}$ & $\begin{array}{c}\text { PS cycle } \\
(\mathbf{s})\end{array}$ & Shots & $\begin{array}{c}\text { Number of } \\
\text { protons }\end{array}$ \\
\hline 1 & 12 & +67.5 & 124.82 & 14.4 & 315 & $8.507 \times 10^{10}$ \\
& 10 & +7.5 & 45.62 & & 179 & $3.702 \times 10^{11}$ \\
2 & 3 & +7.5 & 16.77 & 14.4 & 153 & $3.028 \times 10^{11}$ \\
3 & 6 & +7.5 & 61.85 & 14.4 & 129 & $1.297 \times 10^{12}$ \\
4 & 10 & +7.5 & 45.62 & 14.4 & 139 & $1.638 \times 10^{12}$ \\
5 & 11 & +7.5 & 68.76 & 14.4 & 128 & $9.997 \times 10^{11}$ \\
6 & 11 & +7.5 & 68.76 & 14.4 & 126 & $1.576 \times 10^{12}$ \\
7 & 3 & +7.5 & 16.77 & 19.2 & 126 & $1.219 \times 10^{12}$ \\
8 & 3 & +22.5 & 27.04 & 19.2 & 3 & $3.276 \times 10^{10}$ \\
& 3 & +22.5 & 27.04 & & 112 & $1.060 \times 10^{12}$ \\
\hline
\end{tabular}

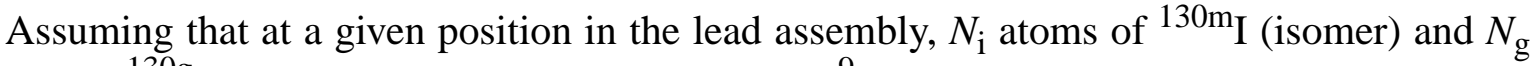

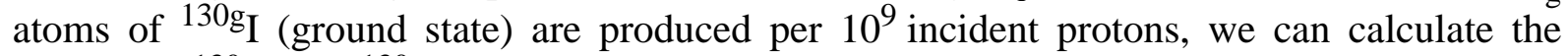
number of ${ }^{130 \mathrm{~m}_{\mathrm{I}} \text { and }}{ }^{130 \mathrm{~g}} \mathrm{I}$ atoms present at the end of irradiation in the following way.

\subsection{Direct production of ${ }^{130 \mathrm{~m}_{\mathrm{I}}}$}

The total number of ${ }^{130 \mathrm{~m}}$ I atoms present at the end of the irradiation is given by:

$$
N\left({ }^{130 \mathrm{~m}} \mathrm{I}\right)_{\mathrm{end}}=N_{\mathrm{i}} \sum_{n=1}^{n=N} X_{\mathrm{n}} \times e^{-\lambda_{\mathrm{m}}\left(t_{\mathrm{end}}-t_{\mathrm{n}}\right)}
$$

where $X_{\mathrm{n}}$ is the number of protons (in units of $10^{9}$ protons) in beam shot $n$, $\lambda_{\mathrm{m}}=1.28360 \times 10^{-3} \mathrm{~s}^{-1}$ is the decay constant of ${ }^{130 \mathrm{~m}} \mathrm{I}, t_{\mathrm{end}}$ is the time of the last PS shot, and $t_{\mathrm{n}}$ is the time of shot number $n$. Figure 98(a) is an example of the time evolution of the contribution of each PS shot to the number of ${ }^{130 \mathrm{~m}_{\mathrm{I}}}$ atoms directly produced by neutron capture and still present at the end of the irradiation.
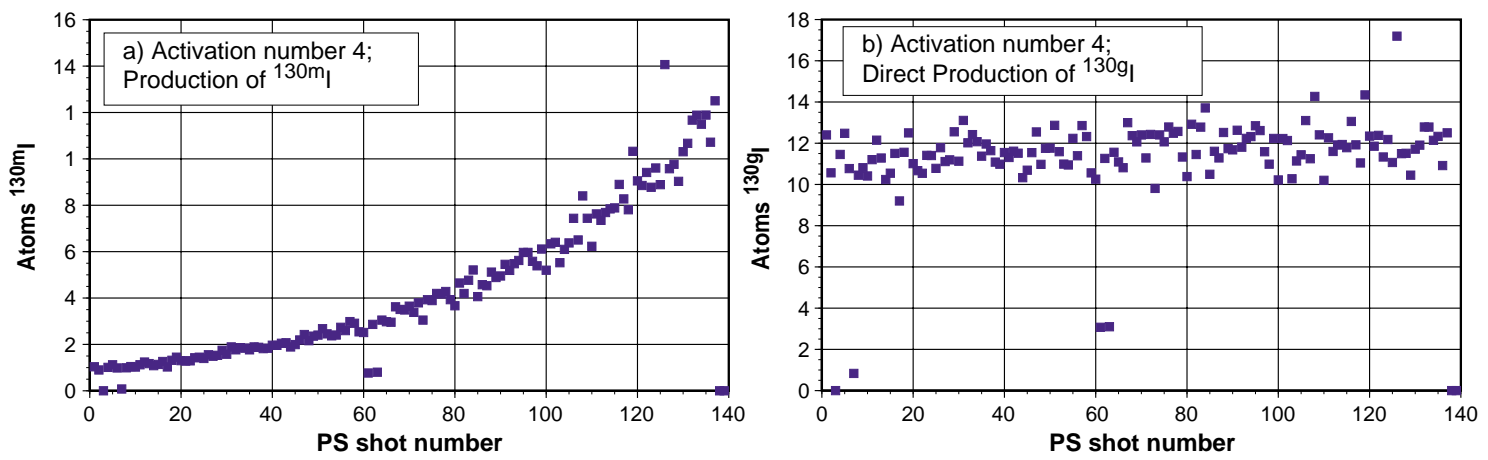

Fig. 98: a) Quantity $X_{\mathrm{n}} \times e^{-\lambda_{\mathrm{m}}\left(t_{\mathrm{end}}-t_{\mathrm{n}}\right)}$ versus PS shot number $(n)$. This quantity multiplied by $N_{\mathrm{i}}$ represents the number of atoms of ${ }^{130} \mathrm{~m}_{\mathrm{I}}$ produced by shot $n$ and still present at the end of irradiation; b) Quantity $X_{\mathrm{n}} \times e^{-\lambda_{\mathrm{g}}\left(t_{\mathrm{end}}-t_{\mathrm{n}}\right)}$ versus PS shot number $(n)$. This quantity multiplied by $N_{\mathrm{g}}$ represents the number of atoms of ${ }^{130 \mathrm{~g}}$ I produced by shot $n$ and still present at the end of irradiation.

\subsection{Direct production of ${ }^{130 \mathrm{~g}_{\mathrm{I}}}$}

The total number of ${ }^{130 \mathrm{~g}}$ I atoms present at the end of the irradiation is given by:

$$
N\left({ }^{130 \mathrm{~g}} \mathrm{I}\right)_{\mathrm{end}}=N_{\mathrm{g}} \sum_{n=1}^{n=N} X_{\mathrm{n}} \times e^{-\lambda_{\mathrm{g}}\left(t_{\mathrm{end}}-t_{\mathrm{n}}\right)}
$$


where $\lambda_{\mathrm{g}}=1.55777 \times 10^{-5} \mathrm{~s}^{-1}$ is the decay constant of ${ }^{130 \mathrm{~g}} \mathrm{I}$. Figure $98(\mathrm{~b})$ shows an example

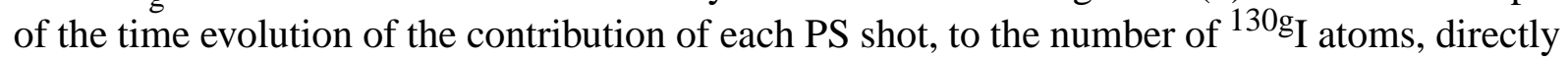
produced by neutron capture and still present at the end of the irradiation.

\subsection{Indirect production of ${ }^{130 \mathrm{~g}_{\mathrm{I}}}$}

Since the ${ }^{130 \mathrm{~m}} \mathrm{I}$ isomer has a relatively short half-life and since it decays through internal

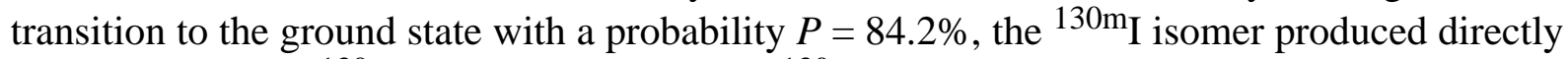

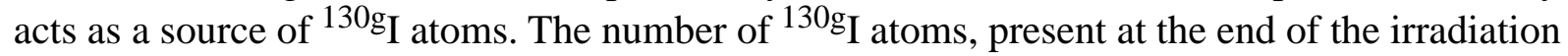
and due to isomer production and decay is given by:

$$
N\left({ }^{130 \mathrm{~m} \rightarrow \mathrm{g}} \mathrm{I}\right)_{\text {end }}=P \times N_{\mathrm{i}} \times \frac{\lambda_{\mathrm{m}}}{\lambda_{\mathrm{g}}-\lambda_{\mathrm{m}}} \sum_{n=1}^{n=N} X_{\mathrm{n}} \times\left[e^{-\lambda_{\mathrm{m}}\left(t_{\mathrm{end}}-t_{\mathrm{n}}\right)}-e^{-\lambda_{\mathrm{g}}\left(t_{\mathrm{end}}-t_{\mathrm{n}}\right)}\right]
$$

The numerical value of the factor $P \times \lambda_{\mathrm{m}} /\left(\lambda_{\mathrm{g}}-\lambda_{\mathrm{m}}\right)$ is -0.85234 . Figure 99 (a) illustrates the

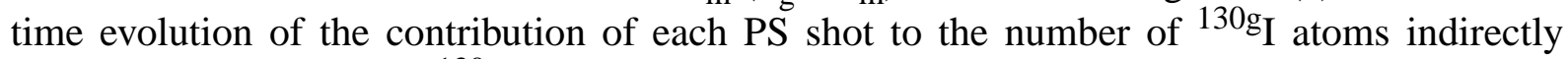
produced by the decay of ${ }^{130 \mathrm{~m}} \mathrm{I}$ and still present at the end of the irradiation.
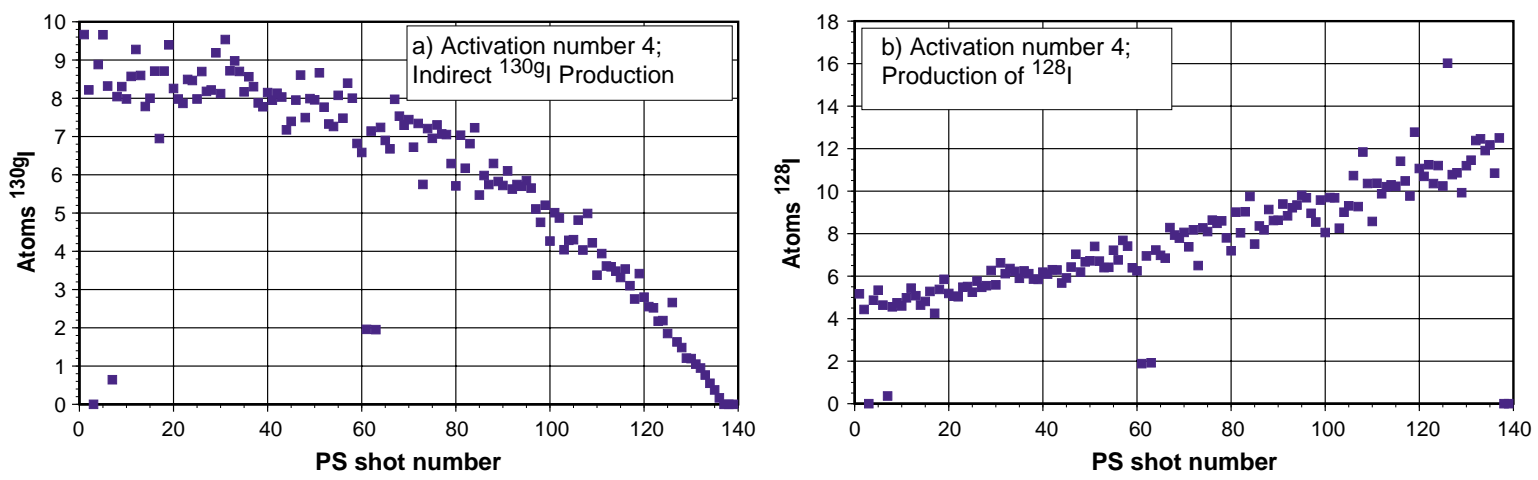

Fig. 99: a) Plot of $P \times\left[\lambda_{\mathrm{m}} /\left(\lambda_{\mathrm{g}}-\lambda_{\mathrm{m}}\right)\right] \times X_{\mathrm{n}} \times\left[e^{-\lambda_{\mathrm{m}}\left(t_{\mathrm{end}}-t_{\mathrm{n}}\right)}-e^{-\lambda_{\mathrm{g}}\left(t_{\mathrm{end}}-t_{\mathrm{n}}\right)}\right]$ versus PS shot number $(n)$,

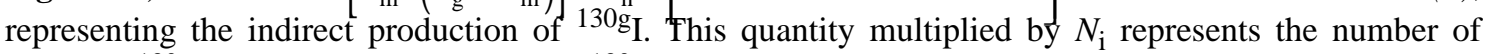
atoms of ${ }^{130 \mathrm{~g}}$ I due to shot $\mathrm{n}$ produced by ${ }^{130 \mathrm{~m}} \mathrm{I}$ decay and still present at the end of irradiation; $\mathrm{b}$ ) Plot of the quantity $X_{\mathrm{n}} \times e^{-\lambda_{\mathrm{k}}\left(t_{\mathrm{end}}-t_{\mathrm{n}}\right)}$ versus PS shot number $(n)$. This quantity multiplied by $N_{\mathrm{k}}$ represents the number of atoms of ${ }^{128}$ I produced by shot $n$ and still present at the end of irradiation.

\subsection{Production of ${ }^{128} \mathrm{I}$}

The number of ${ }^{128} \mathrm{I}$ atoms, present at the end of the irradiation is given by:

$$
N\left({ }^{128} \mathrm{I}\right)_{\mathrm{end}}=N_{\mathrm{k}} \sum_{n=1}^{n=N} X_{\mathrm{n}} \times e^{-\lambda_{\mathrm{k}}\left(t_{\mathrm{end}}-t_{\mathrm{n}}\right)}
$$

where $\lambda_{\mathrm{k}}=4.62283 \times 10^{-4} \mathrm{~s}^{-1}$. Figure 99(b) shows an example of the time evolution of the contribution of each PS shot to the number of ${ }^{128} \mathrm{I}$ atoms produced by neutron capture and still present at the end of the irradiation.

The quantities:

$$
\begin{aligned}
& A \equiv N\left({ }^{130 \mathrm{~m}} \mathrm{I}\right)_{\mathrm{end}} / N_{\mathrm{i}} ; \quad B \equiv N\left({ }^{130 \mathrm{~g}} \mathrm{I}\right)_{\mathrm{end}} / N_{\mathrm{g}} ; \\
& C \equiv N\left({ }^{130 \mathrm{~m} \rightarrow \mathrm{g}}\right)_{\mathrm{end}} / N_{\mathrm{i}} ; \quad D \equiv N\left({ }^{128} \mathrm{I}\right)_{\mathrm{end}} / N_{\mathrm{k}}
\end{aligned}
$$

characterize a given irradiation and are calculated for each run (Table 15).

The statistical errors are negligible. The main systematic error comes from the absolute beam calibration uncertainty $(5 \%)$. 
Table 15: Beam integrals $(A, B, C$ and $D)$ calculated for each irradiation run. These beam integrals

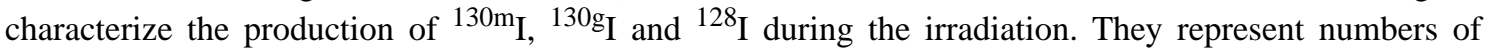
protons in units of $10^{9}$ protons.

\begin{tabular}{c|c|c|c|c}
\hline Activation & $\boldsymbol{A}$ & $\boldsymbol{B}$ & $\boldsymbol{C}$ & $\boldsymbol{D}$ \\
\hline 1 & 107 & 394 & 218 & 216 \\
2 & 118 & 299 & 153 & 206 \\
3 & 508 & 1279 & 656 & 880 \\
4 & 612 & 1620 & 857 & 1090 \\
5 & 387 & 986 & 509 & 677 \\
6 & 614 & 1554 & 800 & 1068 \\
7 & 403 & 1199 & 696 & 763 \\
8 & 395 & 1076 & 579 & 714 \\
\hline
\end{tabular}

\subsubsection{Gamma spectra analysis}

\subsection{Procedure and calibration}

The iodine sample was quickly (within $3 \mathrm{~min}$ ) taken to the CERN/TIS Ge counter system, where the activity of the various gamma lines of interest was measured. Several short counting periods $(600,600$ and 900 seconds) were performed at the beginning to allow us to detect the effect of the short decay time of ${ }^{130 \mathrm{~m}} \mathrm{I}$. Then, longer countings were made to detect, with high statistics, the ${ }^{130 \mathrm{~g}} \mathrm{I}$ at longer times where it dominates the sample activity.

Several calibrations of the Ge counter were made using a ${ }^{152} \mathrm{Eu}$ source and also a ${ }^{133} \mathrm{Ba}$ source. The activity of the ${ }^{152} \mathrm{Eu}$ is known to $\pm 5 \%$.

\subsection{Correction for extended source geometry and gamma absorption}

The Ge calibration is performed with a naked point-like source, therefore, we need to calculate corrections to take into account that (a) the iodine sample is an extended source of $\gamma \mathrm{s}$ and (b) that the $\gamma$ s produced can be absorbed in the iodine sample itself as well as in the shuttle material. Absorption in the Ge counter material (aluminium window, etc.) is common to the calibration procedure and to the data taking.

The correction was obtained by a simulation performed using both MCNP [66] and FLUKA codes [20]. The results are given in Table 16. They are found to be small $(\leq 5 \%)$.

Table 16: Efficiencies obtained from point-like source calibration, for positions $D_{1}$ and $D_{2}$, and relative corrections to the efficiencies for extended source geometry and absorption obtained with MCNP

\begin{tabular}{c|c|c|c|c}
\hline Energy $(\mathbf{k e V})$ & $\begin{array}{c}\text { Eff. at } \mathbf{D}_{\mathbf{1}} \\
(\boldsymbol{\%})\end{array}$ & $\begin{array}{c}\text { Corr. at } \mathbf{D}_{\mathbf{1}} \\
(\boldsymbol{\%})\end{array}$ & $\begin{array}{c}\text { Eff. at } \mathbf{D}_{\mathbf{2}} \\
(\boldsymbol{\%})\end{array}$ & $\begin{array}{c}\text { Corr. at } \mathbf{D}_{\mathbf{1}} \\
(\boldsymbol{\%})\end{array}$ \\
\hline 417.93 & 1.25 & 4.96 & 0.401 & 4.47 \\
443 & 1.16 & 4.55 & 0.377 & 4.08 \\
526.5 & 0.949 & 3.53 & 0.315 & 3.13 \\
536.1 & 0.931 & 3.44 & 0.309 & 3.05 \\
586.37 & 0.849 & 3.03 & 0.283 & 2.67 \\
668.57 & 0.752 & 2.52 & 0.251 & 2.19 \\
739.5 & 0.693 & 2.19 & 0.230 & 1.89 \\
1156.9 & 0.497 & 1.16 & 0.159 & 0.95 \\
\hline
\end{tabular}




\subsection{Correction for gamma overlap}

We have computed the corrections to the Ge counter efficiency, using (a) the overlap probability between the various gamma lines [Table 17(a), 17(b) \& 17(c)], and (b) the angular correlations between the various gammas Refs. [70] and [71]. To do this we used a combination of MCNP to obtain the $\gamma$ spectra in the Ge counter, including Compton scattering and resolution effects with a specially written Monte Carlo to simulate the various decay chains.

Table 17: Probability (\%) of simultaneous emission of other gammas, when a given gamma (first column) is emitted: a) ${ }^{\left.130 \mathrm{~g}_{\mathrm{I}}, \mathrm{b}\right)}{ }^{130 \mathrm{~m}_{\mathrm{I}} \text { and c) }{ }^{128} \mathrm{I}}$

\begin{tabular}{|c|c|c|c|c|c|c|}
\hline a) & $418 \mathrm{keV}$ & $536 \mathrm{keV}$ & $586 \mathrm{keV}$ & $668 \mathrm{keV}$ & $739 \mathrm{keV}$ & $1157 \mathrm{keV}$ \\
\hline $418 \mathrm{keV}$ & - & 100 & 0 & 100 & 100 & 0 \\
\hline $536 \mathrm{keV}$ & 34.98 & - & 1.48 & 97.34 & 83.57 & 11.54 \\
\hline $586 \mathrm{keV}$ & 0 & 100 & - & 0 & 0 & 0 \\
\hline $668 \mathrm{keV}$ & 35.95 & 100 & 0 & - & 85.85 & 11.86 \\
\hline $739 \mathrm{keV}$ & 41.88 & 100 & 0 & 100 & - & 0 \\
\hline $1157 \mathrm{keV}$ & 0 & 100 & 0 & 100 & 0 & - \\
\hline b) & $536 \mathrm{keV}$ & $586 \mathrm{keV}$ & & c) & $443 \mathrm{keV}$ & $526 \mathrm{keV}$ \\
\hline $536 \mathrm{keV}$ & - & 7.83 & & $443 \mathrm{keV}$ & - & 9.36 \\
\hline $586 \mathrm{keV}$ & 100 & - & & $526 \mathrm{keV}$ & 100 & - \\
\hline
\end{tabular}

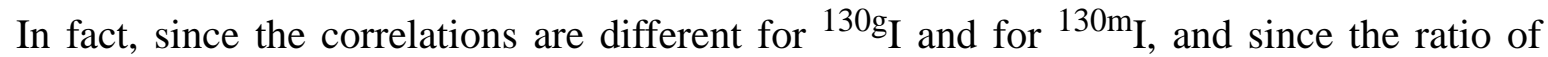

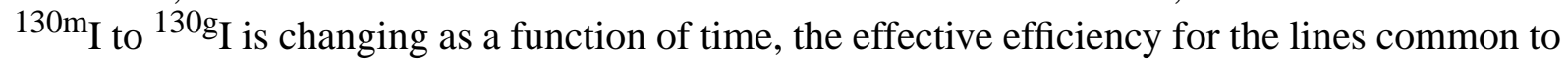
${ }^{130 \mathrm{~g}} \mathrm{I}$ and ${ }^{130 \mathrm{~m}} \mathrm{I}$ is also changing as a function of time. This was taken into account properly in our evaluation of the contribution of each line. The overlap corrections computed for all the $\gamma$ lines used in this analysis [Table 18(a), 18(b) \& 18(c)] were used to correct the Ge counter efficiencies determined from single $\gamma$ data. These corrections are small, they reach at most $6 \%$.

Table 18: Overlap corrections to the various $\gamma$ lines of: (a) ${ }^{130 \mathrm{~g}}$; (b) ${ }^{130 \mathrm{~m}} \mathrm{I}$ and (c) ${ }^{128} \mathrm{I}$

\begin{tabular}{|c|c|c|c|}
\hline${ }^{130 g_{I}}$ & $\begin{array}{l}\text { (a) } \gamma \text { energy } \\
(\mathrm{keV})\end{array}$ & $\begin{array}{c}\text { Correction for position } D_{1} \\
(\%)\end{array}$ & $\begin{array}{c}\text { Correction for position } \mathrm{D}_{2} \\
(\%)\end{array}$ \\
\hline & $\begin{array}{c}418 \\
536 \\
586 \\
668 \\
739 \\
1157\end{array}$ & $\begin{array}{l}6.20 \pm 0.6 \\
4.58 \pm 0.4 \\
1.88 \pm 0.2 \\
4.71 \pm 0.5 \\
4.86 \pm 0.5 \\
3.99 \pm 0.4\end{array}$ & $\begin{array}{c}2.20 \pm 0.2 \\
1.52 \pm 0.15 \\
0.62 \pm 0.06 \\
1.53 \pm 0.15 \\
1.55 \pm 0.15 \\
1.39 \pm 0.14\end{array}$ \\
\hline \multirow[t]{2}{*}{${ }^{130} \mathrm{~m}_{\mathbf{I}}$} & $\begin{array}{l}\text { (b) } \gamma \text { energy } \\
(\mathrm{keV})\end{array}$ & $\begin{array}{c}\text { Correction for position } D_{1} \\
(\%)\end{array}$ & $\begin{array}{c}\text { Correction for position } \mathrm{D}_{2} \\
(\%)\end{array}$ \\
\hline & $\begin{array}{l}536 \\
586\end{array}$ & $\begin{array}{c}0.146 \pm 0.015 \\
2.88 \pm 0.28\end{array}$ & $\begin{array}{c}0.0389 \pm 0.004 \\
0.84 \pm 0.08\end{array}$ \\
\hline \multirow[t]{2}{*}{${ }^{128}{ }_{I}$} & $\begin{array}{l}\text { (c) } \gamma \text { energy } \\
(\mathrm{keV})\end{array}$ & $\begin{array}{c}\text { Correction for position } D_{1} \\
(\%)\end{array}$ & $\begin{array}{c}\text { Correction for position } \mathrm{D}_{2} \\
(\%)\end{array}$ \\
\hline & $\begin{array}{l}443 \\
586\end{array}$ & $\begin{array}{c}0.164 \pm 0.016 \\
1.753 \pm 0.17\end{array}$ & $\begin{array}{c}0.0588 \pm 0.006 \\
0.00665 \pm 0.0006\end{array}$ \\
\hline
\end{tabular}




\subsection{Systematics in shuttle positioning}

It was possible to monitor the relative position of the shuttle, using the $39.5 \mathrm{keV}$ gamma emitted by ${ }^{129}$ I with a $7.52 \%$ branching ratio. The maximum spread between rates observed for the $39.5 \mathrm{keV}$ line is $2.6 \%$ at $\mathrm{D}_{1}$ and $1.13 \%$ at $\mathrm{D}_{2}$, which, when transformed into a spread in position, gives respectively 0.6 and $0.5 \mathrm{~mm}$ displacements expected for $\mathrm{D}_{1}=43 \mathrm{~mm}$ and $\mathrm{D}_{2}=93 \mathrm{~mm}$. The absolute position has an error of $1 \mathrm{~mm}$. Therefore, the overall systematic error in the shuttle position is estimated to be $1.1 \mathrm{~mm}$.

\subsubsection{Peak analysis}

The number of counts in the peaks was determined in three ways: (a) using the standard algorithm available from the CERN/TIS Ge system (Inter gamma Analysis), which has the advantage of being very robust; (b) using an algorithm used also for the rabbit analysis [61] (see Section 7.1), and (c) making a fit with an interactive software (PAW), in cases of ambiguity of results from (a) and (b). This allowed us to compare the methods, and gave us a way to correct problems and estimate the systematic errors. In all cases the $\gamma_{\text {s }}$ of interest are sufficiently separated from one another (Fig. 100) to have a reasonably good measurement of the number of counts.

A practical overall test of the peak fitting performance, the estimation of the Ge counter acceptance, the effect of $\gamma$ overlap and the determination of the $\gamma$-line branching ratio is obtained by verifying that all the $\gamma$-lines of one element give the same activity. This is indeed

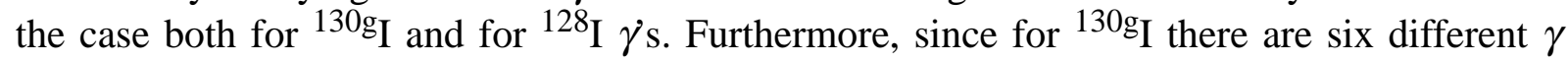
lines, the spread between the activities of these lines, averaged over the eight activations, is a good measure of the systematic error. We find a standard deviation of $4 \%$, which we use in our systematic error analysis.

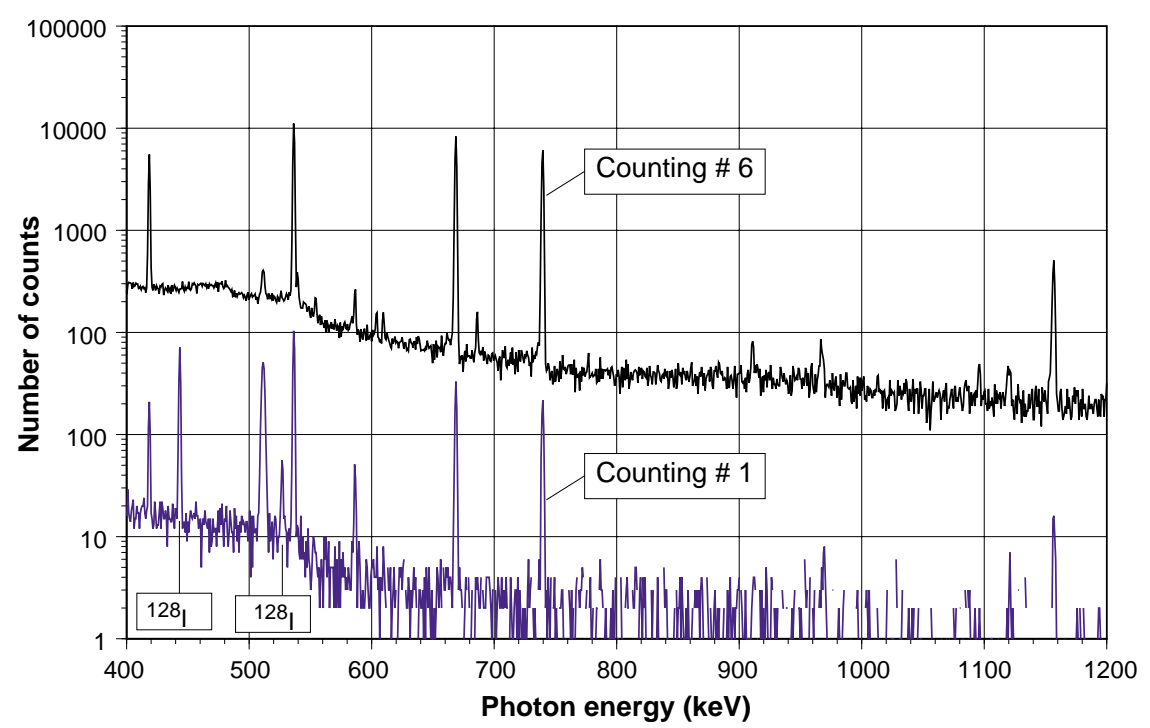

Fig. 100: Comparison of first and sixth countings for activation number 7 in hole 3 at $z=7.5 \mathrm{~cm}$, showing the disappearance of the ${ }^{128} \mathrm{I}$ peaks $\left(443\right.$ and $526 \mathrm{keV}$ ) after nine half-lives of ${ }^{128} \mathrm{I}$ (the rate of the sixth counting is multiplied by 10 to avoid overlap of spectra).

\subsubsection{Calculation of capture rates}

We define the origin of time as the end of the irradiation time (time of the last PS shot). At

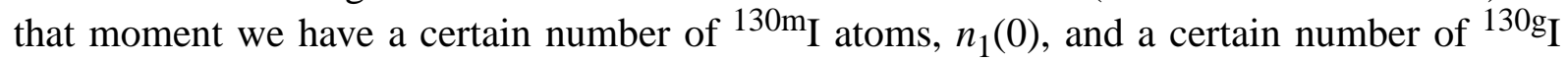
atoms, $n_{2}(0)$, which can be expressed as: 


$$
\begin{aligned}
& n_{1}(0)=A N_{\mathrm{i}} \\
& n_{2}(0)=B N_{\mathrm{g}}+C N_{\mathrm{i}}
\end{aligned}
$$

whith $A, B, C, N_{\mathrm{i}}$ and $N_{\mathrm{g}}$ are the quantities defined in Section 7.2.2.1.

From the above initial conditions we can calculate $n_{1}(t)$ and $n_{2}(t)$, the numbers of ${ }^{130 \mathrm{~m}} \mathrm{I}$ and

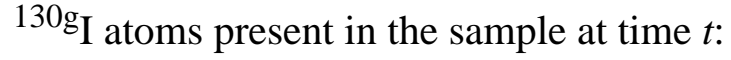

$$
\begin{aligned}
& n_{1}(t)=A N_{\mathrm{i}} e^{-\lambda_{\mathrm{m}} t} \\
& n_{2}(t)=N_{\mathrm{i}}\left[\frac{A P \lambda_{\mathrm{m}}}{\lambda_{\mathrm{g}}-\lambda_{\mathrm{m}}} e^{-\lambda_{\mathrm{m}} t}+\left(C-\frac{A P \lambda_{\mathrm{m}}}{\lambda_{\mathrm{g}}-\lambda_{\mathrm{m}}}\right) e^{-\lambda_{\mathrm{g}} t}\right]+N_{\mathrm{g}} B e^{-\lambda_{\mathrm{g}} t}
\end{aligned}
$$

From these relations we can predict the number of $\gamma$ s due to the decay of species $\mathrm{x}$ with a decay constant $\lambda_{\mathrm{x}}$ observed in the Ge counter, for a given counting period, which started at $t=$ age and lasted $t_{\mathrm{c}}$ seconds:

$$
N_{\gamma} \equiv\left[\int_{\text {age }}^{\text {age }+t_{\mathrm{c}}} \lambda_{\mathrm{x}} n_{\mathrm{x}}(t) \mathrm{d} t\right] \mathrm{Br}_{\gamma} \varepsilon_{\gamma}
$$

where $\mathrm{Br}_{\gamma}$ and $\varepsilon_{\gamma}$ are respectively the branching ratio and the Ge counter acceptance for the $\gamma$ line considered ( $\varepsilon_{\gamma}$ includes the correction for $\gamma$ overlap in the decay chains). Applying the

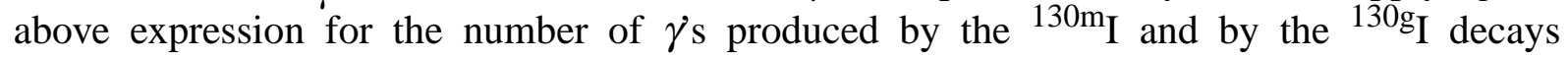
respectively we obtain the following equations:

$$
\begin{aligned}
N_{\gamma_{\mathrm{i}}}= & A N_{\mathrm{i}}\left[e^{-\lambda_{\mathrm{m}} \text { age }}-e^{-\lambda_{\mathrm{m}}\left(\mathrm{age}+t_{\mathrm{c}}\right)}\right] \mathrm{Br}_{\gamma_{\mathrm{i}}} \varepsilon_{\gamma_{\mathrm{i}}} \\
N_{\gamma_{\mathrm{g}}}= & \left\{\frac{\lambda_{\mathrm{g}} N_{\mathrm{i}} P A}{\lambda_{\mathrm{g}}-\lambda_{\mathrm{m}}}\left[e^{-\lambda_{\mathrm{m}} \text { age }}-e^{-\lambda_{\mathrm{m}}\left(\mathrm{age}+t_{\mathrm{c}}\right)}\right]\right. \\
& \left.+\left[N_{\mathrm{i}}\left(C-\frac{\lambda_{\mathrm{m}} P A}{\lambda_{\mathrm{g}}-\lambda_{\mathrm{m}}}\right)+N_{\mathrm{g}} B\right]\left[e^{-\lambda_{\mathrm{g}} \mathrm{age}}-e^{-\lambda_{\mathrm{g}}\left(\mathrm{age}+t_{\mathrm{c}}\right)}\right]\right\} \mathrm{Br}_{\gamma_{\mathrm{g}}} \varepsilon_{\gamma_{g}}
\end{aligned}
$$

For each observed $\gamma$-line, and for each counting period we obtain an equation by equating the number of $\gamma$ s observed in the $\gamma$-peak to the number of $\gamma$ s predicted as a function of $N_{\mathrm{i}}$ and $N_{\mathrm{g}}$, using the two above equations, or only one of them, depending on the case. The two main

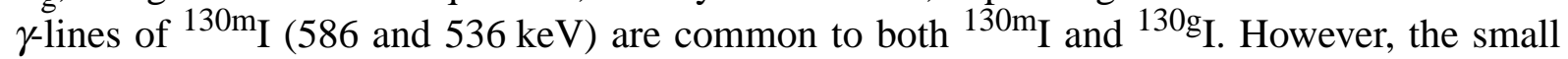
branching ratio for the $586 \mathrm{keV}$ line, $\sim 1.1 \%$, is such that it is mainly the $536 \mathrm{keV}$ line which determines the isomer fraction. In practice, for each Ge counting period, we have a set of seven equations to solve.

For the production of ${ }^{128} \mathrm{I}$, the method is similar, the initial condition at the time of the last PS shot is:

$$
n_{3}(0)=D N_{\mathrm{k}}
$$

The time evolution of the number of ${ }^{128} \mathrm{I}$ atoms is given by:

$$
n_{3}(t)=D N_{\mathrm{k}} e^{-\lambda_{\mathrm{k}} t}
$$

from which we obtain the equation for the number of $\gamma$ s observed during a given Ge counting:

$$
N_{\gamma_{\mathrm{k}}}=D N_{\mathrm{k}}\left[e^{-\lambda_{\mathrm{k}} \mathrm{age}}-e^{-\lambda_{\mathrm{k}}\left(\mathrm{age}+t_{\mathrm{c}}\right)}\right] \mathrm{Br}_{\gamma_{\mathrm{k}}} \varepsilon_{\gamma_{\mathrm{k}}}
$$


In the case of ${ }^{128}$ I there are only two main $\gamma$-lines (442.9 and $\left.526.6 \mathrm{keV}\right)$. The $526 \mathrm{keV}$ line is weak $(\mathrm{Br} \sim 1.2 \%)$, and it is mainly the $442.9 \mathrm{keV}$ line, with a branching ratio of $12.8 \%$, which determines the number of ${ }^{128}$ I atoms.

Some very small corrections were applied to take into account residual activity from the previous irradiation (see Refs. [68] and [8]).

\subsubsection{Experimental results}

\subsubsection{Measurements of the ratio ${ }^{130 m}$ I to ${ }^{130 g}$ I}

It is interesting to note that by measuring the number of ${ }^{130 m_{I}}$ and ${ }^{130 g_{I}}$ atoms separately, we obtain a measurement of the ratio isomer to ground state produced by ${ }^{129} \mathrm{I}$ capture. This ratio is not generally known for all neutron energies. It is given for thermal neutrons as $\sigma\left(\mathrm{n}+{ }^{129} \mathrm{I} \rightarrow{ }^{130 \mathrm{~m}} \mathrm{I}\right)=18.0 \pm 2 \mathrm{~b}$ and $\sigma\left(\mathrm{n}+{ }^{129} \mathrm{I} \rightarrow{ }^{130 \mathrm{~g}} \mathrm{I}\right)=9.0 \pm 1 \mathrm{~b}$ [72], which corresponds to a ratio isomer to ground state of $2.0 \pm 0.315$. This ratio is not expected to vary much with energy below $10 \mathrm{keV}$ [73].

Our simulation shows that in all cases, less than $10 \%$ of all captures occur above a neutron energy of $10^{5} \mathrm{eV}$, therefore, the isomer-to-ground-state ratio should be constant between thermal energies and $10^{5} \mathrm{eV}$.

Indeed, we find that this ratio is constant within errors over the distance range measured [Fig. 101(a)]. If we assume a constant ratio for all the measurements we obtain $1.58 \pm 0.14$, where the error is obtained from the different $\gamma$-lines spread of the measurements $(8.3 \%)$ and from the systematic error from the spread of activities (2 times 4\%). In 1973, P. K. Hopke et al. [74] made a measurement at the MIT reactor where they obtained a ratio of $1.51_{-0.16}^{+0.21}$ which is closer to our result than the BNL value [Fig. 101(b)]. Our measurement of both the ${ }^{130 \mathrm{~m}}$ I and

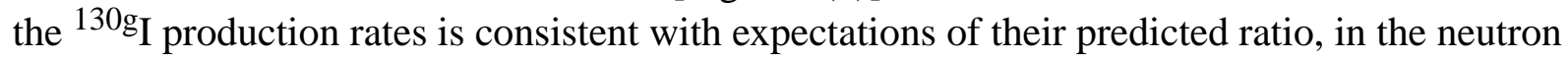
energy range relevant here, and constitutes an important quality test of the data collected in TARC for the transmutation rate of ${ }^{129} \mathrm{I}$.
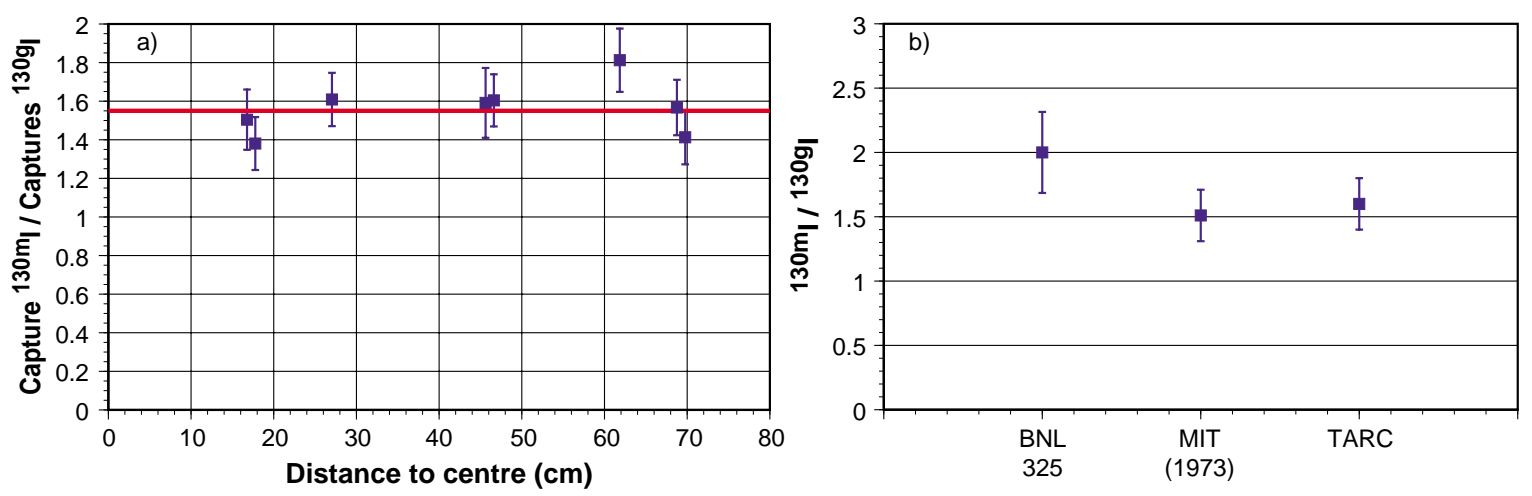

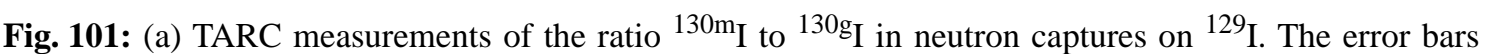

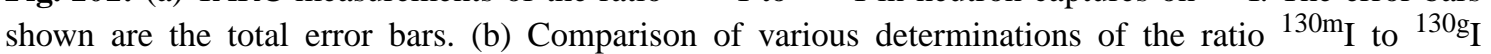
produced by neutron capture on ${ }^{129} \mathrm{I}$.

\subsubsection{Transmutation rates for ${ }^{129} I$ and ${ }^{127} I$}

In total, eight activations were performed at five different positions in the lead volume, spanning distances to the centre of the lead assembly from $16.77 \mathrm{~cm}$ to $68.76 \mathrm{~cm}$. For three of the positions, two activations were performed, at different times, with different beam intensities. For the distance of $68.76 \mathrm{~cm}$, the Ge counting took place at $\mathrm{D}_{1}$ for activation 5 and at $\mathrm{D}_{2}$ for activation 6 . This allows some cross-checks on the reproducibility of the data, which 
is found to be better than $4 \%$ in the case of ${ }^{129}$ I. For each sample activation the number of neutron captures that occurred on ${ }^{129} \mathrm{I}$ and on ${ }^{127} \mathrm{I}$ is given in Table 19 and Table 20, respectively. The results are also shown in Figs. 105 and 107, for ${ }^{129} \mathrm{I}$ and ${ }^{127} \mathrm{I}$, respectively.

Table 19: Measured number of captures on ${ }^{129} \mathrm{I}$, with the corresponding production of ${ }^{130 \mathrm{~m}_{\mathrm{I}}}$ and ${ }^{130 \mathrm{~g}} \mathrm{I}$ atoms, per $10^{9}$ protons incident on the lead assembly. The errors quoted are peak-finding (stat.) errors only.

\begin{tabular}{c|c|c|c|c|c|c}
\hline Activation & $\mathbf{1 3 0 m}_{\mathbf{I}}$ & $\mathbf{1 3 0}_{\mathbf{I} \text { error }}$ & $\mathbf{1 3 0 g}_{\mathbf{I}}$ & $\mathbf{1 3 0 g}_{\mathbf{I}}$ error & $\begin{array}{c}\text { Total } \\
\text { captures }\end{array}$ & $\begin{array}{c}\text { Error on } \\
\text { total }\end{array}$ \\
\hline 1 & 16043 & 596 & 10082 & 518 & 26125 & 790 \\
2 & 19652 & 594 & 13064 & 544 & 32716 & 806 \\
3 & 14414 & 256 & 7953.4 & 226 & 22368 & 341 \\
4 & 16063 & 236 & 10013 & 206 & 26076 & 314 \\
5 & 11682 & 244 & 7454.1 & 217 & 19136 & 326 \\
6 & 10800 & 311 & 7644.2 & 277 & 18444 & 416 \\
7 & 18711 & 556 & 13553 & 490 & 32264 & 741 \\
8 & 18855 & 300 & 11720 & 265 & 30575 & 401 \\
\hline
\end{tabular}

Table 20: Measured number of captures on ${ }^{127} \mathrm{I}$, per $10^{9}$ protons incident on the lead assembly. The errors are peak-finding (stat.) errors only.

\begin{tabular}{c|c|c}
\hline Activation & Total $^{\mathbf{1 2 7}} \mathbf{I}$ capture & Error on total \\
\hline 1 & 14421 & 276 \\
2 & 18923 & 309 \\
3 & 13554 & 138 \\
4 & 15368 & 121 \\
5 & 11660 & 134 \\
6 & 10784 & 182 \\
7 & 18758 & 288 \\
8 & 17922 & 154 \\
\hline
\end{tabular}

\subsubsection{Comparison with the Monte Carlo simulation}

\subsubsection{Neutron capture cross-section}

Both the neutron capture and elastic cross-sections of ${ }^{129} \mathrm{I}$ [Fig. 102(a)] and ${ }^{127} \mathrm{I}$ [Fig. 102(b)] are fairly well known below 2-3 MeV. In the case of TARC we expect to have at least $90 \%$ of neutron captures occurring below a few tens of $\mathrm{keV}$ for ${ }^{129} \mathrm{I}$ and below a few $\mathrm{keV}$ for ${ }^{127} \mathrm{I}$.

The ${ }^{127}$ I neutron capture cross-section is in principle better known than the ${ }^{129} \mathrm{I}$ one, for which the differences between various evaluations result in a difference of up to $12.5 \%$ between JENDL-3.2 [5] and the other data bases while it reaches $30 \%$ for ${ }^{129}$ I. Our data favour slightly the JENDL-3.2/JEF-2.2/BROND evaluations [75] of the ${ }^{129}$ I capture cross-section [Fig. 103(a) \& 103(b)].

\subsubsection{Monte Carlo results}

As expected, the distribution of captures reflects the resonance structure in the capture cross-section [Fig. 104(a) \& 104(b)] the main one being at $40 \mathrm{eV}$ in the case of ${ }^{127} \mathrm{I}$ and $70 \mathrm{eV}$ in the case of ${ }^{129}$ I. The capture energy spectrum is significantly harder than in the case of ${ }^{99} \mathrm{Tc}$, 
where a large fraction of the captures occurs below $25 \mathrm{eV}$. We expect only $20 \%$ of the captures below $50 \mathrm{eV}$ in the case of ${ }^{129} \mathrm{I}$ and $4 \%$ of the captures below $20 \mathrm{eV}$ in the case of ${ }^{127} \mathrm{I}$.
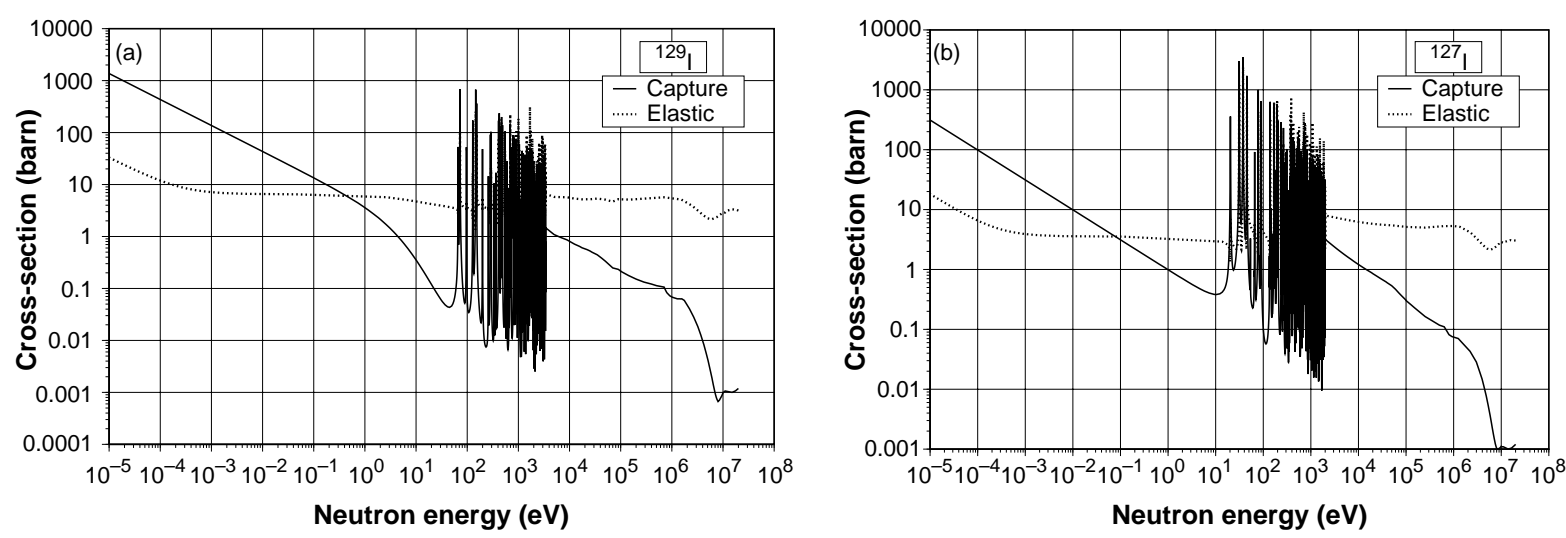

Fig. 102: Neutron elastic and capture cross-sections as a function of neutron kinetic energy (JENDL-3.2 [75]), (a) ${ }^{129}$ I and (b) ${ }^{127}$ I.
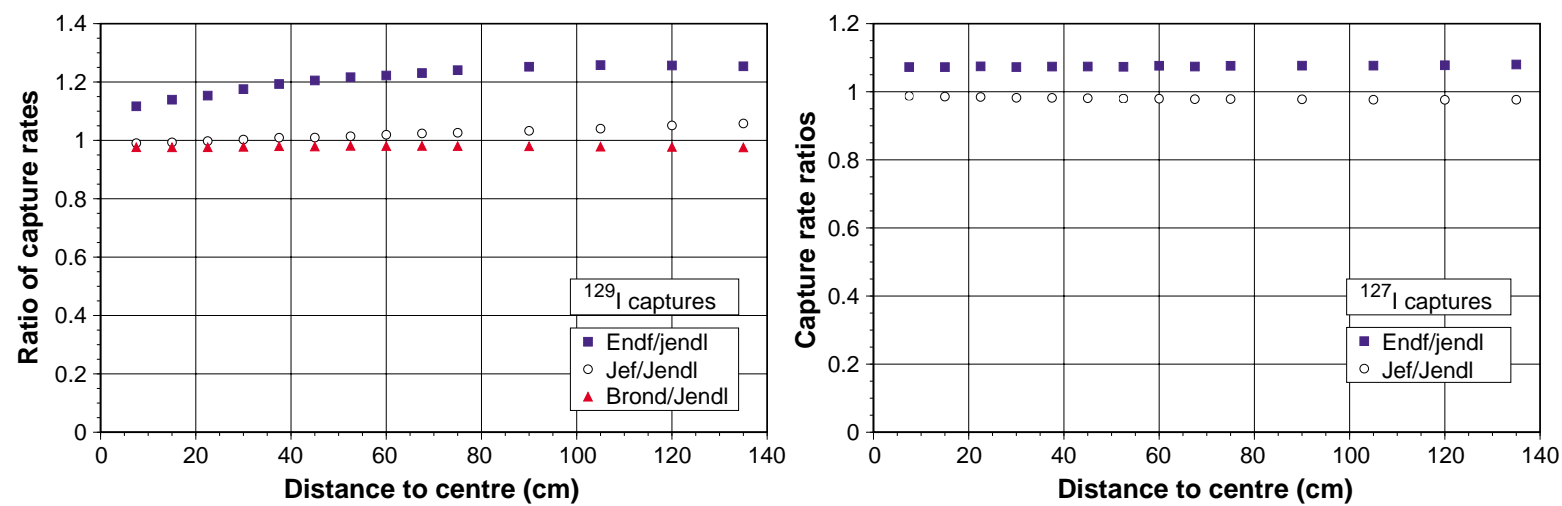

Fig. 103: Ratios of ${ }^{129} \mathrm{I}$ and ${ }^{127} \mathrm{I}$ capture rates as a function of distance to the centre of the lead assembly for three data bases [75] (JEF-2.2, ENDF/B-VI and BROND-2) to JENDL-3.2 used in this analysis.

The detailed Monte Carlo results are shown in Table 21. The statistical errors were obtained from the actual fluctuations in the results obtained from different FLUKA batches processed separately on different Convex processors. A comparison between the Monte Carlo simulation and the data is presented in Figs. 105 to 108.

Table 21: Monte Carlo calculation of the number of captures on the iodine sample. The errors quoted are statistical only.

\begin{tabular}{c|c|c|c|c|c|c|c}
\hline Act. & $\begin{array}{c}\text { Hole } \\
\text { number }\end{array}$ & $\begin{array}{c}\boldsymbol{z} \\
\mathbf{c m})\end{array}$ & $\begin{array}{c}\mathbf{M C} \\
\text { position } \\
\mathbf{c o d e}\end{array}$ & $\begin{array}{c}\mathbf{1 2 9}_{\mathbf{I}} \\
\text { captures }\end{array}$ & $\begin{array}{c}\mathbf{1 2 9}_{\mathbf{I}} \\
\text { Stat. } \\
\text { error }\end{array}$ & $\begin{array}{c}\mathbf{1 2 7} \mathbf{I} \\
\text { captures }\end{array}$ & $\begin{array}{c}\mathbf{1 2 7}_{\mathbf{I}} \\
\text { Stat. } \\
\text { error }\end{array}$ \\
\hline 1 & 10 & 7.5 & 1011 & 23800 & 490 & 13890 & 248 \\
2 & 3 & 7.5 & 311 & 33165 & 743.5 & 18562 & 494 \\
3 & 6 & 7.5 & 611 & 19205 & 316 & 11526 & 160.5 \\
4 & 10 & 7.5 & 1011 & 23800 & 490 & 13890 & 248 \\
5 & 11 & 7.5 & 1111 & 18039 & 407 & 10921 & 404 \\
6 & 11 & 7.5 & 1111 & 18039 & 407 & 10921 & 404 \\
7 & 3 & 7.5 & 311 & 33165 & 743.5 & 18562 & 494 \\
8 & 3 & 22.5 & 312 & 31043 & 1133 & 17169 & 398 \\
\hline
\end{tabular}



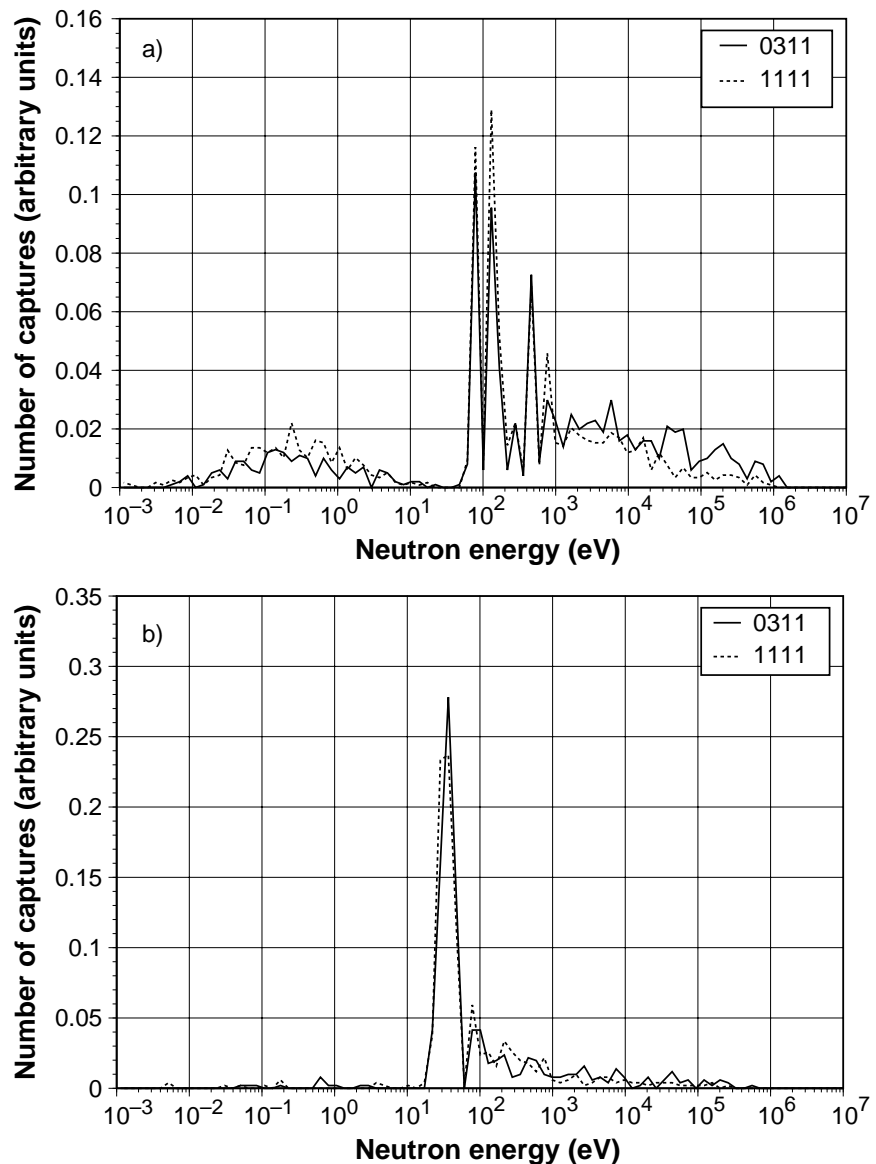

Fig. 104: Neutron energy spectra at capture in (a) ${ }^{129} \mathrm{I}$ and (b) ${ }^{127} \mathrm{I}$ samples. The two curves (0311 and 1111) correspond to extreme measurement positions ( 16.77 and $68.76 \mathrm{~cm}$ from center of lead volume) in the lead volume (Monte Carlo codes defining positions are shown in Table 14) and have been normalized to unit area.

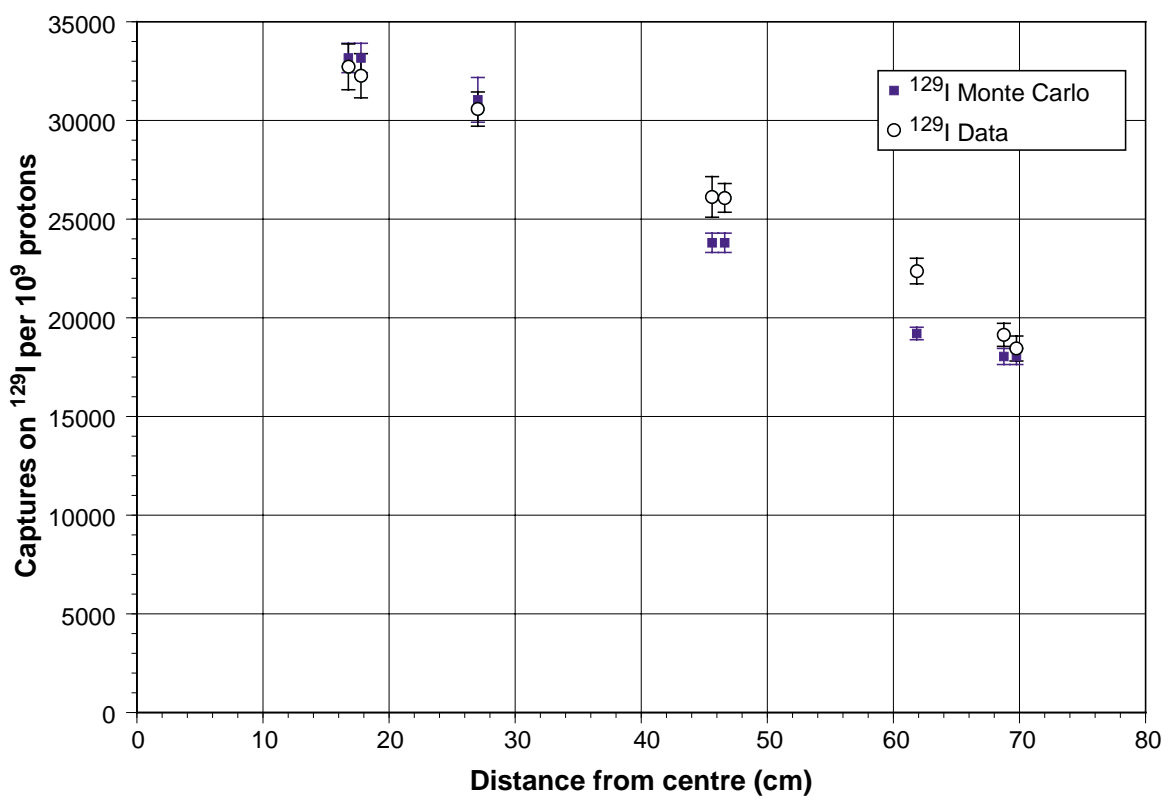

Fig. 105: Comparison of the measured capture rate on ${ }^{129}$ I with the Monte Carlo simulation based on JENDL-3.2. Error bars include point-to-point errors only (statistics and systematic). For the data there is an additional global systematic error of $8.3 \%$. For the Monte Carlo simulation there is an additional global systematic error of $10.8 \%$. 


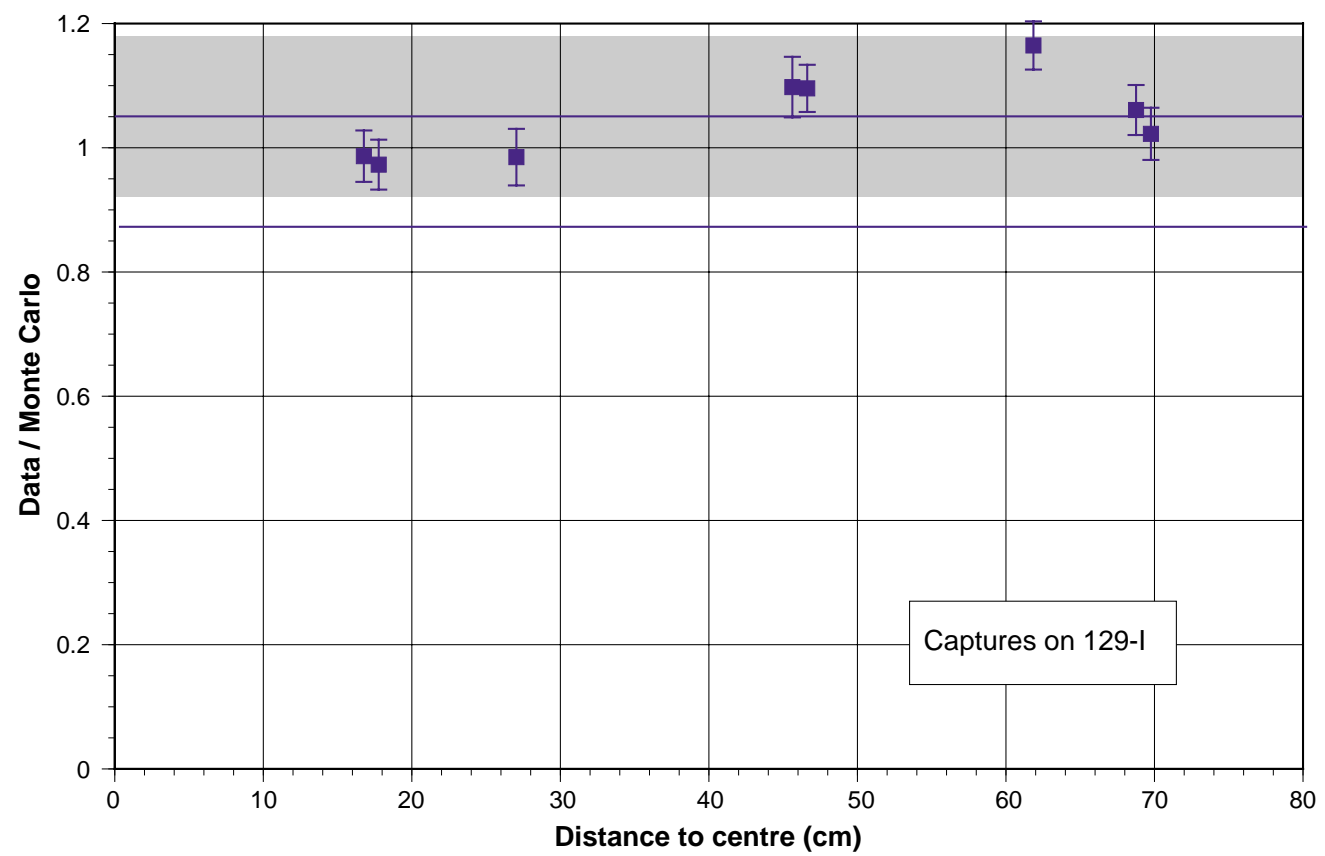

Fig. 106: Ratio of measured to predicted (JENDL-3.2) capture rates on ${ }^{129} \mathrm{I}$ as a function of distance from the centre of the lead assembly. The error bars include point-to-point systematic errors only. The grey band shows the global systematic uncertainty.

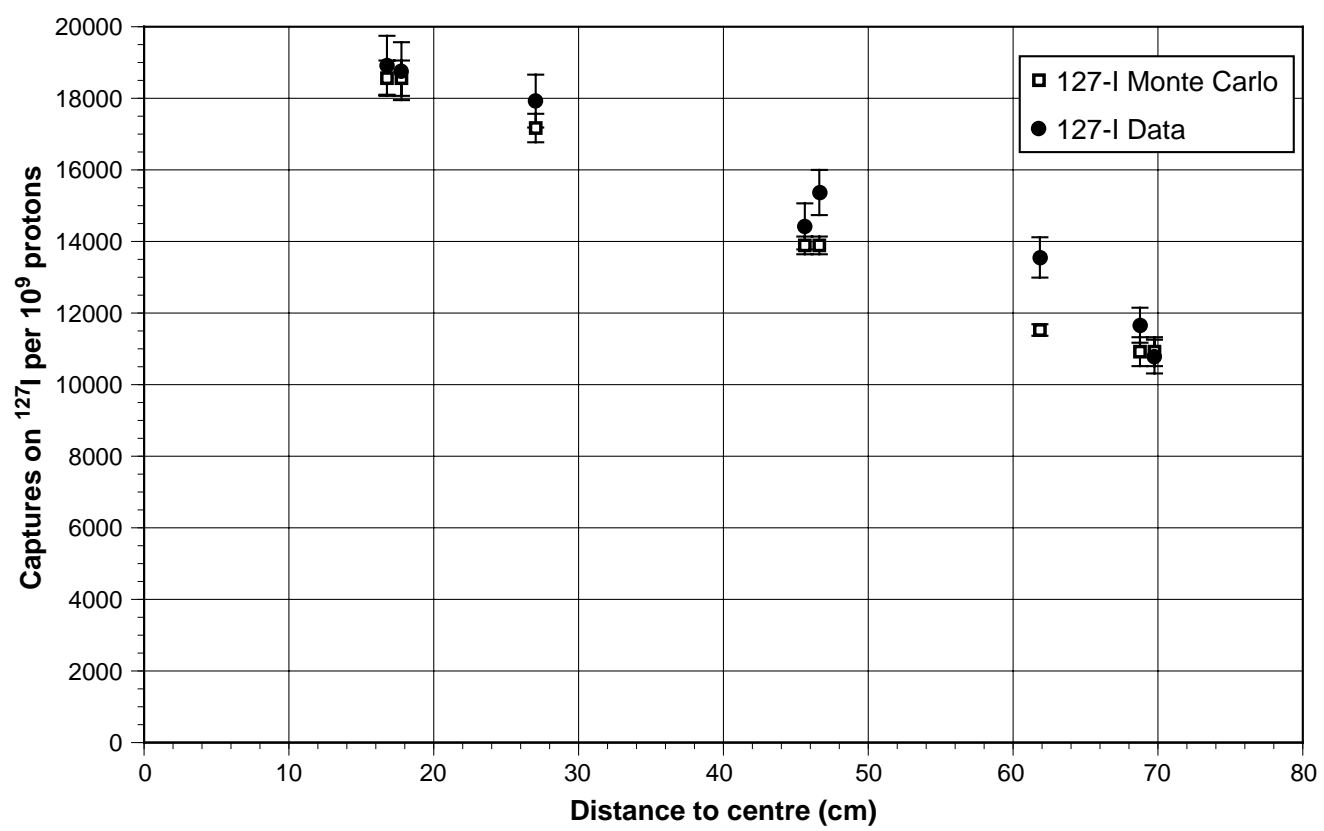

Fig. 107: Comparison of the measured capture rates on ${ }^{127} \mathrm{I}$ with the Monte Carlo simulation based on JENDL-3.2. Error bars include point-to-point errors only (statistics and systematic). For the data there is an additional global systematic error of $8.4 \%$. For the Monte Carlo simulation there is an additional global systematic error of $7.2 \%$.

\subsubsection{Systematic errors}

The neutron capture measurement error is dominated by the systematic errors. The total error in each measurement of the numbers of ${ }^{129} \mathrm{I}$ and ${ }^{127} \mathrm{I}$ captures is $10.5 \%$ and $9.9 \%$ respectively. A more detailed discussion of systematic errors can be found in Ref. [8]. 


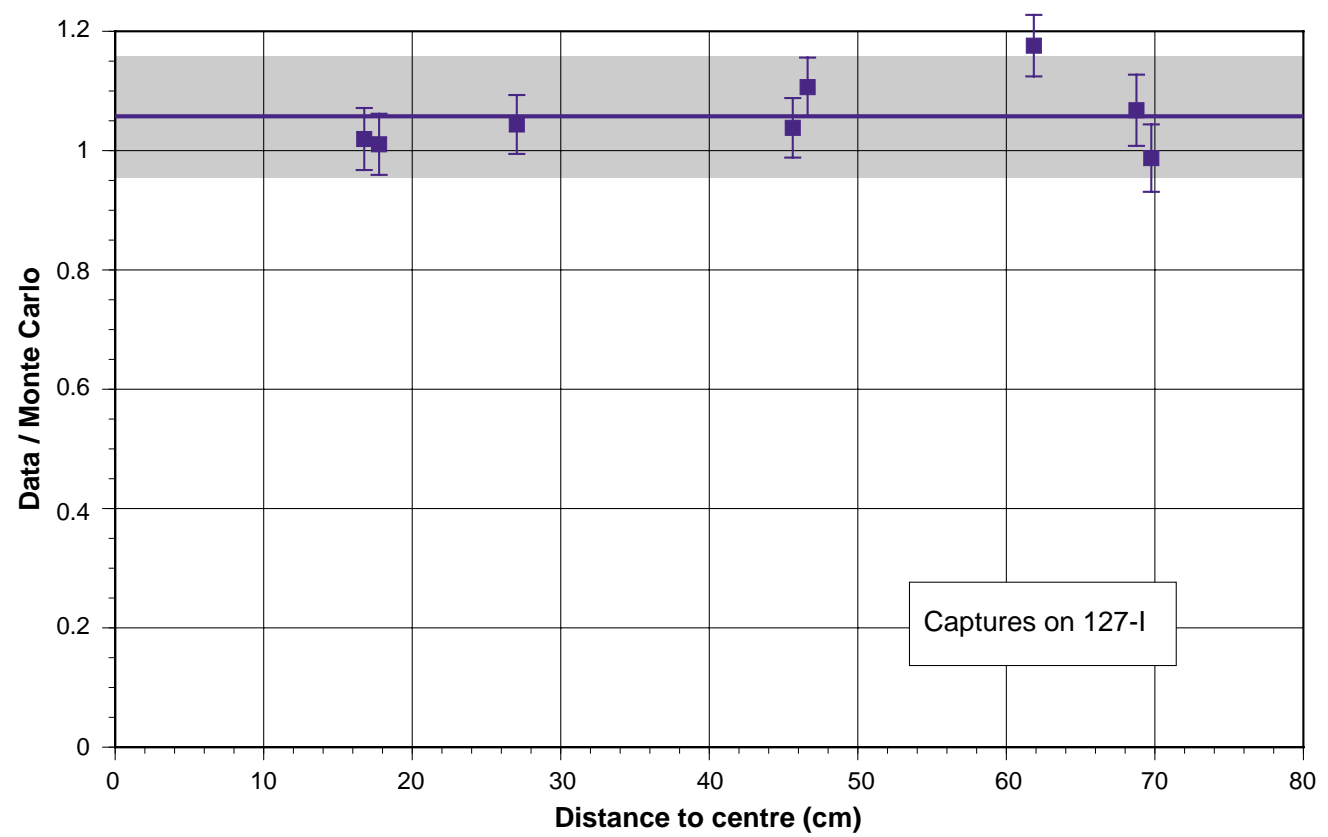

Fig. 108: Ratio of measured to predicted capture rates on ${ }^{127} \mathrm{I}$ as a function of distance from the centre of the lead assembly. The error bars include point-to-point systematic errors only. The grey band shows the global systematic uncertainty.

\subsubsection{Conclusion}

We have measured at five different positions in the TARC lead assembly the neutron capture rates on a sample containing both ${ }^{129} \mathrm{I}$ and ${ }^{127} \mathrm{I}$. We find an excellent agreement between our simulation and the TARC measurements.

This iodine measurement is also an integral part of a validation of the Energy Amplifier simulation, concerning the phenomenology of neutrons in pure lead and the properties of fissions fragments. It is shown here that we are able to simulate the neutron capture history of a very small sample $\left(64.7 \mathrm{mg}\right.$ for ${ }^{129} \mathrm{I}$ and $10.4 \mathrm{mg}$ for $\left.{ }^{127} \mathrm{I}\right)$ placed inside a large assembly of $334 \mathrm{t}$ of pure lead.

We have also obtained a new measurement of the production ratio of ${ }^{130 \mathrm{~m}_{\mathrm{I}}}$ to ${ }^{130 \mathrm{~g}} \mathrm{I}$ in ${ }^{129} \mathrm{I}$ capture, for neutron energies $\leq 10^{5} \mathrm{eV}$.

\subsection{Conclusion on transmutation of long-lived fission fragments}

We have measured at a large number of positions inside the TARC lead volume the integral absolute transmutation rates of ${ }^{99} \mathrm{Tc},{ }^{129}$ I and ${ }^{127}$ I summarized in [Fig. 109(a) \& 109(b)].

With the $\mathrm{CeF}_{3}$ crystal used to calibrate the energy-time relation, we also obtained measurements of the ${ }^{99} \mathrm{Tc}$ differential transmutation rate as a function of neutron energy (Fig. 110).

In all cases, the observed transmutation rates $\left({ }^{99} \mathrm{Tc},{ }^{129} \mathrm{I}\right.$ and $\left.{ }^{127} \mathrm{I}\right)$ agree well with the prediction and follow very closely the decrease of neutron fluence as a function of increasing distances from the centre of the lead volume. This implies that neutron capture at low energies dominates and that the capture rate per unit of fluence varies little within most of the lead volume. 
The excellent agreement between our simulation and the entire TARC data set validates further our simulation. In turn, the correct prediction of integral and differential transmutation rates for ${ }^{99} \mathrm{Tc}$ and ${ }^{129} \mathrm{I}$ validates the efficiency of the TARC method to transmute LLFFs.

In the case of ${ }^{99} \mathrm{Tc}$ we have obtained a complete set of measurements of all the parameters involved in the ARC process: (a) the integral neutron capture rate with the rabbit system; (b) the differential neutron capture rate as a function of neutron energy below $1 \mathrm{keV}$ with the $\mathrm{CeF}_{3}$ detector; (c) the neutron capture cross-section below $1 \mathrm{keV}$. All of this was done in a well-controlled neutron flux which had also been measured precisely using several redundant methods. We can conclude that these transmutation measurements in TARC complete the demonstration of the efficiency of the Adiabatic Resonance Crossing effect to be used for the destruction of the most offending long-lived fission fragments produced in nuclear fission.
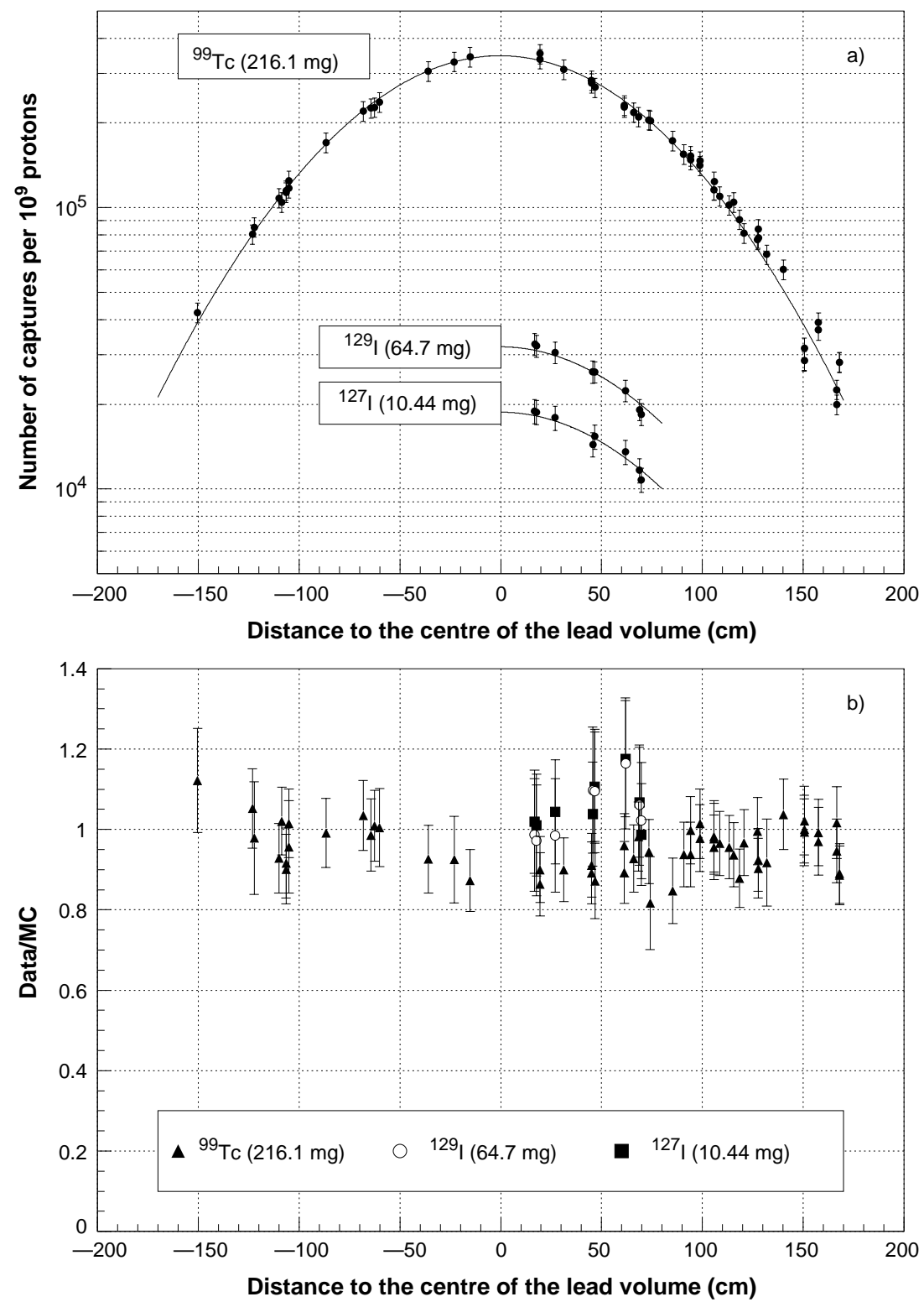

Fig. 109: (a) Measurement of transmutation rates for ${ }^{99} \mathrm{Tc}(216.11 \mathrm{mg}),{ }^{129} \mathrm{I}(64.7 \mathrm{mg})$ and ${ }^{127} \mathrm{I}(10.44 \mathrm{mg})$ samples as a function of distance from the centre of the lead volume. The lines are Gaussian fits to the data; (b) Ratio between data and prediction for the transmutation rates shown in part (a). 


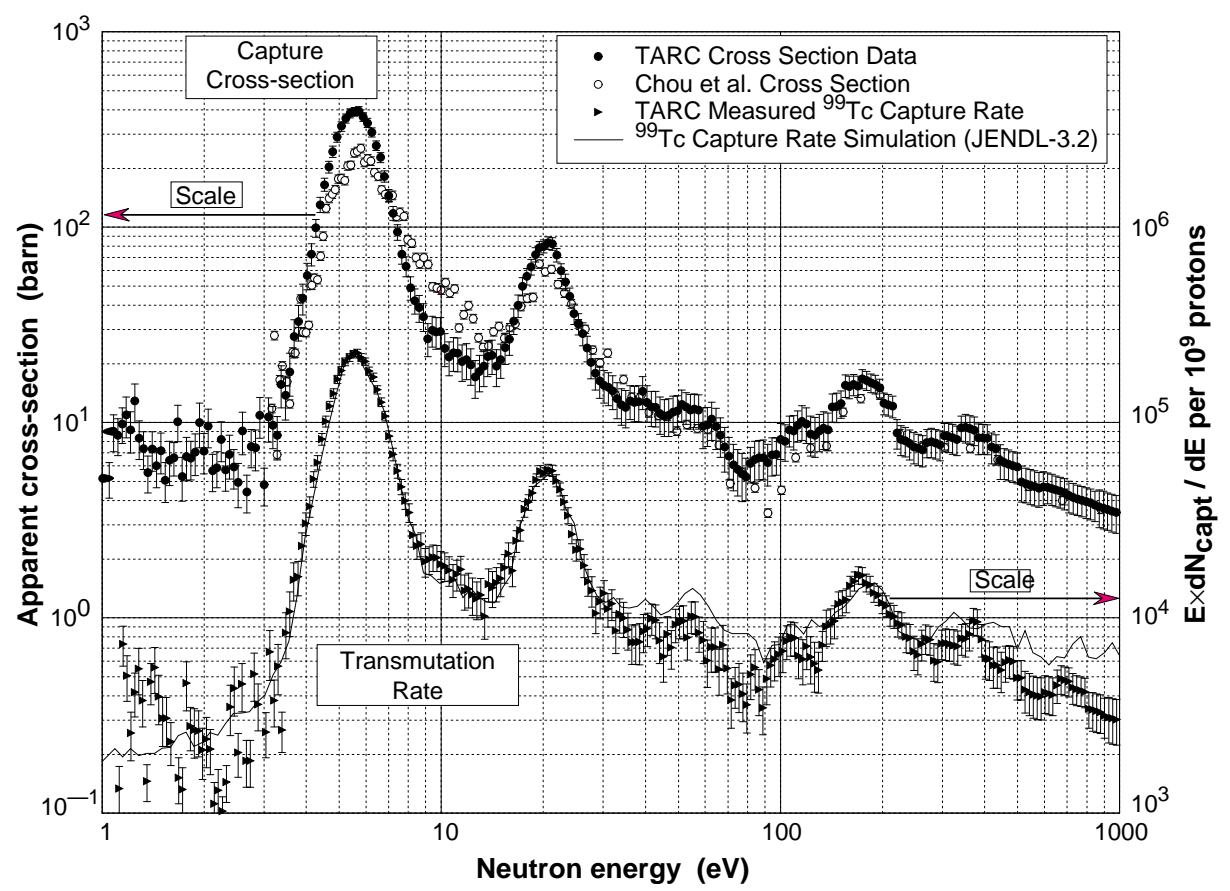

Fig. 110: Measured transmutation rate (hole $10, z=+7.5 \mathrm{~cm}$ ) for a $85 \mathrm{mg}{ }^{99} \mathrm{Tc}$ sample as a function of neutron energy compared to simulation (right-hand scale). Measurement of the ${ }^{99} \mathrm{Tc}$ apparent neutron capture cross-section as a function of neutron energy, and comparison with the previous measurement by Chou et al. [39] extrapolated to zero thickness (left-hand scale).

\section{Other Integral Measurements}

\subsection{Production rates of ${ }^{233} \mathrm{U}$ and ${ }^{239} \mathrm{Pu}$}

We have measured the production rates of ${ }^{233} \mathrm{U}$ from ${ }^{232} \mathrm{Th}$ and of ${ }^{239} \mathrm{Pu}$ from ${ }^{238} \mathrm{U}$ with the use of delayed $\gamma$ spectroscopy. Two sets of metallic foils of ${ }^{232} \mathrm{Th}$ and ${ }^{238} \mathrm{U}$ were irradiated during the same run inside the lead assembly [76].

\subsubsection{Production rate of ${ }^{233} U$}

In a low-intensity neutron flux the transmutation of ${ }^{232} \mathrm{Th}$ to ${ }^{233} \mathrm{U}$ proceeds through the following nuclear reactions:

$$
{ }^{232} \mathrm{Th} \stackrel{(\mathrm{n}, \gamma)}{\longrightarrow}{ }^{233} \mathrm{Th} \stackrel{\beta^{-}(22.3 \mathrm{~min})}{\longrightarrow}{ }^{233} \mathrm{~Pa} \stackrel{\beta^{-}(27 \mathrm{~d})}{\longrightarrow}{ }^{233} \mathrm{U}
$$

In order to measure the production rate of ${ }^{233} \mathrm{U}$, we detected the gammas of ${ }^{233} \mathrm{U}^{*}$ instead of those from the de-excitation of ${ }^{233} \mathrm{~Pa}$, since the half-life of ${ }^{233} \mathrm{~Pa}(27 \mathrm{~d})$ is larger than the half-life of ${ }^{233} \mathrm{Th}$ (22.3 $\mathrm{min}$ ), thus resulting in smaller errors in time measurements, transport of the sample, and gamma counting.

The thorium samples have $99.85 \%$ purity, $88.9 \mu \mathrm{m}$ thickness, $12.7 \mathrm{~mm}$ diameter and an average weight of $132 \mathrm{mg}$ [48]. The foils were activated during run 767 which lasted 8.5 hours for a total of $(2.141 \pm 0.107) \times 10^{13}$ protons [15], [16]. The $311.98 \mathrm{keV}(38.63 \%)$ gamma line is the most direct gamma emitted from the ${ }^{233} \mathrm{U}$ excited states [59] and has a total attenuation coefficient of $0.11 \mathrm{~cm}^{2} / \mathrm{g}$, which results in the absorption of $1.1 \%$ of the $\gamma_{\mathrm{s}}$. The samples were measured with a GeLi counter and analysed with the help of the Inter Technique Gamma Spectrum analysis program. We have used the same calibration of the GeLi detector as that used in triple-foil activation measurements. The activity of the samples was further normalized to $10^{9}$ protons. 
The transmutation rate is calculated as:

$$
P=\frac{R \times t_{1 / 2} \times A}{\ln 2 \times N_{\mathrm{A}} \times m_{\mathrm{f}}}
$$

where $P$ expressed in $\mathrm{g} / \mathrm{g}$ is the quantity of produced element over the quantity of initial sample, $R$ is the normalized activity of the foil in becquerel, $t_{1 / 2}$ is the half-life of the produced element, $A$ its atomic weight, $N_{\mathrm{A}}$ is the Avogadro constant and $m_{\mathrm{f}}$ is the mass of the foil. The ${ }^{232} \mathrm{Th}$ activation data are given in Table 22. The rates of production of ${ }^{233} \mathrm{U}$ are in good agreement with the simulation given an overall systematic error of about $10 \%$ each on data and on Monte Carlo calculations (Fig. 111).

Table 22: ${ }^{232} \mathrm{Th}$ foil positions, activities $(R)$ and transmutation rates $(P)$. The errors are statistical only

\begin{tabular}{c|c|c|c}
\hline Hole & $\begin{array}{c}\boldsymbol{z} \\
(\mathbf{c m})\end{array}$ & $\begin{array}{c}\boldsymbol{R} \\
(\mathbf{B q})\end{array}$ & $\begin{array}{c}\boldsymbol{P} \\
(\mathbf{g} / \mathbf{g}) / \mathbf{1 0} \mathbf{9} \mathbf{p}\end{array}$ \\
\hline 1 & 22.5 & $97 \pm 6$ & $(3.7 \pm 0.2) \times 10^{-17}$ \\
2 & 37.5 & $203 \pm 10$ & $(7.7 \pm 0.4) \times 10^{-17}$ \\
3 & 22.5 & $339 \pm 15$ & $(1.3 \pm 0.6) \times 10^{-16}$ \\
3 & 52.5 & $277 \pm 12$ & $(1.0 \pm 0.5) \times 10^{-16}$ \\
6 & 22.5 & $238 \pm 11$ & $(9.0 \pm 0.4) \times 10^{-17}$ \\
7 & 22.5 & $166 \pm 8$ & $(6.3 \pm 0.3) \times 10^{-17}$ \\
8 & 22.5 & $100 \pm 8$ & $(3.8 \pm 0.3) \times 10^{-17}$ \\
9 & 7.5 & $29 \pm 4$ & $(1.0 \pm 0.2) \times 10^{-17}$ \\
\hline
\end{tabular}

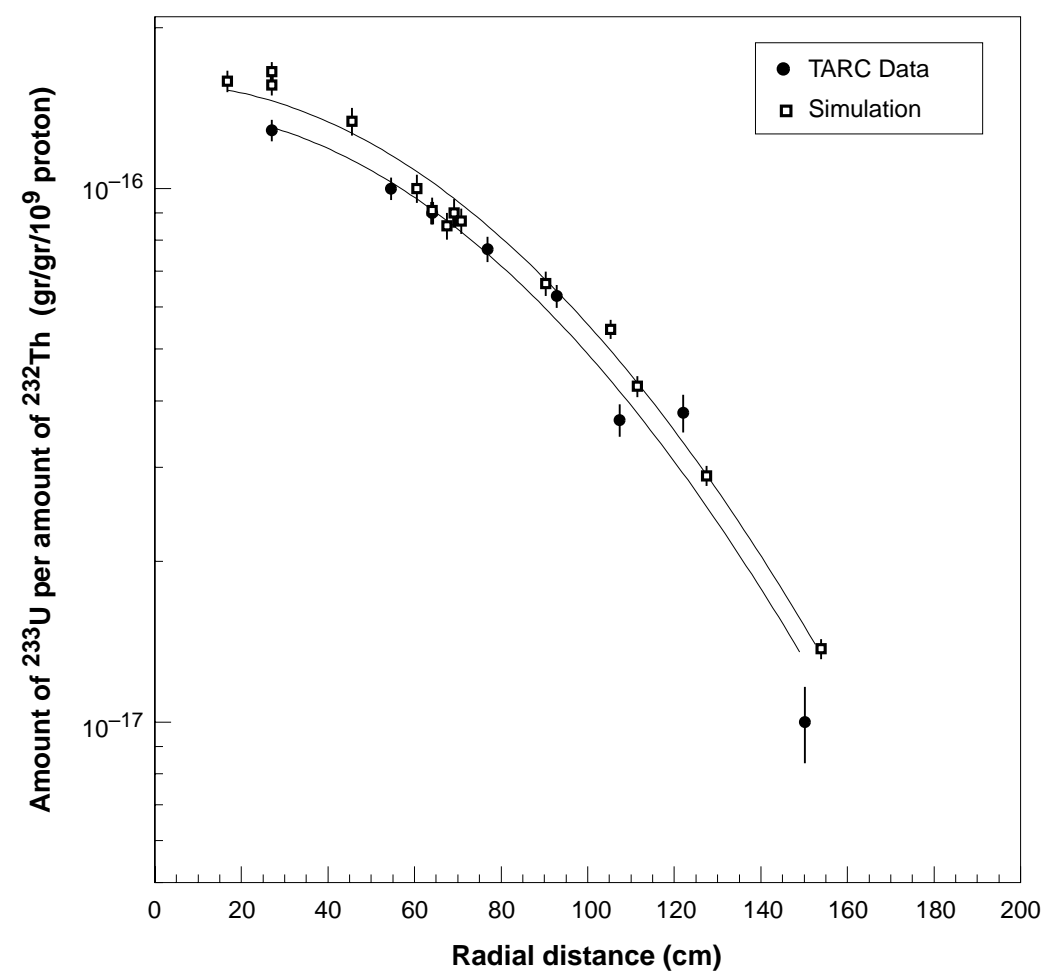

Fig. 111: Production rates for ${ }^{233} \mathrm{U}$ : experimental data and rates calculated from Monte Carlo simulation versus the distance from the centre of the lead volume. The lines are guides to the eye made using Gaussian fits. 


\subsubsection{Production of ${ }^{239} \mathrm{Pu}$}

In a low-intensity neutron flux, the transmutation of ${ }^{238} \mathrm{U}$ into ${ }^{239} \mathrm{Pu}$ proceeds through the following nuclear reactions:

$$
{ }^{238} \mathrm{U} \stackrel{(\mathrm{n}, \gamma)}{\longrightarrow}{ }^{239} \mathrm{U} \stackrel{\beta^{-}(23.45 \mathrm{~min})}{\longrightarrow}{ }^{239} \mathrm{~Np} \stackrel{\beta^{-}(2.36 \mathrm{~d})}{\longrightarrow}{ }^{239} \mathrm{Pu}
$$

Since the half-life of ${ }^{239} \mathrm{~Np}(2.36 \mathrm{~d})$ is larger than the half-life of ${ }^{239} \mathrm{U}(23.5 \mathrm{~min}),{ }^{239} \mathrm{Pu}^{*}$ gammas allow a smaller time measurement error. We used the $277.6 \mathrm{keV}(6.8 \%)$ and $228.18 \mathrm{keV}(11.46 \%)$ gamma lines of ${ }^{239} \mathrm{~Np}^{*}$ which are the most direct gammas emitted during the decay of ${ }^{239} \mathrm{~Np}$ [59].

The samples are depleted uranium foils with $400 \mathrm{ppm}$ of ${ }^{235} \mathrm{U}$ with a purity of $99.23 \%$, a thickness of $127 \mu \mathrm{m}$, and a diameter of $12.7 \mathrm{~mm}$, corresponding to an average weight of $290 \mathrm{mg}$ [48]. The foils were activated in the same run as the ${ }^{232} \mathrm{Th}$ samples (run 767).

Figure 112 shows that the measured transmutation rate of ${ }^{238} \mathrm{U}$ is in good agreement with our Monte Carlo simulation given a systematic error of about $10 \%$ each on data and on Monte Carlo calculation. The ${ }^{238} \mathrm{U}$ activation data are given in Table 23.

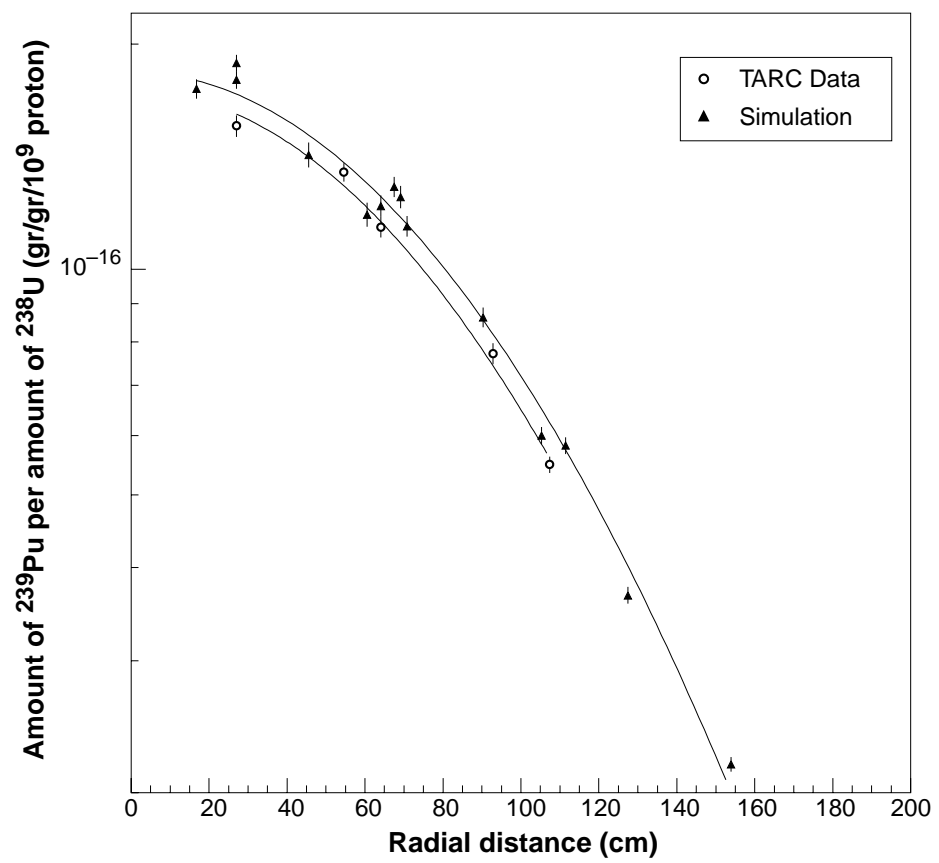

Fig. 112: Production rates for ${ }^{239} \mathrm{Pu}$ : experimental data and rates calculated from Monte Carlo simulation versus the distance from the centre of the lead volume. The lines are guides to the eye made using Gaussian fits.

Table 23: ${ }^{238} \mathrm{U}$ foil positions, activities $(R)$ and transmutation rates $(P)$. The errors are statistical only

\begin{tabular}{c|c|c|c}
\hline Hole & $\begin{array}{c}\boldsymbol{z} \\
(\mathbf{c m})\end{array}$ & $\begin{array}{c}\boldsymbol{R} \\
(\mathbf{B q})\end{array}$ & $\begin{array}{c}\boldsymbol{P} \\
(\mathbf{g} / \mathbf{g}) / \mathbf{1 0} \mathbf{9} \mathbf{p}\end{array}$ \\
\hline 1 & -22.5 & $2762 \pm 66$ & $(5.5 \pm 0.1) \times 10^{-17}$ \\
3 & -22.5 & $7825 \pm 248$ & $(1.6 \pm 0.5) \times 10^{-16}$ \\
3 & -52.5 & $6787 \pm 187$ & $(1.3 \pm 0.4) \times 10^{-16}$ \\
6 & -22.5 & $5734 \pm 173$ & $(1.1 \pm 0.3) \times 10^{-17}$ \\
7 & -22.5 & $3887 \pm 119$ & $(7.7 \pm 0.2) \times 10^{-17}$ \\
\hline
\end{tabular}




\subsubsection{Systematic errors}

We estimate that the uncertainty in the foil position inside the lead hole is less than $1 \mathrm{~cm}$, which results in less than $1 \%$ error in the estimation of the transmutation rate.

The uncertainty on the measured activity $R$ is 7\% [5\% from absolute activity of calibration sources, $3 \%$ for the efficiency at the $\gamma$ energy, $3 \%$ for the estimation of the $\gamma$ peak area, $2 \%$ for the positioning precision $( \pm 1 \mathrm{~mm}$ at $100 \mathrm{~mm})$ and $0.3 \%$ to $0.9 \%$ for $\gamma$ branching ratios, the time measurement error ( $2 \mathrm{~min}$ ) has negligible effect]. The uncertainty on the beam intensity is $5 \%$.

The errors on the physical properties of the sample are negligible. Thus the total systematic error on the transmutation rates is estimated to be $9 \%$ both for ${ }^{233} \mathrm{U}$ and ${ }^{239} \mathrm{Pu}$.

The systematic error on the Monte Carlo prediction is dominated by the uncertainty on the $(\mathrm{n}, \gamma)$ cross-sections which is hard to estimate. We think that in the case of ${ }^{232} \mathrm{Th}(\mathrm{n}, \gamma)$ it is at least $10 \%$ and, according to the BNL tables [27], it is at least $7 \%$ for ${ }^{238} \mathrm{U}(\mathrm{n}, \gamma)$.

Both for ${ }^{233} \mathrm{U}$ and ${ }^{239} \mathrm{Pu}$ production rates measured with a precision of $9 \%$, the results are consistent with the Monte Carlo simulation obtained with an uncertainty of order $10 \%$.

\subsubsection{Protactinium $\left({ }^{233} \mathrm{~Pa}\right)$ half-life}

We checked that the $312 \mathrm{keV} \gamma$ line used in the previous analysis was due entirely to ${ }^{233} \mathrm{~Pa}$ by checking its half-life. The activation of $0.25 \mathrm{~g}$ of ${ }^{232} \mathrm{Th}$ in hole 5 at $z=+7.5 \mathrm{~cm}$ was used for our measurement (runs 1303 to 1325). The detailed experimental conditions are described in Ref. [77]. The evaluation of the number of counts in the $312 \mathrm{keV}$ peak was made with the same methods used in the ${ }^{99} \mathrm{Tc}$ and ${ }^{129}$ I studies [77].

The number of counts in the $\gamma$ peak can be written as:

$$
N_{\gamma} \cong\left(\alpha_{1}+\alpha_{2}\right) e^{-\lambda_{2} \text { age }}\left(1-e^{-\lambda_{2} t_{\mathrm{c}}}\right) N_{\mathrm{c}}^{\mathrm{Th}} \mathrm{Br}_{\gamma} \varepsilon_{\gamma}
$$

where $N_{\mathrm{c}}^{\mathrm{Th}}$ is the number of captures on ${ }^{232} \mathrm{Th}$ per proton, $\lambda_{1}=5.18047 \times 10^{-4} \mathrm{~s}^{-1}\left({ }^{233} \mathrm{Th}\right)$; $\lambda_{2}=2.97131 \times 10^{-7} \mathrm{~s}^{-1}\left({ }^{233} \mathrm{~Pa}\right) ; t_{\mathrm{c}}$ is the counting time and age is the time between the last proton shot and the beginning of a given Ge detector counting period, $\varepsilon_{\gamma}$ and $\mathrm{Br}_{\gamma}(36.8 \%)$ are respectively the efficiency and the branching ratio of the $312 \mathrm{keV} \gamma$-line, $\alpha_{1}$ and $\alpha_{2}$ are constants depending on the beam intensity.

The ratio between two Ge measurements will eliminate the common constants, Eq. (58), $\left(N_{\mathrm{c}}^{\mathrm{Th}}\right.$ is here the same for all countings):

$$
\frac{N_{\gamma_{\mathrm{i}}}}{N_{\gamma_{\mathrm{j}}}}=e^{-\lambda_{2}\left(\mathrm{age}_{\mathrm{i}}-\mathrm{age}_{\mathrm{j}}\right)} \times \frac{1-e^{-\lambda_{2} t_{\mathrm{ci}}}}{1-e^{-\lambda_{2} t_{\mathrm{cj}}}}
$$

This relation is only dependent on measurements from the Ge detector and not on the irradiation. From each equation (58) a value of $\lambda_{2}$ was extracted.

The spread in the values of $\lambda_{2}$ gives us a check of the consistency of our data. Figure 113 shows the results for all calculated $\lambda_{2}$ values in the two different positions $\mathrm{D}_{1}$ and $\mathrm{D}_{2}, \lambda_{2}$ of $0.31 \times 10^{-6} \pm 0.91 \times 10^{-8} \mathrm{~s}^{-1}$ for position $\mathrm{D}_{1}$, and $0.29 \times 10^{-6} \pm 0.51 \times 10^{-8} \mathrm{~s}^{-1}$ for position $\mathrm{D}_{2}$. The average value for $\lambda_{2}$ is introduced in the following expression as a small correction to the number of $\gamma$ counts:

$$
\frac{N_{\gamma}}{1-e^{-\lambda_{2} t_{\mathrm{c}}}}=A e^{-\lambda \text { age }}
$$


with

$$
A \approx\left(\alpha_{1}+\alpha_{2}\right) N_{\mathrm{c}}^{\mathrm{Th}} \mathrm{Br}_{\gamma} \varepsilon_{\gamma}
$$

Exponential fits to the data using Eq. (59) allow us to obtain our best estimate of the of ${ }^{233} \mathrm{~Pa}$ half-life: $27.46 \pm 1.8$ days which is consistent with the accepted world value of $26.96 \pm 0.002$ days.

No attempt was made to perform a high-precision determination of ${ }^{233} \mathrm{~Pa}$ half-life, however, this study is part of a check of the quality of the TARC data.
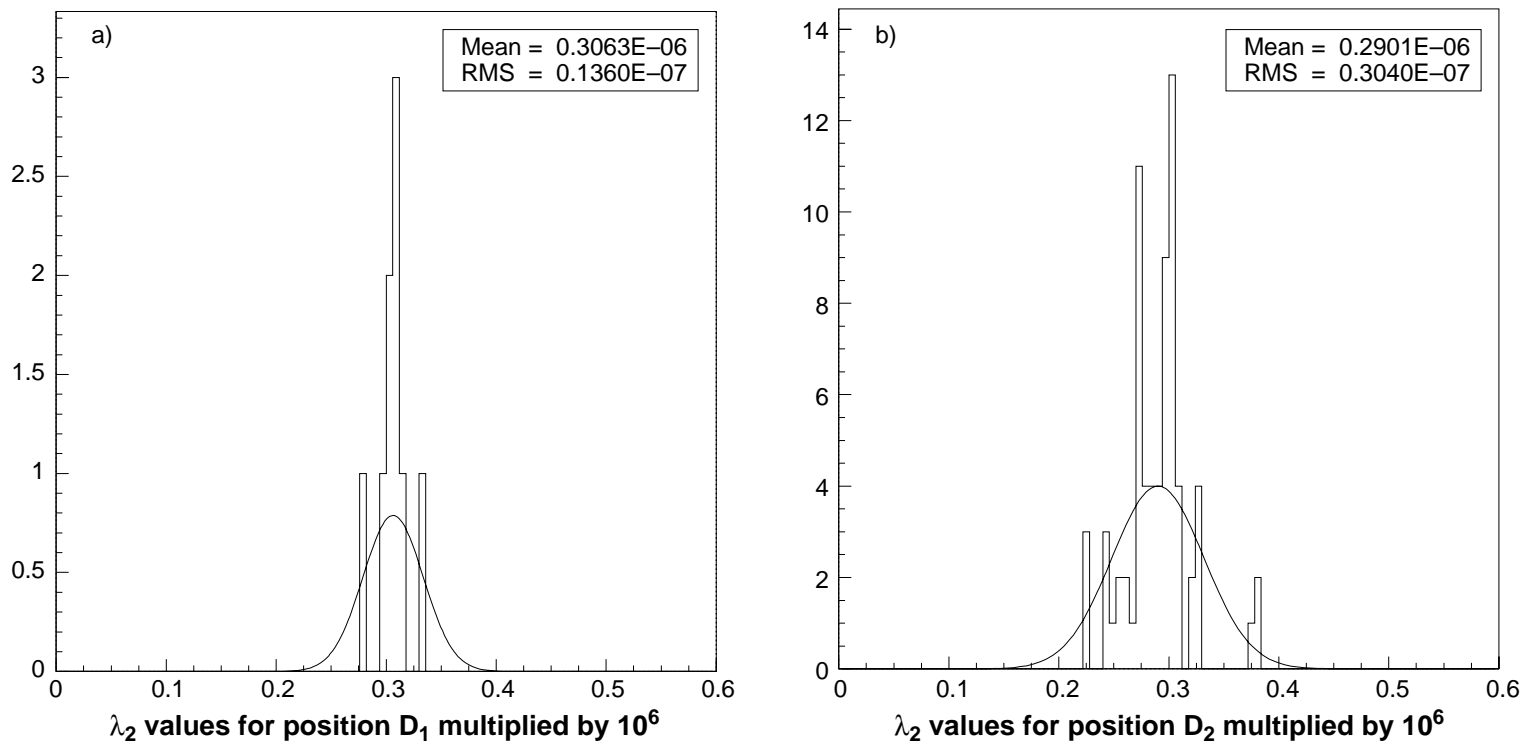

Fig. 113: Distribution of $\lambda$ values for the two different positions $D_{1}$ and $D_{2}$ and for the three methods of peak evolution.

\subsection{The ${ }^{232} \mathrm{Th}(\mathrm{n}, 2 \mathrm{n}){ }^{231} \mathrm{Th}$ reaction rate}

\subsubsection{Motivation}

In the Energy Amplifier project, the contribution to the neutron multiplication from the non-fission reactions is not negligible in particular for the proposed fuel which is ${ }^{232} \mathrm{Th}$. Furthermore, the reactions ${ }^{232} \mathrm{Th}(\mathrm{n}, 2 \mathrm{n}){ }^{231} \mathrm{Th} \rightarrow \beta^{-}+{ }^{231} \mathrm{~Pa}$ and ${ }^{231} \mathrm{~Pa}(\mathrm{n}, \gamma){ }^{232} \mathrm{~Pa} \rightarrow \beta^{-}+{ }^{232} \mathrm{U}$ leading to the production of ${ }^{232} \mathrm{U}$ which is responsible for a large part of the short-term (few centuries) radiotoxicity, while ${ }^{231} \mathrm{~Pa}$ is responsible for the long term radiotoxicity, are of great relevance. We have exploited the opportunity offered by the TARC experiment to measure the $(\mathrm{n}, 2 \mathrm{n})$ reaction rate of ${ }^{232} \mathrm{Th}\left(\int \sigma_{\mathrm{n}, 2 \mathrm{n}}\left(E_{\mathrm{n}}\right) \Phi_{\mathrm{n}}\left(E_{\mathrm{n}}\right) \mathrm{d} E_{\mathrm{n}}, E_{\mathrm{n}} \geq 6 \mathrm{MeV}\right)$ in a lead slowing down spectrometer environment.

The ${ }^{232} \mathrm{Th}(\mathrm{n}, 2 \mathrm{n})$ reaction has been studied in different laboratories [78], but as far as we know, has not been measured with spallation neutrons in lead. The measurement is based on the observation of the gamma rays emitted by ${ }^{231} \mathrm{Th}$ formed by the $\mathrm{n}, 2 \mathrm{n}$ reaction on ${ }^{232} \mathrm{Th}$. As is well known, natural ${ }^{232} \mathrm{Th}$ is an alpha emitter with many gamma rays emitted during this de-excitation. The gamma rays from ${ }^{231} \mathrm{Th}$ are superimposed on the natural gamma-ray background of ${ }^{232} \mathrm{Th}$. The intensity of the gamma rays from ${ }^{231} \mathrm{Th}$ is extracted from the subtraction of this background. This introduces a sizeable uncertainty for the determination of the $n, 2 n$ reaction rate. 
A special experimental set-up was arranged to observe the $25.6 \mathrm{keV}$ gamma-ray transition of ${ }^{231} \mathrm{Th}$. This line has the advantage of a relatively high intensity $(14.5 \%)$, and lies in the gamma-ray region where the contamination is unlikely. The details of this analysis are given in Refs. [8] and [79]. Here we summarized the main elements of the measurement.

The $\mathrm{n}, 2 \mathrm{n}$ energy threshold for ${ }^{232} \mathrm{Th}$ is $6 \mathrm{MeV}$ and the evaluated cross-section is of the order of $3 \mathrm{barn}$ in the neutron energy range between $6 \mathrm{MeV}$ and $14 \mathrm{MeV}$. The two samples used (Table 24) have been placed in hole 3 at $z=+7.5 \mathrm{~cm}$ and $z=0.0 \mathrm{~cm}$ near the centre of the lead volume in order to maximize the component of the spallation neutrons having an energy above $6 \mathrm{MeV}$.

Table 24: Characteristics of the ${ }^{232} \mathrm{Th}$ samples, irradiation positions in the lead volume, and characteristics of the proton beam

\begin{tabular}{c|c|c|c|c|c}
\hline $\begin{array}{c}\text { 232 } \mathbf{T h} \\
\text { sample }\end{array}$ & $\begin{array}{c}\text { Weight } \\
(\mathbf{g})\end{array}$ & Dimension & $\begin{array}{c}\text { Thickness } \\
(\mu \mathbf{m})\end{array}$ & $\begin{array}{c}\text { Proton kinetic energy } \\
(\mathbf{G e V}) \\
\text { Irradiation position }\end{array}$ & $\begin{array}{c}\text { Number of } \\
\text { protons }\left(\mathbf{1 0}^{12}\right)\end{array}$ \\
\hline Sample 1 & 0.158 & $\phi=1.25 \mathrm{~cm}$ & 110.3 & $\begin{array}{c}2.75 \\
(\text { Hole } 3, z=0.0 \mathrm{~cm})\end{array}$ & 4.80 \\
\hline Sample 2 & 0.678 & $3 \mathrm{~cm} \times 2.9 \mathrm{~cm}$ & 66.5 & $\begin{array}{c}2.75 \\
(\text { Hole } 3, z=+7.5 \mathrm{~cm})\end{array}$ & 9.01 \\
\hline
\end{tabular}

\subsubsection{Photon spectra}

The $25.6 \mathrm{keV}$ line can be seen without any ambiguity (Fig. 114), while Fig. 115 shows other peaks of ${ }^{231} \mathrm{Th}$ such as $84.2 \mathrm{keV}$ which are common to ${ }^{232} \mathrm{Th}$ hence harder to separate. Note also the presence of lines at 94.6 and $98.6 \mathrm{keV}$ characteristic of uranium X-rays and probably originating from other high-energy neutron reactions on ${ }^{232} \mathrm{Th}$.

We have checked that the half-life of the $25.6 \mathrm{keV}$ line was consistent with the $25.52 \mathrm{~h}$ expected for ${ }^{231} \mathrm{Th}[80]$.

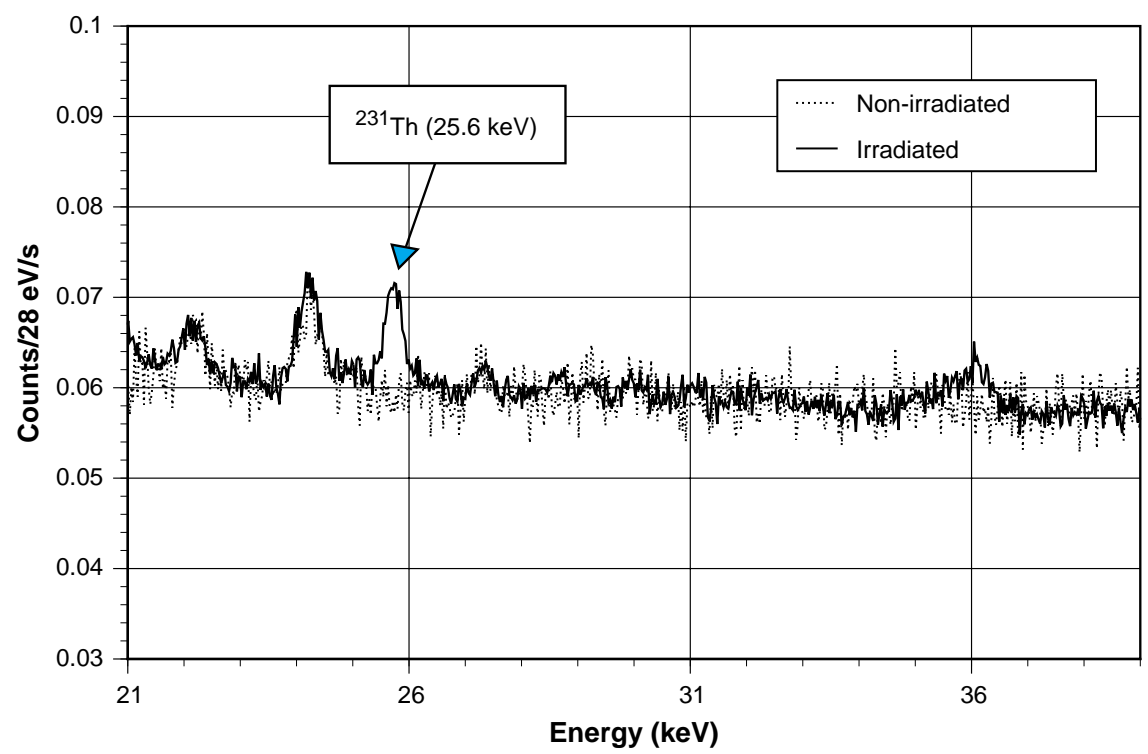

Fig. 114: Observed photon energy distribution from the irradiated and non-irradiated ${ }^{232} \mathrm{Th}$ sample in the energy range $21-39 \mathrm{keV}$. 


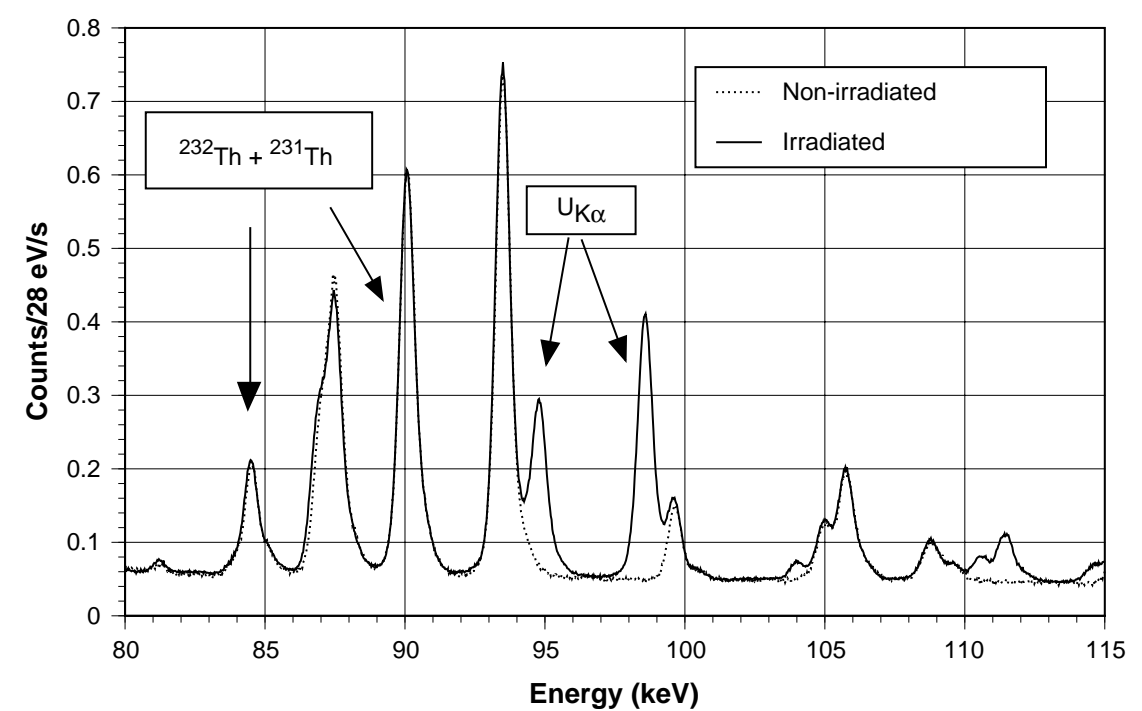

Fig. 115: Observed photon energy distribution from the irradiated and non-irradiated ${ }^{232}$ Th sample in the energy range $80-115 \mathrm{keV}$.

Various sources of background contributing to the $\gamma$ counting obtained with a high-resolution X-ray detector have been studied. Background from the ${ }^{232} \mathrm{Th}$ sample was subtracted from measurements performed before irradiation. The background from ${ }^{233} \mathrm{Th}$ was made negligible by waiting about 10 half-lives $(\sim 4 \mathrm{~h})$ before counting the ${ }^{231} \mathrm{Th} \gamma \mathrm{s}$. The background from bremsstrahlung of $\beta$ 's from ${ }^{231} \mathrm{Th}$ and ${ }^{233} \mathrm{~Pa}$ was made negligible by using a beryllium filter $250 \mu \mathrm{m}$ thick.

Other potential backgrounds such as fissions of ${ }^{232} \mathrm{Th}$ and ${ }^{232} \mathrm{U}, \gamma \mathrm{s}$ from ${ }^{233} \mathrm{~Pa}$ and from ${ }^{233} \mathrm{U}$ were found not to contribute substantially to the $25.6 \mathrm{keV}$ line of interest. Our results for the two irradiations performed are given in Table 25.

For comparison, the results obtained from the $84.2 \mathrm{keV}$ gamma line are also reported in Fig. 116, Fig. 117, and in Table 25. The observed difference between the data (Table 25) is qualitatively consistent with what is expected from the effect of the neutron flux at $z=0.0$ (close to the centre of the spallation source) being greater than the one at $z=+7.5 \mathrm{~cm}$. However, as mentioned above, the $84.2 \mathrm{keV}$ line is not pure and the method used gives a significant uncertainty in the determination of the counts resulting from the de-excitation of the ${ }^{231} \mathrm{Th}$.

Table 25: Measured reaction rates of ${ }^{232} \mathrm{Th}(\mathrm{n}, 2 \mathrm{n}){ }^{231} \mathrm{Th}$ per incident proton and per gram of ${ }^{232} \mathrm{Th}$ using the 25.6 and $84.2 \mathrm{keV}$ lines, for each of the samples indicated (hole 3 )

\begin{tabular}{c|c|c|c}
\hline${ }^{232}$ Th sample & $\boldsymbol{z}$ position & $\mathbf{2 5 . 6} \mathrm{keV}$ line & $\mathbf{8 4 . 2} \mathbf{k e V}$ line \\
\hline $0.678 \mathrm{~g}$ & $7.5 \mathrm{~cm}$ & $(1.32 \pm 0.13) \times 10^{-6}$ & $(2.11 \pm 0.32) \times 10^{-6}$ \\
$0.158 \mathrm{~g}$ & $0.0 \mathrm{~cm}$ & $(6.35 \pm 0.63) \times 10^{-7}$ & $(1.05 \pm 0.16) \times 10^{-6}$ \\
\hline
\end{tabular}

The neutron self-shielding was found negligible in the neutron energy range from $6 \mathrm{MeV}$ to $14 \mathrm{MeV}$.

The systematic error on the measurement was not evaluated with precision. We can, however, conservatively state that they are smaller than $50 \%$. 


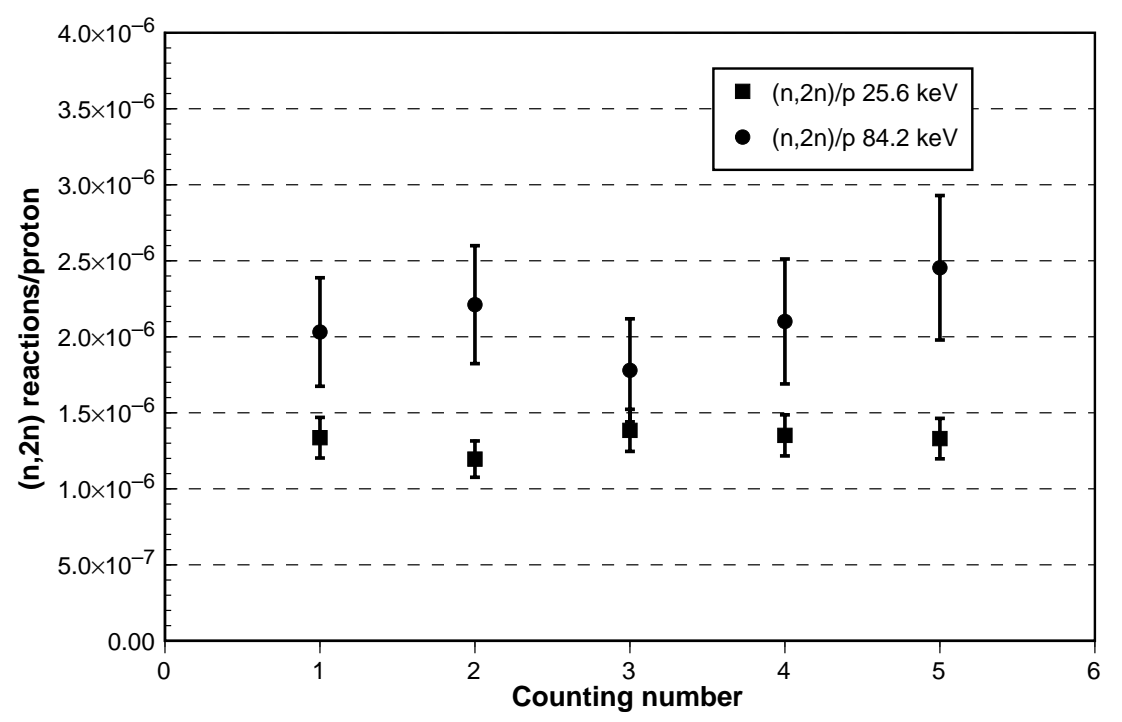

Fig. 116: ${ }^{232} \mathrm{Th}(\mathrm{n}, 2 \mathrm{n}){ }^{231} \mathrm{Th}$ reaction rates obtained from the intensities of the $25.6 \mathrm{keV}$ and $84.2 \mathrm{keV}$ lines, from the $3 \mathrm{~cm} \times 3 \mathrm{~cm}^{232}$ Th sample $(0.678 \mathrm{~g})$ irradiated in hole number 3 at $z=+7.5 \mathrm{~cm}$.

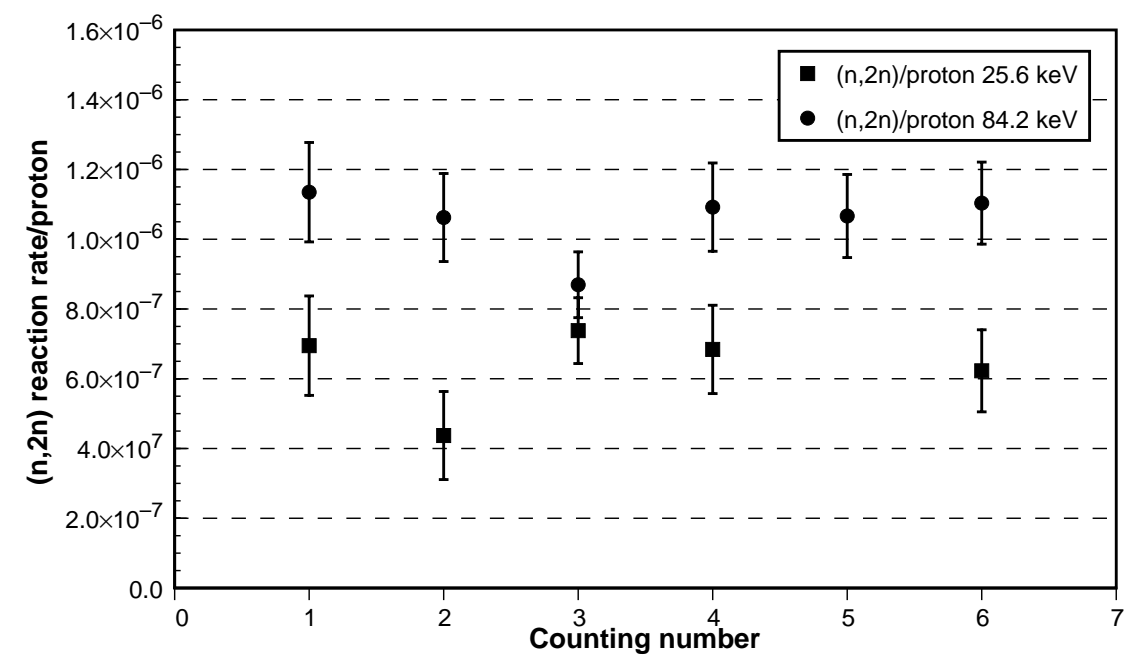

Fig. 117: ${ }^{232} \mathrm{Th}(\mathrm{n}, 2 \mathrm{n}){ }^{231} \mathrm{Th}$ reaction rates obtained from the intensities of the $25.6 \mathrm{keV}$ and $84.2 \mathrm{keV}$ lines, from the $0.158 \mathrm{~g}$ of ${ }^{232} \mathrm{Th}$ sample irradiated in hole number 3 at $z=0.0 \mathrm{~cm}$.

\section{Medical Applications of ARC}

\subsection{General strategy for medical applications of ARC}

\subsubsection{Introduction}

The domain of applications of the ARC method of enhancement of neutron captures is very vast. We will discuss in this section the production of (short-lived) isotopes for medical applications. Radionuclides are extensively used for medical diagnosis applications as 'tracing' elements, i.e. they are directly detectable within the patient under study because of their spontaneous radioactive decays. In order to minimize the integrated radiotoxicity, the half-life of the chosen tracing isotope should be short, ideally not much longer than the examination time. As a consequence, its utilization is limited to a period of a few half-lives from activation, since the radioactivity of the isotope decays exponentially from the moment of production. Another application of growing interest for radionuclides is (cancer) therapy, for which doses significantly larger than those used in diagnostics are required. Most of these 
isotopes must have a relatively short half-life, since they are generally injected or implanted in the patient's body. Today the main sources of supply for these isotopes are nuclear reactors or particle accelerators irradiating a suitable target with a charged-particle beam.

The simplicity of the device (activator) which was proposed by one of us [4] and its relatively modest cost and dimensions are intended to promote 'local' production of short-lived radioisotopes, thus eliminating costly, swift transportation and the consequent need of larger initial inventories and thus extending their practical utilization. This is made possible by the high neutron capture efficiency resulting from the ARC method, which allows the required amount of the radioisotope to be produced with a relatively modest neutron generator.

This method of neutron activation is intended to be a competitive alternative to reactor-driven, neutron capture activation. In addition, several isotopes that are difficult to produce by activation in the (usually thermal) neutron flux of an ordinary reactor can be produced using the broad energy spectrum of the neutrons in the activator extending to high energies and especially designed to make use of the large values of the cross-section at the energy of resonances. This is the case, for instance, in the production of ${ }^{99} \mathrm{~m} \mathrm{Tc}\left({ }^{99} \mathrm{Mo}\right)$, widely used in medicine and which is nowadays generally chemically extracted from the fission fragments of spent nuclear fuel. With the present method, this popular radioisotope can be obtained instead by direct neutron resonant activation of a (natural) molybdenum target with the help of a much simpler and less costly activator driven by a small particle accelerator. Incidentally, the total amount of additional, useless radioactive substances that have to be produced and handled in association with a given amount of this wanted radionuclide is also greatly reduced.

\subsubsection{Selected examples of procedures for an activator}

The procedures to be followed in order to prepare radioactive samples are illustrated by the following practical examples:

(1) A first procedure, suitable for medical examinations (thyroid), consists of activating directly inside our device an already prepared, pharmacological iodine compound. The element is initially available in the most appropriate chemical compound ${ }^{7}$, made from natural iodine (stable isotope ${ }^{127} \mathrm{I}$ ). Shortly before administration, the compound is introduced in the activator driven by a small proton accelerator $(23 \mathrm{MeV}$ and $1 \mathrm{~mA})$ and activated - for instance during a time of the order of one ${ }^{128}$ I half-life $(25 \mathrm{~min})$ or correspondingly less for smaller activation strengths - with the help of the capture reaction ${ }^{127} \mathrm{I}+\mathrm{n} \rightarrow{ }^{128} \mathrm{I}+\gamma$, which transforms natural iodine into the tracing element ${ }^{128}$ I with $\beta^{-}$-decay and a prominent $\gamma$-line at $443 \mathrm{keV}$. There is no chemical 'preparation' between activation and examination. This very simple procedure is becoming practical with the ARC method because of the higher efficiency of neutron capture, which produces the required source strength $(\leq 1 \mathrm{GBq})$ starting from a tiny, initial amount of natural iodine $(\leq 1 \mathrm{~g})$ and using a conventional accelerator of a scale already in wide use within hospitals for other applications (PET, etc.). The feasibility of the production of ${ }^{128}$ I from ${ }^{127}$ I has been confirmed by TARC, using the ${ }^{127}$ I content of the iodine sample used in the transmutation test of ${ }^{129}$ I. The rate of captures measured in TARC is well reproduced by our simulation (Fig. 118). At about $46 \mathrm{~cm}$ from the centre of the lead volume (hole $10, z=+7.5 \mathrm{~cm}$ ) the capture rate is 14900 per $10^{9}$ protons on our

\footnotetext{
7. For instance sodium iodide $(\mathrm{NaI})$.
} 
$10.44 \mathrm{mg}$ sample of ${ }^{127} \mathrm{I}$. These measurements validate the simulation used in this section. Our method makes practical the use of ${ }^{128} \mathrm{I}$ as a tracing element for thyroid diagnosis with a much shorter half-life $(25 \mathrm{~min})$ than the one of currently used iodine isotopes $\left({ }^{131} \mathrm{I}\right.$ and $\left.{ }^{123} \mathrm{I}\right)$ and hence the corresponding important advantage of a much smaller dose to patients 8 .

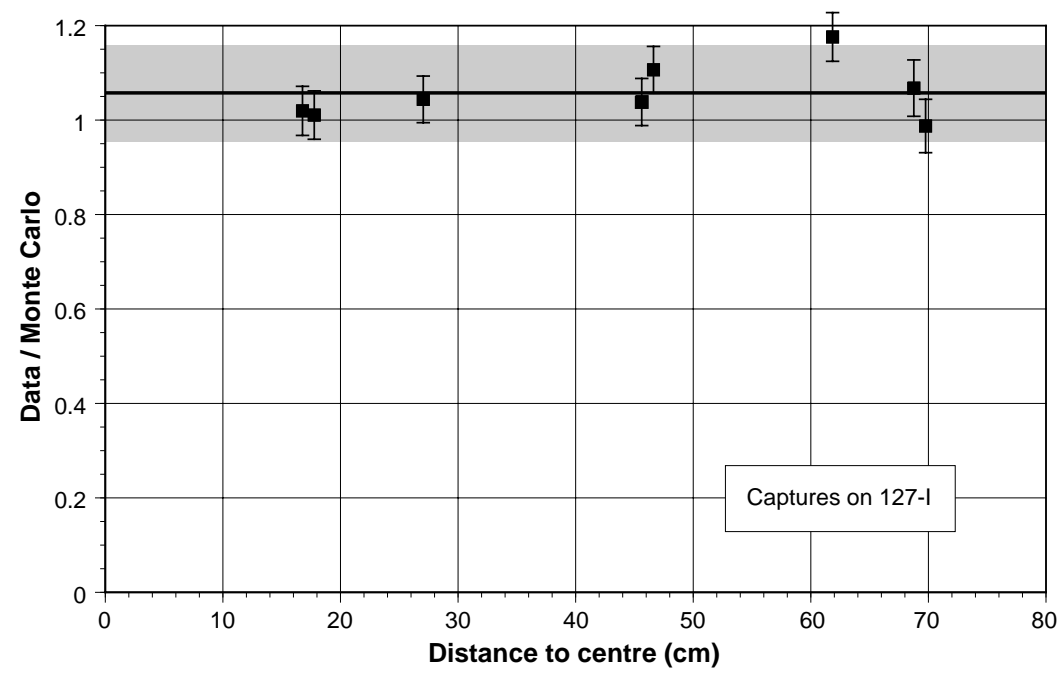

Fig. 118: Ratio of measured to simulated capture rate on our $10.44 \mathrm{mg}{ }^{127} \mathrm{I}$ sample. The grey band represents the size of the common systematic error.

(2) A second example, also tested in TARC (see Section 9.2), illustrates the case in which some (simple) chemical transformation is needed between the activation and the use of the radioactive compound. This is the case for the ${ }^{99 \mathrm{~m}} \mathrm{Tc}$ medical examination, of which many millions are performed annually world-wide. The small sample to be irradiated consists of molybdenum ${ }^{9}$, for instance in the form of $\mathrm{MoO}_{3}$. The appropriate sample of ${ }^{99} \mathrm{Mo}\left(t_{1 / 2}=65 \mathrm{~h}\right)$ is produced with the help of the activator driven by an accelerator and the capture reaction ${ }^{98} \mathrm{Mo}+\mathrm{n} \rightarrow{ }^{99} \mathrm{Mo}+\gamma$. The activated molybdenum sample is then handled according to a generally used procedure: transformed for instance in the form of an appropriate salt, it is captured in an alumina absorber. The production of ${ }^{99 \mathrm{~m}} \mathrm{Tc}$ proceeds inside the absorber through the subsequent $\beta$-decay reaction ${ }^{99} \mathrm{Mo} \rightarrow{ }^{99} \mathrm{~m} \mathrm{Tc}$. The ${ }^{99 \mathrm{~m}} \mathrm{Tc}$ which has a relatively short half-life $\left(t_{1 / 2}=6.01 \mathrm{~h}\right)$ is extracted in the form of $\mathrm{Tc}^{4+}$, for instance by passing a solution of water with a small amount of $\mathrm{NaCl}$ through the Mo sample in the alumina (which remains insoluble). Since only a very small fraction of the compound is activated at each exposure, the molybdenum 'father' can be

8. The current methods of iodine examinations are based on ${ }^{131} \mathrm{I}$, which has a relatively long half-life of eight days and which causes large intake doses for the patients (roughly in the ratio of half-lives (461/1), and ${ }^{123} \mathrm{I}$ which has the shorter half-life of 13.2 hours (31.8 times that of ${ }^{128} \mathrm{I}$ ), but is difficult and costly to obtain since it is normally produced by $30 \mathrm{MeV}$ protons with the $(\mathrm{p}, 2 \mathrm{n})$ reaction on isotopically separated ${ }^{124} \mathrm{Te}$ (natural abundance $4.79 \%$ ). In order to use natural Xe, the reaction is (p,5n) and the energy must be at least $60 \mathrm{MeV}$. The presently proposed method has therefore both a very simple application and leads to much smaller doses to the patient for a given disintegration rate during the examination. We note that the larger doses of the current methods generally hamper extensive application in the case of young subjects and of pregnant women.

9. The isotopic content of ${ }^{98} \mathrm{Mo}$ in natural molybdenum is $24.13 \%$. Isotopic enrichment will be convenient, though not mandatory. 
recycled ${ }^{10}$ flushing it from the alumina absorber and repeatedly re-introducing it in the activator.

(3) Another type of radioisotope used in medicine is extracted from fragments of uranium fission and generally referred to by the generic name of 'fissium'. The increased capture efficiency offered by the ARC method works equally well in the case of neutron capture leading to fission. Fissium can be produced in our activator introducing a small uranium target, possibly enriched with ${ }^{235} \mathrm{U}$, which, as in the previous examples, is strongly activated by primarily resonance-driven captures. The system is not critical and a small amount of fissile target material is sufficient to obtain relatively large amounts of fissium. In the case of activation of short-lived elements, the target must be frequently extracted and reprocessed. This is made extremely easy by the geometry and general conditions of the operation of an activator, when compared with a nuclear reactor. The amount of plutonium produced by the captures in ${ }^{238} \mathrm{U}$ is negligibly small and causes no concern about proliferation.

These cases are examples of the potential of the activator (Fig. 119) and obviously a variety of scenarios are possible, depending on the type of radioisotope and the specific application.

The activator has a geometry close to the TARC experimental set-up [Fig. 119(a) and 119(b)], but with additional improvements to enhance the neutron flux in the energy region of interest described in detail in Ref. [4].

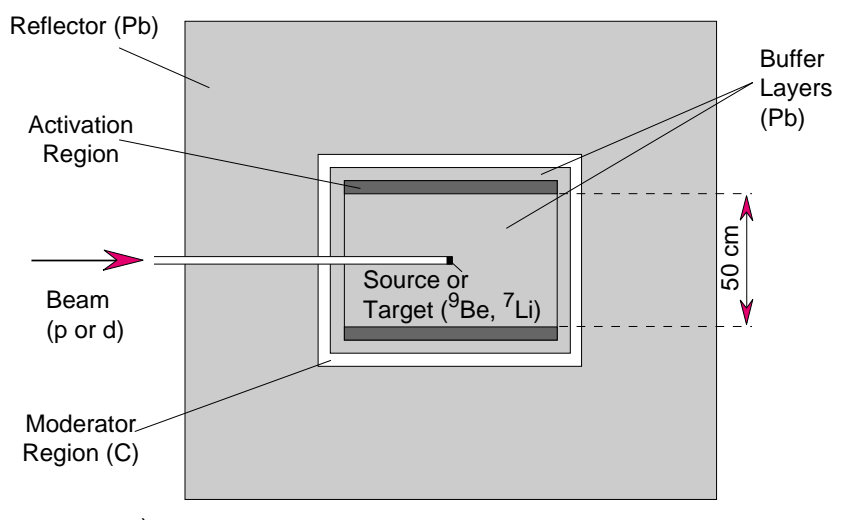

a)

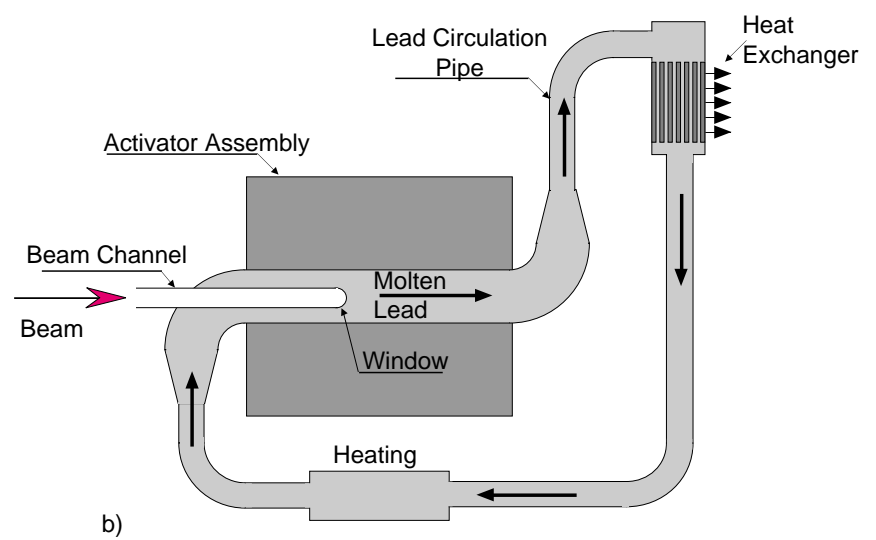

Fig. 119: General layout of the activator (a) for a small target and low-power beam or radioactive source; (b) for high-power beam and spallation neutrons.

10. This is of importance if molybdenum is isotopically enriched. 


\subsubsection{Characterization of the spallation neutron source}

The energy spectrum of the initially produced neutrons is relatively unimportant, since the inelastic processes in the diffuser quickly damp the neutron energy down to about $1 \mathrm{MeV}$, where the lethargic slow-down of the neutrons is taking over. Therefore, the neutron capture efficiency for activation and, more generally, the geometry of the activator are relatively independent of the details of the realization of the source.

In the case of the activation of natural iodine, it is likely that a small sample - of the order of a fraction of a gram - must be activated for each exposure to a level requiring a cyclotron or similar accelerator with a neutron production rate of a few times $10^{13}$ neutrons/second over the full solid angle. This can be obtained with a proton energy of the order of 10-30 MeV and a current of the order of milliamperes, which is also suited for production of isotopes for PET examinations. Therefore a combined facility may be envisaged.

In the case of a large industrial production of radionuclides, like for instance ${ }^{99} \mathrm{Mo}\left({ }^{99 \mathrm{~m}} \mathrm{Tc}\right)$, ${ }^{131} \mathrm{I}$, or of fissium from uranium fissions it may be worth considering similar currents but higher proton energies, in the region of a few hundred $\mathrm{MeV}$. Activation can then be performed within much smaller samples which is a considerable advantage especially in the case of portable ${ }^{99}$ Mo $\left({ }^{99 m} \mathrm{Tc}\right)$ dispensers.

At the other end of the scale, the production of small activation with a simple device using a neutron-emitting radioactive source is worth mentioning, since it might be of interest for applications in which a very weak source $(<<\mathrm{mCi})$ of radioisotopes is needed. This would be cheap and simple to operate.

The overall neutron yield from a thick Be target bombarded with a beam of protons at $E_{\mathrm{p}}=23 \mathrm{MeV}$ is $1.66 \times 10^{14} \mathrm{n} / \mathrm{s} / \mathrm{mA}(E>0.4 \mathrm{MeV})$, corresponding to a neutron flux of $0.654 \times 10^{12} \mathrm{~cm}^{-2} \mathrm{~s}^{-1} \mathrm{~mA}^{-1}$ at $r=20 \mathrm{~cm}$ from the source, according to the formula $\phi(r) \approx S_{0} / 4 \pi D r$, which exhibits the lead enhancement factor $(D=1.01 \mathrm{~cm})$. We note also that the flux is falling like the inverse of the distance $(1 / r)$, more slowly than in empty space where the flux is proportional to the solid angle from the source $\left(1 / r^{2}\right)$. We note that for a current of $10 \mathrm{~mA}$, typical for modern cyclotrons, our system leads to the remarkable flux $6.5 \times 10^{12} \mathrm{~cm}^{-2} \mathrm{~s}^{-1}$, typical of a nuclear reactor. Other target materials can be used, in particular ${ }^{7} \mathrm{Li}$, with comparable yields. The neutron yield is a growing function of the proton energy and for a given beam power is rising proportionally to $E_{0}^{0.886}$. Neutrons can be produced also with other incident particles, in particular deuterons and alphas. For a given incident energy, the forward neutron yield of deuterons is substantially higher than for protons, but, as relevant in our application, the angle integrated flux is comparable to that of protons [81]. The yield for incident $\alpha$-particles is substantially lower. In view of the associated simplicity and their high neutron yield, proton beams seem to be optimal for the present application.

Neutron yields achievable by proton accelerators and different targets are summarized in Fig. 120.

\subsubsection{Performance of a typical activator}

Transmutation rates (Fig. 121) are largely independent of the chemical binding and isotopic composition of the materials inserted in the activator. They are also almost independent of the source geometry and of the process used for the neutron production, provided their energy is initially sufficiently high (>0.4 MeV). More details can be found in Ref. [4]. 


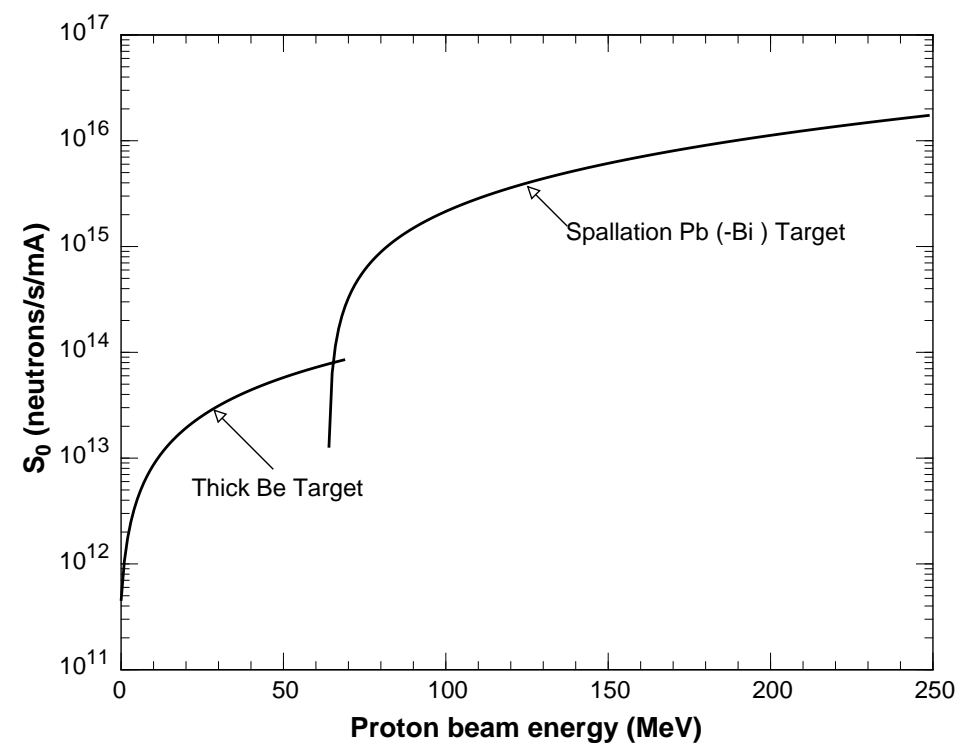

Fig. 120: Neutron yield, $S_{0}$ per $\mathrm{mA}$ of proton current, as a function of the kinetic energy of the proton beam for both a beryllium target and a heavy spallation target (taken from Ref. [4]).

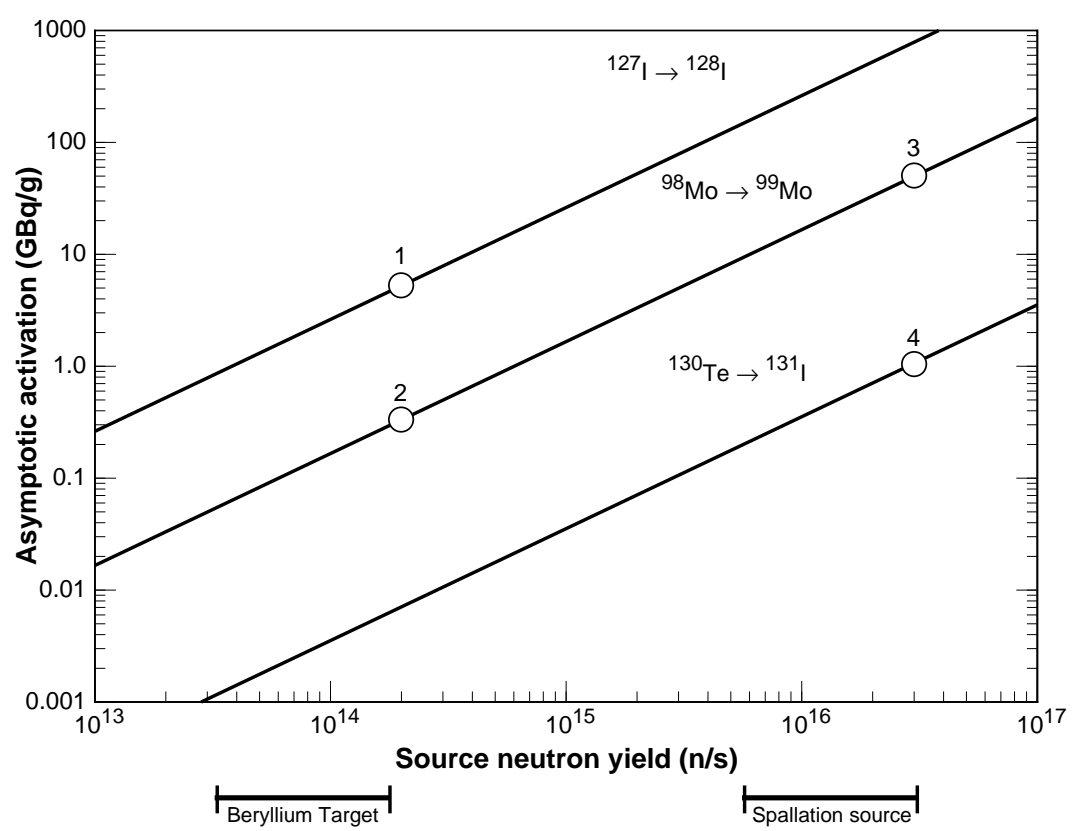

Fig. 121: Asymptotic activated yield for different elements, as a function of the strength $S_{0}$ of the neutron source. Points (1) and (2) correspond to a local production scenario, and points (3) and (4) to a regional industrial scale scenario (taken from Ref. [4]).

\subsection{Measurement of ${ }^{99} \mathrm{~m}$ Tc production rate from natural molybdenum}

\subsubsection{Sample configuration and preparation}

The sample used is a standard commercial $99.985 \%$ pure sample of molybdenum with very few impurities at the $10^{-3}$ level which can be ignored. The molybdenum sample was packaged in a small polyethylene bag to facilitate handling during irradiation in TARC. It had the shape of a thin disk of diameter $12.75 \pm 0.05 \mathrm{~mm}$, a thickness of approximately $0.078 \pm 0.002 \mathrm{~mm}$ for a total weight of $90.3 \pm 0.15 \mathrm{mg}$. The sample contains $21.79 \pm 0.04 \mathrm{mg}$ of ${ }^{99} \mathrm{Mo}$ (Table 26). 
Table 26: Properties of the molybdenum sample irradiated in TARC

\begin{tabular}{c|c|c|c}
\hline Element & $\begin{array}{c}\text { Fraction } \\
(\%)\end{array}$ & $\begin{array}{c}\text { Mass } \\
(\mathbf{m g})\end{array}$ & $\begin{array}{c}\text { Error } \\
(\mathbf{m g})\end{array}$ \\
\hline${ }^{92} \mathrm{Mo}$ & 14.84 & 13.40 & 0.02 \\
${ }^{94} \mathrm{Mo}$ & 9.25 & 8.35 & 0.014 \\
${ }^{95} \mathrm{Mo}$ & 15.92 & 14.375 & 0.02 \\
${ }^{96} \mathrm{Mo}$ & 16.68 & 15.08 & 0.025 \\
${ }^{97} \mathrm{Mo}$ & 9.55 & 8.625 & 0.015 \\
${ }^{98} \mathrm{Mo}$ & 24.13 & 21.79 & 0.036 \\
${ }^{100} \mathrm{Mo}$ & 9.63 & 8.696 & 0.015 \\
\hline Total & $\mathbf{1 0 0 . 0 0}$ & $\mathbf{9 0 . 3}$ & $\mathbf{0 . 1 5}$ \\
\hline
\end{tabular}

\subsubsection{Molybdenum capture and decay schemes}

\subsubsection{Molybdenum transmutation scheme}

Through neutron capture, stable ${ }^{98}$ Mo is transmuted into ${ }^{99}$ Mo (Fig. 122), which decays with a half-life of 65.94 hours into mostly $(\mathrm{Br}=87.5 \%){ }^{99 \mathrm{~m}} \mathrm{Tc}\left(t_{1 / 2}=6.01 \mathrm{~h}\right)$, but also sometimes $(12.5 \%$ of the cases $)$ into ${ }^{99} \mathrm{Tc}\left(t_{1 / 2}=2.111 \times 10^{5} \mathrm{yr}\right) .{ }^{99 \mathrm{~m}} \mathrm{Tc}$ decays almost always (99.996\% of the cases) into the ${ }^{99} \mathrm{Tc}$ ground state through internal transition.

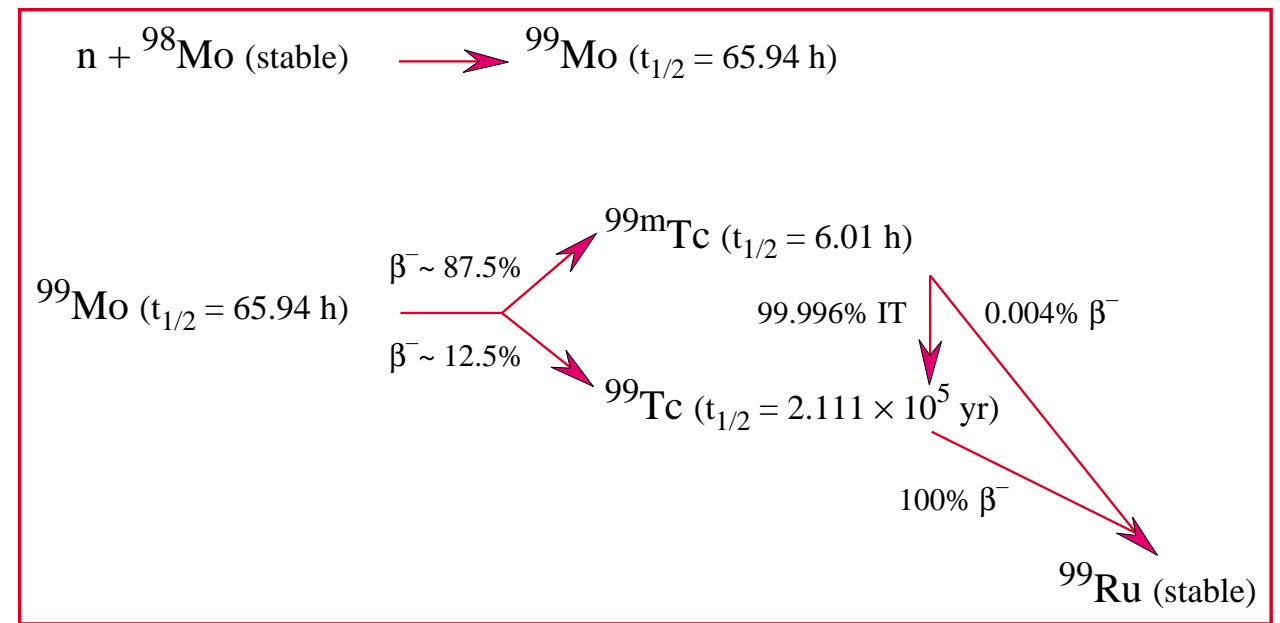

Fig. 122: Neutron capture on ${ }^{98}$ Mo and subsequent decay scheme.

For ${ }^{100} \mathrm{Mo}$ the scheme is simpler (Fig. 123). ${ }^{101} \mathrm{Mo}\left(t_{1 / 2}=14.61 \mathrm{~min}\right)$ is produced which decays $100 \%$ of the time into ${ }^{101} \mathrm{Tc}\left(t_{1 / 2}=14.22 \mathrm{~min}\right)$ which itself decays into stable ${ }^{101} \mathrm{Ru}$.

The ${ }^{92} \mathrm{Mo}$ neutron capture cross-section is relatively small. In our sample of natural molybdenum, ${ }^{92}$ Mo corresponds to only $2.5 \%$ of all the captures. For ${ }^{92}$ Mo, most of the captures (> 77\%) produce ${ }^{93}$ Mo (Fig. 124) which has a long half-life and does not contribute very much to the total sample activity. In less than $23 \%$ of the cases, ${ }^{93} \mathrm{~m}$ Mo is formed. As a result the contribution to the overall sample activity is negligible. It is confirmed by the $\gamma$ spectra where we do not see any significant $\gamma$ peaks from ${ }^{93 \mathrm{~m}} \mathrm{Mo} .{ }^{93}$ Mo has only X-rays, and ${ }^{93 \mathrm{~m}}$ Mo three significant $\gamma_{\mathrm{s}}$ [69]: $263.1 \mathrm{keV}(56.7 \%) ; 684.7 \mathrm{keV}(99.7 \%)$, and $1477.1 \mathrm{keV}$ $(99.1 \%)$. 
All the other isomers $\left({ }^{94} \mathrm{Mo},{ }^{95} \mathrm{Mo},{ }^{96} \mathrm{Mo}\right.$ and $\left.{ }^{97} \mathrm{Mo}\right)$ are irrelevant in this study, since each of them produces, through neutron capture, another stable element. They do not contribute to the activation of the sample. We conclude that the total activation of natural molybdenum should be dominated by the production of ${ }^{99} \mathrm{Mo}$, with a small contribution from the production of ${ }^{101} \mathrm{Mo}$ and a negligible contribution from the production of ${ }^{93} \mathrm{Mo}$ and ${ }^{93} \mathrm{Mo}$. This is indeed confirmed by our measurements.

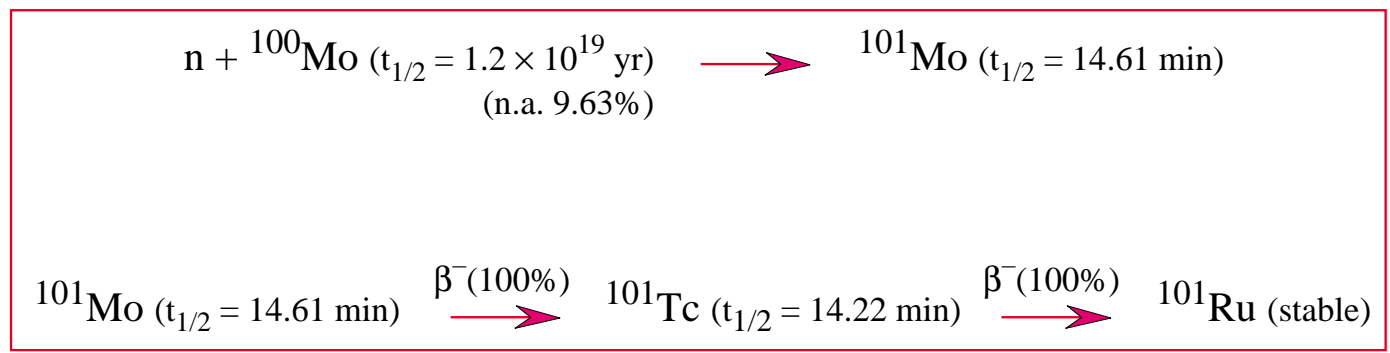

Fig. 123: Neutron capture on ${ }^{100}$ Mo and subsequent decay scheme.

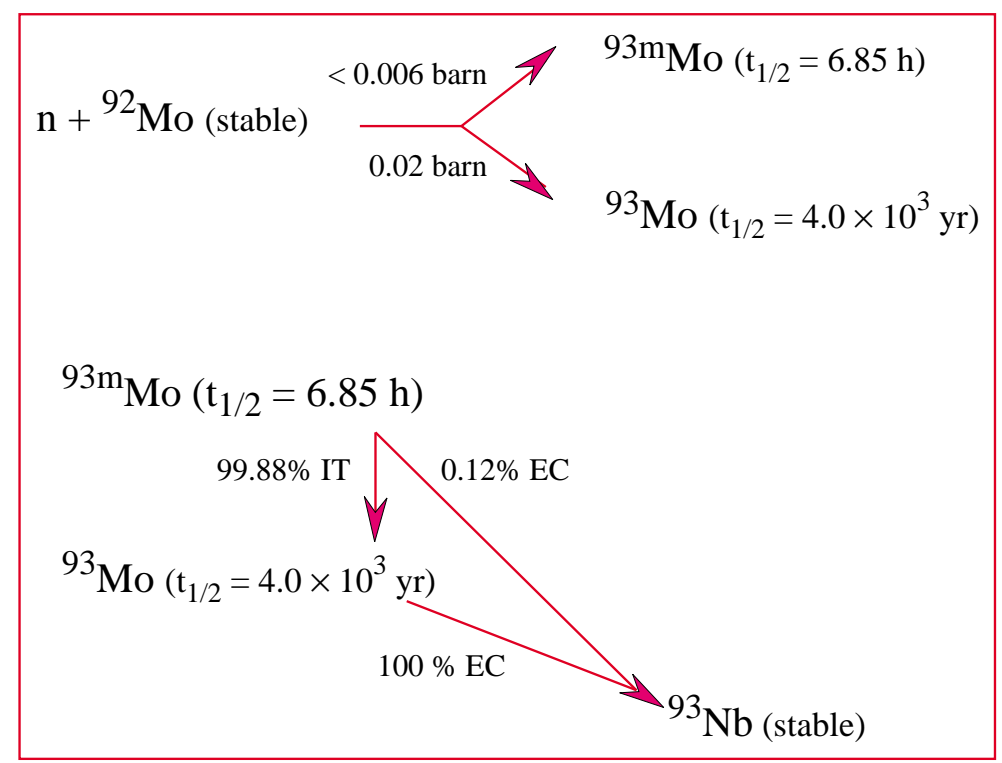

Fig. 124: Neutron capture on ${ }^{92}$ Mo and subsequent decay scheme.

\subsubsection{Other neutron reactions}

Other possible channels with high-energy neutrons include $(n, 2 n)$ reactions which can

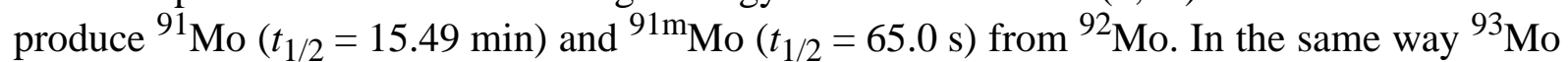
and ${ }^{93 \mathrm{~m}}$ Mo can be produced from ${ }^{94} \mathrm{Mo}$ and finally ${ }^{99} \mathrm{Mo}$ can be produced from ${ }^{100} \mathrm{Mo}$. All these reactions have relatively high neutron energy thresholds $(\geq 10 \mathrm{MeV})$, and represent a very small fraction of the captures, which can be safely neglected. This was checked explicitly with our simulation.

\subsubsection{Gamma branching ratios in molybdenum isotopes}

Tables 27-28 give the values of the branching ratios, for the various gamma lines used to detect the decays of the isotopes of interest. The branching ratio is defined as the probability of producing a given gamma in the decay of the element considered. 
Table 27: $\gamma$ branching ratios in ${ }^{99}$ Mo decay [69]

\begin{tabular}{c|c|c}
\hline${ }^{99}$ Mo lines $(\mathbf{k e V})$ & Branching ratio $(\boldsymbol{\%})$ & Error $(\boldsymbol{\%})$ \\
\hline 140.5 & $90.7 / 6.3(*)$ & $1.8 / 3.3$ \\
181.1 & 6.08 & 0.12 \\
366.5 & 1.16 & 0.04 \\
739.4 & 12.14 & 0.1 \\
777.8 & 4.36 & 0.11 \\
\hline
\end{tabular}

(*) Note on the $140.5 \mathrm{keV} \gamma$ branching ratio: This $\gamma$ comes mainly from the ${ }^{99 \mathrm{~m}} \mathrm{Tc}$ daughter of ${ }^{99} \mathrm{Mo}$. But ${ }^{99}$ Mo also produces this line directly. The first number given in Table 27 (90.7\%) is taken from Ref. [69] which claims that this is the value at 'transient' equilibrium calculated as:

$$
\begin{aligned}
& I_{\mathrm{E}} \equiv \mathrm{Br}_{\text {Equilibrium }}^{\text {Observed }}=I_{\mathrm{D}} \times \frac{\lambda_{\mathrm{D}}}{\lambda_{\mathrm{D}}-\lambda_{\mathrm{P}}} \times \mathrm{Br}\left({ }^{99} \mathrm{Mo} \rightarrow{ }^{99 \mathrm{~m}} \mathrm{Tc}\right) \\
& =0.877 \times \frac{3.2037 \times 10^{-5}}{3.2037 \times 10^{-5}-2.9199 \times 10^{-6}} \times 0.875=0.844
\end{aligned}
$$

where $I_{\mathrm{E}}$ is the $140 \mathrm{keV}$ abundance observed in the case of transient equilibrium,

$$
I_{\mathrm{D}} \equiv \mathrm{Br}^{\left({ }^{99 \mathrm{~m}} \mathrm{Tc}\right)}
$$

is the $140 \mathrm{keV}$ abundance of the same transition in the decay of the daughter alone. $\lambda_{\mathrm{P}}$ and $\lambda_{\mathrm{D}}$ are the decay constants of the parent and daughter respectively, and $\mathrm{Br}$ is the branching ratio for the decay from parent to daughter.

The result is clearly different from the $90.7 \%$ given in the data tables [69]. We suppose that one should add the $140.5 \mathrm{keV} \gamma$ s not coming from ${ }^{99 \mathrm{~m}} \mathrm{Tc}$ according to:

$$
\mathrm{Br}_{\text {Equilibrium }}^{\text {Observed }}=\mathrm{Br}{ }^{\left({ }^{99} \mathrm{Mo}\right)}+\mathrm{Br}\left({ }^{99 \mathrm{~m}} \mathrm{Tc}\right) \times \frac{\lambda_{\mathrm{D}}}{\lambda_{\mathrm{D}}-\lambda_{\mathrm{P}}} \times \mathrm{Br}\left({ }^{99} \mathrm{Mo} \rightarrow{ }^{99 \mathrm{~m}} \mathrm{Tc}\right) .
$$

From this we obtain $\mathrm{Br}^{\left({ }^{99} \mathrm{Mo}\right)}=6.3 \%$. Unfortunately, when we compute that branching ratio directly from the level scheme [63] we get $2.99 \%$. It will be shown later that the $6.3 \%$ value gives an activity more consistent with the other lines, therefore, we use that value, but we attribute a large error to it, namely $6.3 \% \pm 3.3 \%$.

Table 28: $\gamma$ branching ratios in ${ }^{99 \mathrm{~m}} \mathrm{Tc}$ decay [69]

\begin{tabular}{c|c|c}
\hline${ }^{\mathbf{9 9 m}}$ Tc lines (keV) & Branching ratio (\%) & Error (\%) \\
\hline 140.5 & 87.7 & 0.14 \\
142.6 & 0.041 & 0.00025 \\
\hline
\end{tabular}

\subsubsection{Correction for extended source geometry and gamma absorption}

The activity of the various $\gamma$ lines of interest was measured, using the same Ge counter as for the iodine study [68]. The Ge calibration was performed with a naked point-like source, therefore, we need to calculate corrections to take into account that (a) the molybdenum 
sample is an extended source of $\gamma_{\mathrm{s}}$ and (b) that the $\gamma_{\text {s }}$ produced can be absorbed in the molybdenum sample itself. In this case, we removed the plastic holder to perform the $\mathrm{Ge}$ counter measurement (Fig. 125). Absorption in the Ge counter material (aluminium window, etc.) is common to the Ge calibration procedure and to the data taking.

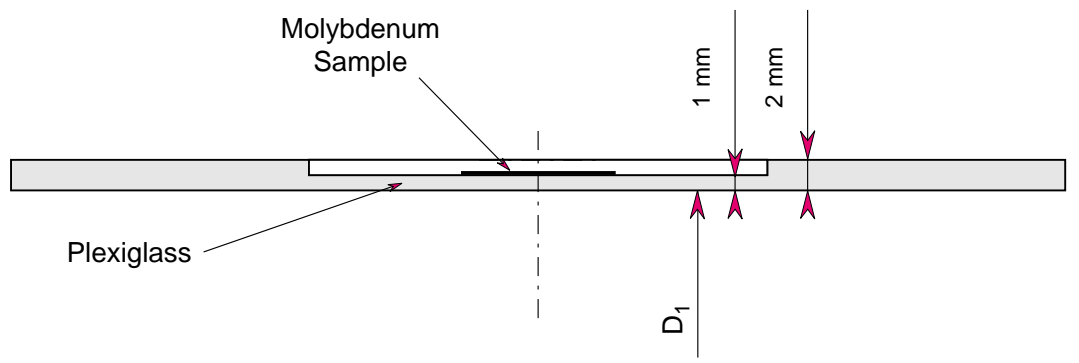

Fig. 125: Details of the set-up used for the measurement of the molybdenum activation, on the TIS Ge counter.

The correction factor, evaluated with the MCNP Monte Carlo code [66], takes into account also the correction needed to go from the point-like source used in the efficiency calibration of the Ge counter, to the extended sample geometry. In both cases the corrections are expected to be small, of only a few per cent. We followed the procedure used in the analysis of the ${ }^{129} \mathrm{I}$ activation [68], where we defined the Ge effective efficiency to be used in the analysis as:

$$
\varepsilon_{\text {eff }} \equiv \varepsilon_{\text {measured }}^{\text {point-like }} \times \frac{\varepsilon_{\text {simulated }}^{\text {sample }}}{\varepsilon_{\text {simulated }}^{\text {point-like }}}
$$

where the correction to the measured point-like efficiency can be defined as:

$$
\text { Correction } \equiv 1-\frac{\varepsilon_{\text {simulated }}^{\text {sample }}}{\varepsilon_{\text {simulated }}^{\text {point-like }}}
$$

The results summarized in Table 29 are taken partially from Ref. [82], for all the $\gamma_{\text {s }}$ of interest here. Note that the systematic uncertainty for the low-energy $\gamma_{\mathrm{s}}$ (below about $500 \mathrm{keV}$ ) may be larger than the canonical $5 \%$ because relatively few points were available in the calibration.

The error on the efficiency correction (Table 29) is estimated to $50 \%$ of the correction but does not matter very much because it is small in absolute value compared to the error on the measured efficiency which suffers from the precision on the activity of the calibration sources $(5 \%)$ and on the interpolation procedure between measured points $(5 \%)$.

\subsubsection{Correction for gamma overlap}

It is apparent from the excited level properties of ${ }^{99} \mathrm{Tc}$, that some of the gamma lines used in this analysis are correlated with one another, both in time and in space. Therefore, there is a certain probability that two or more gammas are recorded together in the same Ge counter. When this happens, the observed energy is not in the peak corresponding to the gammas considered, and the event does not contribute to the peak counting. We estimate, based on a calculation made with MCNP [66], that this effect is smaller than in the case of ${ }^{129}$ I where it was at most $6 \%$. In the following analysis we neglect this effect, since it is small compared to several sources of systematic errors. 
Table 29: Effective $\gamma$ efficiency of the TIS Ge counter used at position $\mathrm{D}_{1}$ (all Ge counter measurements were made at position $\mathrm{D}_{1}$ ). The correction is defined above

\begin{tabular}{c|c|c|c}
\hline Gamma lines (keV) & $\begin{array}{c}\text { Measured } \\
\text { efficiency }(\boldsymbol{\%})\end{array}$ & Correction (\%) & $\begin{array}{c}\text { Effective efficiency } \\
(\%)\end{array}$ \\
\hline 140.5 & 3.39 & 2.5 & 3.30 \\
181.1 & 2.83 & 2.4 & 2.76 \\
191.9 & 2.70 & 2.3 & 2.64 \\
366.5 & 1.43 & 1.9 & 1.41 \\
505.9 & 1.04 & 1.5 & 1.03 \\
590.9 & 0.908 & 1.2 & 0.897 \\
739.4 & 0.729 & 0.8 & 0.723 \\
777.8 & 0.689 & 0.7 & 0.684 \\
1012.5 & 0.530 & 0.37 & 0.530 \\
\hline
\end{tabular}

\subsubsection{General summary of the irradiation data}

The activation was performed in TARC in hole 10 at $z=+7.5 \mathrm{~cm}$ (2172 PS shots, $2.156 \times 10^{13} \pm 3.6 \times 10^{8}$ (stat.) protons. The number of non-empty shots was 2075 and the measured mean distance of the beam impact to the beam axis was $2.84 \mathrm{~mm}$

\subsubsection{Data analysis}

\subsubsection{Production of ${ }^{99}$ Mo and of ${ }^{99 m}$ Tc}

The procedure is totally analogous to the one used for the irradiation of ${ }^{129} \mathrm{I}$ and is described in detail in the corresponding note [68]. The run history for irradiation number 1 is given in Fig. 126.

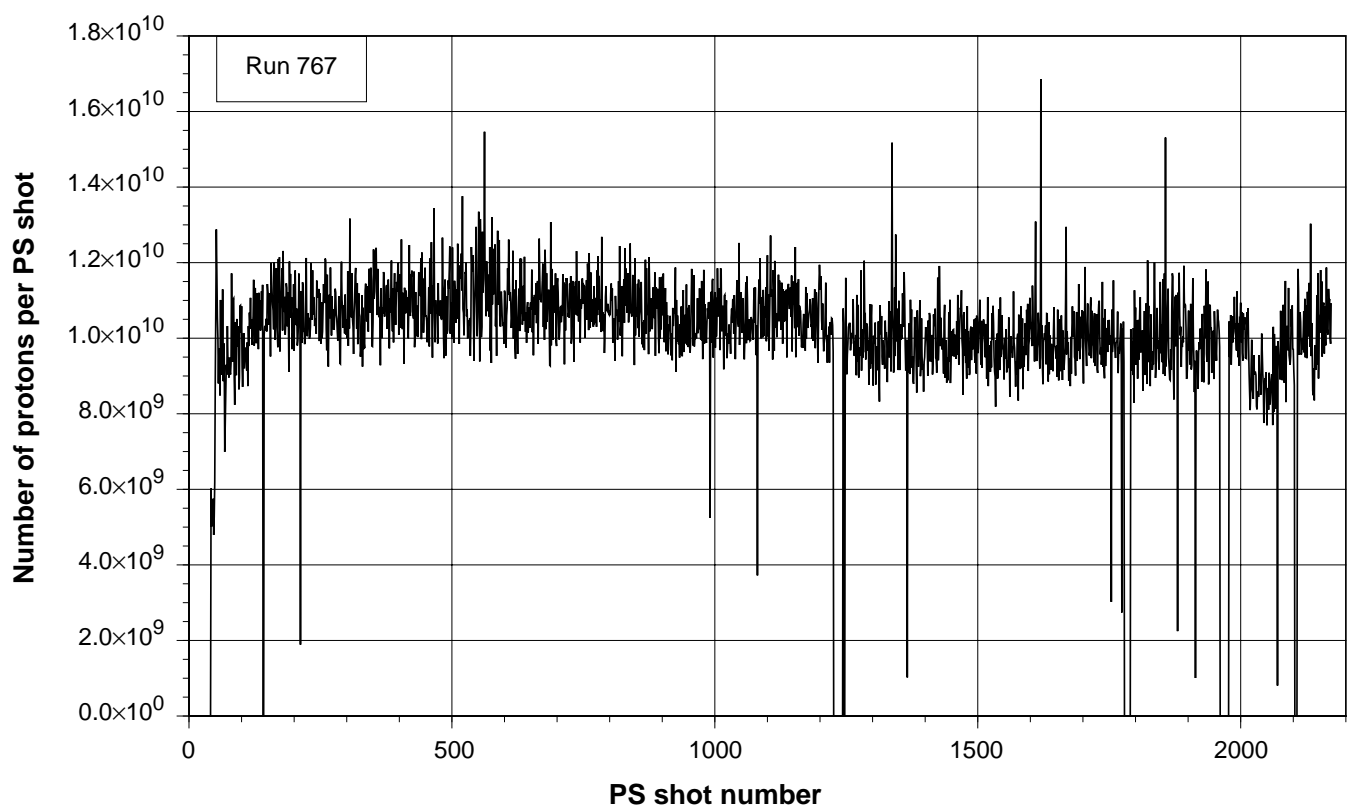

Fig. 126: History of beam intensity for run 767.

Assuming that at a given position in the lead assembly, $N_{\mathrm{i}}$ atoms of ${ }^{99} \mathrm{Mo}$ are produced per $10^{9}$ incident protons, we can calculate the number of ${ }^{99} \mathrm{Mo}$ and of ${ }^{99 \mathrm{~m}} \mathrm{Tc}$ atoms present at the end of irradiation in the following way: 


$$
\begin{gathered}
N\left({ }^{99} \mathrm{Mo}\right)_{\text {end }}=N_{\mathrm{i}} \sum_{n=1}^{n=N} X_{\mathrm{n}} \times e^{-\lambda_{1}\left(t_{\mathrm{end}}-t_{\mathrm{n}}\right)} \\
N\left({ }^{99 \mathrm{~m}} \mathrm{Tc}\right)_{\text {end }}=N_{\mathrm{i}} \frac{\lambda_{1} \times \mathrm{Br}}{\lambda_{2}-\lambda_{1}} \sum_{n=1}^{n=N} X_{\mathrm{n}} \times\left[e^{-\lambda_{1}\left(t_{\mathrm{end}}-t_{\mathrm{n}}\right)}-e^{-\lambda_{2}\left(t_{\mathrm{end}}-t_{\mathrm{n}}\right)}\right]
\end{gathered}
$$

where $X_{\mathrm{n}}$ is the number of protons (in units of $10^{9}$ protons) in PS beam shot $\mathrm{n}, \lambda_{1}=2.91994 \times$ $10^{-6} \mathrm{~s}^{-1}$ is the decay constant of ${ }^{99} \mathrm{Mo}, \lambda_{2}=3.203675 \times 10^{-5} \mathrm{~s}^{-1}$ is the decay constant of ${ }^{99} \mathrm{~m} \mathrm{Tc}, t_{\mathrm{end}}$ is the time of the last PS shot, $t_{\mathrm{n}}$ is the time of shot number $\mathrm{n}, \mathrm{Br}$ is the probability that ${ }^{99 \mathrm{~m}} \mathrm{Tc}$ is formed in the decay of ${ }^{99} \mathrm{Mo}$, taken to be $87.5 \%$. The quantities $A \equiv N\left({ }^{99} \mathrm{Mo}\right)_{\mathrm{end}} / N_{\mathrm{i}}$ which we call the ${ }^{99} \mathrm{Mo}$ beam integral, and $B \equiv N\left({ }^{99 \mathrm{~m}} \mathrm{Tc}\right){ }_{\text {end }} / N_{\mathrm{i}}$ which we call the ${ }^{99 \mathrm{~m}} \mathrm{Tc}$ beam integral characterize a given irradiation and are given in Table 30.

Table 30: Beam integrals $(A, B)$ calculated for each of the processes. These beam integrals characterize the production and decay of ${ }^{99} \mathrm{Mo}$, and ${ }^{99} \mathrm{~m}$ Tc during the irradiation. The errors include the systematic errors coming from the absolute beam calibration (5\%) and the statistical error (but, negligible in all cases).

\begin{tabular}{c|c|c|c|c|c}
\hline Activation & Run & $\boldsymbol{A}$ & $\boldsymbol{\Delta A}$ & $\boldsymbol{B}$ & $\Delta \boldsymbol{B}$ \\
\hline 1 & 767 & 20464.8 & 1000 & 621.46 & 31 \\
\hline
\end{tabular}

\subsubsection{Gamma spectra analysis}

The time measured between the last PS shot and the beginning of Ge counting is 1867 seconds and the measurement lasted 10000 seconds.

The number of counts in the $\gamma$ peaks (Table 31) was determined in three ways: (a) using the standard algorithm available on the Ge system we used (TIS); (b) using an algorithm developed by our group for the rabbit analysis and based on Ref. [61]; and (c) making a fit with interactive software (PAW) [83], in cases of ambiguity of results from (a) and (b). This allowed us to compare the algorithms, and gave us a way to correct technical problems and estimate the systematic errors. Figure 127 shows the Ge gamma spectrum obtained.

Table 31: Peak-finding results for ${ }^{99}$ Mo data (the errors are statistical only)

\begin{tabular}{c|c|c}
\hline $\boldsymbol{E}(\mathbf{k e V})$ & Counts & Error \\
\hline 140.51 & 16154 & 131 \\
181.09 & 1804 & 52 \\
366.42 & 158 & 21 \\
739.5 & 888 & 32 \\
777.91 & 313 & 18 \\
\hline
\end{tabular}

A good technical overall test of (a) the peak-fitting performance; (b) the estimation of the Ge counter acceptance; (c) the effect of $\gamma$ overlap; (d) the determination of the $\gamma$-line branching ratio is obtained by making sure that all the $\gamma$-lines of one element give the same activity. This is indeed the case for ${ }^{99} \mathrm{Mo}$ (Fig. 128). Since, in the case of ${ }^{99} \mathrm{Mo}$, there are five different $\gamma$ lines which we can use, the spread between the activities obtained from these lines, is a good measure of the systematic error. We find a standard deviation of $6 \%$ (Table 31), which is the value we are using in our general error analysis. 


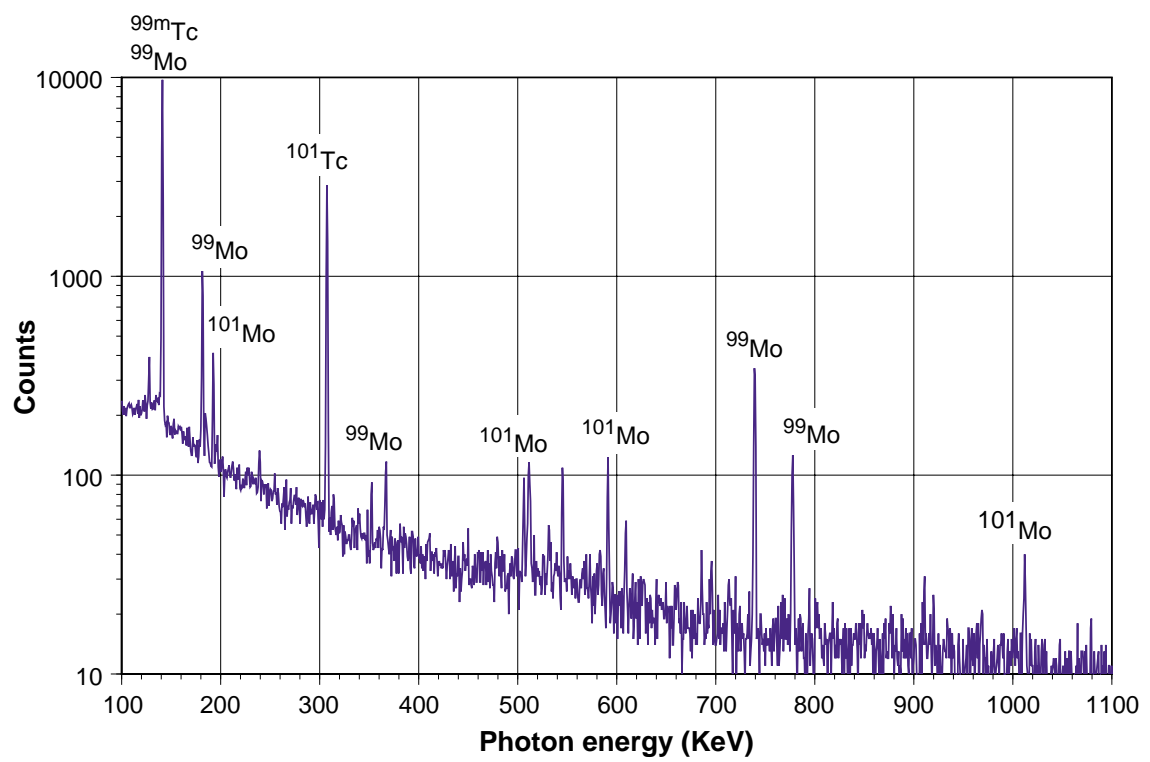

Fig. 127: Ge gamma spectrum taken shortly after the end of irradiation, showing the ${ }^{99} \mathrm{Mo},{ }^{99 \mathrm{~m}} \mathrm{Tc},{ }^{101} \mathrm{Mo}$, and ${ }^{101}$ Tc peaks of interest.

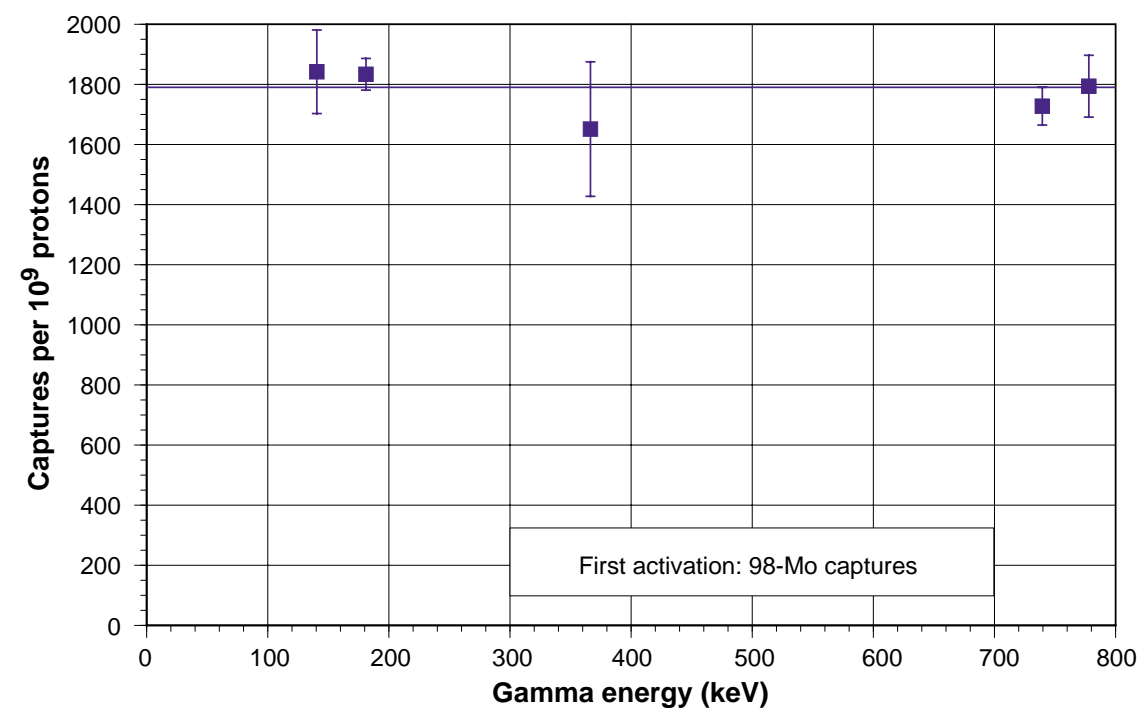

Fig. 128: Measurement of capture rates on ${ }^{98}$ Mo from the activity of each ${ }^{99}$ Mo gamma line (statistical errors only).

\subsubsection{Calculation of the ${ }^{98}$ Mo capture rates}

We call $n_{1}(t)$ and $n_{2}(t)$ the number of ${ }^{99} \mathrm{Mo}$ and ${ }^{99 \mathrm{~m}} \mathrm{Tc}$ atoms at time $t$, and $n_{1}(0)$ and $n_{2}(0)$ the numbers of atoms of these two elements at time $t=0$, defined as the time of the last PS shot in the irradiation run. We have:

$$
\begin{aligned}
& n_{1}(0)=N\left({ }^{99} \mathrm{Mo}\right)_{\text {end }} \equiv A \times N_{\mathrm{i}} \\
& n_{2}(0)=N\left({ }^{99 \mathrm{~m}} \mathrm{Tc}\right)_{\text {end }} \equiv B \times N_{\mathrm{i}}
\end{aligned}
$$


where $A$ and $B$ are the beam integrals given in Table 30. The expressions for $n_{1}(t)$ and $n_{2}(t)$ are given by:

$$
\begin{aligned}
& n_{1}(t)=n_{1}(0) e^{-\lambda_{1} t} \\
& n_{2}(t)=\frac{\lambda_{1} n_{1}(0) \operatorname{Br}}{\lambda_{2}-\lambda_{1}}\left[e^{-\lambda_{1} t}-e^{-\lambda_{2} t}\right]+n_{2}(0) e^{-\lambda_{2} t}
\end{aligned}
$$

where $\mathrm{Br}$ is the fraction of ${ }^{99} \mathrm{Mo}$ decays into ${ }^{99 \mathrm{~m}} \mathrm{Tc}$. With these expressions we can calculate the number of decays for each element in a time window [Age, Age $\left.+T_{\mathrm{c}}\right]$, where Age is the time at which the Ge counting starts, and $T_{\mathrm{c}}$ is the counting time:

$$
\begin{aligned}
& N_{1}^{\text {decay }}=n_{1}(0) e^{-\lambda_{1} A g e}\left[1-e^{-\lambda_{1} T_{\mathrm{c}}}\right] \\
& N_{2}^{\text {decay }}=\frac{\lambda_{2} n_{1}(0) \mathrm{Br}}{\lambda_{2}-\lambda_{1}} e^{-\lambda_{1} A g e}\left[1-e^{-\lambda_{1} T_{\mathrm{c}}}\right]+\left[n_{2}(0)-\frac{\lambda_{1} n_{1}(0) \mathrm{Br}}{\lambda_{2}-\lambda_{1}}\right] e^{-\lambda_{2} A g e}\left[1-e^{-\lambda_{2} T_{\mathrm{c}}}\right]
\end{aligned}
$$

from which we can obtain the number of ${ }^{98}$ Mo captures per $10^{9}$ protons, from the number of Ge counts in a given $\gamma$ peak in the ${ }^{99}$ Mo decays $\left[N_{\text {counts }}(\gamma)\right]$ (except for the $140.5 \mathrm{keV}$ line which has to have special treatment):

$$
N_{\mathrm{i}}=\frac{N_{\text {counts }}(\gamma) e^{\lambda_{1} A g e}}{A \operatorname{Br}_{\gamma} \varepsilon_{\gamma}\left[1-e^{-\lambda_{1} T_{\mathrm{c}}}\right]}
$$

The $140.5 \mathrm{keV} \gamma$ line gets contributions from both ${ }^{99} \mathrm{Mo}$ and ${ }^{99 \mathrm{~m}} \mathrm{Tc}$, and therefore the number of captures per $10^{9}$ protons calculated with this line is a bit more involved:

$$
N_{\mathrm{i}}=\frac{N_{\text {counts }}(140.5)}{A \varepsilon_{\gamma}\left\{\left[\mathrm{Br}_{\gamma}{ }^{99} \mathrm{Mo}+\frac{\mathrm{Br}_{\gamma}{ }^{99 m} T_{\mathrm{c}} \lambda_{2} \mathrm{Br}}{\lambda_{2}-\lambda_{1}}\right] e^{-\lambda_{1} A g e}\left[1-e^{-\lambda_{1} T_{\mathrm{c}}}\right]+\left[\frac{B}{A}-\frac{\lambda_{1} \mathrm{Br}}{\lambda_{2}-\lambda_{1}}\right] \operatorname{Br}_{\gamma}^{99 \mathrm{~m}} T_{\mathrm{c}} e^{-\lambda_{2} A g e}\left[1-e^{-\lambda_{2} T_{\mathrm{c}}}\right]\right\}}
$$

The number of captures obtained from each line is given in Table 32. The average capture rate is found to be $1785 \pm 46$ (stat.) (Table 32). Taking into account, in addition, the systematic errors of $10.7 \%$ (Table 34) we get $N_{\mathrm{i}}=1785 \pm 191$ (tot.), to be compared to the Monte Carlo prediction of $2016 \pm 126$ where the error is statistical only.

Several technical checks of the evaluation of the number of captures are described in Ref. [8].

Table 32: Measured rates of captures on ${ }^{98}$ Mo from each gamma line

\begin{tabular}{c|c|c}
\hline Gamma energy $(\mathbf{k e V})$ & $\boldsymbol{N}_{\mathbf{i}}$ (capt/10 $\mathbf{9}^{\mathbf{p}}$ protons) & Statistical error \\
\hline 140.51 & 1841.9 & 139 \\
181.09 & 1833.6 & 53 \\
366.42 & 1651.3 & 224 \\
739.5 & 1727.5 & 63 \\
777.91 & 1793.9 & 103 \\
Weighted average & 1785 & 46 \\
\hline
\end{tabular}




\subsubsection{Monte Carlo simulation}

\subsubsection{Simulation of capture rates}

The simulation (Table 33) [82] was obtained with the same general procedure used in other similar analyses in the TARC experiment and is based on the JENDL database for molybdenum neutron cross-sections (Fig. 129).
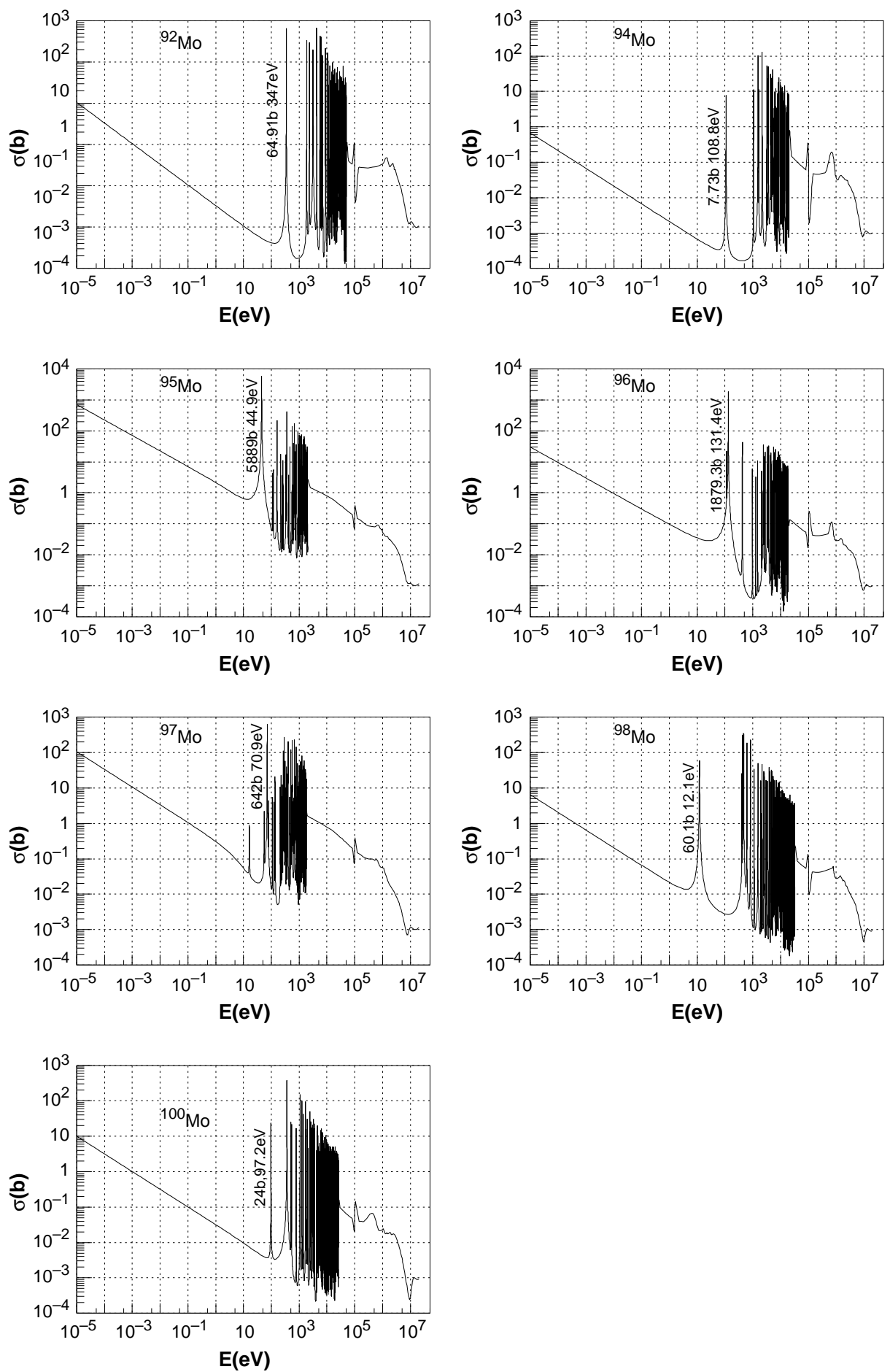

Fig. 129: Neutron capture cross-sections of stable molybdenum isotopes (from JENDL-3.2). 
Table 33: Captures per $10^{9}$ protons, on the various stable isotopes of natural molybdenum contained in the activated sample, as obtained from the TARC simulation

\begin{tabular}{c|c|c|c}
\hline Isotope & Captures per $\mathbf{~ 1 0}^{\mathbf{9}} \mathbf{p}$ & Error (stat.) & Fraction (\%) \\
\hline${ }^{92} \mathrm{Mo}$ & 373.0 & 54 & 1.66 \\
${ }^{94} \mathrm{Mo}$ & 325.4 & 51 & 1.45 \\
${ }^{95} \mathrm{Mo}$ & 13357.1 & 326 & 59.53 \\
${ }^{96} \mathrm{Mo}$ & 3246.0 & 161 & 14.47 \\
${ }^{97} \mathrm{Mo}$ & 2341.3 & 136 & 10.44 \\
${ }^{98} \mathrm{Mo}$ & 2015.9 & 126 & 8.98 \\
${ }^{100} \mathrm{Mo}$ & 777.8 & 79 & 3.47 \\
\hline
\end{tabular}

\subsubsection{Systematic errors}

Table 34 summarizes all the systematic error contributions to the measurement of the ${ }^{98} \mathrm{Mo}$ capture rate and to the Monte Carlo calculation.

Table 34: Sources of systematic errors in the measurement of the number of captures on ${ }^{98} \mathrm{Mo}$ and in their simulation

\begin{tabular}{l|c|c}
\hline Error source & $\begin{array}{c}\text { Error on error } \\
\text { Source }\end{array}$ & $\begin{array}{c}\text { Resulting error on } \\
\mathbf{9 8} \text { Mo captures }\end{array}$ \\
\hline Beam intensity & $5 \%$ & $5 \%$ \\
Ge acceptance (source and systematics of calibration) & $6.4 \%$ & $6.4 \%$ \\
Molybdenum amount & $0.2 \%$ & $0.2 \%$ \\
\hline Total common experimental & & $\mathbf{8 . 1 \%}$ \\
\hline Peak finding and statistics & $2.6 \%$ & $2.6 \%$ \\
Sample position in Ge system & $1.1 \mathrm{~mm}$ & $2.6 \%\left(\mathrm{D}_{1}\right)$ \\
Br. ratio, Ge acceptance, absorption & $5 \%$ text & neglig. \\
$T_{0}$ measurement & $15 \mathrm{~s}$ & $\mathbf{7 \%}$ \\
\hline Total individual experimental & & $\mathbf{1 0 . 7 \%}$ \\
\hline Total experimental & - & $\mathbf{7 \%}$ \\
\hline Monte Carlo 98 Mo capture cross-section(**) & $7 \%$ & $7 \%$ \\
\hline Monte Carlo statistics \& others (***) & & $\mathbf{9 . 9 \%}$ \\
\hline Total Monte Carlo & & \\
\hline
\end{tabular}

(*) Error estimated from spread of activity measurements for various $\gamma$ lines in ${ }^{99}$ Mo decays, which includes part of the first two sources of errors, hence the estimated total of $6 \%$.

(**) As our estimate of the error due to the uncertainty on the neutron capture cross-section on ${ }^{98}$ Mo we take into account the spread of capture rates obtained with the various databases (Fig. 130).

(***) The Monte Carlo error quoted here includes statistical errors and estimates on the error on geometry, impurity contents, method, etc. We do not include the error which comes from the simulation of the neutron flux, but only errors belonging to the simulation of molybdenum properties. From TARC flux measurements we know that the agreement between simulated flux and data is better than $10 \%$ (Fig. 37) on average in the energy range of interest. 


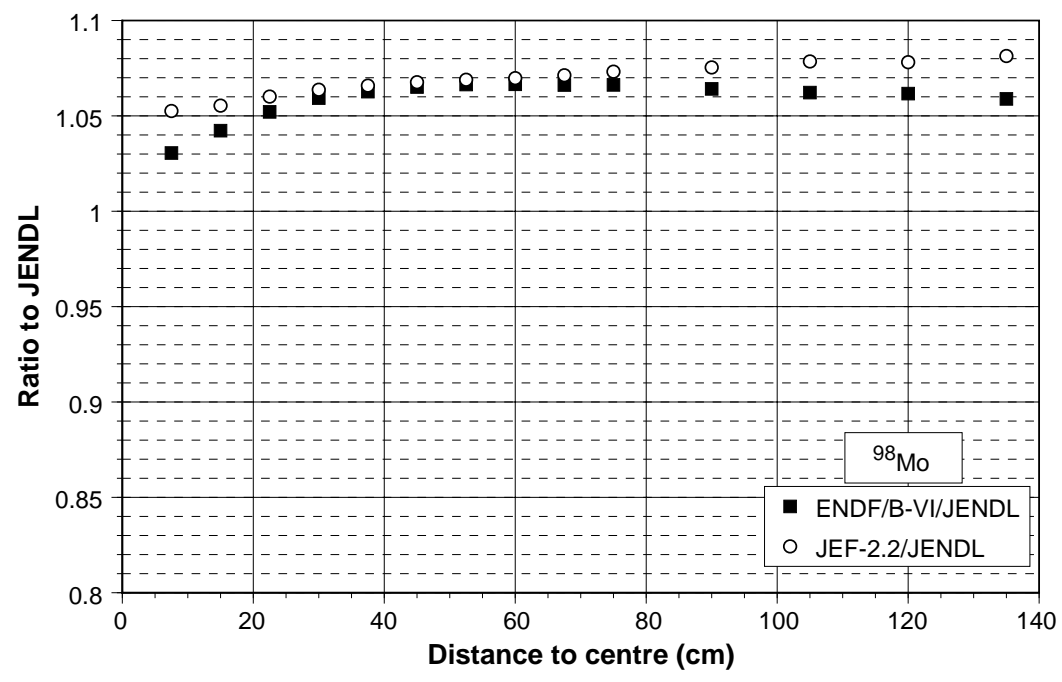

Fig. 130: Ratio of numbers of ${ }^{98}$ Mo captures obtained from various data libraries (ENDF/B-VI \& JEF-2.2) to the JENDL number, as a function of distance to the centre of the lead volume.

\subsubsection{Comparison with the data}

The ${ }^{98}$ Mo measured capture rate $\left(1785 \pm 191\right.$ per $10^{9}$ protons) is well consistent with the prediction (2016 \pm 199 per $10^{9}$ protons) from the TARC simulation (Fig. 131). The average ratio data/Monte Carlo is $0.89 \pm 0.13$.

As for all TARC neutron capture measurements, in the Monte Carlo uncertainty, there is in addition an overall contribution coming from how well the neutron flux is simulated (mainly due to the uncertainty on the spallation process and on the lead cross-sections, see Section 5). This additional uncertainty is about $15 \%$. This makes our measurement clearly more precise than the prediction.

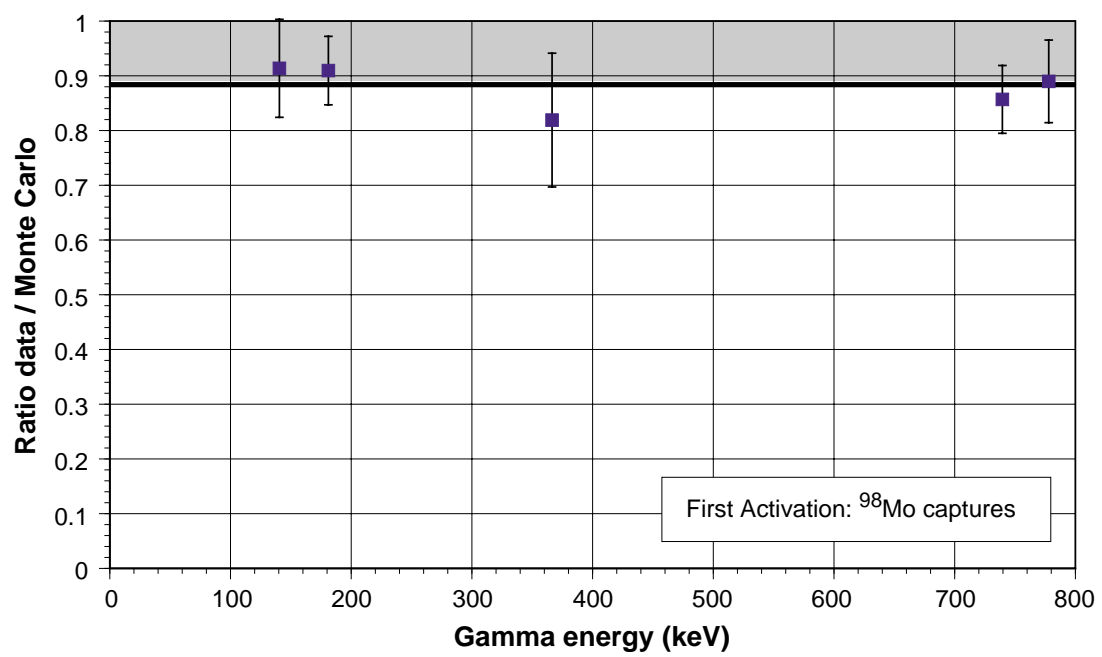

Fig. 131: Comparison between the observed capture rate on ${ }^{98} \mathrm{Mo}$ and the simulation (hole 10, $z=+7.5 \mathrm{~cm}$ ). The values are compared for each of the ${ }^{99} \mathrm{Mo}$ gamma lines used in the analysis. The error bars shown represent the statistical errors of both data and Monte Carlo simulation. The thick horizontal line is the average ratio. The band shows the total common error $(0.11)$ centred on 1 . 


\subsubsection{Various checks on the data}

We have used the various Ge counting measurements, made after a high-intensity irradiation, to verify the quality of some of the basic data used in this analysis. The Ge counting data can be used to study the production of ${ }^{99 \mathrm{~m}} \mathrm{Tc}$ and obtain information on the half-life of the various elements involved.

Using for instance the $181 \mathrm{keV}$ line of ${ }^{99} \mathrm{Mo}$, we can fit the average observed counting time as a function of the age of the sample. We get $t_{1 / 2}=65.7 \mathrm{~h}$ (Fig. 132), in excellent agreement with the $65.94 \mathrm{~h}$ given in the NNDC/BNL library.

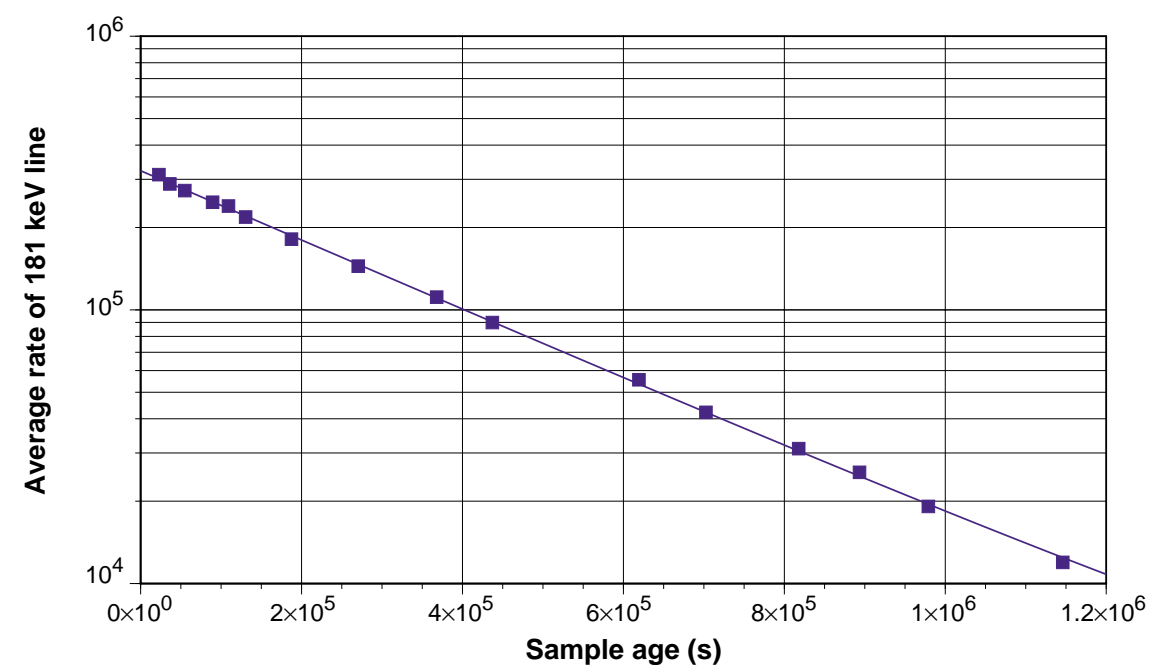

Fig. 132: Determination of the ${ }^{99} \mathrm{Mo}$ half-life, using the $181 \mathrm{keV}$ line. The average counting time is plotted versus the sample age at beginning of counting.

It is clear that the $140.5 \mathrm{keV}$ line is mainly produced by ${ }^{99 \mathrm{~m}} \mathrm{Tc}$. We can estimate the number of ${ }^{99 \mathrm{~m}} \mathrm{Tc}$ atoms present in the sample as a function of time (Fig. 133). The maximum number of ${ }^{99} \mathrm{~m} \mathrm{Tc}$ atoms occurs at time $t_{\max } \sim 5$ hours, given by:

$$
t_{\max }=\frac{\ln \left(\frac{\lambda_{2}}{\lambda_{1}}-\frac{\lambda_{2}\left(\lambda_{2}-\lambda_{1}\right) n_{2}(0)}{\lambda_{1}^{2} n_{1}(0) \mathrm{Br}}\right)}{\lambda_{2}-\lambda_{1}}
$$

and is nicely consistent with what we observe (Fig. 133). The $191 \mathrm{keV}$ line of ${ }^{101}$ Mo was used to obtain a check of the ${ }^{101}$ Mo half-life (Fig. 134). We find $t_{1 / 2}=14 \min 45 \mathrm{~s} \pm 20 \mathrm{~s}$ to be compared to $14 \mathrm{~min} 37 \mathrm{~s}$ given in the NNDC/BNL database.

\subsubsection{Conclusion}

We have verified that ${ }^{99} \mathrm{~m}$ Tc could be produced simply by activation of natural molybdenum producing ${ }^{99}$ Mo from ${ }^{98}$ Mo.

The overall activity of natural molybdenum after activation is dominated by the production of ${ }^{99} \mathrm{Mo}$; the isomers other than ${ }^{98} \mathrm{Mo}$ induce only a small contribution to the overall activity (mainly from ${ }^{101} \mathrm{Mo}$ ).

The rate of neutron capture on ${ }^{98} \mathrm{Mo}$, hence of production of ${ }^{99 \mathrm{~m}} \mathrm{Tc}$, is found to be consistent with the TARC simulation prediction and therefore validates this simulation for this particular process. 


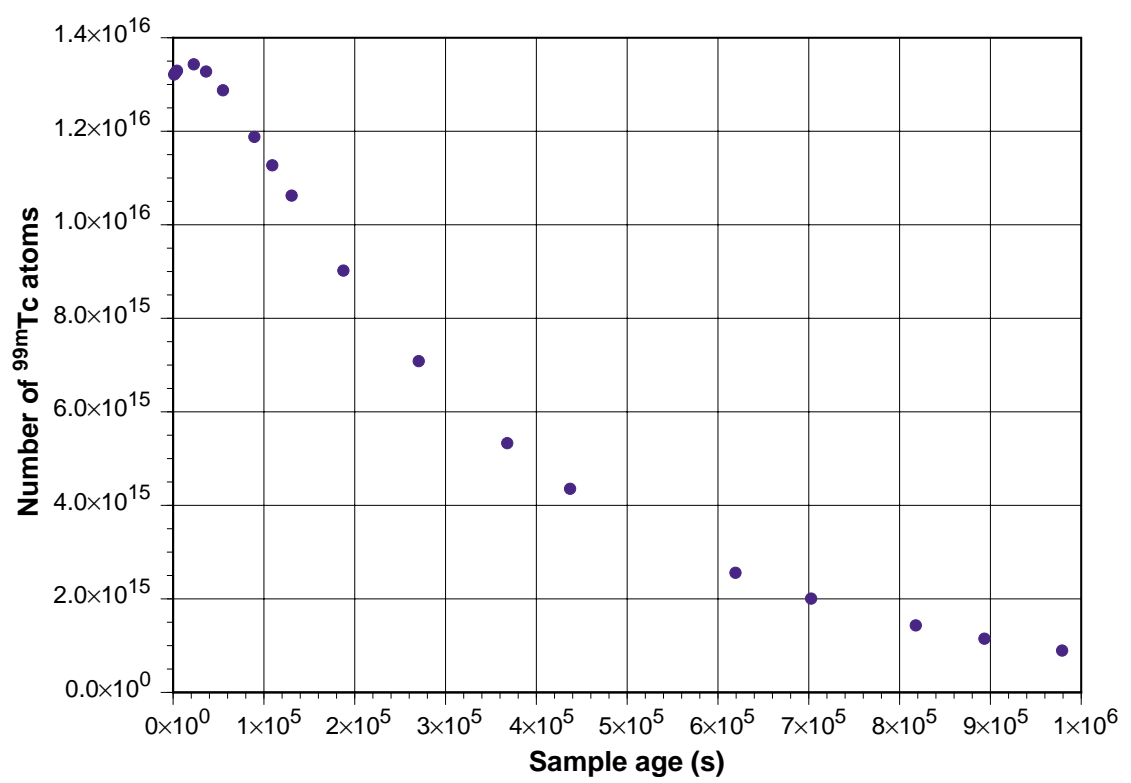

Fig. 133: Number of ${ }^{99 \mathrm{~m}} \mathrm{Tc}$ atoms present in the sample, as a function of time, following the third activation.

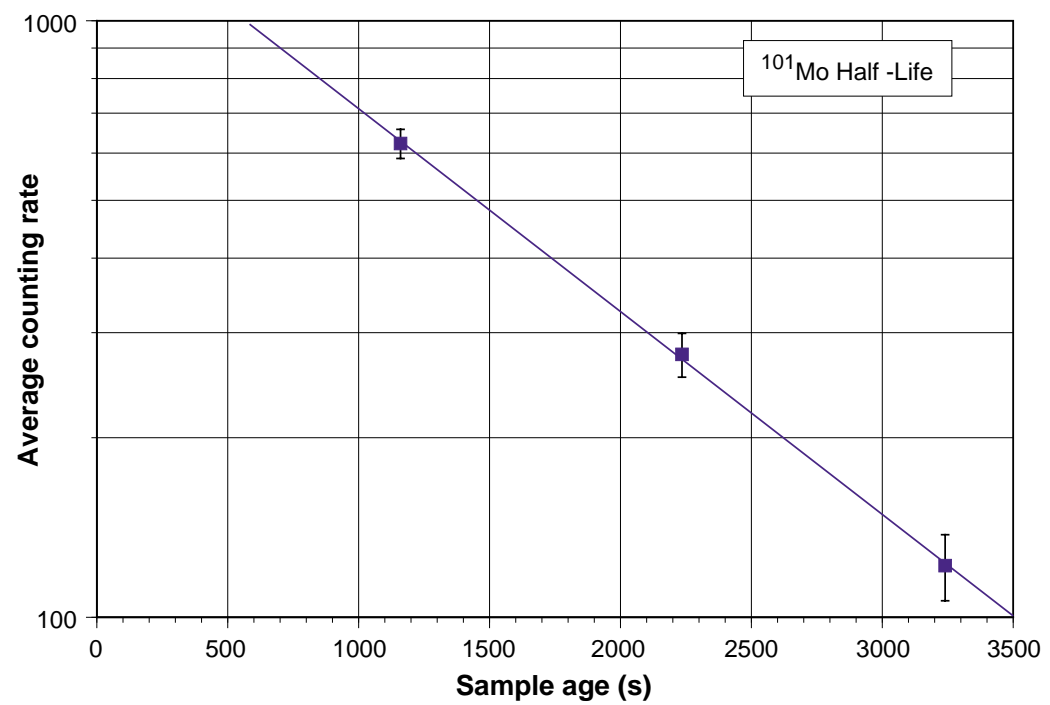

Fig. 134: Determination of ${ }^{101}$ Mo half-life, using the $191 \mathrm{keV}$ line and data from activation number 3.

\section{Energy Deposition in Lead by Neutrons}

\subsection{Introduction}

When a proton beam hits the TARC lead volume, a large number of particles, mainly neutrons, but also photons and charged particles are produced. These particles move around in the lead inducing different scattering processes, some of them producing energy that heats up the lead. The amount of heat generation by neutrons is normally much smaller than the heat deposited by the protons through cascade and ionization, but it is nevertheless very interesting both from the characterization of the spallation and diffusing cascades and from the practical point of view as a direct measurement of total energy deposited at different positions close to the spallation target. Calorimetric measurements of such energy deposition in a lead sample placed inside the TARC assembly were performed in August-September 1996. The energy, E, 
was obtained by a calorimetric method in adiabatic conditions: by measuring the temperature increase, $\Delta T$, exhibited by a sample with known heat capacity, $C(T)$, then:

$$
E=C(T) \Delta T ; \text { assuming } \Delta T<<T \text {. }
$$

At room temperature, $\Delta T$ values are expected to be only some tens of microkelvin for a total heating time of one hour [84]. Therefore, the measurements had to be performed at liquid-helium temperature (about $4 \mathrm{~K}$ ) since the specific heat of lead decreases rapidly as the temperature is lowered. At $4 \mathrm{~K}$ the specific heat of lead is 180 times smaller than the value at room temperature, so the $\Delta T$ values should typically be a few millikelvin. Our experimental set-up (Fig. 135) achieved a temperature resolution of $0.2 \mathrm{mK}$ which allowed reliable measurements of the energy deposition.

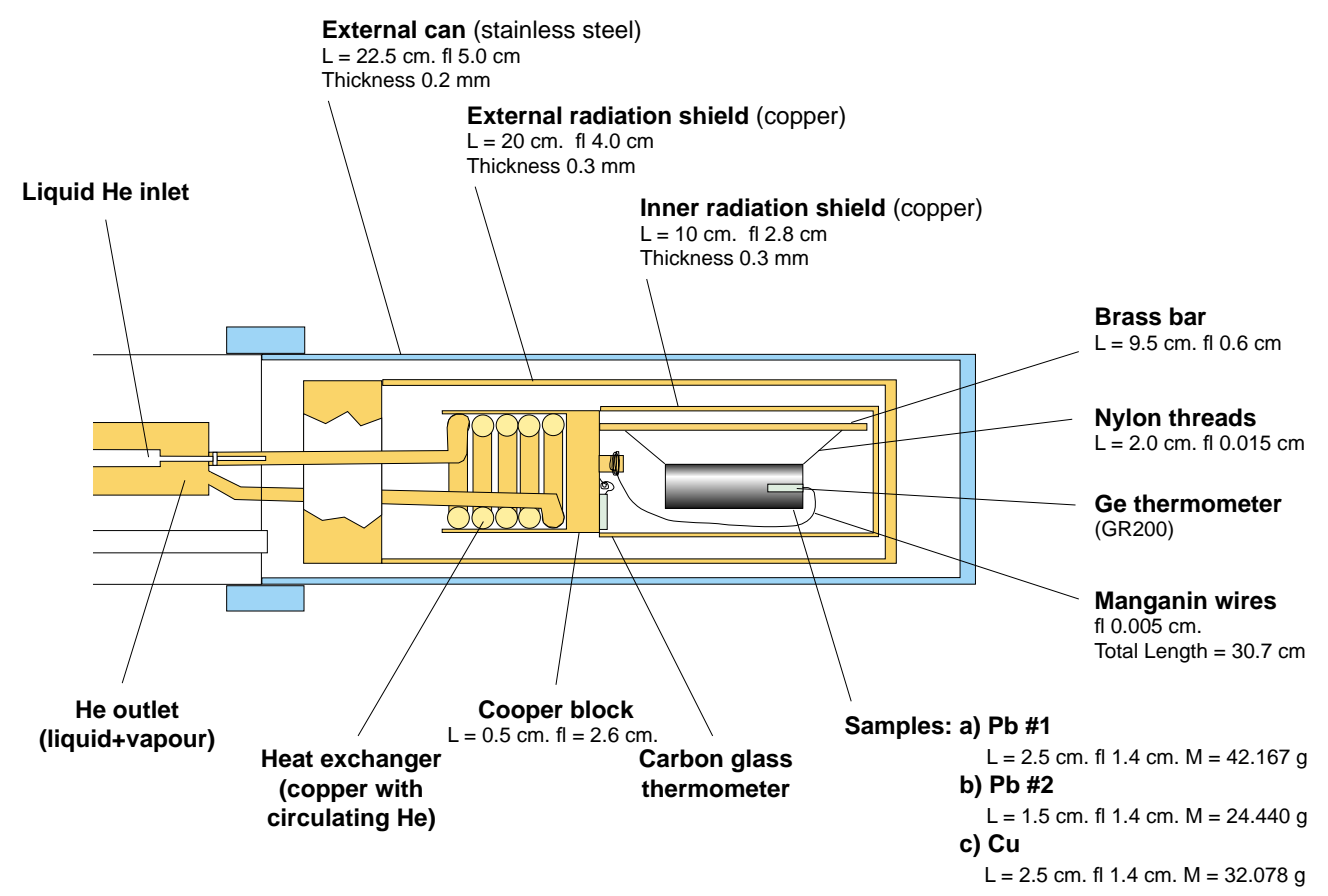

Fig. 135: Schematic description of the different parts and materials of the cryostat.

\subsection{Experimental set-up}

A home-made cryostat (Fig. 135) designed and built in the Laboratorio de Bajas Temperaturas of the Universidad Autónoma de Madrid was used in the measurements. The cryostat is part of a continuous flow system of liquid ${ }^{4} \mathrm{He}$. Liquid helium is transferred from the helium dewar into the cryostat through a double jacket siphon. The helium gas produced by evaporation in the cryostat returns through the external jacket of the siphon, thus refrigerating it. The flux and base temperature are controlled with the aid of a pump and a needle valve. Typical working flows are of $1.25 \mathrm{\ell} / \mathrm{h}$. It takes several hours for the sample to reach low enough temperatures. The base temperature in the experiment was about $4.15 \mathrm{~K}$. Temperature fluctuations of some tenths of kelvin with a period of some tens of seconds can appear in the bath, although normally, the stability is better than $2 \mathrm{mK}$.

As the measurements are performed under adiabatic conditions, it is necessary to get high-quality vacuum; using a turbomolecular pump the level reached was typically $10^{-8}$ bar. Two radiation shields made of copper prevent thermal radiation exchange with the surroundings, the inner one is in good thermal contact with the bath at $4 \mathrm{~K}$. 
The sample hangs from an eccentric brass bar by two nylon threads to keep it under adiabatic conditions; the cooling power of these threads is less than $20 \mathrm{nW} / \mathrm{K}$. The inner shield is also used to cool the sample down to the copper shield temperature $(\sim 4.2 \mathrm{~K})$ : by rotating the whole cryostat, the sample can be put in thermal contact with it. The thermometer is placed inside a hole made in the sample, and clamped by a screw. Its manganin leads are clamped to the thermal bath container at different places. They are finally fixed to the sample with varnish to avoid erroneous measurements of temperature. The cooling power of the leads is less than $20 \mathrm{nW} / \mathrm{K}$. The thermometer Joule heating power is less than $10 \mathrm{nW}$. Two different thermometers were used: a doped germanium chip and a carbon glass resistance. Both are mounted strain-free in a gold plated copper can, with internal atmosphere of ${ }^{4} \mathrm{He}$ and sealed by an epoxy heat sink.

In these quasi-adiabatic conditions, the leak time of the energy between the bath at $4.5 \mathrm{~K}$ to the sample is more than 20 hours. For this reason, the fast temperature fluctuations that eventually appear in the bath are not seen by the sample. So, even when the bath temperature is not very stable, good calorimetric results can be obtained. The resolution in the temperature of the sample is about $0.2 \mathrm{mK}$. As the specific heat of lead at $4 \mathrm{~K}$ is $7 \times 10^{-4} \mathrm{~J} / \mathrm{K} / \mathrm{g}$, this allows one to measure energy productions of the order of $200 \mathrm{~nJ} / \mathrm{g}$.

The specific heat of the sample was measured between $4 \mathrm{~K}$ and $8 \mathrm{~K}$. The estimated error in the region of interest, i.e. below $6 \mathrm{~K}$, is $3 \%$. During the calibration procedure the conditions inside the TARC assembly were simulated: the sample was heated by Joule dissipation pulses in an electrical resistance, which was in good thermal contact with the sample. The amount of energy of these pulses was fixed at similar values to those expected from prior Monte Carlo simulation results.

\subsection{Analysis of the data}

The measurements were mostly performed under adiabatic conditions, although a non-adiabatic experiment was also done. The results in these different conditions are consistent. In what follows, we will distinguish between quasi-adiabatic and non-adiabatic measurements.

\subsubsection{Analysis of the data in quasi-adiabatic conditions}

In the adiabatic experiments we measure the temperature of the sample when protons are injected into the lead assembly, and also both before and after the proton injection period. In Fig. 136, curve 1 shows the evolution of the temperature of the sample versus time in these three regimes. The two small kinks at about 0.57 hours and 1.34 hours, corresponding respectively to the start and end of the beam activation, show that the beam is indeed heating the sample. Nevertheless, even when there is no beam, the temperature changes smoothly. A typical rate in our case was $+0.05 \mathrm{~K}$ per hour.

This indicates the presence of an extra source of heat whose power is about $400 \mathrm{nW}$. This value is bigger than the heating power due to neutrons and other particle interactions in most of the measurements. In the calibration procedure it was realized that the heating rate was proportional to the intensity of mechanical vibrations. These vibrations cannot be reduced to zero, because some of them come from the flux of helium inside the cryostat. The temperature increase of the sample at time $t$ is then:

$$
\Delta T=T(t)-T(0)=\Delta T_{\mathrm{p}}(t)+\Delta T_{\mathrm{v}}(t) .
$$



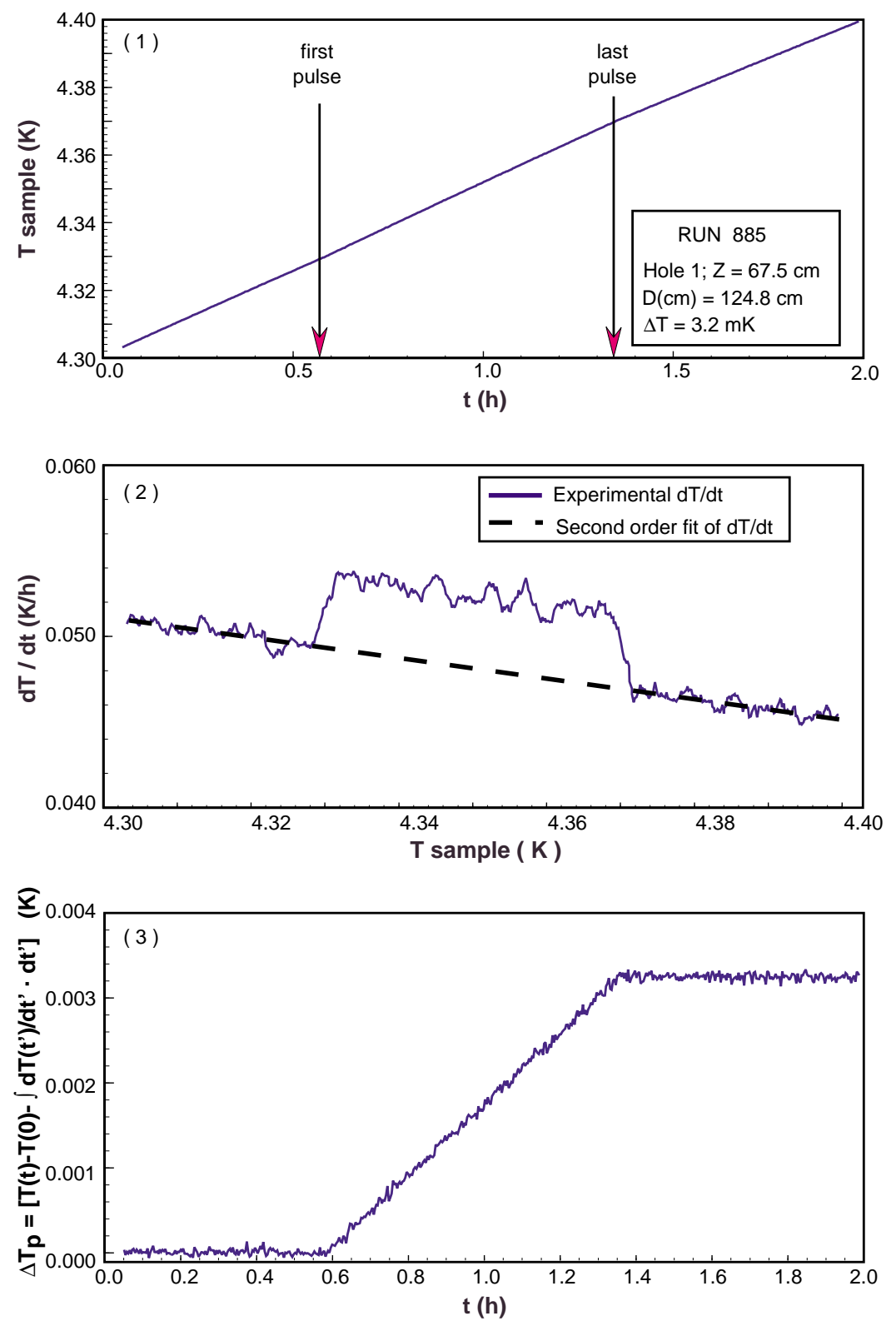

Fig. 136: Analysis of data for an adiabatic measurement: (1) Typical time evolution of the temperature of the sample; (2) Heating rate versus temperature. The dashed line is a second-order polynomial fit of the derivative using the data when the beam is off; (3) Increase in temperature due to the energy released when the proton beam is turned on, after subtraction of the general temperature drift.

$\Delta T_{\mathrm{p}}(t)$ corresponds to the proton pulses, $\Delta T_{\mathrm{v}}(t)$ is due to mechanical vibrations. The net extra heating power seen in the experiment depends on various parameters: the difference in temperature between the bath and the sample, the position of the sample, the mechanical vibrations, the vacuum, and so on.

All these parameters change in every measurement. To obtain $\Delta T_{\mathrm{p}}$ it is necessary to determine and subtract $\Delta T_{\mathrm{v}}$. We have used the following expression for the time evolution of the temperature of the sample:

$$
T(t)=T(0)+\int_{0}^{t} \frac{\mathrm{d} T}{\mathrm{~d} t^{\prime}} \mathrm{d} t^{\prime}
$$


When the beam is off, $\frac{\mathrm{d}\left(T_{\mathrm{p}}(t)\right)}{\mathrm{d} t}=0$ at any time, and $\Delta T_{\mathrm{v}}(t)$ is simply:

$$
\Delta T_{\mathrm{V}}(t)=\int_{0}^{t} \frac{\mathrm{d} T_{\mathrm{OFF}}}{\mathrm{d} t^{\prime}} \mathrm{d} t^{\prime}
$$

where $\mathrm{d} T_{\mathrm{OFF}} / \mathrm{d} t$ means the heating rate when there is no beam. Then, using relation (2) the increase of temperature due to the beam is:

$$
\Delta T_{\mathrm{p}}(t)=T(t)-T(0)-\int_{0}^{t} \frac{\mathrm{d} T_{\mathrm{OFF}}}{\mathrm{d} t^{\prime}} \mathrm{d} t^{\prime} .
$$

In principle the derivative $\mathrm{d} T_{\mathrm{OFF}} / \mathrm{d} t$ is smooth and continuous with $T$. When the beam is off $\mathrm{d} T_{\mathrm{OFF}} / \mathrm{d} t$ can be obtained numerically.

Then, for new situations when the beam is off, it can easily be determined by fitting; on the other hand, for situations with the beam on, the value can be obtained by interpolation. This procedure, i.e. the fit of the heating rate $\mathrm{d} T_{\mathrm{OFF}} / \mathrm{d} t$ when the beam is off versus $T$, is shown in Fig. 136, curve 2. Since $T$ depends on time, $\mathrm{d} T_{\mathrm{OFF}} / \mathrm{d} t=\mathrm{d} T_{\mathrm{OFF}}(T(t)) / \mathrm{d} t$ also depends on time, and the integral in Eq. (75) has to be done numerically.

If we plot the result of this correction against time, we obtain directly the temperature increase of the sample due to the proton source, see Fig. 136, curve 3. In this figure, the goodness of the fit is explicit: before and after the proton pulse interval

$$
\frac{\mathrm{d}\left(\Delta T_{\mathrm{p}}(t)\right)}{\mathrm{d} t} \text { is zero as expected. }
$$

Knowing the specific heat of the sample and the number of protons supplied, we can calculate the energy deposited per gram and per proton using Eq. (72).

\subsubsection{Analysis of the data in non-adiabatic conditions}

It is possible to measure the energy even with the sample in thermal contact with the bath. If the beam is on, the sample temperature rises up to a value determined not only by the energy losses in the sample, but also by the thermal exchange between sample and bath. This depends on the contact area and mass of the sample as main parameters.

Eventually, the sample reaches equilibrium and its temperature is stable. When the proton source stops, the temperature decreases and, following this decay, we can extract the power supplied to the sample. If the cooling power of the contact is linear with the difference of temperatures between bath and sample, $\Delta T(t)$ should be exponential in time. This is a good approximation because the temperature difference is less than $0.1 \%$ :

$$
\Delta T(t)=\Delta T_{0} \exp (-t / \tau) \text { in which } \tau=\mathrm{C} \Delta T_{0} / P,
$$

where $C$ is the heat capacity of the sample and $P$ the heating power of the proton source. The value $C$ is known and $P$ can be obtained by fitting of the experimental data. Since $P$ and the number of protons per second are known, the energy deposited per gram and per proton can be derived.

\subsection{Experimental results}

In all the measurements the protons had a kinetic energy of $2.75 \mathrm{GeV}$ and the beam was provided in the fast extraction mode. The beam intensity was measured by two beam transformers with an absolute precision of 5\%, as already explained in previous sections. 


\subsubsection{Adiabatic measurements}

Measurements were performed in four different holes, at several $z$ positions (Table 35). Three different samples and two different thermometers were used. This was done to test the existence of heating sources different from the protons, and to check possible systematic effects.

Table 35: Experimental results

\begin{tabular}{|c|c|c|c|c|c|c|c|c|c|c|}
\hline $\begin{array}{c}\text { Adia- } \\
\text { batic } \\
\text { data } \\
\text { sample }\end{array}$ & $\begin{array}{l}\text { Thermom. } \\
\text { /screw }\end{array}$ & Run & Hole & $\begin{array}{c}z \\
(\mathbf{c m})\end{array}$ & $\begin{array}{c}D \\
(\mathrm{~cm})\end{array}$ & $\begin{array}{c}T \\
\text { average } \\
(\mathbf{K})\end{array}$ & $\begin{array}{c}C_{\mathrm{p}} \times 10^{4} \\
(\mathrm{~J} / \mathrm{g} / \mathrm{K})\end{array}$ & $\begin{array}{c}\Delta T \\
(\mathbf{m K})\end{array}$ & $\begin{array}{c}\text { No. of } \\
\text { protons } \\
\left(10^{12}\right)\end{array}$ & $\begin{array}{c}E \times 10^{8} \\
(\mathrm{~J} / \mathrm{g}) \text { for } \\
10^{10} \mathrm{p} \\
\Delta E= \\
6 \%\end{array}$ \\
\hline $\mathrm{Big} \mathrm{Pb}$ & Ge/Brass & $\begin{array}{l}763 \\
764 \\
765\end{array}$ & $\begin{array}{c}10 \\
3 \\
3\end{array}$ & $\begin{array}{l}20.0 \\
20.0 \\
20.0\end{array}$ & $\begin{array}{l}49.2 \\
25.0 \\
25.0\end{array}$ & $\begin{array}{l}4.492 \\
4.442 \\
4.531\end{array}$ & $\begin{array}{c}9.77 \\
9.38 \\
10.12\end{array}$ & $\begin{array}{c}5.5 \\
33.0 \\
36.5\end{array}$ & $\begin{array}{l}0.831 \\
0.635 \\
0.743\end{array}$ & $\begin{array}{l}6.48 \\
48.8 \\
49.7\end{array}$ \\
\hline $\mathrm{Big} \mathrm{Pb}$ & $\mathrm{Ge} / \mathrm{Al}$ & $\begin{array}{l}878 \\
866 \\
867 \\
868 \\
869 \\
870 \\
871 \\
872 \\
874 \\
875 \\
877 \\
\\
881 \\
882 \\
883 \\
884 \\
885 \\
\\
886 \\
887 \\
888 \\
890\end{array}$ & $\begin{array}{l}3 \\
3 \\
3 \\
3 \\
3 \\
3 \\
3 \\
3 \\
3 \\
3 \\
3 \\
\\
6 \\
6 \\
6 \\
6 \\
1 \\
\\
10 \\
10 \\
10 \\
10\end{array}$ & $\begin{array}{c}113.0 \\
67.5 \\
52.5 \\
45.0 \\
37.5 \\
30.0 \\
22.5 \\
15.0 \\
7.5 \\
0.0 \\
-6.3 \\
\\
67.5 \\
22.5 \\
7.5 \\
-5.7 \\
67.5 \\
\\
37.5 \\
22.5 \\
7.5 \\
-7.2\end{array}$ & $\begin{array}{c}114.0 \\
69.1 \\
54.6 \\
47.4 \\
40.4 \\
33.5 \\
27.0 \\
21.2 \\
16.8 \\
15.0 \\
16.3 \\
\\
90.3 \\
64.1 \\
60.5 \\
60.3 \\
124.8 \\
\\
58.6 \\
50.3 \\
45.6 \\
45.6\end{array}$ & $\begin{array}{l}4.372 \\
4.423 \\
4.317 \\
4.442 \\
4.418 \\
4.337 \\
4.460 \\
4.483 \\
4.445 \\
4.439 \\
4.479 \\
\\
4.309 \\
4.435 \\
4.352 \\
4.336 \\
4.349 \\
4.325 \\
4.494 \\
4.354 \\
4.322\end{array}$ & $\begin{array}{l}8.85 \\
9.23 \\
8.46 \\
9.38 \\
9.19 \\
8.59 \\
9.53 \\
9.71 \\
9.41 \\
9.36 \\
9.68 \\
\\
8.95 \\
9.33 \\
8.70 \\
8.59 \\
8.66 \\
8.51 \\
9.80 \\
8.72 \\
8.49\end{array}$ & $\begin{array}{c}4.4 \\
20.5 \\
31.5 \\
26.0 \\
47.4 \\
66.2 \\
77.1 \\
86.0 \\
97.8 \\
128.9 \\
115.5 \\
\\
5.0 \\
7.1 \\
8.2 \\
9.2 \\
3.2 \\
\\
9.1 \\
6.8 \\
9.3 \\
11.3\end{array}$ & $\begin{array}{l}1.628 \\
1.814 \\
1.579 \\
1.157 \\
1.581 \\
1.594 \\
1.649 \\
1.501 \\
1.429 \\
1.586 \\
1.440 \\
\\
1.611 \\
1.478 \\
1.508 \\
1.622 \\
2.060 \\
1.496 \\
1.094 \\
1.200 \\
1.382\end{array}$ & $\begin{array}{l}2.39 \\
10.4 \\
16.9 \\
21.1 \\
27.6 \\
35.7 \\
44.6 \\
55.6 \\
64.4 \\
76.0 \\
77.6 \\
\\
2.79 \\
4.48 \\
4.73 \\
4.87 \\
1.35 \\
\\
5.18 \\
6.09 \\
6.76 \\
6.94\end{array}$ \\
\hline $\mathrm{Big} \mathrm{Pb}$ & $\mathrm{C} / \mathrm{Al}$ & $\begin{array}{l}943 \\
944\end{array}$ & $\begin{array}{l}3 \\
3\end{array}$ & $\begin{array}{l}67.5 \\
113\end{array}$ & $\begin{array}{c}69.1 \\
114.0\end{array}$ & $\begin{array}{l}4.366 \\
4.404\end{array}$ & $\begin{array}{l}8.80 \\
9.09\end{array}$ & $\begin{array}{l}6.6 \\
3.2\end{array}$ & $\begin{array}{l}0.579 \\
1.231\end{array}$ & $\begin{array}{c}10.05 \\
2.36\end{array}$ \\
\hline $\begin{array}{c}\text { Small } \\
\mathrm{Pb}\end{array}$ & $\mathrm{Ge} / \mathrm{Al}$ & 954 & 10 & 37.5 & 58.6 & 4.303 & 8.36 & 15.5 & 2.385 & 5.44 \\
\hline $\mathrm{Cu}$ & $\mathrm{Ge} / \mathrm{Cu}$ & $\begin{array}{l}892 \\
917\end{array}$ & $\begin{array}{l}10 \\
10\end{array}$ & $\begin{array}{l}67.5 \\
37.5\end{array}$ & $\begin{array}{l}81.1 \\
58.6\end{array}$ & $\begin{array}{l}4.819 \\
5.062\end{array}$ & $\begin{array}{l}1.37 \\
1.53\end{array}$ & $\begin{array}{l}8.6 \\
2.3\end{array}$ & $\begin{array}{l}0.153 \\
0.035\end{array}$ & $\begin{array}{c}7.7 \\
10.2\end{array}$ \\
\hline $\begin{array}{c}\text { Non } \\
\text { Adia- } \\
\text { batic } \\
\text { data } \\
\text { sample }\end{array}$ & $\begin{array}{l}\text { Thermom. } \\
\text { /screw }\end{array}$ & Run & Hole & $\begin{array}{c}z \\
(\mathbf{c m})\end{array}$ & $\begin{array}{c}D \\
(\mathbf{c m})\end{array}$ & $\begin{array}{c}T \\
\text { average } \\
(\mathrm{K})\end{array}$ & $\begin{array}{c}C_{\mathrm{p}} \times 10^{4} \\
(\mathrm{~J} / \mathrm{g} / \mathrm{K})\end{array}$ & $\begin{array}{c}\Delta T \\
(\mathbf{m K})\end{array}$ & $\begin{array}{c}\text { No. of } \\
\text { protons } \\
\left(\mathbf{1 0}^{12}\right)\end{array}$ & $\begin{array}{c}E \times 10^{8} \\
(\mathrm{~J} / \mathrm{g}) \text { for } \\
10^{10} \mathrm{p} \\
\Delta E= \\
6 \%\end{array}$ \\
\hline $\mathrm{Big} \mathrm{Pb}$ & $\mathrm{Ge} / \mathrm{Brass}$ & 760 & 10 & 20.0 & 49.2 & 4.129 & 3.10 & 1.15 & 7.99 & 6.33 \\
\hline
\end{tabular}


The first lead sample, of $42.167 \mathrm{~g}$, was measured with both thermometers. No discrepancy is seen although germanium and carbon have very different heat cross-sections. The extra heating supplied by the germanium or carbon in the thermometers is negligible. There is no difference between this sample and the second (small) lead sample of $24.440 \mathrm{~g}$. Heating supplied by the varnish, contact grease, manganin leads, copper can or nylon threads by heat conduction can be neglected, because all these elements were the same in both samples.

There is an exponential dependence of the deposited energy with radial distance (Fig. 137). This dependence is suddenly lost for hole 3 near $z=0$. Nevertheless, if we add $4 \mathrm{~cm}$ to the assumed $z$ values, and plot the energy versus $D^{\prime}=\sqrt{x^{2}+y^{2}+(z+4 \mathrm{~cm})^{2}}$, the exponential dependence is recovered for the whole data set (Fig. 137). The decay distances obtained from the exponential fits are: $59 \mathrm{~cm}, 53 \mathrm{~cm}$ and $30 \mathrm{~cm}$ for holes 6,10 and 3, respectively. Hole 3, the closest to the beam, shows a behaviour significantly different from that of the other holes, indicating extra contributions for the energy release in this region of the lead volume (probably the spallation cascade). A spherical symmetry is inferred from the data obtained far away from the beam axis as the measurements in holes 6 and 10 follow a similar radial dependence. At large distances, all curves seem to converge. This is consistent with the fact that far away from the centre of the lead volume, the quasi-spherical behaviour has to be recovered for all directions.

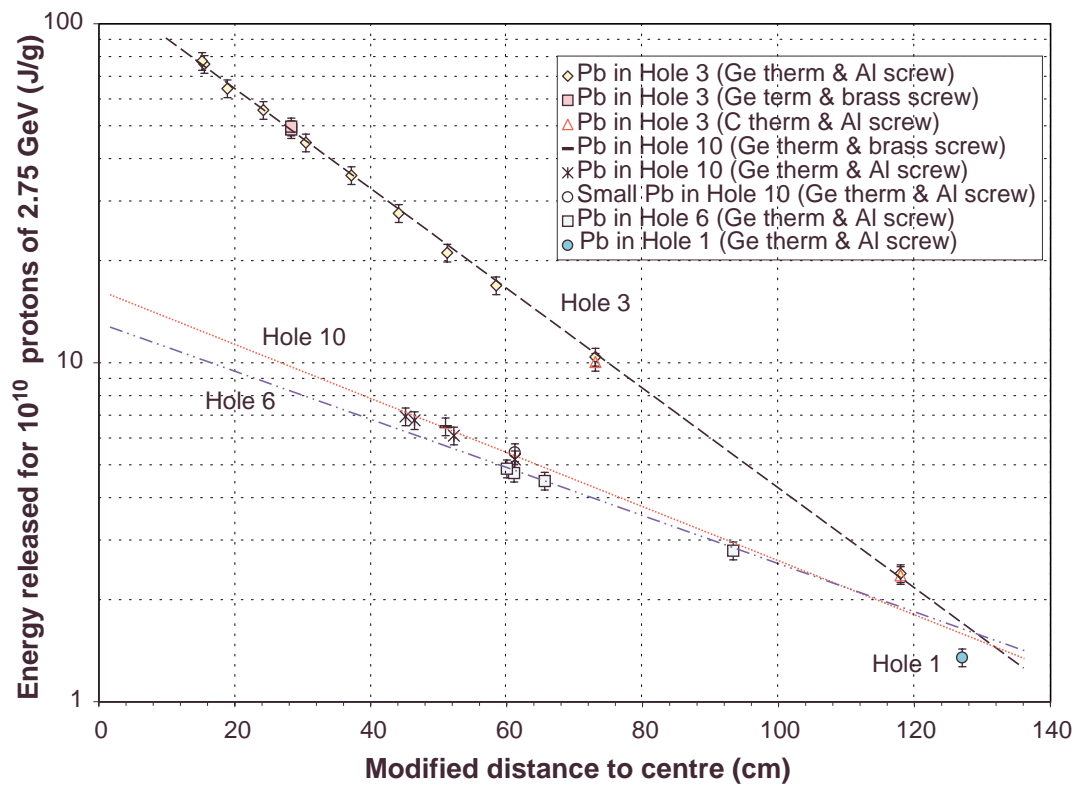

Fig. 137: Experimental data obtained at several holes and $z$ positions. The released energy is plotted versus radial distance to the centre after adding $4 \mathrm{~cm}$ to the assumed $z$ coordinates. Different symbols correspond to different experimental configurations.

Lead becomes superconducting below $7.19 \mathrm{~K}$. This fact is reflected in the specific heat, which is lower than the linear extrapolation of normal lead properties down to $4 \mathrm{~K}$. We do not think that superconductivity had other major influences in the results. Any possible perturbation is relaxed in a few milliseconds, because the temperature increases are small and the pulses are very short. Temperature measurement times are in the range of seconds, so we always measure the temperature when the sample is in equilibrium.

Copper has a much larger heat cross-section. Copper is present in the germanium thermometer, in the screw used to clamp the thermometer, and in the manganin wires: about $150 \mathrm{mg}$ in all. It could then have a significant contribution to the observed energy released in 
the sample. Two measurements were performed in hole 10 with a copper sample (Fig. 137). The specific energy dissipation measured in copper is twice that measured in lead. But, as the mass of copper is 200 times smaller than the lead mass, its effect can be neglected.

To ensure an optimal thermal contact between the sample and the thermometer, the thermometer lead is pressed with a screw. Unfortunately, lead is too soft to be used for this purpose. We have used screws of aluminium and brass $\left({ }^{70} \mathrm{Cu},{ }^{30} \mathrm{Zn}\right)$ of $70 \mathrm{mg}$. There is no appreciable difference in the measurements done with both materials. As the predicted heating for aluminium is much smaller than that of copper, we conclude that the screw has no relevant influence on the measurements. In the case of the copper sample, a copper screw was used. In summary, it is found that the influence of probes in the measured energy can be neglected.

The precision in the determination of positions is about $\pm 0.3 \mathrm{~cm}$. The accuracy in the measurement of the increase of temperature is $0.2 \mathrm{mK}$. The specific heat data have estimated errors of around $3 \%$. The uncertainty on the beam intensity is $5 \%$. It follows that the precision in the determination of the specific energy in the adiabatic measurements is typically $6 \%$. Nevertheless, when the increase in temperature is only of a few millikelvin, the error rises to $10 \%$.

\subsubsection{Contact or non-adiabatic measurements}

The analysis of the non-adiabatic measurement is illustrated in Fig. 138. The evolution of the bath temperature [Fig. 138(a)] shows that the stability of temperature is within about $2 \mathrm{mK}$. In these continuous flux systems there are also fast fluctuations of a few tenths of millikelvin. The evolution of the sample temperature [Fig. 138(b)] shows clearly the turning off and on of the beam as sudden heating or cooling. Four of these jumps can be seen.

The difference of $2 \mathrm{mK}$ between sample and bath is due to usual calibration errors in absolute temperature. However, the sensitivity in the measurement of relative temperature changes is better than $0.2 \mathrm{mK}$.

We have subtracted the thermal drift in the bath from the sample temperature in order to obtain the heating due only to the beam [Fig. 138(c)]. As the thermal contact is far from perfect (in this case the relaxation time of the sample heat is about 3 minutes), the thermal noise in the bath is damped in the sample. Anyway, if we compare the sample and bath temperatures, we see that the temperature in the sample follows the overall evolution of the bath temperature.

From the exponential fit, the relaxation time is $\tau=167.2 \mathrm{~s}$. Using Eq. (77), the thermal conductivity is then $K=C p M \tau^{-1}=1.86 \times 10^{-4} \mathrm{~W} / \mathrm{K}$. The heating power is $P=K \Delta T_{0}=2.13$ $\times 10^{-7} \mathrm{~W}$. Finally, the energy deposited per gram and per $10^{10}$ protons is $E=P M^{-1} N_{\mathrm{ps}}{ }^{-1}=$ $6.3310^{-8} \mathrm{~J} / \mathrm{g} / 10^{10} \mathrm{p}$. Where $N_{\mathrm{ps}}$ is the number of protons per second, in units of $10^{10} \mathrm{p} / \mathrm{s}$. This measurement was done in hole 10 at $z=20 \mathrm{~cm}$. After comparing with the data obtained in quasi-adiabatic conditions in the same position $\left(E=6.48 \times 10^{-8} \mathrm{~J} / \mathrm{g} / 10^{10} \mathrm{p}\right)$, we conclude that the agreement between the two methods is quite reasonable.

There are some disadvantages in the non-adiabatic measurements. First, as the heating observed is always smaller than in the adiabatic case, measurement precision is worse. Only for positions close to the centre of the lead assembly can the temperature increase be resolved with sufficient accuracy. Secondly, as the sample is in mechanical contact with the bath, instabilities can be generated in the sample temperature by both temperature fluctuations and external vibrations. 

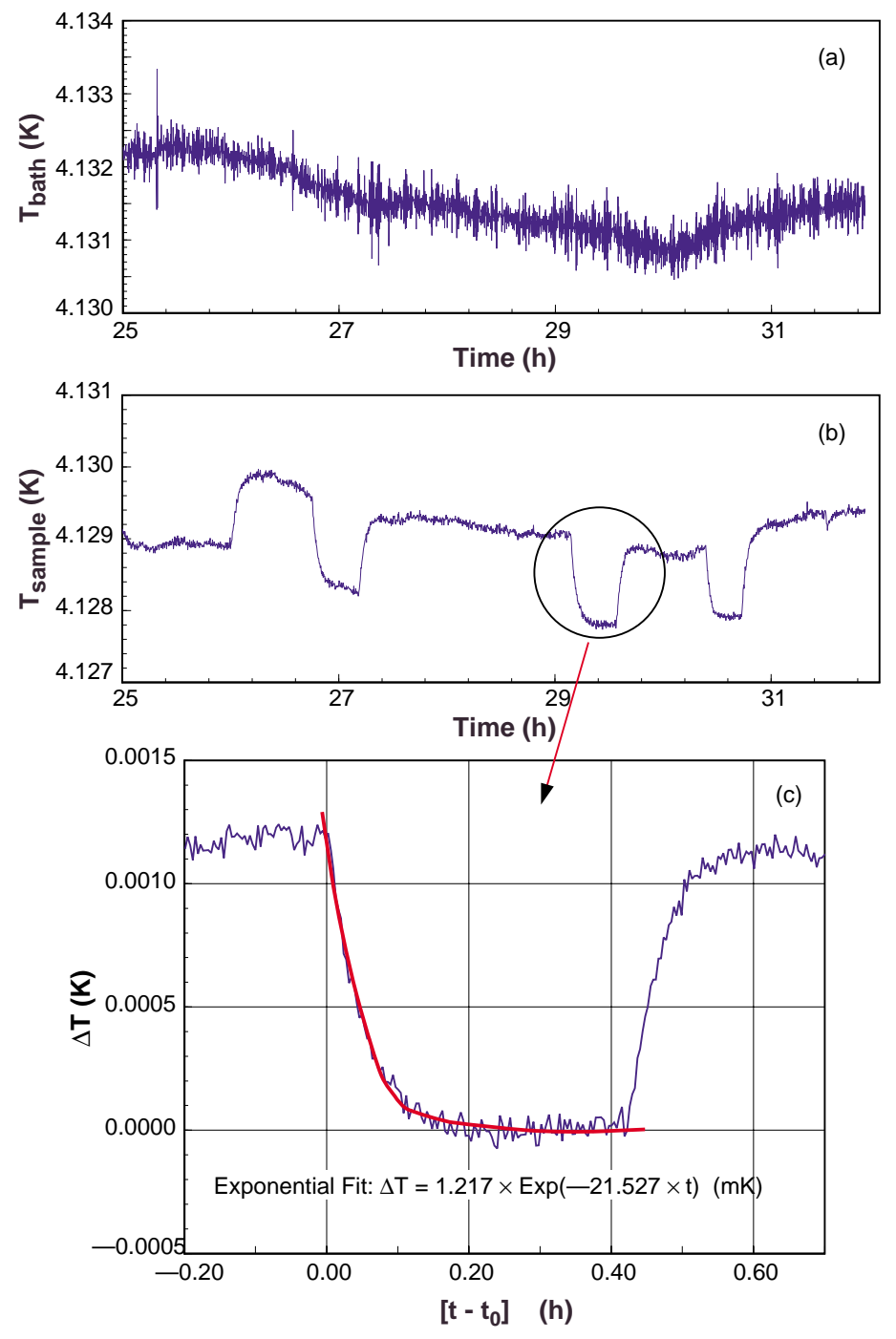

Fig. 138: Non-adiabatic measurement data: (a) Typical time evolution of the temperature of the bath; (b) Time evolution of the temperature of the sample. (Jumps and falls are due to the start and stop of the proton beam.); (c) Exponential fit to the data during one of the sudden falls in temperature. The characteristic time of the exponential fit is the relaxation time of the heat from sample to bath.

\subsection{Monte Carlo simulations}

To compare with the experimental results, a simulation relying on a full Monte Carlo procedure was used. The $2.75 \mathrm{GeV}$ proton beam interacting with the lead volume, the spallation cascade produced, the neutron transport of this cascade through the lead until the thermometer is reached, and the transport of photons, electrons, and positrons produced in the nuclear interactions are fully simulated by the FLUKA code [20].

The energy releases obtained fit the experimental data well within the error bars. This good agreement validates this Monte Carlo simulation's ability to simulate energy depositions.

Special simulations have also been made to study the effects of the surrounding materials on

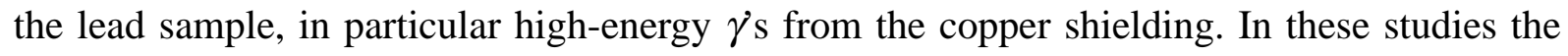
thermometer is simplified to just the lead sample in an empty hole. Figure 139 shows the result of these simulations. Despite the remaining large statistical uncertainty in the Monte Carlo simulation the effect is found to be small. 


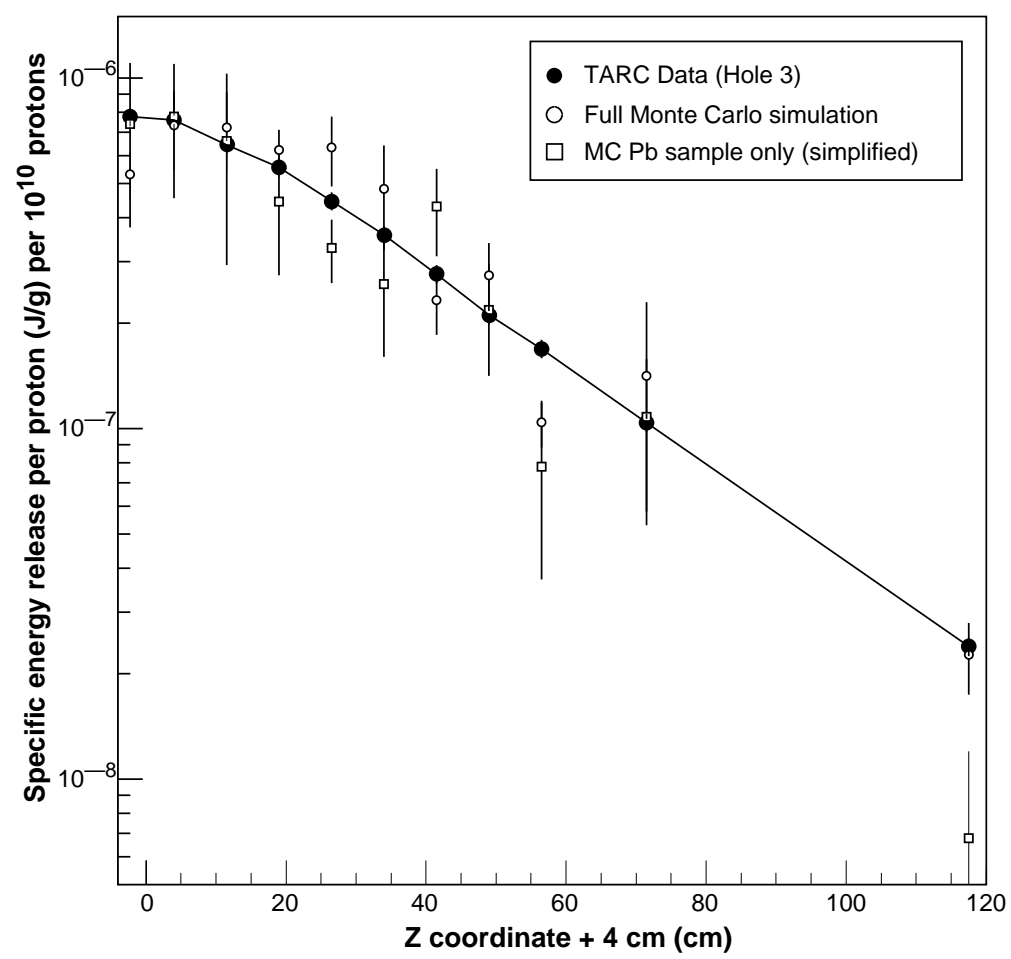

Fig. 139: Comparison of the FLUKA Monte Carlo results with the experimental data for hole 3 . Both the full geometry and the $\mathrm{Pb}$ only simulations are shown.

\subsection{Conclusion}

We have found that the designed thermometer is a highly precise instrument for the measurement of small amounts of deposited energies: its resolution is better than $200 \mathrm{~nJ}$. We exploited the fact that specific heats decrease with decreasing temperature. Going down to liquid-helium temperature, we were able to measure temperature changes that are equivalent to only a few microkelvin at room temperature.

The energy deposited in a small lead sample placed in different holes at several $z$ positions has been measured. The measurements were performed in quasi-adiabatic and non-adiabatic conditions, the results from both methods are found to be in good agreement. The influence of the probes (thermometers, screws or leads) is found to be negligible as a result of measurements taken with different experimental configurations. The results obtained are in good agreement with the FLUKA Monte Carlo simulation.

Hole 3, the one closest to the beam, shows a behaviour different from that of the other holes, indicating extra contributions to the energy release in this region of the lead volume (probably the spallation cascade). A spherical symmetry for long distances seems a good approximation to our data. After shifting by $4 \mathrm{~cm}$ the value of the $z$ parameter, the dependence of energy deposition with radial distance becomes exponential for each hole.

The agreement between experimental data and Monte Carlo results validates the physical parameters used in the simulation. This same validation applies when the program is used to study the energy released in lead by the neutron cascade in the Energy Amplifier. 


\section{Practical scheme for an Incineration Device Based on ARC}

In a system such as the Energy Amplifier (EA) [2] where the TRansUranian elements (TRU) are destroyed by fission, as part of the fuel [3], the long-term radiotoxicity of the waste is dominated by that of long-lived fission fragments (LLFFs). Most of the discussion in this section is reported in more detail in Ref. [4].

We have studied specially ${ }^{99} \mathrm{Tc}$ and ${ }^{129} \mathrm{I}$ which represent about $95 \%$ of the long-term radiotoxicity due to fission fragments

After having developed the necessary tools (the simulation described in Section 3) and collected the necessary facts (TARC data), we are in a position to assess the performance of a practical device to be used for the elimination of LLFFs such as ${ }^{99} \mathrm{Tc}$ and ${ }^{129} \mathrm{I}$. The proposed scenario is mainly based on the fact that outside the core of an Energy Amplifier [2], the neutron flux exhibits the same isolethargic behaviour as in TARC and that neutrons in that area have only a small probability to return to the EA core and be useful for inducing fission. Therefore, in principle, LLFF transmutation could be accomplished in a parasitic mode, without affecting the performance of the EA, implying a minimized cost for the process.

The Monte Carlo simulation validated by TARC has been used to describe the time evolution of neutron fluxes and element compositions in the EA. The EA is cooled with molten lead, which surrounds the core. In this otherwise empty volume (apart from lead), the conditions described for the transmuter [4] develop naturally. This is evidenced by the neutron flux spectra shown in Fig. 140, plotted for various distances above the core considering a small cylindrical volume coaxial to the core and about 1 metre away from the axis. The first five spectra correspond to different vertically segmented levels of the core, starting from the medium plane and rising each time by $15 \mathrm{~cm}$. One can observe a very hard spectrum, which is required for instance in order to fission the TRUs. The subsequent five spectra (6-10) correspond to different vertically segmented levels in the lead surrounding the core, in steps of $40 \mathrm{~cm}$. All spectra are average spectra over the vertical bin. The spectra in the surrounding lead show the characteristic flattening due to the isolethargic condition and enrich dramatically the part of the spectrum which is relevant to transmutation (1-1000 eV). In segments 8 and 9 we have introduced a small, distributed contamination of ${ }^{99} \mathrm{Tc}$ at the density of $2.686 \mathrm{mg} / \mathrm{cm}^{3}$ equivalent to a mass concentration of only $260 \mathrm{ppm}$ with respect to lead. The prominent capture lines corresponding to the leading ${ }^{99} \mathrm{Tc}$ resonances correspond to a strong absorption as indicated by the large drop of the neutron flux at the resonance crossing.

The simulation program was used to study the time evolution of the burning process inside the EA and the subsequent reactions in the transmuter (Fig. 141). The concentration of relevant elements as a function of the burn-up in the EA was obtained for segment $8\left(0.409 \mathrm{~m}^{3}\right)$ in which the ${ }^{99} \mathrm{Tc}$ doping is inserted initially. One can observe directly the large transformation rate of the ${ }^{99} \mathrm{Tc}$ into stable element ${ }^{100} \mathrm{Ru}$, followed by small capture rates into ${ }^{101} \mathrm{Ru}$ and finally ${ }^{102} \mathrm{Ru}$. We remark that all the indicated ruthenium isotopes are stable ${ }^{11}$.

11. The subsequent elements which may be produced by successive captures are also favourable: ${ }^{103}$ Ru decays in $39.3 \mathrm{~d}$ into ${ }^{103} \mathrm{Rh}$ which is stable, ${ }^{104} \mathrm{Ru}$ is stable, while ${ }^{105} \mathrm{Ru}$ decays quickly into the stable ${ }^{105} \mathrm{Pd}$. Also ${ }^{106} \mathrm{Pd}$ is stable, the first long-lived isotope being ${ }^{107} \mathrm{Pd}$, which has a half-life of $6.5 \times 10^{6}$ years. However, its production rate is negligible, taking into account that as many as eight successive neutron captures must occur in the same nucleus to reach ${ }^{107} \mathrm{Pd}$. 
The decay constant for transmutation of ${ }^{99} \mathrm{Tc}$ is about $82 \mathrm{GW} \times$ day/ton (Fig. 141), corresponding to less than three years for the nominal EA power $(1.0 \mathrm{GW}$, thermal). These curves demonstrate the feasibility of elimination of technetium in the periphery of an EA with a reasonable time constant.

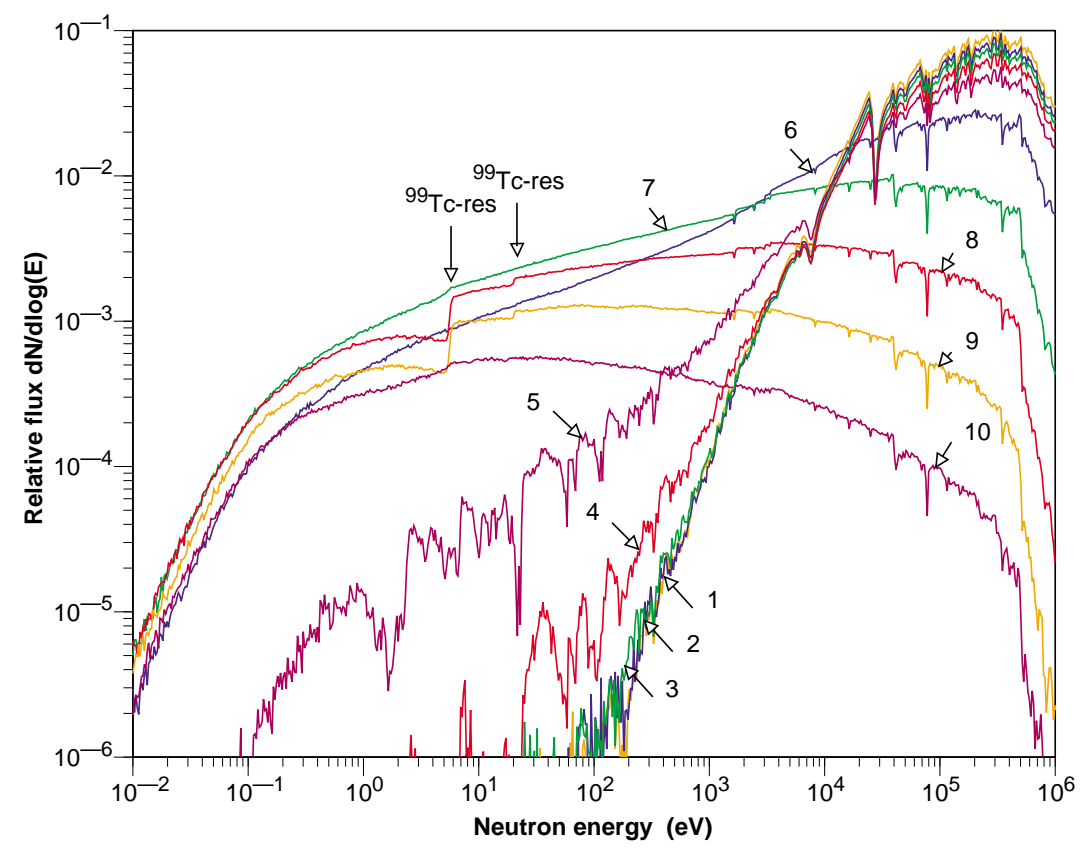

Fig. 140: Neutron spectra plotted at various distances above the EA core for a small cylindrical volume coaxial to the core and about 1 metre away from the axis. The first five spectra (1-5) correspond to different vertically segmented levels of the core, starting from the medium plane and rising each time by $15 \mathrm{~cm}$. The subsequent five spectra (6-10) correspond to different vertically segmented levels in the lead surrounding the core, in steps of $40 \mathrm{~cm}$. In segments 8 and 9 a small, diffused contamination of ${ }^{99} \mathrm{Tc}$ at the density of $2.686 \mathrm{mg} / \mathrm{cm}^{3}$ was introduced.

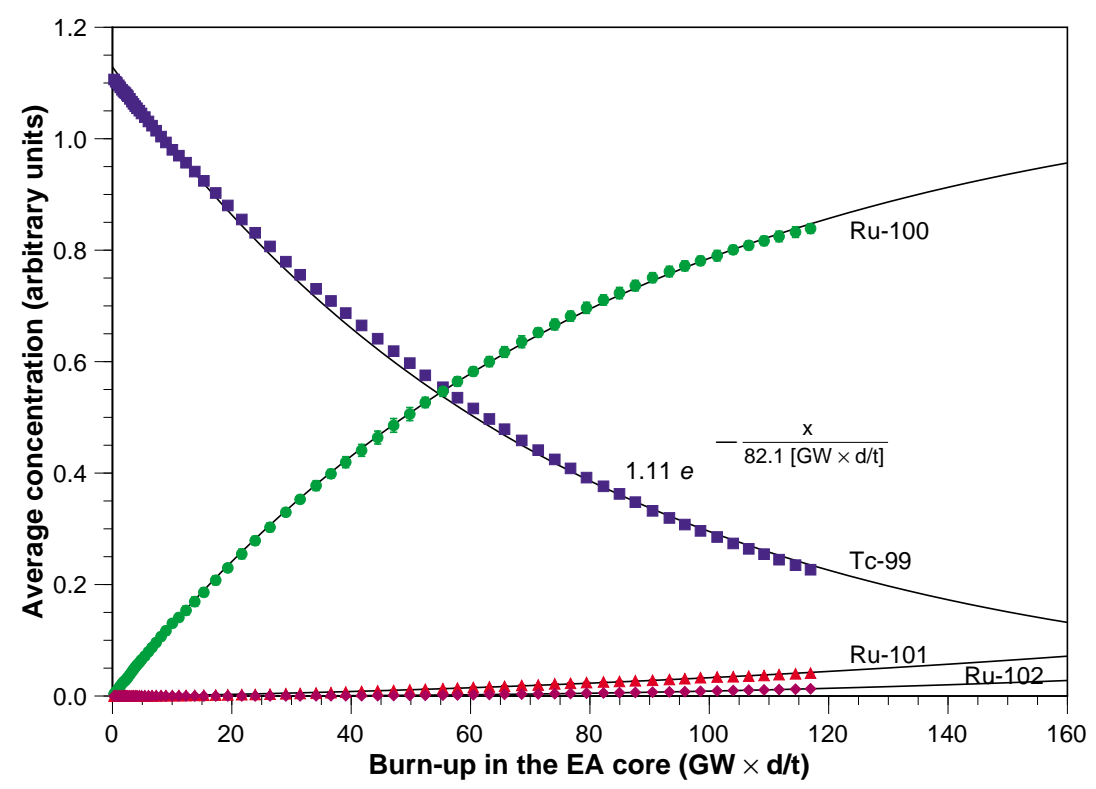

Fig. 141: Concentration of relevant elements as a function of burn-up in segment 8 of Fig. 140. While the ${ }^{99} \mathrm{Tc}$, initially with a density of $2.686 \mathrm{mg} / \mathrm{cm}^{3}$, is rapidly transmuted with a $1 / e$ constant of $82 \mathrm{GW} \mathrm{d} / \mathrm{t}$, the daughter element ${ }^{100} \mathrm{Ru}$ builds up correspondingly. Captures in this second element produce ${ }^{101} \mathrm{Ru},{ }^{102} \mathrm{Ru}$, etc. which are stable and are a small contribution to the stockpile. 
Incidentally, we also remark that if the materials to be transmuted were inserted in the core directly, the transmutation rate would be much smaller, since the neutron flux in the core is concentrated at energies where captures by LLFFs have a small probability.

The performance of the waste transmuter is exemplified in the case of the ${ }^{99} \mathrm{Tc}$ and ${ }^{129} \mathrm{I}$.

For instance, the EA simulation now validated by TARC predicts that, in a $1 \mathrm{GW}_{\text {th }} \mathrm{EA}$, after a burn-up of $100 \mathrm{GW}_{\mathrm{th}} \times \mathrm{d} /$ ton and with an initial load of $270 \mathrm{~kg}$ of ${ }^{99} \mathrm{Tc}$ uniformly diluted in lead at a relative weight concentration of $10^{-3}$ in a dedicated volume of $22.9 \mathrm{~m}^{3}$. The average transmutation rate is $12.3 \mathrm{~kg} / \mathrm{GW}_{\text {th }} \times$ year [4] to be compared to a production rate of $7.5 \mathrm{~kg} / \mathrm{GW} \times$ year in the EA.

A similar simulation was performed for the case of iodine. With a relative weight concentration $1.8 \times 10^{-3}$ for ${ }^{129} \mathrm{I}$ corresponding to an initial mass of $430 \mathrm{~kg}$ one obtains a ${ }^{129} \mathrm{I}$ transmutation rate of $8.8 \mathrm{~kg} / \mathrm{GW}_{\text {th }} \times$ year to be compared to a production rate in the EA of $3.5 \mathrm{~kg} / \mathrm{GW}_{\text {th }} \times$ year.

The fact that the process can take place outside the EA core, in a region where neutrons have a small probability to return to the core and have little chance to contribute to fissions, makes it possible to envisage LLFF transmutation in a parasitic mode, thus minimizing the cost of the process. We find it a very attractive idea to use the available neutrons outside the EA core to reduce the radiotoxicity produced in the overall fuel cycle.

The high confidence level in the proposed scheme is the main consequence of the TARC experiment where a large coherent set of neutron fluence data, over eight orders of magnitude in neutron energies (from thermal to $2 \mathrm{MeV}$ ) and the many measurements performed with ${ }^{99} \mathrm{Tc}$ and ${ }^{129}$ I throughout the lead volume have demonstrated that Adiabatic Resonance Crossing can be used effectively and have validated the innovative simulation used here.

We can conclude that, on the basis of the TARC results, it appears possible to destroy, outside the EA core and in a parasitic mode, very large amounts of ${ }^{99} \mathrm{Tc}$ or ${ }^{129} \mathrm{I}$ at a rate exceeding the production rate, thereby making it practical to reduce correspondingly the existing stockpile of LLFFs.

\section{General conclusion of the TARC study}

We have obtained with TARC a large coherent set of neutron fluence data over eight orders of magnitude in neutron energies (from thermal to $2 \mathrm{MeV}$ ). In the energy region below $10 \mathrm{keV}$, this was done with three different detector techniques, each with an absolute precision of the order of $15 \%$. The spatial distribution of the neutron fluence within the lead volume has been mapped in detail over distances of up to $1.9 \mathrm{~m}$ from the centre.

At low energies (below $\sim 10 \mathrm{keV}$ ) and up to a radius of $1.5 \mathrm{~m}$, the neutron fluence, in good approximation, has spherical symmetry and behaves approximately in the same way as if produced by a point-like source, even though neutron production through the spallation process is neither point-like nor isotropic. This is, of course, precisely what is expected from the small elastic collision length in lead and also from the fact that the lead volume is sufficiently large that edge effects do not constrain the shape of the neutron cloud. The $\mathrm{d} F / \mathrm{d}[\ln (E)]$ distribution decreases very slowly, only one order of magnitude when the neutron energy decreases by four orders of magnitude (from $10 \mathrm{keV}$ to $1 \mathrm{eV}$ ). This is the first important element in the demonstration of the efficiency of ARC. 
The many transmutation measurements performed with ${ }^{99} \mathrm{Tc},{ }^{129} \mathrm{I}$, and ${ }^{127} \mathrm{I}$ throughout the lead volume provide self-consistency checks (reproducibility of measurements, control of systematics) and confirm the validity of ${ }^{99} \mathrm{Tc}$ and ${ }^{129} \mathrm{I}$ cross-section data. From the excellent agreement with the simulation, we conclude that the ARC effect is well understood.

Every effort was made to control carefully the systematics of the various measurements to provide the best possible experimental precision. This implied great care in the construction of the lead assembly, the development of detectors using state-of-the-art techniques and, throughout the experiment, a systematic use of redundant techniques to allow internal cross-checks of the results.

The unique and comprehensive ensemble of measurements reported here, taken under well-controlled conditions, constitutes a very precise validation of the innovative simulation developed by the TARC Collaboration, with which practical schemes for the transmutation of LLFFs were studied. These data will be useful for benchmarking any new simulation needed for the development of future nuclear systems and for related systems such as for the disposal of nuclear waste.

On the basis of the TARC results, it appears possible to destroy, outside the EA core and in a parasitic mode, large amounts of ${ }^{99} \mathrm{Tc}$ or ${ }^{129} \mathrm{I}$ at a rate exceeding the production rate, thereby making it practical to reduce correspondingly the existing stockpile of LLFFs.

The TARC experiment also demonstrates the possibility of producing radioactive elements in particular for medical applications (as shown directly with the ${ }^{127}$ I and natural molybdenum activation measurements), with an accelerator-driven activator, which constitutes an attractive alternative to the production with nuclear reactors.

Several additional physics measurements were carried out as part of the TARC programme as a by-product of the main goal of the experiment. Some of them improve or verify previous nuclear data, others bring into light a number of potential problems. We conclude, for instance, that ${ }^{232} \mathrm{Th}(\mathrm{n}, \gamma),{ }^{186} \mathrm{~W}(\mathrm{n}, \gamma)$ and ${ }^{237} \mathrm{~Np}$ (fission) cross-sections should be re-measured with precision. Measurements concerning the properties of ${ }^{99} \mathrm{Tc},{ }^{100} \mathrm{Tc},{ }^{233} \mathrm{~Pa}$ and ${ }^{99} \mathrm{Mo}$ were performed. The cross-section for ${ }^{99} \mathrm{Tc}(\mathrm{n}, \gamma)$ was measured up to $1 \mathrm{keV}$, as well as the ratio isomer to ground state in the production of ${ }^{130} \mathrm{I}$ in the reaction: ${ }^{129} \mathrm{I}(\mathrm{n}, \gamma)$. Finally, TARC is providing an indirect measurement of spallation neutron production by $2.5 \mathrm{GeV} / c$ ( 60 neutrons per proton) and $3.57 \mathrm{GeV} / c$ (98 neutrons per proton) protons in the thick TARC lead target.

The understanding of the physics related to the exploitation of a high-energy proton beam as an intense source of spallation neutrons and the unique features of 'free' neutron diffusion in a high- $\mathrm{Z}$ medium of very high transparency, both arising from the TARC experiment, have led to the concept and design of a world-wide unique neutron source at CERN, the Time-Of-Flight Facility (TOF). The uniqueness of TOF lies in the availability of a very high neutron flux, of a broad energy range exceeding eight orders of magnitude, and of an unprecedented energy resolution over the whole energy range. These novel features provide great potential for modern experimentation with neutrons, exploiting new ideas in nuclear ADS technology whilst also covering new ground in the fields of nuclear physics, nuclear astrophysics, and nuclear medicine. Its excellent energy resolution and event rates allow practically all cross-section resonances to be resolved as well as permitting the systematic study of relevant neutroninduced reactions in the energy interval between $1 \mathrm{eV}$ to above $250 \mathrm{MeV}$, of almost any nuclide using targets of very modest mass (few milligrams), as needed for radioactive or 
expensive materials such as transuranics and medium to long-lived fission fragments. The TARC experiment showed that ${ }^{232} \mathrm{Th}(\mathrm{n}, \gamma),{ }^{186} \mathrm{~W}(\mathrm{n}, \gamma)$ and ${ }^{237} \mathrm{~Np}$ (fission) cross-sections should obviously be a priority for the TOF facility at CERN. The TOF project makes the sophisticated measuring techniques and the accelerator and infrastructure of CERN accessible to the whole European nuclear data community.

\section{Acknowledgements}

We would like to thank the DGXII of the European Commission for their strong financial support (contract F141-CT96-009) and M. Hugon for his excellent co-ordination of the cluster on P \& T strategy studies and transmutation experiments. We would like to acknowledge the support of the other funding institutions without which this experiment would not have been possible: Centre National de la Recherche Scientifique and IN2P3 in France, Empresa Nacional de Residuos Radiactivos S.A., Comisión Interministerial de Ciencia y Tecnología and the Agrupación Eléctrica para el Desarrollo de la Tecnología Nuclear in Spain.

The TARC experiment was performed successfully and very efficiently thanks to the enthusiastic support of the CERN PS Division. We would like to thank more specifically J.-P. Riunaud, R. Cappi, and L. Durieux. The PS transport group (M. Genolin and his team) did a magnificent job in transporting and helping us assemble our 580 lead blocks. The structure for supporting the lead was designed with the help of N. Dudragne. The PS survey group (L. Grandclement and his group) aligned perfectly the 334 tons of the lead assembly on the beam axis. We are particularly sad to have learned that L. Grandclement passed away recently, and that while he was working for us, he already knew that his days were numbered, nevertheless this did not dampen his enthusiasm towards this experiment. The preparation and organization of the T7 experimental area was done with the precious help of B. Williams and R. Coccoli, both of whom gave us very useful practical advice. Special thanks go to G. Gelato and C. Carter with whom we managed to break the world record for the precision of the beam intensity measurement. We must thank also J. Bergoz who lent us a new version of beam transformer which we commissioned for this experiment.

The TIS Commission also provided us with extremely valuable help ranging from advice regarding the handling of radioactive material to the preparation of special calibration sources. Our thanks go in particular to J. Tuyn, G. Roubaud, J. Wolf, A. Muller and N. Conan.

For the collection of data we used and appreciated the expertise from the CASCADE group in the ECP Division, from whom we obtained an 'off-the-shelf' data acquisition system which turned out to be well adapted to our purpose and also reliable.

M. Schneegans from LAPP provided the $\mathrm{CeF}_{3}$ scintillator and A. Leprêtre from CEA Saclay provided some of the ${ }^{99} \mathrm{Tc}$ targets. A. Ferrari and P. Sala made the latest version of FLUKA available to us, and provided precious help with some aspects of the simulation. We thank Z.X. Zhao from CIAE for the evaluation of the ${ }^{99} \mathrm{Tc}(\mathrm{n}, \gamma)$ cross-section.

We would also like to thank the staff of the CERN Desktop Publishing Service for their help in the preparation of this paper.

Unfortunately, we cannot name here all the many people who contributed in their own way to the success of this experiment, either directly or indirectly, but we wish to thank them all very warmly.

We dedicate this report to the memory of our colleague Alcibiades Apostolakis. 


\section{References}

[1] S. Andriamonje et al., Experimental determination of the energy generated in nuclear cascades by a high energy beam, Phys. Lett. B 348 (1995) 697-709.

[2] C. Rubbia et al., Conceptual design of a fast neutron operated high power energy amplifier, CERN internal report, CERN/AT/95-44 (ET), Sept. 1995; see also C. Rubbia, A high gain energy amplifier operated with fast neutrons, AIP Conf. Proc. 346, Int. Conf. on Accelerator-Driven Tramsmutation Technologies and Applications, Las Vegas, 1994.

[3] C. Rubbia, S. Buono, Y. Kadi and J.A. Rubio, Fast neutron incineration in the energy amplifier as alternative to geological storage: the case of Spain, CERN internal report, CERN/LHC/97-01 (EET), Feb. 1997.

C. Rubbia et al., A realistic plutonium elimination scheme with energy amplifiers and thorium-plutonium fuel, CERN Internal report, CERN/AT/95-53 (ET), Dec. 1995.

[4] C. Rubbia, Resonance enhanced neutron captures for element activation and waste transmutation, CERN internal report, CERN/LHC/97-04 (EET), June 1997.

[5] JENDL Data Base, OECD/NEA Data Bank, Issy-les-Moulineaux, France, 1996.

[6] S. Andriamonje et al., Proposal: Experimental Study of the Phenomenology of Spallation Neutrons in a Large Lead Block, CERN/SPSLC 95-17, SPSLC/P291, 5 May 1995.

[7] H. Arnould et al., Phys. Lett. B 458 (1999) 167-180.

[8] European Commission, Community Research, Project Report, Nuclear Science and Technology, Neutron-Driven Nuclear Transmutation by Adiabatic Resonance Crossing (TARC), EUR 19117 EN (European Commission, Luxembourg, 1999).

CERN Report 99-11, The TARC Experiment (PS211): Neutron-Driven Nuclear Transmutation by Adiabatic Resonance Crossing, J.-P. Revol (Ed.), (CERN, Geneva, 1999).

[9] I. Papadopoulos and K. Komnas, Simulation concerning the suitability of 'EMBECCO' as base of the experimental setup, CERN/LHC/ET/Internal Note 98-02, Nov. 1998.

[10] Calder Industrial Materials Ltd., Chester, United Kingdom.

[11] M. Embid and J.-P. Revol, Determination of the impurity content of the lead assembly for the TARC experiment, CERN/ET/Internal Note 97-01, Jan. 1997.

[12] Britannia Refined Metals Ltd., Gravesend, Kent, United Kingdom.

[13] Bergoz Instrumentation, 01170, Crozet, France. URL: 〈http://www.bergoz.com/>.

[14] J.-P. Revol et al., Beam transformer performance and calibration for the TARC experiment, CERN/ET/Internal Note 99-008, Dec. 1999.

[15] D. Brozzi, M. Embid and J.-P. Revol, Analysis of aluminium foil activation to provide absolute calibration of proton intensities in the TARC experiment, CERN/ET/Internal Note 97-25, May 1997.

[16] M. Embid and J.-P. Revol, Determination of the beam geometry in the fast extraction mode for the TARC experiment, CERN/ET/Internal Note 97-02, Feb. 1997.

[17] A. Abánades et al., Determination of the beam characteristics in the slow extraction mode, CERN/ET/Internal Note 97-08, June 1997. 
[18] A. Abanades et al., Neutron flux measurements in TARC with the ${ }^{3}$ He ionization chambers, CERN/LHC/ET/Internal Note 98-14, Oct. 1998.

[19] CASCADE User's Guide, CERN-ECP Division, FEX Group.

[20] A. Fassó et al., in Intermediate Energy Nuclear Data: Models and Codes, Proceedings of a Specialists' Meeting, Issy-les-Moulineaux (OECD, Paris, 1994), p. 271 and references therein.

A. Fassó, A. Ferrari, J. Ranft, P.R. Sala, G.R. Stevenson and J.M. Zazula, Nucl. Instrum. Methods A 332 (1993) 459, also CERN Internal report, CERN/TIS-RP/93-2/PP (1993).

See also A. Ferrari et al., FLUKA-96 Manual (INFN, Milan, 1996) and FLUKA-97 Manual (private communication from A. Ferrari).

[21] A. Ferrari and P.R. Sala, The physics of high energy reactions, lecture given at the Workshop on Nuclear Reaction Data and Nuclear Reactors Physics, Design and Safety, International Centre for Theoretical Physics, Trieste, 15 April - 17 May 1996.

[22] OECD/NEA Data Bank, Issy-les-Moulineaux, France, 1994.

[23] M. Cobo et al., Notes on the study of the most reliable neutron cross-section data, CERN Internal report, CERN/AT/ET 95-035, Aug. 1995.

[24] R.E. MacFarlane, D.W. Muir and R.M. Boicourt, The NJOY Nuclear Data Processing System: User's Manual, Los Alamos National Laboratory report, LA-9303-M, Vols. I to IV, May 1982.

[25] F. Carminati, PROCESS: A cross-section processing utility, CERN Internal report, CERN/LHC/EET 96-005, Jan. 1996.

[26] F. Carminati, I. Goulas and J.-P. Revol, A general nuclear database for the EET Group, CERN Internal report, CERN/AT/EET 94-022, Aug. 1994. See also 95-008, 95-009, 95-012, 95-026, 95-030 for various updates.

[27] J.K. Tuli, National Nuclear Data Center, Brookhaven National Laboratory, Upton, New York 11973, USA, July 1995.

[28] G. Audi and A.H. Wapstra, The 1995 update to the atomic mass evaluation, Nucl. Phys. A 595 (1995) 409-480.

[29] Final tables of dose coefficients as sent to IAEA for inclusion in the BRSS, National Radiological Protection Board, Chilton, Didcot, Oxon OX11 0RQ, U.K., Mar. 1995.

[30] International Commission on Radiological Protection, ICRP, S-171 16 Stockholm, Sweden, edition 91, 1995.

[31] F. Carminati, I. Goulas and J.-P. Revol, Data for the simulation of heat deposition from nuclear decays and update of the toxicity values, CERN Internal report, CERN/AT/EET 95-009, Mar. 1995.

[32] F. Carminati, Y. Kadi, I. Papadopoulos and C. Rubbia, TARC general-purpose Monte Carlo, CERN Internal report, CERN/LHC/EET 96-011, Apr. 1996.

[33] A. Ashgar et al., Status of lead sample analysis, CERN Internal report, CERN/LHC/EET 96-008, Mar. 1996.

[34] J.-P. Revol, Update on geometric details of the lead assembly and the experimental area for the TARC experiment, CERN Internal report, CERN/LHC/EET 96-029, Mar. 1996. 
[35] C. Rubbia, Relation between time and kinetic energy in a constant lethargy medium, CERN/AT/ET Internal Note 95-010, May 1995.

[36] A.A. Bergman et al., International Conference on the Peaceful Uses of Atomic Energy, 1, Geneva, 1955, p. 642.

[37] A.A. Bergman et al., Sov. Phys. JETP 6 (1958) 6.

[38] M. Sawan and R.W. Conn, Nucl. Sci. Eng. 54 (1974) 127.

[39] Jen-Chang Chou and H. Werle, J. Nucl. Energy, 27 (1973) 811.

[40] A. Ferrari, and P.R. Sala, Background rates in the muon system: recent results and the effect of the tungsten plug, ATLAS Internal Note MUON-NO-090, (1995).

[41] M. Derzon et al., A high pressure ${ }^{3}$ He gas scintillation neutron spectrometer, IEEE Trans. Nucl. Sci. 33 (1986) 247.

[42] J.A. Northrop and J.C. Gursky, Nucl. Instrum. Methods 3 (1958) 207.

[43] J.A. Northrop and R. Nobles, Los Alamos gas counters, Nucleonics, 14, No. 4 (1956) 36; I.R.E. Trans. Nucl. Sci. NS-3, No. 4 (1956) 59.

[44] Ulrich Brackmann, Lambdachrome Laser Dyes Data Sheets (Lambda Physik GmbH, Göttingen, 1994), p. 33.

[45] Photomultipliers and Accessories (Thorn EMI Electron Tubes, Ruislip, UK, 1993).

[46] A. Sayres and S. Wu, Gas scintillation counter, Rev. Sci. Instrum. 28 (1957) 758.

[47] 11-bit ADC 2249 W, Lecroy Research Instrumentation Catalog, p. 91, 1992.

[48] Neutron Activation Foils and Flux Wires (Reactor Experiments Inc., Sunnyvale, Ca., 1965), Catalog 24.

[49] Y.S. Horowitz, Thermoluminescence and Thermoluminescence Dosimetry, Vol. II (CRC Press, Boca Raton, 1984).

[50] G. Grosshoeg, Nucl. Instrum. Methods, 162 (1979) 125.

[51] S. Shalev and J.M. Cutler, Nucl. Sci. Eng. 51 (1973) 52.

[52] O. Buneman et al., Can. J. Res. 27, sec. A (1949) 191.

[53] P. Cennini et al., Nucl. Instrum. Methods, A 345 (1994) 230.

[54] A. Abánades, Medida del espectro neutrónico con una cámara de ionización de ${ }^{3} \mathrm{He}$ en el experimento TARC, Ph.D. thesis (in preparation) Univ. Politécnica de Madrid.

[55] A. Angelopoulos, Neutron flux measurements with Lexan Track-Etch Detectors in the TARC experiment, CERN/LHC/Internal Note 98-07, Nov. 1998.

[56] F. Gunsing et al., Proc. Nuclear Data for Science and Technology, SIF Bologna (1997) p. 1293 and private communication.

[57] The ${ }^{99}$ Tc neutron capture cross-section based on Geel data was evaluated by Z.X. Zhao, using the SAMMY-K1 code ORNL/TM-9179/R3, 1996.

[58] J. Legrand and J. Morel, Phys. Rev. C 8 (1973) 366.

[59] Table of Isotopes, E. Browne et al., Eds C.M. Lederer and V.S. Shirley, Lawrence Berkeley Laboratory (1978). 
[60] S. Andriamonje et al., ${ }^{99}$ Tc capture rate measurements with spallation neutrons in a large lead block, CERN/ET/Internal Note 97-14, May 1997.

[61] M.A. Mariscotti, A method for automatic identification of peaks in the presence of background and its application to spectrum analysis, Nucl. Instrum. Methods, 50 (1967) 309-320.

[62] E. Browne and R.B. Firestone, Table of Radioactive Isotopes, V.S. Shirley (Ed.), Lawrence Berkeley Laboratory (1986).

[63] R.B. Firestone, Table of Isotopes, V.S. Shirley (Ed.), 8th ed. (Wiley, New York, 1996).

[64] G. Berzins, M.E. Bunker and J.W. Starner, Energy levels of ${ }^{100} R u$, Phys. Rev. 187 (1969) 1618.

[65] H. Harada, S. Nakamura, T. Katoh and Y. Ogata, Measurement of thermal neutron cross-section and resonances integral of the reaction ${ }^{99} T c(n, \gamma){ }^{100} T c$, J. Nucl. Sci. Tech. 32, (5) (1995) 395-403.

[66] MCNP A General Monte-Carlo N-Particle Transport Code, Version 4A Judith F. Briesmeister, (Ed.) Los Alamos National Laboratory report, LA-12625-M (1993).

[67] H. Beer et al., Nucl. Instrum. Methods A 337 (1973) 492.

[68] S. Andriamonje et al., Measurement of the neutron capture rate on ${ }^{127}$ I and ${ }^{129}$ I with the TARC experiment, CERN/ET/Internal Note 98-10, Dec. 1998.

[69] U. Rens and W. Westmeier, Atomic Data and Nuclear Data Tables 29 (1983) 193-406.

[70] W.G. Smith et al., Phys. Rev. 114 (1959) 1345.

[71] E.W. Schneider et al., Phys. Rev. C 19 (1979) 1025.

[72] Report on ${ }^{129}$ I neutron capture cross-section, BNL 325, (1973).

[73] S. Ganessan, Ratio ${ }^{130 m} I^{130 g} I$ (private communication).

[74] P.K. Hopke et al., Phys. Rev. C 8 (1973) 745.

[75] JENDL-3.2 (OECD NEA Data Bank, 1996); JEF-2.2 (OECD NEA Data Bank, 1996); ENDF/B-VI (OECD NEA Data Bank, 1996); BROND-2 (OECD NEA Data Bank, 1996). See also J. Cobo et al., Notes on the study of the most reliable neutron cross-section data, CERN/LHC/95-35 (EET), Aug. 1995.

[76] A. Angelopoulos et al., Measurement of production rates of ${ }^{233} U$ and ${ }^{239} U$ in the TARC experiment, CERN/ET/Internal Note 97-024, Oct. 1997.

[77] D. Bundzik et al., Measurement of the neutron capture rate on ${ }^{232} \mathrm{Th}$ and of ${ }^{233} \mathrm{~Pa}$ half-life in the TARC experiment, CERN/ET/Internal Note, 98-013, Oct. 1998.

[78] J.A. Phillips, AERE NP/R 2033 (1956).

J.P. Butler and D.C. Santry, Can. J. Chem. 39 (1961) 689.

R.J. Prestwood and B.P. Bayhurst, Phys. Rev. 121 (1961) 1438.

[79] S. Andriamonje et al., Measurement of the ${ }^{232} T h(n, 2 n)$ reaction rate in the TARC experiment, CERN/ET/Internal Note 99-007, Dec. 1999.

[80] J.K. Tuli, National Nuclear Data Center, BNL (1997). 
[81] M.A. Lone et al., Nucl. Instrum. Methods 143 (1977) 331, see also M.A. Lone et al., Nucl. Instrum. Methods 189 (1981) 515.

[82] V. Lacoste, Simulation of the molybdenum sample irradiation and measurement, EET Internal Note, 98-015, Dec. 1998.

[83] PAW Physics Analysis Workstation, CERN Program library Q121.

[84] R. Fernández and E. González, Evaluation of the heat production in a sample of lead inside the lead assembly, CERN/ET 96-19, July 1996. 


\section{Appendix}

\section{A Neutron Fluence as a Function of Energy}

The first part of the appendix gives the data and errors corresponding to the TARC fluence measurements $(\mathrm{d} F / \mathrm{d}(\ln (E))$ in hole 10 at $z=+7.5 \mathrm{~cm}$ (approximately at a distance of $45.6 \mathrm{~cm}$ from the centre of the lead volume). The fluence is given as neutrons per $\mathrm{cm}^{2}$ per $10^{9}$ protons incident on the lead volume. The proton momentum is either $2.50 \mathrm{GeV} / c$ or $3.57 \mathrm{GeV} / c$, as indicated.

For the ${ }^{6} \mathrm{Li} /{ }^{233} \mathrm{U}$ and ${ }^{3} \mathrm{He} /$ scintillation detector data (Tables $\mathrm{A} 1-\mathrm{A} 4$ ) the binning is isolethargic with a bin width

$$
\Delta[\ln (E)]=\frac{\ln \left(10^{5}\right)-\ln (0.01)}{100}
$$

The energy values given in $\mathrm{eV}$ correspond to the logarithmic mean in the bin defined as:

$$
E_{\mathrm{im}} \equiv e^{\left(0.5 \times\left(\ln \left(E_{\mathrm{i}+1}\right)+\ln \left(E_{\mathrm{i}}\right)\right)\right)}
$$

The first bin starts at $E=10^{-2} \mathrm{eV}$ and the last bin ends at $E=10^{5} \mathrm{eV}$. There are 100 bins in total.

For the ${ }^{3} \mathrm{He} /$ ionization detector data (Table A5), the energy bins are given as the value of the lower edge and the mean value calculated linearly:

$$
E_{\mathrm{im}} \equiv \frac{\left(E_{\mathrm{i}}+E_{\mathrm{i}+1}\right)}{2}
$$

Both the statistical and the systematic errors are given. 
Table A1: ${ }^{3} \mathrm{He} / \mathrm{scintillation} / 2.5 \mathrm{GeV} / \mathrm{c}$

\begin{tabular}{|c|c|c|c|}
\hline $\begin{array}{c}\text { Mean }\left\langle E_{\mathrm{i}}\right\rangle \\
(\mathrm{eV})\end{array}$ & $\begin{array}{c}E \mathrm{~d} F / \mathrm{d} E \\
\left(\mathrm{n} / \mathrm{cm}^{2} / 10^{9} \mathrm{p}\right)\end{array}$ & Stat. error & Syst. error \\
\hline 0.376 & 275278 & 3448 & 37226 \\
\hline 0.442 & 301782 & 3756 & 40810 \\
\hline 0.519 & 340272 & 4157 & 46016 \\
\hline 0.61 & 356588 & 4421 & 48223 \\
\hline 0.716 & 394892 & 4838 & 53404 \\
\hline 0.841 & 429498 & 5256 & 58085 \\
\hline 0.989 & 458291 & 5646 & 61981 \\
\hline 1.161 & 484007 & 6029 & 65460 \\
\hline 1.365 & 506543 & 6422 & 68511 \\
\hline 1.603 & 544825 & 6938 & 73691 \\
\hline 1.884 & 566643 & 7324 & 76646 \\
\hline 2.213 & 616788 & 7971 & 83433 \\
\hline 2.6 & 642024 & 8464 & 86852 \\
\hline 3.055 & 676087 & 9038 & 91466 \\
\hline 3.589 & 719414 & 9673 & 97336 \\
\hline 4.217 & 742831 & 10263 & 100514 \\
\hline 4.955 & 774353 & 10859 & 104792 \\
\hline 5.821 & 827903 & 11755 & 112054 \\
\hline 6.839 & 845510 & 12289 & 114456 \\
\hline 8.035 & 885449 & 13134 & 119885 \\
\hline 9.441 & 915560 & 13871 & 123990 \\
\hline 11.092 & 941733 & 14636 & 127567 \\
\hline 13.032 & 980162 & 15567 & 132814 \\
\hline 15.311 & 1012818 & 16446 & 137288 \\
\hline 17.989 & 1055418 & 17466 & 143123 \\
\hline 21.135 & 1102804 & 18600 & 149624 \\
\hline 24.831 & 1141888 & 19708 & 155017 \\
\hline 29.174 & 1156113 & 20572 & 157055 \\
\hline 34.277 & 1185135 & 21690 & 161127 \\
\hline 40.272 & 1235172 & 23087 & 168088 \\
\hline 47.315 & 1289648 & 24600 & 175696 \\
\hline 55.591 & 1307669 & 25735 & 178382 \\
\hline 65.313 & 1341033 & 27092 & 183211 \\
\hline 76.736 & 1370895 & 28509 & 187623 \\
\hline 90.157 & 1398922 & 29863 & 191857 \\
\hline 105.926 & 1431318 & 31571 & 196778 \\
\hline 124.452 & 1441628 & 32989 & 198759 \\
\hline 146.218 & 1513853 & 35157 & 209411 \\
\hline 171.791 & 1547521 & 36989 & 214898 \\
\hline 201.837 & 1563220 & 38597 & 218059 \\
\hline 237.138 & 1636756 & 41363 & 229518 \\
\hline 278.613 & 1628540 & 42734 & 229762 \\
\hline 327.341 & 1705225 & 45742 & 242287 \\
\hline 384.592 & 1751752 & 48197 & 250941 \\
\hline 451.857 & 1797444 & 50849 & 259929 \\
\hline 530.885 & 1858746 & 53944 & 271730 \\
\hline 623.736 & 1913164 & 56952 & 283196 \\
\hline 732.825 & 1944251 & 59634 & 291933 \\
\hline 860.995 & 1953811 & 62321 & 298177 \\
\hline
\end{tabular}

\begin{tabular}{c|c|r|r}
\hline $\begin{array}{c}\text { Mean }\left\langle\boldsymbol{E}_{\mathbf{i}}\right\rangle \\
(\mathbf{e V})\end{array}$ & $\begin{array}{c}\boldsymbol{E d} \boldsymbol{F} / \mathbf{d} \boldsymbol{E} \\
\left(\mathbf{n} / \mathbf{c m}^{2} / \mathbf{1 0} \mathbf{9}\right)\end{array}$ & Stat. error & Syst. error \\
\hline 1011.58 & 2064894 & 66872 & 320998 \\
\hline 1188.503 & 2070736 & 69862 & 328686 \\
\hline 1396.369 & 2206711 & 75060 & 358571 \\
\hline 1640.59 & 2244527 & 79162 & 374394 \\
\hline 1927.525 & 2341376 & 84198 & 402087 \\
\hline 2264.644 & 2431927 & 89569 & 431292 \\
\hline 2660.725 & 2498864 & 94963 & 459100 \\
\hline 3126.079 & 2645949 & 101803 & 505230 \\
\hline 3672.822 & 2678028 & 106813 & 533181 \\
\hline 4315.188 & 2758465 & 113268 & 574485 \\
\hline 5069.903 & 2878502 & 120584 & 629077 \\
\hline 5956.616 & 2882548 & 126414 & 663089 \\
\hline 6998.413 & 2890662 & 132323 & 701981 \\
\hline 8222.417 & 2994883 & 141359 & 769922 \\
\hline 9660.496 & 3266327 & 154144 & 891239 \\
\hline 11350.091 & 3300371 & 162315 & 958096 \\
\hline 13335.199 & 3237443 & 169011 & 1002119 \\
\hline 15667.5 & 3488670 & 183639 & 1153770 \\
\hline 18407.715 & 3721156 & 198814 & 1317240 \\
\hline 21627.188 & 3758134 & 209985 & 1426233 \\
\hline 25409.74 & 3793469 & 221589 & 1545651 \\
\hline 29853.852 & 4196991 & 244748 & 1838329 \\
\hline 35075.23 & 4477528 & 266618 & 2110672 \\
\hline 41209.82 & 4762069 & 290044 & 2418254 \\
\hline 48417.336 & 4984865 & 312674 & 2729327 \\
\hline 56885.434 & 5477323 & 347075 & 3235861 \\
\hline 66834.586 & 6116790 & 389450 & 3901621 \\
\hline 78523.82 & 6491265 & 424105 & 4472942 \\
\hline 92257.172 & 7726631 & 490180 & 5754467 \\
\hline & & &
\end{tabular}


Table A2: ${ }^{3} \mathrm{He} / \mathrm{Scintillation} / 3.5 \mathrm{GeV} / \mathrm{c}$

\begin{tabular}{|c|c|c|c|}
\hline $\begin{array}{c}\text { Mean }\left\langle E_{\mathbf{i}}\right\rangle \\
(\mathrm{eV})\end{array}$ & $\begin{array}{c}E \mathrm{EdF} / \mathrm{d} E \\
\left(\mathrm{n} / \mathrm{cm}^{2} / 10^{9} \mathrm{p}\right)\end{array}$ & Stat. error & Syst. error \\
\hline 0.376 & 460169 & 6881 & 62229 \\
\hline 0.442 & 506885 & 7514 & 68547 \\
\hline 0.519 & 549528 & 8154 & 74314 \\
\hline 0.61 & 548740 & 8466 & 74209 \\
\hline 0.716 & 594713 & 9165 & 80427 \\
\hline 0.841 & 651083 & 9988 & 88053 \\
\hline 0.989 & 715866 & 10891 & 96816 \\
\hline 1.161 & 739961 & 11506 & 100077 \\
\hline 1.365 & 797973 & 12441 & 107927 \\
\hline 1.603 & 859480 & 13450 & 116250 \\
\hline 1.884 & 911811 & 14340 & 123334 \\
\hline 2.213 & 970610 & 15434 & 131294 \\
\hline 2.6 & 1058194 & 16772 & 143150 \\
\hline 3.055 & 1079046 & 17625 & 145982 \\
\hline 3.589 & 1154850 & 18917 & 156250 \\
\hline 4.217 & 1212281 & 20238 & 164037 \\
\hline 4.955 & 1254628 & 21336 & 169787 \\
\hline 5.821 & 1323898 & 22944 & 179186 \\
\hline 6.839 & 1379847 & 24233 & 186789 \\
\hline 8.035 & 1467118 & 26096 & 198640 \\
\hline 9.441 & 1490151 & 27315 & 201803 \\
\hline 11.092 & 1534421 & 28838 & 207853 \\
\hline 13.032 & 1516054 & 29884 & 205428 \\
\hline 15.311 & 1560426 & 31510 & 211517 \\
\hline 17.989 & 1695097 & 34167 & 229869 \\
\hline 21.135 & 1750221 & 36168 & 237462 \\
\hline 24.831 & 1822483 & 38432 & 247411 \\
\hline 29.174 & 1860997 & 40288 & 252812 \\
\hline 34.277 & 1919743 & 42611 & 261002 \\
\hline 40.272 & 1941352 & 44677 & 264189 \\
\hline 47.315 & 1853578 & 45524 & 252523 \\
\hline 55.591 & 1985524 & 48948 & 270849 \\
\hline 65.313 & 2068154 & 51932 & 282549 \\
\hline 76.736 & 2129360 & 54844 & 291428 \\
\hline 90.157 & 2144374 & 57070 & 294093 \\
\hline 105.926 & 2322807 & 62081 & 319340 \\
\hline 124.452 & 2329668 & 64732 & 321195 \\
\hline 146.218 & 2302510 & 66926 & 318505 \\
\hline 171.791 & 2530406 & 73009 & 351388 \\
\hline 201.837 & 2648351 & 77546 & 369428 \\
\hline 237.138 & 2646419 & 81185 & 371100 \\
\hline 278.613 & 2635380 & 83912 & 371811 \\
\hline 327.341 & 2728743 & 89316 & 387713 \\
\hline 384.592 & 2819625 & 94386 & 403916 \\
\hline 451.857 & 2832283 & 98527 & 409577 \\
\hline 530.885 & 2886461 & 103764 & 421972 \\
\hline 623.736 & 2993124 & 109958 & 443057 \\
\hline 732.825 & 3225018 & 118553 & 484243 \\
\hline 860.995 & 3202944 & 123166 & 488811 \\
\hline
\end{tabular}

\begin{tabular}{c|c|c|c}
\hline $\begin{array}{c}\text { Mean }\left\langle\boldsymbol{E}_{\mathbf{i}}\right\rangle \\
(\mathbf{e V})\end{array}$ & $\begin{array}{c}\boldsymbol{E} \mathbf{d} \boldsymbol{F} / \mathbf{d} \boldsymbol{E} \\
\left(\mathbf{n} / \mathbf{c m}^{\mathbf{2}} / \mathbf{1 0} \mathbf{p}\right)\end{array}$ & Stat. error & Syst. error \\
\hline 1011.58 & 3306183 & 130613 & 513963 \\
\hline 1188.503 & 3373168 & 137634 & 535420 \\
\hline 1396.369 & 3570359 & 147373 & 580152 \\
\hline 1640.59 & 3767851 & 158318 & 628490 \\
\hline 1927.525 & 3892049 & 167565 & 668386 \\
\hline 2264.644 & 3964917 & 176533 & 703161 \\
\hline 2660.725 & 4143703 & 188758 & 761296 \\
\hline 3126.079 & 4288171 & 200048 & 818804 \\
\hline 3672.822 & 4266158 & 208096 & 849369 \\
\hline 4315.188 & 4513817 & 223651 & 940059 \\
\hline 5069.903 & 4614116 & 235655 & 1008384 \\
\hline 5956.616 & 4727511 & 249891 & 1087497 \\
\hline 6998.413 & 4815028 & 263611 & 1169303 \\
\hline 8222.417 & 5082352 & 284245 & 1306567 \\
\hline 9660.496 & 5209888 & 300495 & 1421552 \\
\hline 11350.091 & 5324324 & 318226 & 1545649 \\
\hline 13335.199 & 5279861 & 333159 & 1634330 \\
\hline 15667.5 & 5289359 & 349031 & 1749292 \\
\hline 18407.715 & 5539290 & 374423 & 1960835 \\
\hline 21627.188 & 6070463 & 411945 & 2303774 \\
\hline 25409.74 & 6292250 & 440514 & 2563781 \\
\hline 29853.852 & 7373733 & 500749 & 3229778 \\
\hline
\end{tabular}


Table A3: ${ }^{6} \mathrm{Li} /{ }^{233} \mathrm{U} / 2.5 \mathrm{GeV} / \mathrm{c}$

\begin{tabular}{|c|c|c|c|}
\hline $\begin{array}{c}\operatorname{Mean}\left\langle E_{\mathbf{i}}\right\rangle \\
(\mathrm{eV})\end{array}$ & $\begin{array}{c}E \mathrm{~d} F / \mathrm{d} E \\
\left(\mathrm{n} / \mathrm{cm}^{2} / 10^{9} \mathrm{p}\right)\end{array}$ & Stat. error & Syst. error \\
\hline 0.021 & 15908 & 2296 & 2204 \\
\hline 0.024 & 17993 & 2545 & 2492 \\
\hline 0.029 & 24624 & 3102 & 3411 \\
\hline 0.033 & 38977 & 4069 & 5399 \\
\hline 0.039 & 38936 & 4242 & 5393 \\
\hline 0.046 & 56744 & 5340 & 7860 \\
\hline 0.054 & 51858 & 5209 & 7183 \\
\hline 0.064 & 78687 & 6631 & 10900 \\
\hline 0.075 & 80212 & 6933 & 11111 \\
\hline 0.088 & 92435 & 7721 & 12804 \\
\hline 0.104 & 112138 & 8835 & 15533 \\
\hline 0.122 & 138309 & 10199 & 19159 \\
\hline 0.143 & 147323 & 10941 & 20407 \\
\hline 0.168 & 178602 & 12514 & 24740 \\
\hline 0.197 & 208492 & 14033 & 28881 \\
\hline 0.232 & 231684 & 15343 & 32093 \\
\hline 0.272 & 242909 & 16284 & 33648 \\
\hline 0.320 & 306021 & 13826 & 29984 \\
\hline 0.376 & 310552 & 14432 & 30420 \\
\hline 0.442 & 349617 & 15860 & 34292 \\
\hline 0.519 & 361640 & 16667 & 35494 \\
\hline 0.610 & 372367 & 17347 & 36485 \\
\hline 0.716 & 390333 & 18013 & 38490 \\
\hline 0.841 & 453242 & 18952 & 44399 \\
\hline 0.989 & 502635 & 18568 & 49262 \\
\hline 1.161 & 493583 & 15919 & 48453 \\
\hline 1.365 & 494861 & 12888 & 48492 \\
\hline 1.603 & 482488 & 10717 & 47270 \\
\hline 1.884 & 514136 & 10963 & 50449 \\
\hline 2.213 & 543474 & 13377 & 53391 \\
\hline 2.600 & 627154 & 19953 & 61800 \\
\hline 3.055 & 656274 & 25914 & 64432 \\
\hline 3.589 & 726710 & 28390 & 71219 \\
\hline 4.217 & 724241 & 29453 & 70982 \\
\hline 4.955 & 806797 & 30985 & 79082 \\
\hline 5.821 & 763901 & 25506 & 74898 \\
\hline 6.839 & 737945 & 24175 & 72464 \\
\hline 8.035 & 867885 & 32372 & 85110 \\
\hline 9.441 & 875563 & 29274 & 85930 \\
\hline 11.092 & 829345 & 26276 & 81397 \\
\hline 13.032 & 921048 & 30171 & 90669 \\
\hline 15.311 & 965185 & 33120 & 94758 \\
\hline 17.989 & 1014804 & 32924 & 99699 \\
\hline 21.135 & 889206 & 28541 & 87559 \\
\hline 24.831 & 973931 & 33712 & 95789 \\
\hline 29.174 & 1114639 & 41872 & 109678 \\
\hline 34.277 & 1058564 & 45386 & 104235 \\
\hline 40.272 & 1232387 & 60071 & 121456 \\
\hline 47.315 & 1529422 & 70447 & 150878 \\
\hline 55.590 & 1345060 & 59887 & 132850 \\
\hline
\end{tabular}

\begin{tabular}{c|c|r|r}
\hline $\begin{array}{c}\text { Mean }\left\langle\boldsymbol{E}_{\mathbf{i}}\right\rangle \\
(\mathbf{e V})\end{array}$ & $\begin{array}{c}\boldsymbol{E d} \boldsymbol{F} / \mathbf{d} \boldsymbol{E} \\
\left(\mathbf{n} / \mathbf{c m}^{2} / \mathbf{1 0} \mathbf{p} \mathbf{p}\right)\end{array}$ & Stat. error & Syst. error \\
\hline 65.313 & 1210322 & 57434 & 119781 \\
\hline 76.736 & 1453971 & 67287 & 144068 \\
\hline 90.157 & 1418608 & 66888 & 140843 \\
\hline 105.925 & 1362524 & 63152 & 135945 \\
\hline 124.451 & 1227946 & 66509 & 122624 \\
\hline 146.217 & 1511154 & 81408 & 151257 \\
\hline 171.791 & 1429373 & 78724 & 143870 \\
\hline 201.836 & 1627807 & 86350 & 166021 \\
\hline 237.137 & 1582482 & 87150 & 161064 \\
\hline 278.612 & 1628250 & 91315 & 167587 \\
\hline 327.340 & 1723348 & 99985 & 177132 \\
\hline 384.591 & 1848327 & 113129 & 191310 \\
\hline 451.855 & 2178810 & 132774 & 228913 \\
\hline 530.884 & 2137029 & 130999 & 227725 \\
\hline 623.734 & 2204020 & 130072 & 235396 \\
\hline 732.823 & 1845805 & 121329 & 199994 \\
\hline 860.992 & 1936230 & 130514 & 212977 \\
\hline 1011.577 & 1998776 & 140505 & 224305 \\
\hline 1188.499 & 2311320 & 158478 & 264033 \\
\hline 1396.365 & 2387485 & 165673 & 280557 \\
\hline 1640.586 & 2268294 & 166773 & 272401 \\
\hline 1927.521 & 2483071 & 180262 & 307337 \\
\hline 2264.639 & 2455236 & 186485 & 312542 \\
\hline 2660.720 & 2450272 & 192890 & 323612 \\
\hline 3126.072 & 2766197 & 214243 & 378190 \\
\hline 3672.816 & 2755937 & 221442 & 394280 \\
\hline 4315.182 & 3095936 & 244535 & 462686 \\
\hline 5069.899 & 3118377 & 255526 & 491293 \\
\hline 5956.609 & 3160060 & 387514 & 733096 \\
\hline 6998.403 & 3445465 & 412492 & 843086 \\
\hline 8222.412 & 3091801 & 400721 & 800244 \\
\hline 9660.496 & 3706670 & 455571 & 1017497 \\
\hline 11350.080 & 3717733 & 482881 & 1085017 \\
\hline 13335.170 & 4654344 & 552433 & 1447471 \\
\hline 15667.480 & 3894362 & 517210 & 1293239 \\
\hline 18407.660 & 4508537 & 568926 & 1601695 \\
\hline 21627.140 & 2939137 & 469298 & 1118906 \\
\hline 25409.660 & 4439832 & 589659 & 1813919 \\
\hline 29853.750 & 4631860 & 606093 & 2033568 \\
\hline 35075.100 & 5931119 & 697767 & 2801549 \\
\hline 41209.670 & 9105124 & 885261 & 4631794 \\
\hline 48417.120 & 14277100 & 1135819 & 7828772 \\
\hline 56885.140 & 45261009 & 2044653 & 26773503 \\
\hline 66834.250 & 45816255 & 2084523 & 29256399 \\
\hline 78523.430 & 5828108 & 749593 & 4019784 \\
\hline & & &
\end{tabular}


Table A4: ${ }^{6} \mathrm{Li} /{ }^{233} \mathrm{U} / 3.5 \mathrm{GeV} / \mathrm{c}$

\begin{tabular}{|c|c|c|c|}
\hline $\begin{array}{c}\text { Mean }\left\langle E_{\mathrm{i}}\right\rangle \\
(\mathrm{eV})\end{array}$ & $\begin{array}{c}E \mathrm{E} F / \mathrm{d} E \\
\left(\mathbf{n} / \mathrm{cm}^{2} / 10^{9} \mathbf{p}\right)\end{array}$ & Stat. error & Syst. error \\
\hline 0.075 & 136821 & 13481 & 18952 \\
\hline 0.088 & 147269 & 14511 & 20400 \\
\hline 0.104 & 189792 & 17113 & 26290 \\
\hline 0.122 & 193421 & 17959 & 26793 \\
\hline 0.143 & 261752 & 21715 & 36258 \\
\hline 0.168 & 273486 & 23056 & 37883 \\
\hline 0.197 & 330851 & 26321 & 45830 \\
\hline 0.232 & 387450 & 29543 & 53670 \\
\hline 0.272 & 390250 & 30732 & 54059 \\
\hline 0.320 & 391459 & 31883 & 54226 \\
\hline 0.376 & 495989 & 37146 & 68707 \\
\hline 0.442 & 530785 & 39748 & 73528 \\
\hline 0.519 & 514308 & 40412 & 71246 \\
\hline 0.610 & 639911 & 46296 & 88647 \\
\hline 0.716 & 709795 & 49304 & 98329 \\
\hline 0.841 & 703736 & 48107 & 97492 \\
\hline 0.989 & 790865 & 47406 & 109565 \\
\hline 1.161 & 793205 & 41031 & 109892 \\
\hline 1.365 & 683367 & 30834 & 94678 \\
\hline 1.603 & 810821 & 28306 & 112340 \\
\hline 1.884 & 751001 & 26947 & 104056 \\
\hline 2.213 & 921449 & 35404 & 127679 \\
\hline 2.600 & 1003056 & 51234 & 138995 \\
\hline 3.055 & 1053106 & 66747 & 145941 \\
\hline 3.589 & 1100545 & 71161 & 152527 \\
\hline 4.217 & 1164793 & 76101 & 161447 \\
\hline 4.955 & 1290617 & 79843 & 178907 \\
\hline 5.821 & 1076456 & 61718 & 149239 \\
\hline 6.839 & 1209259 & 62936 & 167677 \\
\hline 8.035 & 1406360 & 83962 & 195042 \\
\hline 9.441 & 1156057 & 68458 & 160363 \\
\hline 11.092 & 1274007 & 66292 & 176768 \\
\hline 13.032 & 1323097 & 73489 & 183633 \\
\hline 15.311 & 1374192 & 80492 & 190790 \\
\hline 17.989 & 1418413 & 79236 & 197009 \\
\hline 21.135 & 1347106 & 71421 & 187194 \\
\hline 24.831 & 1453772 & 83838 & 202129 \\
\hline 29.174 & 1519187 & 99654 & 211362 \\
\hline 34.277 & 1733481 & 118260 & 241361 \\
\hline 40.272 & 1888361 & 151566 & 263162 \\
\hline 47.315 & 1938158 & 161616 & 270386 \\
\hline 55.590 & 1919220 & 145759 & 268075 \\
\hline 65.313 & 2061837 & 152886 & 288412 \\
\hline 76.736 & 2300584 & 172370 & 322354 \\
\hline 90.157 & 2050328 & 163891 & 287859 \\
\hline 105.925 & 2017214 & 156183 & 283868 \\
\hline 124.451 & 2368271 & 187930 & 334174 \\
\hline 146.218 & 2437672 & 210622 & 345059 \\
\hline 171.791 & 2295428 & 203646 & 326127 \\
\hline 201.836 & 2154312 & 203343 & 307399 \\
\hline
\end{tabular}

\begin{tabular}{c|c|c|c}
\hline $\begin{array}{c}\text { Mean }\left\langle\boldsymbol{E}_{\mathbf{i}}\right\rangle \\
(\mathbf{e V})\end{array}$ & $\begin{array}{c}\boldsymbol{E d} \boldsymbol{F} / \mathbf{d} \boldsymbol{E} \\
\left(\mathbf{n} / \mathbf{c m}^{2} / \mathbf{1 0} \mathbf{9} \mathbf{p}\right)\end{array}$ & Stat. error & Syst. error \\
\hline 237.137 & 2369321 & 217854 & 339778 \\
\hline 278.612 & 2460551 & 229689 & 354924 \\
\hline 327.340 & 2280036 & 233981 & 331117 \\
\hline 384.591 & 2756006 & 281549 & 403385 \\
\hline 451.855 & 2745673 & 304728 & 405525 \\
\hline 530.884 & 3279609 & 352961 & 552844 \\
\hline 623.734 & 2695023 & 293074 & 407059 \\
\hline 732.823 & 2715284 & 300159 & 415781 \\
\hline 860.992 & 3005634 & 331130 & 467497 \\
\hline 1011.577 & 3070210 & 355556 & 486105 \\
\hline 1188.499 & 3056556 & 371178 & 493772 \\
\hline 1396.366 & 3084099 & 384940 & 509627 \\
\hline 1640.586 & 3221901 & 405771 & 546065 \\
\hline 1927.522 & 3045267 & 407881 & 530904 \\
\hline 2264.640 & 2995790 & 419121 & 538855 \\
\hline 2660.721 & 4076129 & 508180 & 758821 \\
\hline 3126.073 & 3383133 & 482334 & 653933 \\
\hline 3672.817 & 3637157 & 519923 & 732330 \\
\hline 4315.182 & 3858717 & 557878 & 811939 \\
\hline 5069.900 & 4370950 & 619146 & 964219 \\
\hline 5956.610 & 3494666 & 569171 & 810721 \\
\hline 6998.404 & 3356627 & 568649 & 821348 \\
\hline 8222.412 & 3822169 & 622289 & 989283 \\
\hline 9660.496 & 2886462 & 561498 & 792347 \\
\hline 11350.080 & 3954690 & 695597 & 1154173 \\
\hline 13335.180 & 4563132 & 763981 & 1419105 \\
\hline 15667.480 & 3873365 & 720433 & 1286267 \\
\hline 18407.670 & 4333018 & 778993 & 1539341 \\
\hline 21627.140 & 4400745 & 802051 & 1675327 \\
\hline 25409.670 & 4831128 & 859098 & 1973784 \\
\hline 29853.750 & 5840420 & 950571 & 2564174 \\
\hline 35075.110 & 4162665 & 816448 & 1966224 \\
\hline 41209.680 & 4905666 & 907566 & 2495524 \\
\hline 48417.130 & 4258432 & 866393 & 2335089 \\
\hline 56885.140 & 4116317 & 861217 & 2434949 \\
\hline 66834.250 & 4170700 & 878419 & 2663240 \\
\hline 78523.440 & 5345556 & 1002671 & 3686956 \\
\hline 92256.960 & 5612317 & 1042980 & 4183208 \\
\hline & & &
\end{tabular}


Table A5: ${ }^{3} \mathrm{He} /$ ionization/2.5 GeV/c

\begin{tabular}{c|c|r|r|r}
\hline $\begin{array}{c}\boldsymbol{E}_{\mathbf{i}} / \mathbf{l o w} \\
(\mathbf{e V})\end{array}$ & $\begin{array}{c}\text { Mean }\left\langle\boldsymbol{E}_{\mathbf{i}}\right\rangle \\
(\mathbf{e V})\end{array}$ & $\begin{array}{c}\boldsymbol{E d} \boldsymbol{F} / \mathbf{d} \boldsymbol{E} \\
\left(\mathbf{n} / \mathbf{c m}^{\mathbf{2}} / \mathbf{1 0} \mathbf{p} \mathbf{p}\right)\end{array}$ & $\begin{array}{c}\text { Statistical } \\
\text { error }\end{array}$ & $\begin{array}{c}\text { Systematic } \\
\text { error }\end{array}$ \\
\hline 59500 & 84250 & 4769990 & 585185 & 858598 \\
\hline 109000 & 133750 & 5513695 & 766895 & 992465 \\
\hline 158500 & 183250 & 7339415 & 1079366 & 1321095 \\
\hline 208000 & 232750 & 6917651 & 1154588 & 1176001 \\
\hline 257500 & 282250 & 10017312 & 1627418 & 1702943 \\
\hline 307000 & 331750 & 6167753 & 1283921 & 1048518 \\
\hline 356500 & 381250 & 9621671 & 1816459 & 1635684 \\
\hline 406000 & 430750 & 8704919 & 1812074 & 1392787 \\
\hline 455500 & 480250 & 8429564 & 1884908 & 1348730 \\
\hline 505000 & 529750 & 10091790 & 2221605 & 1614686 \\
\hline 554500 & 579250 & 7095639 & 1910559 & 1135302 \\
\hline 604000 & 628750 & 5036533 & 1670429 & 805845 \\
\hline 653500 & 678250 & 5692027 & 1887832 & 910724 \\
\hline 703000 & 727750 & 12173780 & 3046648 & 1947805 \\
\hline 752500 & 777250 & 2858499 & 1457554 & 457360 \\
\hline 802000 & 851500 & 2481939 & 1043202 & 397110 \\
\hline 901000 & 950500 & 2492927 & 1142402 & 398868 \\
\hline 1000000 & 1081154 & 2233198 & 938652 & 357312 \\
\hline 1162308 & 1256634 & 2491258 & 1047119 & 398601 \\
\hline 1350960 & 1460596 & 959095 & 684930 & 153455 \\
\hline 1570232 & 1697662 & 1814799 & 1063375 & 290368 \\
\hline
\end{tabular}




\section{B Neutron Fluence Distribution in the Lead Volume}

The second part of the appendix gives the distribution in the lead volume of the differential fluence $(\mathrm{d} F / \mathrm{d} E)$ for a selected number of energy bins as a function of the distance to the centre of the lead volume. (This distance has a minus sign when $z$ is a negative value.) In this case only the statistical errors are given. The unit is neutrons per $\mathrm{cm}^{2}$ per eV per $10^{9}$ protons of $3.57 \mathrm{GeV} / c$.

Table B1: ${ }^{6} \mathrm{Li} /{ }^{233} \mathrm{U} / 0.1 \mathrm{eV}$

\begin{tabular}{|c|c|c|}
\hline $\begin{array}{l}\text { Distance } \\
\text { (cm) }\end{array}$ & $\begin{array}{c}\text { Fluence } \\
\left(\mathrm{n} / \mathrm{cm}^{2} / \mathrm{eV} / 10^{9} \mathrm{p}\right)\end{array}$ & Error \\
\hline-187.5 & 178127 & 20916 \\
\hline-164.5 & 208387 & 15236 \\
\hline-154.6 & 252267 & 19480 \\
\hline-132.7 & 652345 & 26868 \\
\hline-124.8 & 721648 & 31621 \\
\hline-121.2 & 695630 & 37039 \\
\hline-111.5 & 861361 & 37286 \\
\hline-107.4 & 951128 & 43864 \\
\hline-105.3 & 992298 & 41004 \\
\hline-97.5 & 1098173 & 43472 \\
\hline-95.2 & 1166686 & 50820 \\
\hline-94.0 & 1075235 & 52536 \\
\hline-90.3 & 1214726 & 59741 \\
\hline-76.9 & 1341157 & 80500 \\
\hline-70.8 & 1143483 & 106866 \\
\hline-70.8 & 1401114 & 60049 \\
\hline-64.1 & 1389402 & 75086 \\
\hline-60.5 & 1479789 & 75960 \\
\hline-58.6 & 1591117 & 69629 \\
\hline-54.6 & 1793428 & 94551 \\
\hline-50.3 & 1734488 & 72263 \\
\hline-45.6 & 1660183 & 71642 \\
\hline-40.4 & 1906804 & 100561 \\
\hline-27.0 & 1998677 & 105661 \\
\hline 27.0 & 1946504 & 108645 \\
\hline 40.4 & 1799165 & 97110 \\
\hline 45.6 & 1965170 & 101310 \\
\hline 46.0 & 1404543 & 214244 \\
\hline 50.3 & 1709736 & 71958 \\
\hline 54.6 & 1479024 & 93239 \\
\hline 58.6 & 1612297 & 68516 \\
\hline 60.5 & 1743914 & 117159 \\
\hline 60.5 & 1638378 & 79586 \\
\hline 67.5 & 1465282 & 64304 \\
\hline 69.1 & 1534629 & 62931 \\
\hline 70.8 & 1367310 & 55589 \\
\hline 70.8 & 1484603 & 73533 \\
\hline 76.9 & 1318944 & 64449 \\
\hline 79.7 & 1307921 & 52070 \\
\hline
\end{tabular}

\begin{tabular}{|c|c|c|}
\hline $\begin{array}{l}\text { Distance } \\
(\mathrm{cm})\end{array}$ & $\begin{array}{c}\text { Fluence } \\
\left(\mathrm{n} / \mathrm{cm}^{2} / \mathrm{eV} / 10^{9} \mathrm{p}\right)\end{array}$ & Error \\
\hline 83.9 & 1048934 & 68685 \\
\hline 85.2 & 1221003 & 59340 \\
\hline 90.3 & 1291466 & 92312 \\
\hline 90.3 & 1157944 & 46655 \\
\hline 90.3 & 1106473 & 61307 \\
\hline 94.0 & 1070874 & 48136 \\
\hline 95.2 & 1101744 & 53235 \\
\hline 102.0 & 992297 & 53476 \\
\hline 105.3 & 844377 & 130788 \\
\hline 105.3 & 925665 & 39518 \\
\hline 111.5 & 891084 & 36844 \\
\hline 112.5 & 859081 & 34146 \\
\hline 117.4 & 815832 & 32958 \\
\hline 120.2 & 725850 & 28554 \\
\hline 124.8 & 671149 & 30435 \\
\hline 127.5 & 730157 & 28978 \\
\hline 127.5 & 603684 & 25423 \\
\hline 127.5 & 532314 & 35862 \\
\hline 131.0 & 556694 & 32606 \\
\hline 133.5 & 609798 & 26898 \\
\hline 137.7 & 540992 & 21415 \\
\hline 143.3 & 480779 & 23472 \\
\hline 144.1 & 445007 & 19050 \\
\hline 144.1 & 350657 & 16957 \\
\hline 145.6 & 469112 & 18525 \\
\hline 150.2 & 242026 & 18278 \\
\hline 153.9 & 348872 & 16337 \\
\hline 154.6 & 251507 & 11483 \\
\hline 154.6 & 269597 & 12693 \\
\hline 154.6 & 243829 & 9988 \\
\hline 164.5 & 198383 & 9593 \\
\hline 164.5 & 242238 & 10798 \\
\hline 164.5 & 279758 & 11411 \\
\hline 165.2 & 216177 & 12280 \\
\hline 171.2 & 184382 & 7422 \\
\hline 187.5 & 106216 & 20669 \\
\hline 187.5 & 159332 & 21266 \\
\hline
\end{tabular}


Table B2: ${ }^{6} \mathrm{Li} /{ }^{233} \mathrm{U} / 1.5 \mathrm{eV}$

\begin{tabular}{|c|c|c|}
\hline $\begin{array}{l}\text { Distance } \\
(\mathrm{cm})\end{array}$ & $\begin{array}{c}\text { Fluence } \\
\left(\mathrm{n} / \mathrm{cm}^{2} / \mathrm{eV} / 10^{9} \mathbf{p}\right)\end{array}$ & Error \\
\hline-187.5 & 52037 & 7250 \\
\hline-164.5 & 60333 & 2796 \\
\hline-154.6 & 73270 & 4200 \\
\hline-150.2 & 75772 & 4035 \\
\hline-132.7 & 172600 & 7786 \\
\hline-124.8 & 208772 & 9666 \\
\hline-121.2 & 208477 & 11622 \\
\hline-111.5 & 262848 & 19126 \\
\hline-111.5 & 251775 & 11478 \\
\hline-107.4 & 285943 & 13805 \\
\hline-105.3 & 269209 & 12593 \\
\hline-97.5 & 291771 & 13215 \\
\hline-95.2 & 352464 & 16428 \\
\hline-94.0 & 340796 & 16835 \\
\hline-90.3 & 362096 & 19401 \\
\hline-90.3 & 366357 & 18540 \\
\hline-76.9 & 443692 & 25199 \\
\hline-70.8 & 374540 & 49984 \\
\hline-70.8 & 402242 & 18332 \\
\hline-67.5 & 481558 & 31378 \\
\hline-67.5 & 477824 & 23938 \\
\hline-64.1 & 445496 & 23782 \\
\hline-64.1 & 420413 & 24629 \\
\hline-60.5 & 475073 & 24555 \\
\hline-58.6 & 489428 & 23622 \\
\hline-54.6 & 479350 & 36273 \\
\hline-54.6 & 540276 & 31094 \\
\hline-50.3 & 524329 & 24304 \\
\hline-50.3 & 515078 & 23643 \\
\hline-45.6 & 538414 & 23636 \\
\hline-40.4 & 573929 & 31042 \\
\hline-27.0 & 563951 & 56170 \\
\hline-27.0 & 610220 & 34302 \\
\hline 27.0 & 597918 & 34339 \\
\hline 40.4 & 546907 & 33215 \\
\hline 45.6 & 536203 & 26304 \\
\hline 46.0 & 435567 & 49354 \\
\hline 50.3 & 498337 & 22665 \\
\hline 54.6 & 517116 & 29952 \\
\hline 58.6 & 455746 & 21531 \\
\hline 60.5 & 491059 & 25864 \\
\hline 60.5 & 474549 & 25428 \\
\hline 60.5 & 484480 & 25025 \\
\hline 67.5 & 415905 & 38030 \\
\hline 67.5 & 433361 & 20431 \\
\hline 69.1 & 422865 & 20274 \\
\hline 70.8 & 388025 & 17357 \\
\hline 70.8 & 434043 & 22920 \\
\hline 70.8 & 412295 & 27045 \\
\hline 76.9 & 364314 & 37569 \\
\hline 76.9 & 395237 & 20274 \\
\hline
\end{tabular}

\begin{tabular}{|c|c|c|}
\hline $\begin{array}{l}\text { Distance } \\
(\mathrm{cm})\end{array}$ & $\begin{array}{c}\text { Fluence } \\
\left(\mathrm{n} / \mathrm{cm}^{2} / \mathrm{eV} / 10^{9} \mathrm{p}\right)\end{array}$ & Error \\
\hline 79.7 & 354786 & 16006 \\
\hline 81.1 & 378500 & 37086 \\
\hline 83.9 & 351309 & 22740 \\
\hline 85.2 & 374363 & 19518 \\
\hline 90.3 & 324092 & 19276 \\
\hline 90.3 & 308537 & 13998 \\
\hline 90.3 & 341238 & 19812 \\
\hline 90.3 & 351774 & 21177 \\
\hline 92.8 & 368103 & 19033 \\
\hline 94.0 & 304353 & 14503 \\
\hline 95.2 & 321900 & 16641 \\
\hline 102.0 & 257844 & 21189 \\
\hline 102.0 & 283505 & 16470 \\
\hline 105.3 & 271252 & 22573 \\
\hline 105.3 & 268307 & 12532 \\
\hline 105.3 & 252442 & 12043 \\
\hline 111.5 & 242475 & 11123 \\
\hline 111.5 & 246286 & 12376 \\
\hline 112.5 & 218185 & 9735 \\
\hline 117.4 & 216957 & 18756 \\
\hline 117.4 & 213167 & 24040 \\
\hline 117.4 & 227283 & 10334 \\
\hline 120.2 & 186689 & 8444 \\
\hline 124.8 & 191253 & 9300 \\
\hline 124.8 & 198983 & 9080 \\
\hline 124.8 & 200761 & 8998 \\
\hline 127.5 & 186511 & 8635 \\
\hline 127.5 & 166697 & 7994 \\
\hline 127.5 & 161090 & 11119 \\
\hline 127.5 & 155814 & 12734 \\
\hline 131.0 & 150208 & 9615 \\
\hline 133.5 & 170289 & 8173 \\
\hline 137.7 & 134541 & 6071 \\
\hline 143.3 & 132267 & 9824 \\
\hline 143.3 & 138269 & 6995 \\
\hline 144.1 & 121826 & 5621 \\
\hline 144.1 & 103701 & 5192 \\
\hline 145.6 & 116794 & 5352 \\
\hline 150.2 & 69495 & 8780 \\
\hline 150.2 & 73972 & 4350 \\
\hline 153.9 & 96561 & 5095 \\
\hline 154.6 & 71825 & 3481 \\
\hline 154.6 & 71305 & 3458 \\
\hline 154.6 & 68455 & 3110 \\
\hline 164.5 & 57475 & 2947 \\
\hline 164.5 & 58911 & 2877 \\
\hline 164.5 & 69183 & 3199 \\
\hline 165.2 & 61544 & 3585 \\
\hline 171.2 & 47362 & 2193 \\
\hline 187.5 & 29628 & 1587 \\
\hline 187.5 & 34947 & 1689 \\
\hline
\end{tabular}


Table B3: ${ }^{6} \mathrm{Li} /{ }^{233} \mathrm{U} / 5 \mathrm{eV}$

\begin{tabular}{|c|c|c|}
\hline $\begin{array}{l}\text { Distance } \\
(\mathbf{c m})\end{array}$ & $\begin{array}{c}\text { Fluence } \\
\left(\mathrm{n} / \mathrm{cm}^{2} / \mathrm{eV} / 10^{9} \mathbf{p}\right)\end{array}$ & Error \\
\hline-187.5 & 19654 & 3775 \\
\hline-164.5 & 23255 & 1093 \\
\hline-154.6 & 28522 & 1666 \\
\hline-150.2 & 29988 & 1721 \\
\hline-132.7 & 66240 & 3049 \\
\hline-124.8 & 81051 & 3790 \\
\hline-121.2 & 82117 & 4661 \\
\hline-111.5 & 105704 & 9652 \\
\hline-111.5 & 97672 & 4424 \\
\hline-107.4 & 110736 & 5439 \\
\hline-105.3 & 104929 & 4826 \\
\hline-97.5 & 112948 & 5058 \\
\hline-95.2 & 139589 & 6534 \\
\hline-94.0 & 135880 & 7070 \\
\hline-90.3 & 144366 & 7872 \\
\hline-90.3 & 148753 & 8028 \\
\hline-76.9 & 173919 & 10862 \\
\hline-70.8 & 151538 & 25055 \\
\hline-70.8 & 152228 & 7128 \\
\hline-67.5 & 183371 & 14961 \\
\hline-67.5 & 192210 & 11922 \\
\hline-64.1 & 178006 & 10140 \\
\hline-64.1 & 171565 & 10552 \\
\hline-60.5 & 190845 & 10178 \\
\hline-58.6 & 198896 & 9008 \\
\hline-54.6 & 198543 & 16385 \\
\hline-54.6 & 221945 & 12552 \\
\hline-50.3 & 207810 & 10763 \\
\hline-50.3 & 208064 & 9314 \\
\hline-45.6 & 214427 & 9596 \\
\hline-40.4 & 234634 & 13543 \\
\hline-27.0 & 219351 & 29333 \\
\hline-27.0 & 246342 & 14200 \\
\hline 27.0 & 246591 & 15097 \\
\hline 40.4 & 224161 & 13512 \\
\hline 45.6 & 214558 & 11421 \\
\hline 46.0 & 178277 & 34321 \\
\hline 50.3 & 200567 & 9076 \\
\hline 54.6 & 204048 & 12301 \\
\hline 58.6 & 187469 & 8673 \\
\hline 60.5 & 195491 & 12339 \\
\hline 60.5 & 190137 & 10444 \\
\hline 60.5 & 193141 & 10628 \\
\hline 67.5 & 159567 & 19067 \\
\hline 67.5 & 171050 & 8002 \\
\hline 69.1 & 172297 & 7824 \\
\hline 70.8 & 151338 & 6762 \\
\hline 70.8 & 171693 & 9521 \\
\hline 70.8 & 164591 & 11145 \\
\hline 76.9 & 173333 & 20852 \\
\hline 76.9 & 155287 & 8340 \\
\hline
\end{tabular}

\begin{tabular}{|c|c|c|}
\hline $\begin{array}{l}\text { Distance } \\
\text { (cm) }\end{array}$ & $\begin{array}{c}\text { Fluence } \\
\left(\mathbf{n} / \mathrm{cm}^{2} / \mathrm{eV} / 10^{9} \mathbf{p}\right)\end{array}$ & Error \\
\hline 79.7 & 139681 & 6195 \\
\hline 81.1 & 149211 & 15392 \\
\hline 83.9 & 139398 & 9484 \\
\hline 85.2 & 147546 & 7751 \\
\hline 90.3 & 127203 & 9142 \\
\hline 90.3 & 120764 & 5280 \\
\hline 90.3 & 135269 & 7885 \\
\hline 90.3 & 135597 & 8838 \\
\hline 92.8 & 144734 & 8759 \\
\hline 94.0 & 119849 & 5903 \\
\hline 95.2 & 124716 & 6937 \\
\hline 102.0 & 103624 & 10581 \\
\hline 102.0 & 109857 & 7022 \\
\hline 105.3 & 104741 & 10277 \\
\hline 105.3 & 104519 & 4950 \\
\hline 105.3 & 97749 & 4845 \\
\hline 111.5 & 93341 & 4274 \\
\hline 111.5 & 94634 & 4839 \\
\hline 112.5 & 82193 & 3672 \\
\hline 117.4 & 85547 & 11095 \\
\hline 117.4 & 85349 & 14371 \\
\hline 117.4 & 88025 & 3608 \\
\hline 120.2 & 70531 & 3157 \\
\hline 124.8 & 73397 & 3470 \\
\hline 124.8 & 78090 & 3603 \\
\hline 124.8 & 78385 & 3459 \\
\hline 127.5 & 69544 & 3096 \\
\hline 127.5 & 64191 & 2978 \\
\hline 127.5 & 60962 & 4475 \\
\hline 127.5 & 59521 & 4677 \\
\hline 131.0 & 57224 & 3867 \\
\hline 133.5 & 64979 & 3196 \\
\hline 137.7 & 50557 & 2232 \\
\hline 143.3 & 47420 & 4926 \\
\hline 143.3 & 52529 & 2765 \\
\hline 144.1 & 46303 & 2133 \\
\hline 144.1 & 39465 & 2013 \\
\hline 145.6 & 43679 & 1946 \\
\hline 150.2 & 26193 & 3588 \\
\hline 150.2 & 27994 & 1728 \\
\hline 153.9 & 36002 & 1892 \\
\hline 154.6 & 27536 & 1363 \\
\hline 154.6 & 26758 & 1340 \\
\hline 154.6 & 26318 & 1193 \\
\hline 164.5 & 21902 & 1139 \\
\hline 164.5 & 21728 & 1135 \\
\hline 164.5 & 25603 & 1154 \\
\hline 165.2 & 23063 & 1463 \\
\hline 171.2 & 17841 & 819 \\
\hline 187.5 & 11053 & 672 \\
\hline 187.5 & 12680 & 641 \\
\hline
\end{tabular}


Table B4: ${ }^{6} \mathrm{Li} /{ }^{233} \mathrm{U} / 10 \mathrm{eV}$

\begin{tabular}{|c|c|c|}
\hline $\begin{array}{l}\text { Distance } \\
(\mathbf{c m})\end{array}$ & $\begin{array}{c}\text { Fluence } \\
\left(\mathrm{n} / \mathrm{cm}^{2} / \mathrm{eV} / 10^{9} \mathbf{p}\right)\end{array}$ & Error \\
\hline-187.5 & 10787 & 2087 \\
\hline-164.5 & 12973 & 682 \\
\hline-154.6 & 16029 & 1040 \\
\hline-150.2 & 16823 & 1044 \\
\hline-132.7 & 37060 & 1781 \\
\hline-124.8 & 45469 & 2252 \\
\hline-121.2 & 46469 & 2928 \\
\hline-111.5 & 59793 & 4664 \\
\hline-111.5 & 54722 & 2772 \\
\hline-107.4 & 61839 & 3443 \\
\hline-105.3 & 59306 & 2869 \\
\hline-97.5 & 63596 & 2944 \\
\hline-95.2 & 79278 & 4101 \\
\hline-94.0 & 77315 & 4362 \\
\hline-90.3 & 82491 & 5048 \\
\hline-90.3 & 84399 & 5004 \\
\hline-76.9 & 97383 & 6723 \\
\hline-70.8 & 87004 & 7565 \\
\hline-70.8 & 83784 & 4240 \\
\hline-67.5 & 103674 & 8171 \\
\hline-67.5 & 109837 & 6506 \\
\hline-64.1 & 101372 & 6318 \\
\hline-64.1 & 98073 & 6950 \\
\hline-60.5 & 109123 & 6420 \\
\hline-58.6 & 115080 & 6008 \\
\hline-54.6 & 114736 & 9192 \\
\hline-54.6 & 129676 & 8399 \\
\hline-50.3 & 119080 & 6141 \\
\hline-50.3 & 120122 & 5597 \\
\hline-45.6 & 121708 & 5960 \\
\hline-40.4 & 136590 & 8962 \\
\hline-27.0 & 121178 & 16016 \\
\hline-27.0 & 141880 & 9266 \\
\hline 27.0 & 144317 & 8974 \\
\hline 40.4 & 130661 & 8990 \\
\hline 45.6 & 123695 & 6426 \\
\hline 46.0 & 103661 & 9005 \\
\hline 50.3 & 115618 & 5571 \\
\hline 54.6 & 114399 & 8418 \\
\hline 58.6 & 110128 & 5254 \\
\hline 60.5 & 112016 & 6757 \\
\hline 60.5 & 109275 & 6325 \\
\hline 60.5 & 109903 & 6871 \\
\hline 67.5 & 91304 & 10339 \\
\hline 67.5 & 97038 & 4866 \\
\hline 69.1 & 100656 & 4916 \\
\hline 70.8 & 85314 & 4015 \\
\hline 70.8 & 97644 & 5502 \\
\hline 70.8 & 92960 & 6999 \\
\hline 76.9 & 93183 & 12350 \\
\hline 76.9 & 87705 & 5109 \\
\hline
\end{tabular}

\begin{tabular}{|c|c|c|}
\hline $\begin{array}{l}\text { Distance } \\
(\mathbf{c m})\end{array}$ & $\begin{array}{c}\text { Fluence } \\
\left(\mathbf{n} / \mathrm{cm}^{2} / \mathrm{eV} / 10^{9} \mathbf{p}\right)\end{array}$ & Error \\
\hline 79.7 & 79562 & 3735 \\
\hline 81.1 & 84859 & 9087 \\
\hline 83.9 & 78723 & 6080 \\
\hline 85.2 & 83401 & 5014 \\
\hline 90.3 & 72673 & 5372 \\
\hline 90.3 & 68564 & 3178 \\
\hline 90.3 & 76771 & 5176 \\
\hline 90.3 & 75967 & 5568 \\
\hline 92.8 & 81769 & 4651 \\
\hline 94.0 & 68056 & 3515 \\
\hline 95.2 & 69807 & 4261 \\
\hline 102.0 & 58161 & 5694 \\
\hline 102.0 & 61592 & 4372 \\
\hline 105.3 & 58092 & 5792 \\
\hline 105.3 & 58780 & 2908 \\
\hline 105.3 & 54528 & 2858 \\
\hline 111.5 & 52244 & 2581 \\
\hline 111.5 & 52313 & 2905 \\
\hline 112.5 & 45462 & 2131 \\
\hline 117.4 & 47536 & 5508 \\
\hline 117.4 & 47586 & 6566 \\
\hline 117.4 & 49418 & 2269 \\
\hline 120.2 & 39064 & 1821 \\
\hline 124.8 & 40854 & 2149 \\
\hline 124.8 & 43521 & 2176 \\
\hline 124.8 & 43647 & 2088 \\
\hline 127.5 & 38148 & 1714 \\
\hline 127.5 & 35901 & 1832 \\
\hline 127.5 & 33429 & 2764 \\
\hline 127.5 & 32990 & 3382 \\
\hline 131.0 & 31792 & 2436 \\
\hline 133.5 & 36060 & 1891 \\
\hline 137.7 & 27950 & 1310 \\
\hline 143.3 & 25418 & 2519 \\
\hline 143.3 & 28993 & 1676 \\
\hline 144.1 & 25652 & 1269 \\
\hline 144.1 & 21769 & 1220 \\
\hline 145.6 & 24058 & 1119 \\
\hline 150.2 & 14371 & 1146 \\
\hline 150.2 & 15479 & 1026 \\
\hline 153.9 & 19639 & 1159 \\
\hline 154.6 & 15312 & 838 \\
\hline 154.6 & 14715 & 845 \\
\hline 154.6 & 14684 & 697 \\
\hline 164.5 & 12111 & 703 \\
\hline 164.5 & 11859 & 672 \\
\hline 164.5 & 13995 & 670 \\
\hline 165.2 & 12605 & 915 \\
\hline 171.2 & 9860 & 472 \\
\hline 187.5 & 6029 & 409 \\
\hline 187.5 & 6884 & 386 \\
\hline
\end{tabular}


Table B5: ${ }^{6} \mathrm{Li} /{ }^{233} \mathrm{U} / 18 \mathrm{eV}$

\begin{tabular}{|c|c|c|}
\hline $\begin{array}{l}\text { Distance } \\
(\mathbf{c m})\end{array}$ & $\begin{array}{c}\text { Fluence } \\
\left(\mathrm{n} / \mathrm{cm}^{2} / \mathrm{eV} / 10^{9} \mathbf{p}\right)\end{array}$ & Error \\
\hline-187.5 & 6356 & 961 \\
\hline-164.5 & 7770 & 395 \\
\hline-154.6 & 9671 & 648 \\
\hline-150.2 & 10087 & 658 \\
\hline-132.7 & 22305 & 1000 \\
\hline-124.8 & 27386 & 1314 \\
\hline-121.2 & 28211 & 1707 \\
\hline-111.5 & 36102 & 2540 \\
\hline-111.5 & 32910 & 1560 \\
\hline-107.4 & 37046 & 1964 \\
\hline-105.3 & 36039 & 1666 \\
\hline-97.5 & 38516 & 1628 \\
\hline-95.2 & 48296 & 2394 \\
\hline-94.0 & 47140 & 2562 \\
\hline-90.3 & 50574 & 3085 \\
\hline-90.3 & 51043 & 3071 \\
\hline-76.9 & 58376 & 4215 \\
\hline-70.8 & 53488 & 4327 \\
\hline-70.8 & 49520 & 2336 \\
\hline-67.5 & 63378 & 4274 \\
\hline-67.5 & 67202 & 3622 \\
\hline-64.1 & 61844 & 3630 \\
\hline-64.1 & 59825 & 4102 \\
\hline-60.5 & 66840 & 3822 \\
\hline-58.6 & 71393 & 3463 \\
\hline-54.6 & 70743 & 5261 \\
\hline-54.6 & 81271 & 5004 \\
\hline-50.3 & 73381 & 3678 \\
\hline-50.3 & 74414 & 3313 \\
\hline-45.6 & 73987 & 3483 \\
\hline-40.4 & 85294 & 5308 \\
\hline-27.0 & 71502 & 7595 \\
\hline-27.0 & 87630 & 5685 \\
\hline 27.0 & 90560 & 5954 \\
\hline 40.4 & 81680 & 5539 \\
\hline 45.6 & 76659 & 3624 \\
\hline 46.0 & 64615 & 5776 \\
\hline 50.3 & 71544 & 3376 \\
\hline 54.6 & 68582 & 5240 \\
\hline 58.6 & 69490 & 3165 \\
\hline 60.5 & 68951 & 3730 \\
\hline 60.5 & 67424 & 3927 \\
\hline 60.5 & 67016 & 4148 \\
\hline 67.5 & 56597 & 6311 \\
\hline 67.5 & 59077 & 2863 \\
\hline 69.1 & 63197 & 2896 \\
\hline 70.8 & 51658 & 2209 \\
\hline 70.8 & 59608 & 3596 \\
\hline 70.8 & 56113 & 4570 \\
\hline 76.9 & 54894 & 6303 \\
\hline 76.9 & 53143 & 3046 \\
\hline
\end{tabular}

\begin{tabular}{|c|c|c|}
\hline $\begin{array}{l}\text { Distance } \\
\text { (cm) }\end{array}$ & $\begin{array}{c}\text { Fluence } \\
\left(\mathrm{n} / \mathrm{cm}^{2} / \mathrm{eV} / 10^{9} \mathrm{p}\right)\end{array}$ & Error \\
\hline 79.7 & 48723 & 2063 \\
\hline 81.1 & 51845 & 5663 \\
\hline 83.9 & 47582 & 3704 \\
\hline 85.2 & 50552 & 2925 \\
\hline 90.3 & 44713 & 2580 \\
\hline 90.3 & 41868 & 1840 \\
\hline 90.3 & 46715 & 3037 \\
\hline 90.3 & 45754 & 3523 \\
\hline 92.8 & 49557 & 2689 \\
\hline 94.0 & 41508 & 2079 \\
\hline 95.2 & 41942 & 2583 \\
\hline 102.0 & 34765 & 3364 \\
\hline 102.0 & 37086 & 2552 \\
\hline 105.3 & 34516 & 3363 \\
\hline 105.3 & 35491 & 1705 \\
\hline 105.3 & 32622 & 1659 \\
\hline 111.5 & 31437 & 1499 \\
\hline 111.5 & 30984 & 1709 \\
\hline 112.5 & 27074 & 1159 \\
\hline 117.4 & $28 \quad 172$ & 2939 \\
\hline 117.4 & 28223 & 3654 \\
\hline 117.4 & 29812 & 1318 \\
\hline 120.2 & 23289 & 1011 \\
\hline 124.8 & 24423 & 1257 \\
\hline 124.8 & 25922 & 1190 \\
\hline 124.8 & 25999 & 1173 \\
\hline 127.5 & 22529 & 1026 \\
\hline 127.5 & 21580 & 1046 \\
\hline 127.5 & 19663 & 1822 \\
\hline 127.5 & 19634 & 1954 \\
\hline 131.0 & 18993 & 1550 \\
\hline 133.5 & 21502 & 1090 \\
\hline 137.7 & 16645 & 707 \\
\hline 143.3 & 14701 & 1414 \\
\hline 143.3 & 17184 & 973 \\
\hline 144.1 & 15277 & 719 \\
\hline 144.1 & 12887 & 753 \\
\hline 145.6 & 14273 & 620 \\
\hline 150.2 & 8467 & 671 \\
\hline 150.2 & 9204 & 676 \\
\hline 153.9 & 11513 & 712 \\
\hline 154.6 & 9145 & 491 \\
\hline 154.6 & 8705 & 484 \\
\hline 154.6 & 8803 & 403 \\
\hline 164.5 & 7190 & 409 \\
\hline 164.5 & 6976 & 379 \\
\hline 164.5 & 8241 & 384 \\
\hline 165.2 & 7400 & 506 \\
\hline 171.2 & 5866 & 268 \\
\hline 187.5 & 3533 & 242 \\
\hline 187.5 & 4036 & 219 \\
\hline
\end{tabular}


Table B6: ${ }^{6} \mathrm{Li} /{ }^{233} \mathrm{U} / 100 \mathrm{eV}$

\begin{tabular}{|c|c|c|}
\hline $\begin{array}{l}\text { Distance } \\
(\mathbf{c m})\end{array}$ & $\begin{array}{c}\text { Fluence } \\
\left(\mathrm{n} / \mathrm{cm}^{2} / \mathrm{eV} / 10^{9} \mathrm{p}\right)\end{array}$ & Error \\
\hline-187.5 & 1257 & 232 \\
\hline-164.5 & 1637 & 101 \\
\hline-154.6 & 2095 & 181 \\
\hline-150.2 & 2100 & 180 \\
\hline-132.7 & 4818 & 261 \\
\hline-124.8 & 5894 & 351 \\
\hline-121.2 & 6242 & 564 \\
\hline-111.5 & 7688 & 951 \\
\hline-111.5 & 7037 & 439 \\
\hline-107.4 & 7789 & 545 \\
\hline-105.3 & 8061 & 442 \\
\hline-97.5 & 8519 & 438 \\
\hline-95.2 & 10821 & 676 \\
\hline-94.0 & 10558 & 712 \\
\hline-90.3 & 11620 & 901 \\
\hline-90.3 & 10888 & 845 \\
\hline-76.9 & 12217 & 1184 \\
\hline-70.8 & 12334 & 1316 \\
\hline-70.8 & 9923 & 631 \\
\hline-67.5 & 14786 & 1543 \\
\hline-67.5 & 15212 & 1217 \\
\hline-64.1 & 13871 & 1084 \\
\hline-64.1 & 13237 & 1239 \\
\hline-60.5 & 15221 & 1191 \\
\hline-58.6 & 17121 & 1055 \\
\hline-54.6 & 16296 & 2031 \\
\hline-54.6 & 20293 & 1740 \\
\hline-50.3 & 17330 & 1135 \\
\hline-50.3 & 17794 & 1034 \\
\hline-45.6 & 16357 & 978 \\
\hline-40.4 & 21007 & 1554 \\
\hline-27.0 & 13977 & 3914 \\
\hline 40.4 & 20184 & 2036 \\
\hline 45.6 & 18474 & 1227 \\
\hline 46.0 & 15769 & 1721 \\
\hline 50.3 & 17059 & 1049 \\
\hline 54.6 & 14299 & 1467 \\
\hline 58.6 & 17890 & 1071 \\
\hline 60.5 & 16155 & 1277 \\
\hline 60.5 & 15908 & 1284 \\
\hline 60.5 & 15019 & 1125 \\
\hline 67.5 & 14015 & 2598 \\
\hline 67.5 & 13224 & 845 \\
\hline 69.1 & 15993 & 944 \\
\hline 70.8 & 11361 & 608 \\
\hline 70.8 & 13493 & 1001 \\
\hline 70.8 & 11989 & 1244 \\
\hline 76.9 & 11636 & 2673 \\
\hline 76.9 & 11672 & 874 \\
\hline 79.7 & 11225 & 600 \\
\hline
\end{tabular}

\begin{tabular}{|c|c|c|}
\hline $\begin{array}{l}\text { Distance } \\
(\mathbf{c m})\end{array}$ & $\begin{array}{c}\text { Fluence } \\
\left(\mathbf{n} / \mathrm{cm}^{2} / \mathrm{eV} / 10^{9} \mathbf{p}\right)\end{array}$ & Error \\
\hline 81.1 & 11806 & 2153 \\
\hline 83.9 & 10268 & 1143 \\
\hline 85.2 & 11098 & 820 \\
\hline 90.3 & 10546 & 849 \\
\hline 90.3 & 9553 & 510 \\
\hline 90.3 & 10405 & 903 \\
\hline 90.3 & 9890 & 1090 \\
\hline 92.8 & 10890 & 838 \\
\hline 94.0 & 9383 & 613 \\
\hline 95.2 & 8936 & 733 \\
\hline 102.0 & 7069 & 1136 \\
\hline 102.0 & 7980 & 774 \\
\hline 105.3 & 6998 & 1132 \\
\hline 105.3 & 7709 & 474 \\
\hline 105.3 & 6816 & 451 \\
\hline 111.5 & 6772 & 411 \\
\hline 111.5 & 6210 & 471 \\
\hline 112.5 & 5635 & 298 \\
\hline 117.4 & 5572 & 1058 \\
\hline 117.4 & 5549 & 1456 \\
\hline 117.4 & 6473 & 332 \\
\hline 120.2 & 4862 & 256 \\
\hline 124.8 & 5114 & 327 \\
\hline 124.8 & 5263 & 324 \\
\hline 124.8 & 5297 & 303 \\
\hline 127.5 & 4539 & 252 \\
\hline 127.5 & 4624 & 270 \\
\hline 127.5 & 3854 & 472 \\
\hline 127.5 & 4039 & 582 \\
\hline 131.0 & 3982 & 453 \\
\hline 133.5 & 4465 & 298 \\
\hline 137.7 & 3469 & 181 \\
\hline 143.3 & 2756 & 428 \\
\hline 143.3 & 3480 & 266 \\
\hline 144.1 & 3162 & 185 \\
\hline 144.1 & 2592 & 202 \\
\hline 145.6 & 2932 & 160 \\
\hline 150.2 & 1676 & 185 \\
\hline 150.2 & 1897 & 197 \\
\hline 153.9 & 2242 & 190 \\
\hline 154.6 & 1907 & 138 \\
\hline 154.6 & 1761 & 132 \\
\hline 154.6 & 1865 & 108 \\
\hline 164.5 & 1465 & 117 \\
\hline 164.5 & 1390 & 96 \\
\hline 164.5 & 1646 & 97 \\
\hline 165.2 & 1446 & 149 \\
\hline 171.2 & 1215 & 73 \\
\hline 187.5 & 687 & 63 \\
\hline 187.5 & 800 & 57 \\
\hline
\end{tabular}


Table B7: ${ }^{6} \mathrm{Li} /{ }^{233} \mathrm{U} / 480 \mathrm{eV}$

\begin{tabular}{|c|c|c|}
\hline $\begin{array}{l}\text { Distance } \\
(\mathbf{c m})\end{array}$ & $\begin{array}{c}\text { Fluence } \\
\left(\mathrm{n} / \mathrm{cm}^{2} / \mathrm{eV} / 10^{9} \mathbf{p}\right)\end{array}$ & Error \\
\hline-187.5 & 262 & 40 \\
\hline-164.5 & 372 & 31 \\
\hline-154.6 & 493 & 57 \\
\hline-150.2 & 464 & 57 \\
\hline-132.7 & 1136 & 71 \\
\hline-124.8 & 1377 & 92 \\
\hline-121.2 & 1509 & 149 \\
\hline-111.5 & 1745 & 266 \\
\hline-111.5 & 1628 & 114 \\
\hline-107.4 & 1760 & 149 \\
\hline-105.3 & 1988 & 126 \\
\hline-97.5 & 2072 & 115 \\
\hline-95.2 & 2660 & 196 \\
\hline-94.0 & 2587 & 219 \\
\hline-90.3 & 2955 & 277 \\
\hline-90.3 & 2468 & 268 \\
\hline-76.9 & 2729 & 305 \\
\hline-70.8 & 3136 & 388 \\
\hline-70.8 & 2108 & 685 \\
\hline-67.5 & 3912 & 532 \\
\hline-67.5 & 3773 & 381 \\
\hline-64.1 & 3403 & 307 \\
\hline-64.1 & 3158 & 346 \\
\hline-60.5 & 3810 & 307 \\
\hline-54.6 & 4100 & 658 \\
\hline-50.3 & 4597 & 330 \\
\hline-50.3 & 4773 & 347 \\
\hline 45.6 & 5030 & 390 \\
\hline 46.0 & 4336 & 540 \\
\hline 50.3 & 4563 & 354 \\
\hline 58.6 & 5307 & 344 \\
\hline 60.5 & 4229 & 393 \\
\hline 60.5 & 4197 & 360 \\
\hline 60.5 & 3683 & 328 \\
\hline 67.5 & 4033 & 862 \\
\hline 69.1 & 4644 & 300 \\
\hline 70.8 & 2733 & 183 \\
\hline 70.8 & 3368 & 315 \\
\hline 70.8 & 2742 & 386 \\
\hline 76.9 & 2802 & 784 \\
\hline 76.9 & 2795 & 265 \\
\hline 79.7 & 2879 & 172 \\
\hline 81.1 & 2976 & 596 \\
\hline 83.9 & 2387 & 292 \\
\hline 85.2 & 2653 & 255 \\
\hline 90.3 & 2803 & 310 \\
\hline 90.3 & 2422 & 137 \\
\hline 90.3 & 2535 & 264 \\
\hline 90.3 & 2330 & 338 \\
\hline 92.8 & 2609 & 239 \\
\hline
\end{tabular}

\begin{tabular}{|c|c|c|}
\hline $\begin{array}{l}\text { Distance } \\
\text { (cm) }\end{array}$ & $\begin{array}{c}\text { Fluence } \\
\left(\mathrm{n} / \mathrm{cm}^{2} / \mathrm{eV} / 10^{9} \mathrm{p}\right)\end{array}$ & Error \\
\hline 94.0 & 2341 & 179 \\
\hline 95.2 & 2058 & 203 \\
\hline 102.0 & 1500 & 322 \\
\hline 102.0 & 1864 & 213 \\
\hline 105.3 & 1501 & 378 \\
\hline 105.3 & 1822 & 125 \\
\hline 105.3 & 1526 & 134 \\
\hline 111.5 & 1589 & 107 \\
\hline 111.5 & 1312 & 142 \\
\hline 112.5 & 1267 & 75 \\
\hline 117.4 & 1144 & 309 \\
\hline 117.4 & 1123 & 377 \\
\hline 117.4 & 1532 & 82 \\
\hline 120.2 & 1097 & 66 \\
\hline 124.8 & 1153 & 90 \\
\hline 124.8 & 1124 & 80 \\
\hline 124.8 & 1140 & 76 \\
\hline 127.5 & 978 & 67 \\
\hline 127.5 & 1077 & 82 \\
\hline 127.5 & 793 & 136 \\
\hline 127.5 & 889 & 203 \\
\hline 131.0 & 902 & 124 \\
\hline 133.5 & 996 & 83 \\
\hline 137.7 & 783 & 45 \\
\hline 143.3 & 542 & 139 \\
\hline 143.3 & 750 & 74 \\
\hline 144.1 & 703 & 49 \\
\hline 144.1 & 553 & 49 \\
\hline 145.6 & 649 & 41 \\
\hline 150.2 & 351 & 55 \\
\hline 150.2 & 420 & 59 \\
\hline 153.9 & 460 & 50 \\
\hline 154.6 & 427 & 36 \\
\hline 154.6 & 381 & 33 \\
\hline 154.6 & 427 & 28 \\
\hline 164.5 & 318 & 37 \\
\hline 164.5 & 296 & 28 \\
\hline 164.5 & 351 & 24 \\
\hline 165.2 & 297 & 38 \\
\hline 171.2 & 271 & 18 \\
\hline 187.5 & 141 & 17 \\
\hline 187.5 & 170 & 15 \\
\hline
\end{tabular}


Table B8: ${ }^{6} \mathrm{Li} /{ }^{233} \mathrm{U} / 1000 \mathrm{eV}$

\begin{tabular}{|c|c|c|}
\hline $\begin{array}{l}\text { Distance } \\
(\mathbf{c m})\end{array}$ & $\begin{array}{c}\text { Fluence } \\
\left(\mathrm{n} / \mathrm{cm}^{2} / \mathrm{eV} / 10^{9} \mathrm{p}\right)\end{array}$ & Error \\
\hline-187.5 & 122 & 24 \\
\hline-164.5 & 182 & 19 \\
\hline-154.6 & 247 & 31 \\
\hline-150.2 & 223 & 33 \\
\hline-132.7 & 571 & 46 \\
\hline-124.8 & 687 & 63 \\
\hline-121.2 & 769 & 89 \\
\hline-111.5 & 853 & 163 \\
\hline-111.5 & 808 & 72 \\
\hline-107.4 & 860 & 90 \\
\hline-105.3 & 1027 & 83 \\
\hline-97.5 & 1061 & 68 \\
\hline-95.2 & 1369 & 111 \\
\hline-94.0 & 1329 & 128 \\
\hline-90.3 & 1554 & 164 \\
\hline-90.3 & 1205 & 161 \\
\hline-76.9 & 1323 & 192 \\
\hline-70.8 & 1647 & 250 \\
\hline-67.5 & 2115 & 311 \\
\hline-67.5 & 1952 & 203 \\
\hline-64.1 & 1749 & 182 \\
\hline-54.6 & 2137 & 405 \\
\hline-50.3 & 2484 & 194 \\
\hline-50.3 & 2594 & 214 \\
\hline-45.6 & 1997 & 192 \\
\hline 45.6 & 2761 & 225 \\
\hline 46.0 & 2392 & 336 \\
\hline 50.3 & 2476 & 181 \\
\hline 58.6 & 3072 & 198 \\
\hline 60.5 & 2266 & 232 \\
\hline 67.5 & 2299 & 461 \\
\hline 69.1 & 2653 & 189 \\
\hline 70.8 & 1389 & 110 \\
\hline 70.8 & 1751 & 185 \\
\hline 70.8 & 1348 & 198 \\
\hline 76.9 & 1436 & 475 \\
\hline 76.9 & 1416 & 149 \\
\hline 79.7 & 1524 & 101 \\
\hline 81.1 & 1557 & 367 \\
\hline 85.2 & 1342 & 156 \\
\hline 90.3 & 1517 & 194 \\
\hline 90.3 & 1297 & 172 \\
\hline 90.3 & 1170 & 200 \\
\hline 92.8 & 1322 & 148 \\
\hline 94.0 & 1217 & 124 \\
\hline 95.2 & 1018 & 127 \\
\hline 102.0 & 930 & 124 \\
\hline 105.3 & 916 & 85 \\
\hline 105.3 & 741 & 88 \\
\hline 111.5 & 795 & 73 \\
\hline
\end{tabular}

\begin{tabular}{c|c|c}
\hline $\begin{array}{c}\text { Distance } \\
(\mathbf{c m})\end{array}$ & $\begin{array}{c}\text { Fluence } \\
\left(\mathbf{n} / \mathbf{c m}^{2} / \mathbf{e V} / \mathbf{1 0} \mathbf{9} \mathbf{p}\right)\end{array}$ & Error \\
\hline 111.5 & 615 & 82 \\
\hline 112.5 & 619 & 46 \\
\hline 117.4 & 525 & 162 \\
\hline 117.4 & 511 & 214 \\
\hline 117.4 & 771 & 51 \\
\hline 120.2 & 537 & 41 \\
\hline 124.8 & 563 & 58 \\
\hline 124.8 & 530 & 50 \\
\hline 124.8 & 540 & 46 \\
\hline 127.5 & 536 & 51 \\
\hline 127.5 & 427 & 102 \\
\hline 131.0 & 442 & 74 \\
\hline 133.5 & 483 & 53 \\
\hline 137.7 & 383 & 27 \\
\hline 143.3 & 356 & 43 \\
\hline 144.1 & 341 & 31 \\
\hline 144.1 & 261 & 30 \\
\hline 145.6 & 314 & 27 \\
\hline 150.2 & 163 & 32 \\
\hline 150.2 & 203 & 36 \\
\hline 153.9 & 212 & 34 \\
\hline 154.6 & 208 & 24 \\
\hline 154.6 & 181 & 20 \\
\hline 154.6 & 211 & 18 \\
\hline 164.5 & 152 & 21 \\
\hline 164.5 & 139 & 17 \\
\hline 164.5 & 166 & 17 \\
\hline 165.2 & 137 & 25 \\
\hline 171.2 & 132 & 12 \\
\hline 187.5 & 80 & 10 \\
\hline & & \\
\hline
\end{tabular}


Table B9: ${ }^{3} \mathrm{He} / \mathrm{scintillation} / 1.5 \mathrm{eV}$

\begin{tabular}{c|c|c}
\hline Distance & $\begin{array}{c}\text { Fluence } \\
\left(\mathbf{n} / \mathbf{c m}^{\mathbf{2}} \mathbf{/ e V / 1 0} \mathbf{~} \mathbf{p}\right)\end{array}$ & Error \\
\hline 16.8 & 665235 & 9979 \\
\hline 40.4 & 593298 & 9474 \\
\hline 45.6 & 602514 & 9483 \\
\hline 69.1 & 387449 & 7658 \\
\hline 81.1 & 420921 & 7966 \\
\hline 98.6 & 331789 & 7105 \\
\hline 105.3 & 275497 & 6362 \\
\hline 113.5 & 218988 & 5755 \\
\hline 124.8 & 207045 & 5607 \\
\hline 153.9 & 115093 & 4100 \\
\hline
\end{tabular}

Table B10: ${ }^{3} \mathrm{He} /$ scintillation $/ 5 \mathrm{eV}$

\begin{tabular}{c|c|c}
\hline Distance & $\begin{array}{c}\text { Fluence } \\
\left(\mathbf{n} / \mathbf{c m}^{\mathbf{2}} / \mathbf{e V} / \mathbf{1 0} \mathbf{p}\right)\end{array}$ & Error \\
\hline 16.8 & 284113 & 4660 \\
\hline 40.4 & 245043 & 4334 \\
\hline 45.6 & 266956 & 4501 \\
\hline 69.1 & 168293 & 3576 \\
\hline 81.1 & 180439 & 3725 \\
\hline 98.6 & 130339 & 3166 \\
\hline 105.3 & 119252 & 3013 \\
\hline 113.5 & 93661 & 2677 \\
\hline 124.8 & 88648 & 2604 \\
\hline 153.9 & 42240 & 1747 \\
\hline
\end{tabular}

Table B11: ${ }^{3} \mathrm{He} /$ scintillation/10 eV

\begin{tabular}{c|c|c}
\hline Distance & $\begin{array}{c}\text { Fluence } \\
\left(\mathbf{n} / \mathbf{c m}^{\mathbf{2}} / \mathbf{e V} / \mathbf{1 0} \mathbf{9} \mathbf{p}\right)\end{array}$ & Error \\
\hline 16.8 & 167763 & 3135 \\
\hline 40.4 & 143894 & 2910 \\
\hline 45.6 & 157657 & 3064 \\
\hline 69.1 & 96559 & 2392 \\
\hline 81.1 & 107418 & 2504 \\
\hline 98.6 & 75270 & 2105 \\
\hline 105.3 & 67229 & 2005 \\
\hline 113.5 & 54325 & 1795 \\
\hline 124.8 & 49735 & 1721 \\
\hline 153.9 & 24108 & 1196 \\
\hline
\end{tabular}

Table B12: ${ }^{3} \mathrm{He} / \mathrm{scintillation} / 18 \mathrm{eV}$

\begin{tabular}{c|c|c}
\hline Distance & $\begin{array}{c}\text { Fluence } \\
\left(\mathbf{n} / \mathbf{c m}^{\mathbf{2}} / \mathbf{e V} / \mathbf{1 0} \mathbf{p}\right)\end{array}$ & Error \\
\hline 16.8 & 105971 & 2071 \\
\hline 40.4 & 90614 & 1921 \\
\hline 45.6 & 100489 & 2019 \\
\hline 69.1 & 59547 & 1556 \\
\hline 81.1 & 66724 & 1647 \\
\hline 98.6 & 47353 & 1383 \\
\hline 105.3 & 41776 & 1301 \\
\hline 113.5 & 32850 & 1151 \\
\hline 124.8 & 30290 & 1107 \\
\hline 153.9 & 14574 & 755 \\
\hline
\end{tabular}

Table B13: ${ }^{3} \mathrm{He} / \mathrm{scintillation} / 100 \mathrm{eV}$

\begin{tabular}{c|c|c}
\hline Distance & $\begin{array}{c}\text { Fluence } \\
\left(\mathbf{n} / \mathbf{c m}^{\mathbf{2}} / \mathbf{e V} / \mathbf{1 0} \mathbf{9} \mathbf{p}\right)\end{array}$ & Error \\
\hline 16.8 & 26393 & 656 \\
\hline 40.4 & 21271 & 588 \\
\hline 45.6 & 24639 & 634 \\
\hline 69.1 & 14014 & 478 \\
\hline 81.1 & 15459 & 502 \\
\hline 98.6 & 10163 & 407 \\
\hline 105.3 & 9156 & 387 \\
\hline 113.5 & 7627 & 353 \\
\hline 124.8 & 6354 & 322 \\
\hline 153.9 & 2970 & 220 \\
\hline
\end{tabular}

Table B14: ${ }^{3} \mathrm{He} /$ scintillation/480 eV

\begin{tabular}{c|c|c}
\hline Distance & $\begin{array}{c}\text { Fluence } \\
\left(\mathbf{n} / \mathbf{c m}^{\mathbf{2}} / \mathbf{e V} / \mathbf{1 0} \mathbf{9}^{\mathbf{p}}\right)\end{array}$ & Error \\
\hline 16.8 & 6932 & 247 \\
\hline 40.4 & 5332 & 217 \\
\hline 45.6 & 6363 & 240 \\
\hline 69.1 & 3500 & 180 \\
\hline 81.1 & 3989 & 188 \\
\hline 98.6 & 2635 & 154 \\
\hline 105.3 & 2244 & 140 \\
\hline 113.5 & 1833 & 125 \\
\hline 124.8 & 1480 & 113 \\
\hline 153.9 & 755 & 79 \\
\hline
\end{tabular}


Table B15: ${ }^{3} \mathrm{He} / \mathrm{scintillation} / 1000 \mathrm{eV}$

\begin{tabular}{|c|c|c|}
\hline Distance & $\begin{array}{c}\text { Fluence } \\
\left(\mathbf{n} / \mathrm{cm}^{2} / \mathrm{eV} / 10^{9} \mathrm{p}\right)\end{array}$ & Error \\
\hline 16.8 & 3746 & 144 \\
\hline 40.4 & 2736 & 124 \\
\hline 45.6 & 3440 & 139 \\
\hline 69.1 & 1835 & 102 \\
\hline 81.1 & 2070 & 108 \\
\hline 98.6 & 1359 & 87 \\
\hline 105.3 & 1092 & 78 \\
\hline 113.5 & 938 & 72 \\
\hline 124.8 & 705 & 61 \\
\hline 153.9 & 316 & 43 \\
\hline
\end{tabular}

Table B16: ${ }^{3} \mathrm{He} / \mathrm{scintillation} / 10000 \mathrm{eV}$

\begin{tabular}{c|c|c}
\hline Distance & $\begin{array}{c}\text { Fluence } \\
\left(\mathbf{n} / \mathbf{c m}^{\mathbf{2}} \mathbf{e V} / \mathbf{1 0} \mathbf{9} \mathbf{~}\right)\end{array}$ & Error \\
\hline 16.8 & 496 & 32 \\
\hline 40.4 & 399 & 28 \\
\hline 45.6 & 603 & 36 \\
\hline 69.1 & 313 & 25 \\
\hline 81.1 & 316 & 26 \\
\hline 98.6 & 200 & 21 \\
\hline 105.3 & 148 & 16 \\
\hline 113.5 & 128 & 16 \\
\hline 124.8 & 82 & 9 \\
\hline 153.9 & 47 & 9 \\
\hline
\end{tabular}

Table B17: ${ }^{3} \mathrm{He} / \mathrm{scintillation} / 50000 \mathrm{eV}$

\begin{tabular}{c|c|c}
\hline Distance & $\begin{array}{c}\text { Fluence } \\
\left(\mathbf{n} / \mathbf{c m}^{\mathbf{2}} \mathbf{/ e V / 1 0} \mathbf{9} \mathbf{p}\right)\end{array}$ & Error \\
\hline 81.1 & 81 & 10 \\
\hline 98.6 & 61 & 8 \\
\hline 105.3 & 27 & 4 \\
\hline 113.5 & 30 & 6 \\
\hline 124.8 & 16 & 5 \\
\hline 153.9 & 9 & 3 \\
\hline
\end{tabular}


Table B18: Triple-foil activation data

\begin{tabular}{|c|c|c|c|c|c|c|}
\hline $\begin{array}{l}\text { Distance } \\
\text { (cm) }\end{array}$ & 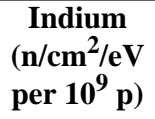 & Error-indium & $\begin{array}{c}\text { Gold } \\
\left(\mathrm{n} / \mathrm{cm}^{2} / \mathrm{eV} \text { per }\right. \\
\left.10^{9} \mathrm{p}\right)\end{array}$ & Error-gold & $\begin{array}{c}\text { Tungsten } \\
\left(\mathrm{n} / \mathrm{cm}^{2} / \mathrm{eV} \text { per }\right. \\
\left.10^{9} \mathrm{p}\right)\end{array}$ & Error-tungsten \\
\hline-127.5 & - & - & - & - & 30992 & 3808 \\
\hline-127.5 & - & - & 71100 & 9609 & 30992 & 3808 \\
\hline-127.5 & 202268 & 38955 & - & - & - & - \\
\hline-90.3 & - & - & - & - & 64198 & 7404 \\
\hline-90.3 & - & - & 138969 & 18979 & 59771 & 7438 \\
\hline-90.3 & 396527 & 49671 & - & - & - & - \\
\hline-27 & - & - & - & - & 103308 & 13138 \\
\hline-27 & - & - & 216533 & 31315 & 94453 & 11985 \\
\hline-27 & 552350 & 78228 & - & - & - & - \\
\hline-27 & 555531 & 78758 & - & - & - & - \\
\hline 16.8 & - & - & 252084 & 31803 & 109949 & 13241 \\
\hline 16.8 & - & - & - & - & 114377 & 14025 \\
\hline 16.8 & 583130 & 79118 & - & - & - & - \\
\hline 60.5 & - & - & - & - & 80433 & 9976 \\
\hline 60.5 & - & - & 219765 & 27277 & 79695 & 9917 \\
\hline 60.5 & 426600 & 61575 & - & - & - & - \\
\hline 67.5 & - & - & - & - & 94453 & 10765 \\
\hline 69.1 & - & - & - & - & 67150 & 8940 \\
\hline 69.1 & - & - & 161592 & 22852 & 75267 & 9128 \\
\hline 69.1 & 386280 & 54658 & - & - & - & - \\
\hline 70.8 & - & - & - & - & 63461 & 8182 \\
\hline 70.8 & - & - & 171288 & 23548 & 74529 & 9067 \\
\hline 70.8 & 424716 & 56162 & - & - & - & - \\
\hline 70.8 & 515799 & 63998 & - & - & - & - \\
\hline 105.3 & - & - & - & - & 45751 & 5444 \\
\hline 105.3 & - & - & - & - & 48702 & 6110 \\
\hline 105.3 & - & - & 100187 & 13942 & 42061 & 5138 \\
\hline 105.3 & 278276 & 36206 & - & - & - & - \\
\hline 153.9 & - & - & - & - & 15496 & 2140 \\
\hline 153.9 & - & - & 42014 & 5300 & 14020 & 2035 \\
\hline 153.9 & 112991 & 14687 & - & - & - & - \\
\hline 153.9 & 97836 & 12999 & - & - & - & - \\
\hline
\end{tabular}

